\title{
VNAP2: \\ A Computer Program for Computation of Two-Dimensional, Time-Dependent, Compressible, Turbulent Flow
}




\section{DISCLAIMER}

This report was prepared as an account of work sponsored by an agency of the United States Government. Neither the United States Government nor any agency Thereof, nor any of their employees, makes any warranty, express or implied, or assumes any legal liability or responsibility for the accuracy, completeness, or usefulness of any information, apparatus, product, or process disclosed, or represents that its use would not infringe privately owned rights. Reference herein to any specific commercial product, process, or service by trade name, trademark, manufacturer, or otherwise does not necessarily constitute or imply its endorsement, recommendation, or favoring by the United States Government or any agency thereof. The views and opinions of authors expressed herein do not necessarily state or reflect those of the United States Government or any agency thereof. 


\section{DISCLAIMER}

Portions of this document may be illegible in electronic image products. Images are produced from the best available original document. 
This work was supported by the Propulsion Aerodynamics Branch of the National Aeronautics and Space Administration, Langley Research Center, Hampton, Virginia.

Edited by Patricia Metropolis

Phulucumpusition by IS-6́ Composing Section

\section{DISCLAIMER}

This report was prepared as an account of work sponsored by an agency of the United States Government. Neither the United States Government nor any agency thereof, nor any of their employees, makes any warranty, express or implied, or assumes any legal liability or responsibility for the accuracy, completeness, or usefulness of any information, apparatus, product, or process disclosed, or represents that its use would not infringe privately owned rights. References herein to any specific commercial product, process, or service by trade name, trademark, manufacturer, or otherwise, does not necessarily constitute or imply its endorsement, recommendation, or favoring by the United States Government or any agency thereof. The views and opinions of authors expressed herein do not necessarily state or reflect those of the United States Government or any agency thereof. 


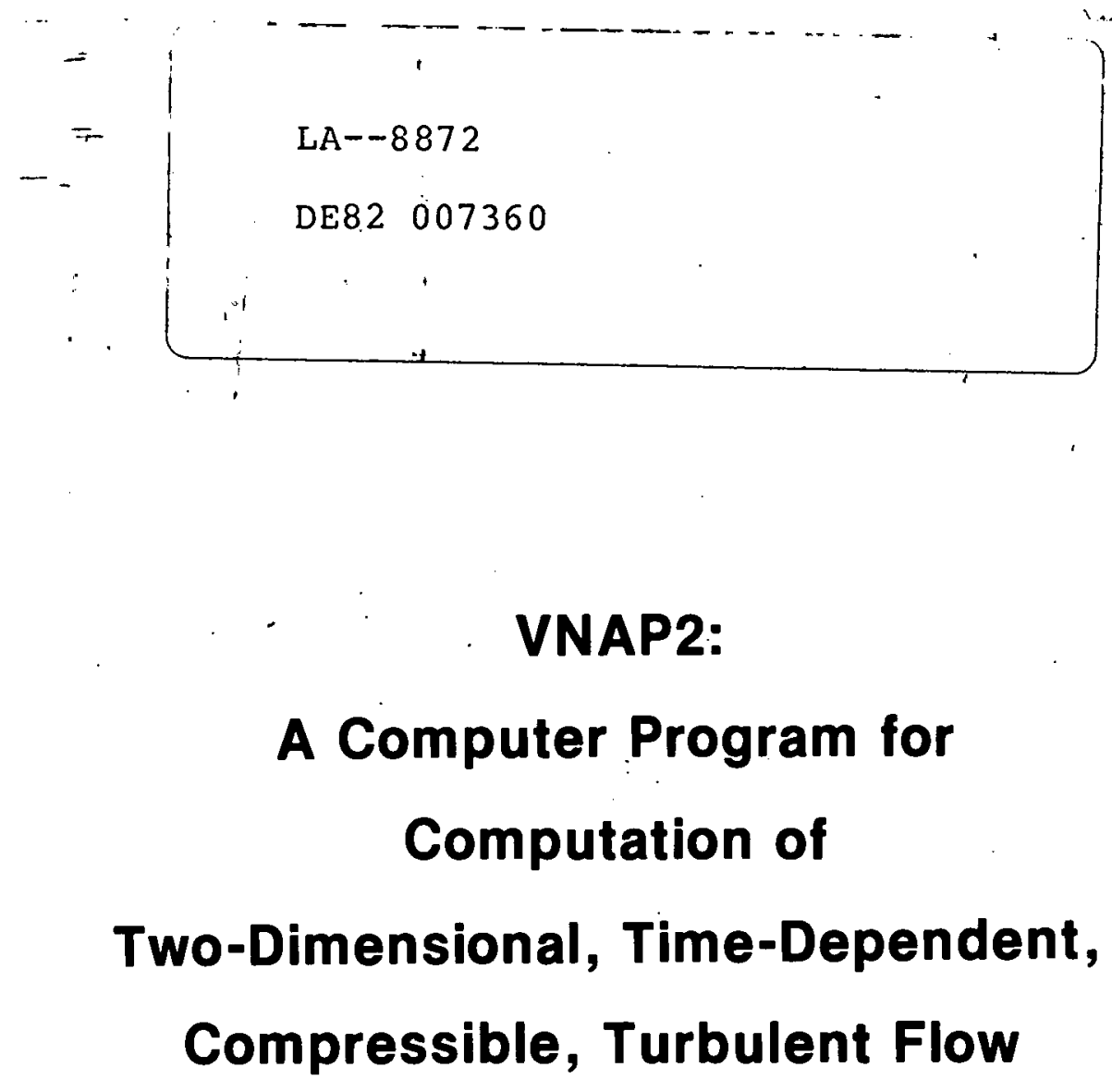

LA-8872

UC-32

Issued: August 1981

Michael C. Cline

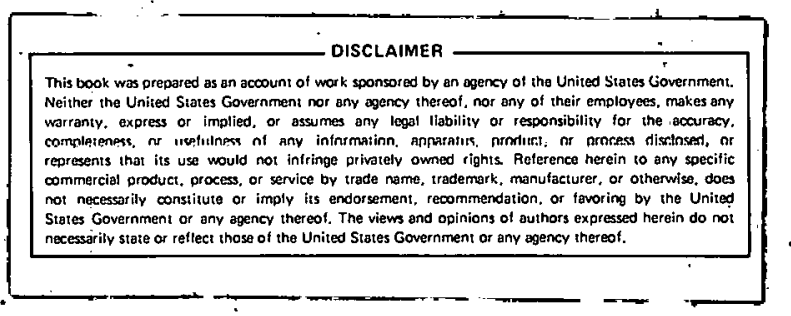

, 


\section{PAGES ii to iii WERE INTENTIONALLY LEFT BLANK}




\section{CONTENTS}

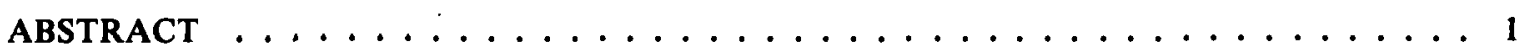

I. THE BASIC METHOD $\ldots \ldots \ldots \ldots \ldots \ldots \ldots \ldots \ldots \ldots \ldots$

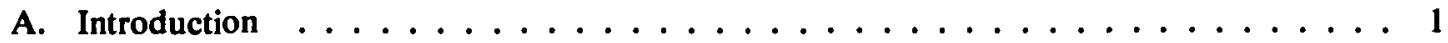

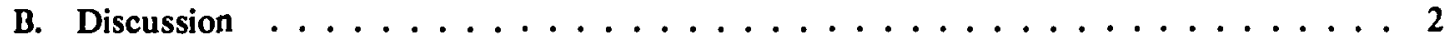

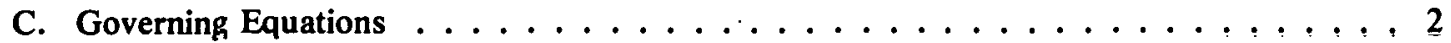

D. Physical and Computational Flow Spaces $\ldots \ldots \ldots \ldots \ldots$

E. . Numerical Method . . . . . . . . . . . . . . . . . . . . . 10

F. Comments on the Calculation of Steady Subsonic Flows $\ldots \ldots \ldots \ldots$

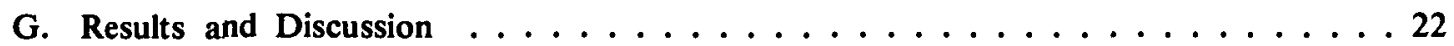

II. DESCRIPTION AND USE OF THE VANP2 PROGRAM $\ldots \ldots \ldots \ldots \ldots \ldots$

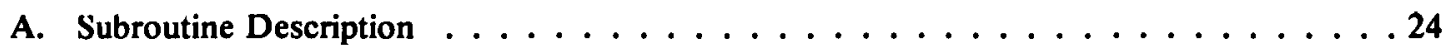

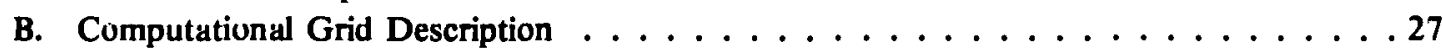

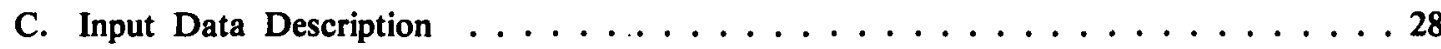

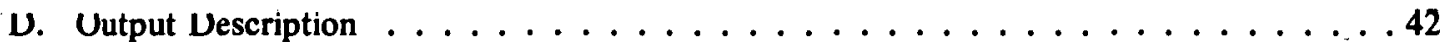

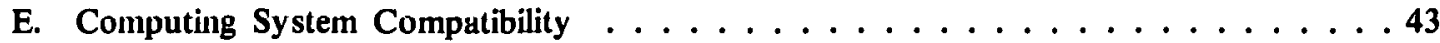

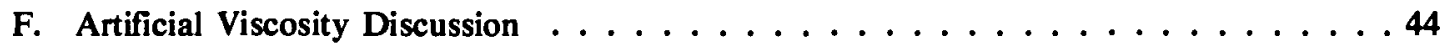

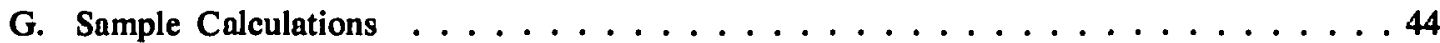

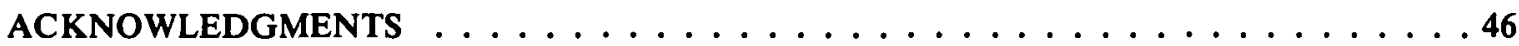

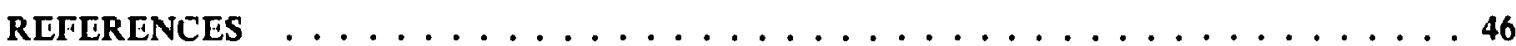

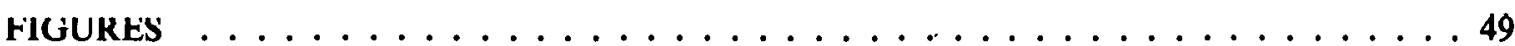

APPENDIX. FORTRAN LISTING OF THE VNAP2 PROGRAM $\ldots \ldots \ldots \ldots$ 


\title{
VNAP2: A COMPUTER PROGRAM FOR COMPUTATION \\ OF TWO-DIMENSIONAL, TIME-DEPENDENT, COMPRESSIBLE, TURBULENT FLOW
}

\author{
by
}

\author{
Michael C. Cline
}

\begin{abstract}
VNAP2 is a computer program for calculating turbulent (as well as laminar and inviscid), steady, and unsteady flow. VNAP2 solves the two-dimensional, timedependent, compressible Navier-Stokes equations. The turbulence is modeled with either an algebraic mixing-length model, a one-equation model, or the Jones-Launder two-equation model. The geometry may be a single- or a dual-flowing stream. The interior grid points are computed using the unsplit MacCormack scheme. Two options to speed up the calculations for high Reynolds number flows are included. The boundary grid points are computed using a reference-plane-characteristic scheme with the viscous terms treated as source functions. An explicit artificial viscosity is included for shock computations. The fluid is assumed to be a perfect gas. The flow boundaries may be arbitrary curved solid walls, inflow/outflow boundaries, or free-jet envelopes. Typical problems that can be solved concern nozzles, inlets, jet-powered afterbodies, airfoils, and free-jet expansions. The accuracy and efficiency of the program are shown by calculations of several inviscid and turbulent flows. The program and its use are described completely, and six sample cases and a code listing are included.
\end{abstract}

\section{THE BASIC METHOD}

\section{A. Introduction}

VNAP2 is a computer program for calculating turbulent (as well as laminar and inviscid), steady, and unsteady flow. VNAP2 is a modified version of the VNAP code discussed in Ref. 1. Like the VNAP code, VNAP2 solves the two-dimensional (2D, nxisymmetric), timc-dcpendent, compressible NavierStokes equations by a second-order-accurate finite-difference method. Unlike the VNAP code, VNAP2 allows arbitrary grid spacing, has two options to speed up the calculations for high Reynolds number flows, contains three different turbulence models, and can solve either single- or dual-flowing strcam geometries. This last option allows the VNAP2 code to compute internal/external flows, such as inlets, and jet-powered afterbodies as well as airfoils.

Because of the variable grid and the options to speed up the calculations for high Reynolds number flows, VNAP2 computes high Reynolds number flows much more efficiently than VNAP. However, fullscale Reynolds numbers $\left(10^{6}-10^{8}\right)$ still require fairly long run times (see Sec. I.G). In addition, 
determination of a reasonable variable grid and selection of the best numerical scheme parameters for high Reynolds number flows require a certain amount of trial and error.

Although the VNAP code replaced the NAP ${ }^{2}$ code, VNAP2 is not necessarily intended to replace the VNAP code. Although VNAP2 can handle all the flows that VNAP is capable of solving, as well as many additional flows, VNAP2 is approximately double the size of VNAP and somewhat more complex. As a result, VNAP2 is more difficult to modify as well as to run on smaller computing systems. For these reasons, many users may prefer to use both codes.

\section{B. Discussion}

The VNAP2 code follows the philosophy of the VNAP code; that is, the boundary grid points are the most important. In addition, except for purely supersonic inflow and outflow, these grid points are generally the most difficult. For these reasons, the construction of boundary grid point routines is not left to the general user, and VNAP2 contains complete and accurate routines for calculating all boundary grid points. Several different boundary conditions are included as options, and all unspecified variables are calculated using a second-order-accurate, reference-plane-characteristic scheme, with the viscous terms treated as source functions. The code also continually checks for subsonic or supersonic flow, as well as inflow or outflow, to apply the correct boundary conditions. Most of the options for inflow and outflow boundary conditions include nonreflecting conditions to accelerate the convergence to steady state.

Like VNAP, VNAP2 employs the unsplit MacCormack scheme ${ }^{3}$ to compute the interior grid points. The governing equations are left in nonconservation form. For flows with thin boundary layers or free shear layers, the small grid spacing required for resolution greatly increases the computer time. To reduce this time, the grid points in the finer parts of the mesh are subcycled. In addition, an explicit modification to the MacCormack scheme (allowing the removal of the speed of sound from the C-F-L condition and thus increasing the time-step size) is also included. An explicit artificial viscosity model stablizes the computations for shock waves.

\section{Governing Equations}

The 2D time-dependent, compressible, Navier-Stokes equations for turbulent flow of a perfect gas can be written as

$$
\begin{aligned}
& \frac{\partial \rho}{\partial t}+u \frac{\partial \rho}{\partial x}+v \frac{\partial \rho}{\partial y}+\rho\left(\frac{\partial u}{\partial x}+\frac{\partial v}{\partial y}+\frac{\varepsilon v}{y}\right) \\
& =\bar{u}\left[\frac{\partial}{\partial \mathbf{x}}\left(\frac{\mu_{\mathrm{T}}}{\rho} \frac{\partial \rho}{\partial \mathbf{x}}\right)+\frac{\partial}{\partial \mathbf{y}}\left(\frac{\mu_{\mathrm{T}}}{\rho} \frac{\partial \rho}{\partial \mathrm{y}}\right)+\frac{\varepsilon \mu_{\mathrm{T}}}{\rho \mathrm{y}} \frac{\partial \rho}{\partial \mathrm{y}}\right] \text {, } \\
& \frac{\partial u}{\partial t}+u \frac{\partial u}{\partial x}+v \frac{\partial u}{\partial y}+\frac{1}{\rho} \frac{\partial p}{\partial x}=\frac{1}{\rho} \frac{\partial}{\partial x}\left[(\lambda+2 \mu) \frac{\partial u}{\partial x}+\lambda \frac{\partial v}{\partial y}\right]+\frac{1}{\rho} \frac{\partial}{\partial y}\left[\mu\left(\frac{\partial v}{\partial x}+\frac{\partial u}{\partial y}\right)\right] \\
& +\frac{\bar{\alpha}}{\rho}\left[u \frac{\partial}{\partial x}\left(\frac{\mu_{\mathrm{T}}}{\rho} \frac{\partial \rho}{\partial \mathrm{x}}\right)+\mathrm{v} \frac{\partial}{\partial \mathrm{y}}\left(\frac{\mu_{\mathrm{T}}}{\rho} \frac{\partial \rho}{\partial \mathrm{x}}\right)\right]+\frac{\varepsilon}{\rho \mathrm{y}}\left[(\lambda+\mu) \frac{\partial \mathrm{v}}{\partial \mathrm{x}}+\mu \frac{\partial \mathrm{u}}{\partial \mathrm{y}}+\frac{\bar{\alpha} \mu_{\mathrm{T}} \mathrm{v}}{\rho} \frac{\partial \rho}{\partial \mathrm{x}}\right] \\
& -\frac{1}{\rho} \frac{2}{3} \frac{\partial p q}{\partial x},
\end{aligned}
$$




$$
\begin{aligned}
& \frac{\partial v}{\partial t}+u \frac{\partial v}{\partial x}+v \frac{\partial v}{\partial y}+\frac{1}{\rho} \frac{\partial p}{\partial y}=\frac{1}{\rho} \frac{\partial}{\partial y}\left[(\lambda+2 \mu) \frac{\partial v}{\partial y}+\lambda \frac{\partial u}{\partial x}\right]+\frac{1}{\rho} \frac{\partial}{\partial x}\left[\mu\left(\frac{\partial v}{\partial x}+\frac{\partial u}{\partial y}\right)\right] \\
& +\frac{\bar{\alpha}}{\rho}\left[v \frac{\partial}{\partial y}\left(\frac{\mu_{T}}{\rho} \frac{\partial \rho}{\partial y}\right)+u \frac{\partial}{\partial x}\left(\frac{\mu_{T}}{\rho} \frac{\partial \rho}{\partial y}\right)\right]+\frac{\varepsilon}{\rho y}\left[(\lambda+2 \mu)\left(\frac{\partial v}{\partial y}-\frac{v}{y}\right)+\frac{\bar{\alpha} \mu_{T} v^{\prime}}{\rho} \frac{\partial p}{\partial y}\right] \\
& -\frac{1}{\rho} \frac{2}{3} \frac{\partial \rho q}{\partial y_{0}}, \\
& \frac{\partial p}{\partial \mathrm{t}}+\dot{\mathrm{u}} \frac{\partial \mathrm{p}}{\partial \mathrm{x}}+\mathrm{v} \frac{\partial \mathrm{p}}{\partial \mathrm{y}}-\mathrm{a}^{2}\left(\frac{\partial \rho}{\partial \mathrm{t}}+\mathrm{u} \frac{\partial \mathrm{p}}{\partial \mathrm{x}}+\mathrm{v} \frac{\partial \mathrm{p}}{\partial \mathrm{y}}\right)=(\gamma-1)\left\{\left(\lambda_{\mathrm{M}}+2 \mu_{\mathrm{M}}\right)\left[\left(\frac{\partial \mathrm{u}}{\partial \mathrm{x}}\right)^{2}+\left(\frac{\partial \mathrm{v}}{\partial \mathrm{y}}\right)^{2}\right]\right. \\
& +\mu_{M}\left[\left(\frac{\partial v}{\partial x}\right)^{2}+\left(\frac{\partial u}{\partial y}\right)^{2}\right]+2 \lambda_{M} \frac{\partial u}{\partial x} \frac{\partial v}{\partial y}+2 \mu_{M} \frac{\partial v}{\partial x} \frac{\partial u}{\partial y}+\frac{\partial}{\partial x}\left(k \frac{\partial T}{\partial x}\right)+\frac{\partial}{\partial y}\left(k \frac{\partial T}{\partial y}\right) \\
& -\bar{\alpha} R T\left[\frac{\partial}{\partial x}\left(\frac{\mu_{T}}{\rho} \frac{\partial \rho}{\partial x}\right)+\frac{\partial}{\partial y}\left(\frac{\mu_{T}}{\rho} \frac{\partial \rho}{\partial y}\right)\right]+\frac{\varepsilon}{y}\left[\left(\lambda_{M}+2 \mu_{M}\right) \frac{v^{2}}{y}+2 \lambda_{M} v\left(\frac{\partial u}{\partial x}+\frac{\partial v}{\partial y}\right)\right. \\
& \left.\left.+\mathrm{k} \frac{\partial \mathrm{T}}{\partial \mathrm{y}}-\frac{\overline{\mathrm{a}} \mathrm{T} \mu_{\mathrm{T}}}{\rho} \frac{\partial \rho}{\partial \mathrm{y}}\right]+\mathrm{pe}\right\},
\end{aligned}
$$

and

$$
p=\rho R T \text {, }
$$

where $\rho$ is the density; $p$ is the pressure; $T$ is the temperature; $u$ and $v$ are the velocity components; $q$ is the turbulence energy; $e$ is the turbulence dissipation rate; $a$ is the speed of sound; $R$ is the gas constant; $\mu=\mu_{M}+\mu_{T} ; \lambda=\lambda_{M}+\lambda_{T} ; \mu_{M}$ and $\lambda_{M}$ are the first and second coefficients of molecular viscosity; $\mu_{\mathrm{T}}$ and $\lambda_{\mathrm{T}}$ are the corresponding turbulent quantities; $\gamma$ is the ratio of specific heats; $k=k_{M}+k_{T} ; k_{M}$ is the coefficient of molecular conductivity; $\mathrm{k}_{\mathrm{T}}$ is the turbulent value; $\mathrm{x}$ and $\mathrm{y}$ are the space coordinates; $\mathrm{t}$ is the time; $\bar{\alpha}$ is a constant; and $\varepsilon$ is 0 for planar flow and 1 for axisymmetric flows. Equations (2)-(4) are written for the two-equation turbulence model. For the mixing-length and one-equation models discussed below, Eqs. (2)-(4) are slightly different. The density gradient terms, premultiplied by the constant $\bar{\alpha}$, on the right-hand side of Eqs. (1)-(4) are from turbulent density fluctuations and are, therefore, zero for laminar flows. Equation (1) is the conservation of mass or continuity equation, Eqs. (2) and (3) are the $x$ and y momentum cquations, respectively, and Eq. (4) is the internal energy equation written in terms of pressure using the equation of state for a perfect gas, Eq. (5). Thus there is a system of five equations for the eight unknowns $u, v, p, \rho, T, \mu_{\mathrm{T}}, \lambda_{\mathrm{T}}$, and $\mathrm{k}_{\mathrm{T}}$ (In the two-equation turbulence model, there are two additional equations for the unknowns $q$ and $e$.) To close this set of equations, the turbulence quantities $\mu_{\mathrm{T}}, \lambda_{\mathrm{T}}$ and $\mathrm{k}_{\mathrm{T}}$ need definition. VNAP2 uses the following three turbulence models to accomplish this.

1. Mixing-Length Turbulence Model. The first model is an algebraic mixing-length model that can be written as

$$
\begin{aligned}
& \mu_{\mathrm{T}}=\rho \ell^{2}\left[\left(\frac{\partial \mathrm{v}}{\partial \mathrm{x}}\right)^{2}+\left(\frac{\partial \mathrm{u}}{\partial \mathrm{y}}\right)^{2}\right]^{1 / 2}, \\
& \lambda_{\mathrm{T}}=\lambda \mu_{\mathrm{T}} / \mu,
\end{aligned}
$$

and 


$$
\mathrm{k}_{\mathrm{T}}=\gamma \operatorname{R}_{\mathbf{T}} /(\gamma-1) \operatorname{Pr}_{\mathbf{T}},
$$

where $\ell$ is the mixing length defined below and $\operatorname{Pr}_{\mathrm{T}}$ is the turbulent $\operatorname{Prandtl}$ number. For free shear layer flows, the model follows Ref. 4 . For monotonic velocity profiles, $\ell$ is defined as

$$
\ell=C_{\mathrm{ML2}} \cdot\left|\mathrm{y}_{2}-\mathrm{y}_{1}\right|
$$

where $\mathrm{C}_{\mathrm{ML2}}$ is a constant and

$$
\begin{aligned}
& y_{1}=y \quad \text { for } \quad \frac{u-u_{L}}{u_{u}-u_{L}}=0.1, \\
& y_{2}=y \quad \text { for } \quad \frac{u-u_{L}}{u_{u}-u_{L}}=0.9,
\end{aligned}
$$

and $u_{L}$ and $u_{U}$ are the lower and upper velocities of a monotonically increasing or decreasing velocity profile. For free shear flows with a velocity profile that has the minimum velocity $u_{M}$ in the interior, $\ell$ is defined as

$$
\ell=\mathrm{C}_{\mathrm{ML1}} \cdot\left|\mathrm{y}_{2}-\mathrm{y}_{1}\right|
$$

where $\mathrm{C}_{\mathrm{ML} 1}$ is a constant and

$$
\begin{aligned}
& y_{1}=y \text { for } \frac{u-u_{L}}{u_{M}-u_{L}}=0.1 \text { and } y<y_{2}, \\
& y_{1}=y \text { for } \frac{u-u_{M}}{u_{U}-u_{M}}=0.9 \text { and } y>y_{2},
\end{aligned}
$$

and

$$
\mathrm{y}_{\mathrm{\eta}}=\mathrm{y} \text { for } \mathrm{u}=\mathrm{u}_{\mathrm{M}} \text {. }
$$

The program continually checks to determine the type of velocity profile present. If $u_{M}$ is within $5 \%$ of the minimum of $u_{L}$ or $u_{U}$, then the monotonic profile is assumed. This check on the size of $u_{M}$ is intended to stop small velocity variations, away from the shear region, from switching the velocity profile type. The $5 \%$ value is arbitrary and can be changed in subroutinc MIXLEN (see Sec. II. A). On the centerline or midplane, Eq. (6) is replaced by

$$
\mu_{\mathrm{T}}=\rho \ell^{3}\left|\frac{\partial^{2} \mathrm{u}}{\partial \mathrm{y}^{2}}\right|
$$

For boundary-layer flows, the Cebeci-Smith' two-layer model is used. In the Intuer layes, $l$ is defined a3

$$
\ell=0.4 y\left[1.0-\exp \left(\frac{-y \sqrt{\rho i_{w}}}{26.0 \mu_{M}}\right)\right]^{\prime},
$$

where $y$ is the distance from the wall and $\tau_{\mathrm{w}}$ is the shear stress at the wall. In the outer layer, Eqs. (6) and (12) are replaced by 


$$
\mu_{\mathrm{T}}=0.0168 \mathrm{pu}_{\mathrm{E}} \delta^{*}\left[1.0+5.5 \frac{\mathrm{y}^{6}}{\delta}\right]^{-1}
$$

where $u_{E}$ is the velocity al the edge of the boundary layer, $\delta$ is the boundary-layer velocity thickness, and $\delta^{*}$ is the boundary-layer displacement thickness given by

$$
\delta^{*}=\int_{0}^{\delta}\left(1-\frac{\rho \mathrm{u}}{\rho_{\mathrm{E}} \mathrm{u}_{\mathrm{E}}}\right) \mathrm{dy} .
$$

The switch from the inner-layer model, given by Eqs. (6) and (12), to the outer-layer model, given by Eq. (13), occurs when the inner $\mu_{\mathrm{T}}$ is greater than the outer value. This model does not employ a relaxation or lag parameter. The values for $\mathrm{C}_{\mathrm{ML} 1}$ and $\mathrm{C}_{\mathrm{ML} 2}$ are 0.125 for planar flows and 0.11 for axisymmetric flows.

For this model, the last term on the right-hand side of Eqs. (2)-(4) vanishes. In addition, the viscosity coefficients $\lambda_{M}$ and $\mu_{M}$ in the first four terms on the right-hand side of Eq. (4) as well as the first two axisymmetric terms, also in Eq. (4), are replaced by $\lambda$ and $\mu$.

2. One-Equation Turbulence Model. This model was developed at Los Alamos National Laboratory by Bart J. Daly. At present, this model has not been extensively proof-tested and, therefore, should be considered experimental. The model attempts to combine the best features of the algebraic mixing-length models and the two-equation models.

This model consists of the following transport equation for the turbulence energy q,

$$
\begin{aligned}
& \frac{\partial \mathrm{q}}{\partial \mathrm{t}}+\mathrm{u} \frac{\partial \mathrm{q}}{\partial \mathrm{x}}+\mathrm{v} \frac{\partial \mathrm{q}}{\partial \mathrm{y}}=\frac{2}{3} \frac{\mathrm{q}}{\rho}\left(\frac{\partial \mathrm{p}}{\partial \mathrm{t}}+\mathrm{u} \frac{\partial \rho}{\partial \mathrm{x}}+\mathrm{v} \frac{\partial \rho}{\partial \mathrm{y}}\right) \\
& +\frac{\lambda_{\mathrm{T}}+2 \mu_{\mathrm{T}}}{\rho}\left[\left(\frac{\partial \mathrm{u}}{\partial \mathrm{x}}\right)^{2}+\left(\frac{\partial \mathrm{v}}{\partial \mathrm{y}}\right)^{2}\right] \\
& +\frac{\mu_{\mathrm{T}}}{\rho}\left[\left(\frac{\partial \mathrm{v}}{\partial \mathrm{x}}\right)^{2}+\left(\frac{\partial \mathrm{u}}{\partial \mathrm{y}}\right)^{2}\right]+\frac{2 \lambda_{\mathrm{T}}}{\rho} \frac{\partial \mathrm{u}}{\partial \mathrm{x}} \frac{\partial \mathrm{v}}{\partial \mathrm{y}}+\frac{2 \mu_{\mathrm{T}}}{\rho} \frac{\partial \mathrm{v}}{\partial \mathrm{x}} \frac{\partial \mathrm{u}}{\partial \mathrm{y}} \\
& +\frac{1}{\rho} \frac{\partial}{\partial \mathrm{x}}\left[\left(\mu_{\mathrm{M}}+\frac{\mu_{\mathrm{T}}}{\sigma_{\mathrm{q}}}\right) \frac{\partial \mathrm{q}}{\partial \mathrm{x}}\right]+\frac{1}{\rho} \frac{\partial}{\partial \mathrm{y}}\left[\left(\mu_{\mathrm{M}}+\frac{\mu_{\mathrm{T}}}{\sigma_{\mathrm{q}}}\right) \frac{\partial \mathrm{q}}{\partial \mathrm{y}}\right]-\frac{2 \mu_{\mathrm{M}} \mathrm{q} \Delta}{\rho S^{2}} \\
& \quad-\frac{2 \bar{\alpha} \mathrm{q}}{3 \rho}\left[\frac{\partial}{\partial \mathrm{x}}\left(\frac{\mu_{\mathrm{T}}}{\rho} \frac{\partial \mathrm{p}}{\partial \mathrm{x}}\right)+\frac{\partial}{\partial \mathrm{y}}\left(\frac{\mu_{\mathrm{T}}}{\rho} \frac{\partial \rho}{\partial \mathrm{y}}\right)\right]+\frac{\varepsilon}{\mathrm{y}}\left[\frac{\lambda_{\mathrm{T}}+2 \mu_{\mathrm{T}}}{\rho} \frac{\mathrm{v}^{2}}{\mathrm{y}}+\frac{2 \lambda_{\mathrm{T}} \mathrm{v}}{\rho}\left(\frac{\partial \mathrm{u}}{\partial \mathrm{x}}+\frac{\partial \mathrm{v}}{\partial \mathrm{y}}\right)\right. \\
& \left.+\frac{\mu}{\rho} \frac{\partial \mathrm{q}}{\partial \mathrm{y}}-\frac{2 \bar{\alpha} \mathrm{q} \mu_{\mathrm{T}}}{\rho^{2}} \frac{\partial \rho}{\partial \mathrm{y}}\right],
\end{aligned}
$$

where

$$
\mathrm{S}=\mathrm{C}_{\mathrm{q}} \ell
$$




$$
\Delta= \begin{cases}5 & \text { for } \frac{S \rho \sqrt{2 q}}{\mu_{M}} \leqslant 5 \\ \frac{S \rho \sqrt{2 q}}{\mu_{M}} & \text { for } \frac{S \rho \sqrt{2 q}}{\mu_{M}}>5,\end{cases}
$$

$\ell$ is the mixing length from the first model, and $c_{q}$ is a constant. The turbulent viscosity $\mu_{\mathrm{T}}$ is defined as

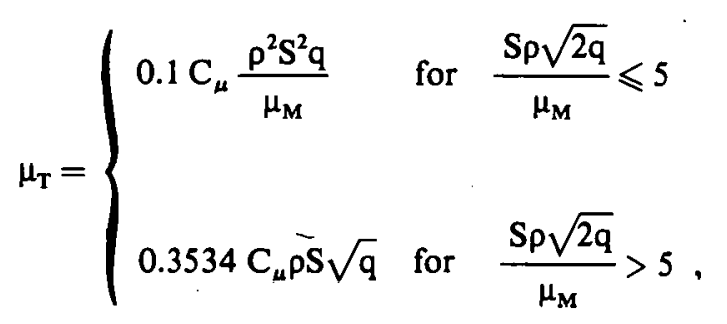

where $C_{q}$ is 17.2 for planar flows and 12.3 for axisymmetric flows and $C_{\mu}=0.09$. The quantities $\lambda_{T}$ and $k_{\mathrm{T}}$ are determined from Eqs. (7) and (8), respectively.

For this model, the last term on the right-hand side of Eq. (4) is replaced with $2 \mu_{M} q \Delta / S^{2}$.

3. Two-Equation, Jones-Launder ${ }^{6-9}$ Turbulence Model. This model employs two transport equations, one for the turbulence energy $q$ and the second for the turbulence dissipation rate $e$. These equations can be written as

$$
\begin{aligned}
& \frac{\partial \mathrm{q}}{\partial \mathrm{t}}+\mathrm{u} \frac{\partial \mathrm{q}}{\partial \mathrm{x}}+\mathrm{v} \frac{\partial \mathrm{q}}{\partial \mathrm{y}}=\frac{\lambda_{\mathrm{T}}+2 \mu_{\mathrm{T}}}{\rho}\left[\left(\frac{\partial \mathrm{u}}{\partial \mathrm{x}}\right)^{2}+\left(\frac{\partial \mathrm{v}}{\partial \mathrm{y}}\right)^{2}\right]+\frac{\mu_{\mathrm{T}}}{\rho}\left[\left(\frac{\partial \mathrm{v}}{\partial \mathrm{x}}\right)^{2}+\left(\frac{\partial \mathrm{u}}{\partial \mathrm{y}}\right)^{2}\right] \\
& +\frac{2 \lambda_{\mathrm{T}}}{\rho} \frac{\partial \mathrm{u}}{\partial \mathrm{x}} \frac{\partial \mathrm{v}}{\partial \mathrm{y}}+\frac{2 \mu_{\mathrm{T}}}{\rho} \frac{\partial \mathrm{v}}{\partial \mathrm{x}} \frac{\partial \mathrm{u}}{\partial \mathrm{y}}+\frac{1}{\rho} \frac{\partial}{\partial \mathrm{x}}\left[\left(\mu_{\mathrm{M}}+\frac{\mu_{\mathrm{T}}}{\sigma_{\mathrm{q}}}\right) \frac{\partial \mathrm{q}}{\partial \mathrm{x}}\right]+\frac{1}{\rho} \frac{\partial}{\partial \mathrm{y}}\left[\left(\mu_{\mathrm{M}}+\frac{\mu_{\mathrm{v}}}{\sigma_{\mathrm{q}}}\right) \frac{\partial \mathrm{q}}{\partial \mathrm{y}}\right] \\
& -\mathrm{e}-\frac{\partial_{\mathrm{\mu}}}{\rho}\left(\frac{\partial \mathrm{q}^{1 / 2}}{\partial \mathrm{x}}+\frac{\partial \mathrm{q}^{1 / 2}}{\partial \mathrm{y}}\right)^{2}+\frac{\varepsilon}{\mathrm{y}}\left[\frac{\lambda_{\mathrm{T}}+2 \mathrm{Il}_{\mathrm{T}}}{\rho} \frac{\mathrm{v}^{2}}{\mathrm{y}}+\frac{\partial \lambda_{\mathrm{T}} \mathrm{v}}{\rho}\left(\frac{\partial \mathrm{u}}{\partial \mathrm{x}}+\frac{\partial \mathrm{v}}{\partial \mathrm{y}}\right)+\frac{1}{\rho}\left(\mu_{\mathrm{M}}+\frac{\mu_{1}}{\sigma_{\mathrm{q}}}\right) \frac{\partial \mathrm{q}}{\partial \mathrm{y}}\right]
\end{aligned}
$$

and

$$
\begin{aligned}
& \frac{\partial \mathrm{e}}{\partial \mathrm{t}}+\mathrm{u} \frac{\partial \mathrm{e}}{\partial \mathrm{x}}+\mathrm{v} \frac{\partial \mathrm{e}}{\partial \mathrm{y}}=\frac{\mathrm{C}_{1} \mathrm{e}}{\mathrm{q}}\left\{\frac{\lambda_{\mathrm{T}}+2 \mu_{\mathrm{T}}}{\rho}\left[\left(\frac{\partial \mathrm{u}}{\partial \mathrm{x}}\right)^{2}+\left(\frac{\partial \mathrm{v}}{\partial \mathrm{y}}\right)^{2}\right]+\frac{\mu_{\mathrm{T}}}{\rho}\left[\left(\frac{\partial \mathrm{v}}{\partial \mathrm{x}}\right)^{2}+\left(\frac{\partial \mathrm{u}}{\partial \mathrm{y}}\right)^{2}\right]\right. \\
& \left.+\frac{2 \lambda_{\mathrm{T}}}{\rho} \frac{\partial \mathrm{u}}{\partial \mathrm{x}} \frac{\partial \mathrm{v}}{\partial \mathrm{y}}+\frac{2 \mu_{\mathrm{T}}}{\rho} \frac{\partial \mathrm{v}}{\partial \mathrm{x}} \frac{\partial \mathrm{u}}{\partial \mathrm{y}}\right\}+\frac{1}{\rho} \frac{\partial}{\partial \mathrm{x}}\left[\left(\mu_{\mathrm{M}}+\frac{\mu_{\mathrm{T}}}{\sigma_{\mathrm{e}}}\right) \frac{\partial \mathrm{e}}{\partial \mathrm{x}}\right] \\
& +\frac{1}{\rho} \frac{\partial}{\partial \mathrm{y}}\left[\left(\mu_{\mathrm{M}}+\frac{\mu_{\mathrm{T}}}{\sigma_{\mathrm{e}}}\right) \frac{\partial \mathrm{e}}{\partial \mathrm{y}}\right]-\frac{\mathrm{C}_{\mathrm{c}} \mathrm{c}}{\mathrm{q}}\left[\mathrm{e}-\frac{2 \mu}{\rho}\left(\frac{\partial \mathrm{q}^{1 / 2}}{\partial \mathrm{x}}+\frac{\partial \mathrm{q}^{1 / 2}}{\partial \mathrm{y}}\right)\right. \\
& +\frac{2 \mu_{\mathrm{M}} \mu_{\mathrm{T}}}{\rho^{2}}\left[\left(\frac{\partial^{2} \mathrm{u}}{\partial \mathrm{x}^{2}}\right)^{2}+\left(\frac{\partial^{2} \mathrm{v}}{\partial \mathrm{x}^{2}}\right)^{2}+\left(\frac{\partial^{2} \mathrm{u}}{\partial \mathrm{y}^{2}}\right)^{2}+\left(\frac{\partial^{2} \mathrm{v}}{\partial \mathrm{y}^{2}}\right)^{2}\right]
\end{aligned}
$$




$$
\begin{aligned}
& +\frac{\varepsilon}{y}\left\{\frac{C_{1} e}{q}\left[\frac{\lambda_{T}+2 \mu_{T}}{\rho} \frac{v^{2}}{y}+\frac{2 \lambda_{T} v}{\rho}\left(\frac{\partial u}{\partial x}+\frac{\partial v}{\partial y}\right)\right]+\frac{1}{\rho}\left(\mu_{M}+\frac{\mu_{T}}{\sigma_{e}}\right) \frac{\partial e}{\partial y}\right. \\
& \left.+\frac{2 y \mu_{M} \mu_{T}}{\rho^{2}}\left[\left(\frac{1}{y} \frac{\partial u}{\partial y}\right)^{2}+\left(\frac{1}{y} \frac{\partial v}{\partial y}\right)^{2}+\frac{2}{y} \frac{\partial u}{\partial y} \frac{\partial^{2} u}{\partial y^{2}}+\frac{2}{y} \frac{\partial v}{\partial y} \frac{\partial^{2} v}{\partial y^{2}}\right]\right\},
\end{aligned}
$$

where

$$
\begin{aligned}
& C_{1}=1.44, \sigma_{q}=1.0, \sigma_{e}=1.3, \\
& C_{2}=\bar{C}_{2}\left[1.0-0.2222 \exp \left(-0.0278 R_{T}^{2}\right)\right], \\
& \text { and } \\
& R_{T}=\rho q^{2} / \mu_{M} e .
\end{aligned}
$$

and

The turbulent viscosity is calculated from

$$
\mu_{\mathrm{T}}=\mathrm{C}_{\mu} \exp \left[-3.4 /\left(1+0.02 \mathrm{R}_{\mathrm{T}}\right)^{2}\right] \rho q^{2} / \mathrm{e}
$$

where $C_{\mu}=0.09$. The quantities $\lambda_{T}$ and $k_{T}$ are determined from Eqs. (7) and (8), respectively. The solid wall boundary condition on $\mathrm{e}$ for this version of the Jones-Launder model is $\partial \mathrm{e} / \partial \mathrm{y}=0$.

For strongly separated flows, this model has two numerical problems. One problem is that the turbulence dissipation rate becomes extremely small near a reattachment point. To overcome this, a lower bound on $q$ and $e$ at a given $y$ was added as an option to VNAP2 in the manner of Coakley and Viegas. ${ }^{10}$ The second problem is associated with the treatment of the convection terms in Eqs. (18) and (19). In the far field where $q \rightarrow 0$, the variations of $q$ and $e$ are such in some problems that extremely large values of $\mu_{\mathrm{r}}$ occur. Using the donor cell scheme in the $\mathrm{x}$ direction and the MacCormack scheme in the $y$ direction removes this problem for all cases tested so far. Also included is the following fourth-order smoothing term added to Eq. (18):

$$
C_{Q}\left(\frac{(|u|+a) \Delta x^{3}}{q}\left|\frac{\partial^{2} q}{\partial x^{2}}\right| \frac{\partial^{2} q}{\partial x^{2}}+\frac{(|v|+a) \Delta y^{3}}{q}\left|\frac{\partial^{2} q}{\partial y^{2}}\right| \frac{\partial^{2} q}{\partial y^{2}}\right),
$$

where $C_{Q}$ is a constant. A similar term, with e replacing $q$ and $C_{E}$ replacing $C_{Q}$, is added to Eq. (19). These smoothing terms were added as a possible alternative to the donor cell differencing. However, at this time, the donor cell differencing appears to be more satisfactory.

4. Artificial Viscosity Model. To stabilize the numerical method for shock wave calculations, an explicit artificial viscosity model is included. This model replaces the explicit fourth-order smoothing : usually employed by MacCormack. ${ }^{11}$ The procedure here is first to calculate artificial viscosity coefficients $\mu_{A}, \lambda_{A}$ and a thermal conductivity coefficient $k_{A}$ and, second, to add these values to the molecular values. These quantities are calculated from the following equations:

$$
\begin{aligned}
& \lambda_{\mathrm{A}}=\mathrm{C} \mathrm{C}_{\lambda} \Delta \mathrm{x} \Delta \mathrm{y} \rho\left|\frac{\partial \mathrm{u}}{\partial \mathrm{x}}+\frac{\partial \mathrm{v}}{\partial \mathrm{y}}+\varepsilon \frac{\mathrm{v}}{\mathrm{y}}\right|, \\
& \mu_{\mathrm{A}}=\mathrm{C}_{\mu 1} \lambda_{\mathrm{A}} / \mathrm{C}_{\lambda},
\end{aligned}
$$

and

$$
\mathbf{k}_{\mathrm{A}}=\gamma \mathrm{R} \mu_{\mathrm{A}} /(\gamma-1) \mathrm{Pr}_{\mathrm{A}},
$$


where $\mathrm{C}, \mathrm{C}_{\lambda}, \mathrm{C}_{\mu 1}$, and $\mathrm{Pr}_{\mathrm{A}}$ are constants, with $\mathrm{Pr}_{\mathrm{A}}$ representing an artificial Prandtl number, and $\Delta \mathrm{x}$ and $\Delta y$ are the mesh spacing. The following artificial density smoothing term also is added to the right-hand side of Eq. (1).

$$
\text { Equation (1) }=\frac{C_{\rho}}{\rho}\left[\frac{\partial}{\partial x}\left(\mu_{\mathrm{A}} \frac{\partial \rho}{\partial \mathrm{x}}\right)+\frac{\partial}{\partial \mathrm{y}}\left(\mu_{\mathrm{A}} \frac{\partial \rho}{\partial \mathrm{y}}\right)+\frac{\varepsilon \mu_{\mathrm{A}}}{\mathrm{y}} \frac{\partial \rho}{\partial \mathrm{y}}\right] \text {, }
$$

where $C_{\rho}$ is a constant. When the divergence of the velocity is greater than zero (expansions), these artificial quantities are set equal to zero.

\section{Physical and Computational Flow Spaces}

Figure 1 shows the physical flow-space geometry, with flow from left to right. The upper boundary, called the wall, can be either a solid boundary, a free-jet boundary, or an arbitrary subsonic (normal to the boundary) inflow/outflow boundary. The lower boundary, called the centerbody, can be either a solid boundary or a plane (line) of symmetry. The geometry can be either a single-flowing stream or, if the dual-flow-space walls are present, a dual-flowing stream. The dual-flow-space walls may begin in the interior and continue to the exit (inlet geometry), may begin at the inlet and terminate in the interior as shown in Fig. 1 (afterbody geometry), or may begin and end in the interior (airfoil geometry). All of the above boundaries may be arbitrary curved boundaries provided the y coordinate is a single value function of $\mathrm{x}$. If the dual-flow-space walls begin or end in the interior, then they must have pointed ends. The points can be very blunt, but there cannot be vertical walls. The left boundary is a subsonic, supersonic, or mixed inflow boundary, whereas the right boundary is a subsonic, supersonic, or mixed outflow boundary or a subsonic inflow/outflow boundary.

The $x, y, t$ physical space is mapped into a rectangular $\zeta, \eta, \tau$ computational space as shown in Fig. 1 . The mapping is carried out in two stages: the first maps the physical space to a rectangular computational space and the second maps the variable grid spacing to a uniform grid spacing. Because the single- and dual-flow-space mappings are different, they will be discussed separately.

1. Single-Flow Space. The $x, y, t$ physical space, with variable grid spacing, is mapped into the $\bar{\zeta}, \bar{\eta}$, $\bar{\tau}$ space, which also has variable grid spacing, by the following transformation:

$$
\bar{\zeta}=\mathbf{x} ; \bar{\eta}=\frac{\mathrm{y} \cdots \mathrm{y}_{\mathrm{c}}}{\mathrm{y}_{\mathrm{w}}-\mathrm{y}_{\mathrm{c}}} ; \bar{\tau}=\mathrm{t},
$$

where $y_{C}$ is a function of $x$ and denotes the centerbody $y$ value and $y_{w}$ is a function of $x$ and $t$ and denotes the wall y value. The quantity $\bar{\eta}$ varies between 0 and 1 . This variable grid $\bar{\zeta}, \bar{\eta}, \bar{\tau}$ space is mapped into a uniform grid $\zeta, \eta, \tau$ space by the following transformation:

$$
\zeta=\zeta(\bar{\zeta}) ; \quad \eta=\eta(\bar{\eta}) ; \quad \tau=\bar{\tau} ;
$$

that is, $\zeta$ is an arbitrary tabular function of $\bar{\zeta}$, etc. Using Eqs. (27) and (28), the derivatives become

$$
\frac{\partial}{\partial \mathbf{x}}=\omega \frac{\partial}{\partial \zeta}+\alpha \frac{\partial}{\partial \eta} ; \quad \frac{\partial}{\partial \mathbf{y}}=\beta \frac{\partial}{\partial \eta} ; \frac{\partial}{\partial t}=\frac{\partial}{\partial \tau}+\delta \frac{\partial}{\partial \eta},
$$


where

$$
\omega=\frac{\mathrm{d} \zeta}{\mathrm{d} \bar{\zeta}} ; \quad \beta=\frac{\mathrm{d} \eta}{\mathrm{d} \bar{\eta}} \frac{1}{\mathrm{y}_{\mathrm{w}}-\mathrm{y}_{\mathrm{c}}} ; \quad \alpha=\beta\left[(\bar{\eta}-1) \frac{\mathrm{d} \mathrm{y}_{\digamma}}{\mathrm{dx}}-\bar{\eta} \frac{\partial \mathrm{y}_{\mathrm{w}}}{\partial \mathrm{x}}\right] ; \quad \delta=-\bar{\eta} \beta \frac{\partial \mathrm{y}_{\mathrm{w}}}{\partial \mathrm{t}}
$$

The derivatives $d \zeta / d \bar{\zeta}$ and $d \eta / d \bar{\eta}$ are computed numerically using differences consistent with the MacCormack scheme.

This results in a physical space grid with the following properties: one set of grid lines is straight and in the $y$ direction with arbitrary spacing in the $x$ direction; the second set of grid lines approximately follows the wall and centerbody contours; the $\Delta y$ spacing of these grid lines is arbitrary at one $x$ location and is proportional to those values at any other $\mathrm{x}$ location; and the proportionality factor is based on the distance between $y_{w}$ and $y_{C}$. For more details on the physical space grid, see the example shown in Fig. 2 as well as the computed results in Sec. I.G.

2. Dual-Flow Space. If part of the flow in the dual-flow-space example is a single-flow space, then the single-flow-space option discussed above is used in that part. In the dual-flow-space section, the procedure is to divide the dual-flow space into two single-flow spaces and then to use the single-flow-space transformations discussed above. Both the upper and lower dual-flow-space walls collapse to the same grid line in the computational space, as shown in Fig. 1. The flow variables at the grid points on the upper dual-flow-space wall are stored in the regular solution array, whereas the lower wall variables are stored in a dummy array. These flow variables are continually switched between the two arrays during the calculation. For the dual-flow-space example, Eq. (27) becomes

$$
\left.\begin{array}{ll}
\bar{\zeta}=x ; \quad \bar{\eta}=c \frac{y-y_{C}}{y_{L}-y_{c}} ; \quad \bar{\tau}=t \quad & \text { for } \quad y_{c} \leqslant y \leqslant y_{L}, \\
\bar{\zeta}=x ; \quad \bar{\eta}=c+(1-c) \frac{y-y_{U}}{y_{w}-y_{U}} ; \quad \bar{\tau}=t \quad \text { for } y_{U} \leqslant y \leqslant y_{w},
\end{array}\right\}
$$

where $y_{L}$ and $y_{U}$ are functions of $x$ and denote the lower and upper dual-flow-space walls, respectively. The parameter $c$ is a constant and equals $\left(y_{L}-y_{C}\right) /\left(y_{w}-y_{U}+y_{L}-y_{C}\right)$ evaluated at a specified $x$ location. For completely dual flows, $\mathrm{c}$ can be evaluated at any $\mathrm{x}$ and in practice is evaluated at the left boundary. However, for flows with both dual- and single-flow-space parts, $c$ must be evaluated at the $x$ location where the dual-flow-space walls either begin or end. This ensures that the single grid line that corresponds to the lower and upper dual-flow-space walls remains continuous as it extends into the single-flow-space section. If the dual-flow-space walls begin and end in the interior, as in the case of a planar airfoil, then the values of $c$ must be equal at both ends of the dual-flow-space walls. This requirement means that if $y_{c}$ and $y_{w}$ are straight horizontal lines, then the airfoil must be at a zero angle of attack. If the upper boundary or wall is the arbitrary inflow/outflow option, then $y_{w}$ can be adjusted to produce an angle of attack. However, if the upper boundary or wall is a fixed solid boundary, as in the case of an airfoil in a wind tunnel, then the angle of attack of the airfoil relative to the wall is fixed. For the axisymmetric case, the airfoil becomes a duct and the angle of attack discussion deals with the duct-axial area variation. For the dual-flow-space example, Eqs. (28) and (29) remain unchanged, and Eq. (30) becomes 


$$
\omega=\frac{d \zeta}{d \bar{\zeta}} ; \quad \beta=\frac{d \eta}{d \bar{\eta}} \frac{c}{y_{L}-y_{c}} ; \quad \alpha=\frac{\beta}{c}\left[(\bar{\eta}-c) \frac{d y_{c}}{d x}-\bar{\eta} \frac{d y_{L}}{d x}\right] ; \quad \delta=0
$$

for $y_{c} \leqslant y \leqslant y_{L}$,

and

$$
\begin{aligned}
& \omega=\frac{\mathrm{d} \zeta}{\mathrm{d} \bar{\zeta}} ; \quad \beta=\frac{\mathrm{d} \eta}{\mathrm{d} \bar{\eta}} \frac{1-\mathrm{c}}{\mathrm{y}_{\mathrm{w}}-\mathrm{y}_{\mathrm{U}}} ; \quad \alpha=\frac{\beta}{1-\mathrm{c}}\left[(\bar{\eta}-1) \frac{d y_{U}}{\mathrm{dx}}-(\bar{\eta}-\mathrm{c}) \frac{\partial \mathrm{y}_{\mathrm{w}}}{\partial \mathrm{x}}\right] ; \\
& \delta=\frac{\beta(\bar{\eta}-\mathrm{c})}{1-\mathrm{c}} \frac{\partial \mathrm{y}_{\mathrm{w}}}{\partial \mathrm{t}} \text { for } \mathrm{y}_{\mathrm{U}} \leqslant \mathrm{y} \leqslant \mathrm{y}_{\mathrm{w}} .
\end{aligned}
$$

3. Transformed Governing Equations. Using Eqs. (27) and (29), the original governing equation can be written in the $\zeta, \eta, \tau$ variables. For example Eq. (1) becomes

$$
\begin{aligned}
& \frac{\partial \rho}{\partial \tau}+u \omega \frac{\partial \rho}{\partial \zeta}+\bar{v} \frac{\partial \rho}{\partial \eta}+\rho\left(\omega \frac{\partial u}{\partial \zeta}+\alpha \frac{\partial u}{\partial \eta}+\beta \frac{\partial v}{\partial \eta}+\frac{\varepsilon v}{y}\right) \\
& =\frac{\bar{\alpha}}{\rho}\left\{\left(\omega \frac{\partial}{\partial \zeta}+\alpha \frac{\partial}{\partial \eta}\right)\left[\mu_{T}\left(\omega \frac{\partial \rho}{\partial \zeta}+\alpha \frac{\partial \rho}{\partial \eta}\right)\right]+\beta \frac{\partial}{\partial \eta}\left(\mu_{T} \beta \frac{\partial \rho}{\partial \eta}\right)+\varepsilon \frac{\mu_{T} \beta}{y} \frac{\partial \rho}{\partial \eta}\right\},
\end{aligned}
$$

where

$$
\left.\begin{array}{ll}
\bar{v}-u \alpha+v \beta+\delta, & \\
y=y_{c}+\eta\left(y_{w}-y_{c}\right) & \text { for the single-liuw space }, \\
y=y_{c}+\frac{\bar{\eta}}{c}\left(y_{L}-y_{c}\right) & \text { for the lower dual-tlow space } \\
y=y_{U}+\frac{\bar{\eta}-c}{1-c}\left(y_{w}-Y_{U}\right) & \text { for the upper dual-flow space }
\end{array}\right\}
$$

and the $u$ and $v$ velocity components are the original values.

\section{E. Numerical Method}

The computational plane grid points are divided into interior and boundary points. The boundary grid points are further divided into left-boundary, right-boundary, wall, centerbody, and dual-flow-space wall points (see Fig. 1).

1. Interior Grid Points. The interior grid points are computed using the unsplit MacCormack scheme discussed in Ref. 3. This scheme is a second-order-accurate, noncentered, two-step, finite-difference scheme. Backward differences are used on the first step, forward differences on the second. The governing equations are left in nonconservation form. As an example of the basic scheme, the finite-difference equations for Eq. (2) for planar $(\varepsilon=0)$, laminar $(\bar{\alpha}=q=0)$ flow are 


$$
\begin{aligned}
& \bar{u}_{L, M}^{N+1}=u_{L, M}^{N}-\Delta t\left[u_{L, M}^{N}\left(\frac{u_{L, M}^{N}-u_{L-1, M}^{N}}{\Delta x}\right)+v_{L, M}^{N}\left(\frac{u_{L, M}^{N}-u_{L, M-1}^{N}}{\Delta y}\right)\right. \\
& \left.+\frac{1}{p_{L, M}^{N}}\left(\frac{p_{L, M}^{N}-p_{L-1, M}^{N}}{\Delta x}\right)\right] \\
& +\frac{\Delta t}{\rho_{L, M}^{N} \Delta x}\left[(\lambda+2 \mu)_{L+1 / 2, M} \cdot\left(\frac{u_{L+1, M}^{N}-u_{L, M}^{N}}{\Delta x}\right)\right. \\
& +\lambda_{L+1 / 2, M}\left(\frac{v_{L 11, M+1}^{N}+v_{L, M+1}^{N}-v_{L+1, M-1}^{N}-v_{L, M-1}^{N}}{4 \Delta y}\right) \\
& -(\lambda+2 \mu)_{L-1 / 2, M}\left(\frac{u_{L, M}^{N}-u_{L-1, M}^{N}}{\Delta x}\right) \\
& \left.-\lambda_{L-1 / 2, M}\left(\frac{v_{L, M+1}^{N}+v_{L-1, M+1}^{N}-v_{L, M-1}^{N}-v_{L-1, M-1}^{N}}{4 \Delta y}\right)\right] \\
& +\frac{\Delta t}{\rho_{L, M}^{N} \Delta y}\left[\mu_{L, M+1 / 2}\left(\frac{v_{L+1, M+1}^{N}+v_{L+1, M}^{N}-v_{L-1, M+1}^{N}-v_{L-1, M}^{N}}{4 \Delta x}\right)\right. \\
& +\mu_{L, M+1 / 2}\left(\frac{u_{L, M+1}^{N}-u_{L, M}^{N}}{\Delta y}\right) \\
& -\mu_{L, M-1 / 2}\left(\frac{v_{L+1, M}^{N}+v_{L+1, M-1}^{N}-v_{L-1, M}^{N}-v_{L-1, M-1}^{N}}{4 \Delta x}\right) \\
& \left.-\mu_{L, M-1 / 2}\left(\frac{u_{L, M}^{N}-u_{L, M-1}^{N}}{\Delta y}\right)\right] \text {, }
\end{aligned}
$$

for the first step and

$$
\begin{aligned}
& u_{L, M}^{N+1}=0.5\left\{u_{L, M}^{N}+\bar{u}_{L, M}^{N+1}-\Delta t\left[\bar{u}_{L, M}^{N+1}\left(\frac{\bar{u}_{L+1, M}^{N+1}-\bar{u}_{L, M}^{N+1}}{\Delta x}\right)\right.\right. \\
& \left.\left.+\bar{v}_{\mathrm{L}, M}^{N+1}\left(\frac{\overline{\mathbf{u}}_{L, M+1}^{N+1}-\bar{u}_{L, M}^{N+1}}{\Delta y}\right)+\frac{1}{\beta_{L, M}^{N+1}}\left(\frac{\overline{\mathbf{p}}_{L+1, M}^{N+1}-\bar{p}_{L, M}^{N+1}}{\Delta x}\right)\right]+Q\right\}
\end{aligned}
$$


for the second step, where the subscripts $L$ and $M$ denote axial and radial grid points, respectively, the superscript $\mathbf{N}$ denotes the time step, the bar denotes values calculated on the first step, and $\mathbf{Q}$ denotes the terms in the last two brackets on the right-hand side of Eq. (36), that is, the viscous terms. Equations (36) and (37) show that all viscous terms are calculated using center differences in the initial-value plane only, so that they are second-order accurate in space but first-order accurate in time. Raising them to second-order accuracy in time requires re-evaluating them using the $\overline{\mathrm{u}}^{\mathrm{N}+1}$ values from the first step. For most problems, this greater accuracy does not seem worth the increased effort.

To improve the computational efficiency for high Reynolds number flows, the grid points in the fine part of the grid may be subcycled. This is accomplished by first computing the grid points in the coarse part of the grid for one time step $\Delta t$. Next, the grid points in the fine grid are calculated $k$ times (where $k$ is an integer) with a time step $\Delta t / k$. The grid points at the edge of the fine grid require a special procedure, because one of their neighboring points is calculated as part of the coarse grid. Except for the first subcycled time step, this point is unknown. However, the values at $t$ and $t+\Delta t$ are known from the coarse grid solution, so that the values between $t$ and $t+\Delta t$ are determined by linear interpolation.

'lo improve the computational efficiency further, a special procedure (called the Quick Solver) is employed to increase the allowable time step in the subcycled part of the grid. This procedure allows the removal of the sound speed from the time-step C-F-L condition. Procedures that accomplish this have been proposed by Harlow and Amsden ${ }^{12}$ and MacCormack. ${ }^{13}$ The procedure of Harlow and Amsden removes the sound speed, in both the $\mathrm{x}$ and $\mathrm{y}$ directions, by an implicit treatment of the mass equation and the pressure gradient terms in the momentum equations. MacCormack's procedure is explicit and removes the sound speed in only one direction. (It also includes an implicit procedure to remove the viscous diffusion restriction from the time-step C-F-L condition.) Because explicit schemes are easier to program for efficient computation on vector computers and because high Reynolds number flows usually require fine grid spacing in only one direction, a procedure similar to MacCormack's was chosen.

MacCormack's procedure is based on the assumption that the velocity component, in the coordinate direction with the fine grid spacing, is negligible compared to the sound speed. This allows the governing equations to be simplified. MacCormack then applies the Method of Characteristics to these simplified equations. However, for flows over bodies with large amounts of curvature as well as many shear flows, this assumption is questionable; and because VNAP2 is intended as a general code for solving a variety of problems, MacCormack's assumption seems too restrictive. Therefore, the main differences between MacCormack's scheme and the one presented below are that this restriction is removed and that the flow in the $\mathrm{y}$ direction is assumed to be subsonic.

The sound speed limitation is associated with the inviscid part of the Navier-Stokes equations. In addition, because the following procedure is used only in the y direction, it can be illustrated by using the following inviscid, one-dimensional (1D) equations

$$
\begin{aligned}
& \frac{\partial \rho}{\partial t}+v \frac{\partial \rho}{\partial y}+\rho \frac{\partial v}{\partial y}-0, \\
& \partial v+v \frac{\partial v}{\partial y}+\frac{1}{\rho} \frac{\partial p}{\partial y}-0, \\
& \partial t
\end{aligned}
$$

and

$$
\frac{\partial p}{\partial t}+v \frac{\partial p}{\partial y}+p \mathbf{u}^{2} \frac{\partial v}{\partial y}=0
$$


where $v$ is the velocity, $\rho$ is the density, $p$ is the pressure, $a$ is the speed of sound, $y$ is distance, $t$ is time, Eq. (38) is the continuity equation, Eq. (39) is the momentum equation, and Eq. (40) is the internal energy equation written in terms of pressure using the equation of state for an ideal gas. The time step for explicit methods used to solve Eqs. (38)-(40) is the C-F-L condition and can be written as $\Delta t \leqslant \Delta y /(|v|$ $+\mathrm{a})$. However, to improve the computational efficiency in the boundary layers, where $\Delta y$ and $v$ are small but a is large, a procedure that allows $\Delta t \leqslant \Delta y /|v|$ is developed. Writing Eqs. (38)-(40) in characteristic form yields

$$
\begin{array}{ll}
\frac{d p}{d t}=a^{2} \frac{d \rho}{d t} & \text { for } \frac{d y}{d t}=v, \\
\frac{d p}{d t}+\rho a \frac{d u}{d t}=0 & \text { for } \quad \frac{d y}{d t}=v+a,
\end{array}
$$

and

$$
\frac{\mathrm{dp}}{\mathrm{dt}}-\rho \mathrm{a} \frac{\mathrm{du}}{\mathrm{dt}}=0 \text { for } \frac{\mathrm{dy}}{\mathrm{dt}}=\mathrm{v}-\mathrm{a}
$$

Therefore, Eq. (41) applies along the flow streamline and Eqs. (42) and (43) apply along Mach lines. Thus, if a time step $\Delta t \leqslant \Delta y /|v|$ is selected for some finite-difference method, the domain of dependence for Eq. (41) is included in the adjacent grid points, but the domain of dependence of Eqs. (42) and (43) is outside the adjacent grid points. This larger domain of dependence can be determined by solving for the intersection of the characteristics of Eqs. (42) and (43) with the initial-value surface. Using these intersection points allows differences to be calculated for the larger domain of dependence in much the same manner as for the adjacent grid points.

The final step is to determine which derivatives in Eqs. (38)-(40) depend on the streamline (the adjacent grid points) and which derivatives depend on the Mach lines (the characteristic initial-value surface intersection points). Following the procedure used by Kentzer ${ }^{14}$ in his boundary condition scheme and replacing the total derivatives along characteristics in Eqs. (41)-(43) with partial derivatives, while denoting the space derivatives in Eq. (42) with bars and Eq. (43) with hats give

$$
\begin{aligned}
& \frac{\partial \rho}{\partial \mathrm{t}}+\mathrm{v} \frac{\partial \rho}{\partial \mathrm{y}}-\frac{1}{\mathrm{a}^{2}}\left(\frac{\partial \mathrm{p}}{\partial \mathrm{t}}+\mathrm{v} \frac{\partial \mathrm{p}}{\partial \mathrm{y}}\right)=0, \\
& \frac{\partial \mathrm{v}}{\partial \mathrm{t}}+\frac{\mathrm{v}+\mathrm{a}}{2} \frac{\partial \overline{\mathrm{v}}}{\partial \mathrm{y}}+\frac{\mathrm{v}-\mathrm{a}}{2} \frac{\partial \hat{\mathrm{v}}}{\partial \mathrm{y}}+\frac{1}{\rho \mathrm{a}}\left(\frac{\mathrm{v}+\mathrm{a}}{2} \frac{\partial \overline{\mathrm{p}}}{\partial \mathrm{y}}-\frac{\mathrm{v}-\mathrm{a}}{2} \frac{\partial \hat{\mathrm{p}}}{\partial \mathrm{y}}\right)=0,
\end{aligned}
$$

and

$$
\frac{\partial \mathrm{p}}{\partial \mathrm{t}}+\frac{\mathrm{v}+\mathrm{a}}{2} \frac{\partial \overline{\mathrm{p}}}{\partial \mathrm{y}}+\frac{\mathrm{v}-\mathrm{a}}{2} \frac{\partial \hat{\mathbf{p}}}{\partial \mathrm{y}}+\rho \mathrm{a}\left(\frac{\mathrm{v}+\mathrm{a}}{2} \frac{\partial \overline{\mathrm{v}}}{\partial \mathrm{y}}-\frac{\mathrm{v}-\mathrm{a}}{2} \frac{\partial \hat{\mathrm{v}}}{\partial \mathrm{y}}\right)=0 .
$$

The derivatives without bars or hats are calculated by the unsplit MacCormack scheme using the adjacent grid points, with backward differences on the predictor step and forward differences on the corrector step. For the bar derivatives the following procedure is employed: first, the values of the dependent variables at the point (denoted by 1 in Fig. 3 ) where the $v+$ a Mach line intersects the initial-value surface $\mathrm{N}$ are determined by linear interpolation; then the bar derivatives, using $\mathrm{v}$ as an example, are evaluated by 


$$
\frac{\partial \bar{v}}{\partial y}=\frac{\left[C_{s} v_{M}^{N}+\left(1-C_{s}\right)\left(v_{M+1}^{N}+V_{M-1}^{N}\right) / 2\right]-v_{1}}{y_{M}-y_{1}}
$$

on the predictor step and

$$
\frac{\partial \bar{v}}{\partial y}=\frac{v_{M}^{N+1}-v_{M-1}^{N+1}}{y_{M}-y_{M-1}}
$$

on the corrector step. The hat derivatives are calculated by

$$
\frac{\partial \hat{v}}{\partial y}=\frac{v_{2}-\left[C_{s} v_{M}^{N}+\left(1-C_{s}\right)\left(v_{M+1}^{N}+v_{M-1}^{N}\right) / 2\right]}{y_{2}-y_{M}}
$$

on the predictor step and

$$
\frac{\partial \hat{\mathbf{v}}}{\partial \mathbf{y}}=\frac{\mathbf{v}_{M+1}^{N+1}-\mathbf{v}_{M}^{N+1}}{\mathbf{y}_{M+1}-\mathbf{y}_{M}}
$$

on the corrector step. The coefficient $C_{s}$ is usually set equal to U.S. If the intersectlon polnts 1 and 2 in Fig. 3 lie outside the computational grid, then reflection is used to obtain flow variables at these points from points inside the grid.

The above analysis used the 1D equations to illustrate the method. The actual equations used are derived from the $\zeta=$ constant reference-plane-characteristic scheme used at the wall boundary. The Mach line compatibility equations, without the viscous and $\zeta$ direction convection source terms, are computed on the large domain as discussed above. The streamline compatibility equation, including all source terms, is computed using the standard MacCormack scheme.

The above procedure for evaluating the terms that depend on the sound speed is only first-order accìrate in space. Using the ideas of the $\lambda$ scheme $^{15}$ could probably produce second-order accuracy. However, this was not done because the procedure is used only in boundary and shear layers where the viscous terms dominate the sound speed terms and, in addition, the $\lambda$ scheme increases the size of the domain ot dependence of the dlfference schente.

2. Left-Boundary Grid Points. The left boundary can only be an inflow boundary. For supersunic inflow, $u, v, p$, and $\rho$ are specified. The temperaluie is determinid from the equation of state. For suhsonic inflow. therc are three different boundary condition options. The first specifies the total pressure $p_{T}$, total temperature $T_{T}$, and flow angle $\theta$ as proposed by Serra. ${ }^{16}$ The second and third, which are discussed by Oliger and Sundström, ${ }^{17}$ specify either $u, v$, and $p$ or $p, v$, and $p$. For a discussion of the relative merits of these boundary conditions, see Sec. I.F. Following the ideas of Moretti and Abbett, ${ }^{18}$ all the unspecified dependent variables are computed using a second-order-accurate, reference-plane-characteristic scheme. In this scheme, the partial derivatives with respect to $\eta$ in the convective terms are computed in the initial-value and solution surfaces using noncentered differencing as in the MacCormack scheme. In the viscous terms, the partial derivatives with respect to $\eta$ are computed as in the interior point scheme and the derivatives with respect to $\zeta$ are calculated using reflection. The cross derivative viscous terms are set equal to zero. These convection and viscous term derivatives are then treated as source terms, and the resulting system of equations is solved in the $\eta=$ constant reference planes using a two-step, two-independent-variable characteristic scheme. The characteristic relation that couples the interior flow to' the boundary is derived following the procedurc of Ref. 1 and can be written as

$$
\mathrm{dp}-\rho \mathrm{adu}=\left(\Psi_{4}+\mathrm{a}^{2} \Psi_{1}-\rho a \psi_{2}\right) \mathrm{d} \tau \text { for } \quad \mathrm{d} \zeta=\omega(\mathrm{u}-\mathrm{a}) \mathrm{d} \tau,
$$


where the first equation is called the compatibility equation and the second is called the characteristic curve equation. The $\psi$ terms follow the definitions in Ref. 1. Equation (51) may be written in finite-difference form by first replacing the differentials by differences along the characterisitic curve. The coefficients are either evaluated in the initial-value plane (first step) or considered as averages of the coefficients evaluated in both the initial-value and solution planes (second step). A discussion of the unit processes and details of the schemes are given in Ref. 1 .

For the $\mathrm{p}_{\mathrm{T}}, \mathrm{T}_{\mathrm{T}}$, and $\theta$ boundary condition, the following equations that relate the stagnation or total conditions to the static conditions are required.

$$
\mathrm{p}_{\mathrm{T}} / \mathrm{p}=\left[1+(\gamma-1) \mathrm{M}^{2} / 2\right]^{\gamma /(\gamma-1)}
$$

and

$$
\mathrm{T}_{\mathrm{T}} / \mathrm{T}=1+(\gamma-1) \mathrm{M}^{2} / 2
$$

where $\gamma$ is the ratio of specific heats, $M$ is the Mach number, $T$ is the temperature, and the subscript $T$ denotes the stagnation or total conditions. The solution procedure is as follows: $M$ is assumed, $p$ and $T$ are calculated from Eqs. (52) and (53), $\rho$ is calculated from the equation of state, $u$ is calculated from Eq. (51), $v$ is calculated from the specified flow angle, a new $M$ is calculated from $u, v, p$, and $\rho$, and the process is continued until the change in $\mathrm{M}$ has converged to $10^{-3}$.

For the $u, v$, and $\rho$ boundary condition, there is only one unspecified variable, $p$, which can be calculated from Eq. (51). Likewise, the $p$, $v$, and $p$ boundary condition has one unspecified variable, $u$, which also can be determined from Eq. (51). In both cases the temperature is determined from the equation of state.

Both the $u, v$, and $\rho$ and the $p, v$, and $\rho$ boundary conditions include a nonreflecting option based on the ideas of the outflow boundary condition of Rudy and Strikwerda. ${ }^{19}$ Rudy and Strikwerda use the following equation

$$
\frac{\partial p}{\partial t}-\rho a \frac{\partial u}{\partial t}+C_{\alpha}\left(p-p_{e}\right)=0
$$

to replace the outflow boundary conditon $p=p_{e}$, where $p_{e}$ is the exit pressure and $C_{\alpha}$ is a constant. The first two terms of Eq. (54) can be interpreted as the 1D compatibility equation on the incoming characteristic (where this characteristic is parallel to the boundary) and the last term is included to asymptotically enforce the specification of the exit pressure. Forcing the incoming characteristic to be parallel to the boundary as if the outflow were sonic removes normal reflections back into the interior. This interpretation of Rudy and Strikwerda's outflow boundary condition allows formulation of a similar procedure for inflow boundaries. Therefore, for the inflow case, using the $\mathrm{p}, \mathrm{v}$, and $\rho$ boundary condition, Eq. (54) becomes

$$
\frac{\partial p}{\partial t}+\rho a \frac{\partial u}{\partial t}+C_{\alpha}\left(p-p_{\downarrow}\right)=0
$$

where $p_{1}$ is the specified inflow pressure. For the $u, v$, and $p$ boundary conditions, Eq. (54) becomes

$$
\frac{\partial \mathrm{u}}{\partial \mathrm{t}}+\frac{1}{\rho \mathrm{a}} \frac{\partial \mathrm{p}}{\partial \mathrm{t}}+\mathrm{C}_{\alpha}\left(\mathrm{u}-\mathrm{u}_{\mathrm{v}}\right)=0
$$

where $u_{1}$ is the specified inflow velocity. Equation (55) or (56) is solved with Eq. (51) to determine $u$ and $\mathrm{p}$ at the inflow boundary. 
For mixed supersonic/subsonic inflow, VNAP2 uses the supersonic boundary condition at grid points where the flow is supersonic and either the $u, v$, and $\rho$ or the $p, v$, and $\rho$ boundary conditions (but not the $\mathrm{p}_{\mathrm{T}}, \mathrm{T}_{\mathrm{T}}$, and $\theta$ boundary conditions) at the subsonic points. VNAP2 allows using the supersonic boundary condition everywhere as an option.

The turbulence model boundary conditions are the specification of $q$ for the one-equation model and $q$ and $e$ for the Jones-Launder two-equation model. The specified values of $q$ and $e$ can be determined following a procedure similar to that of Ref. 4. The value of $q$ is calculated from

$$
\mathrm{q}=\frac{\mu_{\mathrm{T}}|\partial \mathrm{u} / \partial \mathrm{y}|}{0.3 \rho}
$$

where $|\partial u / \partial y|$ and $\rho$ can be determined from the inflow velocity profile and $\mu_{T}$ can be determined by the mixing-length model. The value of $\mathrm{e}$ for the two-equation model can be calculated from Eq. (21). For large $\mathbf{R}_{\mathrm{T}}$, Eq. (21) reduces to

$$
\mu_{\mathrm{T}}=\mathrm{C}_{\mu} \rho \mathrm{q}^{2} / \mathrm{e} \text {, }
$$

which can be easily solved for e, and for small $R_{T}$ a trial and error solution can be used. For some flows this procedure produces values of $q$ that are much lower than the evolved value at the first downstream grid point. However, increasing $q$ to agree with the first downstream grid point value, while adjusting e to keep $\mu_{\mathrm{T}}$ constant, produces little change in the solution. If the $\mathrm{p}_{\mathrm{T}}, \mathrm{T}_{\mathrm{T}}$, and $\theta$ inflow boundary condition is used then a short run can be made, using the mixing-length model, to determine an inflow velocity profile. If the inflow profile is a uniform flow profile, that is, no shearing flow is present, then the inflow values of $q$ and $e$ can be set to some small values so that $\mu_{\mathrm{T}}$ is negligible when compared to the molecular value.

3. Right-Boundary Grid Points. The right boundary can be a supersonic outflow boundary or a subsonic inflow/outflow boundary. This subsonic inflow option is required for internal flows with flow separation at the right boundary. For supersonic outflow, the flow variables are extrapolated. For subsonic outflow, the exit pressure is specified and the remaining variables are calculated using a characteristic scheme similar to the left-boundary scheme. 'The characteristic relations that couple the interior flow to the boundary are derived following the procedure of Ref. 1 and can be written as

$$
\left.\begin{array}{l}
\mathrm{dp}-\mathrm{a}^{2} \mathrm{~d} \rho=\psi_{4} \mathrm{~d} \tau \\
\mathrm{dv}=\mu_{9} \mathrm{~d} d \tau
\end{array}\right\} \quad \text { for } \mathrm{d} \zeta=\omega \mathrm{ud} \tau
$$

and

$$
d p+\rho a d u=\left(\psi_{4}+a^{2} \psi_{1}+\rho a \Psi_{2}\right) d t \quad \text { for } \quad d \zeta=\omega(u+a) d \tau
$$

These equations are written in finite-difference form like those for the left-boundary scheme. The pressure is specified, and the u velocity component is then calculated from Eq. (59); the density from Eq. (57); the $\mathrm{v}$ velocity component from Eq. (58); and the temperature from the equation of state. If subsontic reverse flow (inflow) occurs at the right boundary, inflow boundary conditions must be specified. This is accomplished by leaving $p$ equal to the specified exit pressure, setting $\rho$ equal to the value at the boundary where separation occurred, and setting the flow angle equal to the value obtained by linear interpolation 
between the boundaries. The $\rho$ and $v$ boundary conditions used here are arbitrary and can be changed by modifying subroutine EXITT (see Sec. II.A).

The code includes the nonreflecting outflow boundary condition of Rudy and Strikwerda. ${ }^{19}$ Here, $u$ and $\mathrm{p}$ are calculated from Eqs. (54) and (59); the density from Eq. (57); v from Eq. (58); and T from the equation of state. This nonreflection option is also used when reverse flow occurs.

For mixed supersonic/subsonic outflow, VNAP2 uses the supersonic boundary condition at grid points where the flow is supersonic and the subsonic boundary condition at subsonic points. VNAP2 allows using either the supersonic or subsonic boundary conditions everywhere as an option.

The turbulence model boundary conditions are the extrapolation of $q$ for the one-equation model and $q$ and $\mathrm{e}$ for the Jones Launder two-equation model.

4. Wall Grid Points. The wall boundary can be a free-slip boundary, a free-jet boundary, a no-slip boundary, or a constant pressure inflow/outflow boundary. The constant pressure inflow/outflow boundary is required for external flows.

a. Free-Slip Boundary. For a free-slip boundary, a reference-plane-characteristic scheme is used. Partial derivatives with respect to $\zeta$ in the convective terms are computed in the initial-value and solution surfaces using noncentered differencing as in the MacCormack scheme. All derivatives in the viscous terms arc computed in the initial-value surface only, using centered differencing. The $\eta$ and cross derivatives in the viscous terms are calculated by either reflecting or extrapolating a row of fictitious mesh points outside the flow boundary. These convection and viscous term derivatives are then treated as source terms, and the resulting system of equations is solved in the $\zeta=$ constant reference planes using a two-step, two-independent-variable characteristic scheme.

The characteristic relations that couple the interior flow to the boundary are derived following the procedure of Ref. 1 and can be written as

$$
\left.\begin{array}{l}
\beta d u-\alpha d v=\left(\beta \psi_{2}-\alpha \psi_{3}\right) d \tau \\
d p-a^{2} d \rho=\psi_{4} d \tau
\end{array}\right\} \text { for } d \eta=\bar{v} d \tau
$$

and

$$
\begin{aligned}
& \mathrm{dp}+\rho \alpha \mathrm{a} d u / \alpha^{*}+\rho \beta \mathrm{adv} / \alpha^{*}=\left(\psi_{4}+\mathrm{a}^{2} \psi_{1}+\rho \alpha a \psi_{2} / \alpha^{*}+\rho \beta a \psi_{3} / \alpha^{*}\right) \mathrm{d} \tau \\
& \text { for } \mathrm{d} \eta=\left(\overline{\mathrm{v}}+\alpha^{*} \mathrm{a}\right) \mathrm{d} \tau,
\end{aligned}
$$

where

$$
\alpha^{*}=\left(\alpha^{2}+\beta^{2}\right)^{1 / 2}
$$

These equations are written in finite-difference form like those for the left-boundary scheme.

The boundary condition is that the flow is tangent to the boundary. This can be written as

$$
v=u \tan \theta+\partial y_{w} / \partial t
$$

where $\theta$ is the local boundary angle. The time derivative is present because, in the free-jet option, the wall boundary coordinates are a function of time. Equation (63) is substituted into Eq. (60), and the resulting 
equation is solved for the velocity component $u$. Then the v velocity component is obtained from Eq. (63); the pressure from Eq. (62); the density from Eq. (61); and the temperature from the equation of state.

The turbulence model boundary conditions are the extrapolation of $q$ for the one-equation model and $q$ and $\mathrm{e}$ for the Jones-Launder two-equation model.

This code has an option to improve the accuracy of the calculation of one sharp expansion corner on the wall contour. The flow at this corner must be supersonic and the boundary condition option must be the free-slip boundary with no free jet. The grid point is treated by a special procedure. First, an upstream solution is computed at the corner grid point, using the upstream flow tangency condition as the boundary condition and backward $\zeta$ differences in both initial-value and solution planes. Next, a downstream solution is calculated, using the Prandtl-Meyer exact solution and the stagnation conditions from the upstream grid point. The upstream solution is used when computing wall grid points upstream of the corner grid point as well as the adjacent interior grid point; the downstream solution is used when computing downstream wall grid points.

b. Free-Jet Boundary. The free-jet boundary grid points are computed by the wall routine so that the pressure equals the specified pressure. 'l'his is accomplished by first assuming the shape of the jet boundary and then using the wall routine to calculate the pressure. Next, the jet boundary location is changed slightly and a second pressure is computed. The secant method determines a new jet boundary location. This procedure is then repeated at each grid point until the jet boundary pressure and the ambient pressure agree within some specified tolerance.

When a free-jet calculation is made, the wall exit lip grid point becomes a singularity, so it is treated by a special procedure. First, an upstream solution is computed at the exit grid point, using the flow tangency condition as the boundary condition and backward $\zeta$ differences in both the initial-value and solution planes. Next, a downstream solution is calculated, using the specified pressure as the boundary condition and the stagnation conditions calculated from the upstream grid point. The upstream solution is used in computing wall grid points upstream of the exit grid point and the downstream solution in computing downstream free-jet grid points. A third exit grid point solution for interior grid point calculation is determined as follows. When the upstream solution is subsonic, the two solution Mach numbers are averaged to be less than or equal to one. This Mach number, along with the upstream stagnation temperature and pressure, is then used to calculate the exit grid point solution for computing the interior grid points. When the upstream solution is supersonic, it is used to calculate the interior grid points.

c. No-Slip Boundary. Unlike the VNAP code, VNAP2 uses the characteristic scheme to enforce the no-slip boundary condition. The boundary condition is the vanishing of the velocity components and either the vanishing of the temperature gradient normal to the boundary (adiabatic wall) or the specification of the temperature. The pressure is calculated from Eq. (62) with the normal temperature gradient set equal to zero and the density from Eq. (61). If the vanishing of the normal temperature gradient option is desired, then the temperature can be determined from the equation of state. If the specified wall temperature option is desired, then the pressure is recomputed from the equation of state.

The boundary conditions for the turbulence models are the vanishing of $q$ for the one-equation model and the vanishing of $q$ and the specification of $\mathrm{e}$ so that $\partial \mathrm{e} / \partial \mathrm{y}=0$ for the Jones-Launder two-equation model.

d. Constant Pressure Inflow/Outflow Boundary. The constant pressure inflow/outfluw boundary grid points are also calculated using the characteristic scheme. The pressure is always specified. If the flow across the boundary is outflow, then $\mathrm{u}$ and $\mathrm{v}$ are calculated from Eqs. (60) and (62), and $p$ is calculated from Eq: (61). For inflow, $u$ and $\rho$ are specified and $v$ is calculated from Eq. (62). The actual values of $u$ and $\rho$ specified are the values at the grid point where the left boundary intersects the wall. The 
temperature is determined from the equation of state. A nonreflecting boundary condition option, similar to that used at the right boundary, is employed here.

The turbulence model boundary conditions are the extrapolation of $q$ for the one-equation model and $q$ and $\mathrm{e}$ for the Jones-Launder two-equation model.

5. Centerbody Grid Points. The centerbody boundary can be a free-slip boundary, a no-slip boundary, or a plane (axis) of symmetry. The free-slip and no-slip boundary calculations follow the wall procedure. The characteristic relation that couples the interior flow to the boundary is derived following. the procedure of Ref. 1 and can be written as

$$
\begin{aligned}
& \mathrm{dp}-\rho \alpha \mathrm{adu} / \alpha^{*}-\rho \beta \mathrm{adv} / \alpha^{*}=\left(\psi_{4}+\mathrm{a}^{2} \psi_{1}-\rho \alpha \mathrm{a} \psi_{2} / \alpha^{*}-\rho \beta \mathrm{a} \psi_{3} / \alpha^{*}\right) \mathrm{d} \tau \\
& \text { for } \mathrm{d} \eta=\left(\bar{v}-\alpha^{*} \mathrm{a}\right) \mathrm{d} \tau .
\end{aligned}
$$

Equation (63) becomes

$$
\mathrm{v}=\mathrm{u} \tan \theta \text {. }
$$

The time derivative in Eq. (63) does not appear in Eq. (65) because the centerbody coordinates are not a function of time.

For flows where the centerbody is a plane (axis) of symmetry, the centerbody grid points are computed by the interior point scheme. The boundary condition is flow symmetry.

The turbulence model boundary conditions are the same as the wall boundary for the free-slip and no-slip cases. For the plane (axis) of symmetry case, $q$ and e are specified so that $\partial \mathrm{q} / \partial \mathrm{y}=\partial \mathrm{e} / \partial \mathrm{y}=0$.

6. Dual-Flow-Space Wall Grid Points. The dual-flow-space walls can be either a free-slip or a no-slip boundary. The calculations follow the wall and centerbody procedures. The centerbody equations are used for the upper dual-flow space, and the wall equations, with Eq. (65) replacing Eq. (63), are used for the lower dual-flow-space wall. The turbulence model boundary conditions are the same as the wall and centerbody boundaries.

7. Step Size. The step size $\Delta t$ is determined by

$$
\Delta t=\min \left(\Delta t_{x}, \Delta t_{y}\right)
$$

where

$$
\Delta \mathrm{t}_{\mathrm{x}}=\mathrm{A} /\left[(|\mathrm{u}|+\mathrm{a}) / \Delta \mathrm{x}+\mu / \mathrm{A}_{1} \rho \Delta \mathrm{x}^{2}\right]
$$

and

$$
\Delta \mathrm{t}_{\mathrm{y}}=\mathrm{A} /\left[(|\mathrm{v}|+\mathrm{a}) / \Delta \mathrm{y}+\mu / \mathrm{A}_{1} \rho \Delta \mathrm{y}^{2}\right]
$$

where $A$ and $A_{1}$ are constants that usually equal 0.9 and 0.25 , respectively. For the Quick Solver option, Eq. (68) becomes

$$
\Delta \mathrm{t}_{\mathrm{y}}=\mathrm{A} /\left(|\mathrm{v}| / \Delta \mathrm{y}+\mu / \mathrm{A}_{1} \rho \Delta \mathrm{y}^{2}\right)
$$

These conditions are checked at each grid point in the flow field at each time step. However, these conditions are not checked on the subcycled time steps.

\section{F. Comments on the Calculation of Steady, Subsonic Flows}

Because signals propagate in all directions in subsonic flows, disturbances can reflect inside the computational grid for many time steps and can significantly prolong the convergence to steady state. 
However, in supersonic flows, signals only propagate downstream and are, therefore, swept out of the grid. As a result, supersonic flows generally converge to steady state in fewer time steps than subsonic flows. As an example, consider the following two inviscid accelerating flows: planar subsonic sink flow and planar supersonic source flow. The comptational regions for the subsonic sink and supersonic source flows are enclosed by the dashed lines in Figs. 4 and 5, respectively. The top dashed line is treated as a free-slip wall, the bottom dashed line is the flow midplane, and the left and right dashed lines are inflow and outflow boundaries, respectively. The outflow midplane Mach number for the subsonic case is 0.5 , and the inflow midplane Mach number for the supersonic case is 1.5. The boundary conditions for the subsonic flow are the specification of $p_{T}, T_{T}$, and $\theta$ at the inlet and $p$ at the exit. For the supersonic flow, all inlet variables are specified and all outlet variables are extrapolated. The initial-data surface for both flows is the 1D solution generated by the VNAP2 code. Figure 6 shows the pressure vs number of time steps for both flows. The top curve for both flows gives the solution at an interior grid point near the inflow boundary, and the lower line is a grid point near the outflow boundary. The supersonic flow reaches steady state in around 150 time steps, whereas the subsonic case requires approximately 1200 . For very complex flows, this difference is often greater. Therefore, the following discussion will be concerned with improving the convergence to steady state of subsonic flows.

Figure 7 shows the pressure vs number of time steps for the subsonic sink flow employing different techniques to accelerate the convergence to steady state. Again, the $p_{T}, T_{T}$, and $\theta$ inflow boundary condition is used. The grid point plotted in Fig. 7 is the one near the inlet in Fig. 6. The top curve is for a calculation that started from an initial-data surface consisting of a stationary flow at the stagnation pressure and temperature. At time equal to zero, the pressure at the outflow boundary was dropped from the stagnation value to the sink flow exact solution, thus simulating a bursting diaphragm. The other four calculations started with an initial-data surface generated by the VNAP2 code, which is the 1D solution. The third line from the top shows the solution using the Rudy and Strikwerda ${ }^{19}$ nonreflecting outflow boundary condition. The coefficient $\mathrm{C}_{\alpha}$ (ALE in Namelist BC) in Eq. (54) equals 0.1. (Namelists are given in Sec. II.C.) The fourth curve from the top shows the solution for which all the dependent variables were smoothed in space for the first 500 time steps. This calculation multiplies the value at a grid point by a weighting parameter and adds it to the average of the values of its nearest neighboring grid points multiplied by one minus the weighting parameter. The weighting parameter was 0.5 for the first time step and linearly increased to 1.0 (no smoothing) by the 500th time step (SMP $=0.5, \mathrm{SMPF}=1.0$, and NST $=500$ in Namelist AVL). The bottom curve used the extended-interval time-smoothing option, which stores the solution for all dependent variables on the first time step and then monitors the pressure at a specified grid point on each time step. When this pressure changes direction, the solution at the current time step is averaged with the solution at the first time step. This averaged solution replaces the current time-step solution and, in addition, is stored in place of the first time-step solution. This process is continued for the entire computation (SMPT $=0.5, \mathrm{SMPTF}=0.5, \mathrm{NTST}=0$, and NST $=$ NMAX in Namelist AVL). The diaphragm initiai-data surface solution requires around 1800 time steps to reach steady state, whereas the 1D initial-data surface solution is steady in approximately 1100 times. The nonreflecting and space-smoothing options further increase the convergence to steady state. However, the largest increase is due to the time smoothing, which results in a converged solution in about 400 time stcps. The incrcased convergence rate of the time-smoothed solution over the other options is more pronounced for more complex flows.

Figure 8 shows the pressure vs the number of time steps for the $u, v$, and $\rho$ inflow boundary condition. The diaphragm initial-data surface solution produced results similar to the $1 \mathrm{D}$ curve and, therefore, is not shown. The top three curves correspond to the same options in Fig. 7. The bottom curve is the solution using the nonreflecting inflow option discussed in Sec. I.E.2. The coefficient $C_{a}$ (ALI in Namelist BC) in Eq. (56) equals 0.1 . The top curve of Fig. 8 shows that the $u, v$, and $\rho$ boundary condition trapped the 
initial disturbances in the computational grid. The Rudy and Strikwerda ${ }^{19}$ nonreflecting outflow boundary condition option (not shown) did not significantly improve this result. Note that the Rudy and Strikwerda boundary condition is used in conjunction with the reference-plane-characteristic scheme, which is somewhat different from the numerical procedure they used. Their procedure may produce different results. As Fig. 8 shows, the space- and time-smoothing options, as well as the nonreflecting inflow boundary condition option, all producc steady solutions.

The ID solution, which is used as the initial-data surface, has an outflow Mach number of 0.55 . The sink flow exact solution has midplane and upper wall outflow Mach numbers of 0.5 and 0.42 , respectively. The high 1D solution Mach number was chosen so that the $1 \mathrm{D}$ solution would not approximate the 2D sink flow solution too closely. However, this high Mach number produces a $12 \%$ difference in mass flow between the $1 \mathrm{D}$ solution and the 2D sink flow solution. Because the $u, v$, and $\rho$ inflow boundary condition specifies the 2D sink flow solution mass flow, an expansion wave is produced at the inlet. This expansion wave causes the large drop in pressure, shown in Fig. 8, during the early stages of the calculation. Adjusting the Mach number of the $1 \mathrm{D}$ solution so that the $1 \mathrm{D}$ mass flow closely approximates the mass flow specified by the $u, v$, and $\rho$ boundary condition yields the results shown in Fig. 9 where, except for the top curve, the convergence to steady state is greatly improved.

From the above and other similar results, some general conclusions can be drawn. First of all, for steady, subsonic flows the $p_{T}, T_{T}$, and $\theta$ inflow boundary condition is preferred over the $u, v$, and $\rho$ boundary condition. For subsonic computations that require long run times, the extended-interval time smoothing can significantly reduce computational time. For subsonic/supersonic nozzle flows, the $\mathrm{p}_{\mathbf{T}}, \mathrm{T}_{\mathbf{T}}$, and $\theta$ inflow boundary condition is also preferred, because the mass flow is usually not known in advance. If the $u, v$, and $\rho$ boundary condition is used for steady, subsonic flows, then either the nonreflecting inflow option of space or extended-interval time smoothing should be used. The $u, v$, and $\rho$ inflow boundary condition is useful for unsteady subsonic flows where the user wishes to specify the mass flow. VNAP2 allows only constant values of $u, v$, and $\rho$ to be specified; however, the code could easily be modified to allow time-dependent functions for $u, v$, and $\rho$. The $u, v$, and $\rho$ inflow boundary condition also works well for the subsonic part of the boundary layer in a supersonic flow. In many cases, this subsonic part of the boundary can be treated with supersonic boundary conditions. However, where this practice gives poor results, the $u, v$, and $\rho$ boundary condition is an improvement. The test cases run to date indicate that the $u, v$, and $\rho$ boundary condition produces results more consistent with the supersonic part of the flow than does the $\mathrm{p}_{\mathrm{T}}, \mathrm{T}_{\mathrm{T}}$, and $\theta$ boundary condition. As a result, VNAP2 allows only the $u, v$, and $p$ option at subsonic parts of a mixed subsonic/supersonic inflow.

The $p, v$, and $p$ boundary condition has received little use to date because, in general, it should be used with either the $u$ specified subsonic outflow boundary condition or supersonic outflow. When $p$ is specified as the subsonic outflow boundary condition, some flows are not uniquely defined. For example, if $\mathrm{p}, \mathrm{v}$, and $\rho$ are specitied at the intlow and $\mathrm{p}$ is specified at the outflow for inviscid subsonic flow in a constant area duct, the Mach number would not be uniquely specified. The specified u outflow boundary condition is not incorporated (as originally intended) because there is little use for it. The $p, v$, and $\rho$ boundary condition can be used for subsonic/supersonic nozzle flows because it does not specify the mass flow; however, the $\mathrm{p}_{\mathrm{T}}, \mathrm{T}_{\mathrm{T}}$, and $\theta$ boundary condition is preferred.

In general, the closer the initial-data surface is to the final solution, the faster the solution converges to the steady state. This is also true for viscous flows, where using initial data that approximate all boundary and free-shear layers generally reduces the run time.

Finally, Moretti and I disagre $\mathrm{e}^{20-22}$ on the $u, v$, and $\rho$ subsonic inflow boundary condition. Moretti feels that the $u, v$, and $\rho$ boundary condition is incorrect for a well-posed problem, because disturbances reflected by this boundary condition may remain trapped in the finite-difference grid. Reference 22 lists several published proofs of the correctncss of this boundary condition. As a result of these proofs, I feel that this boundary condition is mathematically correct for a well-posed problem and that the trapping of disturbances is a numerical problem that can be overcome. In addition to these mathematical proofs, the $u, v$, 
and $\rho$ boundary condition satisfies the characteristic compatibility conditions, as does the $p_{T}, T_{T}$, and $\theta$ boundary condition. Both boundary conditions falsify the time-dependent flow by holding quantities fixed that actually vary in time ( $\mathrm{p}_{\mathrm{T}}$ and $\mathrm{T}_{\mathrm{T}}$ are constant only for steady flow). As a result, both cause nonphysical reflections at subsonic boundaries. The $u, v$, and $\rho$ boundary condition causes a reflection that has approximately the same amplitude, whereas the $\mathrm{p}_{\mathrm{T}}, \mathrm{T}_{\mathrm{T}}$, and $\theta$ boundary condition produces a highly damped reflection. These reflection properties differ because they model different upstream conditions-constant mass flow as opposed to constant total pressure-which makes them suitable for different problems. In Ref. 23, Moretti seems to imply that the $u, v$, and $\rho$ boundary condition requires knowledge of the exact solution. Although I specified the exact solution in Ref. 20, as did Moretti. in Ref. 23, the exact solution values of $u, v$, and $\rho$ or $p_{T}, T_{T}$, and $\theta$ are generally not known in advance. (For the special case of inviscid, steady flow, $\mathrm{p}_{\mathrm{T}}$ and $\mathrm{T}_{\mathrm{T}}$, but not $\theta$, are usually known.) Therefore, one specifies his best guess boundary values. The computed solution will satisfy these boundary values as well as the governing equations, and its accuracy will depend on how well these boundary values were estimated. Therefore, I feel that both boundary conditions are correct and that the best choice is problem-dependent.

A second point that concerns this section is Moretti's claim ${ }^{23}$ that the initial-data surface and boundary conditions must be matched so that the transient part of a steady state calculation follows the true transient solution. Although this is the most correct way to formulate problems, it is generally not the most economical. It is true that there are flows where following the true transient solution is very desirable. One such case is the startup of a supersonic wind tunnel. If, for example, the area of the throat downstream of the test section is not large enough to pass the startup shock, then the shock will stand in the test section or nozzle. Beginning a time-dependent calculation of this flow with a purely supersonic initial-data surface will produce the started, all supersonic, steady solution, even though this solution is physically impossible. Beginning this calculation with a 1D subsonic initial-data surface would yield the right solution. However, use of Morelli's recommendation ${ }^{23}$ of the diaphragm initial-data surface, discussed above, provides the right solution without requiring any knowledge of the starting of a supersonic wind tunnel. Thus, there are flows where either hysteresis effects or lack of understanding suggest following Moretti's recommendation. However, for steady, subsonic flows this recommendation can be very expensive. In addition, I have never found a subsonic flow calculation using a time-dependent method where the steady solution depended on the initial-data surface (except for small differences from truncation errors and provided the initial-data surface is subsonic). As a result, I feel that the special procedures discussed above for accelerating the convergence of subsonic flows to their steady state may be used to reduce these lengthy computational times. I have included these last two paragraphs to warn the users of VNAP2 that some of the ideas expressed above are my own and may not be universally accepted as correct procedures.

\section{G. Results and Discussion}

Presented here are three relatively simple flows that are intended to illustrate the three general classes of flows that can be computed with VNAP2: internal, external, and internal/external flows. The data files for these three cases are included at the back of the Fortran listing of the VNAP2 code in the Appendix. The initial-data surfaces for the external and internal/external cases assume solution array sizes of 41 by 25. For the application of VNAP2 to more complex flows, see Ref. 24.

1. Internal, Inviscid Flow. The first case is steady, subsonic/supersonic, inviscid flow in the $45-15^{\circ}$ conical, converging-diverging nozzle shown in Fig. 10 with the flow from left to right. This calculation is also presented in Refs. 1, 2, and 25. The upper boundary is a free-slip wall and the lower boundary is the centerline. The left boundary is a subsonic inflow boundary using the $p_{T}, T_{T}$, and $\theta$ boundary condition. 
The right boundary is a supersonic outflow boundary and, therefore, the variables are extrapolated. The Mach number contours and wall pressure ratio are shown in Fig. 11. The experimental data are those of Cuffel et al. ${ }^{26}$ The computed discharge coefficient is 0.983 , compared with the experimental value of 0.985 . The 21 by 8 uniform computational grid requires 299 time planes and a computation time of $35 \mathrm{~s}$ on the CDC 6600 and $6 \mathrm{~s}$ on the CDC 7600.

Although the Mach number, wall pressure, and throat mass flow results are in good agreement with experiment, the mass flow variation at different axial locations is fairly poor. For example, the mass flow variation between the inlet and throat is $4.5 \%$. If the grid spacing is halved by using a 41 by 15 uniform grid, the mass flow variation between the inlet and throat is $1.4 \%$. Halving the grid spacing again, by using an 81 by 29 uniform grid, produces a mass flow variation between the inlet and throat of $0.1 \%$. Therefore, the mass flow variation appears to go to zero as the grid spacing goes to zero. Some of the error in the coarse grid case may be due to the trapezoidal rule used to evaluate the mass flow integral. However, much of the error is probably due to the large truncation error of the finite-difference equations, owing to the steep gradients in the nozzle throat region. The variation in throat mass flow between the 81 by 29 and 21 by 8 grid cases is $0.25 \%$, whereas between the 81 by 29 and 41 by 15 grid cases it is $0.06 \%$. Therefore, the throat mass flow is fairly good for coarse grid spacings even though the overall mass flow conservation is fairly poor.

This case uses the convergence tolerance option to determine when the steady state has been reached. That is, when the relative change in axial velocity in the throat and downstream regions is less than $0.003 \%$, the flow is assumed to have reached steady state. In general, I have not found this convergence tolerance option to be very useful, because the value of the convergence tolerance depends on the grid spacing and flow conditions and as such is usually not known in advance. One exception to this is the case involving a large parametric study. Here, once the convergence tolerance has been determined by trial and error, it can be used repeatedly in the remaining runs of the parametric study. However, a procedure based on the time of flight of an average fluid particle seems to work more consistently. In this procedure, one sets the total number of time steps so that an average fluid particle will travel through the computational grid a particular number of times. The velocity of an average fluid particle can be estimated from the 1D solution or some other initial-data surface. This average velocity can also be estimated from the numerical solution itself by running the program for a fairly short time and using that solution to estimate the average fluid particle velocity. Use of the restart option allows this run to be contiued to steady state. The time step can be obtained by running the code for one time step (two for viscous flows). Once the average fluid particle velocity and time step have been determined, then the number of time steps required for one trip can be calculated. The last piece of required information is the number of trips made by the average fluid particle through the grid to reach steady state. For supersonic, inviscid flows, three trips are usually sufficient, whereas supersonic, viscous flows require around five. Converging-diverging, supersonic, inviscid nozzle flows usually require around five trips, whereas viscous nozzle flows need around seven. The numbers of trips given above are only rough estimates and should be supplemented by the user's own experiences. In addition, when in doubt as to how many time steps are necessary, always use the restart option.

Finally, for subsonic flows, neither the convergence tolerance nor the time of flight procedure is really effective. The most effective method that $I$ have found is to monitor the static pressure at several spots in the flow (see LPP1, MPP1 in Namelist CNTRL). Provided that an average fluid particle has made at least one trip, then the flow can be assumed to be steady when the pressure is oscillating with an acceptable amplitude about a constant value. Looking at only the amplitude of the oscillation, without regard to whether it occurs about a constant value, is sometimes not sufficient.

2. External, Turbulent Flow. The second case is steady, subsonic, turbulent flow over a boattail afterbody with a solid body simulating the jet exhaust. The geometry is shown in Fig. 12, with the dashed line enclosing the computational region, and the flow is from left to right. This calculation is also 
presented in Ref. 24. The upper boundary is a constant pressure inflow/outflow boundary and the lower boundary is a no-slip wall. The left boundary is a subsonic inflow boundary using the $\mathrm{p}_{\mathrm{T}}, \mathrm{T}_{\mathrm{T}}$, and $\theta$ boundary condition. The values of $\mathrm{p}_{\mathrm{T}}$ and $\mathrm{T}_{\mathrm{T}}$ are determined from an inviscid/boundary-layer solution procedure for the forebody. The right boundary is a subsonic outflow boundary and, therefore, the static pressure is specified. The free-stream Mach number is 0.8 and the Reynolds number, based on the length at the inflow boundary, is $10.5 \times 10^{6}$. For more details on the geometry or experimental data, see Ref. 27 . The turbulence is modeled using the mixing-length model. This calculation employed the subcycling, Quick Solver, and extended-interval time-smoothing options. Figure 13 shows the physical space grid, pressure, and Mach number contours. Figure 14 shows the surface pressure coefficient on the boattail and jet exhaust simulator. Figures 13 and 14 show that the boundary layer remains attached. For cases with separation and exhaust jets, see Ref. 24 . This calculation employs a 40 by 25 variable grid that requires 750 time steps (15 000 subcycled time steps in the boundary layer) and a computation time of 1 $\mathrm{h}$ on the CDC 7600 . Swanson ${ }^{28}$ compared several different formulations of the mixing-length model for computing this case as well as separated cases.

3. Internal/External, Turbulent Flow. The third casc is stcady, subsonic, turbulent flow for a plane jet in a uniform stream. The geometry is shown in Fig. 15 with the dashed line enclosing the computational region, and the flow is from left to right. The upper boundary is a constant pressure inflow/outflow boundary and the lower boundary is the midplane. The dual-flow-space boundaries are no-slip walls. The left boundary is a subsonic inflow boundary using the $u$, $v$, and $\rho$ boundary condition, with the nonreflecting option. The right boundary is a subsonic outflow boundary and, therefore, the static pressure is specified. The jet and external stream have initial Mach numbers of 0.14 and 0.02 , respectively, while the Reynolds number, based on the jet height, is $3.0 \times 10^{4}$. The turbulence is modeled using the mixing-length and Jones-Launder two-equation models. This case, assuming free-slip inflow profiles and a solid free-slip upper boundary and employing the mixing-length turbulence model, was presented in Ref. 1. The physical space grid and Mach number contours for the mixing-length model are shown in Fig. 16. Figure 17 shows the midplane velocity decay for both turbulence models. The subscript $\mathrm{JE}$ denotes the midplane velocity just downstream of the end of the dual-flow-space walls. The increase in the velocity is due to the acceleration of the mean flow caused by the growth of the boundary layer. The experimental data are from Ref. 29. This calculation employs a 41 by 17 variable grid that requires 6000 time steps and a computation time of 24 min (mixing-length model) on the CDC 7600.

This rather lengthy run time, even though a fairly coarse grid spacing was used, is because the flow is almost incompressible. That is, the flow velocity is much smaller than the sound speed. The explicit numerical scheme is limited to time steps so that sound waves travel less than one mesh spacing. (The problem geometry did not allow the use of the Quick Solver option, although some reduction in run time could be made using the subcycle option.) Therefore, many time steps are required before a particle of fluid travels from the inflow to the outflow boundary.

\section{DESCRIPTION AND USE OF THE VNAP2 PROGRAM}

\section{A. Subroutine Description}

The computer program consists of 1 program, 1 function, and 18 subroutines. A complete Fortran listing of the VNAP2 program is included in the Appendix.

1. Program VNAP2. Program VNAP2 initiates a run by reading in the input data. Next, the program title, abstract, and input data descriptions are printed. The program then calls subroutines GE $\emptyset \mathrm{M}, \mathrm{GE} \emptyset \mathrm{MCB}$, and GE $\emptyset \mathrm{MLU}$ to calculate the geometry. If requested, program VNAP2 calls 
subroutine $\emptyset$ NEDIM to calculate the $1 D$, initial-value surface. Program VNAP2 then prints the initial-value surface, which includes a mass flow and momentum thrust calculation made by subroutine MASFL $\emptyset$. Next, subroutine PL $\emptyset$ T is called to plot the data on film. The final part of VNAP2 consists of the time-step loop, which calculates the next time-step size; calls subroutine VISC $\emptyset$ US to calculate the artificial, molecular, and turbulent viscosity-heat conduction terms; calls subroutine QS $\varnothing \mathrm{LVE}$ to calculate the special derivatives used by the Quick Solver package; calls subroutine INTER to compute the interior mesh points; calls subroutine WALL to compute the wall, centerbody, and dual-flow-space wall mesh points; calls subroutine INLET to compute the inlet mesh points; calls subroutine EXITT to compute the exit mesh points; calls subroutine TURBC to set the boundary conditions for the turbulence variables; if requested, calls subroutine SM $\varnothing \varnothing \mathrm{TH}$ to smooth the solution; calls subroutine MASFL $\emptyset$ to compute the mass flow and momentum thrust; prints the solution surface; calls subroutine PLØT to plot the data on film; checks the solution for its convergence to the steady-state solution; and punches (writes) the last solution plane on cards (disc or tape) for restart.

2. Subroutine GEØM. Subroutine GEØM calculates the wall coordinates and slopes for four different wall geometries: a constant area duct wall; a circular-arc, conical wall; and two tabular input walls. In the case of the first tabular wall, a completely general set of wall coordinates is read in. Subroutine GE $\emptyset \mathrm{M}$ then calls subroutine MTLUP, which interpolates for the coordinates. Next, subroutine GE $\emptyset \mathrm{M}$ calls function DIF, which calculates the slopes of the coordinates. For the second tabular wall, the coordinates and slopes are read in.

3. Subroutine GEøMCB. Subroutine GEøMCB calculates the centerbody coordinates and slopes for four different centerbody geometries and is similar to subroutine GE $\emptyset \mathrm{M}$.

4. Subroutine GEøMLU. Subroutine GEøMLU calculates the upper and lower dual-flow-space wall coordinates and slopes for two tabular input geometries. These tabular cases are the same as those in subroutine GEØM.

5. Subroutine MTLUP. Subroutine MTLUP (September 12, 1969) was taken from the National Aeronautics and Space Administration (NASA) Langley program library. This subroutine is called by subroutines GE $\varnothing \mathrm{M}, \mathrm{GE} \emptyset \mathrm{MCB}$, and GE $\emptyset \mathrm{MLU}$ to interpolate the wall, centerbody, and dual-flow-space wall coordinates.

6. Function DIF. Function DIF (August 1, 1968) was also taken from the NASA Langley program

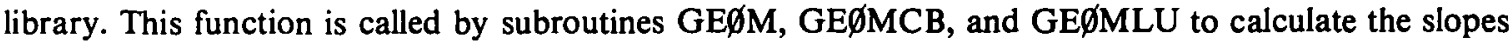
of the wall, centerbody, and dual-flow-space wall coordinates.

7. Subroutine $\emptyset$ NEDIM. Subroutine $\emptyset$ NEDIM is called by program VNAP2 to compute the $1 D$, isentropic initial-value surface. A Newton-Raphson scheme calculates the Mach number for the area ratios, which are determined from the geometry.

8. Subroutine MAP. Subroutine MAP calculates the functions that map the physical plane to a rectangular computational plane. Therefore, this subroutine is called before each mesh point is calculated.

9. Subroutine MASFL $\emptyset$. Subroutine MASFL $\emptyset$ is called by program VNAP2 to calculate the mass flow and momentum thrust for the initial-value and solution surfaces. The trapezoidal rule evaluates the mass flow and momentum thrust integrals.

10. Subroutine PL $\emptyset$ T. Subroutine PL $\emptyset \mathrm{T}$ is called by program VNAP2 to produce velocity vector plots, the physical space grid, and contour plots of density, pressure, temperature, Mach number, 
turbulence energy, and dissipation rate, using the SC-4020 microfilm recorder. The SC -4020 recorder uses a 1022 by 1022 array of plotting points or coordinates on each film frame. The origin is the upper left corner of the array. The coordinates to be plotted by the SC-4020 recorder are assumed to be integer constants. The first section sets up the plot size by setting the maximum left (XXL), right (XR), top (YT), and bottom (YB) coordinates in the physical space. Then the film frame coordinates and scaling factors are determined with the plot beginning at 900 , instead of 1022 , to allow for labeling.

The next section generates the velocity vector plot. First, the maximum velocity is determined to scale the plot, which is done so that the maximum velocity vector is $0.9 \Delta \mathrm{x}$, where $\Delta \mathrm{x}$ is the average value. Subroutine ADV (Los Alamos system routine) advances the film one frame. Then the velocity vector is calculated in fixed point film frame coordinates. Subroutine DRV (Los Alamos system routine) draws a line between the points (IX1, IY1) and (IX2, IY2), after which subroutine PLT (Los Alamos system routine) plots a plus sign at the point (IX 1, IY1). Subroutine LINCNT (Los Alamos system routine) skips down 58 lines. (Each printed line height equals 16 film frame points.) The routine then returns to set up the plot size for the next velocity vector plot if IVPTS $>1$, or goes on to the next section if IVPTS $\leqslant 1$.

The next section resets the plot size for the contour plots in case the different scaled velocity vector plots were requested (IVPTS $>1$ ).

The next section fills the plotting array called CQ with the following variables: density $\left(\mathrm{lbm} / \mathrm{ft}^{3}\right.$ or $\mathrm{kg} / \mathrm{m}^{3}$ ), pressure (psia or $\mathrm{kPa}$ ), temperature $\left({ }^{\circ} \mathrm{R}\right.$ or $\mathrm{K}$ ), and Mach number.

The next section determines the plotting line quantities using the formula

$\mathrm{CQ}_{\mathrm{K}}=\mathrm{CQ}_{\mathrm{MIN}}+0.1 \mathrm{~K}\left(\mathrm{CQ}_{\mathrm{MAX}}-\mathrm{CQ}_{\mathrm{MIN}}\right)$

where $\mathrm{K}$ goes from one to nine. This section also labels the frames.

The next section determines the location of each contour line segment and plots it. The contour line segment defined by the film frame coordinates (IX1, IY1) and (IX2, IY2) is drawn by subroutine DRV. Subroutine PLT plots an $L$ on the low contour $(K=1)$ and an $H$ on the high contour $(K=9)$. .

The last section draws the geometry boundaries for the contour plots. The upper boundary is specified by $\mathrm{YW}$, the lower by $\mathrm{YCB}$, the upper dual-flow-space boundary by $\mathrm{YU}$, and the lower dual-flow-space boundary by YL. Next, the routine returns to the section that fills the plotting array CQ for the next contour plot.

11. Subroutine SWITCH. Subroutine SWITCH switches the solution values from the solution array to the dummy array when dual-flow-space boundaries are present. The dummy array is required because the two dual-flow-space walls collapse to one grid line in the computational plane.

12. Subroutine VISCØUS. Subroutine VISC ØUS calculates the artificial viscosity terms for shock computations using a velocity gradient viscosity coefficient. It also calculates the molecular viscosity terms in the Navier-Stokes equations. In addition, this subroutine calculates the various turbulence terms in the Navier-Stokes equations, as well as the turbulence energy and dissipation rate equations.

13. Subroutine SMффTH. Subroutine SM $\varnothing \mathrm{TH}$ is called hy program VNAP2. to add either space or time numerical smoothing to stabilize the calculations for nonuniform initial-data surfaces or to accelerate the convergence to steady state. The physically correct molecular viscous terms (with a large viscosity coefficient) could also be used; however, they are much slower and cannot be reduced or turned off during a run.

14. Subroutine MIXLEN. Subroutine MIXLEN is called by subroutine VISC $\varnothing U$ US to calculate the shear layer width or the boundary layer thickness and kinematic displacement thickness for the mixing-length model (ITM = 1). These parameters also determine the length scale used by the turbulence energy model $(\mathrm{ITM}=2)$. 
15. Subroutine TURBC. Subroutine TURBC is called by program VNAP2 to set the boundary conditions for the turbulence energy, $Q$, and the dissipation rate, $E$.

16. Subroutine INTER. Subroutine INTER is called by program VNAP2 to calculate the interior mesh points. The conservation of mass, momenta, internal energy, turbulence energy, and dissipation rate equations are solved by the MacCormack second-order, finite-difference scheme. Subroutine INTER also contains part of the Quick Solver package. Special values of the derivatives $u_{\eta}, v_{\eta}$, and $p_{\eta}$, calculated by subroutine QS $\emptyset \mathrm{LVE}$, are used in special forms of the governing equations to allow an increased time step.

17. Subroutine WALL. Subroutine WALL is called by program VNAP2 to compute the wall, centerbody, dual-flow-space walls, free-jet boundary, and sharp expansion corner mesh points. This subroutine uses a second-order, reference-plane-characteristic scheme and also controls the interpolation process for locating the free-jet boundary. Subroutine WALL also contains part of the Quick Solver package that allows an increased time step. However, this subroutine does not use the special derivatives calculated by subroutine QS $\emptyset \mathrm{LVE}$.

18. Subroutine INLET. Subroutine INLET is called by program VNAP2 to compute the inlet mesh points. If the flow is subsonic, a second-order, reference-plane-characteristic scheme is employed, whereas specification of the boundary conditions is used for supersonic flow. This subroutine also checks the Mach number to determine which boundary condition should be used at each mesh point. In addition, subroutine INLET contains part of the Quick Solver package and uses the special derivatives calculated by subroutine QS $\emptyset \mathrm{LVE}$.

19. Subroutine EXITT. Subroutine EXITT is called by program VNAP2 to calculate the exit mesh points. It uses a second-order, reference-plane-characteristic scheme when the flow is subsonic and extrapolation when the flow is supersonic. This subroutine also checks the Mach number to determine which boundary condition should be used at each mesh point. In addition, subroutine EXITT contains part of the Quick Solver package and uses the special derivatives calculated by subroutine QS $\emptyset$ LVE.

20. Subroutine QS $\emptyset$ LVE. Subroutine QS $\emptyset$ LVE, which is part of the Quick Solver package, calculates the partial derivatives $u_{\eta}, v_{\eta}$, and $p_{\eta}$ that are used in subroutines INTER, INLET, and EXITT. These special derivatives are calculated from the domain of dependence defined by the characteristics through the solution point and, therefore, allow an increased time step.

\section{B. Computational Grid Description}

The computational grid for the single-flow-space example is shown in Fig. 18. The grid is rectangular with equal spacing in the $\zeta$ and $\eta$ directions, although $\Delta \zeta$ and $\Delta \eta$ are not in general equal. The grid spacing $(\Delta x, \Delta y)$ in the physical space does not have to be equal.

The dual-flow-space grid (Fig. 19) is the same as the single-flow-space grid except for an extra row of grid puints ( $M=$ MDFS and $L$ between LDFSS and LDFSF). The solution values at these extra grid points are stored in arrays UL, VL, PL, R $\emptyset \mathrm{L}, \mathrm{QL}$, and EL. During the calculation, subroutine SWITCH exchanges these values continually with the values in the solution arrays $U, V, P, R \emptyset, Q$, and $E$ for $M=$ MDFS and $L$ between LDFSS and LDFSF. For reading in initial-data values, the values in UL, VL, PL, $R \emptyset L, Q L$, and EL arrays correpond to the lower dual-flow-space wall, whereas values in the U, V, P, R $\emptyset$, $\mathrm{Q}$, and $\mathrm{E}$ arrays for $\mathrm{M}=$ MDFS and $\mathrm{L}$ between LDFSS and LDFSF correspond to the upper dual-flow-space wall.

The computational grid for the subcycled grid option is shown in Fig. 20. The code advances the solution one time step in the large spacing grid points (from $M=1$ to $M V C B-1$ and from $M=M V C T$ 
+1 to MMAX) and then subcycles the small spacing grid points (from M = MVCB to MVCT). In this way, the small time step requirement of the small spacing grid points (small spacing in the physical plane) is not forced on the large spacing grid points.

The flow is assumed to enter from the left and exit on the right. In addition, flow may enter or exit the wall (see IWALL in Namelist BC).

\section{Input Data Description}

The program input data are entered by a title card and 10 namelists: CNTRL, IVS, GEMTRY, GCBL, BC, AVL, RVL, TURBL, DFSL, and VCL. The title card and each namelist are described below. The program will continue reading in data decks and executing them until a flle mark is encountered. After each data deck is executed, the defaull values für the input data arc rcstored before the next data deck is read in.

1. Title Card. The first card of each data deck is a title card consisting of 80 alphanumeric characters that identify the job. This card must always be the first card of the data deck, even if no information is specified on the card. The 10 namelists must appear in the order in which they are discussed below.

2. Namelist CNTRL. This namelist reads in the parameters that control the overall logic of the program.

LMAX

MMAX

NMAX

NPRINT

TCONV

FDT

FDTI
An integer specifying the number of mesh points in the $x$ direction with a maximum value specified by a PARAMETER statement (see Sec. II.E.1). No default value is specified.

An integer specifying the number of mesh points in the y direction with a maximum value specified by a PARAMETER statement (see Sec. II.E.1). No default value is specifled.

An integer specifying the inaximum number of time stepsi. For NMAX $=0$, only the initial-data surface is computed and printed (provided NPRINT $>0$ ). The default value is 0 .

An integer specifying the amount of output desired. For NPRINT $=\mathrm{N}$, every Nth solution plane, plus the initial-data and final solution planes, is printed. For NPRINT $=-\mathrm{N}$, every $\mathrm{Nth}$ solution plane, plus the final solution plane, is printed. For NPRINT $=0$, only the final solution plane is printed. The default value is 0 . Specifies the axial velocity steady-state convergence tolerance in percentage. If equal to zero, the cunvergente is nul cliecked. This parametcr is a funotion of the problem as well as of grid spacing and, thercforc, should be used carefully. The default value is 0.0 .

The parameter A in Eqs. (67)-(69) that premultiplies the allowable C-F-L time step. It is desirable to use as large a value of FDT as possible without causing the computation to become unstable. Values as large as 1.3 have been used successfully for shock-free flows, but smaller values are required for flows with shocks (see Sec. II.F). The defalut value is 0.9 .

The same as FDT, except it applies on the first time step only. Because the viscous contribution to the time-step limitation is not used on the first time step, FDTI may be used to get the calculation started with a small time step, without having to use this small value for the entire calculation. Some flows may require a small time step for the first few steps owing to initial gradients in the flow variables. This is often 
FDT 1

VDT

VDT1

GAMMA

RGAS

TSTøP

IUI

IUø

IPUNCH

NPLOT

LPP 1,MPP 1

LPP2,MPP2

LPP3,MPP3 true for viscous flows when the Quick Solver option is used. For this situation, make a short run with small enough values of FDT or FDT1 so that the code will run. Then use the restart option (see IPUNCH) to continue the run with more desirable values of FDT or FDT1. For any long running problem, it is usually worth experimenting with FDT and FDT1 (as well as VDT and VDT1) to make sure that optimum values are being used. The default value is FDT.

The same as FDT, except it applies only in the subcycled part of the mesh. That is, FDT1 is used from $M=$ MVCB to $M=$ MVCT (see Namelist VCL). The default value is 1.0 .

The parameter $A_{1}$ in Eqs. (67)-(69) that premultiplies the viscous part of the time-step equation, whereas FDT premultiplies the entire time step. Increasing VDT increases the time step. The default value is 0.25 .

The same as VDT, except it applies only in the subcycled part of the mesh. That is, VDT1 is used from $M=M V C B$ to $M=$ MVCT (see Namelist VCL). The default value is 0.25 , although values larger than 1.0 have been used in free-shear layers.

Denotes the ratio of specific heats. The default value is 1.4.

Denotes the gas constant in $\mathrm{lbf}-\mathrm{ft} / \mathrm{lbm}-{ }^{\circ} \mathrm{R}$ if English units are used, or $\mathrm{J} / \mathrm{kg}-\mathrm{K}$ if metric units are used. The default value is 53.35 .

Specifies the physical time, in seconds, at which the computations will be stopped. The default value is 1.0 .

An integer specifying the type of units to be used for the input quantities. IF $I U 1=1$, English units are assumed; if IUI $=2$, metric units are assumed. In using any default values, make sure the values correspond to the proper units. The default value is 1 . The same as IUI except for output quantities. IF IU $\emptyset=3$, both English and metric units are printed. The default value is 1 .

An integer which, if nonzero, punches (writes) the last solution plane on cards (disc or tape) for restart. The default value is 0 .

An integer which, if greater than or equal to zero, plots both velocity vectors and contours of density, pressure, temperature, Mach number, turbulence energy, and dissipation rate on an SC-4020 microfilm recorder. For NPLOT $=\mathrm{N}$, all $\mathrm{Nth}$ solution planes, plus the initial-data and final solution plane, are plotted. For NPLOT $=0$, only the final solution plane is plotted. The default value is -1 .

Three sets of integers that specify three grid points (the first point is $L=L P P 1, M=$ MPP1) for which the pressure is printed at each time step. When MPP1(MPP2 or MPP3) $=$ MDFS $\neq 0$ (Namelist DFSL), the upper dual-flow-space wall value is printed. This pressure history is very useful for determining when subsonic flows have reached steady state. If $L P P 1<0$, the pressure at each subcycled grid point (see MVCB and MVCT in Namelist VCL) is also printed. The default values are 0 (no printing).

The remaining parameters in Namelist CNTRL are less important than the parameters given above.: For most flows, these remaining parameters can be left at their default values.

NASM

An integer specifying which part of the flow field is tested for steady-state convergence. For NASM $=0$, the entire flow field is tested. For NASM $=1$, the transonic and supersonic (throat region to exit) regions are tested. The default value is 1 .

NAME An integer that, when nonzero, causes the 10 namelists to be printed in addition to the regular output. The default value is 0 .

NCØNVI An integer specifying how many times the convergence tolerance TC $\emptyset \mathrm{NV}$ must be satisfied on consecutive time steps before the solution is considered to have converged. The default value is 1 . 
IUNIT

PLØW

$\mathrm{R} \emptyset \mathrm{L} \emptyset \mathrm{W}$

IVPTS

An integer that, when equal to zero, causes the program to use either English or metric units (see IUI and IU $\emptyset$ ). For IUNIT $=1$, a nondimensional set of units is used. The default value is 0 .

If the pressure becomes negative during a calculation, it is set equal to PL $\emptyset \mathrm{W}$ in psia or $\mathrm{kPa}$. The default value is 0.01 .

If the density becomes negative during a calculation, it is set equal to $R \varnothing L \emptyset W$ in $\mathrm{lb} / \mathrm{ft}^{3}$ or $\mathrm{kg} / \mathrm{m}^{3}$. The default value is 0.0001 .

An integer that controls the scaling of the velocity vector plots. IVPTS $=1$ produces onc plot with the maximum vector cqual to $0.9 \Delta \mathrm{x}$, wherc $\Delta \mathrm{x}$ is the average value. IVPTS $=2$ produces the above plot and a second plot where the maximum vector is $1.9 \triangle \mathrm{x}$, and so on. The default value is 1 .

3. Namelist IVS. This namelist specifies the flow variable for the initial-data surface. N1D

$\mathrm{U}(\mathrm{L}, \mathrm{M}, 1)$

$\mathrm{V}(\mathrm{L}, \mathrm{M}, \mathbf{1})$

$\mathbf{P}(\mathrm{L}, \mathrm{M}, \mathbf{1})$

$\overline{\mathbf{R}} \emptyset(\mathbf{L}, \mathbf{M}, 1)$

$\mathrm{Q}(\mathrm{L}, \mathrm{M}, 1)$

$\mathrm{E}(\mathrm{L}, \mathrm{M}, 1)$

$\mathrm{UL}(\mathrm{L}, 1)$
An integer specifying the type of initial-data surface desired. For N1D $=0$, a 2D initial-data surface is read in. A value of $U, V, P$, and $R \emptyset$ (discussed below) must be read in for all mesh points from $L=1$ to LMAX and from $M=1$ to MMAX. In addition, for dual-flow-space examples, values of UL, VL, PL, and RøL (discussed below) must be read in for all mesh points from $L=$ LDFSS to LDFSF. For the single-equation turbulence model, a value of $Q$, along with $Q L$ for the dual-flow-space example, may be read in. For the two-equation model, a value of $E$, along with EL for the dual-flow-space example, may also be read in. If the arrays $Q$ and QL and the arrays E and EL are not read in, they are set equal to FSQ and FSE (Namelist TURBL), respectively. Values of $Q$ and $E$ may be read in for either NID $=0$ or $\mathrm{N} 1 \mathrm{D} \neq 0$. For $\mathrm{N} 1 \mathrm{D} \neq 0$, a $1 \mathrm{D}$ data surface is computed internally.

The following combinations are possible:

$$
\left.\begin{array}{ll}
\text { N1D }=-2 \text { subsonic } \\
\text { N1D }=-1 \text { supersonic } \\
\text { N1D }=1 \text { subsonic-sonic-supersonic } \\
\text { N1D }=2 \text { subsonic-sonic-subsonic } \\
\text { N1D }=3 \text { supersonic-sonic-supersonic } \\
\text { N1D }=4 \text { supersonic-sonic-subsonic }
\end{array}\right\} \quad \begin{aligned}
& \text { see RSTAR } \\
& \text { No } \\
& \text { additional } \\
& \text { data are } \\
& \text { needed. }
\end{aligned}
$$

The default value is 1 .

An array denoting the $x$-direction velocity component in $f t / \varepsilon$ or $m / \varepsilon$. For $N 1 D=0$, $U(L, M, 1)$ must be read in for cases from $L=1$ to LMAX and from $M=1$ to MMAX. For N1D $\neq 0, U(L, M, 1)$ is not read in. No default values are specified.

An array denoting the $y$-direction velocity component in $\mathrm{ft} / \mathrm{s}$ or $\mathrm{m} / \mathrm{s}$. See $\mathrm{U}(\mathrm{L}, \mathrm{M}, 1)$ for additional information. No default values are specified.

An array denoting the pressure in psia or $\mathbf{k P a}$. See $\mathrm{U}(\mathrm{L}, \mathrm{M}, 1)$ for additional information. No default values are specified.

An array denoting the density in $\mathrm{lbm} / \mathrm{ft}^{3}$ or $\mathrm{kg} / \mathrm{m}^{3}$. See $\mathrm{U}(\mathrm{L}, \mathrm{M}, 1)$ for additional information. No detault values are specitied.

An array denoting the turbulence energy in $\mathrm{ft}^{2} / \mathrm{s}^{2}$ or $\mathrm{m}^{2} / \mathrm{s}^{2}$. See $\mathrm{U}(L, M, 1)$ for additional information. The default value is FSQ(M) in Namelist TUR.BL.

An array denoting the dissipation ratc in $\mathrm{ft}^{2} / \mathrm{s}^{3}$ or $\mathrm{m}^{2} / \mathrm{s}^{3}$. Sec $\mathrm{U}(\mathrm{L}, \mathrm{M}, 1)$ for additional information. The default value is FSE(M) in Namelist TURBL.

An array denoting the $x$-direction velocity component in $\mathrm{ft} / \mathrm{s}$ or $\mathrm{m} / \mathrm{s}$ and corresponding to the lower dual-flow-space wall. The values for the upper dual-flow-space wall are read in by U(L,MDFS,1). For N1D $=0$ and MDFS $\neq 0$, $\mathrm{UL}(\mathrm{L}, 1)$ must be read in for cases from $L=$ LDFSS to LDFSF. For N1D $\neq 0$ or MDFS $=0, U L(L, 1)$ is not read in. No default values are specified. 


\begin{abstract}
$\mathrm{VL}(\mathrm{L}, 1) \quad$ An array denoting the $y$-direction velocity component in $\mathrm{ft} / \mathrm{s}$ or $\mathrm{m} / \mathrm{s}$. See $\mathrm{UL}(\mathrm{L}, 1)$ for additional information. No default values are specified.

$\mathrm{PL}(\mathrm{L}, 1) \quad$ An array denoting the pressure in psia or $\mathrm{kPa}$. See $\mathrm{UL}(\mathrm{L}, 1)$ for additional information. No default values are specified.

$\mathrm{R} \emptyset \mathrm{L}(\mathrm{L}, 1) \quad$ An array denoting the density in $\mathrm{lbm} / \mathrm{ft}^{3}$ or $\mathrm{kg} / \mathrm{m}^{3}$. See $\mathrm{UL}(\mathrm{L}, 1)$ for additional information. No default values are specified.

QL(L, 1) An array denoting the turbulence energy in $\mathrm{ft}^{2} / \mathrm{s}^{2}$ or $\mathrm{m}^{2} / \mathrm{s}^{2}$. See UL(L,1) for additional information. The default value is FSQL in Namelist TURBL.

EL(L,1) An array denoting the dissipation rate in $\mathrm{ft}^{2} / \mathrm{s}^{3} \mathrm{or} \mathrm{m}^{2} / \mathrm{s}^{3}$. See UL(L,1) for additional information. The default value is FSEL in Namelist TURBL.

RSTAR, If N1D $=-1$ or -2 , either RSTAR for planar or RSTARS for axisymmetric flow RSTARS must be read in. RSTAR is the area per unit depth or height (in in. or $\mathrm{cm}$ ) where the Mach number is unity. RSTARS is the area divided by $\pi$ that is the radius squared (in in. ${ }^{2}$ or $\mathrm{cm}^{2}$ ) where the Mach number is unity. No default values are specified.

If the restart option is to be used, the initial run must be made with IPUNCH $\neq 0$ in CNTRL, thereby causing a new IVS Namelist deck to be punched or written on disc or tape. The new IVS Namelist replaces the one used initially and includes two additional parameters, NSTART and TSTART, which denote, respectively, the time step and the physical time where the solution was restarted.

When N1D $\neq 0$, the initial data are calculated using 1D isentropic theory. However, the $x$ and $y$ velocity components are adjusted while the magnitude is kept constant and the flow angle is satisfied. The flow angles are linearly interpolated between the slope of the wall and the centerbody. For the dual-flow-space example, the Mach number is assumed to be equal in both flow spaces at a given value of $x$. However, the flow angles are interpolated between the centerbody and the lower dual-flow-space boundary for the lower space and between the upper dual-flow-space boundary and the wall for the upper space.
\end{abstract}

4. Namelist GEMTRY. This namelist specifies the parameters that define the wall contour.

NDIM

NGEøM

XI

RI

RT

XE

RCI

RCT

ANGI

ANGE

XWI

YWI

NWPTS

IINT
An integer denoting the flow geometry. For NDIM $=0,2 \mathrm{D}$ planar flow is assumed, and for NDIM = 1, axisymmetric flow is assumed. The default value is 1 .

An integer specifying one of four different wall geometries. A discussion of these four cases follows the definitions of the additional parameters in this namelist. No default value is specified.

The $\mathrm{x}$ coordinate, in in. or $\mathrm{cm}$, of the wall inlet. No default value is specified.

The $y$ coordinate, in in. or $\mathrm{cm}$, of the wall inlet. No default value is specified.

The y coordinate, in in. or $\mathrm{cm}$, of the wall throat. No default value is specified.

The $\mathrm{x}$ coordinate, in in. or $\mathrm{cm}$, of the wall or free-jet exit. No default value is specified.

The radius of curvature, in in. or $\mathrm{cm}$, of the wall inlet. No default value is specified. The radius of curvature, in in. or $\mathrm{cm}$, of the wall throat. No default value is specified. The angle, in degrees, of the converging section. No default value is specified. The angle, in degrees, of the diverging section. No default value is specified.

A ID array of nonequally spaced $x$ coordinates in in. or $\mathrm{cm}$. No default values are specified.

A 1D array of $y$ coordinates, in in. or $\mathrm{cm}$, corresponding to the $\mathrm{x}$ coordinates in array XWI. No default values are specified.

An integer specifying the number of entries in arrays XWI and YWI. The maximum value is specified by a PARAMETER statement (see Sec. II.E.1). No default value is specified.

An integer specifying the order of interpolation used. The maximum value is 2 . The default value is 2 . 
IDIF An integer specifying the order of differentiation used. The maximum value is 5 . The default value is 2 .

YW A ID array of $\mathrm{y}$ coordinates, in in. or $\mathrm{cm}$, which correspond to LMAX $\mathrm{x}$ coordinates, given by XP in Namelist VCL. No default values are specified.

NXNY A 1D array (floating point) of the negative of the wall slopes corresponding to the elements of YW. No default values are specified.

JFLAG An integer that, when equal to 1 , denotes that a free-jet calculation is to be carried out and, when equal to -1 , denotes that a supersonic sharp expansion corner is present on the wall. These two options are allowed only for the free-slip wall boundary condition. Many free-jet flows contain shocks and will, therefore, require artificial viscosity (see Namelist AVL). The default value is 0 (no free jet and no sharp expansion corner).

LJET An integer that, when JFLAG $=1$, denotes the first mesh point of the free-jet boundary (the last wall mesh point is LJET -1 ). However, when JFLAG $=-1$, LJET is the next mesh point downstream of the sharp expansion corner (the corner mesh point is LJET - 1). The program assumes that either the wall ends exactly at LJET - $1($ JFLAG $=1)$ or the sharp expansion corner is located exactly at LJET 1) (JFLAG $=-1)$. Also, for the sharp expansion corner case (JFL $\Lambda G=-1)$, the slope of the wall at the corner (LJET - 1) should be the upstream value. The program does not allow both a sharp expansion corner and a free-jet calculation. In addition LJET must be $>2$ and $<$ LMAX -1 . No default value is given.

The following is a discussion of the four different wall geometries considered by this program.

a. Constant Area Duct $(N G E \emptyset M=1)$. The parameters XI,RI (radius of the duct) and XE must be specified.

b. Circular-Arc, Conical Wall $(N G E \emptyset M=2)$. The geometry for this case is shown in Fig. 21. The parameters XI, RI, RT, XE, RCI, RCT, ANGI, and ANGE are specified. The $x$ coordinate of the throat and the radius of the exit are computed internally.

c. General Wall (NGE $\emptyset M=3)$. An arbitrary wall contour is specified by tabular input. NWPTS xand $y$-coordinate pairs are specified by the arrays XWI and YWI, respectively. The tabular data need not be equally spaced. From the specified values of NWPTS, XWI, YWI, IINT, and IDIF, the program uses IINT-order interpolation to obtain LMAX $\mathrm{y}$ coordinates that correspond to the $\mathrm{x}$ coordinates given by $X P$ in Namelist VCL. Next, IDIF-order differentiation is used to obtain the wall slope at these LMAX points.

d. General Wall (NGEDM=4). An arbitrary wall contour is specified by tabular input. LMAX y coordinates and the negative of their slopes are specified by the arrays YW and NXNY, respectively. These $\mathrm{y}$ coordinates correspond to the LMAX $\mathrm{x}$ coordinates given by XP in Namelist VCL. XI and XE also must be read in.

5. Namelist GCBL. This namelist specifies the parameters that define the centerbody geometry. If no centerbody is present, this namelist is left blank but must still be present in the data deck.

NGCB An integer that, when nonzero, specifies one of four different centerbody geometries. A discussion of these four cases will follow the definitions of the additional parameters in this namelist. The default value is 0 .

RICB The y coordinate, in in. or $\mathrm{cm}$, of the centerbody inlet. No default value is specified. RTCB The y coordinate, in in. or $\mathrm{cm}$, of the centerbody maximum radius. No default value is specified. 
RCICB The radius of curvature, in in. or $\mathrm{cm}$, of the centerbody inlet. No default value is specified.

RCTCB The radius of curvature, in in. or $\mathrm{cm}$, of the centerbody maximum radius. No default value is specified.

ANGICB The angle, in degrees, of the converging section. No default value is specified.

ANGECB The angle, in degrees, of the diverging section. No default value is specified.

$\mathrm{XCBI}$

A $1 \mathrm{D}$ array of nonequally spaced $\mathrm{x}$ coordinates in in. or $\mathrm{cm}$. No default values are specified.

YCBI A 1D array of $y$ coordinates, in in. or $\mathrm{cm}$, corresponding to the $\mathrm{x}$ coordinates in array XCBI. No default values are specified.

NCBPTS . An integer specifying the number of entries in arrays XCBI and YCBI. I ne maximum value is specified by a PARAMETER statement (see Sec. II.E.1). No default value is specified.

IINTCB An integer specifying the order of interpolation used. The maximum value is 2 . The default value is 2 .

IDIFCB An integer specifying the order of differentiation used. The maximum value is 5 . The default value is 2 .

YCB A 1D array of $y$ coordinates, in in. or $\mathrm{cm}$, which correspond to LMAX $x$ coordinates given by XP in Namelist VCL. The default values are 0.0 .

NXNYCB The 1D array (floating point) of the negative of the centerbody slopes corresponding to the elements of YCB. The default values are 0.0 .

The following is a discussion of the four different centerbody geometries considered by this program.

a. Cylindrical Centerbody ( $N G C B=2)$. The parameter RICB (radius of the centerbody) must be specified.

b. Circular-Arc, Conical Centerbody $(N G C B=2)$. The geometry for this case is shown in Fig. 22. The parameters RICB, RTCB, RCICB, RCTCB, ANGICB, and ANGECB are specified. The $x$ coordinate of the maximum radius and the radius of the exit are computed internally.

c. General Centerbody $(N G C B=3)$. An arbitrary centerbody contour is specified by tabular input. NCBPTS $x$ - and $y$-coordinate pairs are specified by the arrays XCBI and YCBI, respectively. The tabular data need not be equally spaced. From the specified values of NCBPTS, XCBI, YCBI, IINTCB, and IDIFCB, the program uses IINTCB-order interpolation to obtain LMAX y coordinates that correspond to the $\mathrm{x}$ coordinates given by XP in Namelist VCL. Next, IDIFCB-order differentiation is used to obtain the centerbody slope at these LMAX points.

d. General Centerbody (NGCB =4). An arbitrary centerbody contour is specified by tabular input. LMAX y coordinates and the negatives of their slopes are specified by the arrays YCB and NXNYCB, respectively. These y coordinates correspond to the LMAX $x$ coordinates given by XP in Namelist VCL.

6. Namelist BC. This namelist specifies the flow boundary conditions for all computational boundaries. NSTAG

An integer that, when nonzero, denotes that variable total pressure PT, variable total temperature TT, and variable flow angle THETA (all discussed below) have been specified. If NSTAG $\neq 0$, then a value for PT, TT, and THETA must be specified at all the points from $M=1$ to MMAX, even if one or two of the variables are constant or some grid points are not used (ISUPER $=2$ or 3 ). If $N S T A G=0$, only the first value for each of the three arrays needs to be specified. The default value is 0 .

PT(M) A 1D array denoting the stagnation pressure, in psia or $\mathrm{kPa}$, across the inlet (see ISUPER). This array is used to calculate the $1 \mathrm{D}$ initial-data surface as well as the inflow conditions for ISUPER $=0,2$, or 3 . No default values are specified. 
TT(M)

THETA(M)

PTL

TTL

THETAL

PE(M)

PEL

UI(M)

VI(M)

PI(M)

Rดा(M)

UIL

VIL

PIL

Røít

I'W

TCB

TL

TU

ISUPER
A 1D array denoting the stagnation temperature, in ${ }^{\circ} \mathrm{R}$ or $\mathrm{K}$, across the inlet (see ISUPER). This array is used to calculate the $1 \mathrm{D}$ initial-data surface as well as the inflow conditions for ISUPER $=0,2$, or 3 . No default values are specified.

A ID array denoting the flow angle, in degrees, across the inlet (see ISUPER). The default value is THETA(1) $=0.0$, which is meaningful only when NSTAG $=0$.

Denotes the stagnation pressure, in psia or $\mathrm{kPa}$, at the point where the lower dual-flow-space wall intersects the inlet (see Namelist DFSL). The upper dual-flow-space wall value is read in by PT(MDFS). If NSTAG $=0$ or MDFS $=0$ or LDFSS $\neq 1$, then PTL is not read in. No default value is specified.

The same as PTL, except denotes the stagnation temperature in ${ }^{\circ} \mathbf{R}$ or $\mathrm{K}$.

The same as PTL, except denotes the flow angle is degrees.

A $1 \mathrm{D}$ array denoting the pressure, in psia or $\mathrm{kPa}$, to which the flow is exiting. This pressure is used to compute the flow exit conditions when the flow is subsonic, the free-jet boundary location when a free-jet calculation is requested, or the wall inflow-outflow boundary when IWALL $=1$. The free-jet or wall inflow/outflow boundary pressure is assumed to be constant and equal to PE(MMAX). Subroutine WALL could be modified to allow PE to be a function of $x$ or $t$. This array starts with the centerline or centerbody value and ends with the wall value. If the exit pressure is constant, only the first value of the array needs to be read in. The default value is 14.7 .

Denotes the pressure, in psia or $\mathrm{kPa}$, to which the flow is exiting at the point where the lower dual-flow-space wall intersects the exit (see Namelist DFSL). The upper dual-flow-space wall value is read in by PE(MDFS). If MDFS $=0$ or LDFSF $\neq$ LMAX, PEL is not read in. No default value is specified.

A 1D array denoting the $x$ velocity, in $\mathrm{ft} / \mathrm{s}$ or $\mathrm{m} / \mathrm{s}$, across the inlet (see ISUPER). This array, as well as the arrays VI, PI, and R $\emptyset \mathrm{l}$ below, starts with the centerline or centerbody value and ends with the wall value. Values must be specified for points from $M=1$ to MMAX even if some grid points are not used (ISUPER = 2 or 3). No default values are specified.

The same as UI, except y velocity.

The same as UI, except denotes pressure in psia or $\mathrm{kPa}$.

The same as $\mathrm{UI}$, except denotes density in $\mathrm{lbm} / \mathrm{ft}^{3}$ or $\mathrm{kg} / \mathrm{m}^{3}$.

Denotes the $\mathrm{x}$ velocity in $\mathrm{ft} / \mathrm{s}$ or $\mathrm{m} / \mathrm{s}$ at the point where the lower dual-flow-space wall intersects the inlet (see Namelist DFSL). The upper dual-flow-space wall value is read in by UI(MDFS). For MDFS $=0$ or LDFSS $\neq 1$, UIL is not read in. See ISUPER for additional information. No default value is specified.

The same as UIL, except y velocity.

The same as UIL, except denotes pressure in psia or $\mathrm{kPa}$.

The same as UIL, except denotes density in $1 \mathrm{bm} / \mathrm{ft}^{3}$ or $\mathrm{kg} / \mathrm{m}^{3}$.

A ID array denoting the wall temperalure in ${ }^{\circ} \mathrm{R}$ or $\mathrm{K}$ corresponding to the $\mathrm{x}$ mesh points. If $T W$ is not specified, the wall is assumed to be adiabatic.

The same as TW, cxcept denotes centerbody temperature.

The same as TW, except denotes lower dual-flow-space wall (see Namelist DFSL). If $\mathrm{MDFS}=0, \mathrm{TL}$ is not read in.

The same as TW, except denotes upper dual-flow-space wall (see Namelist DFSL). If $\mathrm{MDFS}=0$, $\mathrm{TU}$ is not read in.

An integer that specifies whether the inlet flow is subsonic, supersonic, or both. ISUPER may have the following values: 

ISUPER $=0$ Subsonic inflow with PT, TT, and THETA as the specified quantities.

ISUPER = 1 Subsonic, supersonic, or mixed inflow with UI, VI, PI, and R $\emptyset \mathrm{I}$ as the specified quantities. For subsonic flow, PI is only an initial guess if INBC $=0$, and UI is only an initial guess if INBC $\neq 0$.

ISUPER $=2$ Subsonic, supersonic, or mixed inflow between the centerbody and lower dual-flow-space wall with UI, VI, PI, and RØI as the specified quantities. For subsonic flow, PI is only an initial guess if INBC $=0$, and $U I$ is only an initial guess if INBC $\neq 0$. ISUPER $=2$ is subsonic inflow between the upper dual-flow-space wall and the wall with PT, TT, and THETA as the specified quantities.

ISUPER $=3$ The same as ISUPER $=2$, except subsonic and subsonic, supersonic or mixed sides are switched.

INBC An integer that specifies whether $u$ or $p$ will be the inflow boundary condition for ISUPER $\neq 0$. If INBC $=0, u$ is the boundary condition and $p$ is calculated. If INBC $\neq 0$, the reverse is true. The default value is 0 .

IWALL An integer that denotes whether the wall is a solid boundary (includes free-jet option) or a constant pressure inflow/outflow boundary that is fixed with respect to time.

IWALL $=0 \quad$ Specifies a solid or free-jet boundary.

IWALL $=1 \quad$ Specifies a constant pressure $[\mathrm{PE}(\mathrm{MMAX})]$ boundary. When there is inflow across this constant pressure boundary, $u$ and $\rho$ are set equal to the wall-inlet value. This option cannot be used with JFLAG $\neq 0$ in Namelist GEMTRY. The default value is 0 .

IWALL $\emptyset$ An integer that, when not equal to 0 , forces linear extrapolation of the pressure at the wall for the IWALL = 1 case. This option is useful when a shock wave exits the wall boundary or when the flow normal to the boundary is supersonic outflow. The default value is 0 .

IINLET An integer that, when not equal to 0 , forces specification of all variables as the inflow boundary condition regardless of the Mach number. It applies only when ISUPER $\neq 0$. The default value is 0 .

IEXITT An integer that, when not equal to 0 , forces either extrapolation (IEXITT $=1$ ) or specified pressure (IEXITT $=2$ ) as the outflow boundary condition regardless of the Mach number. The default value is 0 .

IEX An integer that denotes the type of extrapolation to be used for supersonic outflow. IEX $=0$ denotes zeroth-order extrapolation, and IEX $=1$ denotes linear extrapolation. The default value is 1 .

IVBC An integer that specifies whether extrapolation or reflection is used to determine the viscous terms at boundaries. IVBC $=0$ specifies reflection, IVBC $=1$ specifies linear extrapolation, and IVBC $=2$ specifies zeroth-order extrapolation. Reflection is always used at the centerline or midplane. The adiabatic wall boundary condition (that is, TW, TCB, TL, and TU not specified) requires $\mathrm{IVBC}=0$. The default value is 0 .

NøSLIP An integer that, when equal to zero, specifies free-slip walls whereas NøSLIP = 1 specifies no-slip $(\mathrm{u}=\mathrm{v}=0)$ walls for all solid boundaries. The no-slip boundary condition is not enforced at the wall when IWALL $\neq 0$. The default value is 0 . 
DYW A parameter that specifies the maximum change that is allowed on each time step in the free-jet boundary location. The default value is 0.001 , that is, $0.1 \%$ maximum change per time step.

IAS An integer that, if not equal to zero, causes the upper and lower dual-flow-space wall slopes to be set equal to the average of the two slopes. This occurs only at the point or points where the two dual-flow-space walls intersect. That is, for LDFSS $\neq 1$, the slopes at LDFSS will be set equal to their average. Also, if LDFSF $\neq$ LMAX, the same occurs. The default value is 0 .

ALI The coefficient $C_{\alpha}$ in Eqs. (55) and (56). This coefficient controls the nonreflecting inflow boundary condition employed at the left boundary. Any nonzero value will activate the nonreflecting option; however, values of approximately 0.1 appear to work well for many problems. Specifyling ALI $\neq 0.0$ for the $P_{T}, T_{T}$, and $\theta$ buundarl $y$ coildition or supersonic inflow has no effect. The defuult value is 0.0 .

ALE

The coefficient $\mathrm{C}_{\alpha}$ in Eq. (54). This coefficient controls the nonreflecting inflow and outflow boundary condition at the right boundary. See ALI for further details. Specifying A I.F. $\neq n \cap$ fnr supersnnic nutflow has no effecl. Tlie deraull yalue is 0.0 , ALW The coefficient $C_{\alpha}$ in Eq. (54). This coefficient controls the nonreflecting inflow and outflow boundary condition at the wall boundary. See ALI for further details. Specifying ALW $\neq 0$ when IWALL $=0$ (Namelist BC) has no effect. The default value is 0.0 .

7. Namelist AV.L. This namelist specifies the parameters that determine the artificial viscosity used to stabilize the calculations for shocks and control the space- and time-smoothing options. For flows without shocks or where space or time smoothing is not desired, this namelist is left blank. See Sec. II.F for additional information.

CAV Denotes the artificial viscosity premultiplier C in Eq. (23). See Sec. II.F for typical values. The default value is 0.0 .

XMU Denotes the coefficient $\mathrm{C}_{\mu 1}$ in Eq. (24) in the artificial viscosity model. A nondimensional valuc is uscd. The default value is 0.4 .

XLA Denotes the cocfficient $C_{\lambda}$ in Eq. (23) in the aritificial viscosity model. A nondimensional value is used. The default value is 1.0.

PRA Denotes the coefficient $\operatorname{Pr}_{\mathrm{A}}$ in Eq. (25) in the artificial viscosity model and represents an artificial Prandtl number. The default value is 0.7 .

XR $\emptyset \quad$ Denotes the coefficient $C_{\rho}$ in Eq. (26) in the artificial viscosity model. The default value is 0.6 .

LSS, Integors that opeoify the $x$ mesh points at which the addition of the artificial

LSF viscosity will begin (LSS) and cnd (LSF). These parameters can significantly reduce the run timc for inviscid flows where a shock occupies only a small part of the flow. The default values are LSS $=1$ and LSF - 999.

MSS,

MSF The same as LSS and LSF, except that these specify the y mesh points at which the addition of the artificial viscosity begins (MSS) and ends (MSF). The default values are $\mathrm{MSS}=1$ and $\mathrm{MSF}=999$.

IDIVC An integer that, when not equal to 0 , bypasses the check on the sign of the velocity divergence in the artificial viscosity model. That is, the artiticial viscosity will be nonzero for both expansions and compressions. This improves some complex multiple shock interactions, but also increases the smearing of expansions. The default value is 0 .

ISS An integer that, when not equal to 0 , adds the sound speed gradient to the velocity divergence in Eq. (23). For ISS = 1, the sound speed gradient is added to the 
velocity divergence only if the velocity divergence is $<0$. For ISS $=2$, the sound speed gradient is always added. This term improves contact surface calculations (see Sec. I.F). The default value is 0 .

Denotes the Mach number below which no artificial viscosity for shock calculations is added to the solution. This option is useful for moderate-to-high Reynolds number, steady flow, where the artificial viscosity swamps the molecular and turbulent viscosities in the boundary layer. By setting SMACH equal to $\sim 0.5$, the artificial viscosity is zero for most of the subsonic part of the boundary layer. See Sec. I.F for additional details. The default value is 0.0 .

An integer denoting the time step at which a small amount of numerical space or time smoothing is stopped. Smoothing is employed on the regular time steps and not the subcycled steps (see Namelist VCL). This smoothing may be required to stabilize the calculations for very nonuniform or impulsively started initial-data surfaces. Some initial smoothing in space causes subsonic flows to reach steady state faster, but this is not the case for transonic and supersonic flows. Time smoothing also causes subsonic flows to converge to steady state faster. When using the restart option, make sure NST is set equal to zero unless additional smoothing is desired. If additional smoothing is desired on a restart, make sure that the values of SMP or SMPT on the restart equal the final values of the previous run (see SMP and SMPT discussion below). The default value is 0 (no smoothing).

SMP A parameter that, along with NST and SMPF, controls the amount of space smoothing (provided NST $\neq 0$ ). SMP must be between 0.0 and 1.0. The dependent variables are smoothed by the following formula: $\mathrm{u}_{\mathrm{L}, \mathrm{M}}=\mathrm{SMP}^{*} \mathrm{u}_{\mathrm{L}, \mathrm{M}}+(1.0-$ SMP $)^{*}\left(u_{L+1, M}+u_{L, M+1}+u_{L-1, M}+u_{L, M-1}\right) / 4.0$. The value of SMP changes on each time step by the following replacement formula:

SMP $=$ SMP + (SMPF - SMP $) /$ NST,

where the underlined SMP denotes the original input value. The inlet $(L=1)$ and exit $(L=L M A X)$ columns of grid points are not smoothed. The default value is 1.0. A parameter that, along with NST and SMP, controls the amount of space smoothing (see SMP for details). SMPF must be between 0.0 and 1.0. The default value is 1.0 .

SMPT A parameter that, along with NST and SMPTF, controls the amount of time smoothing or relaxation (provided NST $\neq 0$ ). The dependent variables are smoothed by the following formula:

$\mathrm{u}_{\mathrm{L}, \mathrm{M}}^{\mathrm{N}+1}=\mathrm{SMPT}^{*} \mathrm{u}_{\mathrm{L}, \mathrm{M}}^{\mathrm{N}+1}+(1.0-\operatorname{SMPT})^{*} \mathrm{u}_{\mathrm{L}, \mathrm{M}}^{\mathrm{N}}$

The value of SMPT changes on each time step by the following replacement formula:

SMPT $=$ SMPT $+($ SMPTF $-\underline{\text { SMPT }}) /$ NST, where the underlined SMPT denotes the original input value. Where some initial space smoothing followed by longer duration time smoothing is desired, flows can be computed using the restart option. The default value is 1.0 .

SMPTF A parameler thal, alung willi NST and SMPT, controls the amount of time smoothing (see SMPT for details). The default value is 1.0 .

NTST An integer that specifies the interval of time steps over which the solution is time smoothed (provided NST $\neq 0$ and SMPT $\neq 1.0$ ). For example, if NTST $=10$, then after every 10 time steps the solution at the current time step $\mathrm{N}$ is time averaged with the solution at time step $\mathrm{N}-10$. This averaged solution is then stored and used to average with the solution at $\mathrm{N}+10$. For $\mathrm{NTST}=0$, the code monitors the 
pressure at the $L=L P P 1$ and $M=$ MPP1 grid point (Namelist CNTRL) and time smooths when this pressure changes direction. If LPP 1 and MPP 1 are not specified and NTST $=0$, there is no time smoothing. This extended-interval time smoothing usually improves the convergence to steady state of subsonic flows. To use this option with NTST $=0$ or $>1$, the arrays US, VS, PS, RøS, QS, and ES must be dimensioned for LMAX and MMAX, while arrays ULS, VLS, PLS, RøLS, QLS, and ELS must be dimensioned for LMAX. These arrays are located in Common AV. The default value is 1 .

An integer that, when not equal to 0 , causes the viscous-turbulence terms, turbulence energy, and dissipation rate (or length scale) to be printed at the solution planes specified by NPRINT. IAV $=2$ causes the viscous terms for each subcycled time step to be printed (provided MVCB and MVCT in Namelist VCL are nonzero). The default value is 0 .

8. Namclist RVL. This namclist specifies llie real or mulecular viscostry parameterts. fór inviscid flows, this namelist is left blank.

CMU, These parameters specify the molecular viscosity $\mu$ by the following equation:

EMU

$\mu=\mathrm{CMU} \cdot \mathrm{T}^{\mathrm{EMU}}$,

where $T$ is the temperature in ${ }^{\circ} \mathrm{R}$ or $\mathrm{K}$. The units of $\mu$ are $\mathrm{lbf}-\mathrm{s} / \mathrm{ft}^{2}$ or Pa-s. The default values are 0.0 .

CLA, These parameters specify the second coefficient of viscosity $\lambda$ by the following ELA equations:

$\lambda=\mathrm{CLA} \cdot \mathrm{T}^{\mathrm{ELA}}$,

where $\mathrm{T}$ is the temperature in ${ }^{\circ} \mathrm{R}$ or $\mathrm{K}$. The units of $\lambda$ are $\mathrm{lbf}-\mathrm{s} / \mathrm{ft}^{2}$ or Pa-s. The default values are 0.0 .

CK, Thesc parameters specify the thermal conductivity $\mathrm{k}$ by the following equation:

EK

$\mathrm{k}-\mathrm{CK} \cdot \mathrm{T}^{\mathrm{EK}}$

where $\mathrm{T}$ is temperature in ${ }^{\circ} \mathrm{R}$ or $\mathrm{K}$. The units of $\mathrm{k}$ are $\mathrm{lbf} / \mathrm{s}-{ }^{\circ} \mathrm{R}$ or $\mathrm{W} / \mathrm{m}-\mathrm{K}$. The he default values are 0.0 .

9. Namelist TURBL. This namelist specifies the turbulence model parameters. For laminar as well as inviscid flows, it is left blank. For turbulent flows, Namelist RVL cannot be blank.

ITM

An integer that, when nonzero, specifies one of three different turbulence models. ITM $=1$ specifies a mixing-length model; ITM $=2$ specifies a one-equation, turbulence energy model; and ITM $=3$ specilies a two-equation, turbulence energydissipation-rate model. The default value is 0 .

IMLM

An integer, required for ITM $=1$ or 2 , that specifies whether the flow is a free shear layer (IMLM $=1$ ) or a boundary-layer flow (IMLM = 2). This information is required because the equations for the mixing length (ITM $=1$ ) and the length scale of the one-equation model (ITM $=2$ ) are different depending on whether the flow is a free shear or boundary layer. For single-flow spaces, the shear-layer option assumes either that the boundaries are free slip or that the lower boundary is a symmetry boundary and the wall must be a constant pressure inflow/outflow 
CML1, CML2

CAL

$\mathrm{CQL}$

CQMU

$\mathrm{C} 1, \mathrm{C} 2$, SIGQ,SIGE

BFST

FSQ(M)

FSE(M)

FSQL

FSEL

QLøW boundary. The boundary-layer option assumes one no-slip boundary, which is either a centerbody or a wall, but not both. For dual-flow spaces (see Namelist DFSL), the dual-flow-space walls are assumed to be no-slip boundaries, but the lower boundary must be a symmetry boundary and the wall must be a constant pressure inflow/outflow boundary. The program then uses the boundary-layer option between the dual-flow-space walls and the shear-layer option elsewhere, regardless of IMLM. Therefore, for dual-flow spaces IMLM does not need to be specified. The default value is 1 .

These coefficients, defined in Eqs. (9) and (10) and required for ITM $=1$ or 2, are used in the shear-layer option (for IMLM $=1$ or for dual-flow spaces). The mixing length, used in both ITM $=1$ and 2 , is calculated by multiplying the shear-layer thickness by these coefficients. CML2 is for velocity profiles where the minimum velocity is in the flow interior, and CML1 is for monotonic profiles. The default values for both coefficients are 0.125 for planar flows and 0.11 for axisymmetric flow.

Denotes the coefficient $\bar{\alpha}$ in the governing equations, Eqs. (1)-(4). This coefficient controls the effect of variable density for all three turbulence models. The recommended and default value is 1.0 .

This coefficient, which is $\mathrm{C}_{\mathrm{q}}$ in Eq. (15) and required by ITM = 2, is multiplied by the mixing length to obtain the length scale used in the one-equation model. The default value is $\mathbf{1 7 . 2}$ for planar flows and $\mathbf{1 2 . 3}$ for axisymmetric flow.

This coefficient, which is $\mathrm{C}_{\mathrm{u}}$ in Eqs. (17) and (21) and required by ITM $=2$ or 3, premultiplies the expression for the turbulent viscosity in the one- and two-equation models. The recommended and default value is 0.09 .

Coefficients, which are $C_{1}, \bar{C}_{2}, \sigma_{q}$, and $\sigma_{e}$, respectively, in Eq. (20) and required by ITM $=3$, for the two-equation, turbulence energy-dissipation-rate model. The recommended and default values are 1.44, 1.8, 1.0, and 1.3, respectively.

A parameter, required by ITM $=3$, that sets a lower bound for $q$ and $e$ in the twoequation model by the following relation:

$\mathrm{q}_{\mathrm{L}, \mathrm{M}} \geqslant \mathrm{BFST}^{*} \mathrm{FSQ}(\mathrm{M})$

$\mathrm{e}_{\mathrm{L}, \mathrm{M}} \geqslant \mathrm{BFS}^{\prime *} \mathrm{FSE}^{\mathrm{F}}(\mathrm{M})$,

where FSQ and FSE are detined below. A value between 0.0 and 1.0 is necessary for some separated flows. If MDFS $\neq 0$ and $\mathrm{L}<$ LDFSS or $\mathrm{L}>$ LDFSF (Namelist DFSL), then BFST is set to zero. The default value is 0.0 .

A 1D array that denotes the inlet or free-stream turbulence energy level (ITM $=2$ or 3) in $\mathrm{ft}^{2} / \mathrm{s}^{2}$ or $\mathrm{m}^{2} / \mathrm{s}^{2}$. This array, as well as the array FSE, starts with the centerline or centerbody value and ends with the wall value. The default value is 0.0001 .

The same as FSQ, except that the dissipation rate level $(\mathrm{ITM}=3)$ is given in $\mathrm{ft}^{2} / \mathrm{s}^{3}$ or $\mathrm{m}^{2} / \mathrm{s}^{3}$. The default value is 0.1 .

Denotes the inlet or free-stream turbulence energy levcl (ITM $=2$ or 3$)$ in $\mathrm{ft}^{2} / \mathrm{s}^{2}$ or $\mathrm{m}^{2} / \mathrm{s}^{2}$ at the point where the lower dual-flow-space wall intersects the inlet (see Namclist DFSL). The upper dual-flow-space wall is read in by FSQ(MDFS). For MDFS $=0$ or LDFSS $\neq 1$, FSQL is not read in. The default value is 0.0001 .

The same as FSQL, except that the dissipation rate level $\left(\right.$ ITM = 3) is given in $\mathrm{ft}^{2} / \mathrm{s}^{3}$ or $\mathrm{m}^{2} / \mathrm{s}^{3}$. The default value is 0.1 .

If during a calculation the turbulence energy (ITM $=2$ or 3 ) becomes less than or cqual to QL $\varnothing \mathrm{W}$, it is set equal to QL $\varnothing \mathrm{W}$. The default value is 0.0001 . 
ELøW The same as $Q L \emptyset W$ except for the dissipation rate $($ ITM = 3). The default value is 0.1 .

LPRINT, Integers that, when greater than zero, cause the convection, production, dissipation, MPRINT and diffusion terms of the turbulence energy (ITM $=2$ or 3) and dissipation rate $(\mathrm{ITM}=3)$ to be printed for $\mathrm{L}=\mathrm{LPRINT}, \mathrm{M}=$ MPRINT at every time step. The axisymmetric terms are not included. The default value is 0 .

PRT Denotes the turbulent Prandtl number in Eq. (8). The turbulent viscosity $\mu_{\mathrm{T}}$ is calculated by the turbulence model, after which the turbulent conductivity $\mathrm{k}_{\mathrm{T}}$ is calculated from PRT. The default value is 0.9 .

STBQ, Denote the coefficients $C_{Q}$ and $C_{E}$, respectively, in Eq. (22). These coefficients STBE control the fourth-order smoothing for the two-equation model (ITM = 3). This smoothing may improve the results for strongly scparated flow3. The default values are 0.0 (no smoothing).

10. Namelist DFSL. This namelist specities the dual-flow-space walls. For single-flow-space examples, this namelist is left blank.

MDFS

An integer that, when nonzero, specifies the $M$ row of grid points along which the dual-flow-space walls are positioned. MDFS cannot be set equal to 2 or MMAX - 1. The default value is 0 .

LDFSS, Integers that specify the $\mathrm{x}$ grid points where the dual-flow-space walls start and end, LDFSF respectively. LDFSS and LDFSF cannot be set equal to 2 or LMAX - 1 , respectively. The default values are 0 .

NDFS An integer specifying one of two different dual-flow-space wall geometries. A discussion of these two cases follows the definitions of the additional parameters in this namelist. No default value is specified.

YU, 1D arrays of $y$ coordinates in in. or $\mathrm{cm}$, which correspond to the LMAX $x$ YL coordinates given by XP in Nãmelist VCL. YU denules the upper dual-nuw-space NXNYU, wall and YL denotes the lower. The default values are 0.0 .

NXNYL 1D arrays (floating point) of the negative of the dual-flow-space wall slopes corresponding to the elemento of $Y U$ and $\mathrm{YL}$, respectively. The default values are 0.0 .

XUI, ID arrays of nonequally spaced $x$ coordinates in in. or $\mathrm{cm}$. XUI corresponds to the XLI upper dual-flow-space wall and XLI corresponds to the lower. No default values are specified.

YUI, 1D arrays of $y$ coordinates in in. or $\mathrm{cm}$, corresponding to the $\mathrm{x}$ coordinates in arrays YLI XUI and XLI, respectively. No default values are specified.

NUPTS, Integers specifying the number of entries in arrays XUI-YUI and XLI-YLI, NLPTS respectively. The maximum value is specified by a PARAMETER statement (see Sec. II.E.1). No default values are specified.

IINTDFS An integer specifying the order of interpolation used. The maximum value is 2 . The default value is 2 .

IDIFDFS An integer specifying the order of differentiation used. The maximum value is 5 . The default value is 2 .

The following is a discussion of the two different dual-flow-space wall geometries considered by this program. If the dual-flow-space walls begin in the interior (LDFSS $\neq 1$ ), the values of $Y L$ and $Y U$ (or YLI and YUI) for $L=$ LDFSS must be equal. The same is true at $L=$ LDFSF if the dual-flow-space walls end in the interior (LDFSF $\neq$ LMAX). If the dual-flow-space walls begin and end in the interior, than the ratio ( $Y L-Y C B) /(Y W-Y C B)$ at $L=L D F S S$ must equal that at $L=L D F S F$. The angle of attack of the dual-flow-space walls can be varied somewhat by changing the shape of the centerbody and wall. However, if the centerbody and wall shapes are fixed, then the angle of attack cannot be varied. 
a. General Dual-Flow-Space Wall $(N D F S=1)$. An arbitrary dual-flow-space wall contour is specified by tabular input. NUPTS $\mathrm{x}$ and $\mathrm{y}$ coordinate pairs are specified by the arrays XUI and YUI, respectively. NLPTS $x$ and $y$ coordinate pairs are specified by the arrays XLI and YLI, respectively. The tabular data need not be equally spaced. From the specified values of NUPTS, XUI, YUI, NLPTS, XLI, YLI, IINTDFS, and IDIFDFS, the program uses IINTDFS-order interpolation to obtain (LDFSF LDFSS + 1) upper and lower dual-flow-space wall y coordinates that correspond to the (LDFSF LDFSS +1$) \times$ coordinates given by XP(LDFSS) to XP(LDFSF) in Namelist VCL. Next, IDIFDFSorder differentiation is used to obtain the upper and lower dual-flow-space wall slopes at these (LDFSF LDFSS +1 ) points.

b. General Dual-Flow-Space Wall $(N D F S=2)$. An arbitrary wall contour is specified by tabular input. (LDFSF - LDFSS + 1) y coordinates and the negative of their slopes are specified by the arrays YU and NXNYU for the upper dual-flow-space wall and YL and NXNYL for the lower, respectively. The y coordinates correspond to the (LDFSF - LDFSS +1 ) $x$ coordinates given by XP(LDFSS) to XP(LDFSF) in Namelist VCL.

11. Namelist VCL. This namelist specifies the variable grid coordinates as well as the parameters that control the subcycle and Quick Solver options. For equal or uniform grid spacing, this namelist is left blank.

The subcycle option allows the part of the mesh with the small grid spacing to be computed for many time steps with the required small time step, whereas the rest of the mesh is calculated only one time step. The Quick Solver option can be used with the subcycle option to increase the time step in the small grid part of the mesh and, therefore, reduce the number of time steps or subcycles. The Quick Solver allows the increased time step by a procedure that removes the sound speed from the usual C-F-L stability condition. The Quick Solver assumes the flow in the y direction is subsonic.

IST An integer that, when nonzero, specifies that both the $\mathrm{x}$ and $\mathrm{y}$ coordinates will have variable grid spacings. When IST $=0$, the program will generate equally spaced values of XP and YI. The default value is 0 .

XP

A 1D array that denotes the $x$ coordinate grid spacing. The elements of XP begin with the inlet $(L=1)$ and extend to the outlet ( $L=L M A X)$. The first element $\operatorname{XP}(1)$ must equal XI [or XWI(1)] of Namelist GEMTRY and XP(LMAX) must equal XE [or XWI(NWPTS)]. For IST $=0$, the default values of XP consist of LMAX equally spaced grid points. For IST $\neq 0$, no default values are given.

YI

A 1D array that specifies the y coordinate grid spacing at the inlet or $x=X P(1)$ column of grid points. The elements of YI begin with the centerline or centerbody and extend to the wall. If $\overline{M D F S} \neq 0$ and LDFSS $=1$ (Namelist DFSL), then YI(MDFS) must equal $Y U(1)$ and a value of $Y I=Y L(1)$ is not read in. The grid spacing for the columns corresponding to $\mathrm{x}=\mathrm{XP}(2), \mathrm{XP}(3), \ldots, \mathrm{XP}(\mathrm{LMAX})$ is proportional to the YI spacings. For IST $=0$, the default values of YI consist of MMAX equally spaced grid points. For IST $\neq 0$, no default values are given.

MVCB, Integers that, when nonzero, denote which grid points will be subcycled. The MVCT subcycled grid points are $M=M V C B$ to MVCT for all $L$. The restrictions are MVCB $\neq 2$, MVCT $\neq$ MMAX -1 , and MVCT $>$ MVCB +1 . Where dual-flowspace walls are present, MVCB $\neq$ MDFS +1 and MVCT $\neq$ MDFS -1 . Finally, if the subcycled grid points extend on each side of the dual-flow-space walls, MVCB < MDFS -1 and MVCT $>$ MDFS +1 . The default values are 0 .

NVCMI An integer that, when nonzero, specifies the number of times the small spacing grid points are subcycled. If $\mathrm{NVCMI}=0$, the program determines the value internally. NVCMI must be an odd integer for indexing reasons. See NIQSS and NIQSF for additional details. The default value is 0 . 
IQS

NIQSS, NIQSF

CQS ILLQS SQS
An integer that, when nonzero, specifies the Quick Solver option. This option assumes that the flow in the $y$ direction is subsonic. Also, if MVCT = MMAX, then the wall boundary must be a no-slip solid wall (IWALL $=0$ and NøSLIP $=1$ in Namelist $B C$ ). If $M V C B=1$, then the centerbody boundary must be a no-slip solid wall (NGCB $=1$ in Namelist GCBL and NøSLIP =1). If dual-flow-space walls are present (see Namelist DFSL), the Quick Solver assumes that the subcycled grid points extend on each side of the dual-flow-space walls; that is, MVCB $<$ MDFS $<$ MVCT. The default value is 0 .

Integers that, when nonzero, denote at which time step $\mathrm{N}$ the Quick Solver will start (NIQSS) and stop (NIQSF). If NIQSS $>1$ and NVCMI is nonzero, then the program internally calculates the number of times to subcycle the small spacing grid points for $\mathrm{N}<\mathrm{NIQSS}$ and uses NVCMI when $\mathrm{N} \geqslant$ NIQSS. The default values are NIQSS $=2$ and NIQSF $=$ NMAX in Namelist CNTRL.

A parameter that specifies the convergence tolerance for the iteration that locates the characteristic intersection points in the Quick Solver. The default value is 0.001 . An integer that specifies the maximum number of iteratione allowed in locating the characteristic intersection points in the Quick Solver. The default value is 30 .

The coefficient $\mathrm{C}_{s}$, in Eqs. (47) and (49), that controls the amount of numerical smoothing necessary to stabilize the Quick Solver. The recommended and default value is 0.5 .

\section{Output Description}

Program output consists of printed output, film plots, and punched cards (disc or tape file) for restart. The first two pages (or first three pages in the tabular input geometry case) of output include the program title, abstract, list of control parameters, fluid model, flow geometry, nozzle geometry, boundary conditions, artificial viscosity, molecular viscosity, turbulence model, and variable grid parameters.

Following the title pages is the initial-data surface. Before each initial-data surface, a page is printed that gives the mass flow, ratio of mass flow to inlet $(\mathrm{L}=1)$ mass flow, exit momentum thrust, and ratio of momentum thrust to inlet momentum thrust for $L=1$ to LMAX. These data are either data that have been read in or a ID solution that has been computed by the program. All units are given. For planar flow, the mạss flow uniț are lowm/in $\mathrm{lbf} / \mathrm{in}$. or $\mathrm{N} / \mathrm{cm}$.

After the initial-data surface has been printed, the solution surfaces are printed. Before each solution surface, a page is printed that gives the mass flow, ratio of mass flow to inlet $(\mathrm{L}=1)$ mass flow, exit momentum thrust, and ratio of momentum thrust to inlet momentum thrust for $L=1$ to LMAX. After. the mass flow page, the solution surfaces are printed. These surfaces have the same format as the initialdata surface. Each solution surface gives the flow field for a certain value of time. At the top of each solution surface page is the number of time steps $N$, the time, the time step, the number of subcycles NVCM, and the subcycled Courant number CNUMS. At the top right of each page are two pairs of numbers enclosed in parentheses. These give the grid points where the limiting time step was found. The one on the right is for the subcycled grid. As many solution planes as desired may be printed by varying the input data.

If requested (IAV $\neq 0$ ), artificial viscosity, molecular viscosity, and turbulence parameters are printed before each solution plane. QUT denotes the $x$ momentum equation right-hand-side terms in $\mathrm{ft} / \mathrm{s}$ or $\mathrm{m} / \mathrm{s}$, QVT denotes the y momentum equation right-hand-side terms in $\mathrm{ft} / \mathrm{s}$ or $\mathrm{m} / \mathrm{s}$, QPT denotes the internal energy equation right-hand-side terms in psia or $\mathrm{kPa}$, and $\mathrm{QR} \emptyset \mathrm{T}$ denotes the continuity equation righthand-side terms in $\mathrm{lbm} / \mathrm{ft}^{3}$ or $\mathrm{kg} / \mathrm{m}^{3}$. AVMUR and TLMUR are the ratios of artificial and turbulent viscosities to the laminar value, respectively, $Q$ is the turbulence energy at the $N-1$ time step in $\mathrm{ft}^{2} / \mathrm{s}^{2}$ or 
$\mathrm{m}^{2} / \mathrm{s}^{2}$, and $\mathrm{E}$ is the dissipation rate at the $\mathrm{N}-1$ time step in $\mathrm{ft}^{2} / \mathrm{s}^{3}$ or $\mathrm{m}^{2} / \mathrm{s}^{3}$. QQT is the turbulence energy equation right-hand-side terms in $\mathrm{ft}^{2} / \mathrm{s}^{2}$ or $\mathrm{m}^{2} / \mathrm{s}^{2}$, QET is the dissipation rate equation right-hand-side terms in $\mathrm{ft}^{2} / \mathrm{s}^{3}$ or $\mathrm{m}^{2} / \mathrm{s}^{3}$, and TML is the mixing-length (ITM =1) or length scale (ITM =2) in in. or $\mathrm{cm}$. The parameters for the upper dual-flow-space wall are printed on the last page of the viscous printout. At the end of the viscosity parameters are the grid points whose viscous terms limit the time-step size in the $\mathrm{x}$ and $\mathrm{y}$ directions. Also printed is the ratio of the $\mathrm{y}$ terms to the $\mathrm{x}$ terms. The larger this ratio, the more restrictive the $y$ direction terms become in limiting the time step size. If LPRINT and MPRINT are read in, the turbulence energy and dissipation rate convection, production, dissipation, and diffusion terms (not including axisymmetric terms) are also printed in internal units. Also, film plots with the units of the printed output are made for each requested time step. When the computation is stopped because the flow has satisfied the convergence tolerance, the physical time equals TST $\emptyset$, or the maximum number of time steps has been reached, the final solution plane is always printed and plotted.

\section{E. Computing System Compatibility}

1. Deck Set-Up. The deck begins with the common deck called MCC, followed by the main program called VNAP2 and the remaining function and subroutines. The common deck is preceeded by the card * $\varnothing$ MDECK,MCC, beginning in column 1. This common deck is separated from the main program VNAP2 by the card "DECK,VNAP2, also beginning in column 1. Any routine that uses the common deck MCC has the card ${ }^{*}$ CALL,MCC, beginning in column 1, at the location where the common deck should be in that routine. The CDC routine UPDATE will place the common deck in each routine containing a ${ }^{*} \mathrm{CALL}, \mathrm{MCC}$ card. This simplifies making changes to the COMMON statements as well as array sizes (see below). For computing systems without an UPDATE or comparable routine, remove the ${ }^{*}$ CØMDECK,MCC and *DECK,VNAP2 cards and replace all *CALL,MCC cards with the common deck, MCC.

2. Array Sizes. This version of the program allows for a maximum of $41 \mathrm{x}$ and $25 \mathrm{y}$ mesh points. These values are set by use of a PARAMETER statement, which is the first card in the common deck MCC. In this PARAMETER statement, LI $\geqslant$ LMAX, MI $\geqslant$ MMAX, LII $=\mathrm{LI}+1$, and MII $=\mathrm{MI}+$ 1. MQS $\geqslant$ MVCT sets the Quick Solver array sizes. When the Quick Solver is not being used (IQS $=0$ ), then MQS can be set equal to one to reduce the amount of storage. LTS = LI and MTS = MI set the extended-interval time-smoothing array sizes. When the extended-interval time smoothing is not being used (NTST $=1$ or NST $=0$ ), then LTS and MTS can be set equal to one to reduce the amount of storage. By using the routine UPDATE, discussed above, the array sizes may be changed by changing the one PARAMETER statement card. For computing systems that do not allow a PARAMETER statement, remove the PARAMETER statement and replace the integers LI, MI, LI1, MI1, MQS, LTS, and MTS. in the common block, as well as the two cards defining LD and MD (following the NAMELIST statements in program VNAP2) with the desired values.

3. Film Plotting. The subroutine PLøT discussion in Sec. II.A describes the Los Alamos National Laboratory system routines used by this code. For other computing systems, the Los Alamos routines in subroutine PL $\emptyset \mathrm{T}$ will have to be replaced by comparable routines. On the other hand, if velocity vector and contour plots are not needed, then subroutine PLøT can be replaced by a dummy subroutine.

4. Single-Subscripted Arrays. Unlike VNAP, VNAP2 contains no single subscripting of arrays that are dimensioned with multiple subscripts, because most current Fortran compilers generate nearly as efficient a code with either single or multiple subscripts. For example, the single subscript version of VNAP2 was approximately 1 to $2 \%$ faster than the multiple subscript version using the CDC FTN 4.8 compiler. This small increase in efficiency did not seem to be worth the added complexity. 


\section{F. Artificial Viscosity Discussion}

The artificial viscosity model contains many parameters. However, in most cases the user needs to be concerned with only two, CAV and FDT. CAV controls the overall amount of smoothing and FDT controls the time step. If the space oscillations (that is, oscillations from point to point in the same time plane) are too large, then increase CAV. If the shock is too smeared, then decrease CAV. However, if the time oscillations (that is, oscillations at the same space point in different time planes) are too large, then decrease FDT. Increases in CAV often require decreases in FDT, whereas decreases in CAV often allow increases in FDT. For computation efficiency one uses large values of FDT and, therefore, small values of CAV. In calculations where FDT is too large, the solution usually "blows up" in less than 10 time steps. For calculations where CAV is too small, the solution usually takes longer to blow up. If FDT is smaller than necessary and CAV is larger than required, the solution will not blow up but, instead, will be inaccurate and inefficient. However, there is a lower limit of $\mathrm{FD}$ ( below which space osclllations will appear. The code includes an artificial viscosity contribution in the time step calculation and, therefore, a given value of FDT will usually suffice for a wide range of CAV.

As an example, an oblique shock produced by supersonic flow (Mach naumber $=3.2$ ) over a $30^{\circ}$ wedge (pressure ratio $=6.84$ ) required a CAV of 1.5 and an FDT of 0.8 . In general, stronger shocks require larger values of $\mathrm{CAV}$ and smaller values of FDT. The opposite is true for weaker shocks.

The artificial viscosity discussed above is intended for shocks and is very small for contact surfaces and zero for expansions. Because of this, if contact surfaces are present, additional smoothing is usually needed. This can be accomplished by using the sound speed gradient option (ISS $\neq 0$ ). For ISS $\neq 0$, the sound speed gradient is added to the velocity divergence. If ISS = 1 and the divergence of the velocity is $<0$, then the sound speed gradient is set equal to zero, which again mainly smooths only shocks. If ISS $=$ 2 , the sound speed gradient is always nonzero, which smooths shocks, contact surfaces, and, unfortunately, expansions. Therefore, for contact surfaces or dual flows with very different densities, use the ISS $=2$ option. The IDIVC $\neq 0$ (ISS $=1$ ) option could also be used, but here both the velocity divergence and the sound speed gradient are nonzero, causing additional smearing of any expansions that may he present.

Another problem concerning the artificial viscosity is the shock wave-boundary layer interaction. Here, the artificial viscosity that is necessary for the shock may swamp the molecular and turbulent viscosities in the boundary layer. To minimize this problem, the artificial viscosity depends on the velocity divergence and not the shear gradients. In addition, $\lambda_{\mathrm{A}}$ and $\mu_{\mathrm{A}}$ are multiplied by the Mach number squared in the subsonic part of the boundary layer. If this is not sufficient, the SMACH option can be used. There are no claims that this artificial viscosity model is the best way to treat shock wave-boundary layer interactions. It is to be hoped that additional work will produce better procedures.

\section{G. Sample Calculations}

1. Case No. 1: Subsonic Constant Area, Supersonic Source Flow. The geometry for this case is shown in Fig. 23 and consists of a constant area duct on top containing subsonic flow and a diverging duct on the bottom containing supersonic source flow. The data deck and printed output are presented in Figs. 24 and 25 , respectively.

a. Namelist CNTRL. This case uses a 21 by 11 mesh, therefore LMAX $=21$ and MMAX $=11$. The maximum number of time steps NMAX is set equal to 500. After 500 time steps, the supersonic flow is steady, but the subsonic flow is still changing slightly. Film plots of the final solution plane are requested by setting NPL $\emptyset \mathrm{T}=500$. A nondimensional set of units is used, so IUNIT $=1$. The gas constant for this nondimensional set of units is 0.01 ; therefore RGAS $=0.01$. So that the calculation will not be stopped before the number of time steps reaches NMAX, TST $\emptyset \mathrm{P}$ is increased to 100.0. The additional parameters are left equal to their default values. 
b. Namelist IVS. An initial-data surface that is subsonic in the upper flow space and supersonic in the lower is desired. Because this is not possible using the internally generated initial data, a general initial-data surface is read in. Therefore, N1D $=0$ and values for the arrays $U, V, P, R \emptyset, U L, V L, P L$, and R $\emptyset \mathrm{L}$ must be read in. All the values are assumed to be constant in each flow space. The additional parameters are left equal to their default values.

c. Namelist GEMTRY. The flow geometry for this case is 2D planar flow; therefore NDIM $=0$. The wall is a constant area duct; therefore $\mathrm{NGE} \emptyset \mathrm{M}=1$. The inlet location XI equals 0.0 , the exit location XE equals 4.0, and the radius RI equals 2.1547 . No other input is required.

d. Namelist GCBL. Because this case has no centerbody, no input is required.

e. Namelist BC. Because the lower flow-space inflow is supersonic and the upper flow space is subsonic, ISUPER $=2$. The stagnation pressure PT, stagnation temperature TT, and exit pressure PE for the upper flow space are $213.514,124.2$, and 180.0 , respectively. Values for the arrays UI, VI, PI, and RøI, as well as the variables UIL, VIL, PIL, and RØIL, are read in for the lower flow space. No other input is required.

f. Namelist $A V L$. Because there are no shocks and the initial data is smooth, no input is required.

g. Namelist $R V L$. Because the flow is inviscid, no input is required.

h. Namelist TURBL. Because the flow is inviscid, no input is required.

i. Namelist DFSL. For this case, the upper and lower dual-flow-space walls are specified by LMAX equally spaced values of $\mathrm{YL}$ and $\mathrm{YU}$ and the corresponding negative of their slopes NXNYL and NXNYU; therefore NDFS $=2$. The dual-flow-space walls begin at the inlet and end at the exit; therefore LDFSS $=1$ and LDFSF $=21$. The dual-flow-space walls correspond to the $M=6$ row of grid points; therefore MDFS $=6$. No other input is required.

j. Namelist VCL. Because a uniform grid is used, no input is required.

2. Case No. 2: Supersonic Source, Subsonic Constant Area Flow. This case is the same as Case No. 1, except that the lower dual-flow space is the subsonic constant area duct and the upper flow space is the supersonic source flow. The geometry is shown in Fig. 26. The data deck and printed output are presented in Figs. 27 and 28, respectively. Because the discussion for this case closely follows that of Case No. 1, it is not included here.

3. Case No. 3: Subsonic Airfoil. The geometry for this case is shown in Fig. 29 and consists of a $10^{\circ}$ double wedge airfoil between two solid walls. The data deck and printed output are presented in Figs. 30 and 31 , respectively.

a. Namelist $C N T R L$. This case uses a 21 by 11 mesh; therefore LMAX $=21$ and MMAX $=11$. The maximum number of time steps NMAX is set equal to 500. Film plots of the final solution plane are requested by setting NPL $\emptyset$ T equal to 500 . A nondimensional set of units is used, so IUNIT $=1$. The gas 
constant for this nondimensional set of units is 0.01 ; therefore RGAS $=0.01$. So that the calculation will not be stopped before the number of time steps reaches NMAX, TST $\emptyset \mathrm{P}$ is increased to 100.0. The additional parameters are left equal to their default values.

b. Namelist IVS. A subsonic initial-data surface is computed by the program, so N1D $=-2$. The Mach number everywhere is set by specifying the height for the area where the Mach number equals 1.0; therefore RSTAR $=0.7464$. No other input is required.

c. Namelist GEMTRY. The flow geometry for this case is $2 \mathrm{D}$ planar flow; therefore NDIM $=0$. The wall is a constant area duct, therefore NGE $\emptyset \mathrm{M}=1$. The inlet location $\mathrm{XI}=0.0$, the exit location $\mathrm{XE}=$ 4.0 , and the radius $R I=1.0$. No other input is required.

d. Namelist $G C B L$. The centerbody is a horizontal wall, and so $\mathrm{NGCB}=1$. The radius $\mathrm{RICB}=0.0$. No other input is required.

c. Namelist $B C$. The stagnation pressurc PT $=213.514$, the stagnation temperature $T T=124.2$, and the exit pressure $\mathrm{PE}=180.0$. No other input is required.

f. Namelist $A V L$. Because there are no shocks and the initial data is smooth, no input is required.

g. Namelist $R V L$. Because the flow is inviscid, no input is required.

h. Namelist TURBL. Because the flow is inviscid, no input is required.

i. Namelist DFSL. For this case, the upper and lower dual-flow-space walls are specified by 11 (LDFSF - LDFSS + 1) equally spaced values of YL and YU and the corresponding negative of their slopes NXNYL and NXNYU; therefore NDFS $=2$. The dual-flow-space walls begin at $L=6$ and end at $\mathrm{L}=16$, therefore LDFSS $=6$ and LDFSF $=16$. The dual-flow-space walls correspond to the $M=6$ row of grid points; therefore MDFS = 6. No other input is required.

j. Namelist $V \mathcal{C} L$. Because a unitorm grid is used, no input is required.

\section{ACKNOWLEDGMENTS}

This work was performed at the Los Alamos National Laboratory, Los Alamos, New. Mexico, and supported by the National Aeronautics and Space Administration, Langley Research Center, Hampton, Virginia, and the United States Department of Energy. The contract monitor was Richard G. Wilmoth of the Langley Research Center Propulsion Aerodynamics Branch. The author wishes to thank Richard G. Wilmoth, Lawrence E. Putnam, and K. Charles Swanson, Jr., of the Langley Research Center Propulsion Aerodynamios Branch, and Bart J. Daly, of the Los Alamos National Laboratory, for their many helpful discussions.

\section{REFERENCES}

1. M. C. Cline, "VNAP: A Computer Program for Computation of Two-Dimensional, TimeDependent, Compressible, Viscous, Internal Flow," Los Alamos Scientific Laboratory report LA-7326 (November 1978). 
2. M. C. Cline, "NAP: A Computer Program for the Computation of Two-Dimensional, TimeDependent, Inviscid Nozzle Flow," Los Alamos Scientific Laboratory report LA-5984 (January 1977).

3. R. W. MacCormack, "The Effect of Viscosity in Hypervelocity Impact Cratering," AIAA Hypervelocity Impact Conference, Cincinnati, Ohio, April 1969, AIAA Paper 69-354.

4. B. E. Launder, A. Morse, W. Rodi, and D. B. Spalding, "The Prediction of Free Shear Flows-A Comparison of the Performance of Six Turbulence Models," Proc. NASA Conf. Free Shear Flows, NASA Langley Research Center, Hampton, Virginia, July 1972 (NASA SP-321, 1973), Vol. I, pp. 361-426.

5. Tuncer Cebeci, A. M. O. Smith, and G. Mosinskis, "Calculation of Compressible Adiabatic Turbulent Boundary Layers," AIAA J. 8, 1974 (1970).

6. W. P. Jones and B. E. Launder, "The Prediction of Laminarization with a Two-Equation Model of Turbulence," Int. J. Heat Mass Transfer 15, 301 (1972).

7. W. P. Jones and B. E. Launder, "The Calculation of Low-Reynolds-Number Phenomena with a Two-Equation Model of Turbulence," Int. J. Heat Mass Transfer 16, 1119 (1973).

8. B. E. Launder and B. I. Sharma, "Application of the Energy-Dissipation Model of Turbulence to the Calculation of Flow Near a Spinning Disc," Lett. Heat Mass Transfer 1, 131 (1974).

9. K. Hanjalic and B. E. Launder, "Contribution Towards a Reynolds-Stress Closure for LowReynolds-Number Turbulence,” J. Fluid Mech. 74, 593 (1976).

10. T. J. Coakley and J. R. Viegas, "Turbulence Modeling of Shock Separated Boundary-Layer Flows," Symp. Turbulent Shear Flows, University Park, Pennsylvania, April 18-20, 1977.

11. C. M. Hung and R. W. MacCormack, "Numerical Solutions of Supersonic and Hypersonic Laminar Compression Corner Flows," AIAA J. 14, 475 (1976).

12. F. H. Harlow and A. A. Amsden, "A Numerical Fluid Dynamics Calculation Method for Ali Flow Specds," J. Comput. Phys. 8, 197 (1971).

13. R. W. MacCormack, "A Rapid Solver for Hyperbolic Systems of Equations," Lect. Notes Phys. 59, 307-317 (1976).

14. C. P. Kentzer, "Discretization of Boundary Conditions of Moving Discontinuities," Lect. Notes Phys. 8, 108-113 (1970).

15. G. Moretti, "The $\lambda$-Scheme," Comput. Fluids 7, 191 (1979).

16. R. A. Serra, "Determination of Internal Gas Flows by a Transient Numcrical Technique," AIAA J. 10,603 (1972).

17. J. Oliger and A. Sundström, "Theoretical and Practical Aspects of Some Initial Boundary Value Problems in Fluid Dynamics," SIAM J. Appl. Math. 35, 419 (1978). 
18. G. Moretti and M. Ábbett, "A Time-Dependent Computational Method for Blunt Body Flows," AIAA J. 4, 2136 (1966).

19. D. H. Rudy and J. C. Strikwerda, "A Nonreflecting Outflow Boundary Condition for Subsonic Navier-Stokes Calculations," J. Comput. Phys. 36, 55 (1980).

20. M. C. Cline, "Stability Aspects of Diverging Subsonic Flow," AIAA J. 18, 534 (1980).

21. G. Moretti, "Comment on Stability Aspects of Diverging Subsonic Flow," AIAA J. 19 (May 1981).

22. M. C. Cline, "Reply by Author to G. Moretti," AIAA J. 19, (May 1981).

23. G. Moretti and M. Pandolfi, "On the Calculation of Subsonic Flows in Ducts," Polytechnic Institutc of New York report M/AL 8U-18 (June 1980).

24. M. C. Cline and R. G. Wilmoth, "Computation of High Reynolds Number Intcrnal/Extcrnal Flows," AIAA 14th Fluid Plasma Dyn. Conf., Palo Alto, California, June 23-25, 1981, AIAA Paper 81-1194.

25. M. C. Cline, "Computation of Steady Nozzle Flow by a Time-Dependent Method," AIAA J. 12, 419 (1974).

26. R. F. Cuffel, L. H. Back, and P. F. Massier, "Transonic Flow-Field in a Supersonic Nozzle with Small Throat Radius of Curvature," AIAA J. 7, 1364 (1969).

27. D. E. Reubush, "Experimental Study of the Effectiveness of Cylindrical Plume Simulators for Predicting Jet-On Boattail Drag of Mach Numbers up to 1.30," NASA TN D-7795, 1974.

28. R. C. Swanson, Jr., "Numerical Solutions of the Navier-Stokes Equations for Transonic Afterbody Flows," NASA Technical Paper 1784 (Decembcr 1980).

29. L. J. S. Bradbury, "The Structure of a Self-Preserving Turbulent Plane Jet," J. Fluid Mech. 23, 31 (1965). 

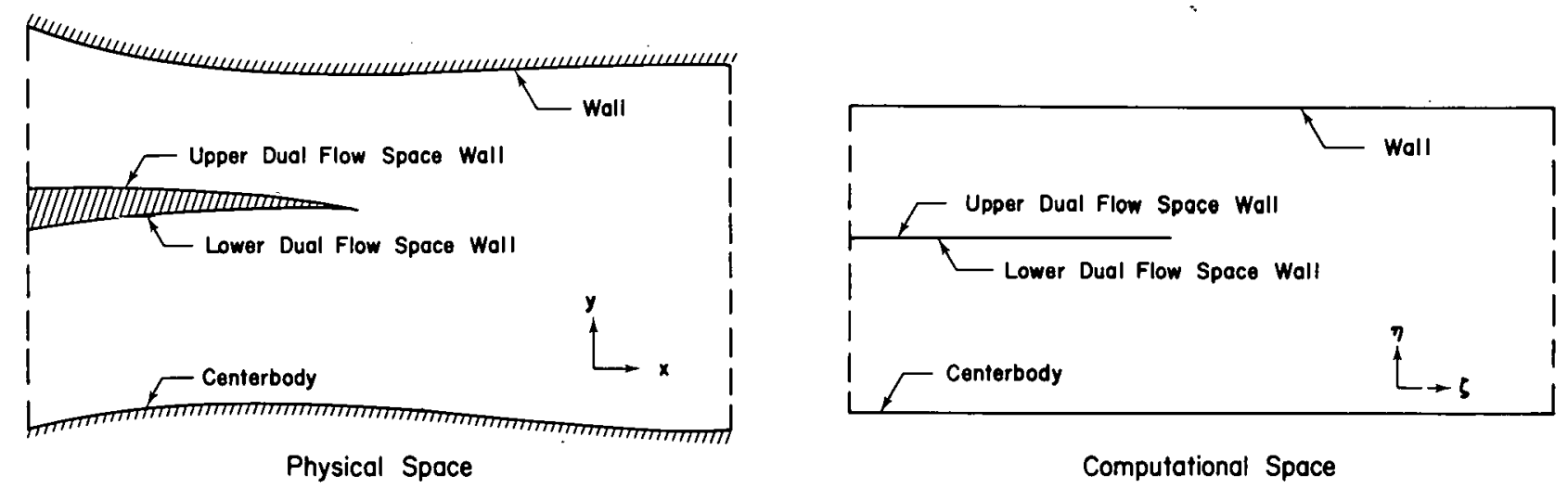

Fig. 1.

Physical and computational spaces.

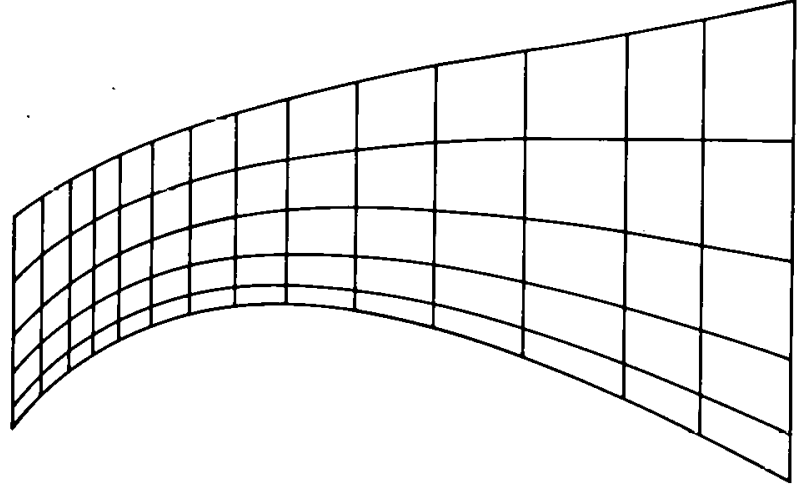

Fig. 2.

Physical space grid.

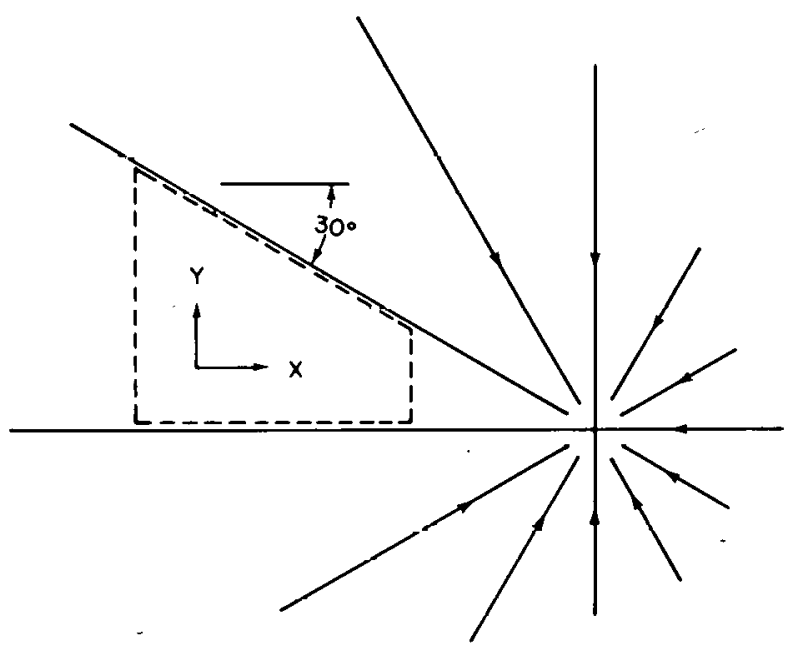

Fig. 4.

Planar subsonic sink flow.

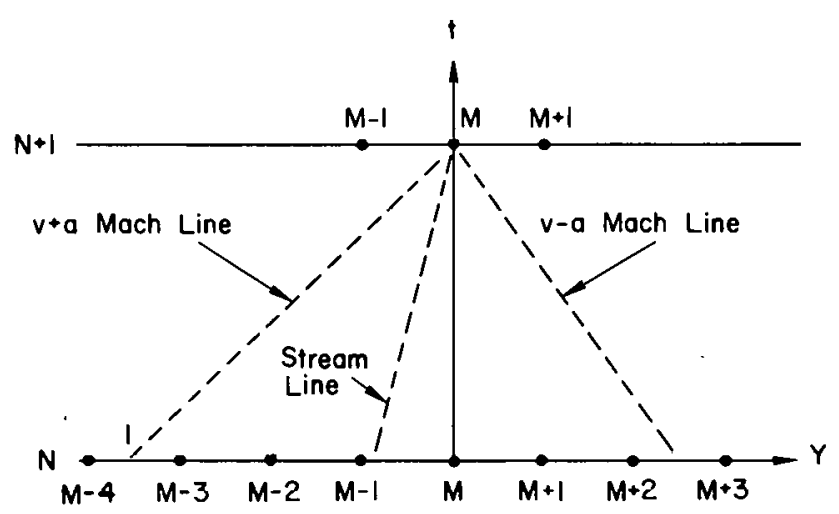

Fig. 3.

Quick Solver characteristic grid.

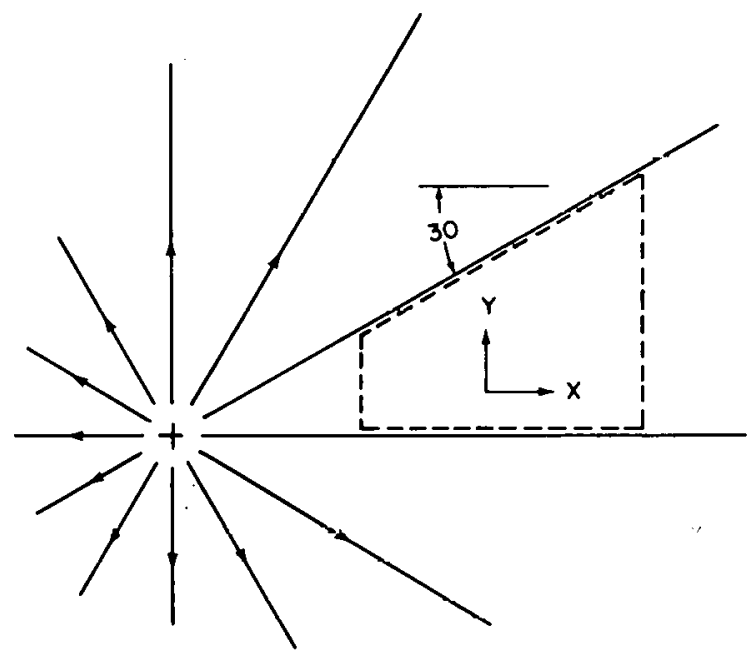

Fig. 5.

Planar supersonic source flow. 


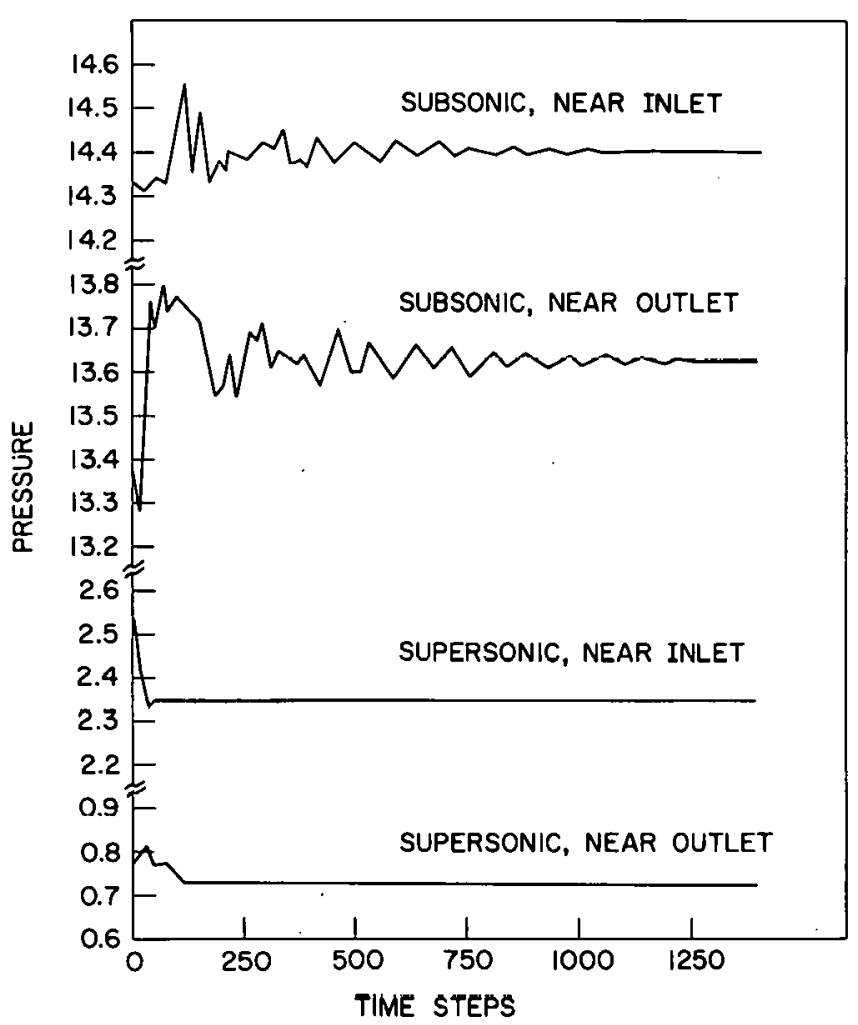

Fig. 6.

Subsonic sink and supersonic source flow solutions.

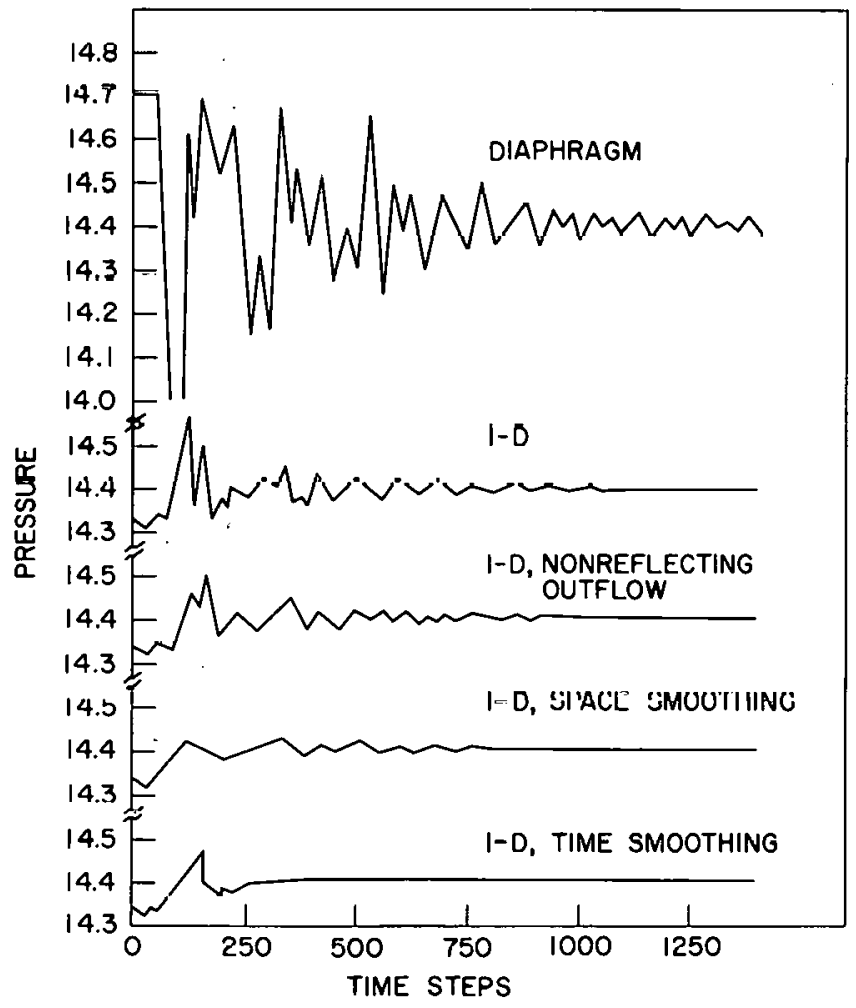

Fig. 7.

Subsonic sink flow with the $\mathrm{p}_{T}, \mathrm{~T}_{\mathrm{T}}$, and $\theta$ inflow boundary condition.

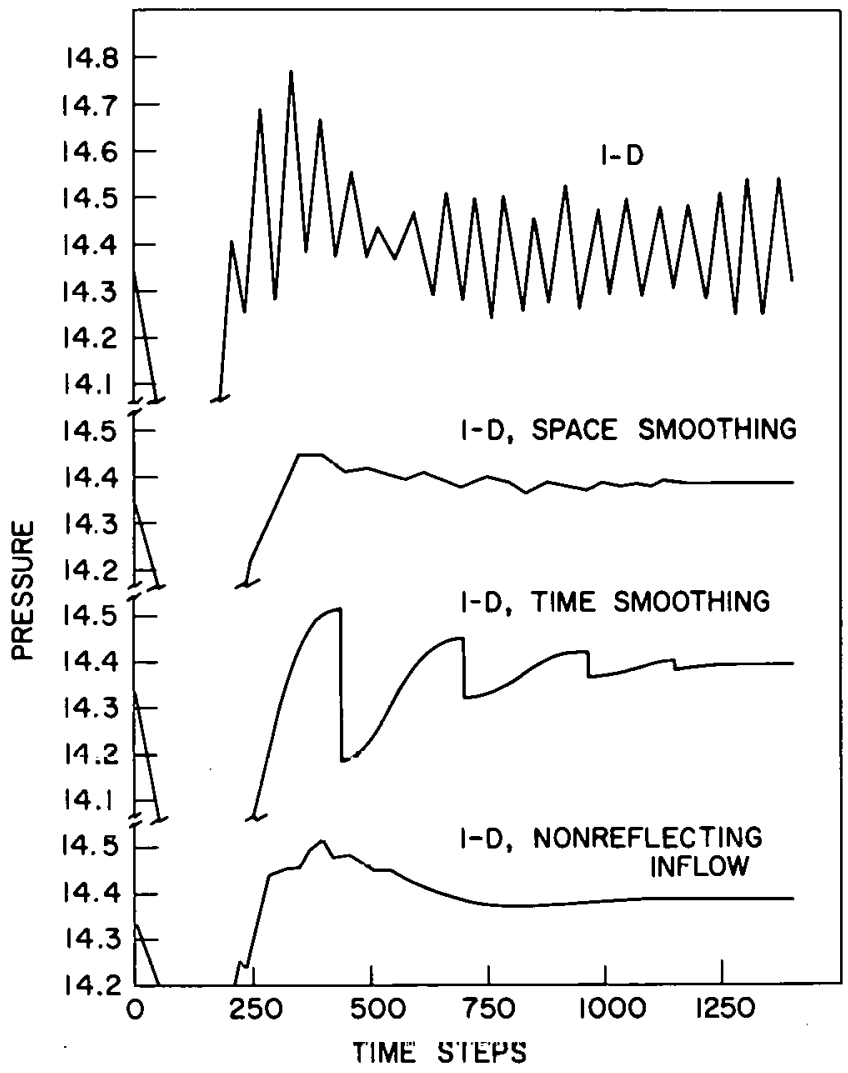

Fig. 8.

Subsonic sink flow with the $u, v$, and $\rho$ inflow boundary condition.

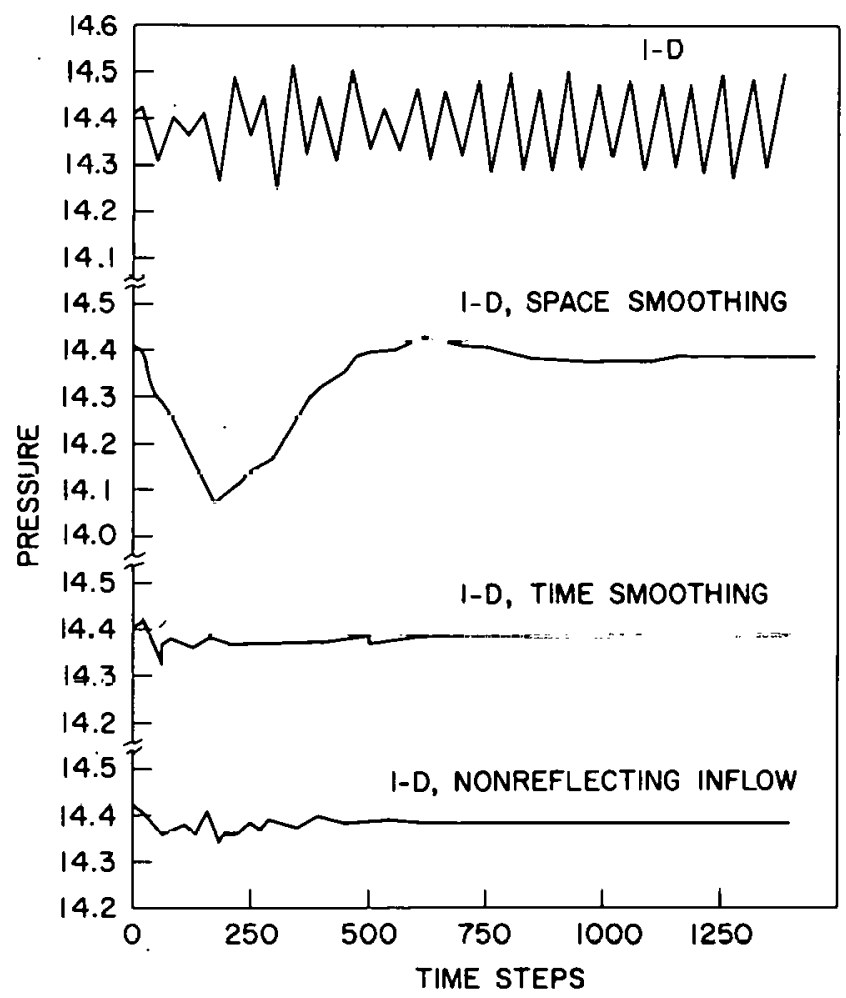

Fig. 9.

Subsonic sink flow with the $u, v$, and $\rho$ inflow boundary condition and matched mass flow, 1D, initial-data surface. 


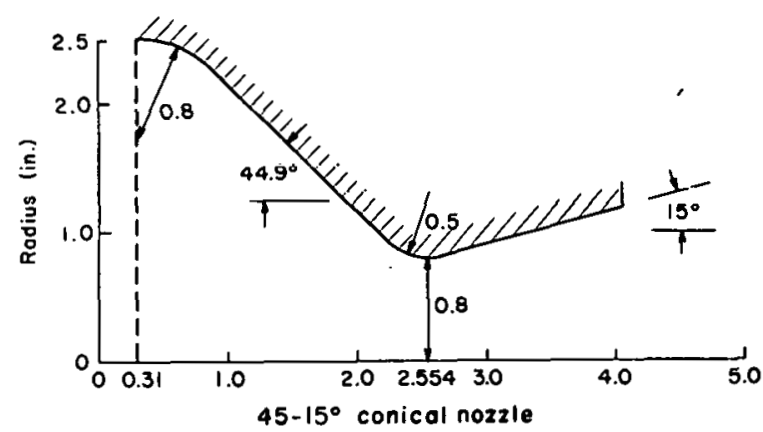

Fig. 10.

Nozzle geometry for Case No. 1.

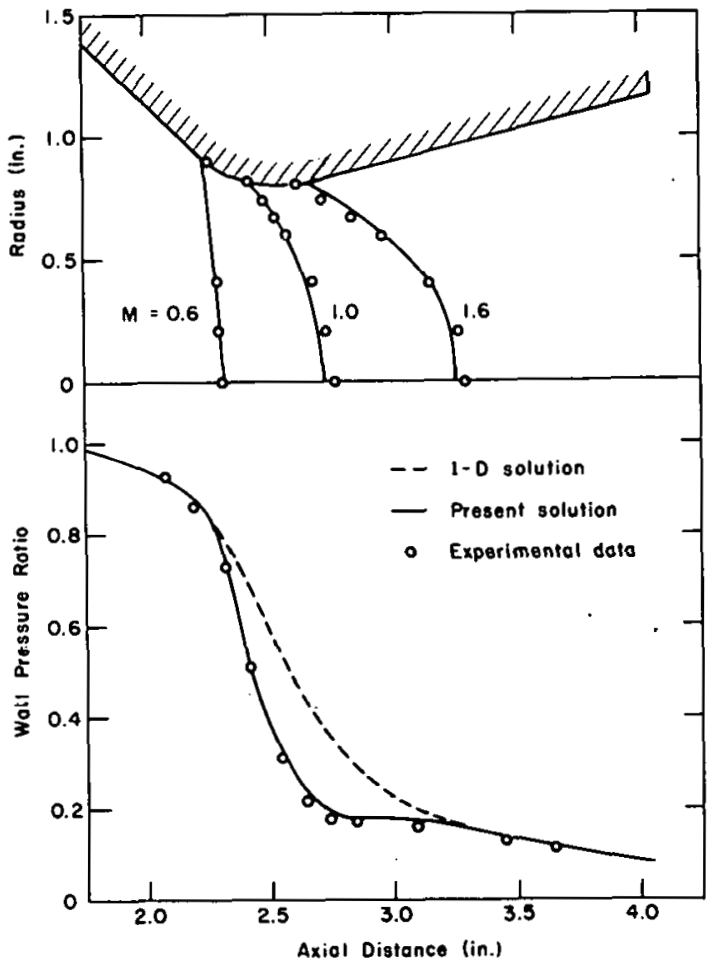

Fig. 11.

Mach number contours (top) and wall pressure ratio for Case No. 1.

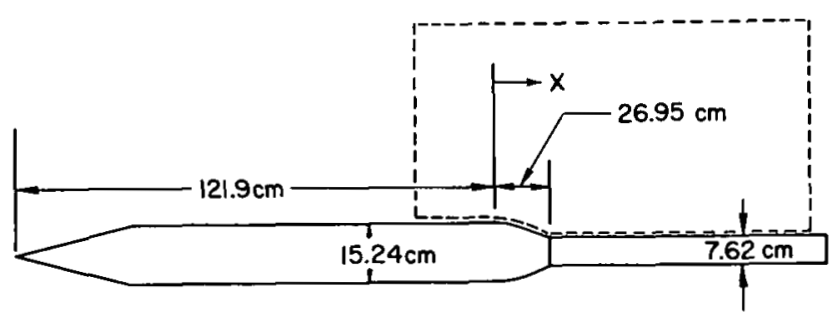

Fig. 12.

Boattail afterbody geometry for Case No. 2.
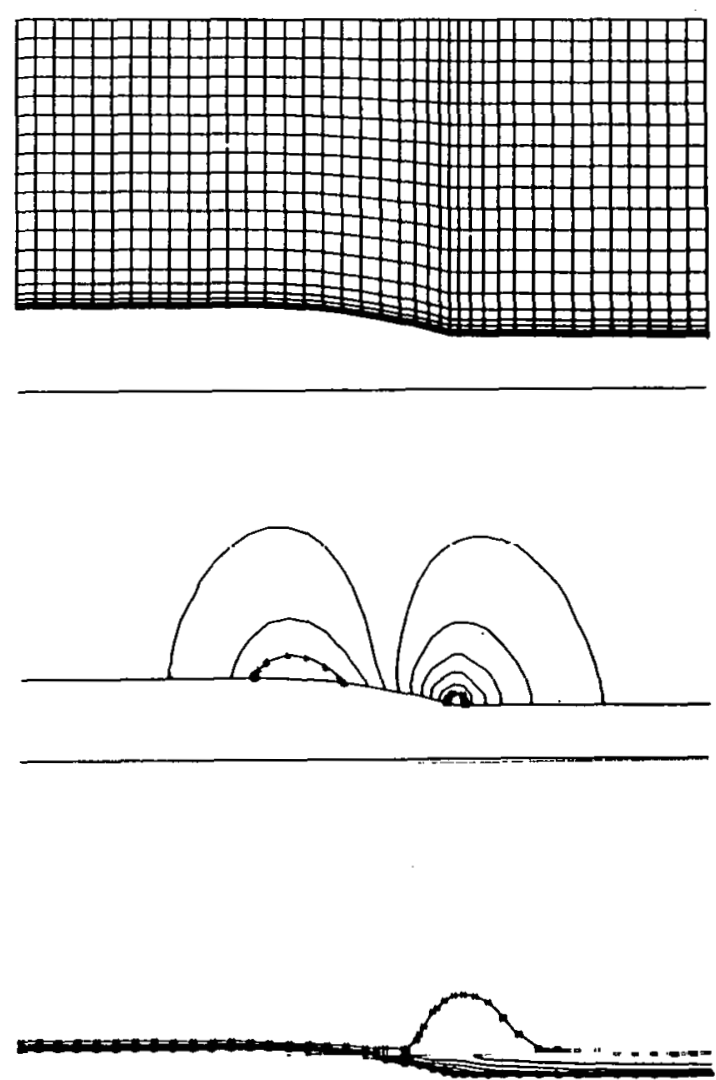

Fig. 13.

Physical space grid (top), pressure (middle), and Mach number contours for Case No. 2. 


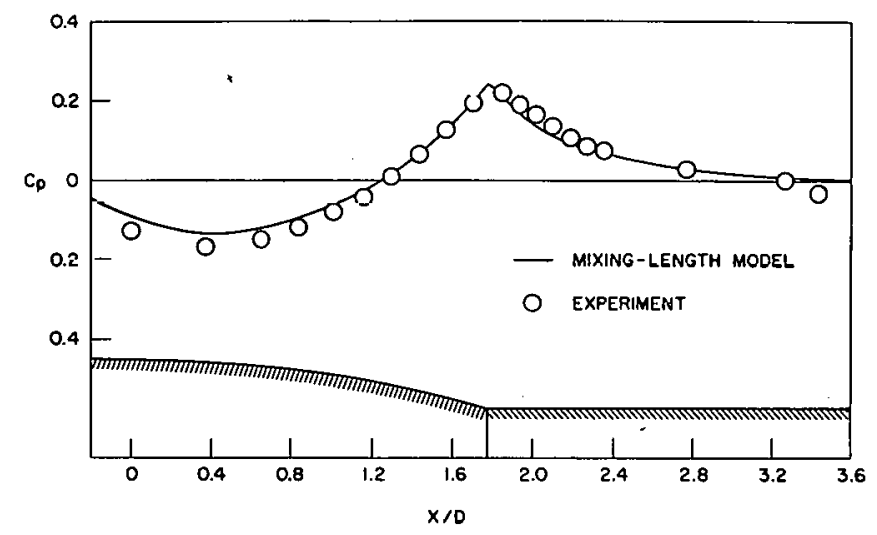

Fig. 14.

Surface pressure coefficient for Case No. 2.

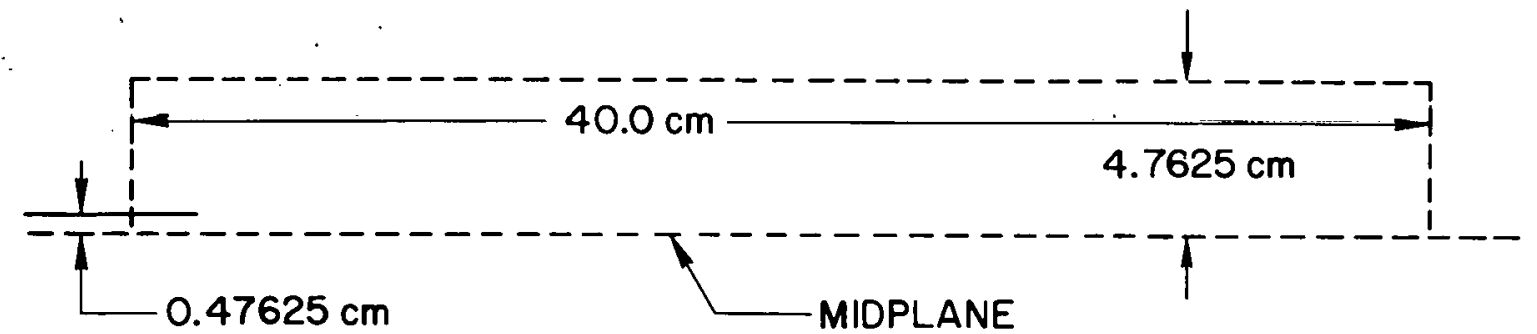

Fig. 15.

Plane jet geometry for Case No. 3.

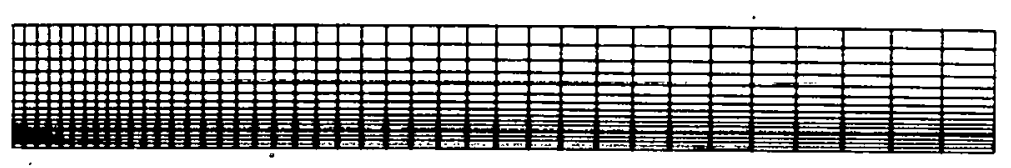

Fig. 16.

Physical space grid (top) and Mach number contours for Case

No. 3. 


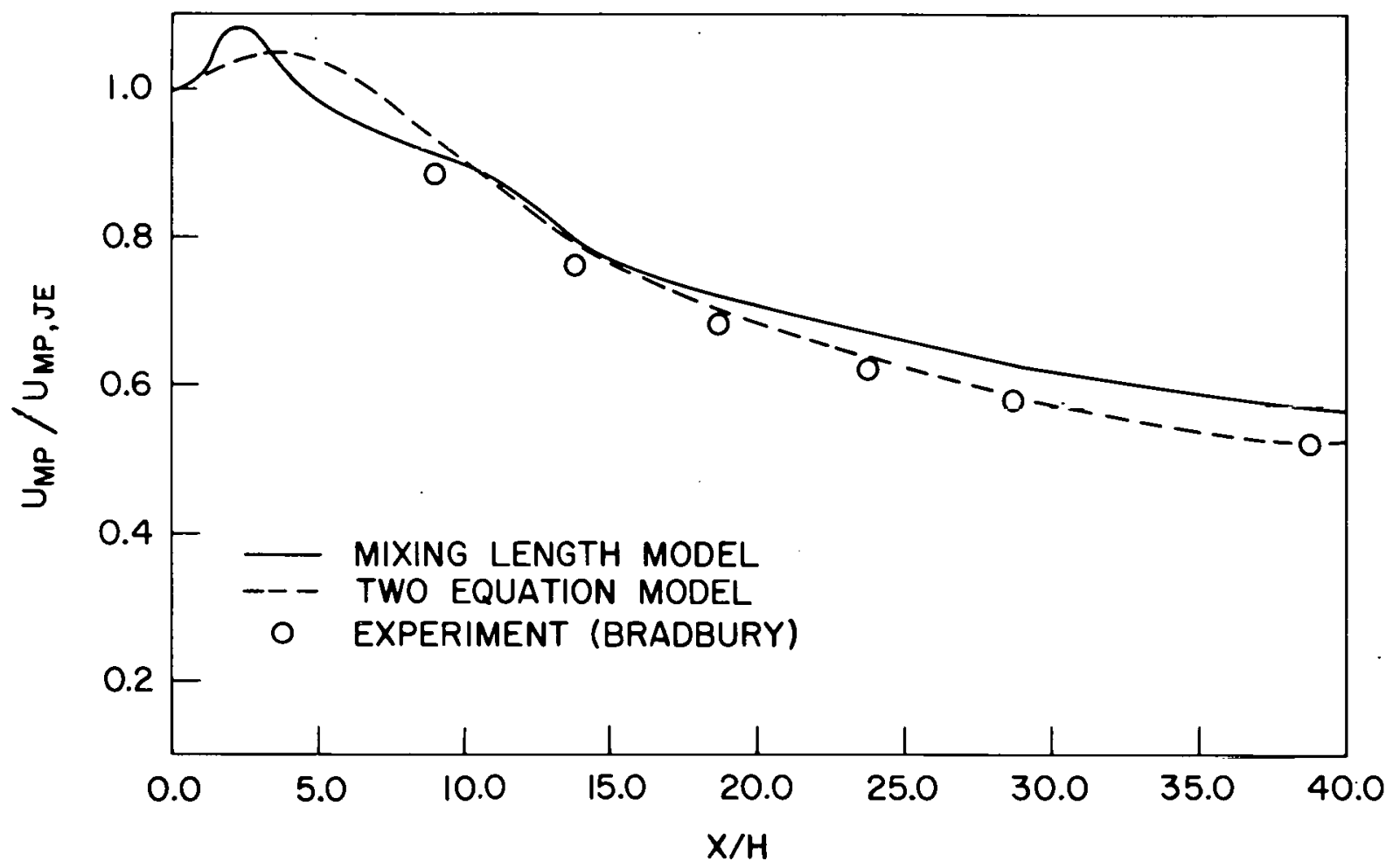

Fig. 17.

Midplane velocity decay for Case No. 3.

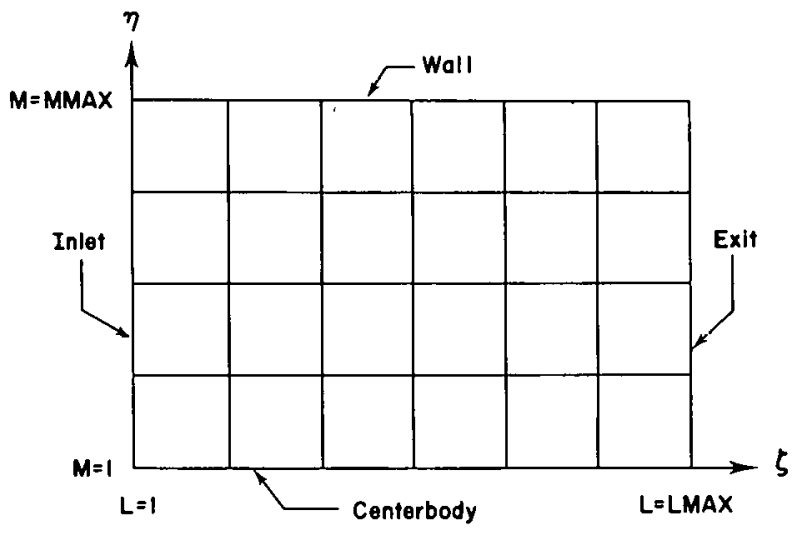

Fig. 18.

Single-flow-space computational grid.

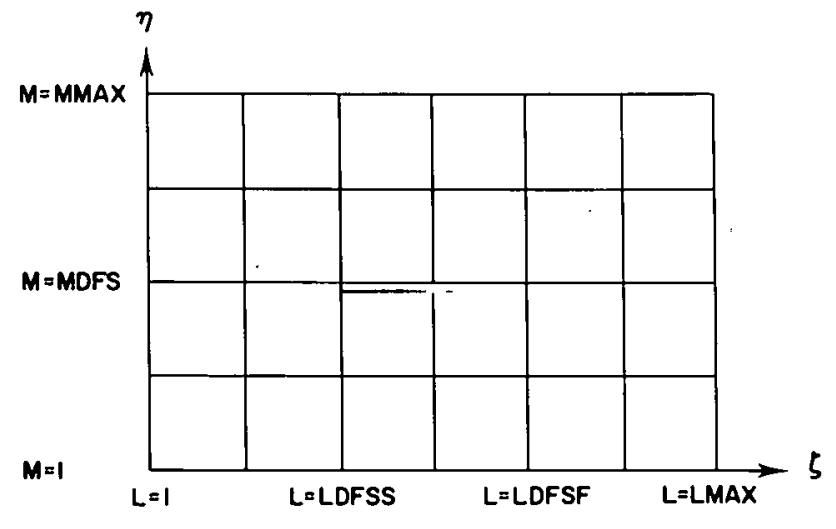

Fig. 19.

Dual-flow-space computational grid. 


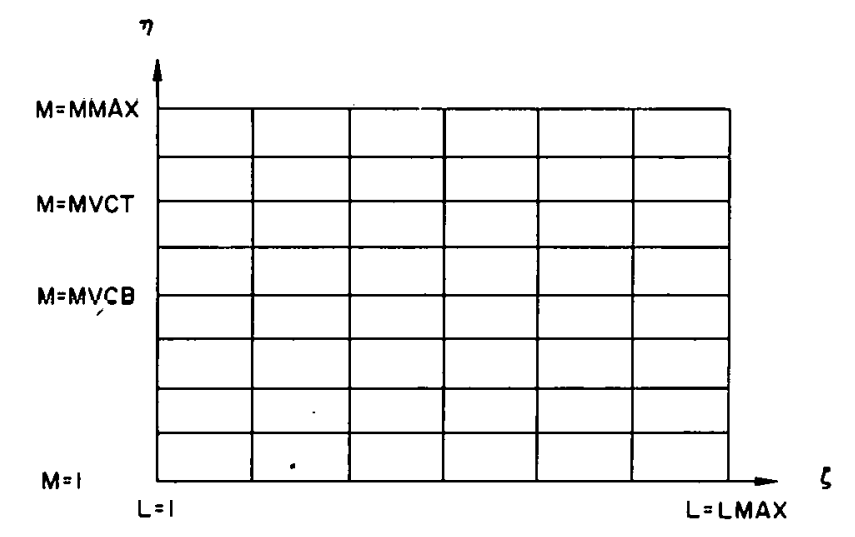

Fig. 20.

Subcycled computational grid.

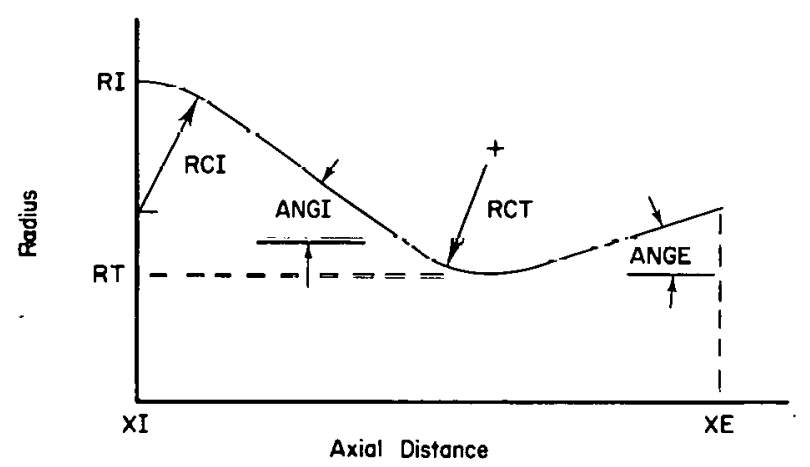

Fig. 21.

Circular-arc, conical wall geometry.

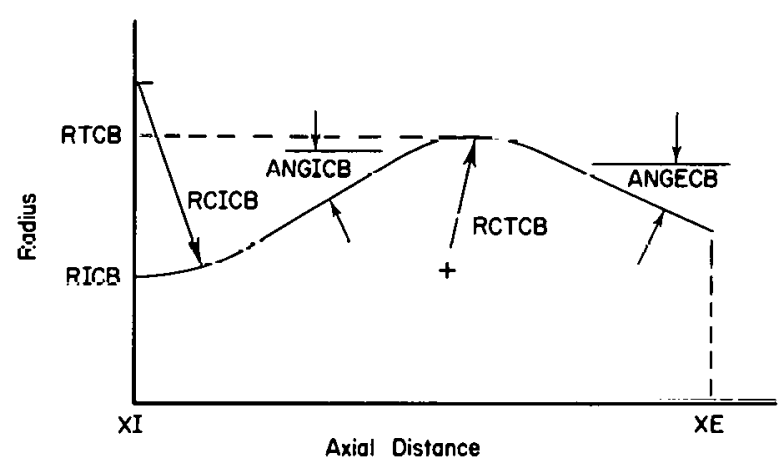

Fig. 22.

Circular-arc, conical centerbody geometry. 


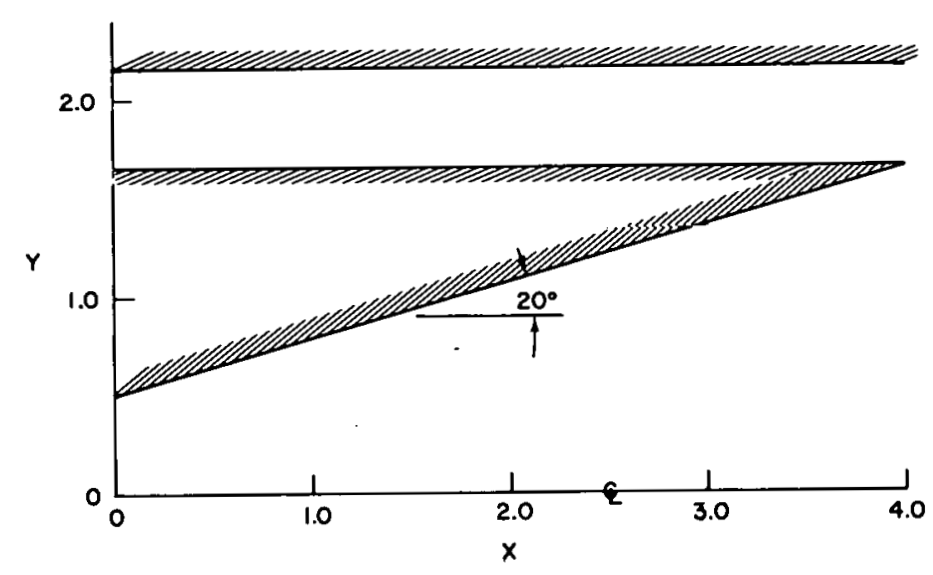

Fig. 23.

Case No. 1 geometry.

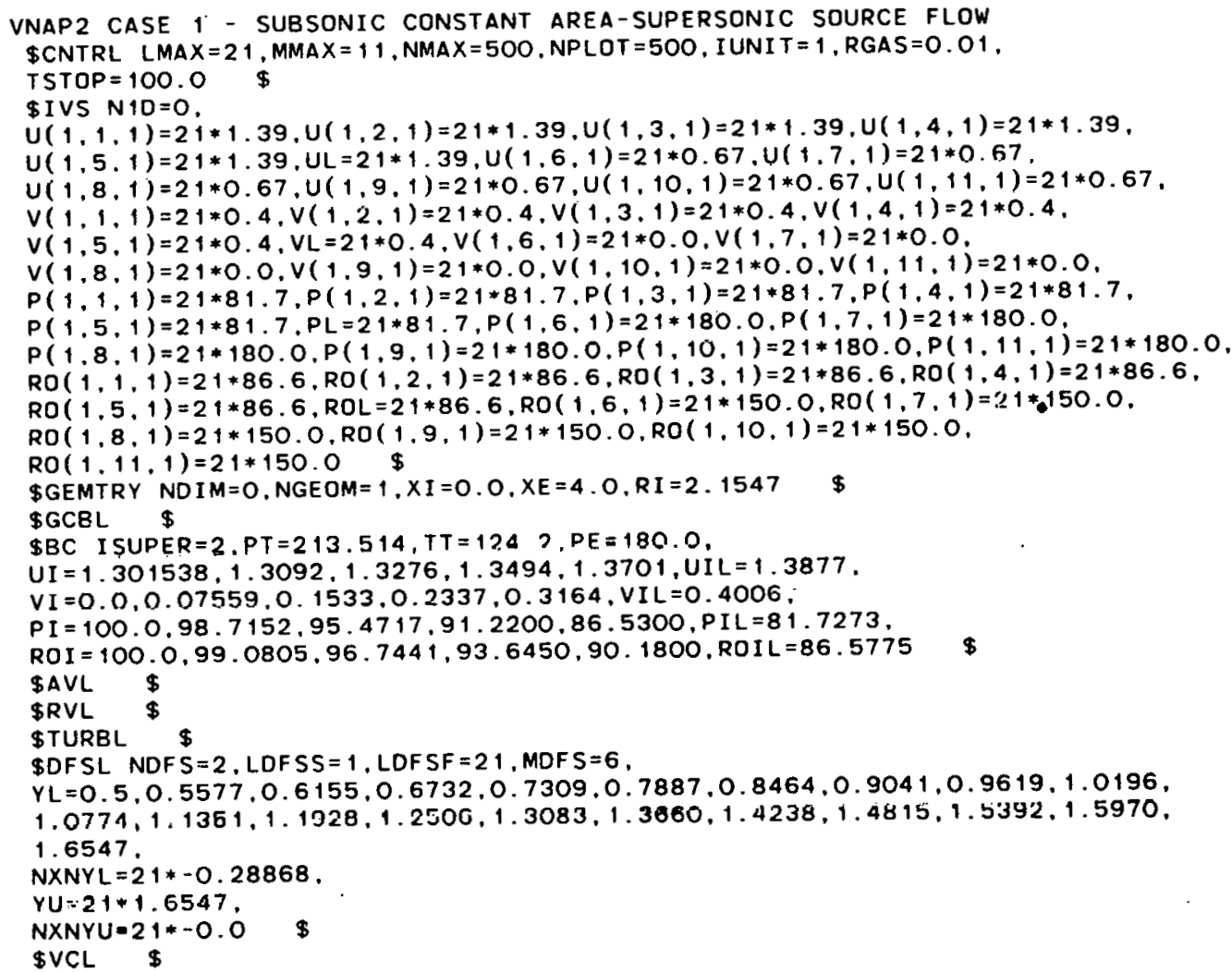

Fig. 24.

Case No. I data deck. 
VNAP2, A COMPUTER PROGRAM FOR THE COMPLTATION OF TWO-DIMENSIONAL, TIME-DEPENDENT, CJMPZESSIBLE. TURBULENT FLOW BY MICHAEL C. CL:NE. T-3 - LOS alamOS NATIONAL LaBORATJRY

PROGRAM ABSTRACT -

THE NAVIER-STOKES EQUATIONS FOR TWO-DIMENSIONAL. TIME-DEPENDENT FLOW ER SOLVED USING THE SECOND-ORDEF, MACCORMACK FINITE-LIFFERENCE SCHEME. ALL BOUNDARY CONDITIGIS ARE COMPUTED USING A SECOND-DRCIER, REFERENCE PLANE, GHARAC-ERISTIC SCHEME WITH THE VISCOUS TERMS TREATED AS SOURCE FUNCTIONS. THE FLUID IS ASSUMED TO BE A PE RFECT GAS. THE STEADY-STATE SOLLTION IS OBTAINED AS THE ASYMPTOIIC SOLUTION =OR LARG TIME THE FLOW BOUNDARIES MAY BE ARBITRAFY CURVED SOLID WALES AS WELL AS IET ENVELOPES. THE GEOMETR* MAY CONSIST OF SINGLE AND DUAL FLOUIVG STREAMS. TURBULENCE EFFECTS ARE MODELED WITH EITHER \& MIXING-LEVGTH, A TURBULENCE ENERGY EOUAT:CN. OR A TURBULENCE ENERGY-DISSIPATION RATE EQUA-IONS NODEL. THIS PROGRAM ALLOWS VARIABLE GFIO SPACING AND INCLUDES OPTIONS TO SPEED UP THE CALCULATION FOR HIGH REYNOLDS NUMBER FLO'NS.

JOB TITLE -

VNAP2 CASE 1 - SUBSONIC CONSTANT AFEA-SUPERSONIC SOURCE FLOW

CCNTROL. PARAMETERS -

\begin{tabular}{|c|c|c|c|c|c|c|c|c|c|c|}
\hline $\begin{array}{l}\operatorname{LMAX}=21 \\
\text { IUI }=\uparrow \\
\text { RSTAR }=\end{array}$ & $\begin{array}{l}\text { MMAX }=11 \\
\text { IUD }=1 \\
0.000000\end{array}$ & $\begin{array}{l}\text { NMAX }=500 \\
\text { IVPTS }=1 \\
\text { RSTARS }=\end{array}$ & $\begin{array}{l}\text { NPR I NT = } \\
\text { NCIONVI = } 1 \\
0 . \text { DOOOOOD }\end{array}$ & 0 & $\begin{array}{l}\text { NPLOT }= \\
\text { TSTOP = } \\
\text { PLOW }=\end{array}$ & $\begin{array}{l}500 \\
.10 E+03 \\
0100\end{array}$ & $\begin{array}{l}\text { FDT }=.90 \\
\text { N1D }=0 \\
\text { ROLOW }=\end{array}$ & $\begin{array}{l}\text { FDT } i=100 \\
\text { TCORV }=6.000 \\
.0001 .50\end{array}$ & $\begin{array}{l}\text { FDT I }=.90 \\
\text { NASM }=1 \\
\text { VDT }=.25\end{array}$ & $\begin{array}{l}\text { IPUNCH }=0 \\
\text { IUNI T }=1 \\
\text { VDT } 1=.25\end{array}$ \\
\hline
\end{tabular}

FLUID MODEL -

THE RATIO OF SPECIFIC HEATS, GAMMA $=1.4000$ AND THE GAS CONSTANT, $R=$. OMTO $(F T-L B F / L B M-R)$

FLOW GEOMETRY -

TWO-DIMENSIONAL, PLANAR FLOW HAS BEEN SPECJFIED

DUCT GEOMETRY -

A CONSTANT AREA DUCT HAS BEEN SPECI =IED BY XI $=0.0000$ (IN), RI $=2.1547$ (IN), AND XE $=4.0000$ (IN)

Fig. 25.

Casa No. 1 output. 
DUAL FLOW SJACE BOUNDARY GEQMETRY -

GENERAL BOUNDARIES HAVE BEEN SPECIFIED BY THE FOLLOWING PARAMETERS,

\begin{tabular}{|c|c|c|c|c|c|}
\hline L & $X P(I N J)$ & $Y L(I N)$ & SLOPEL & $\mathrm{YU}(\mathrm{IN})$ & SLOPEU \\
\hline 4 & 0.0000 & .5000 & .2887 & 1.6547 & 0.0000 \\
\hline 2 & 2000 & .5577 & .2887 & 1.6547 & 0.0000 \\
\hline 3 & 4000 & .6155 & .2887 & 1.6547 & 0.0000 \\
\hline 4 & 6000 & .6732 & .2887 & 1.6547 & 0.0000 \\
\hline 5 & .8000 & .7309 & .2887 & 1.6547 & 0.0000 \\
\hline 6 & 1.0000 & .7887 & .2887 & 1.6547 & 0.0000 \\
\hline 7 & 1.2000 & .8464 & .2887 & 1.6547 & 0.0000 \\
\hline $\mathbf{B}$ & 1.4000 & .9041 & 2887 & 1.6547 & 0.0000 \\
\hline 9 & 1.6000 & .9619 & 2887 & 1.6547 & 0.0000 \\
\hline 12 & 1.8000 & 1.0196 & .2887 & 1.6547 & 0.0000 \\
\hline 11 & 2.0000 & 1.0774 & .2887 & 1.6547 & 0.0000 \\
\hline 12 & 2.2000 & 1. 1351 & .2887 & 1.6547 & 0.0000 \\
\hline 13 & 2.4000 & 1.1928 & .2887 & 1.6547 & 0.0000 \\
\hline 14 & 2.6000 & 1.2506 & .2887 & 1.6547 & 0.0000 \\
\hline 15 & 2.8000 & 1. 3083 & .2887 & 1.6547 & 0.0000 \\
\hline 16 & 3.0000 & $1.36 \in 0$ & .2887 & 1.6547 & 0.0000 \\
\hline 17 & 3.2000 & 1.4238 & 2887 & 1.6547 & 0.0000 \\
\hline 18 & 3.4000 & 1.4815 & 2887 & 1.6547 & 0.0000 \\
\hline 19 & 3.6000 & 1.5392 & 2887 & 1.6547 & 0.0000 \\
\hline 20 & 3.8000 & 1.5970 & .2887 & 1.6547 & 0.0000 \\
\hline 21 & 4.0000 & 1.6547 & .2887 & 1.6547 & 0.0000 \\
\hline
\end{tabular}

Fig. 25. (cont) 
BOUNDAFY CONDITIONS -

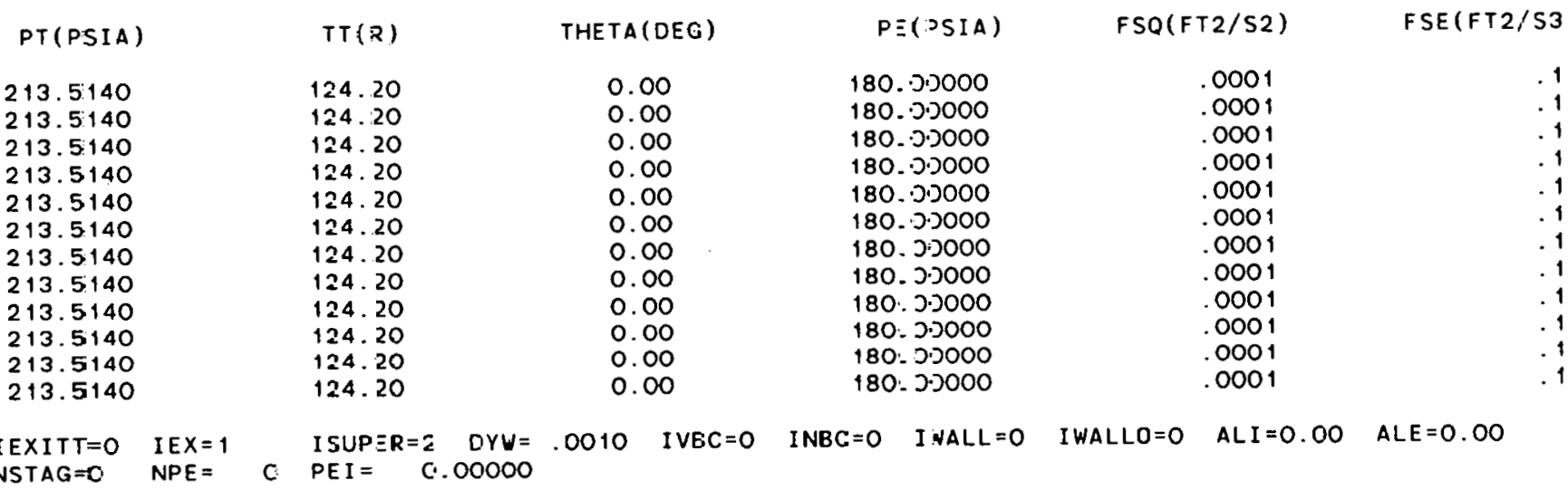
$A L W=0.00$ NSTAG=0 NPE $=$ C PEI $=$ C. .00000

FREE-SLI? WALLS ARE SPECIFIED

ADIABATI: UPPER WALL IS SPECIFIED

ADIABATIZ LOWER DUAL FLOW SFACE BOUNDARY IS SPECIFIED

ADIABATI: UPPER DUAL FLOW SFACE BOIJNJARY IS SPECIFIED

ARTIFICAL VISCOSITY -

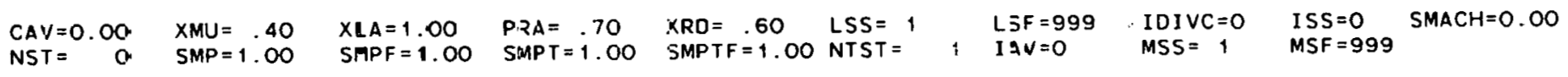

MOLECULAR VISCOSITY -
$\mathrm{CMU}=0$.
$\left(L B F-S / F^{-2}\right) \quad$ CLA $=0$.
(LBF-S/FT2: $\quad C K=0$.
(LBF:S-R) EMU $=0.00 \quad E L A=0.00 \quad E K=0.00$

TURBULENCE MODEL -

NO MODEL IS SPECIFIED

VARIABLE GRID PARAMETERS -

IST $=0 \quad M V C B=0 \quad M V C T=0 \quad I O S=0 \quad$ NIOSS $=2 \quad$ NIOSF $=0 \quad N V C A I=0 \quad I-L O S=30 \quad S O S=.50 \quad$ COS $=.001$

\begin{abstract}
***** EXPECT FILN OUTPUT FOR $N=$ O*****
\end{abstract}
$\begin{array}{lllll}N= & 10, & T= & .67077118 & \text { SECONDS } \\ N= & 20, & T= & 1.35994032 & \text { SECONDS } \\ N= & 30, & T= & 1.92866943 & \text { SECONDS } \\ N= & \angle 0, & T= & 2.52996808 & \text { SECONDS } \\ N= & 50, & T=3.11792749 & \text { SECONDS } \\ N= & 60, & T=3.69420884 & \text { SECONDS } \\ N= & 70, & T=4.25870480 & \text { SECONDS } \\ N= & 80, & T=4.82666822 & \text { SECONDS }\end{array}$
$D T=.06533051$ SECONDS, $\quad$ NVCM $=1, \quad$ CNUMS $=1.00,(5,2),(0,0)$

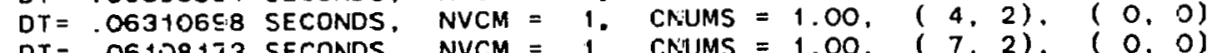
DT $=.061 .28173$ SECONDS, $\quad$ NVCM $=1$. CNUMS $=1.00, \quad(7,2) \cdot(0,0)$ DT $=.05947960$ SECONDS, $\quad$ NVCM $=1$. CNUMS $=1.00, \quad(9,2), \quad(0,0)$

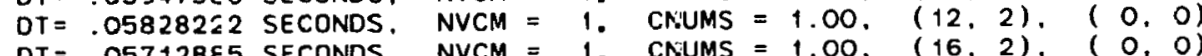
OT $=.057128 E 5$ SECONOS, $\quad$ NVCM $=1, \quad$ CNUMS $=1.00, \quad(16,2), \quad(0,0)$ DT $=.05753545$ SECONDS, $\quad$ NVCM $=1, \quad$ CNUMS $=1.00, \quad(20,2), \quad\left(\begin{array}{ll}0 & 0\end{array}\right)$ 


\begin{tabular}{|c|c|c|c|c|c|c|c|c|c|c|c|c|c|c|c|c|}
\hline 0 & 90. & $T=$ & $\begin{array}{l}5.40287119 \\
5.97823303\end{array}$ & . & $T=$ & 53144 & 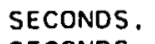 & Aus & & 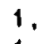 & CNUMS & $=1.00$ & 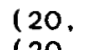 & 2). & o. & 0) \\
\hline $\begin{array}{l}N= \\
N=\end{array}$ & $\begin{array}{l}100 . \\
110 .\end{array}$ & $\begin{array}{l}T= \\
T=\end{array}$ & $\begin{array}{l}5.97823303 \\
6.55370146\end{array}$ & IDS . & $\begin{array}{l}D T= \\
D T=\end{array}$ & $\begin{array}{l}55439 \\
54307\end{array}$ & SECONDS, & NVCM & & $1.2 \mathrm{~s}$ & CNUMS & 1.00 & (20. & & o, & 0) \\
\hline$N=$ & 120. & $T=$ & $\begin{array}{l}6.55370146 \\
7.12915448\end{array}$ & SECONDS & $\begin{array}{l}D T= \\
D T=\end{array}$ & $\begin{array}{l}.05754307 \\
.05754566\end{array}$ & $\begin{array}{l}\text { SECONDS, } \\
\text { SECONDS }\end{array}$ & $\begin{array}{l}\text { NVCM } \\
\text { NVCM }\end{array}$ & $=$ & 1. & CNUMS & $=1.00$. & (20, & 2). & o. & 0) \\
\hline$N=$ & 130. & $T=$ & 7.70460850 & SECONDS, & $\mathrm{DT}=$ & $\begin{array}{l}.05754566 \\
.05754561\end{array}$ & $\begin{array}{l}\text { SECONDS, } \\
\text { SECONDS, }\end{array}$ & $\begin{array}{l}\text { NVCM } \\
\text { NVCM }\end{array}$ & $=$ & 1. & $\begin{array}{l}\text { CNUMS } \\
\text { CNUMS }\end{array}$ & $\begin{array}{l}=1.00 . \\
=1.00 .\end{array}$ & $\begin{array}{l}(20 . \\
(20 .\end{array}$ & 2). & o. & b) \\
\hline$N=$ & 140 & $\mathrm{~T}$ & 6455 & SECONDS, & $D T=$ & .05754551 & SECONDS, & NVCM & $=$ & 1. & CNUMS & $=1.00$ & (20. & 2). & 0. & $\begin{array}{l}\text { 0) } \\
\text { 0) }\end{array}$ \\
\hline$N=$ & 150. & $T=$ & 8.85551982 & NDS, & $D T=$ & .05754552 & SECONDS. & JVCM & $=$ & 1. & CNUMS & 1.00 & (20. & & 0 & 0) \\
\hline$N=$ & 160. & $T=$ & 477 & NDS, & $D T=$ & .05754549 & SECONDS. & NVCM & $=$ & 1. & CNUMS & $=1.00$. & 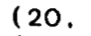 & & 0 . & o) \\
\hline$N=$ & 170. & $T=$ & 10.00642973 & SECONDS, & $\mathrm{DT}=$ & .05754550 & SECONDS. & VCM & $=$ & 1. & IUMS & 1.00 & (20. & 2 & 0. & b) \\
\hline$N=$ & 180 & $T=$ & 69 & & $\mathrm{DT}=$ & .05754550 & CONDS & VCM & $=$ & 1. & NUMS & $=1.00$. & $(20$ & & 0. & \\
\hline $\begin{array}{l}N= \\
N=\end{array}$ & 1 & $=$ & 11.15733965 & os. & $\mathrm{DT}=$ & .05754550 & SECONDS. & VCM & $=$ & 1 & & 1.00 & (20. & & 0. & 0) \\
\hline $\begin{array}{l}N= \\
N=\end{array}$ & $\tau$ & $T=$ & 11.732 & DS. & $D T=$ & 54550 & CONDS & VCM & $=$ & 1 & CNUMS & $=1.00$. & (20, & & 0 . & \\
\hline $\begin{array}{l}N= \\
N=\end{array}$ & & $T$ & 12.30824958 & SECONDS, & $D T=$ & .05754550 & SECONDS, & VCM & $=$ & 1 , & UMS & $=1.00$. & & & 0 . & 0) \\
\hline $\mathbf{N}=$ & 220 & $T=$ & 455 & os. & $\mathrm{DT}=$ & 4550 & & VCM & $=$ & 1 & MS & $=1.00$. & 20. & 2), & 0. & 0) \\
\hline$N=$ & 230 & $=$ & 13. & & $D T=$ & 550 & SECC & IVCM & $=$ & 1. & CNUMS & $=4.0$ & 20. & & 0. & ) \\
\hline$N=$ & 2 & $=$ & 14. & NDS, & $\mathrm{DT}=$ & .05754550 & SEC & NVCM & $=$ & 1. & & $=1 . \mathrm{C}$ & 20 . & & 0. & o) \\
\hline$N=$ & 50 . & $T$ & 14. & Ds. & $D T=$ & 550 & 5 & VCM & $=$ & 1. & JMS & $=1$. & . & & 0. & ) \\
\hline$N=$ & 2 & $T$ & 15. & S, & $\mathrm{DT}=$ & 550 & ECONDS, & CM & $=$ & 1. & & & & & 0 & 0) \\
\hline$N=$ & 270. & $T=$ & 15.7 & DS. & $D T=$ & 50 & 5. & $C M$ & - & 1. & UMS & $=1.00$. & 20 & & 0 . & ) \\
\hline$N=$ & 2 & $T=$ & 16. & & $D T=$ & 50 & SEC & IVCM & $=$ & 1. & JMS & $=$ & & & 0 , & ) \\
\hline$N=$ & $29 C$ & $T=$ & 16. & os. & $D T=$ & 1550 & SECO & NVCM & $=$ & 1. & IUMS & $=1.00$, & 20 . & 2) & 0. & ) \\
\hline$N=$ & 100 & $T=$ & 17. & DS & $D T=$ & 1550 & SEC & NVCM & $=$ & 1. & CNUMS & 1. & م2 & & 0. & ) \\
\hline$N=$ & 310. & $T=$ & 18. & DS, & $\mathrm{DT}=$ & 1550 & SEC & NVCM & $=$ & 1. & IMS & $=1.00$. & 20 & & 0 , & ) \\
\hline$N=$ & 320 & $T=$ & 19 & DS. & $\mathbf{D} T=$ & 550 & SECO & IVCM & $=$ & 1, & IMS & $=1$. & ( 20 & & 0. & )) \\
\hline$N=$ & 330. & $T=$ & 19. & $\mathrm{~s}$ & $D T=$ & 50 & SECO & JVCM & $=$ & 1. & MS & 1.0 & $(20$ & & 0. & ) \\
\hline & 40. & $T=$ & 112 & SE & $\mathrm{DT}=$ & 550 & SEC & VCM & $=$ & 1. & MS & $=1$. & $(20$, & 2 & 0 , & 0) \\
\hline$N=$ & 350. & $\mathbf{T}=$ & 20.3 & SE & $D T=$ & 50 & SEC & IVCM & $=$ & 1. & JMS & 1.00 & (20. & 2 & 0. & 0) \\
\hline N & 3 & $T=$ & 20 & DS. & $D T=$ & 550 & SECC & IVCM & s & 1. & & & & & 0 , & $?$ \\
\hline$N=$ & 370. & $T=$ & 21.5 & IDS, & $D T=$ & 50 & SEC & JVCM & $=$ & 1. & JMS & 1.00 & 0 & & 0 , & ) \\
\hline$N=$ & 3 & $T=$ & 22. & & $D T=$ & 550 & SECC & NVCM & & 1. & JMS & $=1$ & $(20$ & & 0 . & 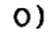 \\
\hline $\mathrm{N}$ & 390. & $T=$ & 22. & SEO & $\mathrm{DT}=$ & 550 & SEC & NVCM & & 1. & JMS & & 130 & & 0 & o) \\
\hline$N=$ & 400. & $T=$ & 23. & & $\mathrm{DT}=$ & 550 & SECO & NVCM & - & 1. & CNUMS & $=1$. & & & 0 , & 0) \\
\hline$N=$ & 410. & $T=$ & 23.8 & SE: & $D T=$ & .057 & SECONDS. & NVCM & $=$ & 1. & & & & & 0 . & ) \\
\hline$N=$ & 420. & $T=$ & 24.3 & SE & $D T=$ & & SECC & NVCM & & 1. & MS & $=1.0$ & $(20$ & & 0 . & o) \\
\hline$N=$ & 430 & $T=$ & 24.9 & SEC & $D T=$ & 1550 & SECONDS & NVCM & & 1. & & & & & & (0) \\
\hline$N=$ & 440. & $T=$ & 25.54371376 & SEC & $D T=$ & & & & & & IMS & & & & 0. & o) \\
\hline$N=$ & 450. & $T=$ & 26.1 & SECOND & $D T=$ & .05754550 & SECONDS & NVCM & $=$ & 1 & CNUMS & $=1$ & & & 0 . & 0) \\
\hline$N=$ & 460 & $T=$ & 26.69462369 & SECOND & $D T=$ & .05754550 & SEC & NVCM & & & CNUMS & & & & 0. & ) \\
\hline$N=$ & 470. & $T=$ & 27.270 & & $D T=$ & .05754550 & SEC & NVCM & & 1 & CNUMS & & & & 0. & o) \\
\hline$N=$ & 480 & $T=$ & 27.84553362 & SECONDS & $D T=$ & .05754550 & SECONDS & NVCM & & 1. & $\mathrm{CI}$ & & & & & - \\
\hline $\mathrm{N}$ & 490. & $T=$ & 28.420 & & $D T=$ & 550 & SE & NVCM & & 1. & CNUMS & $=1.0$ & $(20$ & 21,5 & 0 , & 0) \\
\hline$N=$ & 500. & $T=$ & 28.99644355 & SECOND & $D T=$ & .05754550 & SECONDS, & NVCM & 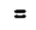 & & CNUMS & $=1.00$. & $\ln$ & & 0 & 0) \\
\hline
\end{tabular}

Fig. 25. (cont) 
MASS FLOW AND THRUST: CALCULA-ION, $N=500$

$\begin{array}{rrrrr}\mathrm{L} & \text { MF (LBM;S) } & \text { MF/MF I } & T(L B F) & T / T I \\ 1 & 112.31686 & 1.0000 & 116.3781 & 1.0000 \\ 2 & 11 \Xi .59665 & 1.0114 & 131.6404 & 1.1 \Xi 11 \\ 3 & 114.00741 & 1.0151 & 140.5393 & 1.2076 \\ 4 & 114.20202 & 1.0168 & 146.8395 & 1.2617 \\ 5 & 114.31913 & 1.0178 & 151.7055 & 1.3036 \\ 6 & 114.40858 & 1.0186 & 155.6649 & 1.3376 \\ 7 & 114.46970 & 1.0192 & 158.9635 & 1.3659 \\ 8 & 114.51786 & 1.0196 & 161.7840 & 1.3902 \\ 9 & 114.56<52 & 1.0200 & 164.2508 & 1.4114 \\ 10 & 114.59610 & 1.0203 & 166.4094 & 1.4299 \\ 11 & 114.62785 & 1.0206 & 168.3425 & 1.4465 \\ 12 & 114.64830 & 1.0208 & 170.0656 & 1.4613 \\ 13 & 114.66<77 & 1.0209 & 171.6242 & 1.4447 \\ 14 & 114.68<57 & 1.0211 & 173.0564 & 1.4870 \\ 15 & 114.69615 & 1.0212 & 174.3582 & 1.4982 \\ 16 & 114.70792 & 1.0213 & 175.5579 & 1.5085 \\ 17 & 114.71788 & 1.0214 & 176.6759 & 1.5181 \\ 18 & 114.73992 & 1.0215 & 177.7096 & 1.5270 \\ 19 & 114.71499 & 1.0214 & 178.6491 & 1.5351 \\ 20 & 114.89694 & 1.0229 & 179.9148 & 1.5460 \\ 21 & 114.86499 & 1.0227 & 180.7273 & 1.5529\end{array}$

Fig. 25. (cont) 
SOLUTION SURFACE NO. $500-$ TIME $=28.99644355$ SECONDS $($ DELTA $T=.05754550$, NVCM $=1$, CNUMS $=1.00,(20,2),($ O, O))

\begin{tabular}{|c|c|c|c|c|c|c|c|c|c|c|}
\hline$L$ & M & $\stackrel{x}{(I N)}$ & $\begin{array}{l}Y \\
(I N)\end{array}$ & $\underset{(F / S)}{U}$ & $\begin{array}{c}V \\
(F / S)\end{array}$ & $\begin{array}{c}P \\
(P S I A)\end{array}$ & $\begin{array}{c}\mathrm{RHO} \\
(\mathrm{LBM} / \mathrm{FT} 3)\end{array}$ & $\begin{array}{l}\text { VMAG } \\
(F / S)\end{array}$ & $\begin{array}{l}\text { MACH } \\
\text { NO }\end{array}$ & $\begin{array}{l}T \\
(R)\end{array}$ \\
\hline 1 & 1 & 0.0000 & 0.0000 & 1.3015 & 0.0000 & 100.00000 & 100.000000 & 1.3015 & 1.1000 & 100.0000 \\
\hline 1 & 2 & 0.0000 & .1000 & 1.3092 & .0756 & 98.71520 & 99.080500 & 1.3114 & 1.1104 & 99.6313 \\
\hline 1 & 3 & 0.0000 & .2000 & 1.3276 & .1533 & 95.47170 & 96.744100 & 1.3364 & 1.1370 & 98.6848 \\
\hline 1 & 4 & 0.0000 & .3000 & 1.3494 & .2337 & 91.22000 & 93.645000 & 1.3695 & 1.1727 & 97.4104 \\
\hline 1 & 5 & 0.0000 & 4000 & 1.3701 & .3164 & 86.53000 & 90.180000 & 1.4062 & 1.2132 & 95.9525 \\
\hline 1 & 6 & 0.0000 & .5000 & 1.3877 & 4006 & 81.72730 & 86.577500 & 1.4444 & 1.2564 & 94.3979 \\
\hline 1 & 6 & 0.0000 & 1.6547 & .6441 & 0.0000 & 179.92863 & 152.129942 & .6441 & .5006 & 118.2730 \\
\hline 1 & 7 & 0.0000 & 1.7547 & .6441 & 0.0000 & 179.92863 & 152.129942 & .6441 & .5006 & 118.2730 \\
\hline 1 & 8 & 0.0000 & 1.8547 & .6441 & 0.0000 & 179.92863 & 152.129942 & $644 t$ & .5006 & 118.2730 \\
\hline 1 & 9 & 0.0000 & 1.9547 & .6441 & 0.0000 & 179.92863 & 152.129942 & .6441 & .5006 & 118.2730 \\
\hline 1 & 10 & 0.0000 & 2.0547 & .6441 & 0.0000 & 179.92863 & 152.129942 & .6441 & .5006 & 118.2730 \\
\hline 1 & 11 & 0.0000 & 2.1547 & .6441 & 0.0000 & 179.92863 & 152.129942 & .6441 & .5006 & 118.2730 \\
\hline 2 & 1 & .2000 & 0.0000 & 1.5437 & 0.0000 & 70.42400 & 77.366711 & 1.5437 & 1.3675 & 91.0262 \\
\hline 2 & 2 & .2000 & .1115 & 1.5460 & .0941 & 69.84010 & 76.921446 & 1.5488 & 1.3738 & 90.7941 \\
\hline 2 & 3 & .2000 & .2231 & 1.5485 & .1857 & 68.51261 & 75.925220 & 1.5596 & 1.3876 & 90.2370 \\
\hline 2 & 4 & .2000 & .3346 & 1.5515 & .2765 & 66.53859 & 74.403717 & 1.5759 & 1.4084 & 89.4291 \\
\hline 2 & 5 & .2000 & .4462 & 1.5540 & .3651 & 64.12068 & 72.501138 & 1.5963 & 1.4345 & 88.4409 \\
\hline 2 & 6 & .2000 & .5577 & 1.5492 & .4472 & 62.00111 & 70.846121 & 1.6125 & 1.4568 & 87.5152 \\
\hline 2 & 6 & .2000 & 1.6547 & .6441 & 0.0000 & 179.92401 & $152 .+27155$ & .6441 & .5006 & 118.2721 \\
\hline 2 & 7 & .2000 & 1.7547 & .6441 & .0000 & 179.92401 & 152.127155 & .6441 & .5006 & 118.2721 \\
\hline 2 & 8 & .2000 & 1.8547 & .6441 & -.0000 & 179.92401 & 152.127155 & .6441 & .5006 & 118.2721 \\
\hline 2 & 9 & .2000 & 1.9547 & .6441 & .0000 & 179.92401 & 152.127155 & .6441 & .5006 & 118.2721 \\
\hline 2 & 10 & .2000 & 2.0547 & .6441 & -.0000 & 179.92401 & 152.127155 & .6441 & .5006 & 118.2721 \\
\hline 2 & 11 & .2000 & 2. 1547 & .6441 & 0.0000 & 179.92401 & 152.127155 & .6441 & .5006 & 118.2721 \\
\hline 3 & 1 & .4000 & 0.0000 & 1.6850 & 0.0000 & 54.68094 & 64.419078 & 1.6850 & 1.5457 & 84.8831 \\
\hline 3 & 2 & .4000 & 1231 & 1.6841 & .1023 & 54.44593 & 64.240050 & 1.6872 & 1.5489 & 84.7539 \\
\hline 3 & 3 & .4000 & .2462 & 1.6807 & .2019 & 53.77164 & 63.725641 & 1.6928 & 1.5575 & 84.3799 \\
\hline 3 & 4 & .4000 & .3693 & 1.6756 & .2990 & 52.69850 & 62.868180 & 1.7021 & 1.5712 & 83.8238 \\
\hline 3 & 5 & .4000 & .4924 & 1.6689 & .3913 & 51.31118 & 61.731773 & 1.7142 & 1.5891 & 83.1196 \\
\hline 3 & 6 & .4000 & .6155 & 1.6572 & .4784 & 49.91357 & 60.590319 & 1.7248 & 1.6061 & 82.3788 \\
\hline 3 & 6 & .4000 & 1.6547 & .6441 & 0.0000 & 179.92725 & $152.129+08$ & .6441 & .5006 & 118.2727 \\
\hline 3 & 7 & .4000 & 1.7547 & .6441 & -.0000 & 179.92725 & 152.129108 & .6441 & .5006 & 118.2727 \\
\hline 3 & 8 & .4000 & 1.8547 & .6441 & -.0000 & 179.92725 & 152.129108 & 6441 & .5006 & 118.2727 \\
\hline 3 & 9 & .4000 & 1.9547 & .6441 & .0000 & 179.92725 & 152.129108 & .6441 & .5006 & 118.2727 \\
\hline 3 & 10 & .4000 & 2.0547 & .6441 & .0000 & 179.92725 & 152.129108 & .6441 & .5006 & 118.2727 \\
\hline 3 & 11 & .4000 & 2.1547 & .6441 & 0.0000 & 179.92725 & 152.129108 & .6441 & .5006 & 118.2727 \\
\hline 4 & 1 & .6000 & 0.0000 & 1.7836 & 0.0000 & 44.63367 & 55.651536 & 1.7836 & 1.6832 & 80.2020 \\
\hline 4 & 2 & .6000 & .1346 & 1.7814 & 1065 & 44.53166 & 55.579614 & 1.7845 & 1.6850 & 80.1223 \\
\hline 4 & 3 & .6000 & .2693 & 1.7752 & .2108 & 44.14704 & 55.288347 & 1.7877 & 1.6908 & 79.8487 \\
\hline 4 & 4 & .6000 & .4039 & 1.7657 & .3123 & 43.51038 & 54.770628 & 1.7931 & 1.7002 & 79.4411 \\
\hline 4 & 5 & .6000 & .5386 & 1.7544 & .4090 & 42.57997 & 53.982336 & 1.8015 & 1.7143 & 78.8776 \\
\hline 4 & 6 & .6000 & .6732 & 1.7390 & .5020 & 41.49522 & 53.054077 & 1.8100 & 1.7297 & 78.2131 \\
\hline 4 & 6 & .6000 & 1.6547 & .6441 & 0.0000 & 179.93296 & 152.132558 & .6441 & .5005 & 118.2738 \\
\hline 4 & 7 & .6000 & 1.7547 & .6441 & -.0000 & 179.93296 & 152.132558 & .6441 & .5005 & 118.2738 \\
\hline 4 & 8 & .6000 & 1.8547 & .6441 & .0000 & 179.93296 & 152.132558 & .6441 & .5005 & 118.2738 \\
\hline 4 & 9 & .6000 & 1.9547 & .6441 & .0000 & 179.93296 & 152.132558 & .6441 & .5005 & 118.2738 \\
\hline 4 & 10 & .6000 & 2.0547 & .6441 & -.0000 & 179.93296 & 152.132558 & .6441 & .5005 & 118.2738 \\
\hline 4 & 11 & .6000 & 2.1547 & .6441 & 0.0000 & 179.93296 & 152.132558 & .6441 & .5005 & 118.2738 \\
\hline 5 & 1 & .8000 & 0.0000 & 1.8584 & 0.0000 & 37.61577 & 49.212201 & 1.8584 & 1.7965 & 76.4359 \\
\hline
\end{tabular}


SOLUTION SURFACE NO. $500-$ TIME $=28.9954435 E$ SECONDS (DELTA $T=.05754550$, NVCM $=1$, CNUMS $=1.00,(20,2)$, 0.0$)$ )

\begin{tabular}{|c|c|c|c|c|c|c|c|c|c|c|}
\hline L & M & $\stackrel{x}{(I N)}$ & $\stackrel{Y}{Y}$ & $\underset{(F / S)}{U}$ & $\begin{array}{c}v \\
(F / S)\end{array}$ & $\begin{array}{c}P \\
(P S I A)\end{array}$ & $\begin{array}{c}\mathrm{RHO} \\
(\mathrm{LBM} / \mathrm{FT} 3)\end{array}$ & $\begin{array}{l}\text { VMAG } \\
(F / S)\end{array}$ & $\begin{array}{l}\text { MACH } \\
\text { NO }\end{array}$ & $\begin{array}{l}T \\
(R)\end{array}$ \\
\hline 5 & 2 & .8000 & .1462 & 1.8556 & .1090 & 37.56076 & 49.178536 & 1.8588 & 1.7976 & 76.3763 \\
\hline 5 & 3 & .8000 & .2924 & i.8481 & .2165 & 37.30861 & 48.990486 & 1.8607 & 1.8020 & 76.1548 \\
\hline 5 & 4 & .8000 & 4385 & $\because .8364$ & .3215 & 36.87358 & 48.631677 & 1.8643 & 1.8095 & 75.8221 \\
\hline 5 & 5 & .8000 & .5847 & i. 8226 & .4228 & 36.16039 & 49.005901 & 1.8710 & 1.8220 & 75.3249 \\
\hline 5 & 6 & .8000 & .7309 & $\therefore .8047$ & .5210 & 35.25577 & 47.196082 & 1.8784 & 1.8368 & 74.7006 \\
\hline 5 & 6 & .8000 & 1.6547 & .6440 & 0.0000 & 179.94062 & 152.137183 & .6440 & .5005 & 118.2752 \\
\hline 5 & 7 & .8000 & 1.7547 & .6440 & -.0000 & 179.94062 & 152.137183 & .6440 & .5005 & 118.2752 \\
\hline 5 & 8 & .8000 & 1.8547 & .6440 & .0000 & 179.94062 & 152.137183 & .6440 & .5005 & 118.2752 \\
\hline 5 & 9 & .8000 & 1.9547 & .6440 & -.0000 & 179.94062 & 152.137183 & .6440 & .5005 & 118.2752 \\
\hline 5 & 10 & .8000 & 2.0547 & .6440 & -.0000 & 179.94062 & $152.137+83$ & .6440 & .5005 & 118.2752 \\
\hline 5 & 11 & .8000 & 2.1547 & .6440 & 0.0000 & 179.94062 & 152.137183 & .6440 & .5005 & 118.2752 \\
\hline 6 & 1 & 1.0000 & 0.0000 & $\cdot .9181$ & 0.0000 & 32.42246 & 44.234596 & 1.9181 & 1.8935 & 73.2966 \\
\hline 6 & 2 & 1.0000 & .1577 & $\cdot .9152$ & .1109 & 32.37947 & 44.209019 & 1.9184 & 1.8945 & 73.2418 \\
\hline 6 & 3 & 1.0000 & .3155 & $\cdot 9070$ & .2209 & 32.18301 & 44.061170 & 1.9197 & 1.8984 & 73.0417 \\
\hline 6 & 4 & 1.0000 & .4732 & $\cdot .8942$ & .3290 & 31.83592 & 43.767295 & 1.9226 & 1.9052 & 72.7391 \\
\hline 6 & 5 & 1.0000 & .6310 & .8790 & .4343 & 31.23841 & $43.223+65$ & 1.9285 & 1.9172 & 72.2724 \\
\hline 6 & 6 & 1.0000 & .7887 & $\cdot .8592$ & .5367 & 30.45887 & 42.495530 & 1.9351 & 1.9318 & 71.6755 \\
\hline 6 & 6 & 1.0000 & 1.6547 & .6440 & 0.0000 & 179.94731 & 152.141222 & .6440 & .5004 & 118.2765 \\
\hline 6 & 7 & 1.0000 & 1.7547 & .6440 & .0000 & 179.94731 & 152.141222 & .6440 & .5004 & 118.2765 \\
\hline 6 & 8 & 1.0000 & 1.8547 & .6440 & .0000 & 179.94731 & 152.141222 & .6440 & .5004 & 118.2765 \\
\hline 6 & 9 & 1.0000 & 1.9547 & .6440 & .0000 & 179.94731 & 152.141222 & .6440 & 5004 & 118.2765 \\
\hline 6 & 10 & 1.0000 & 2.0547 & .6440 & -.0000 & 179.94731 & 152.141222 & .6440 & .5004 & 118.2765 \\
\hline 6 & 11 & 1.0000 & 2.1547 & .6440 & 0.0000 & 179.94731 & 152.141222 & .6440 & .5004 & 118.2765 \\
\hline 7 & 1 & 1.2000 & 0.0000 & 1.9676 & 0.0000 & 28.41421 & 40.241246 & 1.9676 & 1.9789 & 70.6097 \\
\hline 7 & 2 & 1.2000 & .1693 & 1.9646 & .1127 & 28.36953 & 40.210997 & 1.9679 & 1.9801 & 70.5517 \\
\hline 7 & 3 & 1.2000 & .3386 & I. 9562 & .2248 & 28.19273 & 40.072029 & 1.9691 & 1.9840 & 70.3551 \\
\hline 7 & 4 & 1.2000 & .5078 & 1.9429 & .3356 & 27.88247 & 39.798801 & 1.9717 & 1.9908 & 70.0586 \\
\hline 7 & 5 & 1.2000 & .6771 & I. 9266 & .4443 & 27.35629 & 39.301382 & 1.9772 & 2.0029 & 69.6064 \\
\hline 7 & 6 & 1.2000 & .8464 & 1. 9055 & .5501 & 26.67300 & 38.638731 & 1.9833 & 2.0174 & 69.0318 \\
\hline 7 & 6 & 1.2000 & 1.6547 & .6440 & 0.0000 & 179.94594 & 152.140394 & .6440 & .5004 & 118.2762 \\
\hline 7 & 7 & 1.2000 & 1.7547 & .6440 & .0000 & 179.94594 & 152.140394 & .6440 & .5004 & 118.2762 \\
\hline 7 & 8 & 1.2000 & 1.8547 & .6440 & .0000 & 179.94594 & 152.140394 & .6440 & 5004 & 118.2762 \\
\hline 7 & 9 & 1.2000 & 1.9547 & .6440 & .0000 & 179.94594 & 152.140394 & .6440 & .5004 & 118.2762 \\
\hline 7 & 10 & 1.2000 & 2.0547 & .6440 & .0000 & 179.94594 & 152.140394 & .6440 & .5004 & 118.2762 \\
\hline 7 & 11 & 1.2000 & 2.1547 & .6440 & 0.0000 & 179.94594 & 152.140394 & .6440 & .5004 & 118.2762 \\
\hline 8 & 1 & 1.4000 & 0.0000 & 2.0096 & 0.0000 & 25.21985 & 36.945647 & 2.0096 & 2.0557 & 68.2620 \\
\hline 8 & 2 & $\therefore .4000$ & .1808 & 2.0068 & .1144 & 25.16963 & 36.906817 & 2.0100 & 2.0571 & 68.1978 \\
\hline 8 & 3 & $\therefore .4000$ & .3616 & 1.9982 & .2285 & 24.99772 & 36.763487 & 2.0112 & 2.0614 & 67.9960 \\
\hline 8 & 4 & $i .4000$ & .5425 & 1.9847 & .3418 & 24.70364 & 36.492815 & 2.0139 & 2.0687 & 67.6945 \\
\hline 8 & 5 & 1.4000 & .7233 & 1.9676 & .4532 & 24.22841 & 36.027167 & 2.0192 & 2.0809 & 67.2504 \\
\hline 8 & 6 & 1.4000 & .9041 & 1.9453 & .5616 & 23.62296 & 35.419115 & 2.0248 & 2.0954 & 66.6955 \\
\hline 8 & 6 & 1.4000 & 1.6547 & .6440 & 0.0000 & 179.94038 & 152.137043 & .6440 & .5004 & 118.2752 \\
\hline 8 & 7 & 1.4000 & 1.7547 & .6440 & -.0000 & 179.94038 & 152.137043 & .6440 & .5004 & 118.2752 \\
\hline 8 & 8 & 1.4000 & 1.8547 & .6440 & -.0000 & 179.94038 & 152.137043 & .6440 & .5004 & 118.2752 \\
\hline 8 & 9 & 1.4000 & 1.9547 & .6440 & -.0000 & 179.94038 & 152.137043 & .6440 & .5004 & 118.2752 \\
\hline 8 & 10 & 1.4000 & 2.0547 & .6440 & .0000 & 179.94038 & 152.137043 & .6440 & .5004 & 118.2752 \\
\hline 8 & 11 & 1.4000 & 2.1547 & 6440 & 0.0000 & 179.94038 & 152.137043 & .6440 & .5004 & 118.2752 \\
\hline 9 & 1 & 1.6000 & 0.0000 & 2.0461 & 0.0000 & 22.61059 & 34.166358 & 2.0461 & 2.1257 & 66.1779 \\
\hline 9 & 2 & 1.6000 & .1924 & 2.0433 & .1162 & 22.55543 & 34.119462 & 2.0466 & 2.1274 & $6 E .1072$ \\
\hline
\end{tabular}

Fig. 25. (cont) 
SOLUTION SURFACE NO. $500-$ TIME $=28.99644355$ SECONDS (DELTA $T=.05754550$, NVCM $=1$, CNUMS $=1.00,(20,2),(0.0)$ )

\begin{tabular}{|c|c|c|c|c|c|c|c|c|c|c|}
\hline L & $M$ & $\stackrel{x}{(I N)}$ & 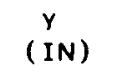 & $\underset{(F / S)}{U}$ & $\begin{array}{c}v \\
(F / S)\end{array}$ & $(P \dot{S} I A)$ & $\begin{array}{c}\text { RHO } \\
\text { (LBM/FT3) }\end{array}$ & $\begin{array}{l}\text { VMAG } \\
(F / S)\end{array}$ & $\begin{array}{l}\text { MACH } \\
\text { NO }\end{array}$ & $\begin{array}{c}T \\
(R)\end{array}$ \\
\hline 9 & 3 & 1.6000 & .3848 & 2.0348 & .2321 & 22.38412 & 33.968206 & 2.0480 & 2. 1322 & 65.8973 \\
\hline 9 & 4 & 1.6000 & .5771 & 2.0210 & .3476 & 22.09955 & 33.694880 & 2.0507 & 2.1401 & 65.5873 \\
\hline 9 & 5 & 1.6000 & .7695 & 2.0034 & .4613 & 21.66517 & 33.254731 & 2.0558 & 2.1526 & 65.1491 \\
\hline 9 & 6 & 1.6000 & .9619 & 1.9801 & .5716 & 21.12425 & 32.693800 & 2.0610 & 2.1670 & 64.6124 \\
\hline 9 & 6 & 1.6000 & 1.6547 & .6440 & 0.0000 & 179.93258 & 152.132332 & .6440 & .5005 & 118.2737 \\
\hline 9 & 7 & 1.6000 & 1.7547 & .6440 & -.0000 & 179.93258 & 152.132332 & .6440 & .5005 & 118.2737 \\
\hline 9 & 8 & 1.6000 & 1.8547 & .6440 & -.0000 & 179.93258 & 152.132332 & .6440 & .5005 & 118.2737 \\
\hline 9 & 9 & 1.6000 & 1.9547 & .6440 & .0000 & 179.93258 & 152.132332 & .6440 & .5005 & 118.2737 \\
\hline 9 & 10 & 1.6000 & 2.0547 & .6440 & .0000 & 179.93258 & 152.132332 & .6440 & .5005 & 118.2737 \\
\hline 9 & 11 & 1.6000 & 2. 1547 & .6440 & 0.0000 & 179.93258 & 152.132332 & .6440 & .5005 & 118.2737 \\
\hline 10 & 1 & 1.8000 & 0.0000 & 2.0783 & 0.0000 & 20.43660 & 31.781310 & 2.0783 & 2. 1904 & 64.3038 \\
\hline 10 & 2 & 1.8000 & .2039 & 2.0756 & 1181 & 20.37869 & 31.728703 & 2.0789 & 2. 1924 & 64.2279 \\
\hline 10 & 3 & 1.8000 & .4078 & 2.0670 & .2357 & 20.20832 & 31.570578 & 2.0804 & 2.1976 & 64.0100 \\
\hline 10 & 4 & 1.8000 & .6118 & 2.0531 & .3530 & 19.93247 & 31.295166 & 2.0832 & 2.2061 & 63.6918 \\
\hline 10 & 5 & 1.8000 & .8157 & 2.0349 & .4686 & 19.53324 & 30.877874 & 2.0881 & 2.2189 & 63.2597 \\
\hline 10 & 6 & 1.8000 & 1.0196 & 2.0108 & .5805 & 19.04665 & 30.358132 & 2.0929 & 2.2332 & 62.7398 \\
\hline 10 & 6 & 1.8000 & 1.6547 & .6440 & 0.0000 & 179.92792 & 152.129500 & 5440 & .5005 & 118.2729 \\
\hline 10 & 7 & 1.8000 & 1.7547 & .6440 & -.0000 & 179.92792 & 152.129500 & .6440 & .5005 & 118.2729 \\
\hline 10 & 3 & 1.8000 & 1.8547 & .6440 & .0000 & 179.92792 & 152.129500 & .6440 & .5005 & 118.2729 \\
\hline 10 & 9 & 1.8000 & 1.9547 & .6440 & .0000 & 179.92792 & 152.129500 & .6440 & .5005 & 118.2729 \\
\hline 10 & 10 & 1.8000 & 2.0547 & .6440 & .0000 & 179.92792 & 152.129500 & .6440 & .5005 & 118.2729 \\
\hline 10 & 11 & 1.8000 & 2.1547 & .6440 & 0.0000 & 179.92792 & 152.129500 & .6440 & .5005 & 118.2729 \\
\hline 11 & 1 & 2.0000 & 0.0000 & 2.1071 & 0.0000 & 18.59719 & 29.707043 & $2: 1071$ & 2.2507 & 62.6019 \\
\hline 11 & 2 & 2.0000 & .2155 & 2.1043 & .1199 & 18.53893 & 29.651490 & 2.1077 & 2.2529 & 62.5228 \\
\hline 11 & 3 & 2.0000 & .4310 & 2.0957 & .2392 & 18.37146 & 29.489336 & 2. 1093 & 2.2586 & 62.2987 \\
\hline 11 & 4 & 2.0000 & .6464 & 2.0816 & 3582 & 18.10560 & 29.214569 & 2.1122 & 2.2676 & 61.9746 \\
\hline 11 & 5 & 2.0000 & .8619 & 2.0629 & 4752 & 17.73778 & 28.818925 & 2.1169 & 2.2805 & 61.5491 \\
\hline 11 & 6 & 2.0000 & 1.0774 & 2.0382 & .5884 & 17.29735 & 28.335426 & 2. 1214 & 2.2948 & 61.0450 \\
\hline 11 & 6 & 2.0000 & 1.6547 & .6440 & 0.0000 & 179.92841 & 152.129794 & .6440 & .5005 & 118.2730 \\
\hline 11 & 7 & 2.0000 & 1.7547 & .6440 & -.0000 & 179.92841 & 152.129794 & .6440 & .5005 & 118.2730 \\
\hline 11 & 8 & 2.0000 & 1.8547 & 6440 & -.0000 & 179.92841 & 152.129794 & 6440 & 5005 & 118.2730 \\
\hline 11 & 9 & 2.0000 & 1.9547 & 6440 & -.0000 & 179.92841 & 152.129794 & .6440 & .5005 & 118.2730 \\
\hline 11 & 10 & 2.0000 & 2.0547 & .6440 & -.0000 & 179.92841 & 152.129794 & .6440 & .5005 & 118.2730 \\
\hline 11 & 11 & 2.0000 & 2. 1547 & .6440 & 0.0000 & 179.92841 & 152.129794 & .6440 & .5005 & 118.2730 \\
\hline 12 & 1 & 2.2000 & 0.0000 & 2. 1330 & 0.0000 & 17.02108 & 27.883243 & 2.1330 & 2.3073 & 61.0441 \\
\hline 12 & 2 & 2.2000 & .2270 & 2.1303 & .1216 & 16.96446 & 27.827205 & 2.1337 & 2.3096 & 60.9636 \\
\hline 12 & 3 & 2.2000 & .4540 & 2.1215 & .2426 & 16.80203 & 27.664177 & 2.1353 & 2.3157 & 60.7357 \\
\hline 12 & 4 & 2.2000 & .6811 & 2.1072 & .3630 & 16.54782 & 27.393221 & 2.1382 & 2.3251 & 60.4084 \\
\hline 12 & 5 & 2.2000 & .9081 & 2.0881 & .4813 & 16.20857 & 27.018521 & 2.1428 & 2.3382 & 59.9906 \\
\hline 12 & 6 & 2.2000 & 1.1351 & 2.0628 & 5955 & 15.80773 & 26.567110 & 2.1470 & 2.3524 & 59.5011 \\
\hline 12 & 6 & 2.2000 & 1.6547 & .6440 & 0.0000 & 179.93270 & 152.132432 & .6440 & .5004 & 118.2737 \\
\hline 12 & 7 & 2.2000 & 1.7547 & .6440 & -.0000 & 179.93270 & 152.132433 & .6440 & .5004 & 118.2737 \\
\hline 12 & 8 & 2.2000 & 1.8547 & .6440 & -.0000 & 179.93270 & 152.132433 & .6440 & .5004 & 118.2737 \\
\hline 12 & 9 & 2.2000 & 1.9547 & .6440 & -.0000 & 179.93270 & 152.132433 & .6440 & 5004 & 118.2737 \\
\hline 12 & 10 & 2.2000 & 2.0547 & .6440 & -.0000 & 179.93270 & 152.132433 & .6440 & .5004 & 118.2737 \\
\hline 12 & 11 & 2.2000 & 2.1547 & .6440 & 0.0000 & 179.93270 & 152.132432 & .6440 & .5004 & 118.2737 \\
\hline 13 & 1 & 2.4000 & 0.0000 & 2. 1567 & 0.0000 & 15.65648 & 26.265270 & 2. 1567 & 2.3608 & $59.609 \uparrow$ \\
\hline 13 & 2 & 2.4000 & 2386 & 2.1538 & .1233 & 15.60287 & 26.210640 & 2.1573 & 2.3631 & 59.5288 \\
\hline 13 & & 2.4000 & & & .2458 & 15.44724 & 26.049531 & 2. 1589 & 2.3695 & 59.2995 \\
\hline
\end{tabular}


SOLUTION SURFACE NO. $500-$ TIME $=28.99644355$ SECONDS $($ DELTA $T=.05754550$, NVCM $=1, \mathrm{CNJMS}=1.00$. $(20,2)$, 0 , O))

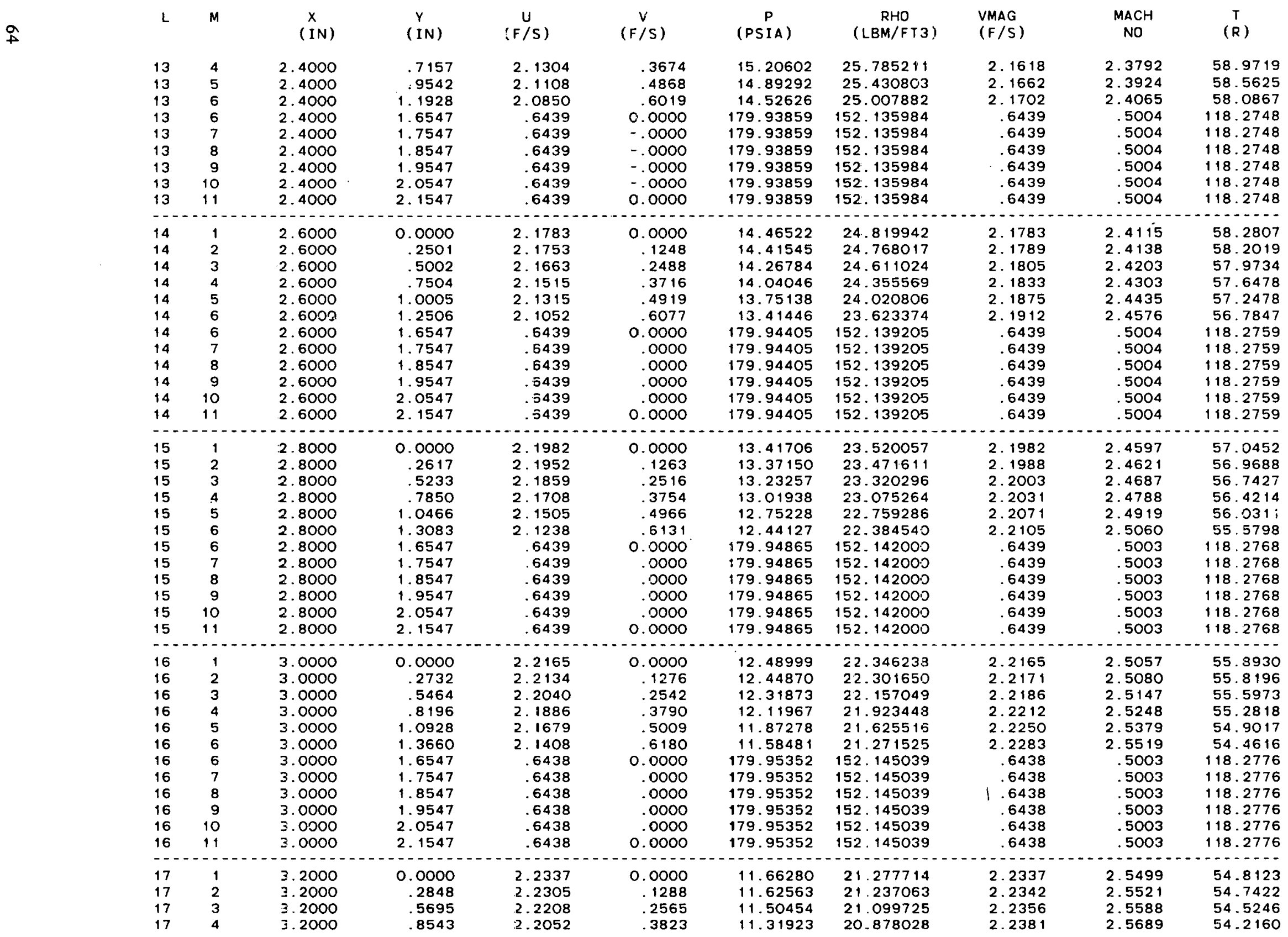

Fig. 25. (cont) 
SOLUTION SURFACE. NO. $500-$ TIME $=28.99644355$ SECONDS $(D E L T A T=.05754550$, NVCM $=1$, CNUMS $=1.00$. $(20,2)$, , O $)$ )

\begin{tabular}{|c|c|c|c|c|c|c|c|c|c|c|}
\hline L & M & $\stackrel{x}{(I N)}$ & $\begin{array}{l}Y \\
(I N)\end{array}$ & $\underset{(F / S)}{U}$ & $\begin{array}{c}V \\
(F / S)\end{array}$ & $\begin{array}{c}P \\
(P S I A)\end{array}$ & $\begin{array}{c}\text { RHO } \\
(L B M / F T 3)\end{array}$ & $\begin{array}{l}\text { VMAG } \\
(F / S)\end{array}$ & $\begin{array}{c}\text { MACH } \\
\text { NO }\end{array}$ & $\begin{array}{c}T \\
(R)\end{array}$ \\
\hline 17 & 5 & 3.2000 & 1.1390 & 2.1841 & .5049 & 11.09066 & 20.596966 & 2.2417 & 2.5819 & 53.8461 \\
\hline 17 & 6 & 3.2000 & 1.4238 & 2.1567 & .6226 & 10.82254 & 20.260958 & 2.2448 & 2.5959 & 53.4158 \\
\hline 17 & 6 & 3.2000 & 1.6547 & .6438 & 0.0000 & 179.96004 & 152.148947 & .6438 & .5003 & 118.2789 \\
\hline 17 & 7 & 3.2000 & 1.7547 & 6438 & .0000 & 179.96004 & 152.148947 & .6438 & .5003 & 118.2789 \\
\hline 17 & 8 & 3.2000 & 1.8547 & .6438 & .0000 & 179.96004 & 152.148947 & .6438 & 5003 & 118.2789 \\
\hline 17 & 9 & 3. 2000 & 1.9547 & .6438 & .0000 & 179.96004 & 152.148947 & .6438 & .5003 & 118.2789 \\
\hline 17 & 10 & 3. 2000 & 2.0547 & .6438 & -.0000 & 179.96004 & 152.148947 & .6438 & .5003 & 118.2789 \\
\hline 17 & 1.1 & 3. 2000 & 2.1547 & .6438 & 0.0000 & 179.96004 & 152.148947 & .6438 & .5003 & 118.2789 \\
\hline 18 & 1 & 3.4000 & 0.0000 & 2.2494 & 0.0000 & 10.92756 & 20.309865 & 2.2494 & 2.5917 & 53.8042 \\
\hline 18 & 2 & 3.4000 & .2963 & 2.2461 & .1300 & 10.89422 & 20.273038 & 2.2498 & 2.5939 & 53.7375 \\
\hline 18 & 3 & 3.4000 & .5926 & 2.2362 & .2587 & 10.78171 & 20.143218 & 2.2511 & 2.6005 & 53.5253 \\
\hline 18 & 4 & 3.4000 & .8889 & 2.2203 & .3853 & 10.60953 & 19.933551 & 2.2535 & 2.6106 & 53.2245 \\
\hline 18 & 5 & 3.4000 & 1. 1852 & 2.1989 & .5085 & 10.39790 & 19.668722 & 2.2569 & 2.6234 & 52.8652 \\
\hline 18 & 6 & 3.4000 & 1.4815 & 2.1712 & 6268 & 10. 14847 & 19.350539 & 2.2598 & 2.6373 & 52.4454 \\
\hline 18 & 6 & 3.4000 & 1.6547 & .6437 & 0.0000 & 179.96894 & 152.154211 & .6437 & .5003 & 118.2806 \\
\hline 18 & 7 & 3.4000 & 1.7547 & 6437 & -.0000 & 179.96894 & 152.154211 & .6437 & .5003 & 118.2806 \\
\hline 18 & 8 & 3.4000 & 1.8547 & .6437 & -.0000 & 179.96894 & 152.154211 & .6437 & 5003 & 118.2806 \\
\hline 18 & 9 & 3.4000 & 1.9547 & 6437 & -.0000 & 179.96894 & 152.154211 & 6437 & 5003 & 118.2806 \\
\hline 18 & 10 & 3.4000 & 2.0547 & 6437 & -.0000 & 179.96894 & 152.154211 & .6437 & 5003 & 118.2806 \\
\hline 18 & 11 & 3.4000 & 2.1547 & .6437 & 0.0000 & 179.96894 & 152.154211 & .6437 & .5003 & 118.2806 \\
\hline 19 & 1 & 3.6000 & 0.0000 & 2.2648 & 0.0000 & 10.25387 & 19.407756 & 2.2648 & 2.6334 & 52.8339 \\
\hline 19 & 2 & 3.6000 & .3078 & 2.2614 & 1310 & 10.22406 & 19.374567 & 2.2652 & 2.6354 & 52.7705 \\
\hline 19 & 3 & 3.6000 & .6157 & 2.2513 & .2608 & 10.11976 & 19.252252 & 2.2664 & 2.6420 & 52.5640 \\
\hline 19 & 4 & 3.6000 & .9235 & 2.2351 & 3881 & 9.96008 & 19.054503 & 2.2686 & 2.6519 & 52.2716 \\
\hline 19 & 5 & 3.6000 & 1.2314 & 2.2134 & 5120 & 9.76366 & 18.804465 & 2.2719 & 2.6646 & 51.9220 \\
\hline 19 & 6 & 3.6000 & 1.5392 & 2.1853 & 6309 & 9.52893 & 18.499550 & 2.2746 & 2.6785 & 51.5090 \\
\hline 19 & 6 & 3.6000 & 1.6547 & .6437 & 0.0000 & 179.97978 & 152.160790 & .6437 & .5002 & 118.2826 \\
\hline 19 & 7 & 3.6000 & 1.7547 & 6437 & -.0000 & 179.97978 & 152.160790 & 6437 & 5002 & 118.2826 \\
\hline 19 & 8 & 3.6000 & 1.8547 & .6437 & -.0000 & 179.97978 & 152.160790 & .6437 & .5002 & 118.2826 \\
\hline 19 & 9 & 3.6000 & 1.9547 & .6437 & -.0000 & 179.97978 & 152.160790 & .6437 & .5002 & 118.2826 \\
\hline 19 & 10 & 3.6000 & 2.0547 & .6437 & -.0000 & 179.97978 & 152.160790 & .6437 & .5002 & 118.2826 \\
\hline 19 & 11 & 3.6000 & 2. 1547 & .6437 & 0.0000 & 179.97978 & 152.160790 & .6437 & 5002 & 118.2826 \\
\hline 20 & 1 & 3.8000 & 0.0000 & 2.2785 & 0.0000 & 9.69812 & 18.639909 & 2.2785 & 2.6697 & 52.0288 \\
\hline 20 & 2 & 3.8000 & .3194 & 2.2750 & .1319 & 9.67116 & 18.609777 & 2.2788 & 2.6716 & 51.9681 \\
\hline 20 & 3 & 3.8000 & 6388 & 2.2647 & .2626 & 9.57404 & 18.494487 & 2.2799 & 2.6781 & 51.7670 \\
\hline 20 & 4 & 3.8000 & 9582 & 2.2483 & .3905 & 9.42515 & 18.307660 & 2.2820 & 2.6879 & 51.4820 \\
\hline 20 & 5 & 3.8000 & 1.2776 & 2.2263 & .5150 & 9.24183 & 18.071130 & 2.2850 & 2.7005 & 51.1414 \\
\hline 20 & 6 & 3.8000 & 1.5970 & 2.1979 & 6345 & 9.01826 & 17.776265 & 2.2876 & 2.7144 & 50.7320 \\
\hline 20 & 6 & 3.8000 & 1.6547 & .6437 & 0.0000 & 179.99068 & 152.167457 & .6437 & .5002 & 118.2846 \\
\hline 20 & 7 & 3.8000 & 1.7547 & .6437 & -.0000 & 179.99068 & 152.167457 & .6437 & .5002 & 118.2846 \\
\hline 20 & 8 & 3.8000 & 1.8547 & 6437 & -.0000 & 179.99068 & 152.167457 & .6437 & .5002 & 118.2846 \\
\hline 20 & 9 & 3.8000 & 1.9547 & 6437 & .0000 & 179.99068 & 152.167457 & .6437 & 5002 & 118.2846 \\
\hline 20 & 10 & 3.8000 & 2.0547 & .6437 & 0000 & 179.99068 & 152.167457 & .6437 & 5002 & 118.2846 \\
\hline 20 & 11 & 3.8000 & 2. 1547 & .6437 & 0.0000 & 179.99068 & 152.167457 & .6437 & .5002 & 118.2846 \\
\hline 21 & 1 & 4.0000 & 0.0000 & 2.2921 & 0.0000 & 9. 14238 & 17.872062 & 2.2921 & 2.7085 & $5 t .1546$ \\
\hline 21 & 2 & 4.0000 & 3309 & 2.2886 & .1327 & 9.11825 & 17.844986 & 2.2924 & 2.7104 & 51.0970 \\
\hline 21 & 3 & 4.0000 & 6619 & 2.2781 & .2643 & 9.02832 & 17.736723 & 2.2934 & 2.7168 & 50.9018 \\
\hline 21 & 4 & 4.0000 & 9928 & 2.2615 & .3930 & 8.89021 & 17.560817 & 2.2953 & 2.7265 & 50.6253 \\
\hline 21 & 5 & 4.0000 & 1. 3238 & 2.2391 & .5180 & 8.71999 & 17.337795 & 2.2982 & 2.7389 & 50.2947 \\
\hline
\end{tabular}


SOLUTION SURFACE NO. $500-$ TIME $=28.99644355$ SECCNOS $($ DELTA T $=.05754550$, NVCM $=1$, CNUMS $=1.00$, $(20,2)$, $(0,0)$ )

\begin{tabular}{|c|c|c|c|c|c|c|c|c|c|c|}
\hline $\mathbf{L}$ & $M$ & $\stackrel{x}{(I N)}$ & $\stackrel{Y}{(I N)}$ & $\stackrel{4}{(F ; S)}$ & $\begin{array}{c}v \\
(F / S)\end{array}$ & $\begin{array}{c}P \\
\text { (PSIA) }\end{array}$ & $\begin{array}{c}\text { RHO } \\
\text { (LEM/FT3) }\end{array}$ & $\begin{array}{l}\text { VMAS } \\
(\equiv / 5)\end{array}$ & $\begin{array}{l}\text { N.ACH } \\
\text { NO }\end{array}$ & $\begin{array}{l}T \\
(R)\end{array}$ \\
\hline 21 & 6 & 4.0000 & 1.6547 & 2.2104 & .6381 & 8. 50758 & 17.052979 & 2.3007 & 2.7529 & 49.8891 \\
\hline 21 & 6 & 4.0000 & 1.6547 & $.643 E$ & 0.0000 & 180.00000 & 152.173097 & .5136 & .5002 & 118.2863 \\
\hline 21 & 7 & 4.0000 & 1.7547 & $.643 E$ & .0000 & 180.00000 & 152.173097 & .5436 & .5002 & 118.2863 \\
\hline 21 & 8 & 4.0000 & 1.8547 & $.643 E$ & .0000 & 180.00000 & 152.173097 & .5436 & .5002 & 118.2863 \\
\hline $2 \uparrow$ & 9 & 4.0000 & 1.9547 & $.643 f$ & .0000 & 180.00000 & 152.173097 & .5436 & .5002 & 118.2863 \\
\hline 21 & 10 & 4.0000 & 2.0547 & $.643 E$ & .0000 & 180.00000 & 152.173097 & .5436 & .5002 & 118.2863 \\
\hline 21 & 11 & 4.0000 & 2. 1547 & .6436 & 0.0000 & 180.00000 & 152.173097 & .5136 & .5002 & 118.2863 \\
\hline
\end{tabular}

Fig. 25. (cont) 


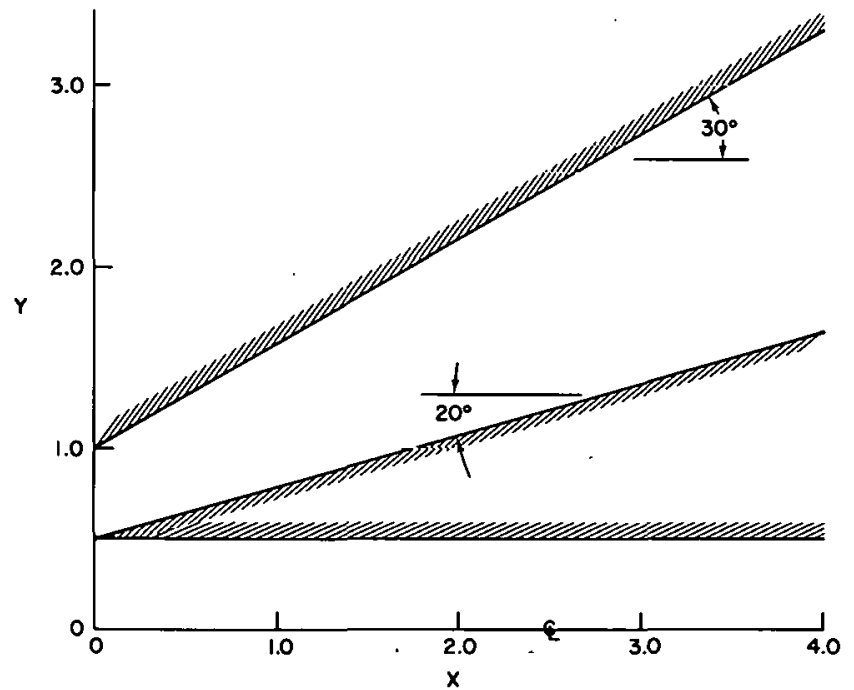

Fig. 26.

Case No. 2 geometry.

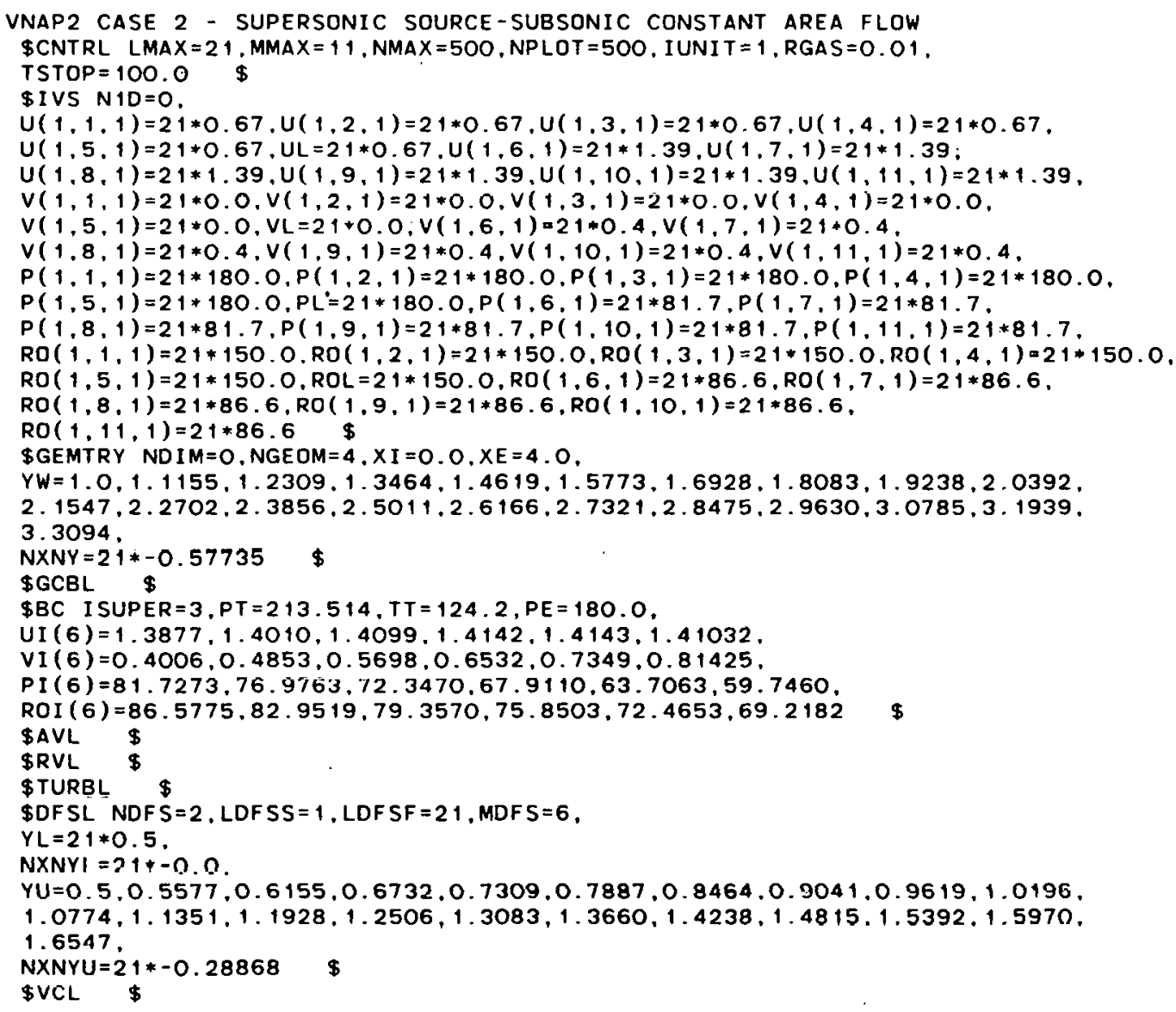

Fig. 27.

Case No. 2 data deck. 
VNAP2. A COMPUTER PRGgRAM FOF THE COMPUTATION OF TWO-DIMENSIONAL. TIME-DEPENDENT. COMPRESSI3LE, TURBULENT FLOW By MICHAEL C. CLINE, T-3 - LOj alamos NATIONAL LABORATCRY

PROGRAM ABSTRACT -

THE NAVIER-STCIKES EQUATIONS FOR TW'D-DIMEVSIONAL. TIME-DEPENOENT FLOW ARE SOLVED USING THE SECOND-ORDER, MACCORMACK FINITE-DIFFERENCE SCTEME. ALL BOUNDARY CONDITIONS ARE COMPUTED USING A SECOND-OFDER, REFERENCE PI.ANE CHARACTERISTI SCHEME WITH THE VISCOUS TERMS IREATED AS SOURCE FUNCTIONS. - THE FLUID' IS ASSUMED TO BE A PERFECT GAS. THE STEADY-STAAE SOLUTJON IS OBTAINED AS THE ASYMPTCTIC SOLUTION FOR LARGE TINE. THE FLOW BOUNDARIES MAY BE AFEITRARY CURVED SOLID WALLS AS WELL AS JET ENVELOPES. THE GEOMETRY MAY CJNSIST OF SINGLE AND DUAL FLOWING STREAMS. TURBULENCE EFFECTS ARE MCDELED WITH EITHER A MIXING-LENGTH. A TURBULENCE ENERSY EOUATION, OR. A TURBULENCE ENERGY-DISEIPATION RATE EOUATIONS MOCEL. THIS PROGRAM ALLOWS VARIABLE GRID SFACING AND INCLUDES OPTIONS TO SPEED UP THE CALCULATION FOR HIGH REYNOLDS NUMBER FLOWS.

JOB TITLE -

VNAP2 CASE 2 - SUPERSONIC SOURCE-SIJBSCNIC CONSTANT AREA FLOW

CONTROL PARAMETERS -

\begin{tabular}{|c|c|c|c|c|c|c|c|c|c|}
\hline $\begin{array}{l}\operatorname{LMAX}=21 \\
\text { IUI }=1 \\
\text { RSTAR }=\end{array}$ & $\begin{array}{l}\text { MiAAX }=11 \\
\text { IJO = } 1 \\
0.000000\end{array}$ & $\begin{array}{l}\text { NMAX }=500 \\
\text { I VPTS }=1 \\
\text { RSTARS }=\end{array}$ & $\begin{array}{l}\text { WPRINT }=10 \\
\text { WCONVI }=1 \\
0.00000 \mathrm{C}\end{array}$ & $\begin{array}{l}\text { NPLOT }= \\
\text { TSTOP = } \\
\text { PLOW = }\end{array}$ & $\begin{array}{l}500 \\
.10 E+03 \\
.0100\end{array}$ & $\begin{array}{l}\text { FDT }=.90 \\
N 1 C=0 \\
\text { ROLOW }=\end{array}$ & $\begin{array}{l}\text { FDT } 1=1.00 \\
\text { TCONV }=0.000 \\
.000100\end{array}$ & $\begin{array}{l}\text { FDT } I=.90 \\
\text { NASM }=1 \\
\text { VDT }=.25\end{array}$ & $\begin{array}{l}\text { IPUNCH }=0 \\
\text { IUNI T }=1 \\
\text { VOT } 1=.25\end{array}$ \\
\hline
\end{tabular}

FLUID MODEL -

THE RATIO OF SPECIFIC HEATS. GIMMA $=\cdot .4000$ AND THE GAS CONSTANT, $R=.0100(\mathrm{FT}-\mathrm{LBF} / \mathrm{LBM-R})$

FLOW GEOMETRY -

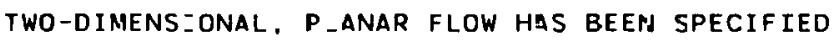

Fig. 28.

Case No. 2 output. 
DUCT GEOMETRY -

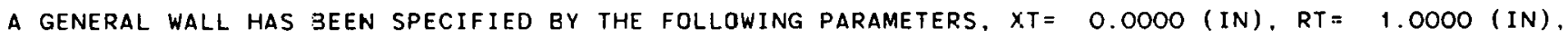

L
1
2
3
1
5
5
7
8
9
10
11
12
13
14
15
16
17
18
19
20
21

$X P(I N)$
0.0000
.2000
.4000
.6000
.8000
1.0000
1.2000
1.4000
1.6000
1.8000
2.0000
2.2000
2.4000
2.6000
2.8000
3.0000
3.2000
3.4000
3.6000
3.8000
4.0000

YW(IN)

SLOPE

1.0000

1. 1155

1.2309
1.3464

1. 3464

1. 4619

1.5773
1.6928

1.6928

1.8083
$1.923 \mathrm{~B}$

2.0392

2. 1547

2. 2702

2. 3856

2. 5011

2.6166

2.7321

2.8475

2.9630

2.9630

3.0785

3.3094
3.309

.5773

.5773
.5773

.5773
.5773

.5773
.5773

.5773

5773

.5773

.5773

.5773

5773

5773

.5773
.5773

.5773

.5773

.5773

5773

.5773

5773

5773

Fig. 28. (cont) 
DUAL FLOW SPAEE BDLNDARY GEONETRY -

GENERAL BOUNDARIES HAVE: BEEN SPECIFIE[ BY THE FOLLOWING PARAMETERS,

\begin{tabular}{|c|c|c|c|c|c|}
\hline L & $X P(I N)$ & $\because($ IN) & SLGPEL & YU(IN) & SLOPEU \\
\hline 1 & 0.0000 & .5000 & 0.0000 & .5300 & .2887 \\
\hline 2 & 2000 & .5000 & 0.0000 & .5577 & .2887 \\
\hline 3 & 4000 & .5000 & 0.0000 & .6 .155 & .2887 \\
\hline 4 & .6000 & .5000 & 0.0000 & $.67 \approx 2$ & .2887 \\
\hline 5 & 8000 & 5000 & 0.0000 & $.73<9$ & .2887 \\
\hline 6 & 1.0000 & .5000 & 0.0000 & $.78 \varepsilon 7$ & .2887 \\
\hline 7 & 1.2000 & .5000 & 0.0000 & $.84 € 4$ & .2887 \\
\hline 8 & 1.4000 & .5000 & 0.0000 & $.90<1$ & .2887 \\
\hline 9 & 1.6000 & .5000 & 0.0000 & $.96 \cdot 9$ & 2887 \\
\hline 10 & 1.8000 & .5000 & 0.0000 & 1. C196 & 2887 \\
\hline 11 & 2.0000 & .5000 & 0.0000 & 1. $67: 4$ & 2887 \\
\hline 12 & 2.2000 & .5000 & 0.0000 & 1.1351 & 2887 \\
\hline 13 & 2.4000 & .5000 & 0.0000 & 1. 1928 & .2887 \\
\hline 14 & 2.6000 & .5000 & 0.0000 & 1.2506 & 2887 \\
\hline 15 & 2.8000 & .5000 & 0.0000 & 1.5083 & 2887 \\
\hline 16 & 3.0000 & .5000 & 0.0000 & 1. 3660 & 2887 \\
\hline 17 & 3.2000 & .5000 & 0.0000 & 1. $<238$ & 2887 \\
\hline 18 & 3.4000 & .5000 & 0.0000 & $1 .<815$ & 2887 \\
\hline 19 & 3.6000 & .5000 & 0.0000 & 1.5332 & 2887 \\
\hline 20 & 3.8000 & .5000 & 0.0000 & $1.597 \mathrm{C}$ & 2887 \\
\hline 21 & 4.0000 & .5000 & 0.0000 & 1.6577 & .2887 \\
\hline
\end{tabular}

Fig. 28 (cont) 
DUUNDARY CONDITIONS -

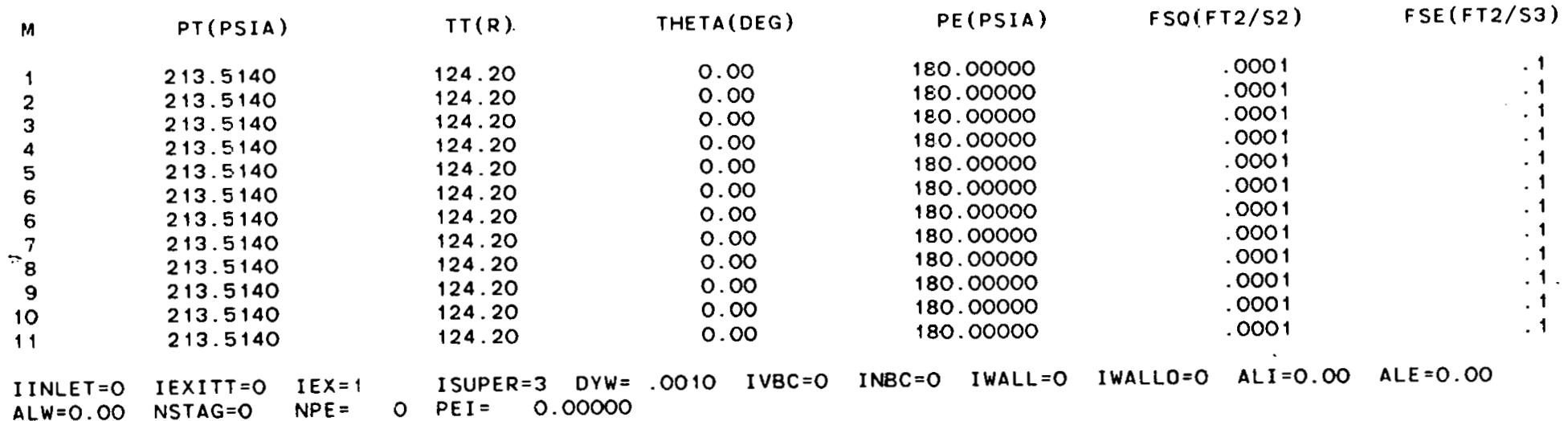

FREE-SLIP WALLS ARE SPECIFIED

ADIABATIC UPPER WALL IS SPECIFIED

ADIABATIC LOWER DUAL FLOW SPACE BOUNDARY IS SPECIFIED

ADIABATIC UPPER DUAL FLOW SPACE BOUNDÁRY IS SPECIFIED

ARTIFICAL VISCOSITY -

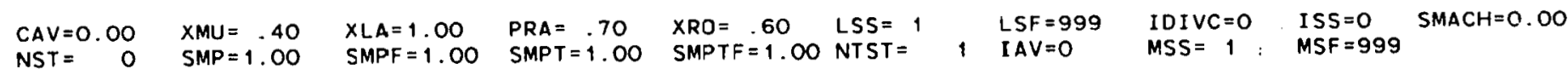

MOLECULAR VISCOSITY -

CMU $=0 . \quad(L B F-S / F T 2) \quad C L A=0 . \quad$ (LBF-S/FT2) $\quad C K=0 . \quad$ (LBF/S-R) $\quad E M U=0.00 \quad E L A=0.00 \quad E K=0.00$

TURBULENCE MOC-EL -

NO NODEL IS SPECIFIED

VARIABLE GRID PARAMETERS -

IST $=0 \quad M V C B=0 \quad$ MVCT $=0 \quad$ IOS $=0 \quad$ NIOSS $=2 \quad$ NIQSF $=0 \quad$ NVCMI $=0 \quad I L L Q S=30 \quad$ SQS $=\quad .50 \quad$ CQS $=.001$

EXPECT FILM OUTPUT FOR $\mathrm{N}=\quad 0 * * * * *$

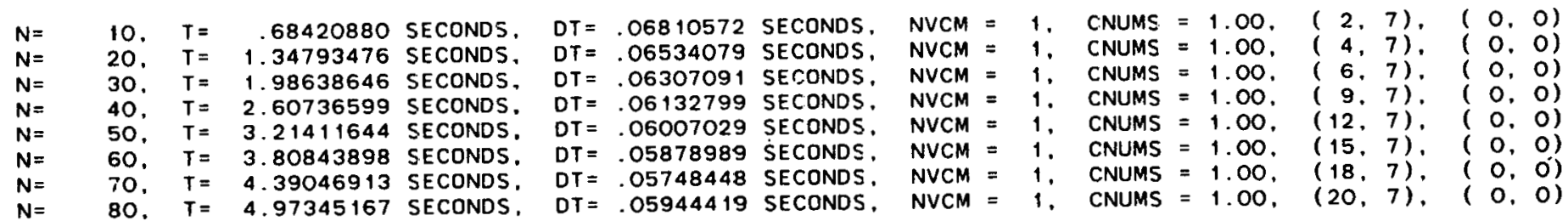


90, $T=5.57260436$ SECONDS. 100, $T=6.17059463$ SECONDS, $\begin{aligned} & \text { 110. } T=6.76882330 \text { SECONDS. } \\ & 120 .\end{aligned}=7.36700424$ SECONDS. 120, $T=7.36700424$ SECONDS $130, T=7.96519419$ SECONDS 150, $T=9.16157502$ SECONDS 160. $T=9.75976499$ SECONDS. 170. $T=10.35795503$ SECONDS 180. $T=10.95614503$ SECONDS 190. $T=11.55433504$ SECONDS 200. $T=12.15252505$ SECONDS $210, T=12.75071507$ SECONDS 220, $T=13 . \tau 4890508$ SECONDS $230, \quad T=13.04709509$ SECONDS 240. $T=14.54528510$ SECONDS 250. $T=15 . i 4347511$ SECONDS $260, T=15 . .4156512$ SECONDS $270 . \quad T=16.33985513$ SECONDS
280. 290. $T=17.53623516$ SECONDS 300. $T=18.13442517$ SECONDS $T=18.73261518$ SECONDS $T=19.33080519$ SECONDS $330 . T=19.92899520$ SECONDS $340, T=20.52718522$ SECONDS 350, $T=21 \quad 12537523$ SECONDS 360. $T=21.72356524$ SECONDS 370, $T=22.32175525$ SECONDS 380. $T=22.91994526$ SECONOS 390. $T=23.51813527$ SECONDS $400, \quad T=24.11632528$ SECONDS $410 . \quad T=24.71451530$ SECONDS $430, \quad T=25.31089532$ SECONDS $440, T=26.50908533$ 5ECONDS $450, T=27.40727534$ SECONDS $460, T=27.70546535$ SECONDS $470, T=28.30365536$ SECONDS $480 . T=28.90184538$ SECONDS $490 . \quad T=29.50003539$ SECONDS 500. $T=30.09822540$ SECONDS.

$D T=.05987051$ SECONDS $\mathrm{DT}=.05982064$ SECONDS $D T=.05981464$ SECONDS DT $=.05982018$ SECONDS $\mathrm{OT}=.05981884 \mathrm{SECONDS}$ $\mathrm{DT}=.05981901$ SECONDS $T=.05981902$ SECONDS $O T=.05981899$ SECONDS DT $=.05981900$ SECONDS DT $=05981900$ SECONDS, DT $=.05981900$ SECONDS $\mathrm{DT}=.05981900$ SECONDS $\mathrm{DT}=.05981900 \mathrm{SECONDS}$ $D T=.05981900$ SECONDS $\mathrm{DT}=.05981900$ SECONDS $D T=.05981900$ SECONDS DI $=.05981900$ SECONDS

DI $=.05981900$ SECONDS

$D 1=.05981900$ SECONDS DI $=.05981900$ SECONDS $D^{T}=.05981900$ SECONDS $D^{-}=.05981900$ SECONDS

$\mathrm{D}^{-}=.05981900$ SECONDS $D^{-}=.05981900$ SECONDS $D^{-}=.05981900$ SECONDS $D^{-}=.05981900$ SECONDS $D^{-}=.05981900$ SECONDS $D^{T}=.05981900$ SECONDS. $\mathrm{DT}=.0598: 900$ SECONDS DT $=.05981900$ SECONDS DI $=.05981900$ SECONDS $D r=.05981900$ SECONDS. Dr $=.05981900$ SECONDS. $\mathrm{DT}=.05981900$ SECONDS. $D T=.05981900$ SECONDS $\mathrm{DT}=.05981900$ SECONDS. $\mathrm{DT}=.05981900$ SECONDS. $\mathrm{DT}=05981900$ SECONDS

NVCM $=1, \quad$ CNUMS $=1.00, \quad(19,7), \quad(0,0)$ NVCM $=1, \quad$ CNUMS $=1.00 . \quad(20,7), \quad(0,0)$ NVCM $=1, \quad$ CNUMS $=1.00, \quad(20,7), \quad(0,0)$ NVCM $=1$, CNUMS $=1.00,(20,7) \cdot(0,0)$ NVCM $=1, \quad$ CNUMS $=1.00, \quad(20,7) . \quad(0,0)$ NVCM $=1$. CNUMS $=1.00, \quad(20,7), \quad(0,0)$ NVCM $=1, \quad$ CNUMS $=1.00 . \quad(20,7), \quad(0,0)$ NVCM $=1, \quad$ CNUMS $=1.00, \quad(20,7),(0,0)$ NVCM $=1, \quad$ CNUMS $=1.00, \quad(20,7), \quad(0,0)$ NVCM $=1, \quad$ CNUMS $=1.00 . \quad(20,7), \quad(0,0)$ NVCM $=1, \quad$ CNUMS $=1.00, \quad(20,7), \quad(0,0)$ NVCM $=1$, CNUMS $=1.00, \quad(20,7), \quad(0,0)$ NVCM $=1, \quad$ CNUMS $=1.00 . \quad(20,7) .(0,0)$ NVCM $=1 . \quad$ CNUMS $=1.00 . \quad(20,7),(0 ., 0)$ NVCM $=1, \quad$ CNUMS $=1.00, \quad(20,7), \quad(0,0)$ NVCM $=1$, CNUMS $=1.00,(20,7) \cdot(\mathrm{C}, 0)$ NVCM $=1, \quad$ CNUMS $=1.00, \quad(20,7), \quad(0,0)$ NVCM $=1, \quad$ CNUMS $=1.00, \quad(20,7), \quad(0,0)$
NVCM $=1, \quad$ CNUMS $=1.00, \quad(20,7), \quad(0,0)$ NVCM $=1, \quad$ CNUMS $=1.00, \quad(20,7), \quad(0,0)$ NVCM $=1, \quad$ CNUMS $=1.00 . \quad(20,7) . \quad(0,0)$ NVCM $=1, \quad$ CNUMS $=1.00, \quad(20,7), \quad(0,0)$ NVCM $=1 . \quad$ CNUMS $=1.00 . \quad(20,7), \quad(0,0)$ NVCM $=1, \quad$ CNUMS $=1.00, \quad(20,7),(0,0)$ NVCM $=1, \quad$ CNUMS $=1.00,(20,7),(0,0)$ NVCM $=1, \quad$ CNUMS $=1.00, \quad(20,7) . \quad(0,0)$ NVCM $=1$, CNUMS $=1.00,(20,7),(0,0)$ NVCM $=1, \quad$ CNUMS $=1.00, \quad(20,7),(0,0)$ NVCM $=1, \quad$ CNUME $=1.00,(20,7) \cdot(0,0)$ NVCM $=1 . \quad$ CNUME $=1.00,(20,7) \cdot(0,0)$ NVCM $=1, \quad$ CNUME $=1.00,(20,7),(0,0)$ NVCM $=1, \quad$ CNUME $=1.00, \quad(20,7), \quad(0,0)$ NVCM $=1, \quad$ CNUME $=1.00, \quad(20,7), \quad(0,0)$ NVCM $=1, \quad$ CNUMS $=1.00, \quad(20,7), \quad(0,0)$
NVCM $=1, \quad$ CNUMS $=1.00, \quad(20,7), \quad(0,0)$ NVCM $=1, \quad$ CNUMS $=1.00 . \quad(20,7), \quad(0,0)$ NVCM $=1, \quad$ CNUMS $=1.00 . \quad(20,7),(0,0)$. NVCM $=1, \quad$ CNUMS $=1.00 . \quad(20,7), \quad(0,0)$ NVCM $=1, \quad$ CNUMS $=1.00, \quad(20,7),(0,0)$ NVCM $=1, \quad$ CNUME $=1.00 .(20.7),(0,0)$ NVCN $=1, \quad$ CNUMS $=1.00 . \quad(20,7),(0,0)$ NVCN $=1, \quad$ CNUMS $=1.00 . \quad(20,7), \quad(0,0)$ NVCM $=1, \quad$ CNUMS $=1.00, \quad(20,7), \quad(0,0)$ NVCM $=1, \quad$ CNUMS $=1.00 . \quad(20,7),(0,0)$ 
MASS FLOW AND THRUST CALCULATION, $N=500$

$\begin{array}{rrrrr}L & M F(L B M / S) & M F / M F I & T(I-B F) & T / T I \\ 1 & 103.66476 & 1.0000 & 108.4936 & 1.0000 \\ 2 & 104.17112 & 1.0049 & 116.7201 & 1.0758 \\ 3 & 104.38358 & 1.0069 & 122.2641 & 1.1269 \\ 4 & 104.52396 & 1.0083 & 126.4869 & 1.1658 \\ 5 & 104.62213 & 1.0092 & 129.8680 & 1.1970 \\ 6 & 104.68099 & 1.0098 & 132.6420 & 1.2226 \\ 7 & 104.73904 & 1.0104 & 135.0156 & 1.2445 \\ 8 & 104.78580 & 1.0108 & 137.0625 & 1.2633 \\ 9 & 104.81792 & 1.0111 & 138.8408 & 1.2797 \\ 10 & 104.84414 & 1.0114 & 140.4132 & 1.2942 \\ 11 & 104.86594 & 1.0116 & 141.8178 & 1.3072 \\ 12 & 104.88962 & 1.0119 & 143.0932 & 1.3189 \\ 13 & 104.90506 & 1.0120 & 144.2406 & 1.3295 \\ 14 & 104.91802 & 1.0121 & 145.2872 & 1.3391 \\ 15 & 104.93230 & 1.0122 & 146.2559 & 1.3481 \\ 16 & 104.94652 & 1.0124 & 147.1501 & 1.3563 \\ 17 & 104.94665 & 1.0124 & 147.9603 & 1.3638 \\ 18 & 104.96477 & 1.0125 & 148.7347 & 1.3709 \\ 19 & 104.95407 & 1.0124 & 149.4413 & 1.3774 \\ 20 & 105.09699 & 1.0138 & 150.3916 & 1.3862 \\ 21 & 105.08073 & 1.0137 & 151.0062 & 1.3918\end{array}$

Fig. 28. (cont) 
SOLUTION SURFACE NO. $500-$ TIME $=30.09822540$ SECONDS (DELTA T $=.05981900$, NVCM $=1$, CNUMS $=1.00 .(20,7),(0.0)$ )

P
(PSIA)

RHO
(LBM/FT3)

VMAG
$(F / S)$

NO

\begin{tabular}{|c|c|c|c|c|c|c|c|c|c|c|}
\hline 1 & 1 & 0.0000 & 0.0000 & 6440 & 0.0000 & 179.93650 & 152.134697 & .6440 & .5005 & 118.2745 \\
\hline 1 & 2 & 0.0000 & .1000 & 6440 & 0.0000 & 179.93650 & 152.134697 & .6440 & 5005 & 118.2745 \\
\hline 1 & 3 & 0.0000 & .2000 & .6440 & 0.0000 & 179.93650 & 152.134697 & .6440 & 5005 & 118.2745 \\
\hline 1 & 4 & 0.0000 & 3000 & .6440 & 0.0000 & 179.93650 & 152.134697 & .6440 & 5005 & 118.2745 \\
\hline 1 & 5 & 0.0000 & .4000 & .6440 & 0.0000 & 179.93650 & 152.134697 & .6440 & 5005 & 118.2745 \\
\hline 1 & 6 & 0.0000 & .5000 & .6440 & 0.0000 & 179.93650 & 152.134697 & .6440 & .5005 & 118.2745 \\
\hline 1 & 6 & 0.0000 & .5000 & $\cdot .3877$ & .4006 & 81.72730 & 86.577500 & 1.4444 & 1.2564 & 94.3979 \\
\hline 1 & 7 & 0.0000 & .6000 & $\cdot .4010$ & .4853 & 76.97630 & 82.951900 & 1.4827 & 1.3008 & 92.7963 \\
\hline 1 & 8 & 0.0000 & .7000 & $\therefore 4099$ & .5698 & 72.34700 & 79.357000 & 1.5207 & 1.3460 & 91.1665 \\
\hline 1 & 9 & 0.0000 & .8000 & 1.4142 & .6532 & 67.91100 & 75.850300 & 1.5578 & 1.3914 & 89.5329 \\
\hline 1 & 10 & 0.0000 & .9000 & 1.4143 & .7349 & 63.70630 & 72.465300 & 1.5938 & 1.4367 & 87.9128 \\
\hline 1 & 11 & 0.0000 & 1.0000 & 1.4103 & .8142 & 59.74600 & 69.218200 & 1.6285 & 1.4814 & 86.3154 \\
\hline 2 & 1 & .2000 & 0.0000 & 6440 & 0.0000 & 179.94450 & 152.139524 & .6440 & 5005 & \\
\hline 2 & 2 & .2000 & .1000 & .6440 & .0000 & 179.94450 & 152.139524 & .6440 & .5005 & $\begin{array}{l}118.2760 \\
118.2760\end{array}$ \\
\hline 2 & 3 & 2000 & .2000 & .6440 & .0000 & 179.94450 & 152.139524 & .6440 & .5005 & 118.2760 \\
\hline 2 & 4 & .2000 & .3000 & .6440 & .0000 & 179.94450 & 152.139524 & .6440 & .5005 & 118.2760 \\
\hline 2 & 5 & .2000 & .4000 & .6440 & .0000 & 179.94450 & 152. 139524 & .6440 & .5005 & 118.2760 \\
\hline 2 & 6 & .2000 & .5000 & .6440 & 0.0000 & 179.94450 & 152.139524 & .6440 & 5005 & 118.2760 \\
\hline 2 & 6 & 2000 & .5577 & 1.5603 & .4504 & 60.90799 & 69.921220 & 1.6240 & 1.4706 & 87.1095 \\
\hline 2 & 7 & .2000 & .6693 & 1.5558 & .5415 & 58.14545 & 67.677034 & 1.6474 & 1.5021 & 85.9161 \\
\hline 2 & 8 & .2000 & .7808 & 1.5494 & .6294 & 55.33358 & 65.350453 & 1.6723 & 1.5360 & 84.6721 \\
\hline 2 & 9 & .2000 & .8924 & 1.5405 & .7154 & 52.45968 & 62.925772 & 1.6985 & 1.5722 & 83.3676 \\
\hline 2 & 10 & .2000 & 1.0039 & 1.5301 & .7992 & 49.55768 & 60.424814 & 1.7262 & 1.6110 & 82.0155 \\
\hline 2 & 11 & .2000 & 1.1155 & 1.5141 & .8741 & 47.12494 & 58.310333 & 1.7483 & 1.6436 & 80.8175 \\
\hline 3 & 1 & .4000 & 0.0000 & .6440 & 0.0000 & 179.95138 & 152.143681 & .6440 & .5005 & 118.2773 \\
\hline 3 & 2 & .4000 & .1000 & .6440 & -.0000 & 179.95138 & 152.143681 & .6440 & .5005 & 118.2773 \\
\hline 3 & 3 & .4000 & .2000 & .6440 & -.0000 & $179.95 \nmid 38$ & 152.143681 & .6440 & 5005 & 118.2773 \\
\hline 3 & 4 & .4000 & .3000 & 6440 & -.0000 & 179.95138 & 152.143681 & .6440 & 5005 & 118.2773 \\
\hline 3 & 5 & .4000 & 4000 & .6440 & -.0000 & 179.95138 & 152.143681 & 6440 & 5005 & 118.2773 \\
\hline 3 & 6 & .4000 & .5000 & .6440 & 0.0000 & 179.95138 & 152.143681 & .6440 & .5005 & 118.2773 \\
\hline 3 & 6 & .4000 & .6155 & 1.6730 & .4830 & 48.48676 & 59.307674 & 1.7413 & 1.6276 & 81.7546 \\
\hline 3 & 7 & .4000 & .7386 & 1.6605 & .5787 & 46.56392 & 57.657919 & 1.7584 & 1.6538 & 80.7589 \\
\hline 3 & 8 & .4000 & .8617 & 1.6462 & .6700 & 44.59378 & 55.936316 & 1.7773 & 1.6823 & 79.7224 \\
\hline 3 & 9 & .4000 & .9847 & 1.6299 & .7585 & 42.53974 & 54.104554 & 1.7978 & 1.7135 & 78.6251 \\
\hline 3 & 10 & .4000 & 1.1078 & 1.6122 & .8423 & 40.48913 & 52.240680 & 1.8190 & 1.7462 & 77.5050 \\
\hline 3 & 11 & .4000 & 1.2309 & ^. 5902 & .9181 & 38.68948 & 50.592252 & 1.8362 & 1.7746 & 76.4731 \\
\hline 4 & 1 & .6000 & 0.0000 & .6440 & 0.0000 & 179.95091 & 152.143398 & 6440 & .5005 & 118.2772 \\
\hline 4 & 2 & .6000 & .1000 & .6440 & .0000 & 179.95091 & 152.143398 & 6440 & .5005 & 118.2772 \\
\hline 4 & 3 & .6000 & .2000 & .6440 & -.0000 & 179.95091 & 152.143398 & 6440 & 5005 & 118.2772 \\
\hline 4 & 4 & 6000 & .3000 & .6440 & .0000 & 179.95091 & 152.143398 & 6440 & 5005 & 118.2772 \\
\hline 4 & 5 & 6000 & .4000 & .6440 & .0000 & 179.95091 & 152.143398 & 6440 & 5005 & 118.2772 \\
\hline 4 & 6 & .6000 & .5000 & .6440 & 0.0000 & 179.95091 & 152.143398 & 6440 & 5005 & 118.2772 \\
\hline 4 & 6 & 6000 & 6732 & $\uparrow .7563$ & .5070 & 40.07869 & 51.712182 & 1.8280 & 1.7549 & 77.5034 \\
\hline 4 & 7 & 6000 & 8078 & 1.7392 & .6061 & 38.61233 & 50.391387 & 1.8418 & 1.7782 & 76.6249 \\
\hline 4 & 8 & 6000 & .9425 & 1.7201 & .6999 & 37.13224 & 49.034950 & 1.8570 & 1.8036 & 75.7261 \\
\hline 4 & 9 & .6000 & 1.0771 & 1.6988 & 7899 & 35.59385 & 47.596833 & 1.8735 & 1.8310 & 74.7820 \\
\hline 4 & 10 & 6000 & 1.2118 & 1.6764 & .8746 & 34.03739 & 46.115423 & 1.8908 & 1.8601 & 73.8091 \\
\hline 4 & 11 & .6000 & 1.3464 & 1.6503 & .9528 & 32.58363 & 44.719000 & 1.9056 & 1.8868 & 72.8631 \\
\hline 5 & 1 & .8000 & 0.0000 & .6440 & 0.0000 & 179.94718 & 152.141143 & .6440 & 5005 & 118.2765 \\
\hline
\end{tabular}

Fig. 28. (cont) 
SOLUTICN SURFACE NO. $500-$ TIME $=30.09822540$ SECONDS. $($ DELTA T $=.05981900$, NVCM $=1$, CNUMS $=1.00 .(20.7)$. $(0.0)$ )

\begin{tabular}{|c|c|c|c|c|c|c|c|c|c|c|}
\hline $\mathbf{L}$ & M & $\stackrel{x}{(I N)}$ & $\stackrel{Y}{(I N)}$ & $\begin{array}{c}U \\
(F / S)\end{array}$ & $\begin{array}{l}\cdot V / S) \\
(F / S)\end{array}$ & $\begin{array}{c}P \\
(P S I A)\end{array}$ & $\begin{array}{c}\text { RHO } \\
\text { ( LBM/FT3) }\end{array}$ & $\begin{array}{l}\text { VMAG } \\
(F / S)\end{array}$ & $\begin{array}{l}\text { MACH } \\
\text { NO }\end{array}$ & $\begin{array}{c}T \\
(R)\end{array}$ \\
\hline 5 & 2 & .8000 & 1000 & .6440 & .0000 & 179.94718 & 152.141143 & .6440 & 5005 & 118.2765 \\
\hline 5 & 3 & .8000 & 2000 & .6440 & .0000 & 179.94718 & 152.141143 & .6440 & .5005 & 118.2765 \\
\hline 5 & 4 & .8000 & .3000 & .6440 & .0000 & 179.94718 & 152.141143 & .6440 & .5005 & 118.2765 \\
\hline 5 & 5 & .8000 & .4000 & .6440 & .0000 & 179.94718 & 152.141143 & .6440 & .5005 & 118.2765 \\
\hline 5 & 6 & .8000 & .5000 & .6440 & 0.0000 & 179.94718 & 152.141143 & .6440 & .5005 & 118.2765 \\
\hline 5 & 6 & .8000 & .7309 & 1.8217 & .5259 & 33.98808 & 45.937772 & 1.8961 & 1.8631 & 73.9872 \\
\hline 5 & 7 & .8000 & .8771 & 1.8016 & .6274 & 32.81371 & 44.833210 & 1.9077 & 1.8846 & 73.1906 \\
\hline 5 & 8 & .8000 & 1.0233 & 1.7793 & .7228 & 31.65328 & 43.724326 & 1.9205 & 1.9077 & 72.3928 \\
\hline 5 & 9 & .8000 & 1.1695 & 1.7546 & .8143 & 30.44179 & 42.543676 & 1.9344 & 1.9327 & 71.5542 \\
\hline 5 & 10 & .8000 & 1.3157 & 1.7289 & .9006 & 29.18547 & 41.297156 & 1.9494 & 1.9598 & 70.6719 \\
\hline 5 & 11 & .8000 & 1.4619 & 1.6998 & .9814 & 27.95671 & 40.064681 & 1. 9627 & 1.9858 & 69.7789 \\
\hline 6 & 1 & 1.0000 & 0.0000 & .6441 & 0.0000 & 179.94134 & 152.137620 & 6441 & 5005 & $118 \quad 2754$ \\
\hline 6 & 2 & 1.0000 & .1000 & .6441 & .0000 & 179.94134 & 152.137620 & $\begin{array}{l}.6441 \\
.6441\end{array}$ & .5005 & $\begin{array}{l}118.2754 \\
118.2754\end{array}$ \\
\hline 6 & 3 & 1.0000 & .2000 & .6441 & .0000 & 179.94134 & 152.137620 & .6441 & .5005 & 118.2754 \\
\hline 6 & 4 & 1.0000 & .3000 & .6441 & .0000 & 179.94134 & 152.137620 & .6441 & .5005 & 118.2754 \\
\hline 6 & 5 & 1.0000 & .4000 & .6441 & -.0000 & 179.94134 & 152.137620 & .6441 & 5005 & 118.2754 \\
\hline 6 & 6 & 1.0000 & .5000 & .6441 & 0.0000 & 179.94134 & 152.137620 & .6441 & 5005 & 118.2754 \\
\hline 6 & 6 & 1.0000 & .7887 & 1.8752 & .5413 & 29.37709 & 41.375081 & 1.9518 & 1.9577 & 71.0019 \\
\hline 6 & 7 & 1.0000 & .9464 & 1.8529 & .6446 & 28.40590 & 40.425452 & 1.9619 & 1.9780 & 70.2674 \\
\hline 6 & 8 & 1.0000 & 1.1041 & 1.8283 & .7414 & 27.46269 & 39.489769 & 1.9729 & 1.9995 & 69.5438 \\
\hline 6 & 9 & 1.0000 & 1.2619 & 1.8012 & .8343 & 26.46292 & 38.477828 & 1.9851 & 2.0230 & 68.7745 \\
\hline 6 & 10 & 1.0000 & 1.4196 & 1.7731 & .9224 & 25.40415 & 37.386397 & 1.9987 & 2.0492 & 67.9502 \\
\hline 6 & 11 & 1.0000 & 1.5773 & 1.7415 & 1.0055 & 24.34057 & 36.276694 & 2.0109 & 2.0748 & 67.0970 \\
\hline 7 & 1 & 1.2000 & 0.0000 & .6441 & 0.0000 & 179.93970 & 152.136628 & .6441 & .5005 & 118.2751 \\
\hline 7 & 2 & 1.2000 & .1000 & .6441 & -.0000 & 179.93970 & 152.136628 & .6441 & .5005 & 118.2751 \\
\hline 7 & 3 & 1.2000 & .2000 & .6441 & -.0000 & 179.93970 & 152.136628 & .6441 & .5005 & 118.2751 \\
\hline 7 & 4 & 1.2000 & .3000 & .6441 & -.0000 & 179.93970 & 152.136628 & .6441 & .5005 & 118.2751 \\
\hline 7 & 5 & 1.2000 & .4000 & .6441 & -.0000 & 179.93970 & 152.136628 & .6441 & .5005 & 118.2751 \\
\hline 7 & 6 & 1.2000 & 5000 & .6441 & 0.0000 & 179.93970 & 152.136628 & 6441 & .5005 & 118.2751 \\
\hline 7 & 6 & 1.2000 & .8464 & 1.9201 & .5543 & 25.77387 & 37.670133 & 1.9986 & 2.0420 & 68.4199 \\
\hline 7 & 7 & 1.2000 & 1.0157 & 1.8962 & .6590 & 24.95046 & 36.836126 & 2.0074 & 2.0615 & 67.7337 \\
\hline 7 & 8 & 1.2000 & 1.1850 & 1.8698 & .7569 & 24.15841 & 36.023058 & 2.0172 & 2.0818 & 67.0637 \\
\hline 7 & 9 & 1.2000 & 1.3542 & 1.8409 & .8514 & 23.30152 & 35.124801 & 2.0283 & 2.1047 & 66.3392 \\
\hline 7 & 10 & 1.2000 & 1.5235 & 1.8109 & .9413 & 22.38400 & 34.144972 & 2.0409 & 2.1304 & 65.5558 \\
\hline 7 & 11 & 1.2000 & 1.6928 & 1.7774 & 1.0262 & 21.45017 & 33.134878 & 2.0523 & 2.1558 & 64.7359 \\
\hline 8 & 1 & 1.4000 & 0.0000 & .6441 & 0.0000 & 179.94290 & 152.138561 & .6441 & .5005 & 118.2757 \\
\hline 8 & 2 & 1.4000 & .1000 & .6441 & -.0000 & 179.94290 & 152.13856 & .6441 & .5005 & 118.2757 \\
\hline 8 & 3 & 1.4000 & .2000 & .6441 & -.0000 & 179.94290 & 152.138561 & .6441 & .5005 & 118.2757 \\
\hline 8 & 4 & 1.4000 & .3000 & .6441 & -.0000 & 179.94290 & 152.138561 & .6441 & .5005 & 118.2757 \\
\hline 8 & 5 & 1.4000 & 4000 & .6441 & -.0000 & 179.94290 & 152.138564 & .6444 & 5005 & 118.2757 \\
\hline 8 & 6 & 1.4000 & .5000 & .6441 & 0.0000 & 179.94290 & 152.138561 & .6441 & .5005 & 118.2757 \\
\hline 8 & 6 & 1.4000 & .9041 & 1.9586 & .5654 & 22.88582 & 34.595375 & 2.0386 & 2.1183 & 66.1528 \\
\hline 8 & $7-$ & 1.4000 & 1.0849 & 1.9333 & .6713 & 22.17336 & 33.850018 & 2.0466 & 2.1371 & 65.5047 \\
\hline 8 & 8 & 1.4000 & 1.2658 & 1.9056 & .7704 & 21.48936 & 33.125244 & 2.0555 & 2.1568 & 64.8731 \\
\hline 8 & 9 & 1.4000 & 1.4466 & 1.8753 & .8664 & 20.73441 & 32.307548 & 2.0658 & 2.1794 & 64.1782 \\
\hline 8 & 10 & 1.4000 & 1.6275 & 1.8437 & 9579 & 19.92505 & 31.414473 & 2.0777 & 2.2048 & 63.4264 \\
\hline 8 & 11 & 1.4000 & 1.8083 & 1.8086 & 1.0442 & 19.09689 & 30.488485 & 2.0883 & 2.2301 & 62.6364 \\
\hline 9 & 1 & 1.6000 & 0.0000 & .6440 & 0.0000 & 179.94924 & 152.142382 & .6440 & .5005 & 118.2769 \\
\hline 9 & 2 & 1.6000 & .1000 & .6440 & -.0000 & 179.94924 & 152.142382 & .6440 & .5005 & 118.2769 \\
\hline
\end{tabular}


SOLUTION SURFACE NO. $500-$ TIME $=30.09822540$ SECONDS (OELTA $T=.05981900$, NVCM $=1$, CNUMS $=1.00,(20,7) .(0.0))$

\begin{tabular}{|c|c|c|c|c|c|c|c|c|c|c|}
\hline L & M & $\begin{array}{l}X \\
(I N)\end{array}$ & $\begin{array}{l}Y \\
(I N)\end{array}$ & $\begin{array}{c}U \\
(F / S)\end{array}$ & $\begin{array}{c}V \\
(F / S)\end{array}$ & $\begin{array}{c}P \\
(P S I A)\end{array}$ & $\begin{array}{c}\text { RHO } \\
(L B M / F T 3)\end{array}$ & $\begin{array}{l}\text { VMAG } \\
(F / S)\end{array}$ & $\begin{array}{c}\text { MACH } \\
\text { NO }\end{array}$ & $\begin{array}{c}T \\
(R)\end{array}$ \\
\hline 9 & 3 & 1.6000 & .2000 & 6440 & -.0000 & 179.94924 & 152.142382 & .6440 & 5005 & 118.2769 \\
\hline 9 & 4 & 1.6000 & .3000 & .6440 & -.0000 & 179.94924 & 152.142382 & 6440 & 5005 & 118.2769 \\
\hline 9 & 5 & 1.6000 & .4000 & .6440 & -.0000 & 179.94924 & 152.142382 & 6440 & 5005 & 118.2769 \\
\hline 9 & 6 & 1.6000 & .5000 & .6440 & 0.0000 & 179.94924 & 152.142382 & .6440 & 5005 & 118.2769 \\
\hline 9 & 6 & 1.6000 & .9619 & 1.9921 & .5751 & 20.52185 & 31.997014 & 2.0734 & 2.1881 & 64.1368 \\
\hline 9 & 7 & 1.6000 & 1. 1543 & 1.9657 & .6821 & 19.89505 & 31.321326 & 2.0807 & 2.2065 & 63.5192 \\
\hline 9 & 8 & 1.6000 & 1.3467 & 1.9370 & .7824 & 19.29097 & 30.561937 & 2.0890 & 2.2259 & 62.9151 \\
\hline 9 & 9 & 1.6000 & 1.5390 & 1.9055 & .8798 & 18.61319 & 29.905045 & 2.0988 & 2.2484 & 62.241 \\
\hline 9 & 10 & 1.6000 & 1.7314 & 1.8725 & .9727 & 17.89087 & 29.083375 & 2.1100 & 2.2737 & 61.515 \\
\hline 9 & 11 & 1.6000 & 1.9238 & 1.8361 & 1.0600 & 17.15044 & 28.229734 & 2.1201 & 2.2988 & 60.753 \\
\hline 10 & 1 & 1.8000 & 0.0000 & 6440 & 0.0000 & 179.95680 & 152.146960 & 6440 & .5004 & 118.278 \\
\hline 10 & 2 & 1.8000 & .1000 & 6440 & -.0000 & 179.95680 & 152.146960 & 6440 & 5004 & 148.278 \\
\hline 10 & 3 & 1.8000 & 2000 & 6440 & -.0000 & 179.95680 & 152.146960 & 6440 & 5004 & 118.278 \\
\hline 10 & 4 & 1.8000 & .3000 & 6440 & -.0000 & 179.95680 & 152.146960 & .6440 & .5004 & 118.278 \\
\hline 10 & 5 & 1.8000 & .4000 & 6440 & -.0000 & 179.95680 & 152.146960 & 6440 & .5004 & 118.278 \\
\hline 10 & 6 & 1.8000 & 5000 & 6440 & 0.0000 & 179.95680 & 152.146960 & .6440 & .5004 & 118.278 \\
\hline 10 & 6 & 1.8000 & 1.0196 & 2.0216 & .5836 & 18.55307 & 29.768315 & 2. 1042 & 2.2526 & 62.324 \\
\hline 10 & 7 & 1.8000 & 1.2235 & 1.9944 & 6917 & 17.99419 & 29.148766 & 2.1109 & 2.2707 & 61.732 \\
\hline 10 & 8 & 1.8000 & 1.4274 & 1.9647 & 7931 & 17.45156 & 28.539665 & 2.1187 & 2.2899 & 61.148 \\
\hline 10 & 9 & 1.8000 & 1.6314 & 1.9322 & 8919 & 16.83548 & 27.831693 & 2.1282 & 2.3126 & 60.4903 \\
\hline 10 & 10 & 1.8000 & 1.8353 & 1.8981 & 9859 & 16.18554 & 27.071089 & 2.1388 & 2.3378 & 59.789 \\
\hline 10 & 11 & 1.8000 & 2.0392 & 1.8605 & 1.0742 & 15.51887 & 26.280284 & 2.1483 & 2.3628 & 59.051 \\
\hline 11 & 1 & 2.0000 & 0.0000 & 6439 & 0.0000 & 179.96285 & 152.150622 & .6439 & .5004 & 118.279 \\
\hline 11 & 2 & 2.0000 & .1000 & .6439 & 0000 & 179.96285 & 152.150622 & .6439 & .5004 & 118.279 \\
\hline 11 & 3 & 2.0000 & .2000 & .6439 & .0000 & 179.96285 & 152.150622 & .6439 & .5004 & 118.279 \\
\hline 11 & 4 & 2.0000 & .3000 & 6439 & .0000 & 179.96285 & 152.150622 &.$\in 439$ & 5004 & 118.279 \\
\hline 11 & 5 & 2.0000 & .4000 & 6439 & .0000 & 179.96285 & 152.150622 & .6439 & 5004 & 148.279 \\
\hline 11 & 6 & 2.0000 & .5000 & 6439 & 0.0000 & 179.96285 & 152.150622 &.$€ 439$ & .5004 & 118.279 \\
\hline 11 & 6 & 2.0000 & 1.0774 & 2.0480 & 5912 & 16.88929 & 27.832515 & 2.1316 & 2.3127 & 60.681 \\
\hline 11 & 7 & 2.0000 & 1.2929 & 2.0200 & .7003 & 16.38558 & 27.259293 & 2.1379 & 2.3305 & 60.110 \\
\hline 11 & 8 & 2.0000 & 1.5083 & 1.9895 & .8029 & 15.89210 & 26.690556 & 2. 1454 & 2.3498 & 59.542 \\
\hline 11 & 9 & 2.0000 & 1.7238 & 1.9562 & 9029 & 15.32754 & 26.024145 & 2.1545 & 2.3726 & 58.897 \\
\hline 11 & 10 & 2.0000 & 1.9392 & 1.9210 & 9978 & 14.73910 & 25.316975 & 2.1647 & 2.3977 & 58.218 \\
\hline 11 & 11 & 2.0000 & 2.1547 & 1.8824 & 1.0868 & 14.13497 & 24.581054 & 2.1737 & 2.4226 & 57.503 \\
\hline 12 & 1 & 2.2000 & $0.000 \mathrm{C}$ & .6439 & 0.0000 & 179.96793 & 152.153669 & $\in 439$ & .5004 & 118.280 \\
\hline 12 & 2 & 2.2000 & $.100 \mathrm{G}$ & .6439 & .0000 & 179.96793 & 152.153669 & 6439 & .5004 & 118.280 \\
\hline 12 & 3 & 2.2000 & .2000 & 6439 & .0000 & 179.96793 & 152.153669 & .6439 & .5004 & 118.280 \\
\hline 12 & 4 & 2.2000 & $-300 \mathrm{C}$ & .6439 & .0000 & 179.96793 & 152.153669 & .6439 & .5004 & 118.280 \\
\hline 12 & 5 & 2.2000 & .4000 & 6439 & .0000 & 179.96793 & 152.153669 & 6439 & .5004 & $118.28 i$ \\
\hline 12 & 6 & 2.2000 & .5000 & 6439 & 0.0000 & 179.96793 & 152.153669 &.$\epsilon .439$ & 5004 & 118.280 \\
\hline 12 & 6 & 2.2000 & $1.135 \uparrow$ & 2.0718 & .5981 & 15.46617 & 26.133668 & $2 . \$ 564$ & 2.3690 & 59.18 \\
\hline 12 & 7 & $2.200 \mathrm{C}$ & 1.3624 & 2.0430 & .7082 & 15.00826 & 25.599550 & $2 . ; 62 \Xi$ & 2.3867 & 58.627 \\
\hline 12 & 8 & $2.200 \mathrm{C}$ & 1.5894 & 2.0118 & .8119 & 14.55552 & 25.064617 & $2 .{ }^{4} 695$ & 2.4061 & 58.072 \\
\hline 12 & 9 & $2.200 \mathrm{C}$ & 1.8162 & 1.9777 & 9129 & 14.03541 & 24.434968 & $2.78 \Xi$ & 2.4291 & 57.439 \\
\hline 12 & 10 & $2.200 \mathrm{C}$ & 2.0432 & 1.9416 & 1.0086 & 13.49995 & 23.775252 & $2 . \cdot 880$ & 2.4540 & 56.78 \\
\hline 12 & 11 & $2.200 \mathrm{C}$ & 2.2702 & 1.9023 & 1.0983 & 12.94929 & 23.087544 & $2 . \cdot 96 \epsilon$ & 2.4788 & 56.087 \\
\hline 13 & 1 & 2.4000 & 0.0000 & .6439 & 0.0000 & 179.97290 & 152.156657 & .6439 & .5004 & 118.28 \\
\hline 13 & 2 & $2.400 C$ & .1000 & .6439 & .0000 & 179.97290 & 152.156657 & .6439 & 5004 & 118.28 \\
\hline 13 & 3 & 2.400 & 2000 & 6439 & 0000 & 179.97290 & 152.156657 & 6439 & 5004 & 118.28 \\
\hline
\end{tabular}

Fig. 28. (cont) 


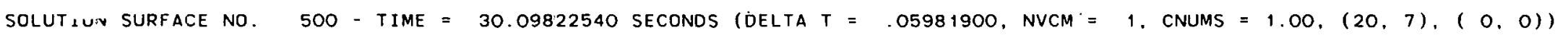

\begin{tabular}{|c|c|c|c|c|c|c|c|c|c|c|}
\hline $\mathrm{L}$ & M & $\begin{array}{l}x \\
(I N)\end{array}$ & $\stackrel{Y}{(I N)}$ & $\begin{array}{c}U \\
(F / S)\end{array}$ & $\begin{array}{c}v \\
(F / S)\end{array}$ & $\begin{array}{c}P \\
\text { (PSIA) }\end{array}$ & $\begin{array}{c}\text { RHO } \\
\text { (LBM/FT3) }\end{array}$ & $\begin{array}{l}\text { VMAG } \\
(F / S)\end{array}$ & $\begin{array}{l}\text { MACH } \\
\text { NO }\end{array}$ & $\begin{array}{c}T \\
(R)\end{array}$ \\
\hline 13 & 4 & 2.4000 & .3000 & .6439 & .0000 & 179.97290 & 152.156657 & 6439 & 5004 & 118.2813 \\
\hline 13 & 5 & 2.4000 & .4000 & .6439 & .0000 & 179.97290 & 152.156657 & .6439 & 5004 & 118.2813 \\
\hline 13 & 6 & 2.4000 & 5000 & .6439 & 0.0000 & 179.97290 & 152.156657 & .6439 & 5004 & 118.2813 \\
\hline 13 & 6 & 2.4000 & 1. 1928 & 2.0934 & .6043 & 14.23589 & 24.629139 & 2.1789 & 2.4222 & 57.8010 \\
\hline 13 & 7 & 2.4000 & 1.4314 & 2.0640 & .7154 & 13.81672 & 24.128653 & 2.1845 & 2.4397 & 57.2627 \\
\hline 13 & 8 & 2.4000 & 1.6699 & 2.0321 & .8202 & 13.39878 & 23.623069 & 2.1914 & 2.4592 & 56.7191 \\
\hline 13 & 9 & 2.4000 & 1.9085 & 1.9973 & .9221 & 12.91781 & 23.026793 & 2.1999 & 2.4823 & 56.0990 \\
\hline 13 & 10 & 2.4000 & 2.1470 & 1.9604 & 1.0185 & 12.42841 & 22.409512 & 2.2092 & 2.5072 & 55.4604 \\
\hline 13 & 11 & 2.4000 & 2.3856 & 1.9203 & 1.1087 & 11.92377 & 21.764301 & 2.2174 & 2.5319 & 54.7859 \\
\hline 14 & 1 & 2.6000 & 0.0000 & .6438 & 0.0000 & 179.97908 & 152.160424 & .6438 & .5003 & 118.2824 \\
\hline 14 & 2 & 2.6000 & .1000 & .6438 & -.0000 & 179.97908 & 152.160424 & .6438 & 5003 & 118.2824 \\
\hline 14 & 3 & 2.6000 & .2000 & .6438 & -.0000 & 179.97908 & 152.160424 & 6438 & .5003 & 118.2824 \\
\hline 14 & 4 & 2.6000 & .3000 & .6438 & -.0000 & 179.97908 & 152.160424 & .6438 & .5003 & 118.2824 \\
\hline 14 & 5 & 2.6000 & .4000 & .6438 & .0000 & 179.97908 & 152.160424 & .6438 & .5003 & 118.2824 \\
\hline 14 & 6 & 2.6000 & .5000 & .6438 & 0.0000 & 179.97908 & 152.160424 & .6438 & .5003 & 118.2824 \\
\hline 14 & 6 & 2.6000 & 1.2506 & 2.1132 & .6100 & 13. 16282 & 23.286586 & 2.1994 & 2.4725 & 56.5253 \\
\hline 14 & 7 & 2.6000 & 1.5007 & 2.0831 & .7221 & 12.77693 & 22.815539 & 2.2047 & 2.4900 & 56.0010 \\
\hline 14 & 8 & 2.6000 & 1.7508 & 2.0506 & .8278 & 12.38946 & 22.336234 & 2.2114 & 2.5095 & 55.4680 \\
\hline 14 & 9 & 2.6000 & 2.0009 & 2.0152 & .9306 & 11.94346 & 21.770772 & 2.2197 & 2.5328 & 54.8601 \\
\hline 14 & 10 & 2.6000 & 2.2510 & 1.9776 & 1.0275 & 11.49447 & 21.191776 & 2.2286 & 2.5574 & 54.2403 \\
\hline 14 & 11 & 2.6000 & 2.5011 & 1.9368 & 1.1182 & 11.02983 & 20.584401 & 2.2364 & 2.5821 & 53.5834 \\
\hline 15 & 1 & 2.8000 & 0.0000 & .6438 & 0.0000 & 179.98692 & 152.165171 & .6438 & .5003 & 118.2839 \\
\hline 15 & 2 & 2.8000 & .1000 & .6438 & -.0000 & 179.98692 & 152.165171 & .6438 & .5003 & 118.2839 \\
\hline 15 & 3 & 2.8000 & .2000 & .6438 & -.0000 & 179.98692 & 152.165171 & 6438 & .5003 & 118.2839 \\
\hline 15 & 4 & 2.8000 & .3000 & .6438 & -.0000 & 179.98692 & 152.165171 & .6438 & .5003 & 118.2839 \\
\hline 15 & 5 & 2.8000 & 4000 & .6438 & .0000 & 179.98692 & 152.165171 & .6438 & .5003 & 118.2839 \\
\hline 15 & 6 & 2.8000 & .5000 & .6438 & 0.0000 & 179.98692 & 152.165171 & .6438 & .5003 & 118.2839 \\
\hline 15 & 6 & 2.8000 & 1.3083 & 2.1313 & .6153 & 12.21939 & 22.080390 & 2.2184 & 2.5203 & 55.3405 \\
\hline 15 & 7 & 2.8000 & 1.5700 & 2.1007 & .7283 & 11.86240 & 21.6 .35351 & 2.2234 & 2.5377 & 54.8288 \\
\hline 15 & 8 & 2.8000 & 1.8316 & 2.0677 & .8349 & 11.50196 & 21.179933 & 2.2299 & 2.5574 & 54.3059 \\
\hline 15 & 9 & 2.8000 & 2.0933 & 2.0316 & .9384 & 11.08743 & 20.643063 & 2.2379 & 2.5807 & 53.7102 \\
\hline 15 & 10 & 2.8000 & 2.3549 & 1.9934 & 1.0359 & 10.67390 & 20.098480 & 2.2464 & 2.6053 & 53.1080 \\
\hline 15 & 11 & 2.8000 & 2.6166 & 1.9520 & 1.1270 & 10.24396 & 19.524511 & 2.2540 & 2.6299 & 52.4672 \\
\hline 16 & 1 & 3.0000 & 0.0000 & .6437 & 0.0000 & 179.99548 & 152.170292 & .6437 & .5002 & 118.2856 \\
\hline 16 & 2 & 3.0000 & .1000 & .6437 & -.0000 & 179.99548 & 152.170292 & .6437 & .5002 & 118.2856 \\
\hline 16 & 3 & 3.0000 & .2000 & .6437 & -.0000 & 179.99548 & 152.170292 & .6437 & .5002 & 118.2856 \\
\hline 16 & 4 & 3.0000 & 3000 & .6437 & -.0000 & 179.99548 & 152.170292 & 6437 & .5002 & 118.2856 \\
\hline 16 & 5 & 3.0000 & 4000 & .6437 & -.0000 & 179.99548 & 152.170292 & .6437 & .5002 & 118.2856 \\
\hline 16 & 6 & 3.0000 & .5000 & .6437 & 0.0000 & 179.99548 & 152.170292 & .6437 & .5002 & 118.2856 \\
\hline 16 & 6 & 3.0000 & 1.3660 & 2.1481 & .6201 & 11.38500 & 20.991480 & 2.2358 & 2.5658 & 54.2363 \\
\hline 16 & 7 & 3.0000 & 1.6392 & 2.1170 & .7340 & 11.05352 & 20.569916 & 2.2406 & 2.5833 & 53.7364 \\
\hline 16 & 8 & 3.0000 & 1.9124 & 2.0834 & 8415 & 10.71752 & 20.136775 & 2.2469 & 2.6030 & 53.2236 \\
\hline 16 & 9 & 3.0000 & 2.1857 & 2.0467 & .9457 & 10.33154 & 19.626696 & 2.2546 & 2.6263 & 52.6402 \\
\hline 16 & 10 & 3.0000 & 2.4589 & 2.0079 & 1.0436 & 9.94958 & 19.113580 & 2.2629 & 2.6507 & 52.0550 \\
\hline 16 & 11 & 3.0000 & 2.7321 & 1.9659 & 1.1350 & 9.55056 & 18.570203 & 2.2701 & 2.6753 & 51.4295 \\
\hline 17 & 1 & 3.2000 & 0.0000 & .6437 & 0.0000 & 180.00297 & 152.174806 & .6437 & .5002 & 118.2870 \\
\hline 17 & 2 & 3.2000 & .1000 & .6437 & -.0000 & 180.00297 & 152.174806 & .6437 & .5002 & 118.2870 \\
\hline 17 & 3 & 3.2000 & .2000 & .6437 & -.0000 & 180.00297 & 152.174806 & .6437 & .5002 & 118.2870 \\
\hline 17 & 4 & 3.2000 & .3000 & .6437 & $-: 0000$ & 180.00297 & 152.174806 & .6437 &. .5002 & 118.2870 \\
\hline
\end{tabular}


SOLUTION SURFACE NO. $500-$ TIME $=30.09822540$ SECONCS $($ DELTA T $=.05981900$, NVCM $=1$, CNUMS $=1.00,(20,7),($ O. O) $)$

\begin{tabular}{|c|c|c|c|c|c|c|c|c|c|c|}
\hline $\mathbf{L}$ & M & $\begin{array}{l}X \\
(I N)\end{array}$ & $\stackrel{Y}{Y}$ & $\begin{array}{c}J \\
(F ! S)\end{array}$ & $\begin{array}{c}v \\
(F / S)\end{array}$ & $\begin{array}{c}P \\
(P S I A)\end{array}$ & $\begin{array}{c}\text { RHO } \\
\text { ( LBM/FT3) }\end{array}$ & $\begin{array}{l}\text { VMAG } \\
(F / S)\end{array}$ & $\begin{array}{l}\text { MACH } \\
\text { NO }\end{array}$ & $\begin{array}{c}T \\
(R)\end{array}$ \\
\hline 17 & 5 & 3.2000 & 4000 & .6437 & -.0000 & 180.00297 & 152.174806 & 6437 & .5002 & 118.2870 \\
\hline 17 & 6 & 3.2000 & 5000 & .6437 & 0.0000 & 180.00297 & 152.174806 & 6437 & .5002 & 118.2870 \\
\hline 17 & 6 & 3.2000 & 1.4238 & 2.1638 & .6246 & 10.64075 & 20.000878 & 2.2521 & 2.6095 & 53.2014 \\
\hline 17 & 7 & 3.2000 & 1.7085 & 2.1322 & .7393 & 10.33162 & 19.600098 & 2.2567 & 2.6270 & 52.7121 \\
\hline 17 & 8 & 3.2000 & 1.9933 & 2.0980 & .8477 & 10.01753 & 19.187340 & 2.2628 & 2.6467 & 52.2091 \\
\hline 17 & 9 & 3.2000 & 2.2780 & 2.0609 & .9524 & 9.65735 & 18.702112 & 2.2703 & 2.6701 & 51.6378 \\
\hline 17 & 10 & 3.2000 & 2.5628 & 2.0215 & 1.0507 & 9.30312 & 18.217098 & 2.2782 & 2.5944 & 51.0681 \\
\hline 17 & 11 & 3.2000 & 2.8475 & 1.9791 & 1.1426 & 8.93058 & 17.699921 & 2.2852 & 2.7190 & 50.4555 \\
\hline 18 & 1 & 3.4000 & 0.0000 & .6436 & 0.0000 & 180.00750 & 152.177594 & .6436 & .5002 & 118.2878 \\
\hline 18 & 2 & 3.4000 & .1000 & .6436 & .0000 & 180.00750 & 152.177594 & .6436 & .5002 & 118.2878 \\
\hline 18 & 3 & 3.4000 & .2000 & .6436 & .0000 & 180.00750 & 152.177594 & .6436 & .5002 & 118.2878 \\
\hline 18 & 4 & 3.4000 & .3000 & .6436 & -.0000 & 180.00750 & 152.177594 & .6436 & .5002 & 118.2878 \\
\hline 18 & 5. & 3.4000 & .4000 & .6436 & -.0000 & 180.00750 & 152.177594 & .6436 & .5002 & 118.2878 \\
\hline 18 & 6 & 3.4000 & .5000 & .6436 & 0.0000 & 180.00750 & 152.177594 & .6436 & .5002 & 118.2878 \\
\hline 18 & 6 & 3.4000 & 1.4815 & 2.1781 & 6288 & 9.97767 & 19.101643 & 2.2670 & 2.6510 & 52.2346 \\
\hline 18 & $T$ & 3.4000 & 1.7778 & 2.1460 & .7443 & 9.68915 & 18.720764 & 2.2714 & 2.6684 & 51.7561 \\
\hline 18 & $8:$ & 3.4000 & 2.0741 & 2.1114 & .8534 & 9.39553 & 18.327845 & 2.2773 & 2.6882 & 51.2637 \\
\hline 18 & 9 & 3.4000 & 2.3704 & 2.0737 & 9586 & 9.05890 & 17.865995 & 2.2845 & 2.7115 & 50.7047 \\
\hline 18 & 10 & 3.4000 & 2.6667 & 2.0338 & 1.0573 & 8.72981 & 17.407209 & 2.2922 & 2.7356 & 50.1505 \\
\hline 18 & 11 & 3.4000 & 2.9630 & 1.9909 & 1.1495 & 8.38247 & 16.916345 & 2.2989 & 2.7601 & 49.5525 \\
\hline 19 & 1 & 3.6000 & 0.0000 & .6436 & 0.0000 & 180.00804 & 152.177928 & .6436 & .5001 & 118.2879 \\
\hline 19 & 2 & 3.6000 & .1000 & .6436 & .0000 & 180.00804 & 152.177928 & .6436 & .5001 & $1+8.2879$ \\
\hline 19 & 3 & 3.6000 & .2000 & .6436 & -.0000 & 180.00804 & 152.177928 & .6436 & .5001 & 118.2879 \\
\hline 19 & 4 & 3.6000 & .3000 & .6436 & -.0000 & 180.00804 & 152.177928 & .6436 & .5001 & 118.2879 \\
\hline 19 & 5 & 3.6000 & 4000 & .6436 & -.0000 & 180.00804 & 152.177928 & .6436 & 5001 & 118.2879 \\
\hline 19 & 6 & 3.6000 & .5000 & .6436 & 0.0000 & 180.00804 & 152.177928 & .6436 & 5001 & 118.2879 \\
\hline 19 & $\epsilon$ & 3.6000 & 1.5392 & 2. 1922 & 6328 & 9.37246 & 18.267047 & 2.2817 & 2.6922 & 51.3080 \\
\hline 19 & 7 & 3.6000 & 1.8471 & 2. 1597 & .7490 & 9.10049 & 17.901453 & 2.2859 & 2.7096 & 50.8366 \\
\hline 19 & $\varepsilon$ & 36000 & 2.1549 & 2.1246 & 8588 & 8.82515 & 17.526411 & 2.2916 & 2.7294 & 50.3534 \\
\hline 19 & ؟ & 3.6000 & 2.4628 & 2.0864 & 9645 & 8.51013 & 17.086474 & 2.2986 & 2.7527 & 49.8062 \\
\hline 19 & 10 & 3.6000 & 2.7706 & 2.0461 & 1.0636 & 8.20309 & 16.650830 & 2.3060 & 2.7767 & 49.2654 \\
\hline 19 & 11 & 3.6000 & 3.0785 & 2.0026 & 1.1562 & 7.87531 & 16. 179224 & 2.3125 & 2.8013 & 48.6754 \\
\hline 20 & 1 & 3.8000 & 0.0000 & 6436 & 0.0000 & 180.00500 & 152.176053 & .6436 & .5002 & 118.2873 \\
\hline 20 & $z$ & 3.8000 & .1000 & 6436 & -.0000 & 180.00500 & 152.176053 & 6436 & 5002 & 118.2873 \\
\hline 20 & 3 & 3.8000 & .2000 & 6.436 & -.0000 & 180.00500 & 152.176053 & 6436 & 5002 & 118.2873 \\
\hline 20 & $<$ & 3.8000 & .3000 & .6436 & -.0000 & 180.00500 & 152.176053 & .6436 & .5002 & 118.2873 \\
\hline 20 & 5 & 3. 8000 & .4000 & .6436 & -.0000 & 180.00500 & 152.176053 & .6436 & .5002 & 118.2873 \\
\hline 20 & 6 & 3.8000 & .5000 & .6436 & 0.0000 & 180.00500 & 152.176053 & .6436 & 5002 & 118.2873 \\
\hline 20 & 6 & 3.8000 & 1.5970 & $=2047$ & 6364 & 8.87248 & 17.555816 & 2.2947 & 2.7280 & 50.5387 \\
\hline 20 & $?$ & 3.8000 & 1.9164 & 2.1718 & .7532 & 8.61417 & 17.203403 & 2.2987 & 2.7455 & 50.0725 \\
\hline 20 & $\mathbf{8}$ & 3.8000 & 2.2358 & 2.1363 & .8635 & 8.35413 & 16.843914 & $2: 3043$ & 2.7653 & 49.5973 \\
\hline 20 & 9 & 3.8000 & 2.5551 & 2.0978 & .9696 & 8. 05694 & 16.422722 & 2.3110 & 2.7886 & 49.0597 \\
\hline 20 & 10 & 3.8000 & 2.8745 & 2.0570 & 1.0691 & 7.76865 & 16.007691 & 2.3182 & 2.8124 & 48.5307 \\
\hline 20 & 11 & 3.8000 & 3.1939 & 2.0130 & 1. 1622 & 7.45564 & 15.550579 & 2.3245 & 2.8372 & 47.9445 \\
\hline 21 & 1 & 4.0000 & 0.0000 & .6436 & 0.0000 & 180.00000 & 152.173018 & .6436 & 5002 & 118.2864 \\
\hline 21 & 2 & 4.0000 & .1000 & .6436 & -.0000 & 180.00000 & 152.173018 & .6436 & 5002 & 118.2864 \\
\hline 21 & 3 & 4.0000 & .2000 & .6436 & -.0000 & 180.00000 & 152.173018 & .6436 & 5002 & 118.2864 \\
\hline 21 & 4 & 4.0000 &. .3000 & .6436 & -.0000 & 180.00000 & 152.173018 & .6436 & 5002 & 118.2864 \\
\hline 21 & 5 & 4.0000 & 4000 & 6436 & 0000 & 180.00000 & 152.173018 & 6436 & 5002 & 2864 \\
\hline
\end{tabular}

Fig. 28. (cont) 
SOLUTION SURFACE NO. $500-$ TIME $=30.09822540$. SECONDS $($ DELTA $T=.05981900$. NVCM $=1$, CNUMS $=1.00$, $(20$. 7$)$, $(0.0)$ )

$\begin{array}{rrrrr}L & M & \begin{array}{c}X \\ (I N)\end{array} & \begin{array}{l}Y \\ (I N)\end{array} & \begin{array}{l}U \\ (F / S)\end{array} \\ 21 & 6 & 4.0000 & .5000 & .6436 \\ 21 & 6 & 4.0000 & 1.6547 & 2.2172 \\ 21 & 7 & 4.0000 & 1.9856 & 2.1839 \\ 21 & 8 & 4.0000 & 2.3166 & 2.1481 \\ 21 & 9 & 4.0000 & 2.6475 & 2.1091 \\ 21 & 10 & 4.0000 & 2.9785 & 2.0679 \\ 21 & 11 & 4.0000 & 3.3094 & 2.0234\end{array}$

$V$
$(F / S)$
0.0000
.6401
.7574
.8683
.9748
1.0746
1.1682

$\begin{array}{cc}\text { P } & \text { RHO } \\ \text { (PSIA) } & \text { (LBM/FT3) } \\ & \\ 80.00000 & 152.173018 \\ 8.37249 & 16.844586 \\ 8.12785 & 16.505354 \\ 7.88311 & 16.161417 \\ 7.60375 & 15.758970 \\ 7.33421 & 15.364551 \\ 7.03598 & 14.921935\end{array}$

VMAG
$(\mathrm{F} / \mathrm{S})$

MACH
NO

$T$
$(\mathbf{R})$

***** EXPECT FILM OUTPIT FOR $\mathrm{N}=500 * * * * *$

Fig. 28. (cont) 


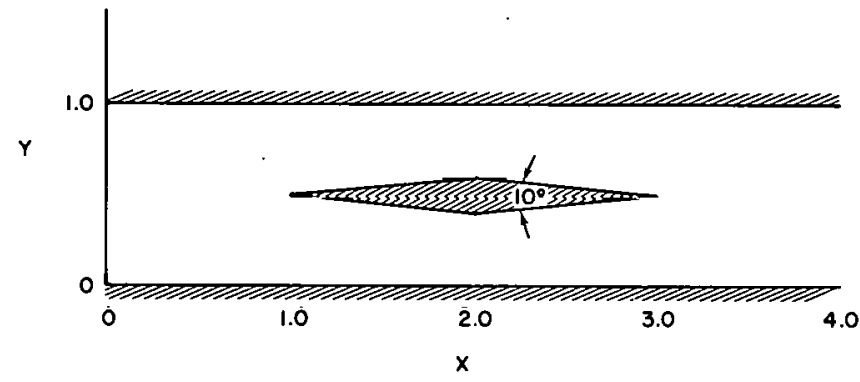

Fig. 29.

Case No. 3 geometry.

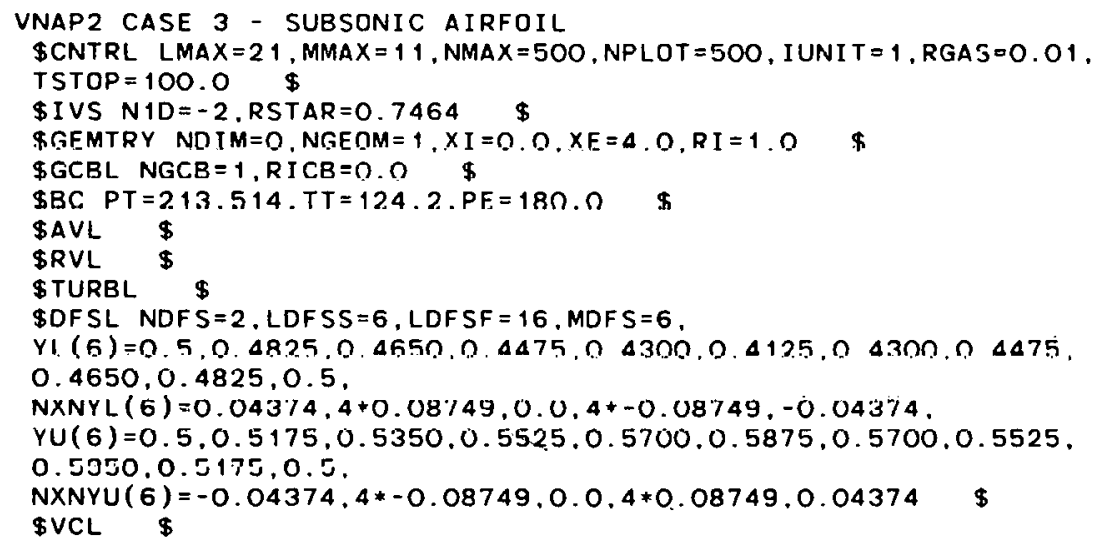

Fig. 30.

Case No. 3 data deok. 
VNAP2. A COMPUTER PROGRAM FOR THE COMPUTATION OF TWO-DIMENSIONAL, TIME-DEPENDENT, COMPRESSIBLE, TURBULENT FLOW by michael C. CLine, T-3 - los alamos national laboratory

PROGRAM AESTRACT -

THE NAVIER-STOKES EQUATIONS FOR TWO-DIMENSIONAL. TIME-DEPENDENT FLOW ARE SOLVED USING THE SECOND-OF:DER, MACCORMACK FINITE-DIFFERENCE SCHEME. ALL BOUNDARY CONDITIONS ARE COMPUTED USING A SECOND-ORDER, REFERENCE PLANE CHARACTERISTIC SCHEME WITH THE VISCOUS TERMS TREATED AS SOURCE FUNCTIONS. THE FLUID IS ASSUMED TO BE A PERFECT GAS. THE STEADY-STATE SOLUTION IS OBTAINED AS THE ASYMFTOTIC SOLUTION FOR LARGE TIME. THE FLOW BOUNDARIES MAY BE ARBITRARY CURVED SOLID WALLS AS WELL AS JET ENVELOPES. THE GEOMETRY MAY CONSIST OF SINGLE AND DUAL FLOWING STREAMS. TURBULENCE EFFECTS ARE MODELED WITH EITHER A MIXING-LENGTH, A TURBULENCE ENERGY EQUATION. OR A TURBULENCE ENERGY-DISSIPATION RATE EOUATIDNS MODEL. THIS PROGRAM ALLOWS VARIABLE GRID SPACING AND INCLUDES OPTIONS TO SPEED UP THE CALCULATION FOR HIGH REYNOLDS NUMBER FLOWS.

JOB TITLE -

VNAP2 CASE 3 - SUBSONIC AIRFOIL

CONTROL PARAMETERS -

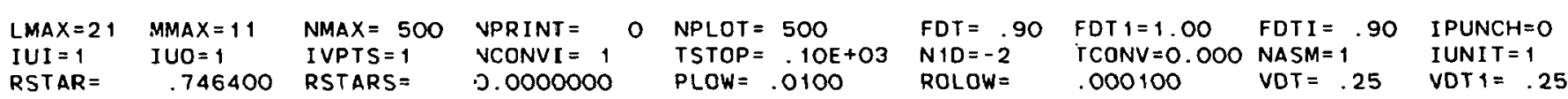

FLUID MODEL -

THE RATIO OF SPECIFIC HEATS, GAMMA $=1.4000$ AND THE GAS CONSTANT, $R=.0100(\mathrm{Ft}-$ LBF $/$ LBM-R)

FLOW GEDMETRY -

two-dimensional. planar flów has been specified

DUCT GEOMETRY

A CONSTANT AREA DUCT HAS BEEN JPECIFIED BY $X I=0.0000$ (IN), RI = 1.0000. (IN), AND XE $=4.0000$ (IN)

A CYLINDRICAL CENTERBODY HAS BEEN SPECIFIED BY XICB $=0.0000($ IN), RICB $=0.0000$ (IN), AND XECB = 4.0000 (IN)

Fig. 31.

Case No. 3 output. 
DUAL FLOV SPACE BOUNDARY GEOMETRY -

GENERAL BOIJNDARIES HAVE BEEN SFECIFIED BY THE FOLLOWING PARAMETERS,

\begin{tabular}{|c|c|c|c|c|c|}
\hline $\mathbf{L}$ & $X P(I N)$ & $Y L(I N)$ & SLOPEL & YU(IN) & SLOPEU \\
\hline 6 & 1.0000 & .5000 & -.0437 & $.50 c 0$ & .0437 \\
\hline 7 & 1.2000 & .4825 & -.0875 & .5175 & .0875 \\
\hline 8 & 1.4000 & .4650 & -.0875 & .5350 & .0875 \\
\hline 9 & 1.6000 & .4475 & -.0875 & $.55 \div 5$ & .0875 \\
\hline 10 & 1.8000 & .4300 & -.0875 & .5700 & .0875 \\
\hline 11 & 2.0000 & .4125 & 0.0000 & .5875 & 0.0000 \\
\hline 12 & 2.2000 & .4300 & .0875 & .57010 & -.0875 \\
\hline 13 & 2.4000 & .4475 & .0875 & $.55: 5$ & -.0875 \\
\hline 14 & 2.6000 & 4650 & .0875 & .5350 & -.0875 \\
\hline 15 & 2.8000 & .4825 & .0875 & .5175 & -.0875 \\
\hline 16 & 3.0000 & .5000 & .0437 & .5000 & -.0437 \\
\hline
\end{tabular}

Fig. 31. (cont) 
JUNDARY CONDITIONS -

\begin{tabular}{|c|c|c|c|c|c|c|c|c|c|}
\hline M & PT (PSIA) & & & $T T(R)$ & THETA (DEG) & PE(PSIA) & F5Q(F & $\mathrm{T} 2 / \mathrm{S} 2)$ & FSE C F T 2 \\
\hline 1 & 213.5140 & & & 124.20 & 0.00 & 180.00000 & & .0001 & \\
\hline 2 & 213.5140 & & & 124.20 & 0.00 & 180.00000 & & .0001 & \\
\hline 3 & 213.5140 & & & 124.20 & 0.00 & 180.00000 & & .0001 & \\
\hline 4 & 213.5140 & & & 124.20 & 0.00 & 180.00000 & & .0001 & \\
\hline 5 & 213.5140 & & & 124.20 & 0.00 & 180.00000 & & .0001 & \\
\hline 6 & 213.5140 & & & 124.20 & 0.00 & 180.00000 & & .0001 & \\
\hline 7 & 213.5140 & & & 124.20 & 0.00 & 180.00000 & & .0001 & \\
\hline 8 & 213.5140 & & & 124.20 & 0.00 & 180.00000 & & .0001 & \\
\hline 9 & 213.5140 & & & 124.20 & 0.00 & 180.00000 & & .0001 & \\
\hline 10 & 213.5140 & & & 124.20 & 0.00 & 180.00000 & & .0001 & \\
\hline 11 & 213.5140 & & & 124.20 & 0.00 & 180.00000 & & .0001 & \\
\hline $\begin{array}{l}\text { IINLET }=0 \\
A L W=0.00\end{array}$ & IEXITT $=0$ & IEX $=1$ & & I SUPER $=0 \quad$ DYW $=$ & .0010 IVBC $=0$ & INBC $=0 \quad$ I WALL $=0$ & IWALLO $=0$ & $\Delta L I=0.00$ & $A L E=0.00$ \\
\hline$A L W=0.00$ & NSTAG $=0$ & $N P E=$ & 0 & PEI $=0.00000$ & & & & & \\
\hline
\end{tabular}

ADIABATIC UPPER WALL IS SOECIFIED

ADIABATIC LOWER CENTER3ODV IS SPECIFIED

ADIABATIC LOWER DUAL FLOW SPACE BOUNDARY IS SPECIFIED

ADIABATIC UPPER DUAL FLOW SPACE BOUNDARY IS SPECIFIED

ARTIFICAL VISCOSITY -

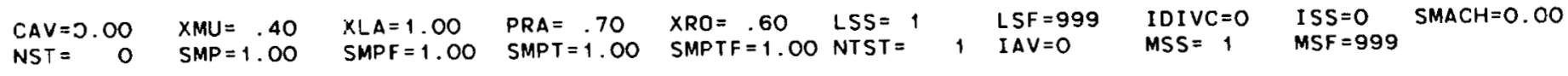

MOLECULAR VISCJSITY -
CMU $=0$.
(LBF-S/FT2! CLA $=0$.
(LBF-S/FT2) CK $=0$.
$(L B F / S-R) \quad E M U=0.00 \quad E L A=0.00$
$E K=0.00$

TURBULENCE MODEL -

NO MODEL IS SPECIFIED

VARIABLE GRID PARAMETERS -

IST $=0 \quad M V C B=0 \quad M V C T=0 \quad I Q S=0 \quad N I O S S=2 \quad N I O S \bar{F}=0 \quad N V C M I=0 \quad I L L O S=30 \quad S O S=.50 \quad$ COS $=.001$

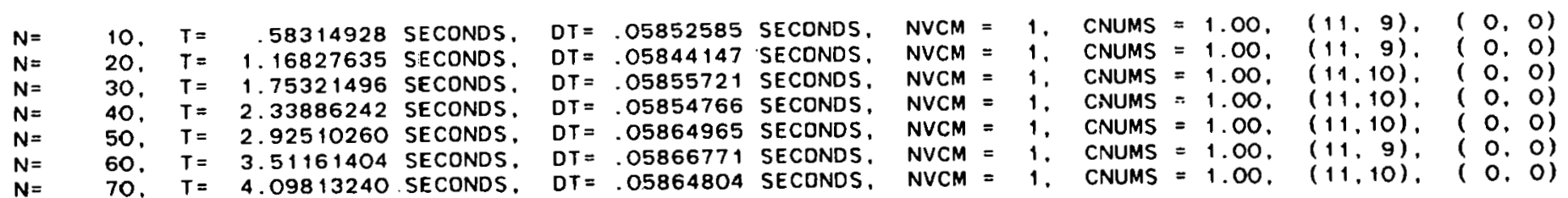

Fig. 31. (cont) 


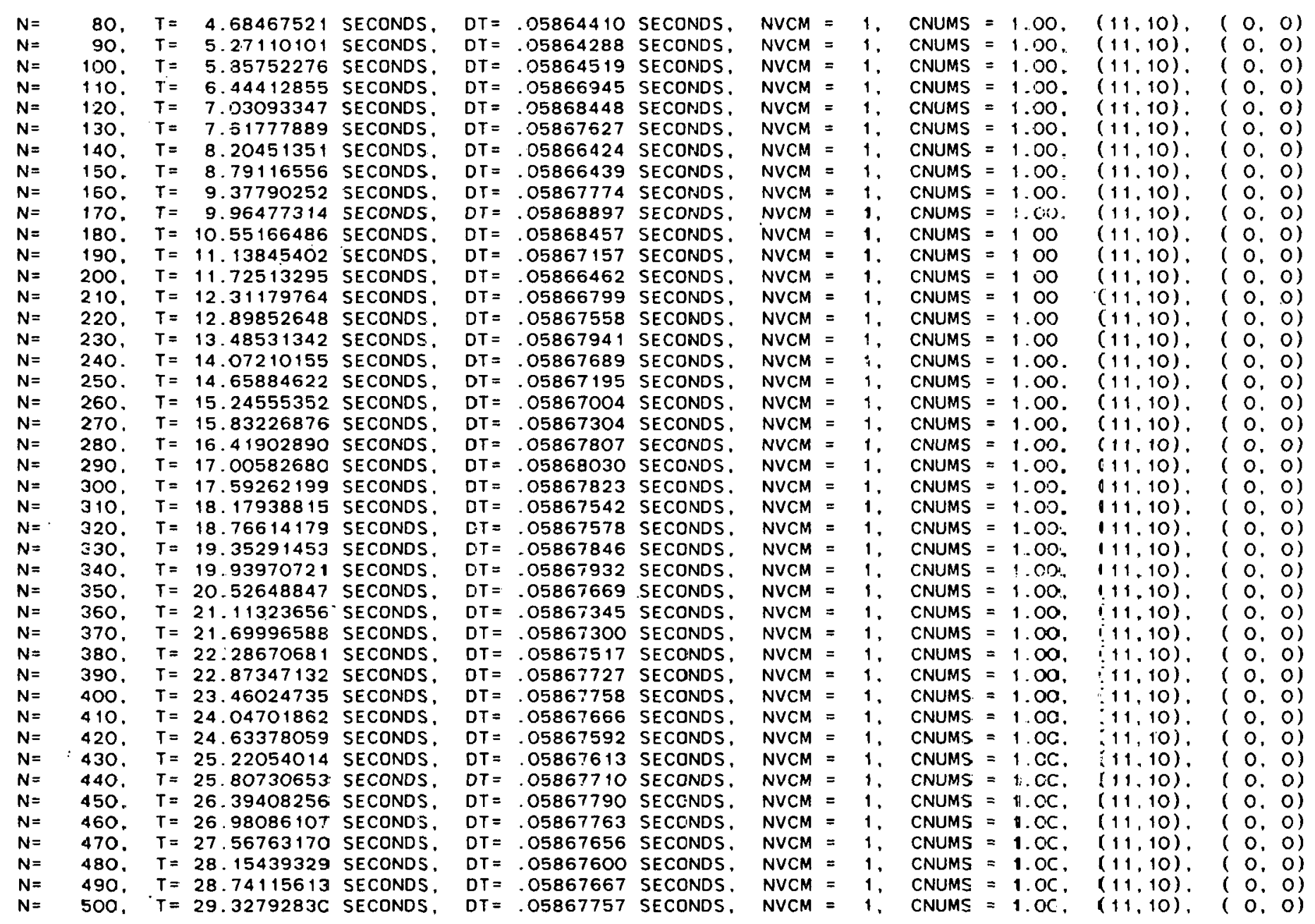

Fig. 31. (cont) 
MASS FLOW AND THRUST CALCULATION, $N=500$

$\begin{array}{rr}\mathrm{L} & \text { MF }(\text { LBM/S }) \\ 1 & 97.97247 \\ 2 & 97.98057 \\ 3 & 97.95771 \\ 4 & 98.04827 \\ 5 & 97.71521 \\ 6 & 98.04291 \\ 7 & 97.76694 \\ 8 & 98.06552 \\ 9 & 97.52895 \\ 10 & 98.74291 \\ 11 & 97.73069 \\ 12 & 97.85483 \\ 13 & 97.56375 \\ 14 & 98.01990 \\ 15 & 97.29910 \\ 16 & 98.11851 \\ 17 & 97.79844 \\ 18 & 98.01869 \\ 19 & 97.87349 \\ 20 & 97.97664 \\ 21 & 97.89335\end{array}$

$$
\begin{array}{r}
\text { MF/MF I } \\
1.0000 \\
1.0001 \\
.9998 \\
1.0008 \\
.9974 \\
1.0007 \\
.9979 \\
1.0009 \\
.9955 \\
1.0079 \\
.9975 \\
.9988 \\
.9958 \\
1.0005 \\
.9931 \\
1.0015 \\
.9982 \\
1.0005 \\
.9990 \\
1.0000 \\
.9992
\end{array}
$$$$
\text { T ( LBF) }
$$

63.0912

1.0000

63. 1015

1. 0002

$63.1755 \quad 1.0998$

$62.8246 \quad .9958$

$\begin{array}{ll}63.3069 & 1.0034 \\ 65.8924 & 1.0444\end{array}$

69.9632

$73.2858-1.1616$

79.8388

83.8832

$73.8832 \quad 1.3296$

$73.0532-1.2480$

73.0532

65.927341 .1084

$\begin{array}{ll}65.2744 & 1.0346 \\ 63.4184 & 1.0052\end{array}$

$62.7905 \quad .9952$

$63.2089 \quad 1.0019$

$62.9277 \quad .9974$

$63.1262 \quad 1.0006$

62.9648

.9980

Fig. 31. (cont) 
SOLUTION SURFACE NO. $500-$ TIME $=29.32792830$ SECONOS (DELTA T $=.05867757$, VVCM $=1$, CNUMS $=1.00,(11,10)$, 0 , 0))

$\stackrel{(I N)}{(\text { IN })} \quad(F, S)$

$(\mathrm{F} / \mathrm{S})$

(PSIA)

(LBM/FT3)

VMAG
$(E / S)$

MACH
NO

(R)

\begin{tabular}{|c|c|c|c|c|c|c|c|c|c|c|}
\hline 1 & 1 & 0.0000 & 0.0000 & .6441 & 0.0000 & 179.92963 & 152.130548 & .6441 & .5006 & 118.2732 \\
\hline 1 & 2 & 0.0000 & 1000 & .6442 & 0.0000 & 179.92510 & 152.127812 & .6442 & .5006 & 118.2723 \\
\hline 1 & 3 & 0.0000 & 2000 & .6440 & 0.0000 & 179.93776 & 152.135456 & .6440 & .5005 & 118.2747 \\
\hline 1 & 4 & 0.0000 & 3000 & .6438 & 0.0000 & 179.95468 & 152.145675 & .6438 & .5003 & 118.2779 \\
\hline 1 & 5 & 0.0000 & 4000 & .6438 & 0.0000 & 179.96019 & 152.149000 & .6438 & .5003 & 118.2789 \\
\hline 1 & 6 & 0.0000 & 5000 & .6435 & 0.0000 & 179.98871 & 152.166225 & .6435 & .5000 & 118.2843 \\
\hline 1 & 7 & 0.0000 & .6000 & 6438 & 0.0000 & 179.95754 & 152.147399 & .6438 & .5003 & 118.2784 \\
\hline 1 & 8 & 0.0000 & .7000 & 6440 & 0.0000 & 179.93879 & 152.136077 & .6440 & .5005 & 118.2749 \\
\hline 1 & 9 & 0.0000 & .8000 & 6441 & 0.0000 & 179.93403 & 152.133201 & .6441 & .5005 & 118.2740 \\
\hline 1 & 10 & 0.0000 & .9000 & 6443 & 0.0000 & 179.91313 & 152.120584 & .6443 & .5007 & 118.2701 \\
\hline 1 & 11 & 0.0000 & 1.0000 & 6443 & 0.0000 & 179.90967 & 152.118493 & .6443 & .5007 & 118.2694 \\
\hline 2 & 1 & .2000 & 0.0000 & 6442 & 0.0000 & 179.93163 & 152.132487 & .6442 & .5006 & 118.2730 \\
\hline 2 & 2 & .2000 & .1000 & 6443 & -.0001 & 179.93517 & 152.134726 & .6443 & .5007 & 118.2736 \\
\hline 2 & 3 & .2000 & .2000 & .6441 & -.0001 & 179.94171 & 152.138639 & .6441 & .5005 & 118.2748 \\
\hline 2 & 4 & .2000 & .3000 & .6439 & -.0001 & 179.95268 & 152.145235 & .6439 & .5004 & 118.2769 \\
\hline 2 & 5 & .2000 & .4000 & .6438 & -.0000 & 179.96177 & 152.150775 & .6438 & .5003 & 118.2786 \\
\hline 2 & 6 & .2000 & . 5000 & .6433 & .0001 & 179.96011 & 152.149066 & .6433 & .4999 & 118.2788 \\
\hline 2 & 7 & .2000 & .6000 & .6439 & .0001 & 179.95908 & 152.148965 & .6439 & .5004 & 118.2782 \\
\hline 2. & 8 & .2000 & -7000 & .6441 & .0001 & 179.94327 & 152.139524 & .6441 & .5005 & 118.2752 \\
\hline 2 & 9 & .2000 & .8000 & .6441 & .0001 & 179.92701 & 152.129707 & .6441 & .5005 & 118.2721 \\
\hline 2 & 10 & .2000 & .9000 & .6444 & .0000 & 179.92106 & 152.126143 & .6444 & .5008 & 118.271 \\
\hline 2 & 11 & .2000 & 1.0000 & .6444 & 0.0000 & 179.91326 & 152.121433 & .6444 & .5008 & 118.2695 \\
\hline 3 & 1 & $.400 \mathrm{C}$ & 0.0000 & .6444 & 0.0000 & 179.84896 & 152.081505 & .6444 & .5008 & 118.2583 \\
\hline 3 & 2 & .4000 & .1000 & .6442 & -.0001 & 179.85251 & 152.083603 & .6442 & .5007 & 118.2590 \\
\hline 3 & 3 & .4000 & .2000 & .6439 & -.0002 & 179.88470 & 152.103070 & .6439 & .5004 & 118.2650 \\
\hline 3 & 4 & .4000 & .3000 & .6436 & -.0002 & 179.92894 & 152.129802 & .6436 & .5002 & 118.2733 \\
\hline 3 & 5 & .4000 & .4000 & .6432 & -.0000 & $179.98890^{\circ}$ & 152.166007 & .6432 & .4998 & 118.2846 \\
\hline 3 & 6 & .4000 & .5000 & -6435 & .0001 & 180.04365 & 152.199378 & .6435 & .5000 & 118.2946 \\
\hline 3 & 7 & .4000 & .6000 & -6439 & .0003 & 179.99410 & 152.169190 & .6439 & .5004 & 118.2855 \\
\hline 3 & 8: & .4000 & .7000 & .6439 & .0004 & 179.92717 & 152.128717 & .6439 & .5004 & 118.2730 \\
\hline 3 & 9 & .4000 & 8000 & .6442 & .0003 & 179.87968 & 152.100037 & .6442 & .5007 & 118.2641 \\
\hline 3 & 10 & .4000 & 9000 & .6445 & .0001 & 179.84139 & 152.076893 & .6445 & .5009 & 118.2569 \\
\hline 3 & 11 & .4000 & 1.0000 & .6445 & 0.0000 & 179.82842 & 152.069065 & .6445 & .5009 & 118.2544 \\
\hline 4 & 1 & .6000 & 0.0000 & .6455 & 0.0000 & 179.83587 & 152.075105 & .6455 & .5017 & 118.2546 \\
\hline 4 & 2 & .6000 & 1000 & .6455 & -.0009 & 179.85532 & 152.087029 & .6455 & .5017 & 118.2582 \\
\hline 4 & 3 & .6000 & 2000 & .6449 & -.0016 & 179.91666 & 152.124012 & .6449 & .5012 & 118.2697 \\
\hline 4 & 4 & .6000 & 3000 & .6429 & -.0018 & 180.02353 & 152.188501 & .6439 & .5003 & 118.2898 \\
\hline 4 & 5 & .6000 & 4000 & .6432 & -.0015 & 180.13405 & 152.255283 & .6432 & .4998 & 118.3105 \\
\hline 4 & $E$ & .6000 & .5000 & .6413 & .0004 & 180.24852 & 152.323238 & .6413 & .4982 & 118.3329 \\
\hline 4 & 7 & .6000 & .6000 & .6429 & .0017 & 180.18237 & 152.284226 & .6429 & .4995 & 118.3198 \\
\hline 4 & $\varepsilon$ & .6000 & .7000 & .6448 & .0019 & 180.04020 & 152.198552 & .6448 & .5011 & 118.2930 \\
\hline 4 & g & .6000 & .8000 & 6452 & .0017 & 179.90938 & 152,119568 & .6452 & .5015 & 118.2684 \\
\hline 4 & 10 & .6000 & .9000 & .6459 & .0009 & 179.83438 & 152.074321 & .6459 & .5020 & 118.2543 \\
\hline 4 & 11 & .6000 & 1.0000 & .6458 & 0.0000 & 179.78844 & 152.046557 & .6458 & .5020 & 118.2456 \\
\hline 5 & - & .8000 & 0.0000 & .6483 & 0.0000 & 179.27175 & 151.732123 & .6483 & .5041 & 118.1502 \\
\hline 5 & 2 & .8000 & .1000 & .6477 & $-.00+4$ & 179.30708 & 151,753332 & .6477 & .5036 & 118.1569 \\
\hline 5 & 3 & .8000 & .2000 & .6456 & -.0025 & 179.45714 & 151.844019 & .6456 & .5019 & 118.1852 \\
\hline 5 & 4 & 8000 & 3000 & .6422 & -.0029 & 179.70017 & 151,990842 & 6422 & .4992 & 118.230 \\
\hline & 5 & & & .6370 & -.0014 & $180 \quad 14007$ & 152256542 & 6370 & & \\
\hline
\end{tabular}

Fig. 31. (cont) 


\begin{tabular}{|c|c|c|c|c|c|c|c|c|c|c|}
\hline$L$ & $M$ & $\begin{array}{l}X \\
(\text { IN })\end{array}$ & $\stackrel{Y}{(I N)}$ & $\underset{(F / S)}{U}$ & $\begin{array}{c}V \\
(F / S)\end{array}$ & $\begin{array}{c}P \\
(P S I A)\end{array}$ & $\begin{array}{c}\text { RHO } \\
(\text { LBM/FT3) }\end{array}$ & $\begin{array}{l}\text { VMAG } \\
(F / S)\end{array}$ & $\begin{array}{c}\text { MACH } \\
\text { NO }\end{array}$ & $\begin{array}{c}T \\
(R)\end{array}$ \\
\hline 5 & 6 & $.9000^{\circ}$ & .5000 & .6328 & .0007 & 180.60786 & 152.540245 & .6328 & .4915 & 118.4001 \\
\hline 5 & 7 & .8000 & .6000 & .6379 & .0022 & 180.16143 & 152.269756 & .6379 & .4956 & 118.3173 \\
\hline 5 & $\mathbf{B}$ & .8000 & .7000 & .6426 & .0033 & 179.72030 & 152.003133 & .6426 & .4994 & 118.2346 \\
\hline 5 & $\boldsymbol{\theta}$ & .8000 & .8000 & .6461 & .0027 & 179.44078 & 151.834197 & .6461 & .5023 & 118.1821 \\
\hline 5 & 10 & .8000 & .9000 & .6484 & .0014 & 179.26674 & 151.729012 & .6484 & .5041 & 118.1493 \\
\hline 5 & 11 & .8000 & 1.0000 & .6494 & 0.0000 & 179.18449 & 151.679309 & .6494 & .5049 & 118.1338 \\
\hline 6 & 1 & 1.0000 & 0.0000 & .6553 & 0.0000 & 178.69533 & 151.386362 & .6553 & .5098 & 118.0393 \\
\hline 6 & 2 & 1.0000 & .1000 & .6540 & -.0059 & 178.76880 & 151.431231 & .6541 & .5088 & 118.0528 \\
\hline 6 & 3 & 1.0000 & .2000 & .6505 & -.0116 & 179.07243 & 151.614839 & .6506 & .5060 & 118.1101 \\
\hline 6 & 4 & 1.0000 & .3000 & .6449 & -.0175 & 179.58106 & 151.922426 & .6451 & .5015 & 118.2058 \\
\hline 6 & 5 & 1.0000 & .4000 & .6359 & -.0224 & 180.45083 & 152.448094 & .6363 & .4942 & 118.3687 \\
\hline 6 & 6 & 1.0000 & .5000 & .6225 & -.0272 & 181.43926 & 153.039413 & .6231 & .4837 & 118.5572 \\
\hline 6 & 6 & 1.0000 & .5000 & .6237 & .0273 & 181.25469 & 152.934071 & .6243 & .4846 & 118.5182 \\
\hline 6 & 7 & 1.0000 & .6000 & .6376 & .0227 & 180.17262 & 152.279217 & .6380 & 4957 & 118.3173 \\
\hline 6 & 8 & 1.0000 & .7000 & .6464 & .0170 & 179.44205 & 151.838009 & .6466 & .5027. & 118.1799 \\
\hline 6 & 9 & 1.0000 & .8000 & .6520 & .0113 & 178.92438 & 151.525080 & .6521 & .5072 & 118.0824 \\
\hline 6 & 10 & 1.0000 & .9000 & .6554 & .0057 & 178.64862 & 151.358340 & .6554 & .5099 & 118.0302 \\
\hline 6 & 11 & 1.0000 & 1.0000 & .6568 & 0.0000 & 178.56632 & 151.308482 & .6568 & .5110 & 118.0147 \\
\hline$\cdot 7$ & 1 & 1.2000 & 0.0000 & .6804 & 0.0000 & 176.36226 & 149.972133 & .6804 & .5303 & 117.5967 \\
\hline 7 & 2 & 1.2000 & .0965 & .6794 & -.0108 & 176.43367 & 150.016652 & .6795 & .5295 & 117.6094 \\
\hline 7 & 3 & 1.2000 & .1930 & .6771 & -.0220 & 176.68532 & 150.170556 & .6774 & .5278 & 117.6564 \\
\hline 7 & 4 & 1.2000 & .2895 & .6732 & -.0339 & 177.08691 & 150.416278 & .6741 & .5250 & 117.7312 \\
\hline 7 & 5 & 1.2000 & .3860 & .6671 & -.0464 & 177.71065 & 150.796887 & .6687 & .5206 & 117.8477 \\
\hline 7 & 6 & 1.2000 & .4825 & .6633 & -.0580 & 178.03249 & 150.994017 & .6658 & .5182 & 117.9070 \\
\hline 7 & 6 & 1.2000 & .5175 & .6597 & .0577 & 178.16165 & 151.067225 & .6622 & .5154 & 117.9353 \\
\hline 7 & 7 & 1.2000 & .6140 & .6688 & .0441 & 177.45605 & 150.642222 & .6702 & .5219 & 117.7997 \\
\hline 7 & 8 & 1.2000 & .7105 & .6741 & .0321 & 176.89414 & 150.298820 & .6749 & .5257 & 117.6950 \\
\hline 7 & 9 & 1.2000 & .8070 & .6777 & .0210 & 176.50877 & 150.062813 & .6781 & .5284 & $:: 7.6233$ \\
\hline 7 & 10 & 1.2000 & .9035 & .6799 & .0105 & 176.32830 & 149.952214 & .6800 & .5300 & 117.5897 \\
\hline 7 & 11 & 1.2000 & 1.0000 & .6805 & 0.0000 & 176.28884 & 149.926980 & .6805 & .5304 & 117.5831 \\
\hline 8 & 1 & 1.4000 & 0.0000 & .7162 & 0.0000 & 172.60884 & 147.692533 & .7162 & .5599 & 116.8704 \\
\hline 8 & 2 & 1.4000 & .0930 & .7164 & -.0119 & 172.64040 & 147.712929 & .7165 & .5602 & 116.8756 \\
\hline 8 & 3 & 1.4000 & .1860 & .7154 & -.0238 & 172.68209 & 147.739069 & .7158 & .5596 & 116.8832 \\
\hline 8 & 4 & 1.4000 & 2790 & .7137 & -.0360 & 172.75828 & 147.786599 & .7146 & .5586 & 116.8971 \\
\hline 8 & 5 & 1.4000 & .3720 & .7115 & -.0490 & 172.81628 & 147.824383 & .7132 & .5575 & 116.9065 \\
\hline 8 & 6 & 1.4000 & .4650 & .7096 & -.0621 & 172.99911 & 147.929190 & .7123 & .5567 & 116.9472 \\
\hline 8 & 6 & 1.4000 & .5350 & .7084 & .0620 & 173.19136 & 148.059243 & .7111 & .5557 & 116.9744 \\
\hline 8 & 7 & 1.4000 & .6280 & .7108 & .0488 & 172.91918 & 147.884985 & .7125 & .5569 & 116.9282 \\
\hline 8 & 8 & 1.4000 & .7210 & .7123 & .0360 & 172.81147 & 147.816503 & .7132 & .5575 & 116.9095 \\
\hline 8 & 9 & 1.4000 & .8140 & .7142 & .0237 & 172.75060 & 147.779000 & .7146 & .5586 & 116.8979 \\
\hline 8 & 10 & 1.4000 & .9070 & .7151 & .0118 & 172.73111 & 147.766929 & .7152 & .5591 & 116.8943 \\
\hline 8 & 11 & 1.4000 & 1.0000 & .7153 & 0.0000 & 172.73544 & 147.769380 & .7153 & .5591 & 116.8953 \\
\hline 9 & 1 & 1.6000 & 0.0000 & .7537 & 0.0000 & 168.36449 & 145.080278 & .7537 & .5913 & 116.0492 \\
\hline 9 & 2 & 1.6000 & .0895 & .7527 & -.0114 & 168.33985 & 145.066195 & .7528 & .5906 & 116.0435 \\
\hline 9 & 3 & 1.6000 & .1790 & .7523 & -.0234 & 168.31983 & 145.053813 & .7526 & .5905 & 116.0396 \\
\hline 9 & 4 & 1.6000 & .2685 & .7517 & -.0363 & 168.29635 & 145.039824 & .7525 & .5904 & 116.0346 \\
\hline 9 & 5 & 1.6000 & .3580 & .7505 & -.0504 & 168.28215 & 145.028951 & .7522 & .5902 & 116.0335 \\
\hline 9 & 6 & 1.6000 & .4475 & .7443 & -.0651 & 167.83234 & 144.757824 & .7472 & .5865 & 115.9401 \\
\hline 9 & 6 & .6000 & 5525 & .7440 & .0651 & 167.92753 & 144.804303 & .7469 & .5862 & 115.96 \\
\hline
\end{tabular}


SOLUTION SURFACE NO. $500-$ TIME $=29.32792830$ SECONDS (DELTA $T=.058677 .57$, NVCN $r=1$, CNUMS $=1.00$. $(11,10),(0,0)$,

$\begin{array}{llll}X & Y & U & V \\ (I N) & (I N) & (F / S) & (F / S)\end{array}$

97

\begin{tabular}{rrr}
$\mathbf{9}$ & 7 & 1.6000 \\
$\mathbf{9}$ & $\mathbf{8}$ & 1.6000 \\
$\mathbf{9}$ & 9 & 1.6000 \\
$\mathbf{9}$ & 10 & 1.6000 \\
$\mathbf{9}$ & 11 & 1.6000 \\
\hline & &
\end{tabular}

10

$\begin{array}{lll}10 & 1 & 1.8000 \\ 10 & 2 & 1.8000\end{array}$

$\begin{array}{lll}10 & 3 & 1.8000 \\ 10 & 4 & 1.8000\end{array}$

$\begin{array}{llll}10 & 4 & 1.8000 \\ 10 & 5 & 1.8000\end{array}$

$\begin{array}{lll}10 & 5 & 1 \\ 10 & 6 & 1 \\ 10 & 6 & 1.88 \\ 10 & 7 & 1\end{array}$

$\begin{array}{lll}10 & 6 & 1.8000\end{array}$

$\begin{array}{lll}10 & 8 & 1.8000\end{array}$

$\begin{array}{lll}10 & 9 & 1.8000 \\ 10 & 10 & 1.8000\end{array}$

$\begin{array}{lll}10 & 10 & 1.8000 \\ 10 & 11 & 1.8000\end{array}$

$\begin{array}{lll}11 & 1 & 2.0000\end{array}$

$\begin{array}{lll}11 & 2 & 2.0000 \\ 11 & 3 & 2.0000\end{array}$

$\begin{array}{lll}11 & 3 & 2.0000 \\ 11 & 4 & 2.0000\end{array}$

$\begin{array}{lll}11 & 5 & 2.0000\end{array}$

$\begin{array}{lll}11 & 6 & 2.0000 \\ 11 & 6 & 2.0000\end{array}$

$\begin{array}{lll}11 & 7 & 2.0000\end{array}$

$\begin{array}{lll}11 & 8 & 2.0000\end{array}$

$\begin{array}{rrr}11 & 9 & 2.0000\end{array}$

$\begin{array}{lll}11 & 10 & 2.0000 \\ 11 & 11 & 2.0000\end{array}$

\begin{tabular}{ccccc}
12 & 1 & 2.2000 & 0.0000 & .7964 \\
12 & 2 & 2.2000 & .0860 & .7974 \\
12 & 3 & 2.2000 & .1720 & .860 \\
12 & 4 & 2.2000 & .2580 & .805 \\
12 & 5 & 2.2000 & .3440 & .8153 \\
12 & 6 & 2.2000 & .4300 & .813 \\
12 & 6 & 2.2000 & .5700 & .8224 \\
12 & 7 & 2.2000 & .6560 & .812 \\
12 & 8 & 2.2000 & .7420 & .804 \\
12 & 9 & 2.2000 & .8280 & .7990 \\
12 & 10 & 2.2000 & .9140 & .7966 \\
12 & 11 & 2.2000 & 1.0000 & .7966 \\
\hdashline 13 & 1 & 2.4000 & 0.0000 & .750 \\
13 & 2 & 2.4000 & .0895 & .750 \\
13 & 3 & 2.4000 & .1790 & .7500 \\
13 & 4 & 2.4000 & .2685 & .749 \\
13 & 5 & 2.4000 & .3580 & .746 \\
13 & 6 & 2.4000 & .4475 & .7436 \\
13 & 6 & 2.4000 & .5525 & .7444 \\
13 & 7 & 2.4000 & .6420 & .7483 \\
& & & &
\end{tabular}

,

\begin{tabular}{llll} 
& .6420 & .7513 & .050 \\
0 & .7315 & .7527 & .036 \\
0 & .8210 & .7526 & .023 \\
0 & .9105 & .7527 & .01 \\
0 & 1.0000 & .7532 & 0.000 \\
\hline 0 & 0.0000 & .7992 & 0.000
\end{tabular}

1720

.8008

.8044
.8104

2580
3440

.4300

$.5700 \quad .8401$

$8242 \quad .0721$

$\begin{array}{ll}.7420 & .8075 \\ .8280 & .8075\end{array}$

8010

1.0000

.7972
.7947

0.0000

P RHO

$\begin{array}{cc}\text { RHO } & \text { VMA } \\ (1-B M / F T 3) & (F / S)\end{array}$

MACH

.0506
.0362
.0235
.0116
0.0000

168.05350

144.893533

$168.35166 \quad 145.076201$ $168.44078 \quad 145.130086$

.7530

$0.0000 \quad 164.43271 \quad 142.668435$

$164.43271 \quad 142.668435$

$-.0127$

$-.0264$

-.0419
-.0601

$-.0735$

.0521
.0368

.0236

.0120
0.0000

$\begin{array}{ll}164.04485 & 142.621085 \\ 163.432924\end{array}$

$163.45307 \quad 142.072841$

$162.52985 \quad 141.515621$

$159.64350 \cdot 139.715563$

$\begin{array}{ll}161.05797 & 140.605579\end{array}$

$162.54924 \quad 141.513242$

$163.45810 \quad 142.067836$

$164.09803 \quad 142.459858$

$\begin{array}{rr}164.48294 & 142.696498 \\ 164.69942 & 142.830843\end{array}$

0.0000

142.830843

$\begin{array}{ll}158.81698 & 139.164508\end{array}$

$\begin{array}{ll}158.58480 & 139.025564 \\ 157.73375 & 138.495248\end{array}$

.0012

.0010

$153.71971 \quad 135.978802$

$0.0000 \quad 151.21521 \quad 134.403945$

$\begin{array}{rr}153.40795 & 135.757117\end{array}$

.0001

.0003

$-.0001$

158.01311 138.471736

$159.30354 \quad 138.662628$

$159.95114 \quad 139.467588$ $160.14805 \quad 139.988771$

0.0000

$163.58950 \quad 142.152968$

.0127
.0263

.0419

.0598

.0711

$-.0719$

$1 \in 3.39534142 .042019$

$\begin{array}{rr}162.88680 & 141.733474 \\ 162.07130 & 141.238851\end{array}$

160.77808 14C. 452870

$160.66941 \quad 140.396774$

159.94058 139.929516

-.0520
-.0365

-.0365
-.0231

161.36048

140.801458

$162.49001 \quad 141.487087$

$-.0119$

141.951062 163.64165 42.180759

169.11378 145.548993

$\begin{array}{lll}.0113 & 169.08978 & 145.543276\end{array}$

.0229

169.09601

45.550863

169.11170 145.56697C

.0494

$169.39531 \quad 145.745691$

$69.55218 \quad 145.844506$

$-.0651$

169.18976

145.621219

$-.0502$

$169.11983 \quad 145.574651$

$\begin{array}{ll}.7530 & .5909 \\ .7535 & .5913\end{array}$

$.7535 \quad .5913$

$5908 \quad 116.0187$

$\begin{array}{lll}.7532 & .5908 & 116.0812\end{array}$

$\begin{array}{lll}.7992 & 6291 & 115.2551\end{array}$

$\begin{array}{lll}.8009 & .6305 & 115.2387\end{array}$

$\begin{array}{lll}.8048 & .6338 & 115.1734\end{array}$

$\begin{array}{lll}.8115 & .6394 & 115.0488\end{array}$

$\begin{array}{lll}.8207 & .6472 & 114.8494\end{array}$

$\begin{array}{lll}.8433 & .6668 & 114.2632\end{array}$

$\begin{array}{lll}.8273 & .6533 & 114.5459\end{array}$

$\begin{array}{lll}.8174 & 6446 & 114.8650\end{array}$

$\begin{array}{lll}.8084 & .6369 & 115.0564 \\ .8014 & 6310 & 115.1890\end{array}$

$\begin{array}{lll}.8014 & .6310 & 115.1890 \\ .7973 & .6276 & 115.2677\end{array}$

$\begin{array}{lll}.7973 & .6276 & 115.2677 \\ .7947 & .6255 & 115.3108\end{array}$

$.8394 \quad .6641 \quad 114.1218$

$\begin{array}{lll}.8394 & .6641 & 114.1218 \\ .856 & .6676 & 114.0688\end{array}$

$\begin{array}{lll}.8436 & .6676 & 114.0688 \\ .8510 & .6739 & 113.8911 \\ .8641 & .6853 & 113.5842\end{array}$

$\begin{array}{lll}.8641 & .6853 & 113.5842 \\ .8856 & .7039 & 113.0468\end{array}$

$\begin{array}{lll}.9196 & .7039 & 113.0468 \\ .8996 & .7327 & 112.5080\end{array}$

$\begin{array}{lll}.9196 & .7327 & 112.5080 \\ 8996 & .7152 & 113.0018\end{array}$

$\begin{array}{lll}.8996 & .7152 & 113.0018 \\ .8692 & .6894 & 113.5593\end{array}$

$\begin{array}{lll}.8508 & .6894 & 113.5593 \\ .8390 & .6736 & 113.9551\end{array}$

$\begin{array}{lll}.8390 & .6635 & 114.2226 \\ .8323 & 6578 & 114.3567\end{array}$
$\begin{array}{lll}.8323 & .6578 & 114.3567 \\ 8289 & .6550 & 114.4006\end{array}$

7964
.7975

$\begin{array}{lll}.7964 & .6274 & 115.0799 \\ .7975 & .6284 & 115.0331 \\ .8012 & .6317 & 114.9247\end{array}$

$\begin{array}{lll}.8012 & .6317 & 114.9247\end{array}$

$\begin{array}{lll}-8069 & .6366 & 114.7498 \\ -8175 & .6458 & 114.4712\end{array}$

$\begin{array}{lll}-8175 & .6458 & 114.4712 \\ .8161 & .6448 & 114.4395\end{array}$

$\begin{array}{lll}.8161 & .6448 & 114.4395\end{array}$

$\begin{array}{lll}.8255 & .6526 & 114.3008 \\ .8142 & .6428 & 114.6014\end{array}$

$\begin{array}{lll}.8142 & .6428 & 114.6014\end{array}$

$\begin{array}{lll}.8056 & .6353 & 114.8444 \\ .7993 & .6300 & 115.0063\end{array}$

$\begin{array}{lll}.7967 & .6300 & 115.0063 \\ .7927 & 115.0767\end{array}$

$\begin{array}{lll}.7962 & .6277 & 115.0941\end{array}$

$\begin{array}{lll}.7505 & .5883 & 116.1903 \\ .7504 & .5885 & 116.1783\end{array}$

$\begin{array}{lll}.7504 & .5884 & 116.1766\end{array}$

$\begin{array}{lll}.7504 & .5884 & 116.1766 \\ .7504 & .5884 & 11 \epsilon .1745 \\ .7478 & .5862 & 116.2266\end{array}$

$\begin{array}{lll}.7464 & .5851 & 116.2554 \\ .7473 & .5859 & 116.1848\end{array}$

$\begin{array}{lll}.7473 & .5859 & 116.1848 \\ .7500 & .5881 & 116.1740\end{array}$

Fig. 31. (Eont) 
SOLUTION SURFACE NO. $500-$ TIME $=29.32792830$ SECONDS (DELTA T $=.05867757$. NVCM $=1$. CNUMS $=1.00$. $(11,10)$. $($. O) $)$

\begin{tabular}{|c|c|c|c|c|c|c|c|c|c|c|}
\hline L & M & $\stackrel{x}{(I N)}$ & $\stackrel{Y}{\text { (IN) }}$ & $\begin{array}{c}U \\
(F / S)\end{array}$ & $\begin{array}{c}V \\
(F / S)\end{array}$ & $\begin{array}{c}P \\
(P S I A)\end{array}$ & $\begin{array}{c}\text { RHO } \\
(\text { LBM/FT3) }\end{array}$ & $\begin{array}{l}\text { VMAG } \\
(F / S)\end{array}$ & $\begin{array}{l}\text { MACH } \\
\text { NO }\end{array}$ & $\begin{array}{c}T \\
(R)\end{array}$ \\
\hline 13 & 8 & 2.4000 & .7315 & .7492 & -.0363 & 169.08397 & 145.544534 & .7501 & 5881 & 116.1734 \\
\hline 13 & 9 & 2.4000 & .8210 & .7496 & -.0236 & 169.09189 & 145.544310 & 7499 & 5880 & 116.1790 \\
\hline 13 & 10 & 2.4000 & 9105 & .7501 & -.0118 & 169.06066 & 145.521759 & .7502 & .5882 & 116. 175 \\
\hline 13 & 11 & 2.4000 & 1.0000 & .7502 & 0.0000 & 169.05207 & 145.510546 & .7502 & .5883 & 116.1786 \\
\hline 14 & 1 & 2.6000 & 0.0000 & .7164 & 0.0000 & 172.36093 & 147.547241 & .7164 & .5602 & 116.8175 \\
\hline 14 & 2 & 2.6000 & .0930 & .7164 & .0118 & 172.36350 & 147.557870 & .7165 & 5603 & 116.8108 \\
\hline 14 & 3 & 2.6000 & .1860 & .7155 & .0237 & 172.39963 & 147.584491 & .7159 & 5598 & 116.8142 \\
\hline 14 & 4 & 2.6000 & .2790 & .7140 & .0362 & 172.45723 & 147.627584 & .7150 & 5591 & 116.8191 \\
\hline 14 & 5 & 2.6000 & .3720 & .7118 & .0491 & 172.60962 & 147.727886 & .7135 & .5578 & 116.8430 \\
\hline 14 & 6 & 2.6000 & .4650 & .7094 & .0621 & 173.11309 & 148.046439 & .7121 & 5566 & 116.9316 \\
\hline 14 & 6 & 2.6000 & .5350 & .7086 & -.0620 & 173.27541 & 148.124471 & .7113 & 5558 & 116.9796 \\
\hline 14 & 7 & 2.6000 & 6280 & .7102 & -.0481 & 172.98563 & 147.949866 & .7118 & 5563 & 116.9218 \\
\hline 14 & 8 & 2.6000 & .7210 & .7125 & -.0354 & 172.80929 & 147.832618 & .7134 & .5576 & 116.8952 \\
\hline 14 & 9 & 2.6000 & .8140 & .7139 & -.0232 & 172.68210 & 147.749196 & .7142 & 5584 & 116.8752 \\
\hline 14 & 10 & 2.6000 & .9070 & .7149 & -.0116 & 172.60397 & 147.697519 & .7150 & .5590 & 116.8631 \\
\hline 14 & 11 & 2.6000 & 1.0000 & .7151 & 0.0000 & 172.55756 & 147.663132 & .7151 & .5591 & 116.8589 \\
\hline 15 & 1 & 2.8000 & 0.0000 & .6782 & 0.0000 & 176.40221 & 150.004977 & .6782 & 5286 & 117.5976 \\
\hline 15 & 2 & 2.8000 & .0965 & .6774 & .0106 & 176.44090 & 150.038176 & .6775 & .5280 & 117.5973 \\
\hline 15 & 3 & 2.8000 & .1930 & .6747 & .0216 & 176.61634 & 150.149443 & .6751 & 5260 & 117.6270 \\
\hline 15 & 4 & 2.8000 & .2895 & .6703 & .0330 & 176.92 .917 & 150.347476 & .6711 & 5229 & 117.6802 \\
\hline 15 & 5 & 2.8000 & .3860 & .6631 & .0458 & 177.43889 & 150.664840 & .6647 & 5177 & 117.7706 \\
\hline 15 & 6 & 2.8000 & .4825 & .6497 & .0568 & 178.46103 & 151.286038 & .6522 & 5075 & $1+7.9627$ \\
\hline 15 & 6 & 2.8000 & .5175 & .6546 & -.0573 & 177.97915 & 150.991957 & .6571 & 5115 & 117.8733 \\
\hline 15 & 7 & 2.8000 & .6140 & .6651 & -.0441 & 177.31139 & 150.580243 & .6666 & 5192 & 117.7521 \\
\hline 15 & 8 & 2.8000 & .7105 & .6714 & -.0321 & 176.83180 & 150.279437 & .6721 & .5237 & 117.6687 \\
\hline 15 & 9 & 2.8000 & .8070 & .6759 & -.0211 & 176.50801 & 150.076833 & .6762 & .5270 & 117.6118 \\
\hline 15 & 10 & 2.8000 & .9035 & .6787 & -.0106 & 176.31330 & 149.954547 & 6787 & .5290 & 117.5778 \\
\hline 15 & 11 & 2.8000 & 1.0000 & .6799 & $0.0000^{\circ}$ & 176.23057 & 149.898024 & .6799 & .5300 & 117.5670 \\
\hline 16 & 1 & 3.0000 & 0.0000 & .6562 & 0.0000 & 178.57515 & 151.321025 & .6562 & .5105 & 118.0108 \\
\hline 16 & 2 & 3.0000 & .1000 & .6547 & .0058 & 178.65575 & 151.379994 & .6547 & .5094 & 118.0181 \\
\hline 16 & 3 & 3.0000 & .2000 & .6513 & .0114 & 178.97167 & 151.575645 & 6514 & 5066 & 118.0742 \\
\hline 16 & 4 & 3.0000 & .3000 & .6454 & .0166 & 179.48814 & 151.895740 & .6456 & .5019 & 118.1654 \\
\hline 16 & 5 & 3.0000 & .4000 & .6363 & .0222 & 180.44888 & 152.482391 & .6367 & .4947 & 118.3408 \\
\hline 16 & 6 & 3.0000 & .5000 & .6256 & .0274 & 181.29569 & 153.003296 & .6262 & .4862 & $1+8.4914$ \\
\hline 16 & 6 & 3.0000 & .5000 & .6270 & -.0274 & 181.11328 & 152.871307 & .6276 & .4873 & 118.4743 \\
\hline 16 & 7 & 3.0000 & .6000 & .6374 & -.0225 & 180.06779 & 152.246798 & .6378 & .4957 & 118.2736 \\
\hline 16 & 8 & 3.0000 & .7000 & .6465 & -.0175 & 179.32977 & 151.791409 & .6467 & .5028 & 118.1422 \\
\hline 16 & 9 & 3. 0000 & .8000 & .6522 & -.0120 & 178.81648 & 151.475165 & .6523 & .5074 & 118.0500 \\
\hline 16 & 10 & 3.0000 & .9000 & .6557 & -.0061 & 178.54805 & 151.308804 & .6557 & .5101 & 118.0024 \\
\hline 16 & 11 & 3.0000 & 1.0000 & .6572 & 0.0000 & 178.47106 & 151.255431 & .6572 & .5113 & 117.9932 \\
\hline 17 & 1 & 3.2000 & 0.0000 & .6477 & 0.0000 & 179.67603 & 151.986138 & .6477 & .5035 & 118.2187 \\
\hline 17 & 2 & 3.2000 & .1000 & .6470 & .0014 & 179.74705 & 152.039364 & .6470 & .5029 & 118.2240 \\
\hline 17 & 3 & 3.2000 & .2000 & .6448 & .0024 & 179.97430 & 152.181260 & $.644 B$ & .5011 & 118.2631 \\
\hline 17 & 4 & 3.2000 & .3000 & .6419 & .0023 & 180.27633 & 152.371799 & .6419 & .4988 & 118.3134 \\
\hline 17 & 5 & 3.2000 & .4000 & .6347 & .0031 & 181.02479 & 152.830633 & .6347 & 4929 & 118.4480 \\
\hline 17 & 6 & 3.2000 & .5000 & .6312 & .0007 & 181.20769 & 152.940791 & .6312 & .4901 & 118.4822 \\
\hline 17 & 7 & 3.2000 & .6000 & .6375 & -.0024 & 180.65552 & 152.602871 & .6375 & 4952 & 118.3828 \\
\hline 17 & 8 & 3.2000 & .7000 & 6423 & -.0031 & 180.18298 & 152.307114 & .6423 & .4991 & 118.3024 \\
\hline 17 & 9 & 3.2000 & .8000 & .6455 & $-.002 B$ & 179.85039 & 152.100038 & .6455 & .5017 & .118 .2448 \\
\hline
\end{tabular}


SOLUTION SURFACE. NO. $500-$ TIME $=.29 .32792830$ SECONDS $($ DELTA T $=.05867757$, NVCN $=1$. CNUMS $=1.00 .(11,10),(0.0))$

\begin{tabular}{|c|c|c|c|c|c|c|c|c|c|c|}
\hline L & M & $\stackrel{x}{(\text { IN })}$ & $\begin{array}{l}Y \\
(I N)\end{array}$ & $\underset{(F / S)}{J}$ & $\begin{array}{c}v \\
(F / S)\end{array}$ & $\begin{array}{c}P \\
(P S I A)\end{array}$ & $\begin{array}{c}\text { RHO } \\
(\text { LBM/FT3) }\end{array}$ & $\begin{array}{l}\text { VMAG } \\
(F / S)\end{array}$ & $\begin{array}{l}\mathrm{MACH} \\
\mathrm{NO}\end{array}$ & $\stackrel{\top}{(R)}$ \\
\hline $\begin{array}{l}17 \\
17\end{array}$ & $\begin{array}{l}10 \\
11\end{array}$ & $\begin{array}{l}3.2000 \\
3.2000\end{array}$ & $\begin{array}{r}.9000 \\
1.0000\end{array}$ & $\begin{array}{l}.6473 \\
.6478\end{array}$ & $\begin{array}{l}-.0014 \\
0.0000\end{array}$ & $\begin{array}{l}179.69516 \\
179.65637\end{array}$ & $\begin{array}{l}152.002028 \\
151.971562\end{array}$ & $\begin{array}{l}.6473 \\
.6478\end{array}$ & $\begin{array}{l}.5032 \\
.5036\end{array}$ & $\begin{array}{l}118.2189 \\
118.2171\end{array}$ \\
\hline 18 & 1 & 3.4000 & 0.0000 & .6469 & 0.0000 & 179.54338 & 151.906250 & .6469 & .5029 & 118.1935 \\
\hline 18 & 2 & 3.4000 & .1000 & .6465 & .0009 & 179.57099 & 151.933177 & .6465 & .5026 & 118.1908 \\
\hline 18 & 3 & 3.4000 & .2000 & .6456 & .0015 & 179.63098 & 151.974009 & .6456 & .5019 & 118.1985 \\
\hline 18 & 4 & 3.4000 & .3000 & .6444 & .0020 & 179.72757 & 152.040465 & .6444 & .5009 & 118.2104 \\
\hline 18 & 5 & 3.4000 & .4000 & .6429 & .0013 & 179.82734 & 152.107726 & .6429 & .4997 & 118.2237 \\
\hline 18 & 6 & 3.4000 & .5000 & .6414 & -.0004 & 179.82416 & 152.105322 & .6414 & .4985 & 118.2235 \\
\hline 18 & 7 & 3.4000 & .6000 & .6437 & -.0016 & 179.75819 & 152.060802 & .6437 & .5004 & 118.2147 \\
\hline 18 & 8 & 3.4000 & .7000 & .6449 & -.0018 & 179.67170 & 151.998224 & .6449 & .5013 & 118.2064 \\
\hline 18 & 9 & 3.4000 & .8000 & .6458 & -.0015 & 179.59676 & 151.946866 & .6459 & .5021 & 118.1971 \\
\hline 18 & 10 & 3.4000 & .9000 & .6465 & -.0008 & 179.56182 & 151.921583 & .6465 & .5026 & 118.1938 \\
\hline 18. & 11 & 3.4000 & 1.0000 & .6468 & 0.0000 & 179.55297 & 151.909237 & .6468 & .5028 & 118.1975 \\
\hline 19 & 1 & 3.6000 & 0.0000 & .6442 & 0.0000 & 179.98355 & 152.172301 & .6442 & .5006 & 118.2762 \\
\hline 19 & 2 & 3. 6000 & .1000 & .6440 & .0002 & 179.99746 & 152.190994 & .6440 & .5004 & 118.2708 \\
\hline 19 & 3 & 3.6000 & .2000 & .6435 & .0003 & 180.02851 & 152.214380 & .6435 & .5001 & 118.2730 \\
\hline 19 & 4 & 3.6000 & .3000 & .6427 & .0004 & 180.08225 & 152.254990 & .6427 & .4995 & 118.2767 \\
\hline 19 & 5 & 3. 6000 & .4000 & .6417 & .0003 & 180.12635 & 152.288732 & .6417 & .4987 & 118.2795 \\
\hline 19 & 6 & 3.6000 & $.5000^{\circ}$ & .6403 & -.0000 & 180.12693 & 152.288461 & .6403 & .4976 & 118.2801 \\
\hline 19 & 7 & 3.6000 & .6000 & .6423 & -.0003 & 180.08996 & 152.261252 & .6423 & .4991 & 118.2769 \\
\hline 19 & 8 & 3.6000 & .7000 & .6431 & -.0004 & 180.04633 & 152.224539 & .6431 & .4998 & 118.2768 \\
\hline 19 & 9 & 3.6000 & .8000 & .6436 & -.0003 & 180.00328 & 152.192431 & .6436 & .5002 & 118.2735 \\
\hline 19 & 10 & 3.6000 & .9000 & .6441 & -.0002 & 179.98525 & 152.177342 & .6441 & .5005 & 118.2734 \\
\hline 19 & 11 & 3.6000 & 1.0000 & .6442 & 0.0000 & 179.97940 & 152.166774 & .6442 & .5006 & 118.2777 \\
\hline 20 & 1 & 3.8000 & 0.0000 & .6451 & 0.0000 & 179.77278 & 152.045085 & .6451 & .5014 & 118.2365 \\
\hline 20 . & 2 & 3.8000 & .1000 & .6450 & .0001 & 179.77647 & 152.057672 & .6450 & .5013 & 118.2291 \\
\hline 20 & 3 & 3.8000 & .2000 & .6447 & .0002 & 179.78302 & 152.066276 & .6447 & .5011 & 118.2268 \\
\hline 20 & 4 & 3.8000 & .3000 & .6443 & .0002 & 179.78979 & 152.078542 & .6443 & .5008 & 118.2217 \\
\hline 20 & 5 & 3.8000 & 4000 & .6436 & .0001 & 179.79404 & 152.088246 & .6436 & .5003 & 118.2169 \\
\hline 20 & 6 & 3. 8000 & .5000 & .6422 & -.0000 & 179.79208 & 152.086341 & .6422 & .4992 & 118.2171 \\
\hline 20 & 7 & 3. 8000 & .6000 & .6440 & -.0002 & 179.78889 & 152.079376 & .6440 & .5006 & 118.2204 \\
\hline 20 & 8 & 3.8000 & 7000 & .6445 & -.0002 & 179.78175 & 152.064717 & .6445 & .5009 & 118.2271 \\
\hline 20 & 9 & 3. 8000 & .8000 & .6447 & -.0002 & 179.77840 & 152.056602 & 6447 & .5011 & 118.2312 \\
\hline 20 & 10 & 3. 8000 & 9000 & .6450 & -.0001 & 179.77595 & 152.050939 & 6450 & .5013 & 118.2340 \\
\hline 20 & 11 & 3. 8000 & 1.0000 & .6451 & 0.0000 & 179.77676 & 152.044388 & .6451 & .5014 & 118.2397 \\
\hline 21 & 1 & 4.0000 & 0.0000 & .6438 & 0.0000 & 180.00000 & 152.182221 & .6438 & .5003 & 148.2793 \\
\hline 21 & 2 & 4.0000 & .1000 & .6437 & .0001 & 180.00000 & 152.192626 & .6437 & .5002 & 118.2712 \\
\hline 21 & 3 & 4.0000 & .2000 & .6435 & .0002 & 180.00000 & 152.197272 & 6435 & .5001 & 118.2676 \\
\hline 21 & 4 & 4.0000 & .3000 & .6433 & .0002 & 180.00000 & 152.205457 & .6433 & .4999 & 118.2612 \\
\hline 21 & 5 & 4.0000 & .4000 & .6427 & .0001 & 180.00000 & 152.212584 & .6427 & .4995 & 118.2557 \\
\hline 21 & 6 & 4.0000 & .5000 & .6414 & -.0001 & 180.00000 & 152.211907 & .6414 & .4984 & 118.2562 \\
\hline 21 & 7 & 4.0000 & .6000 &.$€ 430$ & -.0002 & 180.00000 & 152.206858 & .6430 & .4997 & 118.2601 \\
\hline 21 & 8 & 4.0000 & .7000 & .6434 & -.0002 & 180.00000 & 152.196505 & .6434 & .5000 & 118.2682 \\
\hline 21 & 9 & 4.0000 & .8000 & .6435 & -.0002 & 180.00000 & 152. 190416 & .6435 & .5001 & 118.2729 \\
\hline 21 & 10 & 4.0000 & .9000 & .6437 & -.0001 & 180.00000 & 152. 186234 & .6437 & .5003 & $118.276 t$ \\
\hline 21 & 11 & 4.0000 & 1.0000 & 6438 & 0.0000 & 180.00000 & 152.179192 & 6438 & 5003 & 118.2816 \\
\hline
\end{tabular}

***** EXPECT FILM OUTPUT FOR $N=500 * * * * *$ 


\section{APPENDIX \\ FORTRAN LISTING OF THE VNAP2 PROGRAM}

Los Alamos Identification No. LP-833

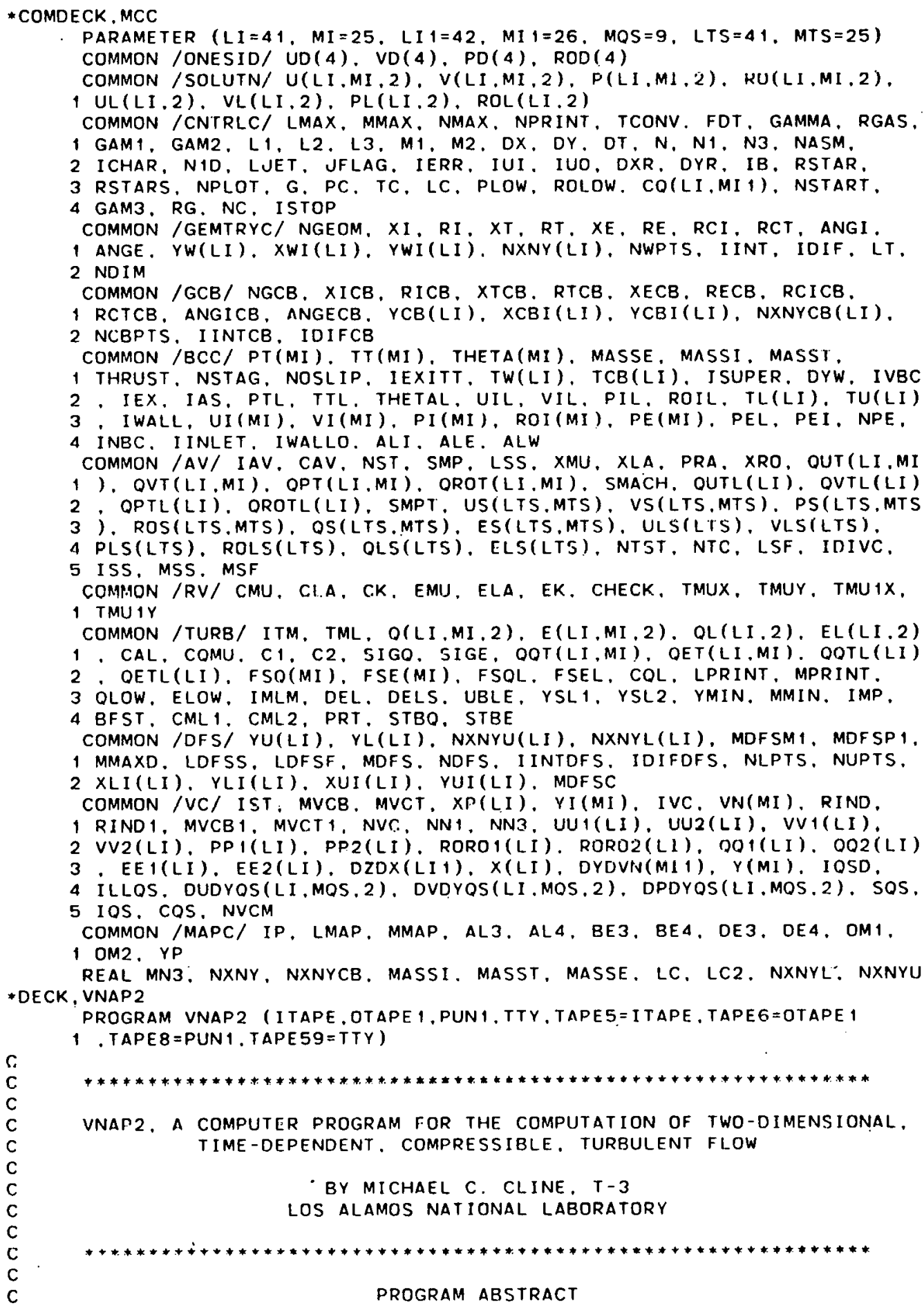




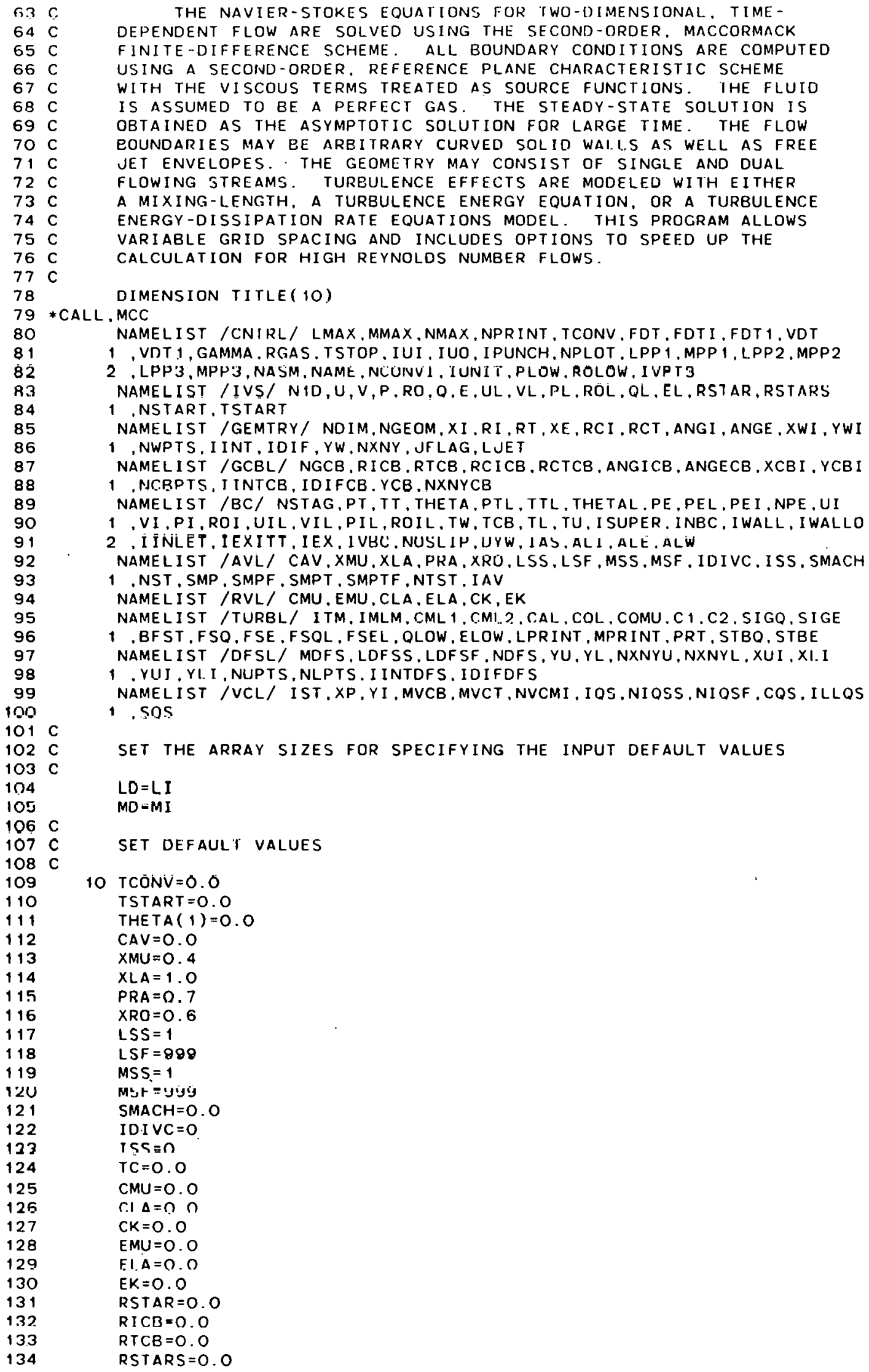




\begin{tabular}{|c|c|}
\hline 135 & $\operatorname{PT}(1)=0.0$ \\
\hline 136 & $\operatorname{TT}(1)=0.0$ \\
\hline 137 & DELSMP $=0.0$ \\
\hline 138 & DSMPT $=0.0$ \\
\hline 139 & $C M L 1=0.0$ \\
\hline 140 & CML 2 $=0.0$ \\
\hline 141 & $P T L=0.0$ \\
\hline 142 & $T T L=0.0$ \\
\hline 143 & THE TAL $=0.0$ \\
\hline 144 & UIL $=0.0$ \\
\hline 145 & $V I L=0.0$ \\
\hline 146 & $P I L=0.0$ \\
\hline 147 & ROIL $=0.0$ \\
\hline 148 & $\operatorname{PE}(1)=14.7$ \\
\hline 149 & $\operatorname{PE}(2)=-1.0$ \\
\hline 150 & $P E L=0.0$ \\
\hline 151 & $P E I=0.0$ \\
\hline 152. & $N P E=0$ \\
\hline 153 & $T M U X=0.0$ \\
\hline 154 & $T M U Y=0.0$ \\
\hline 155 & $B F S T=0.0$ \\
\hline 156 & $C O L=0.0$ \\
\hline 157 & $T M U+X=0.0$ \\
\hline 158 & $T M U \uparrow Y=0.0$ \\
\hline 159 & $A L I=0.0$ \\
\hline 160 & $A L E=0.0$ \\
\hline 161 & AL $W=0.0$ \\
\hline 162 & FDT I $=0.0$ \\
\hline 163 & TSTOP $=1.0$ \\
\hline 164 & CNUMS $=1.0$ \\
\hline 165 & $S M P=1.0$ \\
\hline 166 & $S M P F=1.0$ \\
\hline 167 & $S M P T=1.0$ \\
\hline 168 & SMPTF $=1.0$ \\
\hline 169 & $F D T A=1.0$ \\
\hline 170 & $F D T=0.9$ \\
\hline 171 & $V D T=0.25$ \\
\hline$\$ 7.2$ & VDT $1=0.25$ \\
\hline 173 & NASM $=1$ \\
\hline 174 & $N 1 D=1$ \\
\hline 175 & ND I $M=1$ \\
\hline 176 & IEX $=1$ \\
\hline 177 & NCONVI $=1$ \\
\hline 178 & $I U I=1$ \\
\hline 179 & $I \cup O=1$ \\
\hline 180 & IVPTS $=1$ \\
\hline 181 & $N \vee C M=1$ \\
\hline 182 & NVCM $1=1$ \\
\hline 183 & I $M L M=1$ \\
\hline 184 & $N T S T=1$ \\
\hline 185 & NSTAG $=0$ \\
\hline 186 & NAME $=0$ \\
\hline 187 & I PUNCH $=0$ \\
\hline 188 & $\mathrm{NGCB}=0$ \\
\hline 189 & NMAX $=0$ \\
\hline 190 & $N P R I N T=0$ \\
\hline 191 & $N S T=0$ \\
\hline 192 & $N-O$ \\
\hline 193 & $I E R R=0$ \\
\hline 194 & $J F L A G=0$ \\
\hline 1yb & I SUPER-O \\
\hline 196 & NVCMI $=0$ \\
\hline 197 & IUNI $T=0$ \\
\hline 198 & NOSL IP $=0$ \\
\hline 199 & I INLET $=0$ \\
\hline 200 & IEXITT $=0$ \\
\hline 201 & NSTART $=0$ \\
\hline 202 & I T M $=0$ \\
\hline 203 & $I \wedge S=0$ \\
\hline 204 & IVC $=0$ \\
\hline 205 & $M \vee C B=0$ \\
\hline 206 & $M \vee C T=0$ \\
\hline
\end{tabular}




\begin{tabular}{|c|c|c|}
\hline 207 & & IWALL $=0$ \\
\hline 208 & & I $W A L L O=0$ \\
\hline 209 & & $18=0$ \\
\hline 210 & & LOFSS $=0$ \\
\hline 211 & & LDF SF $=0$ \\
\hline 212 & & MDF $S=0$ \\
\hline 213 & & LPR INT $=0$ \\
\hline 214 & & MPR INT $=0$ \\
\hline 215 & & I $V B C=0$ \\
\hline 216 & & INBC $=0$ \\
\hline 217 & & $\operatorname{LPP} 1=0$ \\
\hline 218 & & $I O S=0$ \\
\hline 219 & & NIOSF $=0$ \\
\hline 220 & & I $A V=0$ \\
\hline 221 & & I ST $=0$ \\
\hline 222 & & LDUF $=0$ \\
\hline 223 & & MUUF $=U$ \\
\hline 224 & & NTC $=0$ \\
\hline 225 & & I INT $=2$ \\
\hline 226 & & $1 D I F=2$ \\
\hline 227 & & I INTCB $=2$ \\
\hline 228 & & ID I FCB $=2$ \\
\hline 229 & & I INTO $\bar{F} \bar{\delta}=\dot{3}$ \\
\hline 230 & & IDIFDF $=2$ \\
\hline 231 & & I LLOS $=30$ \\
\hline 232 & & GAMMA $=1.4$ \\
\hline 233 & & RGAS $=53.35$ \\
\hline 234 & & NPLOT $=-1$ \\
\hline 235 & & $G=32.174$ \\
\hline 236 & & $P C=144.0$ \\
\hline 237 & & $L C=12.0$ \\
\hline 238 & & PLOW $=0.01$ \\
\hline 239 & & ROLOW $=0.0001$ \\
\hline 240 & & $D Y W=0.001$ \\
\hline 241 & & $C A L=1.0$ \\
\hline 242 & & COMU $=0.09$ \\
\hline 213 & & $r .1 \equiv 1 \quad \Delta A$ \\
\hline 244 & & $C 2=1.8$ \\
\hline 245 & & $S I G Q=1.0$ \\
\hline 246 & & $S I G E=1.3$ \\
\hline 247 & & $\bar{S} \cap S=0.5$ \\
\hline 248 & & $C Q S=0.001$ \\
\hline 249 & & NIOSS $=2$ \\
\hline 250 & & $F S O L=0.0001$ \\
\hline 251 & & SI nW=0. חnO1 \\
\hline 252 & & $F S E L=0.1$ \\
\hline 253 & & $E L O W=0.1$ \\
\hline 254 & & $P R I=0.9$ \\
\hline 255 & & $S T B Q=0.0$ \\
\hline 256 & & $S T B E=0.0$ \\
\hline 257 & & ISTOP $=0$ \\
\hline 250 & & $D O \quad 20 M=1, M D$ \\
\hline 259 & & $U I(M)=0.0$ \\
\hline 260 & & $\operatorname{VI}(M)=0.0$ \\
\hline 261 & & $F I(M)=0.0$ \\
\hline 262 & & $\operatorname{ROI}(M)=0.0$ \\
\hline 263 & & $F S O(M)=0.0001$ \\
\hline 264 & & $\operatorname{FSE}(M)=0.1$ \\
\hline 265 & 20 & CONT INUE \\
\hline 286 & & $0030 \mathrm{~L}-1 . \mathrm{LO}$ \\
\hline 267 & & $Y C B(L)=0.0$ \\
\hline 268 & & $Y L(L)=0.0$ \\
\hline 269 & & $Y U(L)-O: O$ \\
\hline 270 & & $\operatorname{NXNYCB}(L)=0.0$ \\
\hline 271 & & $\operatorname{NXNYL}(L)=0.0$ \\
\hline 272 & & $\operatorname{NXNYU}(L)=0.0$ \\
\hline 273 & & $\mathrm{OL}(L .1)=0.0$ \\
\hline 274 & & $E L(L, 1)=0.0$ \\
\hline 275 & & $O L(L .2)=0.0$ \\
\hline 276 & & $E L(L .2)=0.0$ \\
\hline 277 & & $U L(L, 1)=0.0$ \\
\hline 278 & & $V L(L, 1)=0.0$ \\
\hline
\end{tabular}




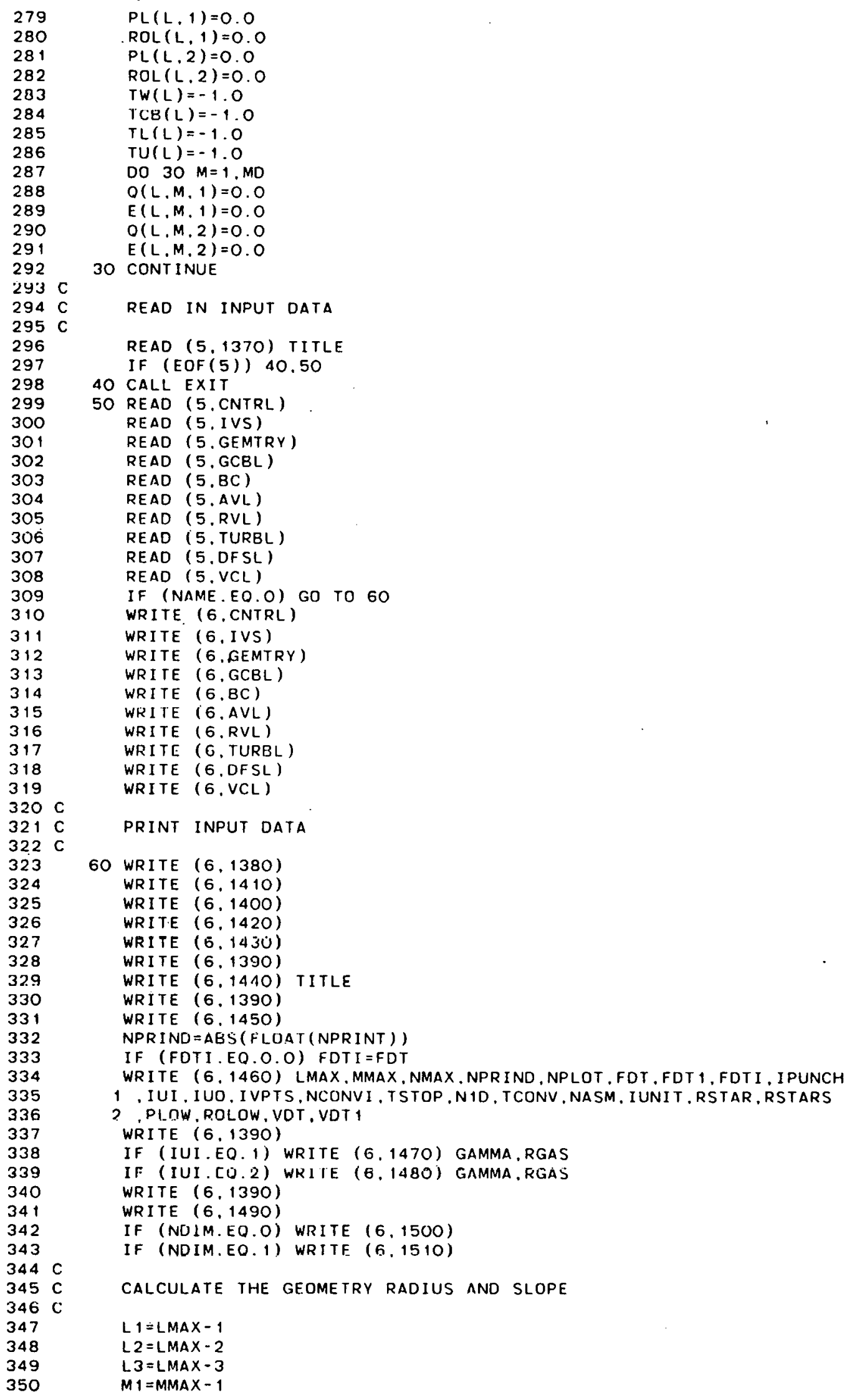




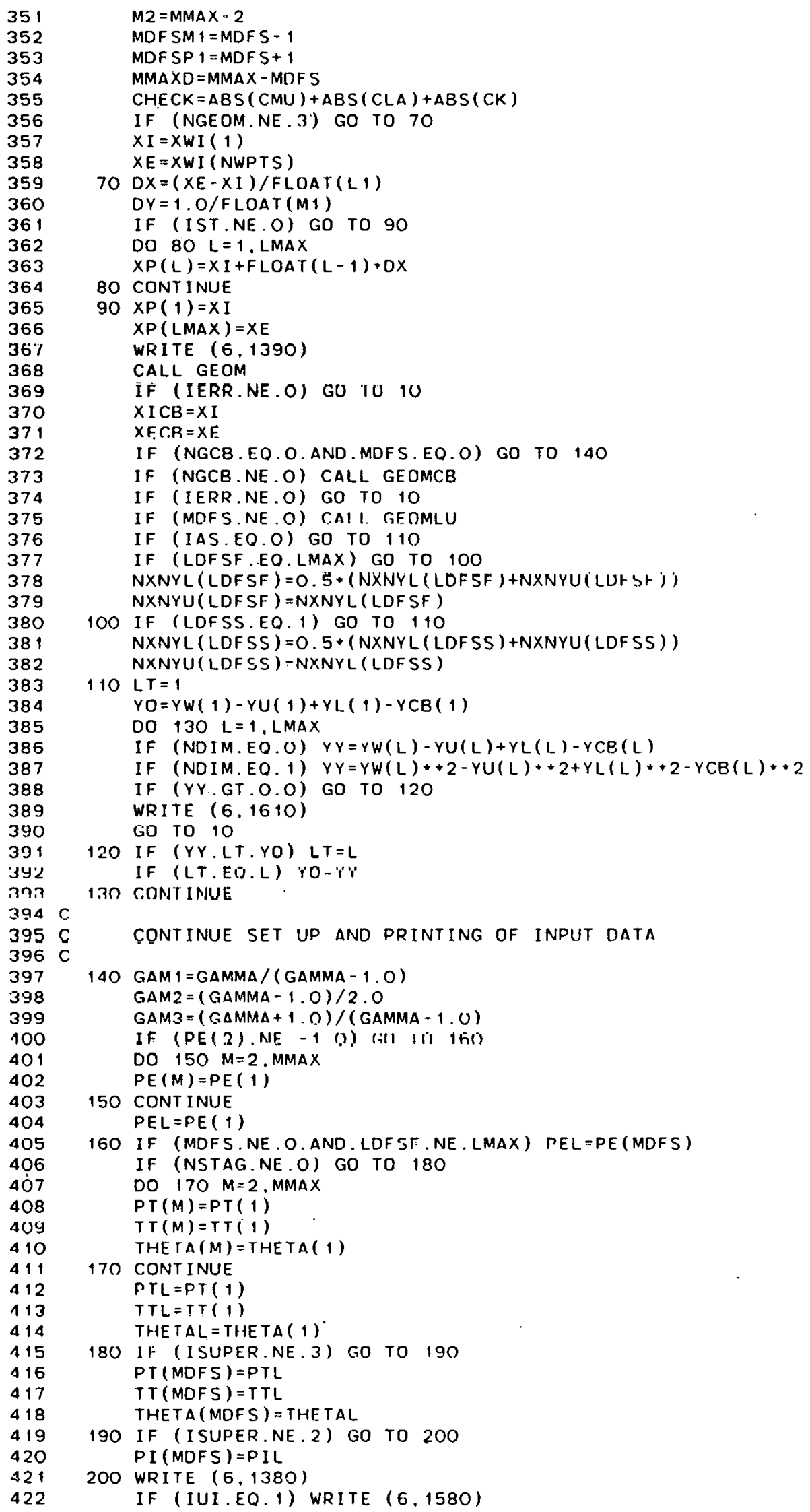




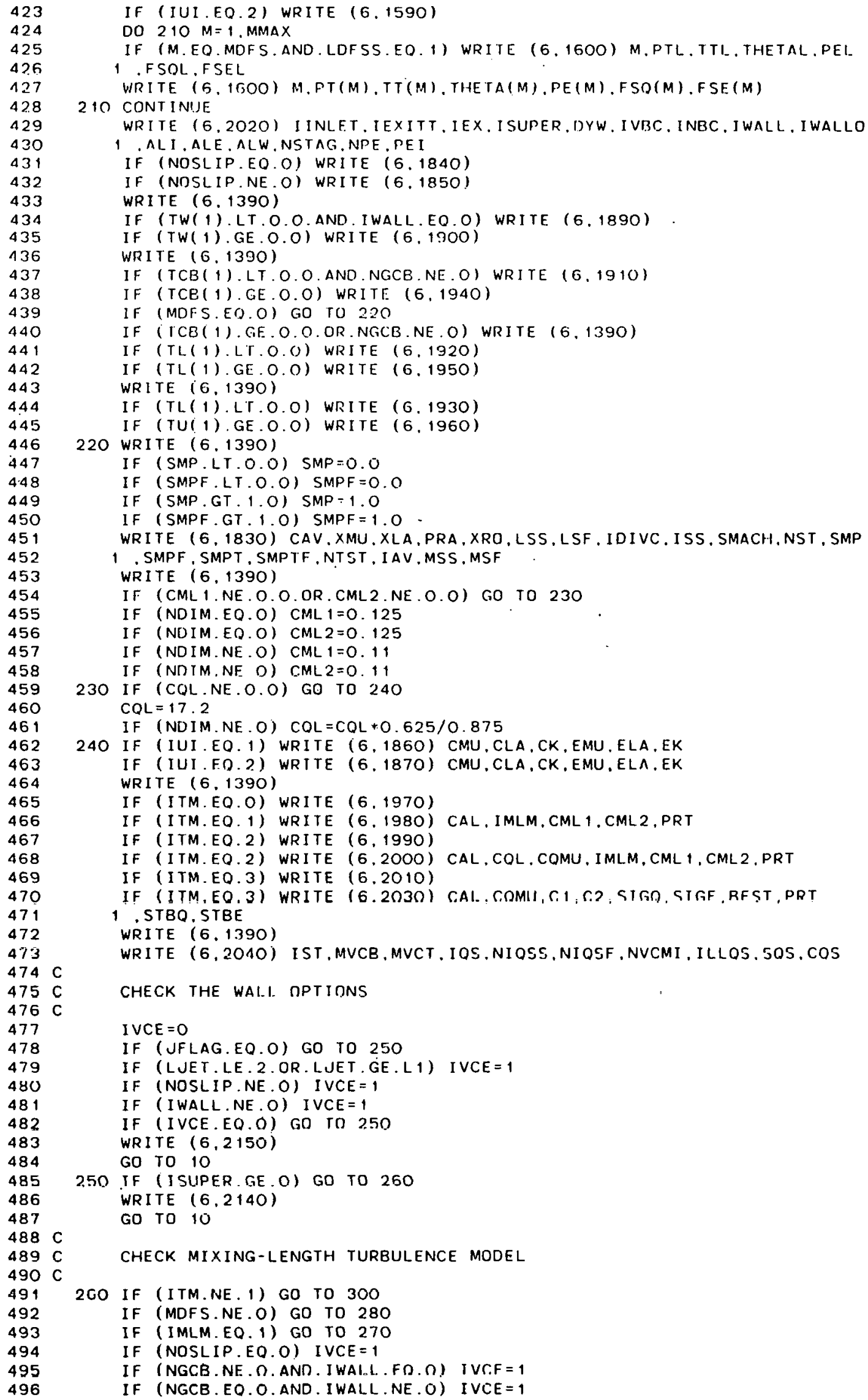

IF (IUI.EO.2) WRITE $(6.1590)$

DO $210 M=1$. MMAX

IF (M.EO.MDFS.AND.LDFSS.EQ. 1) WRITE $(6,1600)$ M.PTL.TTL. THETAL.PEI 1 . FSOL,FSEL

WRITE $(5$, TSOO) M,PT(M),TT(M),THETA(M), PE (M),FSO(M),FSE(M)

210 CONTINUJE

WRITE (6.2020) IINLET. IEXITT. IEX. ISUPER.DYW. IVBC. INBC. IWALL. IWALLO

1 . ALI I ALE, ALW, NSTAG.NPE, PEI

IF (NOSLIP.EO.O) WRITE $(6,1840)$

IF (NOSLIP.NE. O) WRITE $(6,1850)$

WRITE $(6,1390)$

IF (TW( 1) LT O O AND. IWALL, EO O) WRITE $(6,1890)$.

IF (TW(1).GE.O.O) WRITE $(6,1900)$

WRITE $(6,1390)$

IF (TCB ( 1$) . L T, 0.0$. AND. NGCB.NE.O) WRITE $(6.1910)$

IF (TCE (1).GE.O.O) WRITE $(6,1940)$

IF (MDFS.EO.O) GO TO 220

IF (ICB (1).GE . O.O.OR.NGCB.NE.O) WRITE $(6,1390)$

IF (TL(1).LT.O.O) WRITE $(6,1920)$

IF (TL(1).GE.O.O) WRITE $(6,1950)$

WRITE $(6,1390)$

IF (TL(1).LT.0.0) WRITE $(6.1930)$

IF (TU (1).GE.O.O) WRITE $(6,1960)$

220 WRITE $(6.1390)$

IF (SMP.LT.O.O) SMP $=0.0$

IF (SMPF.LT.O.O) SMPF $=0.0$

IF (SMP.GT . 1.0) SMP $=1.0$

IF (SMPF.GT . 1.0) SMPF $=1.0$.

WRITE $(6,1830)$ CAV, XMU, XLA,PRA, XRO, LSS.LSF, IDIVC. ISS, SMACH, NST, SMP

1 . SMPF, SMPT, SMPTF, NTST, IAV.MSS.MSF

WRITE $(6,1390)$

IF (CML1.NE.O.O.OR.CML2.NE.O.O) GO TO 230

IF (NDIM.EQ.O) $\mathrm{CML} 1=0.125$

IF (NOIM.EQ.O) $\mathrm{CML} 2=0.125$

IF (NDIM.NE.O) $\quad \mathrm{CML} 1=0.11$

IF (NDIM.NF O) $C M L 2=0.11$

230 IF (CQL.NE.O.O) GO TO 240

$C Q L=17.2$

IF (NDIM.NE.O) $\mathrm{CQL}=\mathrm{CQL}+0.625 / 0.875$

240 IF (IUI.EO. 1) WRITE $(6,1860)$ CMU,CLA,CK,EMU,ELA,EK

IF (IUI.FD.2) WRTTE $(6,1870)$ CMU,CLA,CK,EMU,ELA.EK

WRITE $(6,1390)$

IF (ITM.EQ.O) WRITE $(6,1970)$

IF (ITM.EO.1) WRITE $(6,1980)$ CAL. IMLM, CML 1.CML 2,PRT

IF (ITM.EO.2) WRITE $(6,1990)$

IF (ITM.EQ.2) WRITE $(6,2000)$ CAL, CQL, COMU, IMLM, CML 1, CML 2, PRT

IF (ITM.EO.3) WRITE $(6,2010)$

IF (ITM,EQ.3) WRITE $(6.2030)$ CAL. SOMII, S., C., SIGR, SIGF .RFST, PRT

1 . STBQ. STBE

WRITE $(6.1390)$

WRITE (6.2040) IST, MVCB, MVCT, IOS.NIOSS, NIOSF, NVCMI, ILLOS. SOS. COS

CHECK THE WALI. RPTIONS

I $V C E=0$

IF (UFLAG.EQ.O) GO TO 250

IF (LJET.LE.2.OR. LJET.GE.L1) IVCE $=1$

IF (NOSLIP.NE.O) IVCE $=1$

IF (IWALL.NE.O) IVCE $=1$

IF (IVCE.EO.O) GO TO 250

WRITE $(6,2150)$

GO TO 10

250 IF (ISUPER GE . O) GO TO 260

WRITE $(6,2140)$

GO TO 10

CHECK MIXING-LENGTH TURBULENCE MODEL

200 IF (ITM.NE. 1) GO TO 300

IF (MDFS.NE.O) GO TO 280

IF (IMLM.EQ.1) GO TO 270

IF (NOSLIP.EQ.O) IVCE $=1$

IF (NGCB.NE. O. AND. I WALLL. FO. O) IVR.F $=1$

IF (NGCB.EQ O. AND. I WALL. NE . O) IVCE $=1$ 


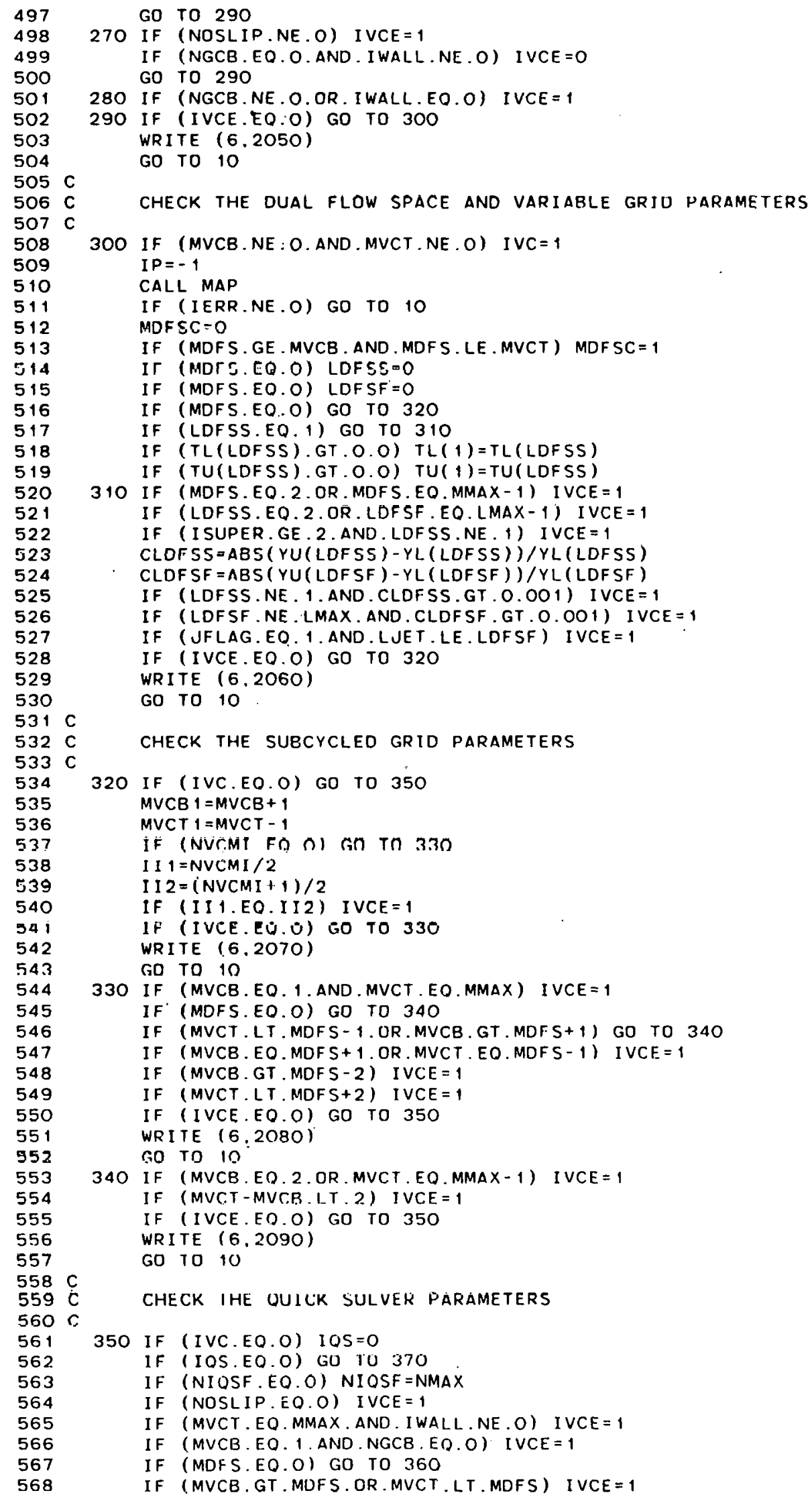




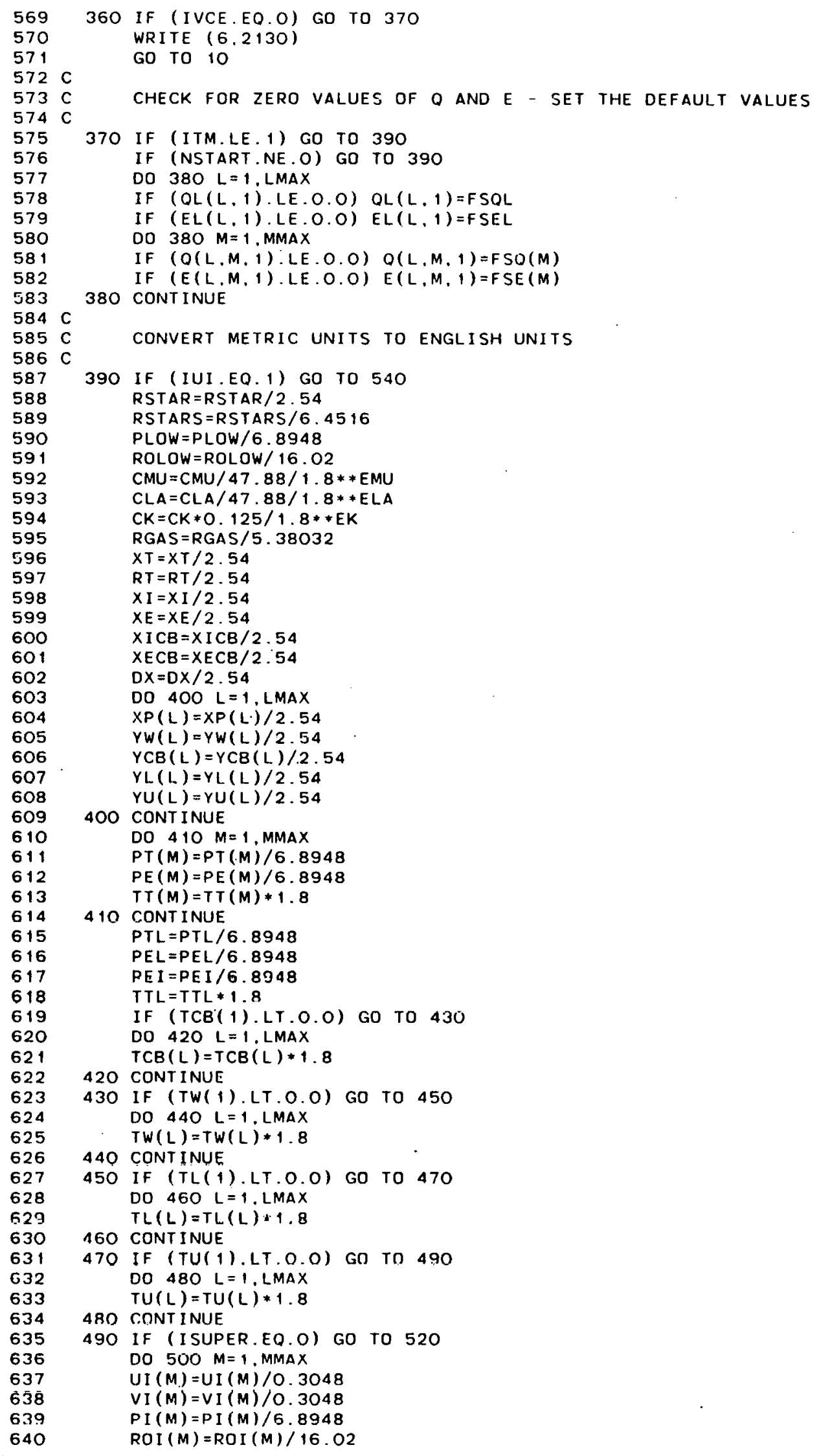




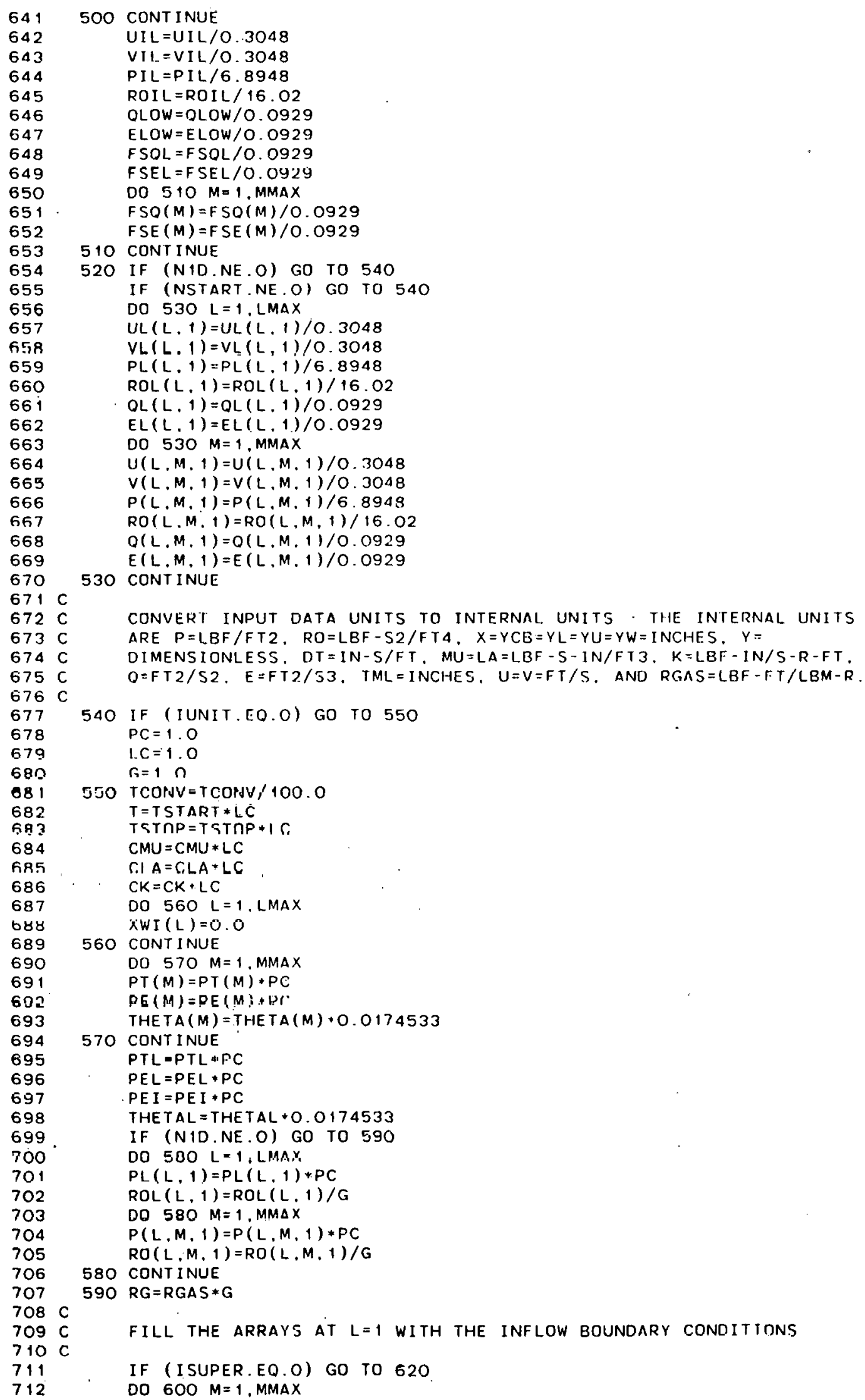




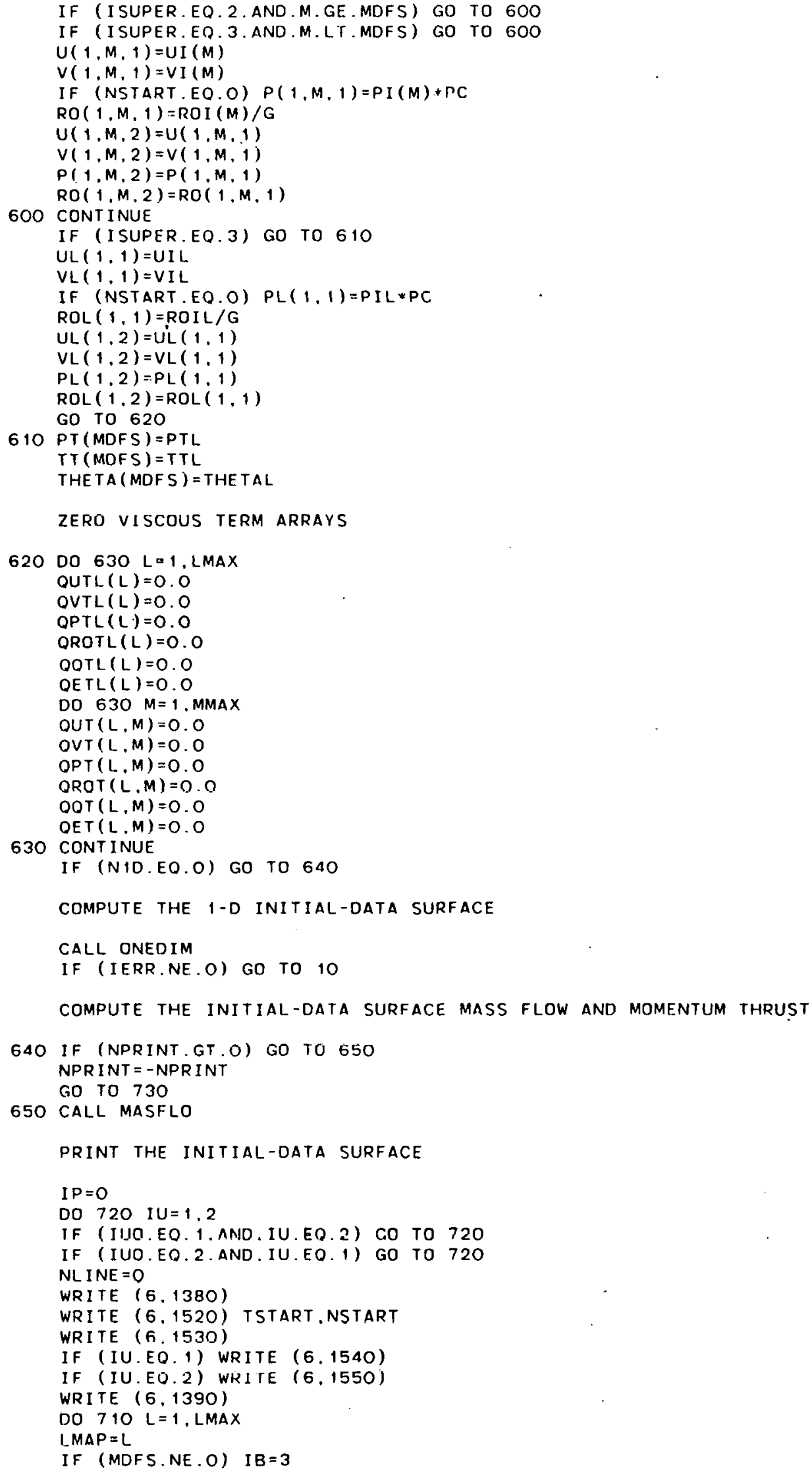




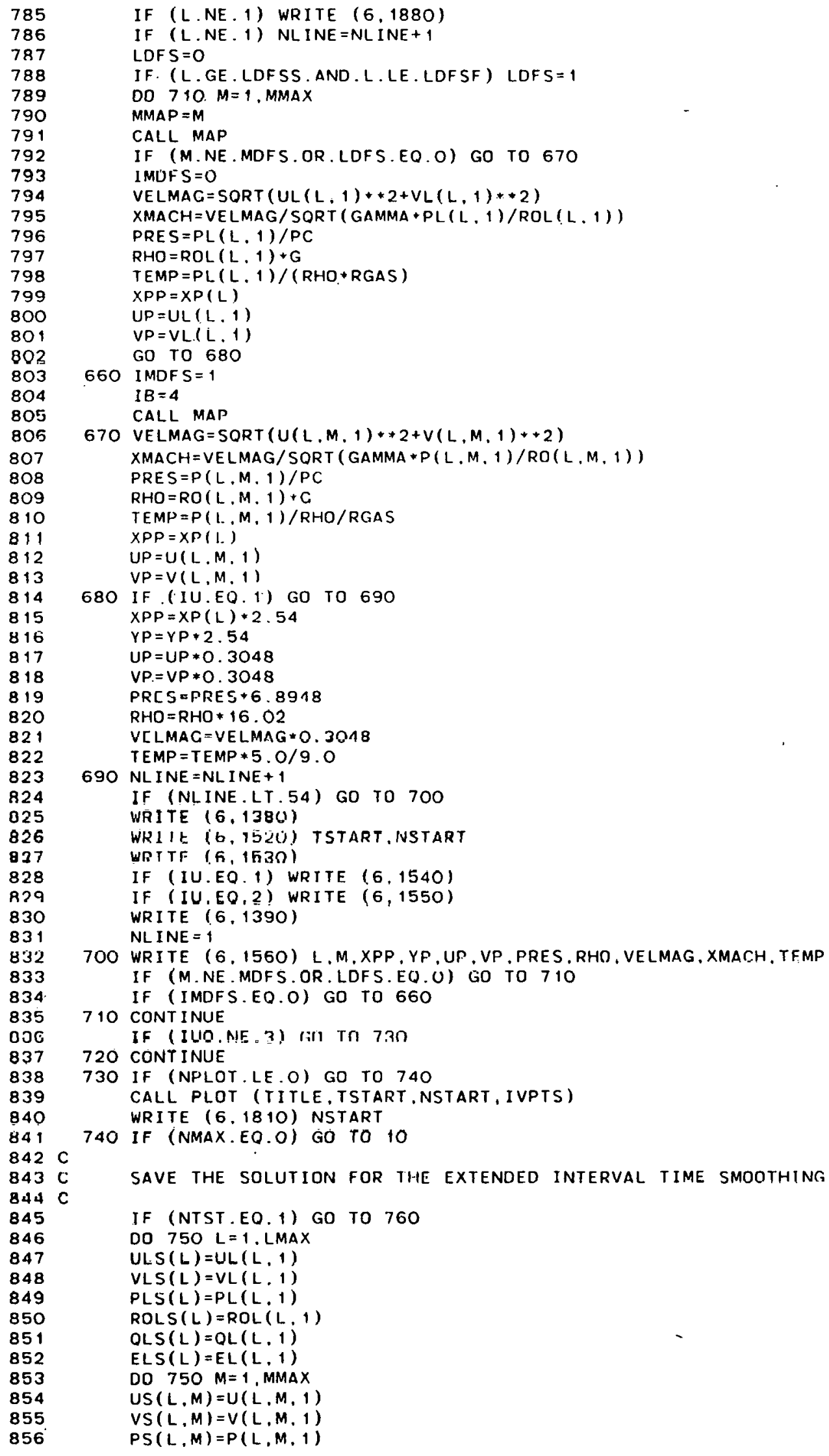




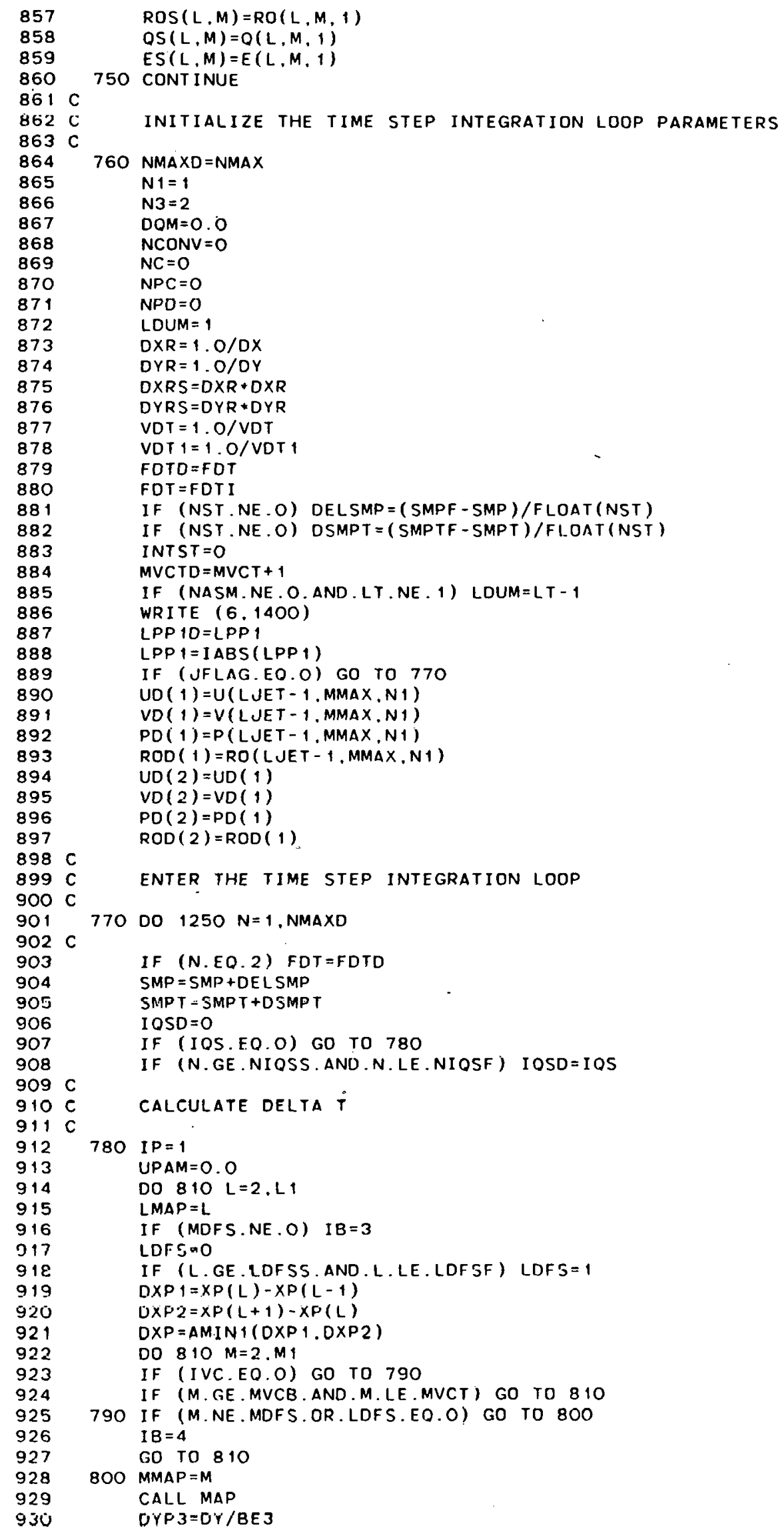




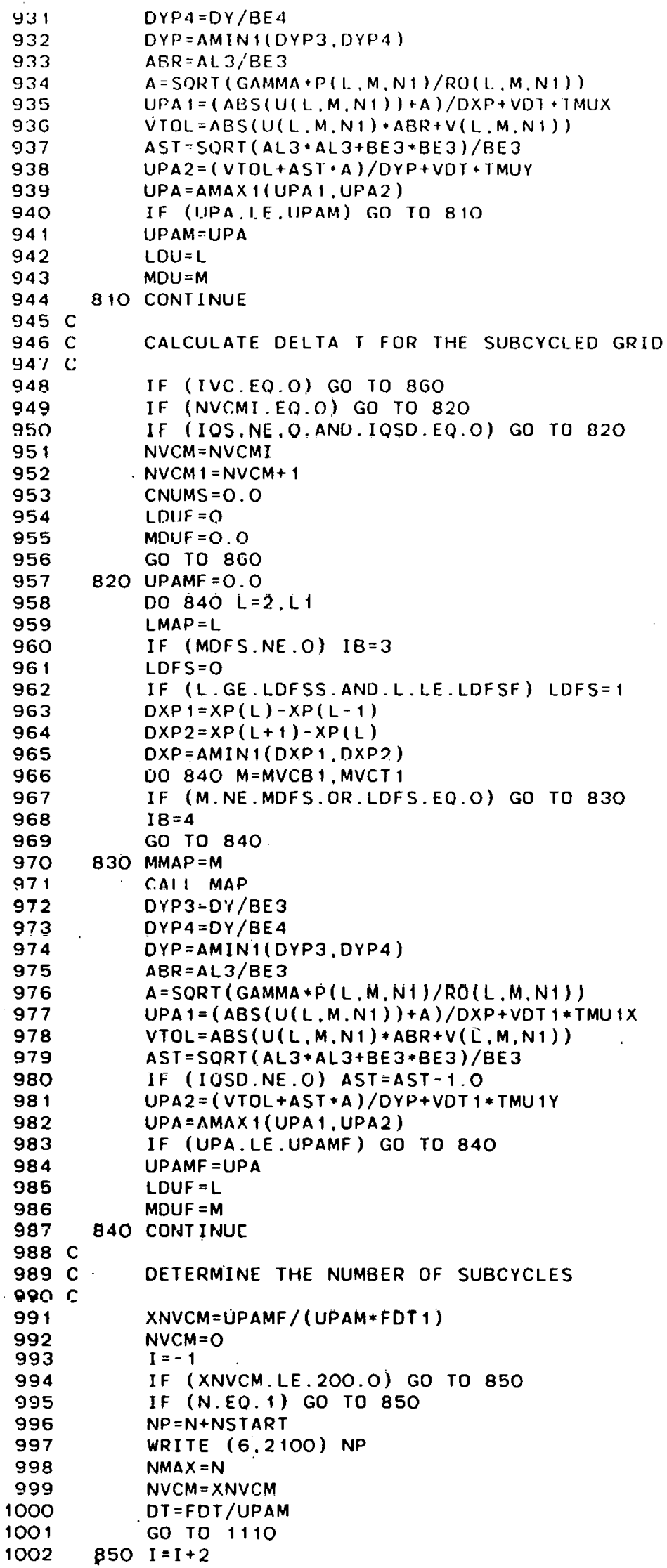




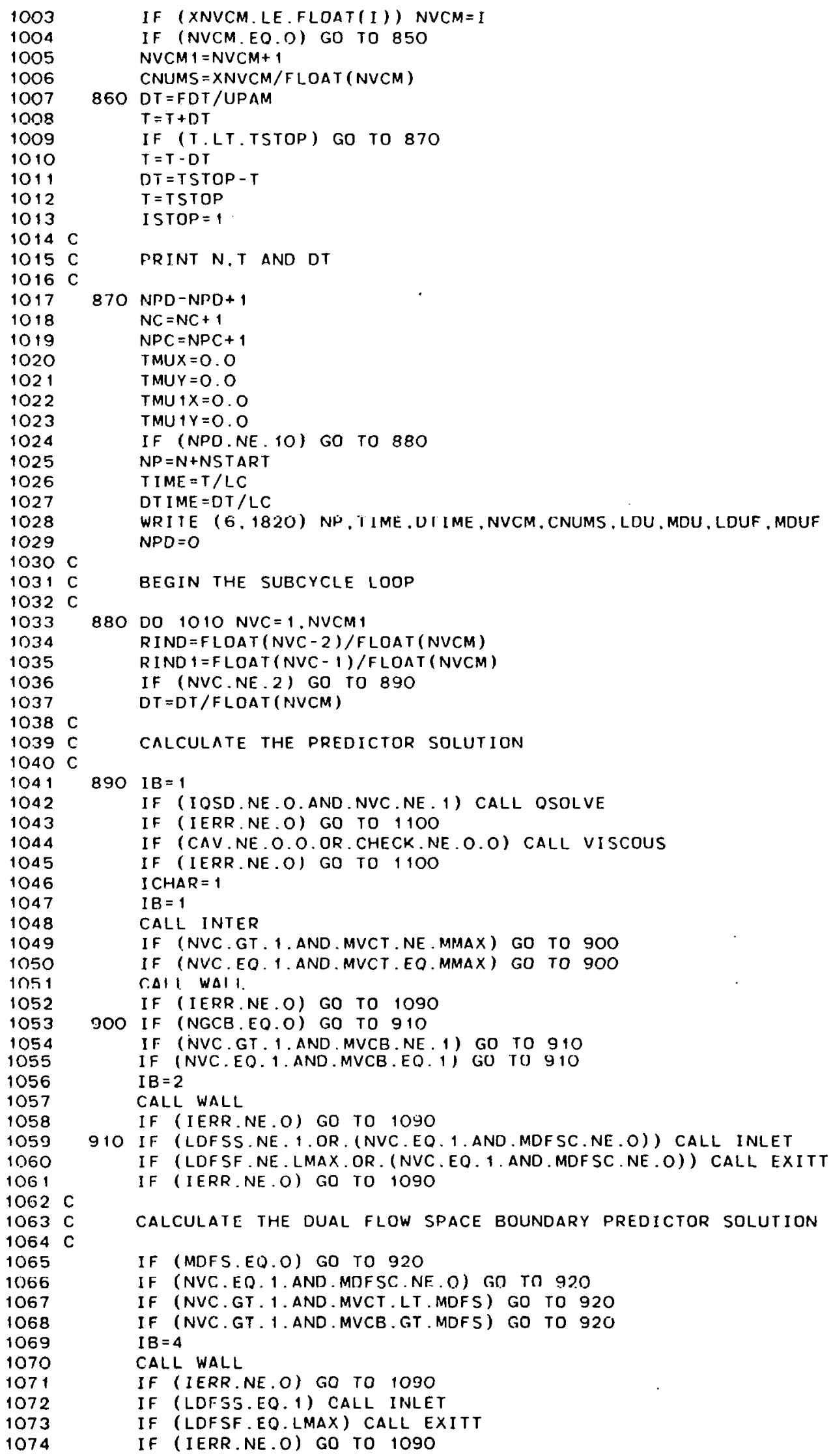




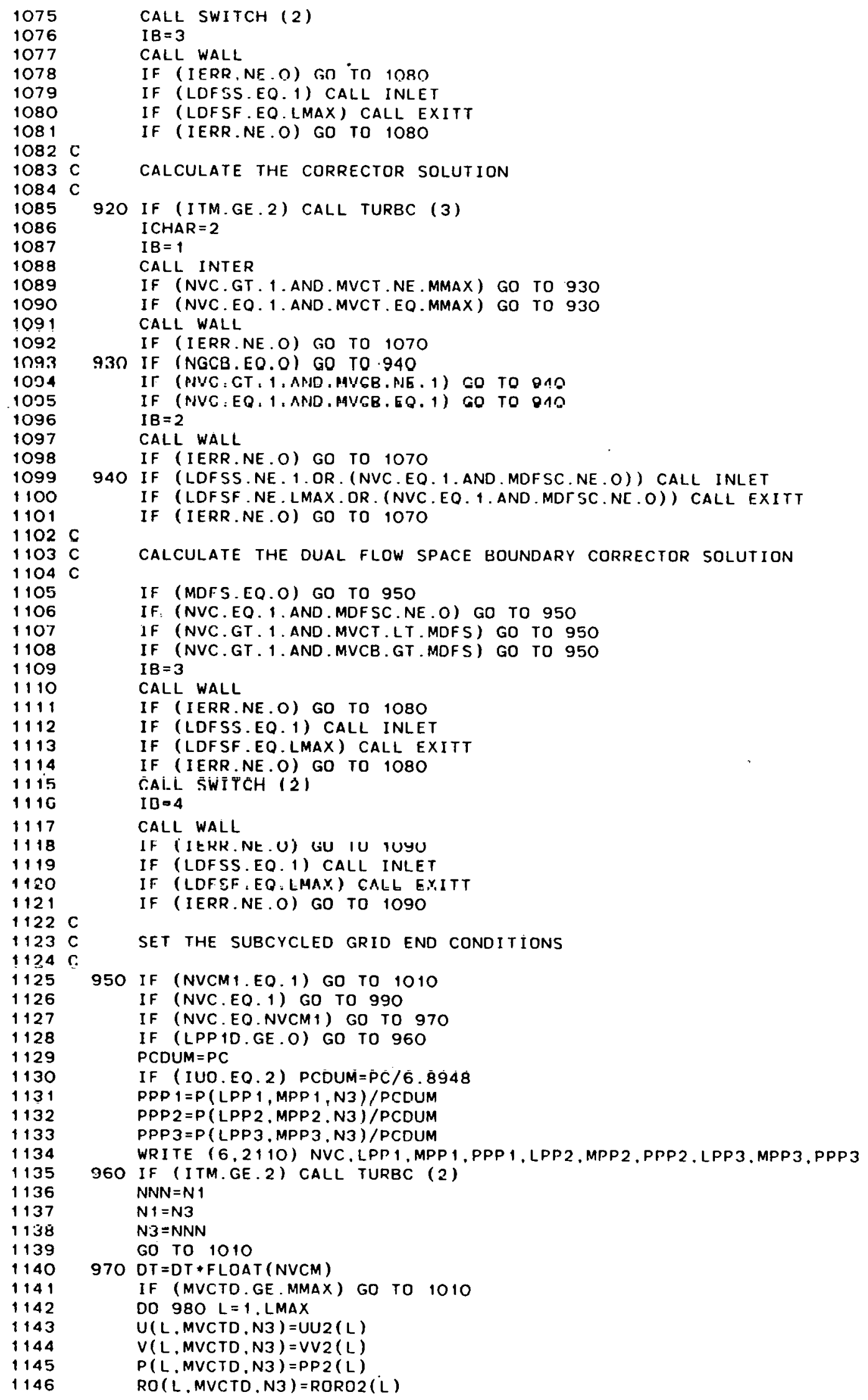




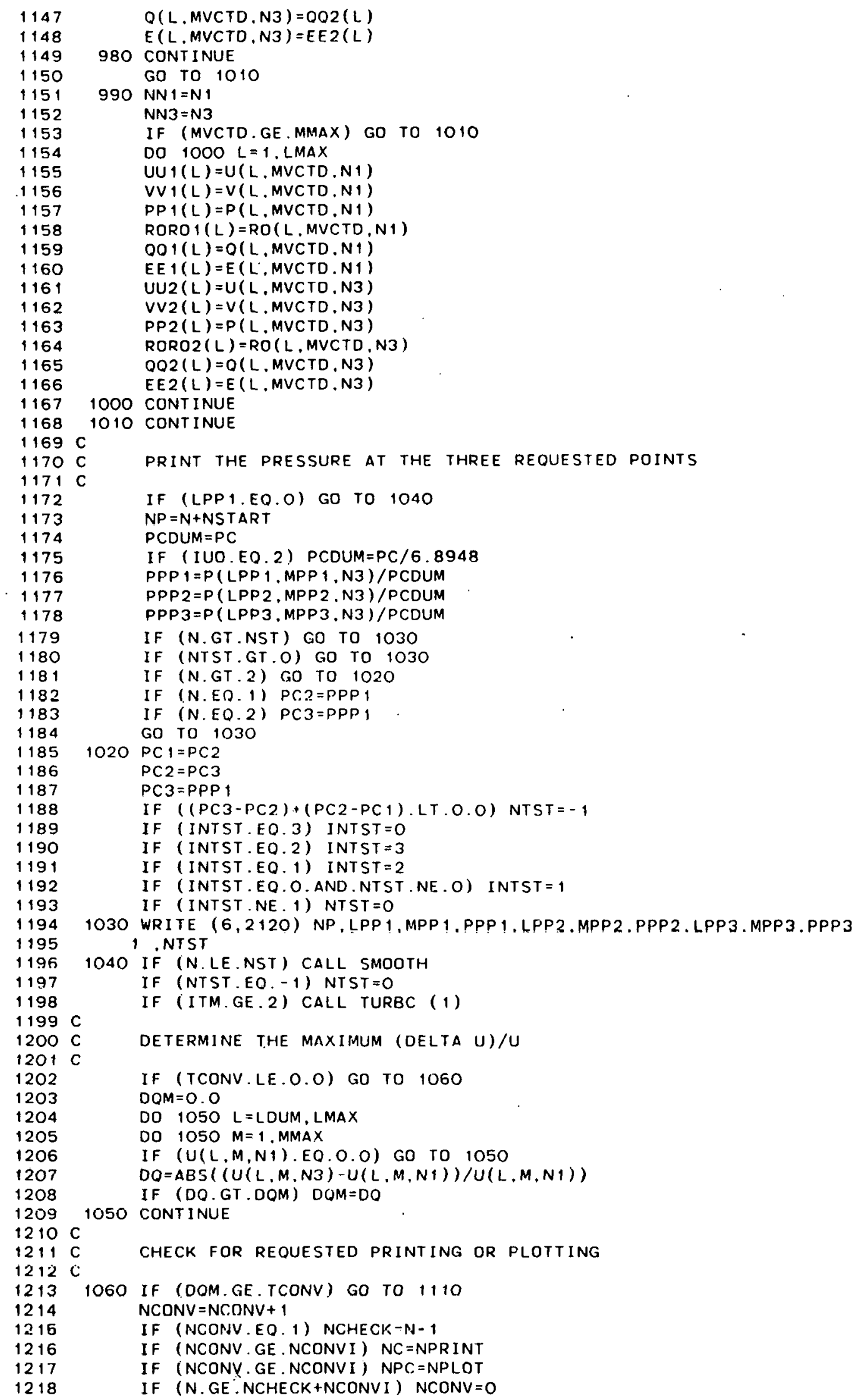




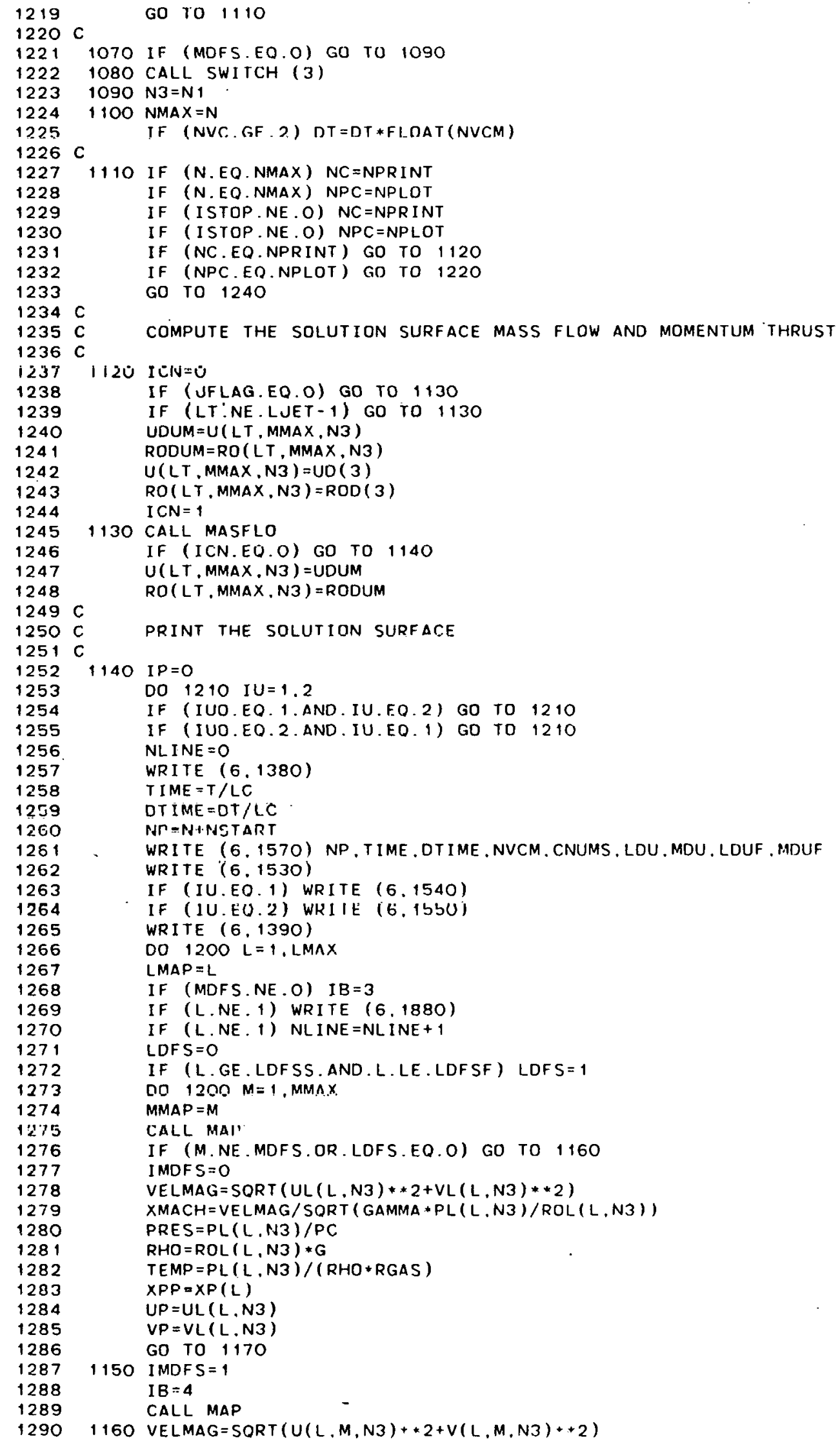




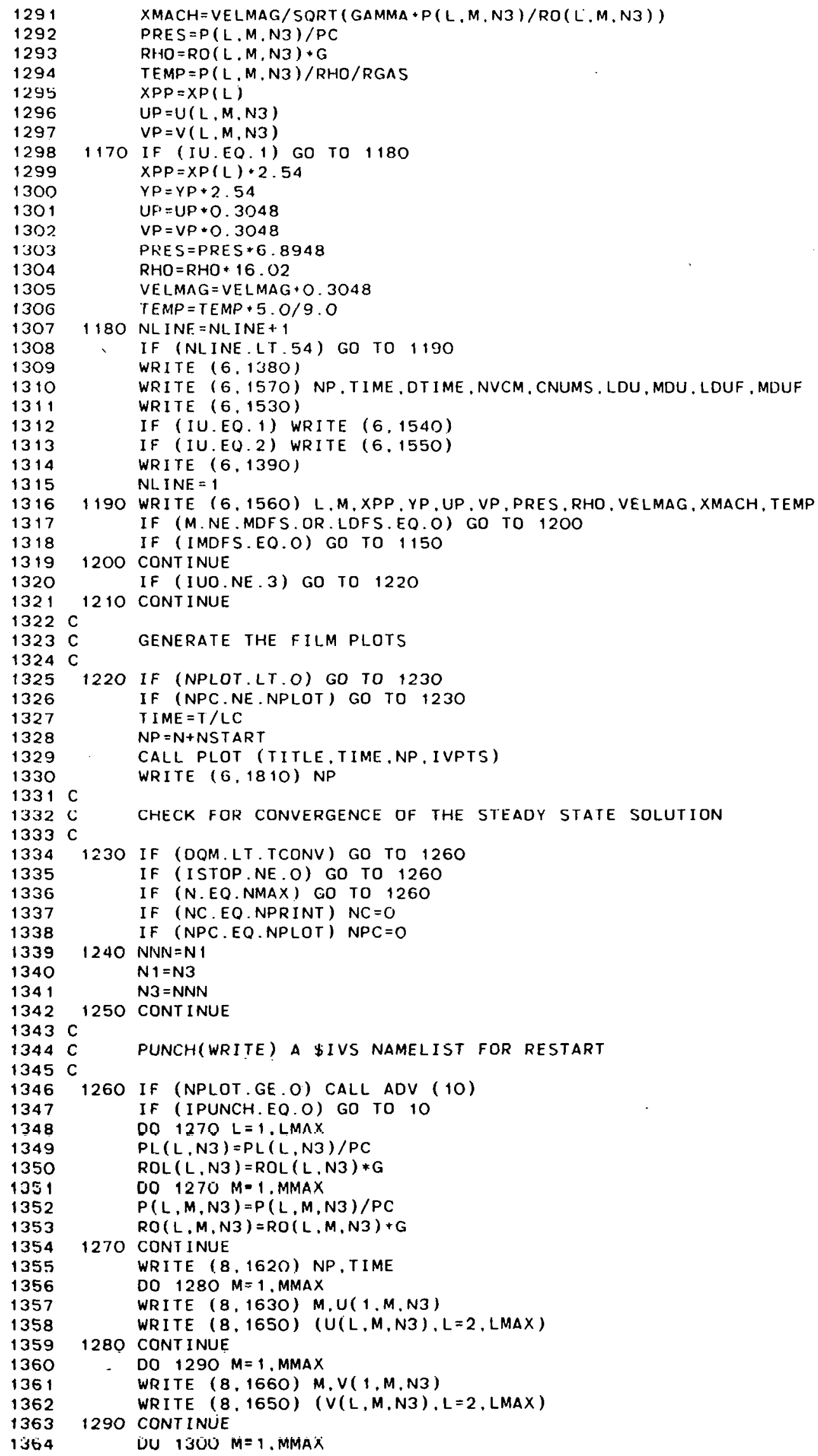


1387

1388

1389

1390

1391

1392

1393

1394

1395

1396

1397

1398

1399

1400

1401

$1402 \mathrm{C}$

$1403 \mathrm{C}$

$1404 \mathrm{C}$

1405

1406

1407

1408

1409

1410

1411

1412

1413

1414

1415

1416

1417

1418

1419

1420

1421

1422

1423

1424

1425

1426

1427

1428

1429

1430

1431

1432

1433

1434

1435

1436
WRITE $(8.1680) \mathrm{M} . P(1 . \mathrm{M}, \mathrm{N} 3)$

WRITE $(8,1700)(P(L, M, N 3), L=2, L M A X)$

1300 CONT INUE

DO $1310 M=1$, MMAX

WRITE $(8,1710) M, R O(1, M, N 3)$

WRITE $(8,1730)(R O(L, M, N 3), L=2 . L M A X)$

1310 CONTINUE

IF (ITM.LE. 1) GO TO 1340

DO $1320 M=1$, MMAX

WRITE $(8,1740)$ M.O(1.M.N3)

WRITE $(8,1760)(Q(L, M, N 3), L=2, L M A X)$

1320 CONT INUE

IF (ITM.EO.2) GO TO 1340

DO $1330 M=1$, MMAX

WRITE $(8,1750) M, E(1, M, N 3)$

WRITE $(8,1760)$ (E (L.M.N3), L =2, LMAX)

$133 U$ LUNTI INUE

1340 IF (MDFS.EO.O) GO TO 1350

LDF SSP $1=$ LDF SS +1

WRITE $(8,1640)$ LDFSS, UL (LDFSS, N3)

WRITE $(8,1650)$ (UL (L,N3). L=LDFSSP 1. LDFSF)

WRITE (8,1670) LDFSS,VL(LDFSS.N3)

WRITE $(8,1650)$ (VL (L,N3), L=LUHSSP 1, LUFSF )

WR ITE $(8,1690)$ LDFSS,PL (LDFSS,N3)

WR ITF $(8,1700)$ (PL (L,N3), L=LDFSSP 1. LDFSF )

WRITE $(8,1720)$ LDFSS.ROL (LDFSS.N3)

WRITE $(8,1730)$ (ROL(L,N3), L=LDFSSP 1, LDFSF)

IF (ITM.LE. 1) GO TO 1350

WRITE $(8,1770)$ LDFSS.OL (LDFSS.N3)

WRITE $(8,1760)$ (OL (L,N3).L=LDFSSP1, LDFSF)

IF (ITM.EO.2) GO TO 1350

WRITE (8,1780) LDFSS,EL(LDFSS,N3)

WRITE $(8,1760) \quad(E L(L . N 3) . L=L O F S S P 1$, LDFSF)

1350 WR ITE $(8,1790)$

NCARDS $=($ LMAX $/ 7+2) *$ MMAX $* 4+2+$ LDF SF - LDFSS

WRITE $(6,1800)$ NCARDS

in tก in

1370 FORMAT (10A8)

1380 FORMAT $(1111)$

1390 FORMAT $(4 \mathrm{H})$

1400 FORMAT (1HO)

1410 FORMAT (1HO.10X.47HVNAP2, A COMPUTER PROGRAM FOR THE COMPUTATION 0 $1.58 \mathrm{HF}$ TWO-DIMENESIONAL, TIME-DEPENDENT, COMPRESSIBLE, TURBULENT.5H 2 FLOW, //37X,57HBY MICHAEI C. CLINE, T-3 - LOS ALAMOS NATIONAL LABO JRATORY)

1420 FORMAT ( IHO, 10X, 18HPROGRAM ABSTRACT - //26X, 17HTHE NAVIER-STOKES, 6 $12 H$ EQUATIONS FOR TWO DIMENSIONAL. TIME DCPCNDENT FLOW ARE SOLVED, 2 1OH USING THE./.21X.62HSECOND-ORDFR. MACCORMACK FINITE-DIFFERENCE 3 SC.HFMF, AI I. ROINNAR . 31HY CONDITIDNS ARE COMPUTED USING./.21X, 13HA - 4 SECOND-ORDE. 62HR, REFERENCE PLANE CHARACTERISTIC SCHEME WITH THE 5VISCOUS TERM, 19HS TREATED AS SOURCE)

1430 FORMAT ( $1 \mathrm{H}, 20 X, 41 \mathrm{HFUNCTIONS.} \mathrm{THE} \mathrm{FLUID} \mathrm{IS} \mathrm{ASSUMED} \mathrm{TO} \mathrm{BE} \mathrm{A.54HPE}$ IRFECT GAS. THE STEADY-STATE SOLUTION IS ORTAINFD AS./.21X.62HTHE 2AJYMPTOTIC GOLUTION TOR LARGE TIMC. TIIC TLOW BOUNDARIES M.3AHAY $B$ 3E ARBITRARY CURVED SOLID WALLS, $/ .21 \times, 62 H A S$ WELL AS JET ENVELOPES. 4 THE GEOMETRY MAY CONSIST OF SINGLE. 36HAND DUAL FLOWING STREAMS. 5TURBULFNCE, /.21X.62HEFFECTS ARE MODELED WITH EITHER A MIXING-LENGT 6H. A TURBULENCE .32HENERGY EQUATION. OR A TURBULENCE./, $21 X, 62$ HENER 7GY-DISSIPATION RATE EQUATIONS MODEL. THIS PROGRAM ALLOWS, 34HVARI 8ABLE GRID SPACING AND INCLLIOES, $.21 \mathrm{X}, 17 \mathrm{H} P$ TIINNS TO SPFFD, 5OHUP TH 9E CALCULATION FOR HIGH REYNOLDS NUMBER FLOWS.)

1440 FORMAT ( 1 HO, 1OX, 11 HJOB TITLE - //21X, 10A8)

1450 FORMAT (1HO, 10X, 2OHCONTROL PARAMETERS - )

1460 FORMAT (1HO, 2OX, 5HLMAX = I $2,2 \mathrm{X}, 5 \mathrm{HMMAX}=, \mathrm{I} 2,3 \mathrm{X}, 5 \mathrm{HNMAX}=$, I 4, 2X, 7HNPR INT $1=.14,2 X, 6 \mathrm{HNPLOT}=. I 4,6 \mathrm{X} .4 \mathrm{HFDT}=, \mathrm{F} 4.2,2 \mathrm{X}, 5 \mathrm{HFDT} 1=, \mathrm{F} 4.2,3 \mathrm{X}, 5 \mathrm{HFDTI}=. \mathrm{F} 4.2$ $2,2 \mathrm{X}, 7 \mathrm{HIPUNCH}=, \mathrm{I} 1, / .21 \mathrm{X}, 4 \mathrm{HIUI}=, 11,4 \mathrm{X}, 4 \mathrm{HIUO}=, 11,5 \mathrm{X}, 6 \mathrm{HIVPTS}=, I 1,4 \mathrm{X}, 7$ $3 \mathrm{HNCONVI}=, 12,4 X, 6 \mathrm{HTSTOP}=, \mathrm{E} 8,2,2 \mathrm{X}, 4 \mathrm{HN} 1 \mathrm{D}=, 12,4 \mathrm{X}, 6 \mathrm{HTCONV}=, \mathrm{F} 5.3 ; \mathrm{X}, 5 \mathrm{HN}$ 4 ASM $=, I 1,5 X, 6 H I U N I T=, I 1, / .21 X, 6 H R S T A R=, F 11,6,2 X, 7 H R S T A R S=, F 13.7,4 X$, $55 \mathrm{HPLOW}=, F 6.4,5 X, 6 \mathrm{HROLOW}=. \mathrm{F} 11.6,5 \mathrm{X}, 4 \mathrm{HVDT}=, F 4.2 .3 \mathrm{X}, 5 \mathrm{HVDT} 1=, \mathrm{F} 4.2)$ 
1470 FORMAT (1HO, 10X, 13HFLUID MODEL $-, / / 2 i x, 36 H T H E$ RATIO OF SPECIFIC HE 1ATS. GAMMA =.F6.4,26H AND THE GAS CONSTANT, $R=. F 9.4,15 H(F T-L B F / L$ 2BM-R ))

1480 FORMAT (1HO, 10X, 13HFLUID MODEL - $/ / 21 \mathrm{X}, 36 \mathrm{HTHE}$ RATIO OF SPECIFIC HE 1ATS, GAMMA $=, F 6.4 .26 \mathrm{H}$ AND THE GAS CONSTANT, $R=, F 9.4 .9 \mathrm{H}(\mathrm{J} / \mathrm{KG}-\mathrm{K}))$ 1490 FORMAT (1HO, 10X. 15HFLOW GEOMETRY -)

1500 FORMAT ( $110.20 X, 47 H T$ TO-DIMENSIONAL. PLANAR FLOW HAS BEEN SPECIFIED 1)

1510 FORMAT (1HO, 20X, 36HAXISYMMETRIC FLOW HAS BEEN SPECIFIED)

1520 FORMAT $(1 \mathrm{H}, 3$ OHINITIAL-DATA SURFACE - TIME $=, F 12.8,8 \mathrm{H}$ SECONDS, $4 \mathrm{H}$ $1(N=.16,1 H))$

1530 FORMAT ( $1 \mathrm{HO}, 11 \mathrm{X}, 1 \mathrm{HL}, 4 \mathrm{X}, 1 \mathrm{HM}, 9 \mathrm{X}, 1 \mathrm{HX}, 10 \mathrm{X}, 1 \mathrm{HY}, 10 \mathrm{X}, 1 \mathrm{HU}, 11 \mathrm{X}, 1 \mathrm{HV}, 12 \mathrm{X}, 1 \mathrm{HP}$, 1 (1X, 3HRHO, 7X, 4HVMAG, 1OX, 4HMACH, $8 X, 1 \mathrm{HT})$

1540 FORMAT ( $1 \mathrm{H}, 25 \mathrm{X}, 4 \mathrm{H}(\mathrm{IN}), 7 \mathrm{X}, 4 \mathrm{H}(\mathrm{IN}), 6 \mathrm{X}, 5 \mathrm{H}(\mathrm{F} / \mathrm{S}), 7 \mathrm{X}, 5 \mathrm{H}(\mathrm{F} / \mathrm{S}), 7 \mathrm{X}, 6 \mathrm{H}$ (PSIA) $1,6 \mathrm{X}, 9 \mathrm{H}(\mathrm{LBM} / \mathrm{FT} 3), 4 \mathrm{X}, 5 \mathrm{H}(\mathrm{F} / \mathrm{S}), 10 \mathrm{X}, 2 \mathrm{HNO}, 8 \mathrm{X}, 3 \mathrm{H}(\mathrm{R}))$

1550 FORMAT ( $1 \mathrm{H}, 25 \mathrm{X}, 4 \mathrm{H}(\mathrm{CM}), 7 \mathrm{X}, 4 \mathrm{H}(\mathrm{CM}), 6 \mathrm{X}, 5 \mathrm{H}(\mathrm{M} / \mathrm{S}), 7 \mathrm{X}, 5 \mathrm{H}(\mathrm{M} / \mathrm{S}), 7 \mathrm{X}, 6 \mathrm{H}$ (KPA) $1,7 X .7 H(K G / M 3), 5 X, 5 H(M / S), 10 X .2 H N O, 8 X, 3 H(K))$

1560 FORMAT ( $1 \mathrm{H}, 7 \mathrm{X}, 2 \mathrm{I} 5,4 \mathrm{~F} 12.4, \mathrm{~F} 13.5, \mathrm{~F} 12.6,3 \mathrm{~F} \mathrm{12.4)}$

1570 FORMAT ( $1 \mathrm{H}$, 2OHSOLUTION SURFACE NO., I6.3H-.7HTIME $=. F 12.8 .20 \mathrm{H}$ IECONDS (DELTA $T=, F 10.8,8 \mathrm{H}, \mathrm{NVCM}=, 13,9 \mathrm{H}, \mathrm{CNUMS}=, F 5.2,3 \mathrm{H},(.12,1$ $2 H, .12,4 H),(, I 2,1 H, .12,2 H) j)$

1580 FORMAT (1HO, 10X, 2 1HBOUNDARY CONDITIONS - .//22X, 1HM, 10X, 8HPT(PSIA), $111 \mathrm{X}, 5 \mathrm{HTT}(\mathrm{R}), 10 \mathrm{X}, 10 \mathrm{HTHETA}$ (DEG), 10X,8HPE (PSIA), $7 \mathrm{X}, 1 \mathrm{HHF}$ SQ(FT2/S2) . $7 \mathrm{X}$ $2.11 \mathrm{HFSE}(\mathrm{FT} 2 / \mathrm{S} 3), 1)$

1590 FDRMAT (1HO. 10X, 2 IHBOUNDARY CONDITIONS - $, / / 22 \mathrm{X}, 1 \mathrm{HM}, 10 \mathrm{X}, 7 \mathrm{HPT}$ (KPA), 1 $12 X, 5 H T T(K), 10 X, 10 H T H E T A(D E G), 10 X, 7 H P E(K P A), 8 X, 10 H F S O(M 2 / S 2), 8 X, 10$ 2 HFSE (M2/S3), /)

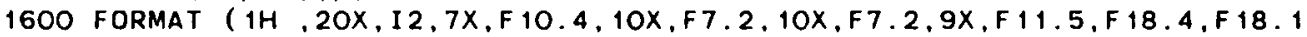
1 )

1610 FORMAT (1HO.51H***** THE RADIUS OF THE CENTERBODY IS LARGER THAN T 1 . 2OHHE WALL RADIUS *****)

1620 FORMAT ( $1 \mathrm{X}, 18 \mathrm{H} \$$ IVS $N 1 D=0$, NSTART $=, 16,8 \mathrm{H}, \mathrm{TSTART}=, \mathrm{F} 14,10,1 \mathrm{H}$, )

1630 FORMAT $(1 X .4 \mathrm{HU}(1, . \mathrm{I} 2,5 \mathrm{H}, 1)=, \mathrm{F} 10.3,1 \mathrm{H}$,

1640 FORMAT $($ IX, 3HUL $($, I $2,5 H, 1)=, F 10.3,1 \mathrm{H}$, )

1650 FORMAT $\left(\left(1 X, 7\left(F 10.3,1 H_{,}\right)\right)\right)$

1660 FORMAT $(1 X, 4 H V(1, I 2,5 H, 1)=, F 10.3,1 H$.

1670 FORMAT $(1 X, 3 \mathrm{HVL}(, \mathrm{I} 2,5 \mathrm{H}, \mathrm{i})=, \dot{F} 10.3, \mathrm{H}, \mathrm{H})$

1680 FORMAT $(1 X, 4 H P(1, \ldots I 2,5 H, 1)=. F 10.4,1 H$.

1690 FORMAT $(1 X, 3$ HPL $(, I 2,5 H, 1)=, F 10.4,1 \mathrm{H}$.

1700 FORMAT $((1 X, 7(F 10,4,1 \mathrm{H}))$,

1710 FORMAT $(1 X .5 H R O(1, \ldots, 5 H, 1)=, F 10.6,1 H$,

1720 FORMAT (1X.4HROL $(, 12,5 \mathrm{H}, 1)=. F 10.6,1 \mathrm{H}, \mathrm{)}$

1730 FORMAT $((1 X, 7(F 10.6,1 H))$.

1740 FORMAT $(1 X, 4 H Q(1, \ldots I 2,5 H, 1)=. E 10.4,1 H$.

1750 FORMAT $(1 X, 4 H E(1,12,5 H, 1)=. E 10.4,1 H$,

1760 FORMAT $\left(\left(, X, 7\left(E, 0.4,1 H_{,}\right)\right)\right)$

1770 FORMAT $(1 X, 3 \mathrm{HQL}(, 12,5 \mathrm{H}, 1)=, \mathrm{E} 10.4,1 \mathrm{H}$,

1780 FORMAT $(1 X, 3$ HEL $(, 12,5 H, 1)=, E 10.4,1 H$,

$1 \%$ FU FURMAT $(1 \mathrm{X}, 1 \mathrm{H} \$)$

1800 FORMAT ( 1 HO, 27H***** EXPECT APPROXIMATELY . I4.2OH PUNCHED CARDS ** 1***)

1810 FORMAT ( $1 \mathrm{HO}, 31 \mathrm{H*****}$ EXPECT FILM OUTPUT FOR $\mathrm{N}=, 16,6 \mathrm{H} * * * * *$ )

1820 FORMAT $(1 \mathrm{H}, 10 \mathrm{X}, 2 \mathrm{HN}=, 16,5 \mathrm{H}, \mathrm{T}=, \mathrm{F} 12.8,14 \mathrm{H}$ SECONDS, $D T=, F 10.8,8 \mathrm{H} \mathrm{S}$ 1ECONDS.9H. $\mathrm{NVCM}=.13 .10 \mathrm{H}$. CNUMS $=. \mathrm{F5} .2 .4 \mathrm{H},(.12,1 \mathrm{H}, .12 .5 \mathrm{H}),($ $2, I 2,1 H,(12,1 H))$

1830 FORMAT ( 1 HO, 10X, 2 1HARTIFICAL VISCOSITY - .//2 1X, 4HCAV = F 4.2.3X, 4HXM $1 U=, F 4.2,3 X, 4 H X L A=, F 4,2,3 X, 4 H P R A=, F 4,2,3 X, 4 H X R O=, F 4.2,3 X, 4 H L S S=, I 2$, $25 X, 4 H L S F=. I 3,3 X, 6 H I D I V C=, I 1,3 X, 4 H I S S=, I 1,3 X, 6 H S M A C H=, F 4.2,1,21 X, 4$ $3 \mathrm{HNST}=, 14,3 \mathrm{X}, 4 \mathrm{HSMP}=, \mathrm{F} 4,2,3 \mathrm{X}, 5 \mathrm{HSMPF}=, F 4,2,2 \mathrm{X}, 5 \mathrm{HSMPT}=, F 4,2,2 \mathrm{X}, 6 \mathrm{HSMPT}$ $4 F=, F 4,2,1 X, 5 H N T S T=. I 4,2 X, 4 H I A V=. I 1,5 X, 4 H M S S=. I 2,4 X, 4 H M S F=. I 3)$

1840 FUKMAT (1HO.20X.29HFREE-SLIP WALLS ARE SPECIFIED)

1850 FORMAT (1HO.20X.27HNO-SLIP WALLS ARE SPECIFIED)

1860 FORMAT ( $1 \mathrm{HO}, 10 \mathrm{X}, 2$ IHMOLECULAR VISCOSITY $-. / / 21 \mathrm{X}, 4 \mathrm{HCMU}=, \mathrm{E} 10.4,18 \mathrm{H}$ (L $1 B F-S / F T 2) \quad C L A=. E 11.4 .17 H(L B F-S / F T 2) \quad C K=. E 10.4,16 H(L B F / S-R) \quad E M$ $2 U=. F 4.2 .6 H \quad E L A=. F 4.2 .5 H \quad E K=. F .4 .2)$

1870 FORMAT ( 1HO. 1OX.21HMOLECULAR VISCOSITY $-. / / 21 \mathrm{X}, 4 \mathrm{HCMU}=. \mathrm{H} 10.4,13 \mathrm{H}$ (P) $1 \mathrm{~A}-\mathrm{S}) \quad C L A=, E 11.4 .12 \mathrm{H}(\mathrm{PA}-\mathrm{S}) \quad \mathrm{CK}=. \mathrm{E} 10.4,14 \mathrm{H}(\mathrm{W} / \mathrm{M}-\mathrm{K}) \quad \mathrm{EMU}=. \mathrm{F} 4.2,6 \mathrm{H}$ $2 E L A=, F 4.2,5 H \quad E K=, F 4.2)$

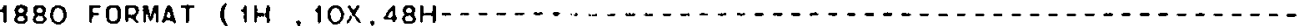
$1-.61 \mathrm{H}-\ldots$ $2.7 \mathrm{H}-\ldots . .-1)$

1890 FORMAT (1H.20X,33HADIABATIC UPPER WALL IS SPECIFIED) 


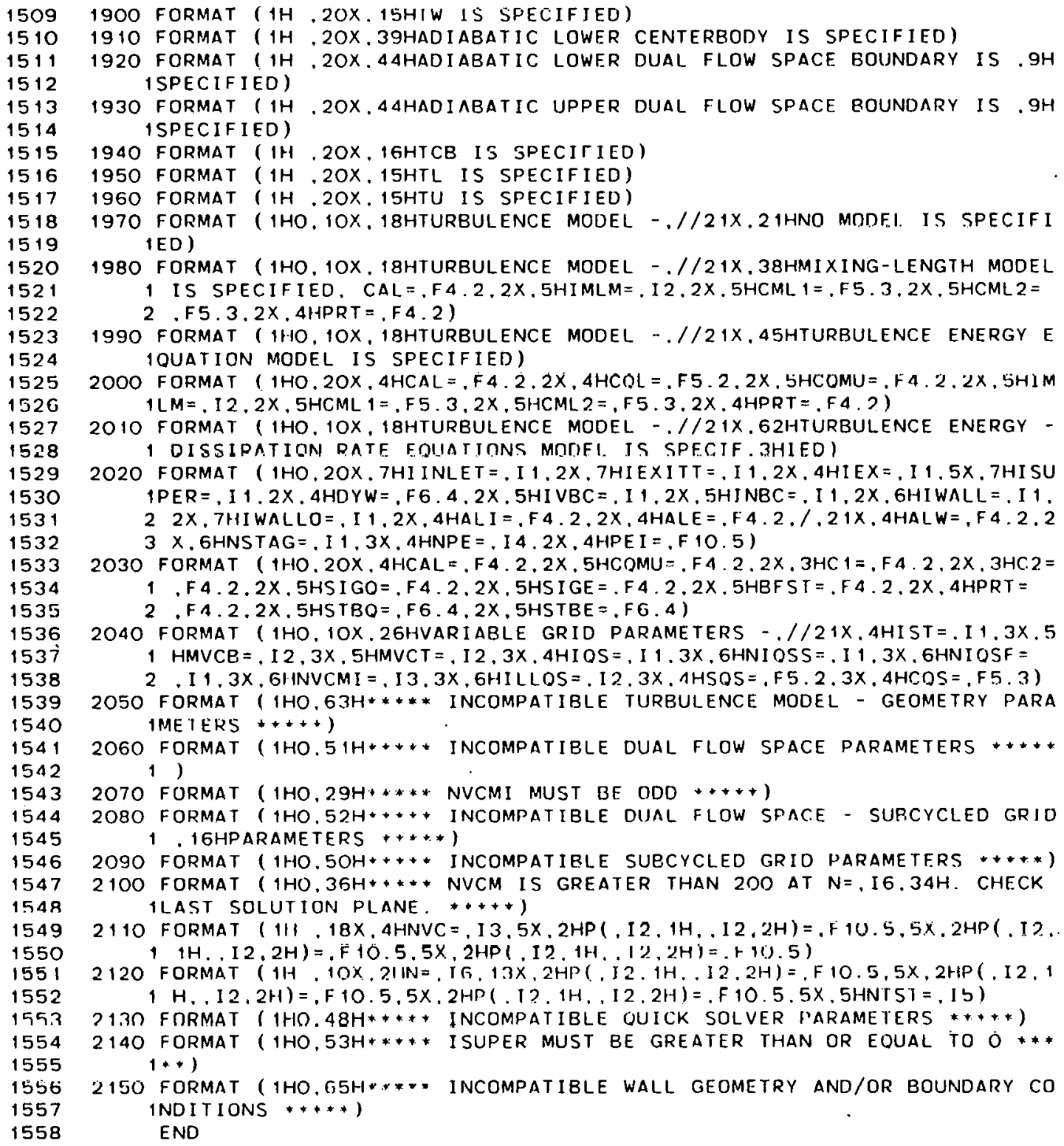




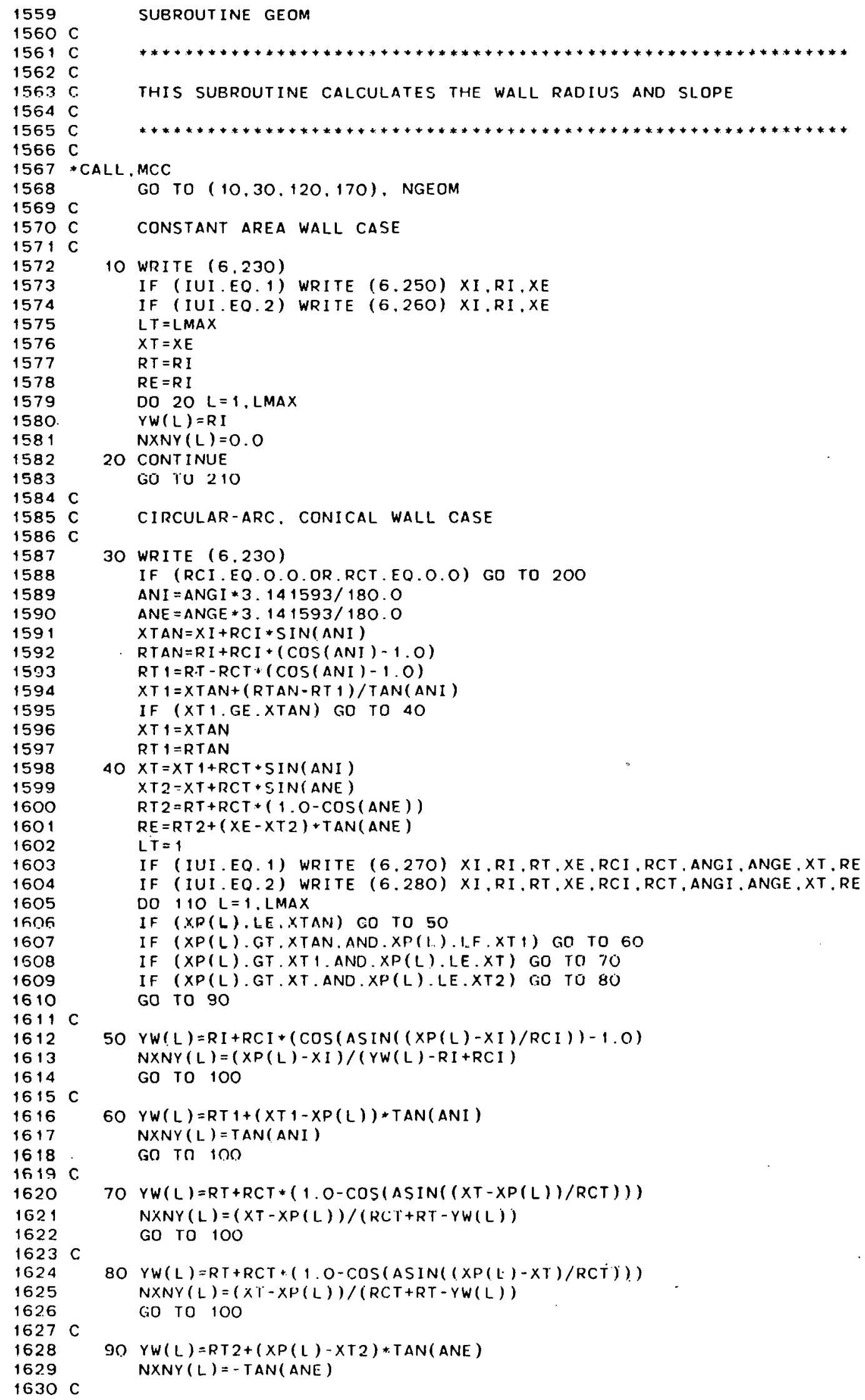




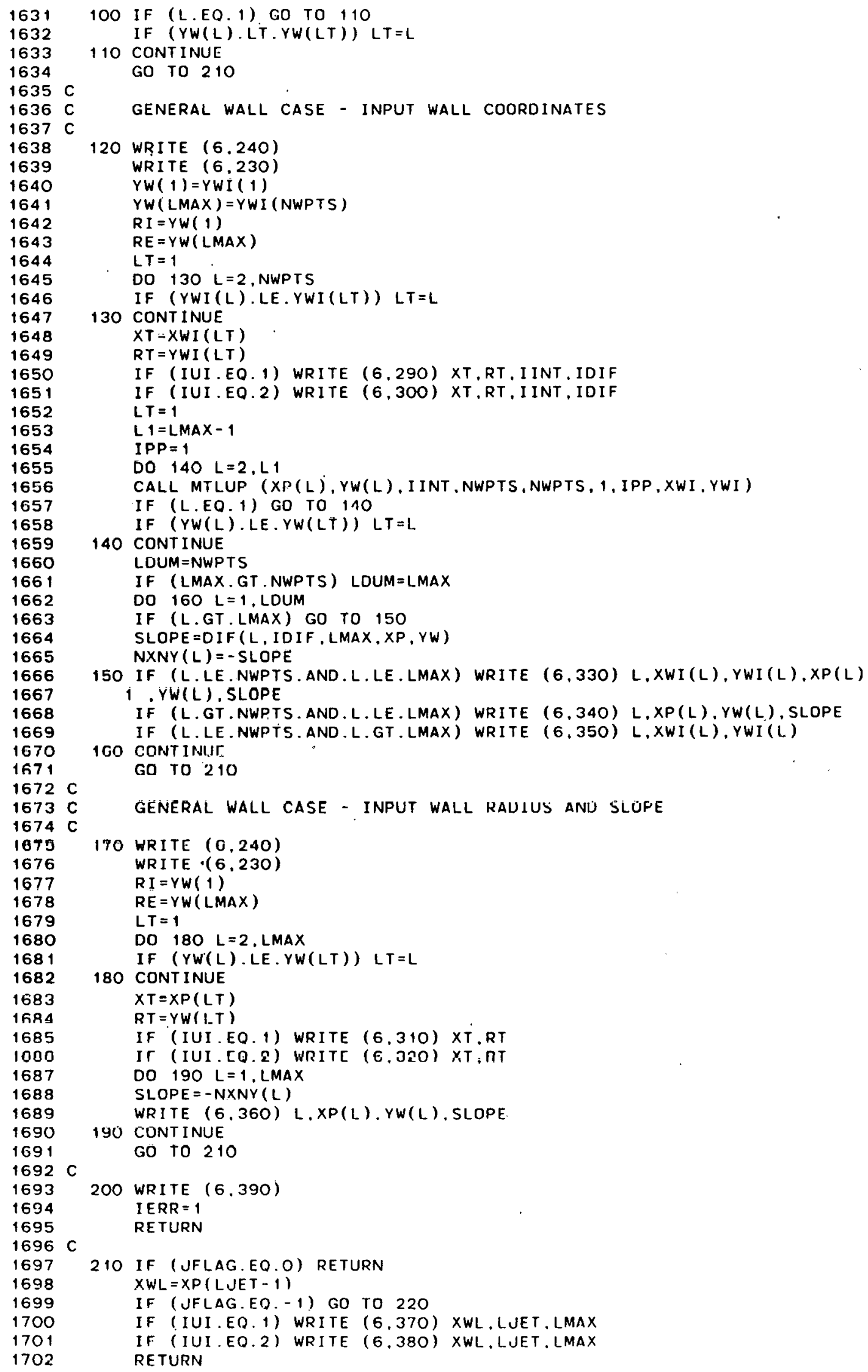


1703

1704

1705

$1706 \mathrm{C}$

$1707 \mathrm{C}$

$1708 \mathrm{C}$

1709

1710

1711

1712

1713

1714

1715

1716

1717

1718

1719

1720

1721

1722

1723

1724

1725

1726

1727

1728

1729

1730

1731

1732

1733

1734

1735

1736

1737

1738

1739

1740

1741

1742

1743

1744

1745

1746

1747

1748

1749

1750

1751

1752

1753

1754

1755

1756

220 IF (IUI.EQ.1) WRITE $(6.400) \times W L$ IF (IUI.EO.2) WRITE $(6.410) \times W L$ RE TURN

\section{FORMAT STATEMENTS}

230 FORMAT (1HO, 10X, 15HDUCT GEOMETRY - )

240 FORMAT (1H1)

250 FURMAT ( 1 HO, 2OX, 46HA CONSTANT AREA DUCT HAS BEEN SPECIFIED BY XI = $1 . F 8.4 .1 O H(I N)$, RI $=. F 8.4 .14 H(I N)$. AND $X E=. F 8.4 .5 H($ IN))

260 FORMAT (1HO.2OX, 46HA CONSTANT AREA DUCT HAS BEEN SPECIFIED BY XI = $1 . F 8.4 .10 \mathrm{H}(\mathrm{CM}), \mathrm{RI}=. \mathrm{F8} .4 .14 \mathrm{H}(\mathrm{CM}), A N D X E=. F 8.4 .5 \mathrm{H}(\mathrm{CM}))$

270 FORMAT ( 1 HO.2OX,56HA CIRCULAR-ARC. CONICAL NOZZLE HAS BEEN SPECIFI $1 E D$ BY $X I=, F 8.4 .10 H(I N), R I=, F 8.4 .6 H(I N), . /, 21 X, 3 H R T=, F 8,4.1 O H(I$ $2 N), X E=. F 8.4 .11 \mathrm{H}(\mathrm{IN}), \mathrm{RCI}=, \mathrm{FB} .4,1 \mathrm{HH}(\mathrm{IN}), \mathrm{RCT}=, \mathrm{FB} .4,12 \mathrm{H}$ (IN), $\triangle \mathrm{ING}$ $3 I=. F 6.2 .7 H(D E G) \ldots / .21 \times .9 H A N D$ ANGE $=. F 6.2 .35 H$ (DEG). THE COMPUTED $V$ \ALUES ARE $X T=, F 8.4 .13 \mathrm{H}$ (IN) AND RE=.F8.4.6H (IN).)

280 FORMAT ( 1 HO, 2OX.56HA CIRCULAR-ARC, CONICAL NOZZLE HAS BEEN SPECIFI $1 \mathrm{ED}$ BY $X I=, F 8.4 .10 \mathrm{H}(\mathrm{CM}), \mathrm{RI}=, F 8.4 .6 \mathrm{H}(\mathrm{CM}) . .1 .21 \times, 3 \mathrm{HRT}=, \mathrm{FB} .4 .1 \mathrm{OH}(\mathrm{C}$ 2M), $X E=. F 8.4,11 \mathrm{H}(\mathrm{CM}), \quad \mathrm{RCI}=. \mathrm{FB} .4,1 \mathrm{H}(\mathrm{CM}), \quad R C T=, F 8.4,12 \mathrm{H}(\mathrm{CM}), \quad A N G$ $3 I=. F 6.2 .7 H(D E G) \ldots / .21 X .9 H A N D$ ANGE $=. F 6.2 .35 H$ (DEG). THE COMPUTED $V$ $\triangle A L U E S$ ARE $X T=. F 8.4,13 \mathrm{H}(\mathrm{CM})$ AND RE $=. F 8.4,6 \mathrm{H}(\mathrm{CM})$.

290 FORMAT (1HO.20X.45HA GENERAL WALL HAS BEEN SPECIFIED BY THE FOLL. 2 1 1HOWING PARAMETERS, $X T=. F 8.4,1 \mathrm{HH}(\mathrm{IN}) . \mathrm{RT}=. \mathrm{F} 8.4,6 \mathrm{H}(\mathrm{IN}), .1,21 \mathrm{X}, \mathrm{SH}$ 2 I INT $=$ I $1,7 \mathrm{H}$, IDIF $=$, I $1,1 \mathrm{H}, 1 / 22 \mathrm{X}, 1 \mathrm{HL}, 10 \mathrm{X}, 7 \mathrm{HXWI}(\mathrm{IN}), 10 \mathrm{X}, 7 \mathrm{HYWI}(\mathrm{IN}), 11$ $3 \times, 6 \mathrm{HXP}(\mathrm{IN}), 11 \times, 6 \mathrm{HYW}(\mathrm{IN}), 12 \times, 5 \mathrm{HSLOPE}, /)$

300 FORMAT ( IHO, 20X, 45HA GENERAL WALL HAS BEEN SPECIFIED BY THE FOLL, 2 1 HHOWING PARAMETERS, $X T=. F 8.4 .10 \mathrm{H}(\mathrm{CM}) . \mathrm{RT}=. \mathrm{F} 8.4 .6 \mathrm{H}(\mathrm{CM}) \ldots .1 .21 \mathrm{X} .5 \mathrm{H}$ 2 I INT $=. I 1,7 \mathrm{H}$, IDIF $=, I 1,1 \mathrm{H}, . / / 22 \mathrm{X}, 1 \mathrm{HL}, 10 \mathrm{X}, 7 \mathrm{HXWI}(\mathrm{CM}), 10 \mathrm{X}, 7 \mathrm{HYWI}(\mathrm{CM}), 11$ $3 \times, 6 H X P(C M), 11 X, 6 H Y W(C M), 12 X, 5 H S L O P E . /)$

310 FORMAT ( IHO, 20X, 45HA GENERAL WALL HAS BEEN SPECIFIED BY THF FOLL, 2 1 IHOWING PARAMETERS, $X T=, F 8.4,1 O H(I N), R T=, F B .4,6 H(I N), / / 22 X, 1 H$ $2 L, 11 X, 6 \mathrm{HXP}(\mathrm{IN}), 11 \mathrm{X}, 6 \mathrm{HYW}(\mathrm{IN}), 12 \times, 5 \mathrm{HSLOPE}, /)$

320 FORMAT ( 1 HO, 2OX, 45HA GENERAL WALL HAS BEEN SPECIFIED BY THE FOLL. 2 1 HOWING PARAMETERS. $X T=, F 8.4 .1 \mathrm{OH}(\mathrm{CM}), \mathrm{RT}=, F 8.4,6 \mathrm{H}(\mathrm{CM}) \ldots / / 22 \mathrm{X}, 1 \mathrm{H}$ $2 \mathrm{~L}, 11 \mathrm{X}, 6 \mathrm{HXP}(\mathrm{CM}), \$ 1 \mathrm{X}, 6 \mathrm{HYW}(\mathrm{CM}), 12 \times, 5 \mathrm{HSLOPE} . /)$

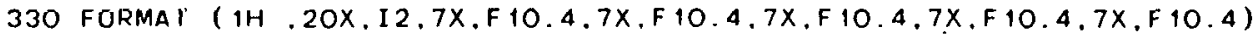

340 FORMAT ( $1 \mathrm{H}, 20 \mathrm{X} .12 .41 \times, F 10.4,7 \times, F 10.4 .7 \times, F 10.4)$

350 FORMAT ( $1 \mathrm{H}, 20 \mathrm{X}, \mathrm{I2}, 7 \mathrm{X}, \mathrm{F} 10.4,7 \mathrm{X}, \mathrm{F} 10.4$ )

360 FORMAT ( $1 \mathrm{H}, 20 \mathrm{X}, 12,7 \mathrm{X}, \mathrm{F} 10.4,7 \mathrm{X}, \mathrm{F} 10.4,7 \mathrm{X}, \mathrm{F} 10.4)$

370 FORMAT (1HO, 20X, 43HA FREE-JET CALCULATION HAS BEEN REOUESTED. $20 H$ 1 THE WALL ENDS AT $X=. F 8.4 .11 H$ (IN). THE./.21X.14HMESH PNINTS $L=$ 2 I $3,6 \mathrm{H}$ TO $\mathrm{L}=.13 .55 \mathrm{H}$ ARE AN INITIAL APPROXIMATION TO THE FREE-JET 3BOUNDARY.)

380 FORMAT ( THO, 2OX, 43HA FREE-JET CALCULATION HAS BEEN REQUESTED. $20 H$ 1 THE WALL ENDS AT $X=. F 8.4 .11 \mathrm{H}$ (CM). THE./.21X, 14HMESH POINTS $L=$ 2 I $3.6 H$ TO $L=.13 .55 H$ ARE AN INITIAL APPROXIMATION TO THE FREE-JET 3BOUNDARY .)

390 FORMAT ( $1 \% 0.44 H * * \ldots$ RCI OR RCT WAS SPECIFIEU AS $2 E R O * * * * *)$

4 OO FORMAT ( 1 HO, 2OX,54HTHE WALL CONTOUR HAS AN EXPANSION CORNER LOCATE 10 AT $X=, F 8.4,6 \mathrm{H}(\mathrm{IN})$.

410 FUKMAT ( THO, 2OX.54HTHE WALL CONTOUR HAS AN EXPANSION CORNER LOCATE 10 AT $X=, F 3.4 .6 \mathrm{H}(\mathrm{CM})$. END 


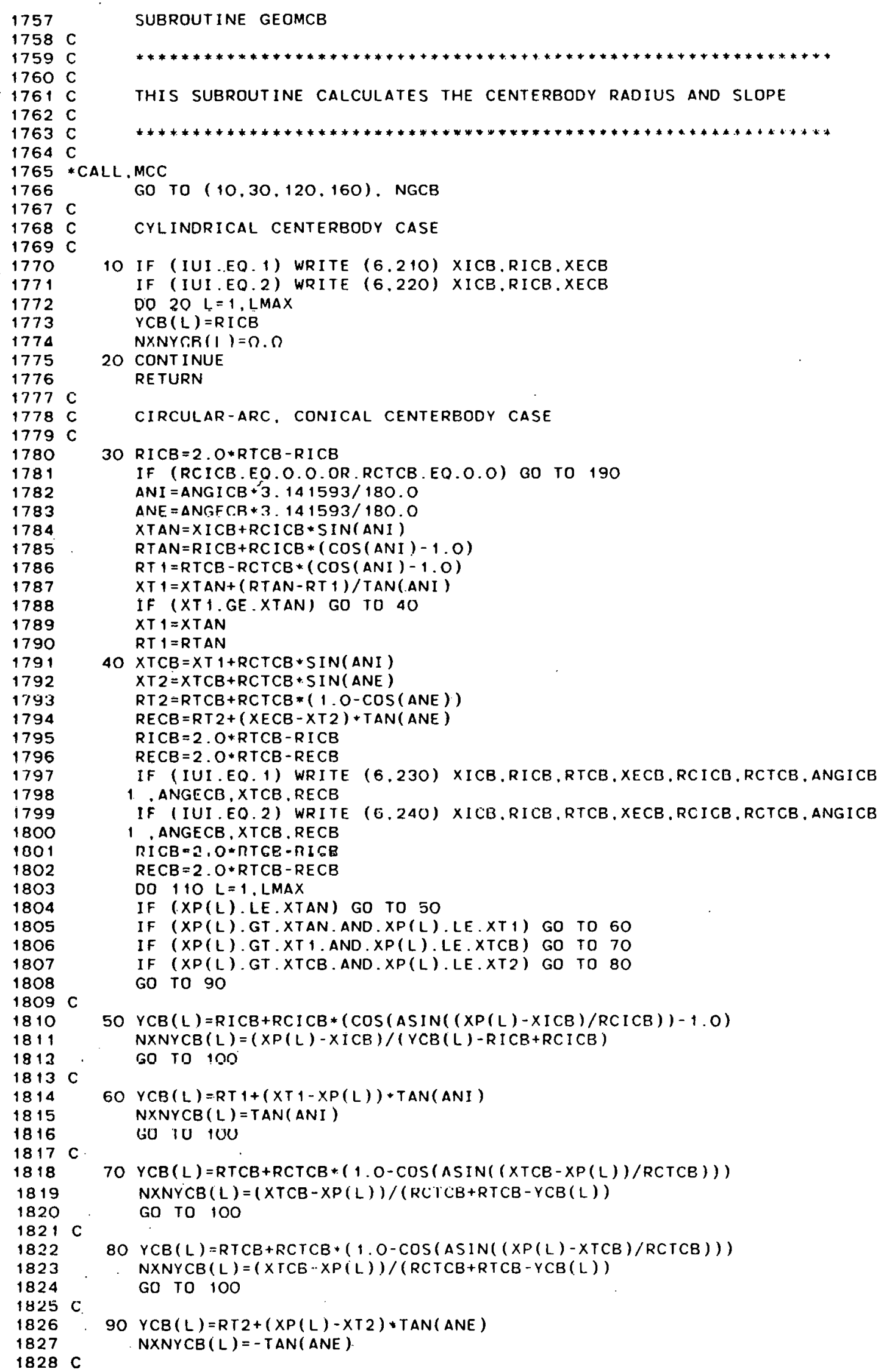

THIS SUBROUTINE CALCULATES THE CENTERBODY RADIUS AND SLOPE 


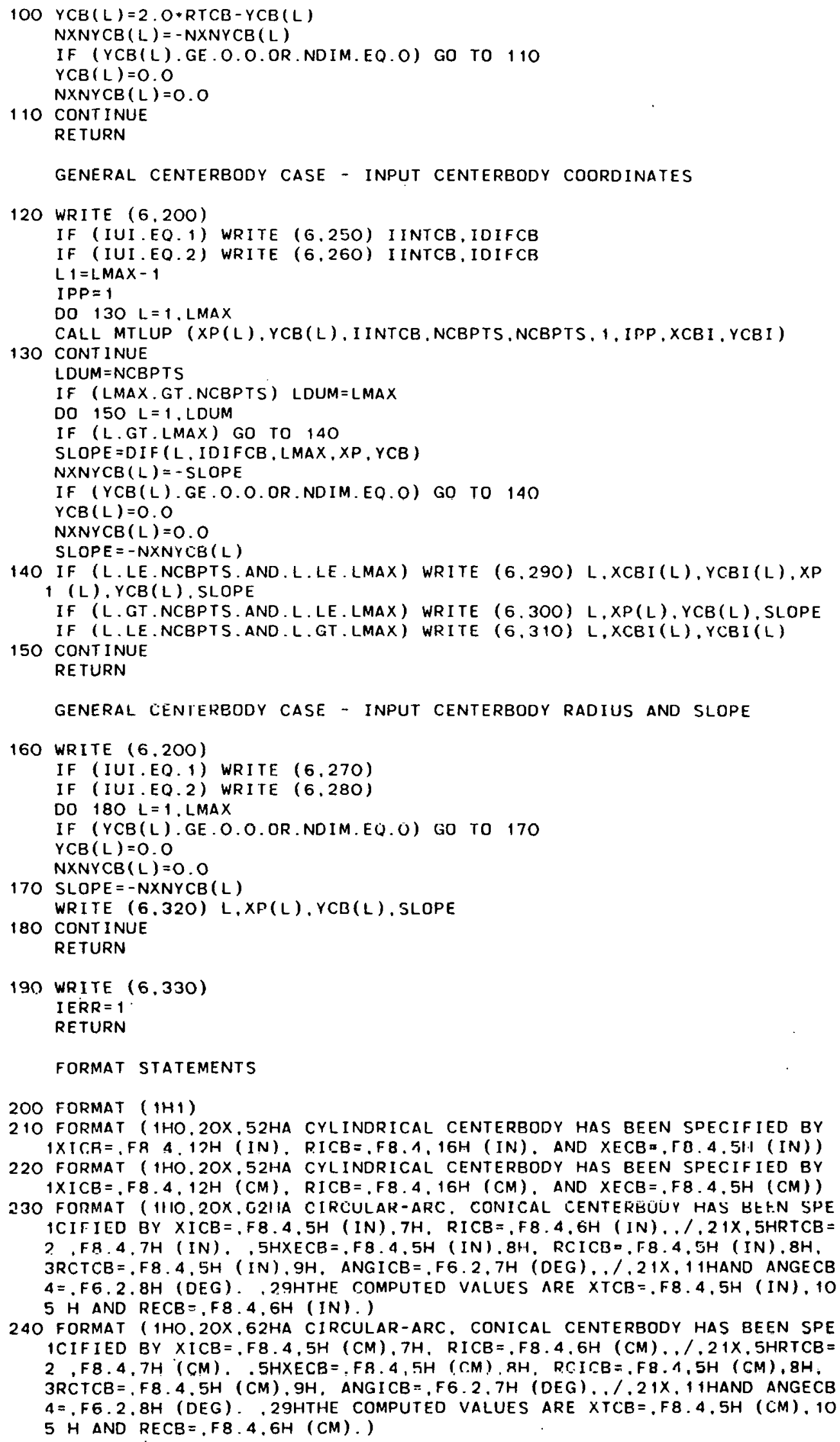
1CIFIED BY XICB =.F8.4,5H (CM),7H, RICB =.F8.4.6H (CM) ../.21X,5HRTCB= $2, F 8.4,7 H(C M)$. . $5 H X E C B=, F B .4,5 H(C M), 8 H, R C I C B=, F 8.4,5 H(C M), 8 H$, $3 R C T C B=. F 8.4 .5 H(C M), 9 H, \quad A N G I C B=, F 6.2,7 H(D E G), 1.21 \times, 11$ HAND ANGECB $4=. F 6.2 .8 H(D E G) . .29 H T H E$ COMPUTED VALUES ARE XTCB $=. F 8.4 .5 \mathrm{H}(\mathrm{CM}), 10$ 5 H AND RECB $=. F 8.4 .6 H(C M)$.) 
1901 1902 1903 1904

1905

1906

1907

1908

1909

1910

1914

1912

1913

1914

1915

1916

1917

1918

1919

1920

250 FORMAT (1HO, 20X,47HA GENERAL CENTERBODY HAS BEEN SPECIFIED BY THE $1.29 \mathrm{HFOLLOWING}$ PARAMETERS. IINTCB $=, 11,9 \mathrm{H}$. IDIFCB $=, 11,1 \mathrm{H}, . / / 22 \mathrm{X}, 1 \mathrm{HL}$ $2,10 X, 8 \mathrm{HXCBI}$ (IN), 10X,8HYCBI (IN), 10X,6HXP(IN), 10X,7HYCB(IN), 11X,5HS $3 L O P E . / 1)$

260 FORMAT (1HO, 20X.47HA GENERAL CENTERBODY HAS BEEN SPECIFIED BY THE 1 .29HFOLLOWING PARAMETERS. I INTCB $=, 11,9 \mathrm{H}$, IDIFCB $=, 11,1 \mathrm{H}, 1 / 22 \mathrm{X}, 1 \mathrm{HL}$ 2 . 10X, 8HXCBI (CM), 10X,8HYCBI (CM), 10X,6HXP(CM), 10X,7HYCB (CM), 11X, 5HS 3 LOPE . /)

270 FORMAT ( IHO. 2OX.47HA GENERAL CENTERBODY HAS BEEN SPECIFIED BY THE 1,2 1HFOLLOWING PARAMETERS.,//22X, 1HI., 12X,6HXP(IN). 10X, 7HYCB(IN), 11 $2 \times$.5HSLOPE. /)

280 FORMAT (1HO, 20X, 47HA GENERAL CENTERBODY HAS BEEN SPECIFIED BY THE 1,2 1 HFOLLOWING PARAMETERS $, / / 22 \mathrm{X}, 1 \mathrm{HL}, 12 \mathrm{X}, 6 \mathrm{HXP}(\mathrm{CM}), 10 \mathrm{X}, 7 \mathrm{HYCB}(\mathrm{CM}), 11$ $2 \times$.5HSLOPE. /)

290 FORMAT (1H .20X.I2,7X,F 10,4,7X,F10.4,7X,F10.4,7X,F 10.4,7X,F 10,4)

300 FORMAT ( $1 \mathrm{H}, 20 X .12 .41 X, F 10.4,7 X, F 10.4 .7 X, F 10.4)$

310 FORMAT (1H, 20X, I2,7X,F 10,4,7X, 10.4$)$

320 FORMAT ( $1 \mathrm{H}, 20 \mathrm{X}, \mathrm{I} 2,7 \mathrm{X}, \mathrm{F} 10,4,7 \mathrm{X}, \mathrm{F} 10.4,7 \mathrm{X}, \mathrm{F} 10.4)$

330 FORMAT ( $1 \mathrm{HO}, 48 \mathrm{H} * * * *$ RCICB OR RCTCB WAS SPECIFIED AS ZERO ****) END 


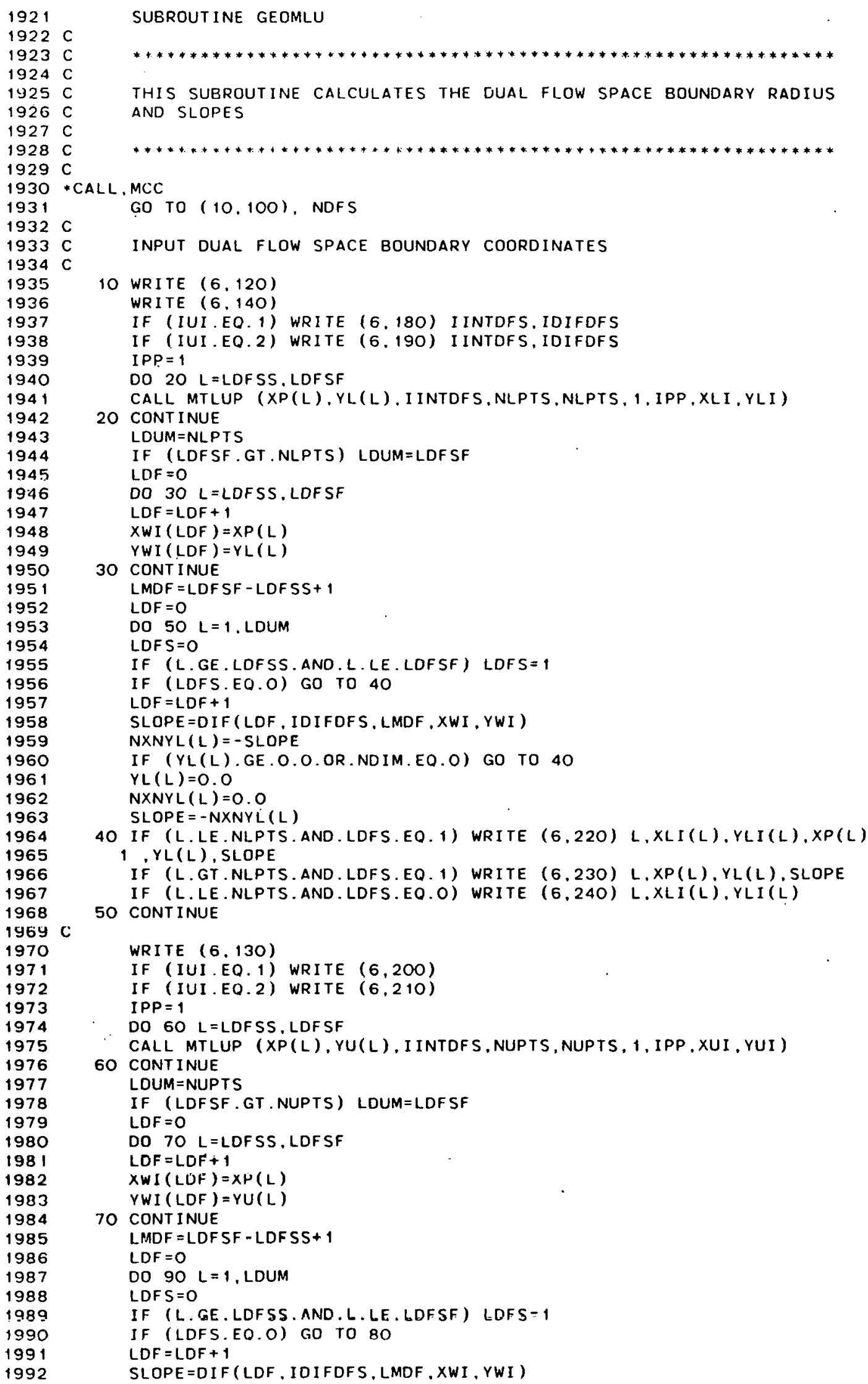

THIS SUBROUTINE CALCULATES THE DUAL FLOW SPACE BOUNDARY RADIUS AND SLOPES 


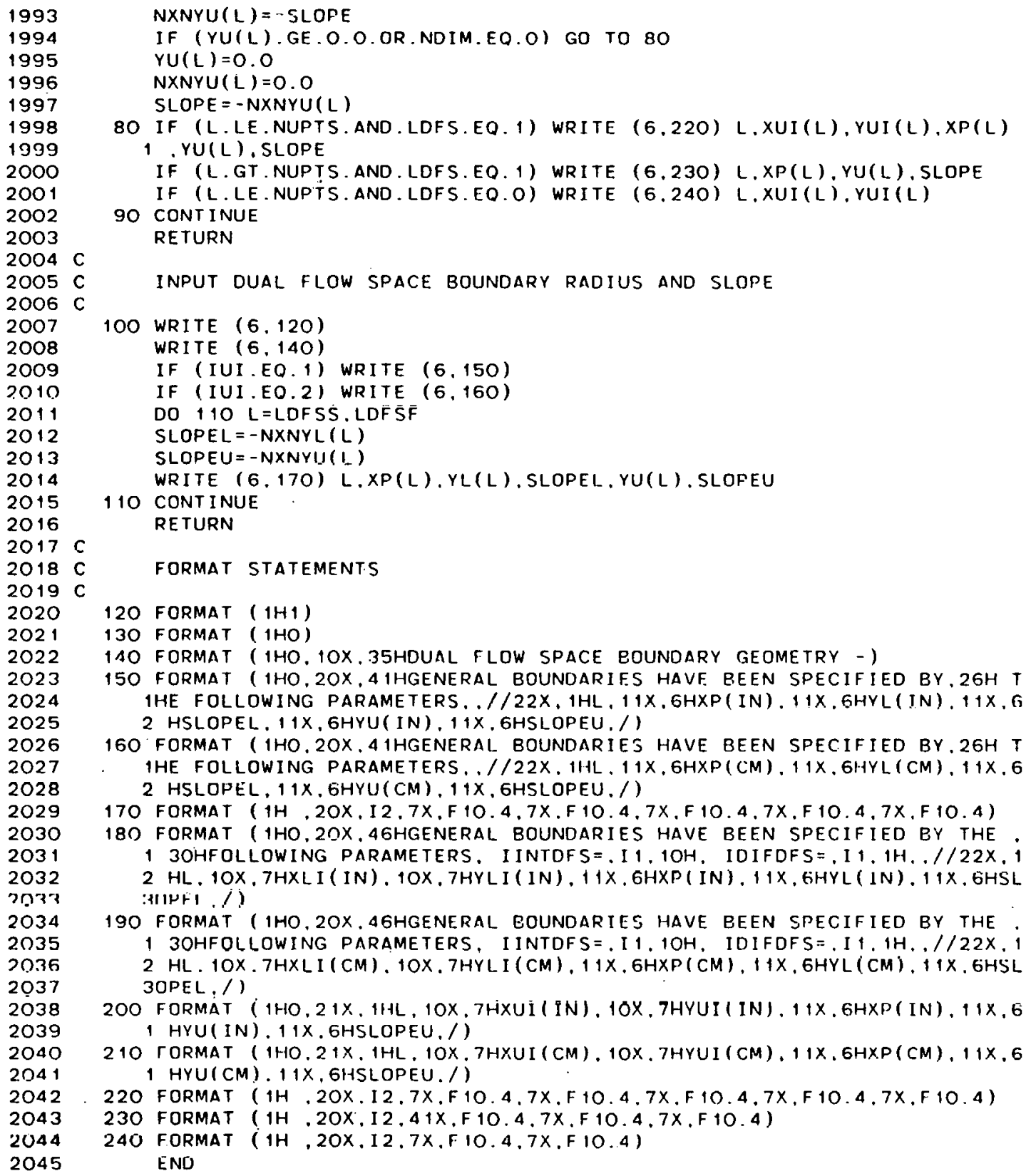

NXNYU(L) $=-$ SLOPE

IF (YU(L).GE.O.O.OR. NDIM.EO.O) GO TO 80

$Y U(L)=0.0$

NXNYU (L) $=0.0$

SLOPE $=-N X N Y U(L)$

80 IF (L.LE.NUPTS. AND.LDFS.EO.1) WRITE (6.220) L.XUI(L), YUI(L),XP(L) 1 . YU(L), SLOPE

IF (L.GT.NUPTS. AND. LOFS.EQ.1) WRITE $(6,230) \mathrm{L}, X P(L), Y U(L)$, SLOPE

IF (L.LE.NUPTS. AND. LDFS.EQ.O) WRITE $(6,240)$ L.XUI (L), YUI(L)

90 CONT INUE

RETURN

100 WRITE $(6,120)$

WRITE $(6,140)$

IF (IUI.EQ.1) WRITE $(6,150)$

IF (IUI.EO.2) WRITE $(6,160)$

DO $110 L=L D F S \dot{S}$. LDF $\bar{S} \bar{F}$

SLOPEL $=-$ NXNYL (L)

SLOPEU $=-N X N Y U(1)$

WRITE $(6,170)$ L.XP(L). YL(L), SLOPEL.YU(L). SLOFEU

110 CONTINUE

RETURN

120 FORMAT (1H1)

130 FORMAT (1HO)

†AO FORMAT ( 1 HO, TOX. 35HDUAL FLOW SPACE BOUNDARY GEOMETRY - )

150 FORMAT ( 1 HO, 2OX, 41 HGENERAL BOUNDARIFS HAVF BEEN SPECIFIED BY, 26H T 1HE FOLLOWING PARAMETERS, . $/ 122 \mathrm{X}, 1 \mathrm{HL}, 11 \mathrm{X}, 6 \mathrm{HXP}(\mathrm{IN}), 11 \mathrm{X}, 6 \mathrm{HYL}(\mathrm{IN}), 1+\mathrm{X}, 6$ 2 HSLOPEL, $11 \mathrm{X}, 6 \mathrm{HYU}(\mathrm{IN}), 14 \mathrm{X}, 6 \mathrm{HSLOPEU}, /)$

160 FORMAT ( IHO.2OX.41HGENERAL BOUNDARIES HAVE BEEN SPECIFIED BY.26H T 1HE FOLLOWING PARAMETERS../122X.1HL.11X.6HXP(CM $, 11 \times, 6 H Y L(C M), 11 X, 6$ 2 HSLOPEL, 11X, 6HYU(CM), $11 \times, 6 H S L O P E U . /)$

170 FORMAT (1H, 2OX.I2.7X,F10.4.7X.F10.4.7X.F 10.4.7X.F 10.4.7X.F10.4)

180 FORMAT (1HO,20X,46HGENERAL BOUNDARIES HAVE BEEN SPECIFIED BY THE 130 HFOLLOWING PARAMETERS. IINTDFS $=.11,10 \mathrm{H}$, IDIFDFS $=.11,1 \mathrm{H}, 1 / 22 \times, 1$ $2 \mathrm{HL} .10 X .7 \mathrm{HXLI}(\mathrm{IN}) .10 \mathrm{X} .7 \mathrm{HYLI}(\mathrm{IN}), 11 \mathrm{X} .6 \mathrm{HXP}(\mathrm{IN}) .11 \mathrm{X}, 6 \mathrm{HYL}(1 \mathrm{~N}) .11 X .6 \mathrm{HSL}$ :IIIHFI., I)

190 FORMAT ( 1HO, 20X.46HGENERAL EOUNDARIES HAVE BEEN SPECIFIED BY THE 13 OHFOLLOWING PARAMETERS, I INTDFS $=.11,10 \mathrm{H}$, IDIFDFS $=. \mathrm{I} 1.1 \mathrm{H}, 1 / 22 \times, 1$ $2 \mathrm{HL}$. 1OX.7HXLI (CM). 1OX.7HYLI (CM), 11X,6HXP(CM), 11X,6HYL (CM), 11X.6HSL 3OPEL : /)

200 FORMAT ( 1 HO, $21 \times, 1 H \mathrm{~L}, 10 \mathrm{X}, 7 \mathrm{HXXI}(\mathrm{IN}), 10 \mathrm{X}, 7 \mathrm{HYUI}(\mathrm{IN}), 1 \mathrm{IX}, 6 \mathrm{HXP}(\mathrm{IN}), 1 \mathrm{X}, 6$ 1 HYU(IN). $11 \times$, GHSLOPEU./)

210 TORMAT (1HO.21X.1HL. 10X.7HXUI (CM).1OX.7HYUI (CM), 1 IX.6HX.P(CM), 11X.6 1 HYU (CM). $11 \times$, GHSLOPEU./)

220 FORMAT (1H,.20X.I2.7X.F10.4.7X.F10.4.7X.F10.4.7X,F10.4.7X.F10.4)

230 FORMAT (1H, 2OX,I2,41X,F10.4.7X,F 10.4,7X,F 10.4)

240 FORMAT (1H, 20X.12.7X,F 10.4.7X.F 10.4) END 


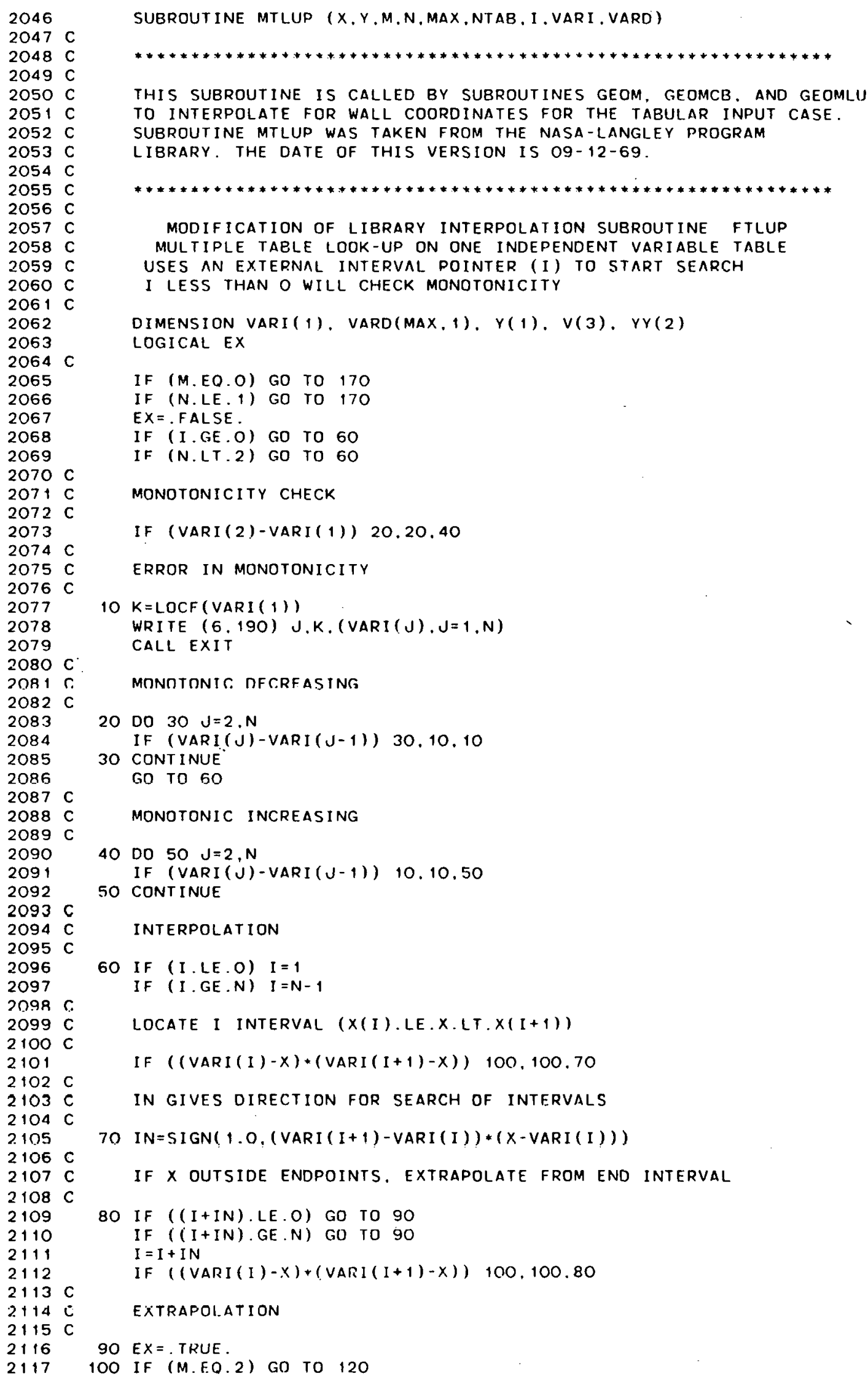

SUBROUTINE MTLUP (X,Y,M,N,MAX,NTAB, I , VARI , VARD)

THIS SUBROUTINE IS CALLED BY SUBROUTINES GEOM, GEOMCB. AND GEOMLU TO INTERPOLATE FOR WALL COORDINATES FOR THE TABULAR INPUT CASE. SUBROUTINE MTLUP WAS TAKEN FROM THE NASA-LANGLEY PROGRAM LIBRARY. THE DATE OF THIS VERSION IS 09-12-69.

MODIFICATION OF LIBRARY INTERPOLATION SUBROUTINE FTLUP MULTIPLE TABLE LOOK-UP ON ONE INDEPENDENT VARIABLE TABLE USES AN EXTERNAL INTERVAL POINTER (I) TO START SEARCH

I LESS THAN O WILL CHECK MONOTONICITY

DIMENSION VARI (1), VARD $(\operatorname{MAX}, 1), Y(1), V(3), Y Y(2)$ LOGICAL EX

IF (M.EQ.O) GO TO 170

IF (N.LE.1) GO TO 170

$E X=. F A L S E$.

IF (I.GE.O) GO TO 60

IF (N.LT.2) GO TO 60

MONOTONICITY CHECK

IF (VARI (2)-VARI (1)) 20.20 .40

ERROR IN MONOTONICITY

$10 K=\operatorname{LOCF}(\operatorname{VARI}(1))$

WRITE $(6,190)$ J.K. (VARI $(J), J=1, N)$

CALL EXIT

MONOTONIS. DFCRFASING

20 DO $30 \mathrm{~J}=2 . \mathrm{N}$

IF (VARI $(J)$-VARI $(J-1)) 30,10,10$

30 CONTINUE

GO TO 60

MONOTONIC INCREASING

40 DO $50 \quad J=2, N$

IF (VARI $(U)$-VARI $(u-1)) \quad 10,10,50$

SO CONTINUE

INTERPOLATION

60 IF (I.LE.O) I $=1$

If $(I, G E, N) I=N-1$

LOCATE I INTERVAL $(X(I)$.LE.X.LT.X $(I+1))$

IF $((\operatorname{VARI}(I)-X) *(\operatorname{VARI}(I+1)-X)) \quad 100,100.70$

IN GIVES DIRECTION FOR SEARCH OF INTERVALS

$70 \operatorname{IN}=S I G N(1.0,(\operatorname{VARI}(I+1)-\operatorname{VARI}(I)) *(X-\operatorname{VARI}(I)))$

IF $X$ OUTSIDE ENDPOINTS. EXTRAPOLATE FROM END INTERVAL

80 IF $((I+I N)$.LE.O $)$ GO TO 90

IF $($ (I+IN).GE.N) GO TO 90

$I=I+I N$

IF $((\operatorname{VARI}(I)-X)+(\operatorname{VAR} I(I+1)-X)) 100,100.80$

EXTRAPOI.ATION

gO EX =.TRUE.

100 IF (M.F.O.2) GO TO 120 


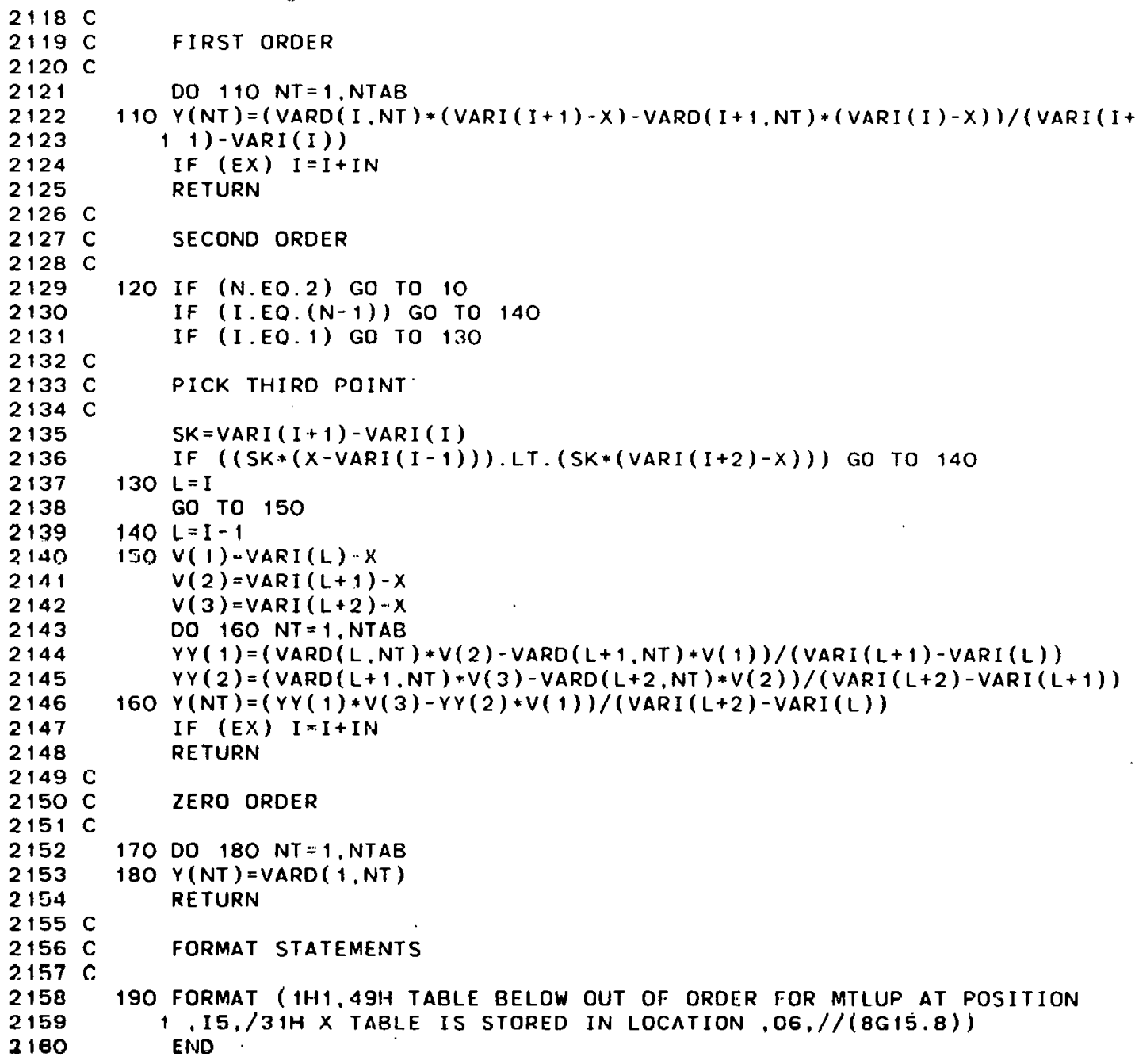




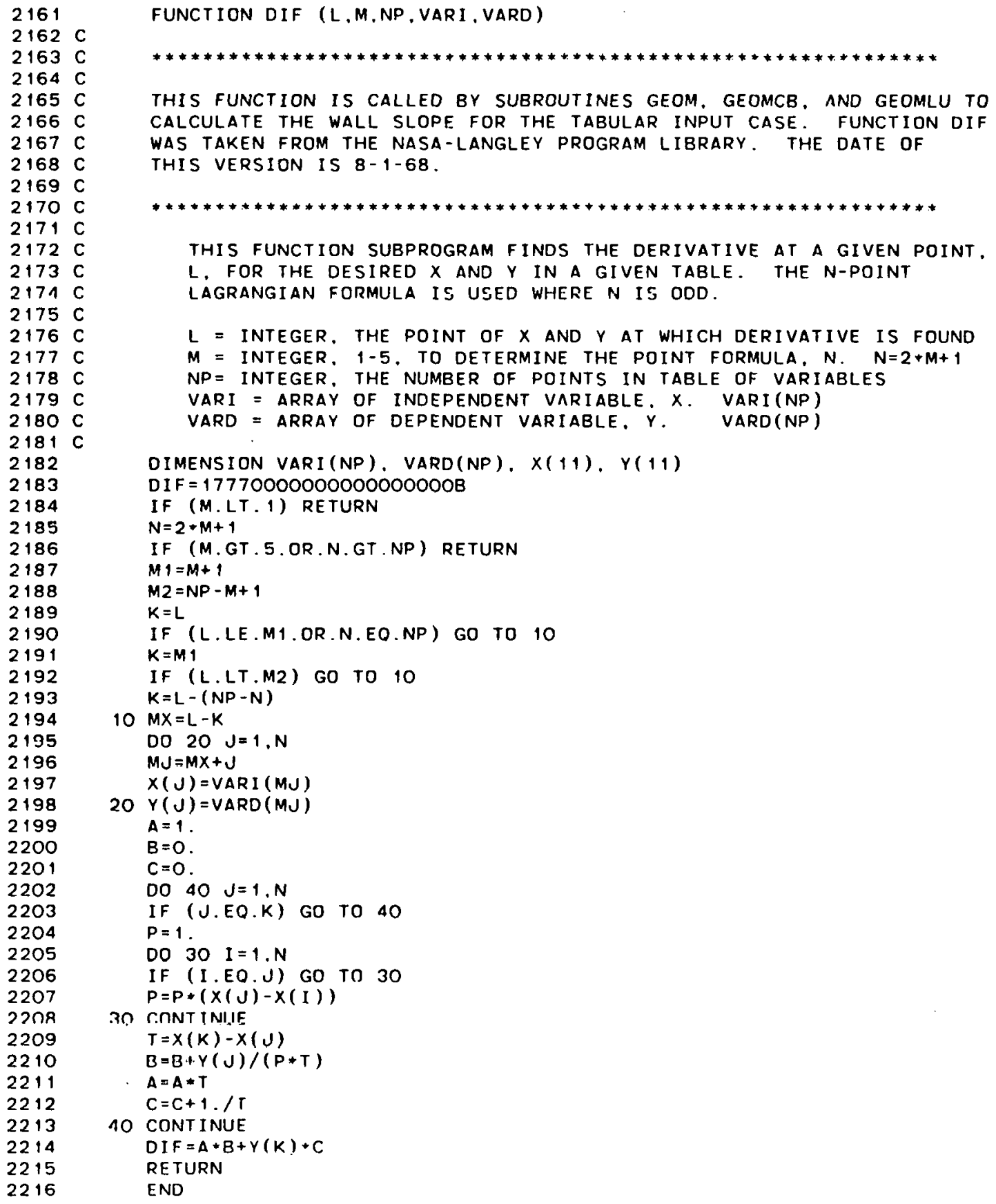

THIS FUNCTION IS CALLED BY SUBROUTINES GEOM, GEOMCB, AND GEOMLU TO CALCULATE THE WALL SLOPE FOR THE TABULAR INPUT CASE. FUNCTION DIF WAS TAKEN FROM THE NASA-LANGLEY PROGRAM LIBRARY. THE DATE OF THIS VERSION IS 8-1-68.

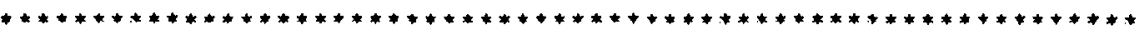

THIS FUNCTION SUBPROgRAM FINDS THE DERIVATIVE AT A GIVEN POINT. L, FOR THE DESIRED $X$ AND Y IN A GIVEN TABLE. THE N-POINT LAGRANGIAN FORMULA IS USED WHERE $N$ IS ODD.

$L=$ INTEGER, THE POINT OF $X$ AND $Y$ AT WHICH DERIVATIVE IS FOUND

$M=$ INTEGER, 1-5, TO DETERMINE THE POINT FORMULA, $N$. $N=2+M+1$

NP = INTEGER, THE NUMBER OF POINTS IN TABLE OF VARIABLES

VARI = ARRAY OF INDEPENDENT VARIABLE. $X$. VARI (NP)

VARD = ARRAY OF DEPENDENT VARIABLE. $Y$. VARD(NP)

DIMENSION VARI (NP), VARD(NP), $X(11), Y(11)$ DIF $=17770000000000000000 B$

IF (M.LT.1) RETURN

$N=2 * M+1$

IF (M.GT.5.OR.N.GT.NP) RETURN

$M 1=M+1$

$M 2=N P-M+1$

$K=L$

IF (L.LE.M1.OR.N.EQ.NP) GO TO 10

$K=M 1$

IF (L.LT,M2) GO TO 10

$K=L-(N P-N)$

$10 M X=L-K$

DO $20 J=1, N$

$M J=M X+J$

$X(J)=V A R I(M J)$

$20 \mathrm{Y}(\mathrm{J})=\operatorname{VARD}(\mathrm{MJ})$

$A=1$.

$B=0$.

$C=0$.

DO $40 \quad J=1, N$

IF (U.EQ.K) Go To 40

$P=1$.

DO $30 I=1 . N$

IF (I.EO.J) GO TO 30

$P=P *(X(J)-X(I))$

3ח R.NNT INITE

$T=X(K)-X(U)$

$B=B+Y(J) /(P+T)$

$A=A * T$

$C=C+1 . / T$

$1 O$ CONT INUE

$D$ IF $=A * B+Y(K) \cdot C$

RETURN

END 


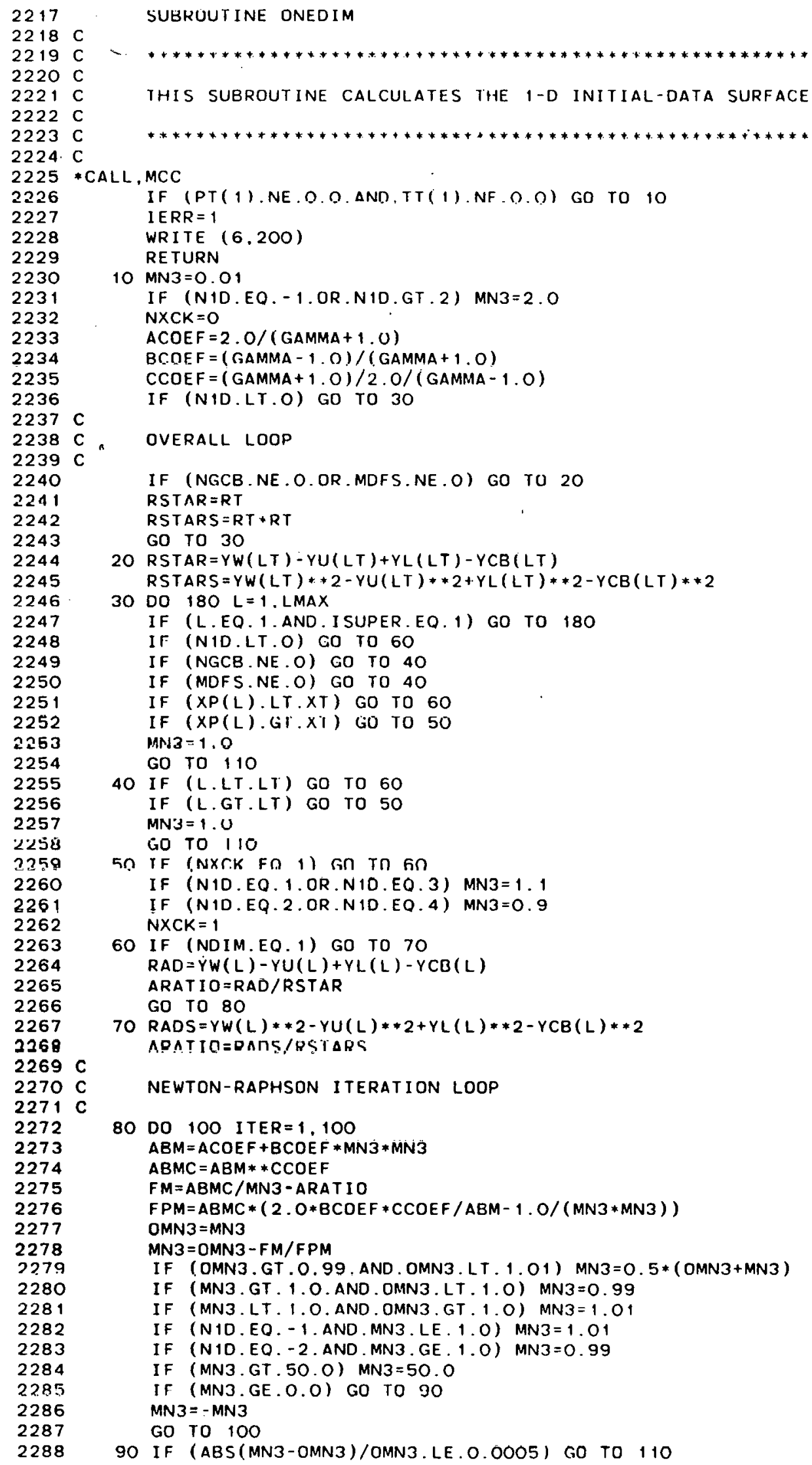




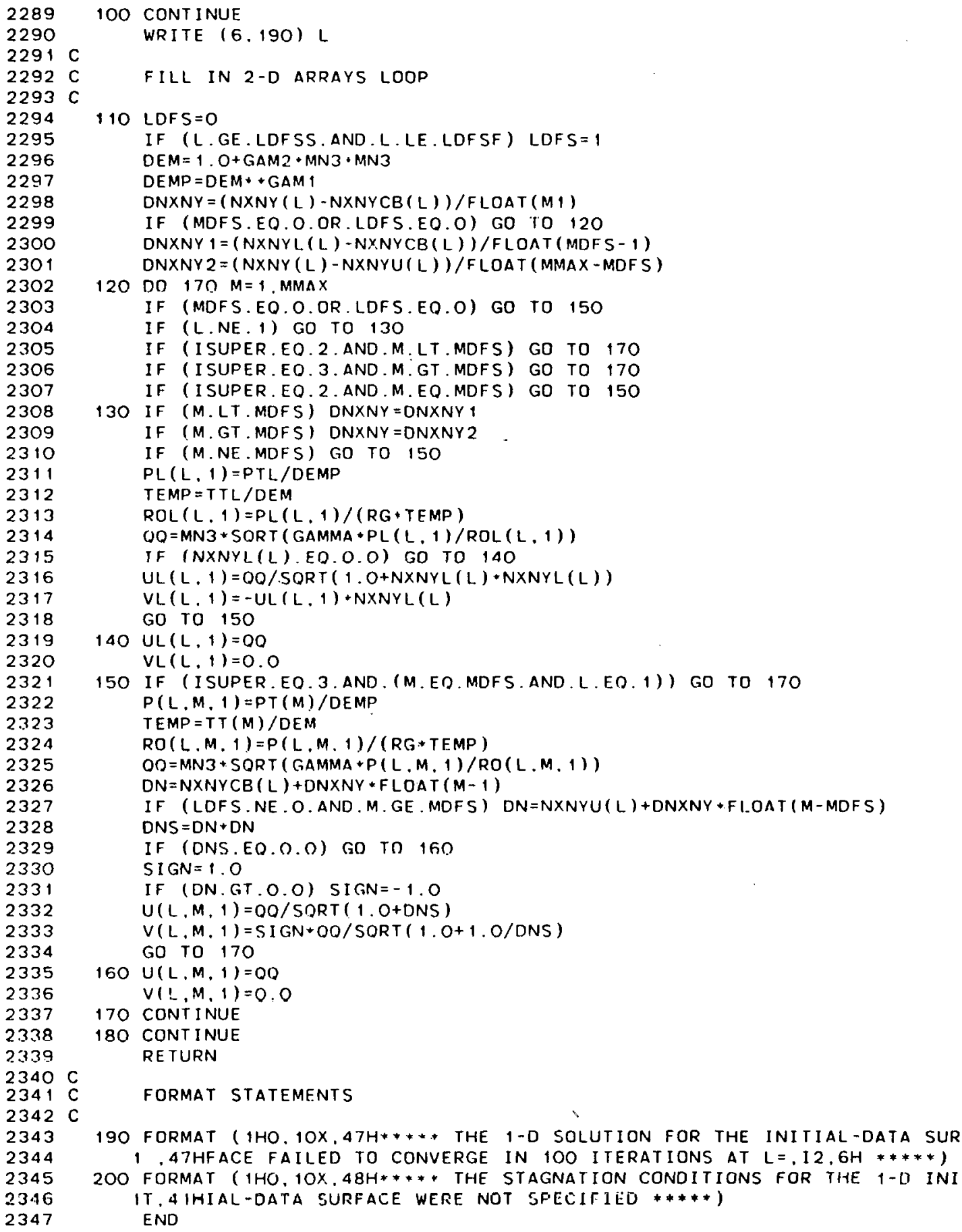




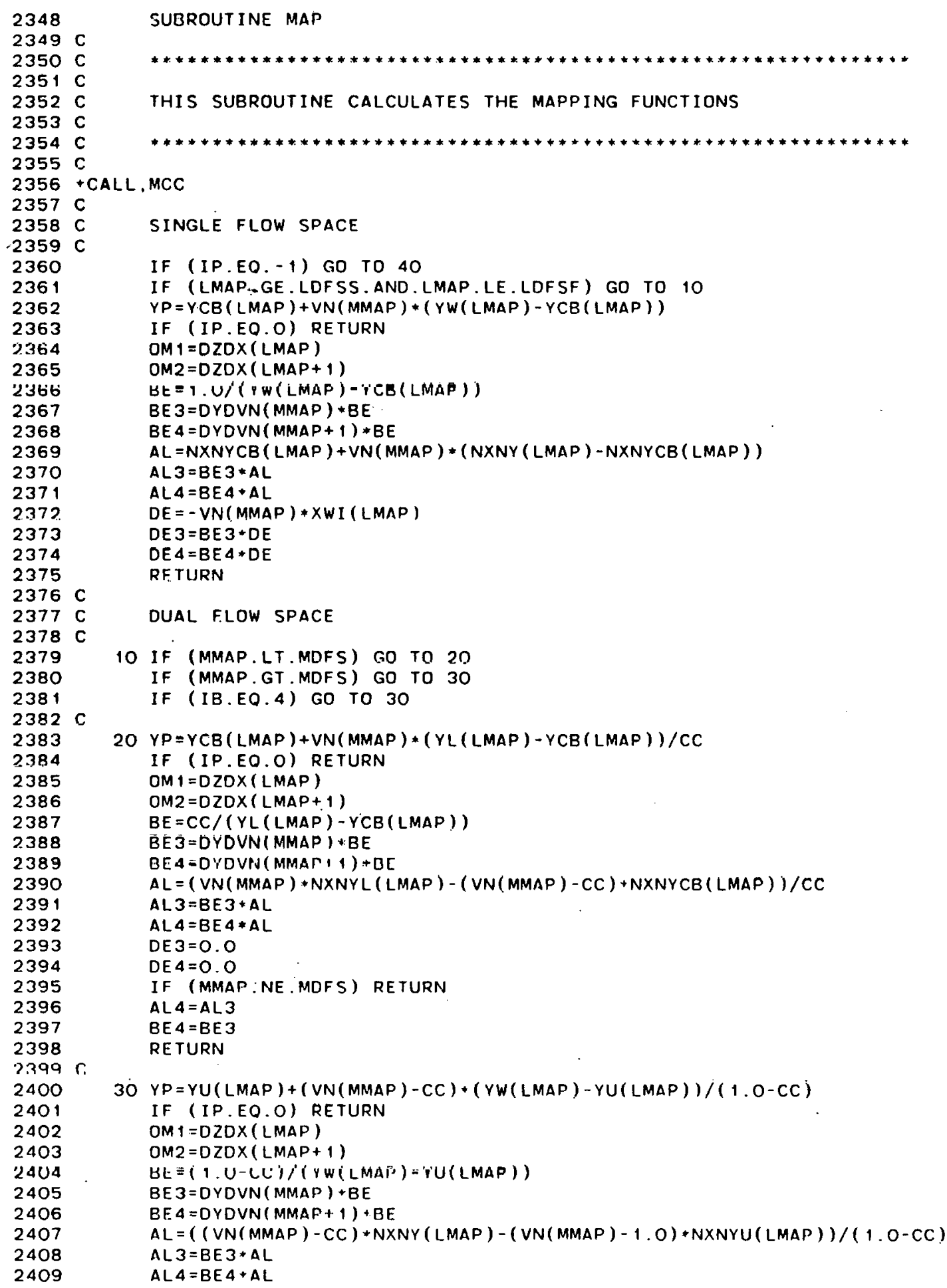




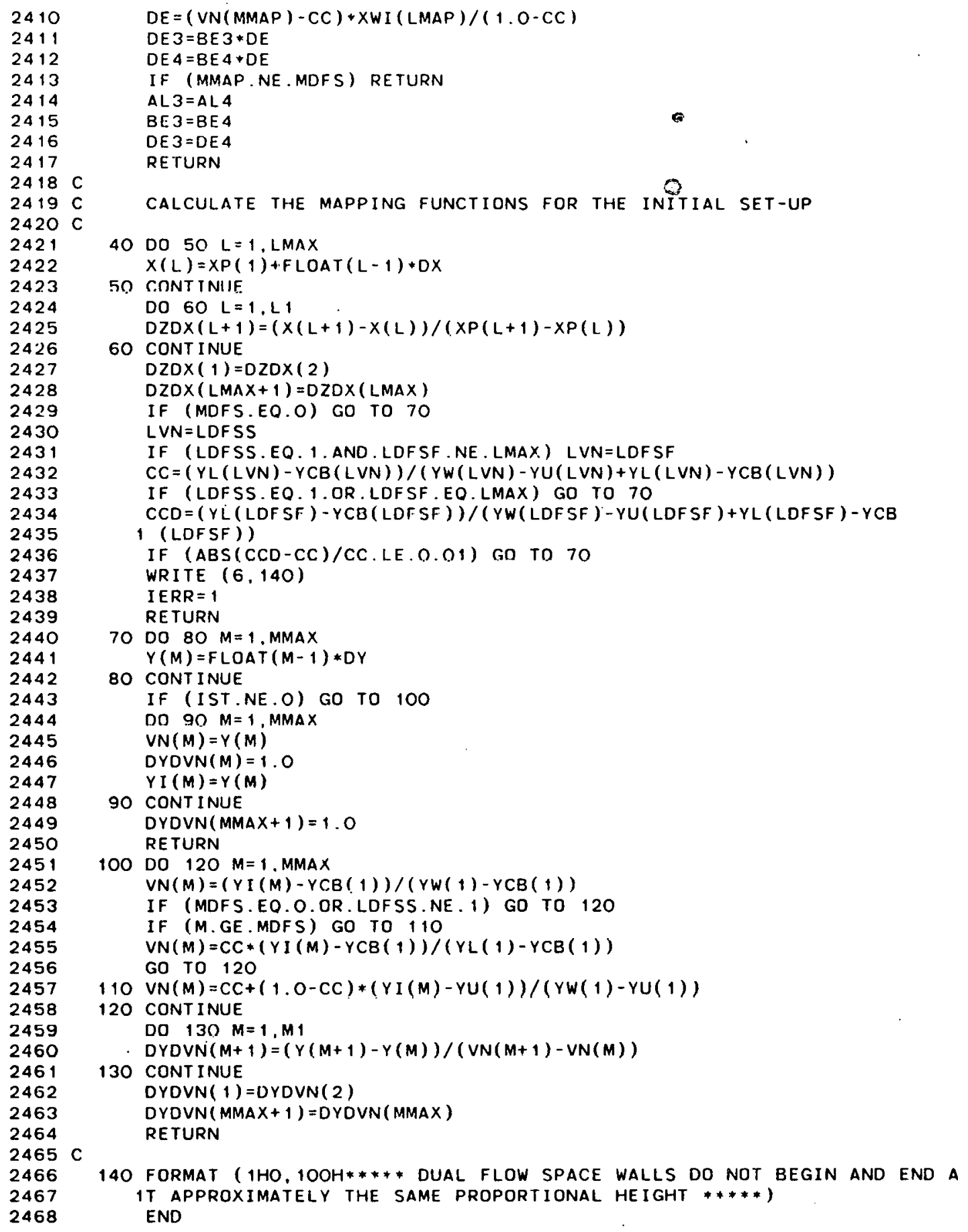




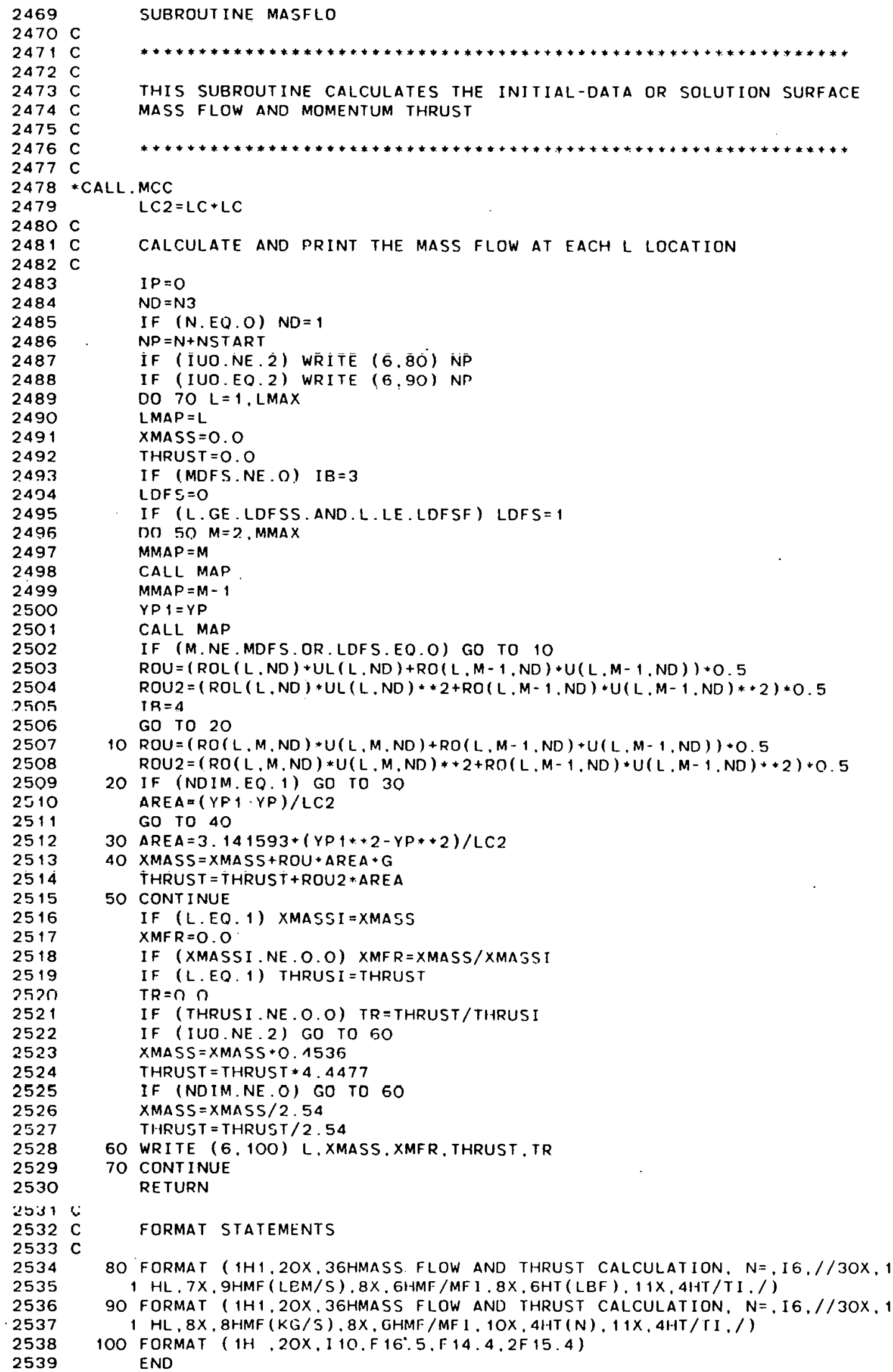




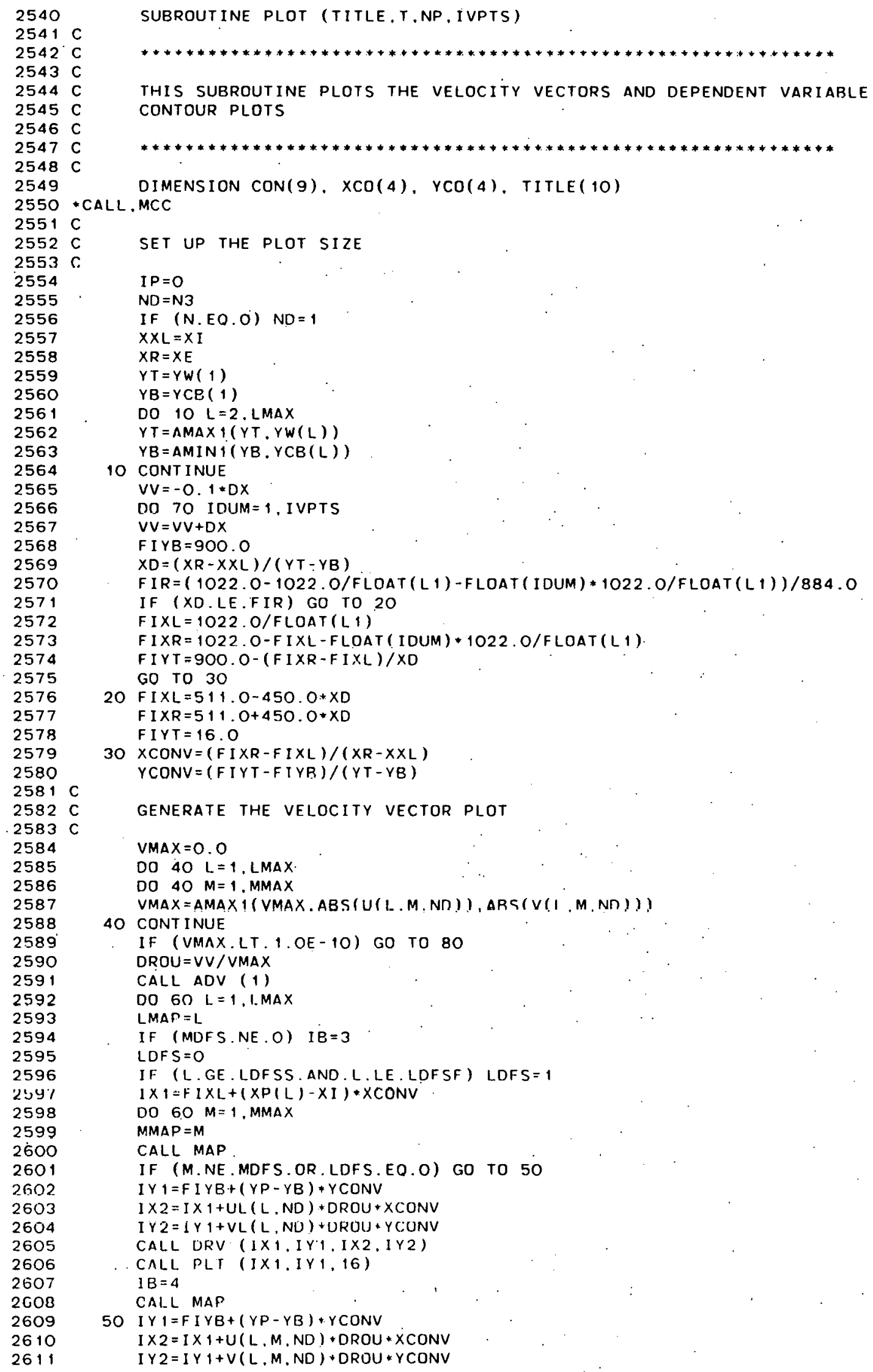




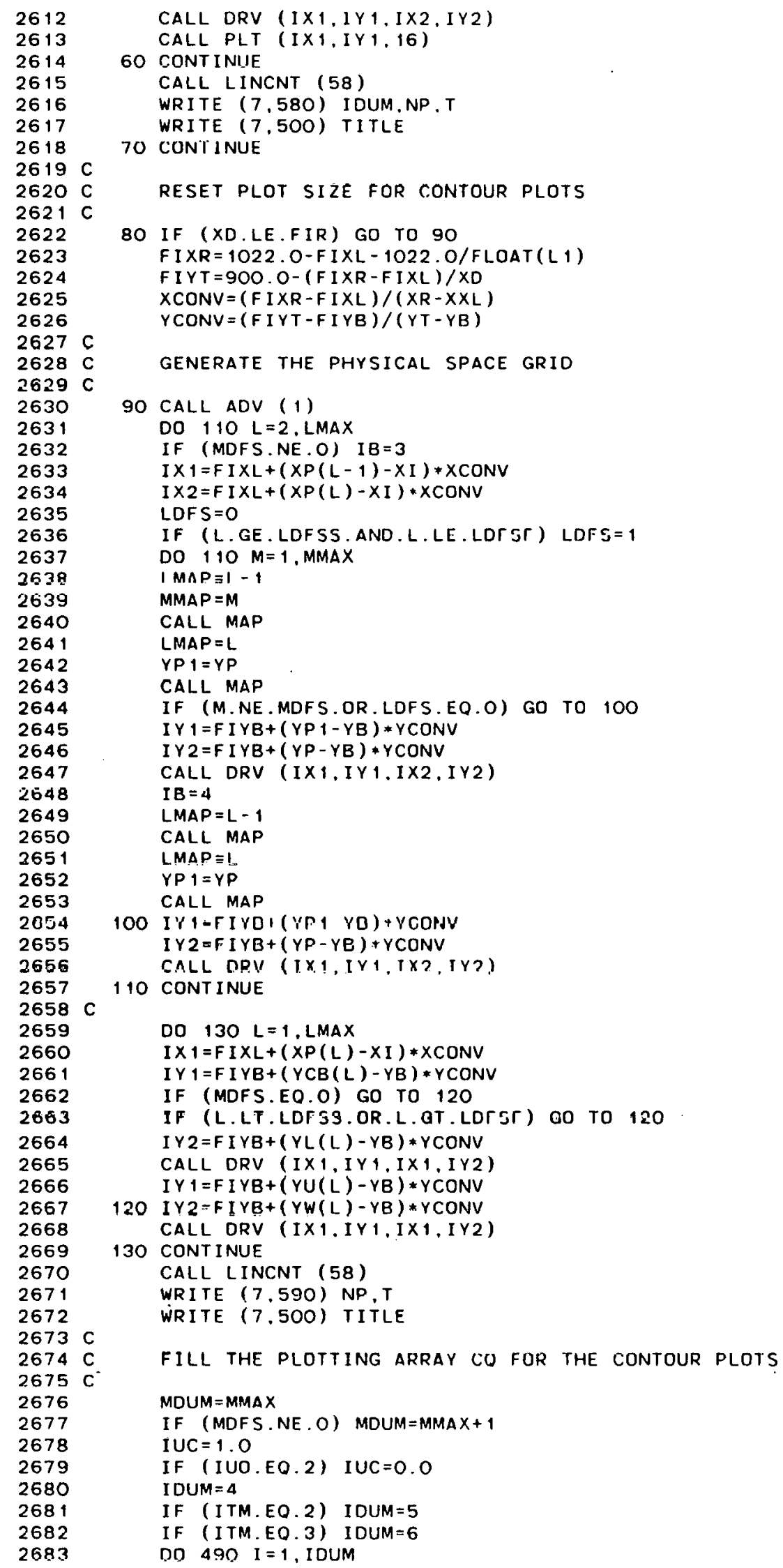




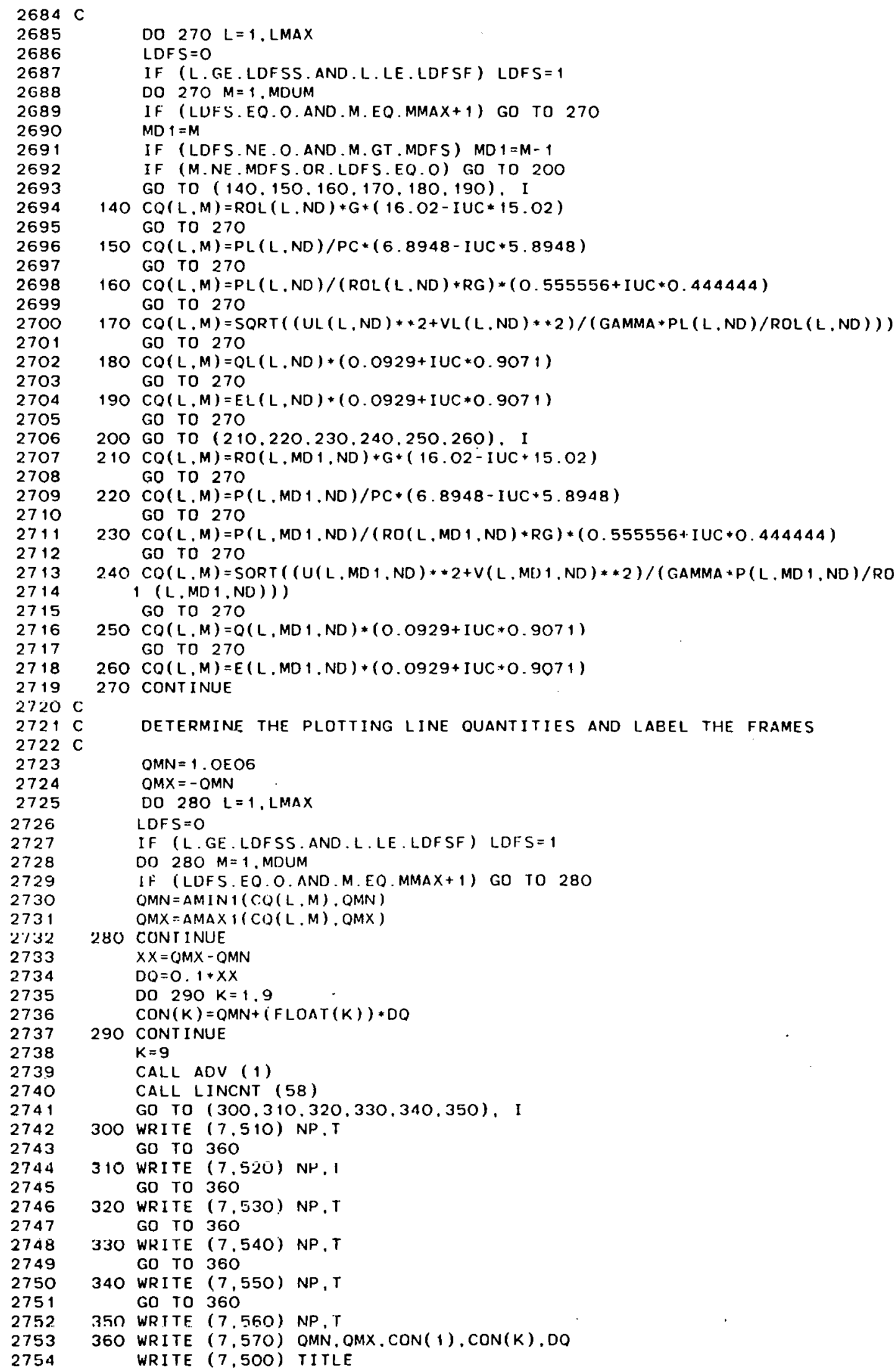




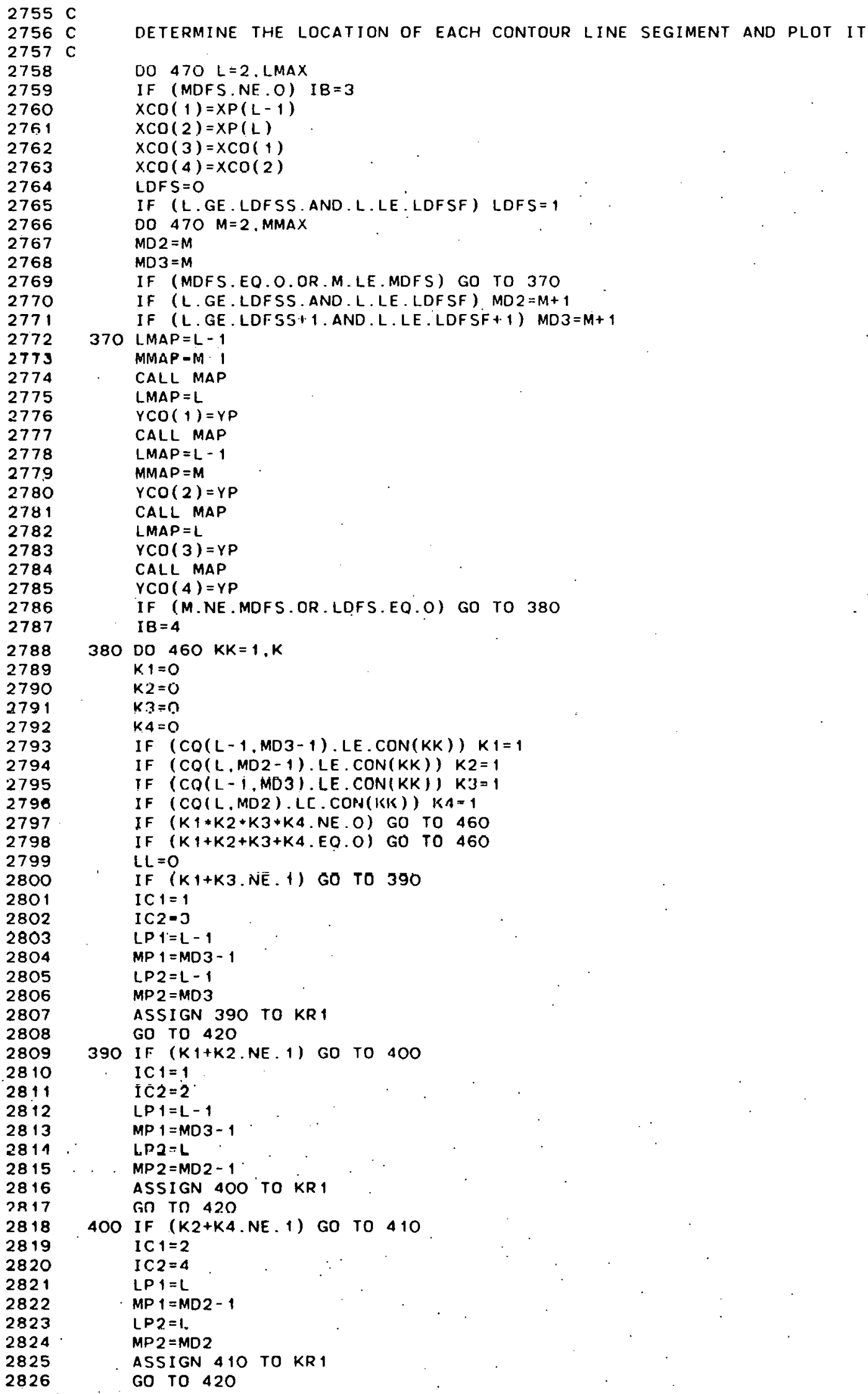




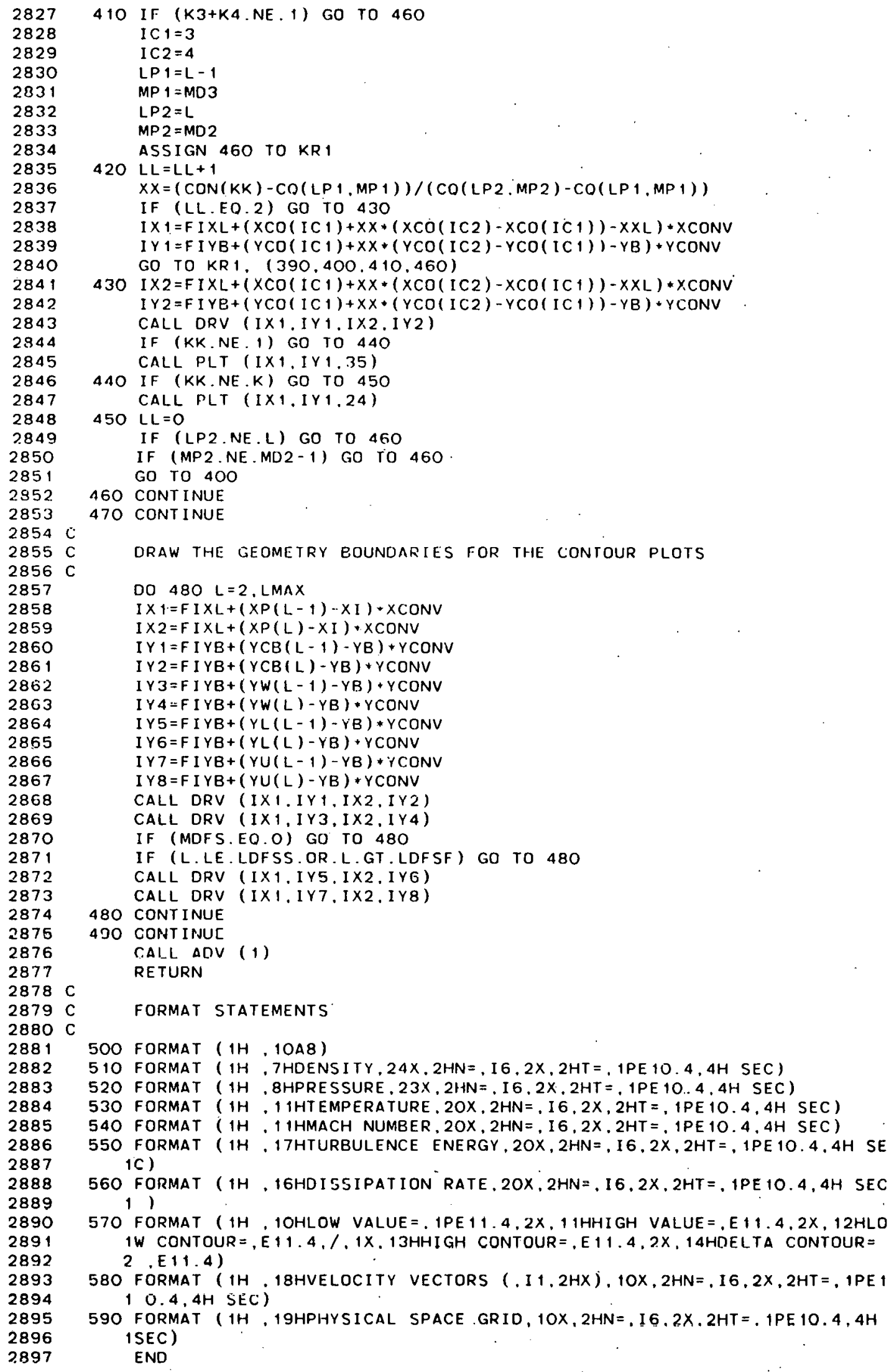




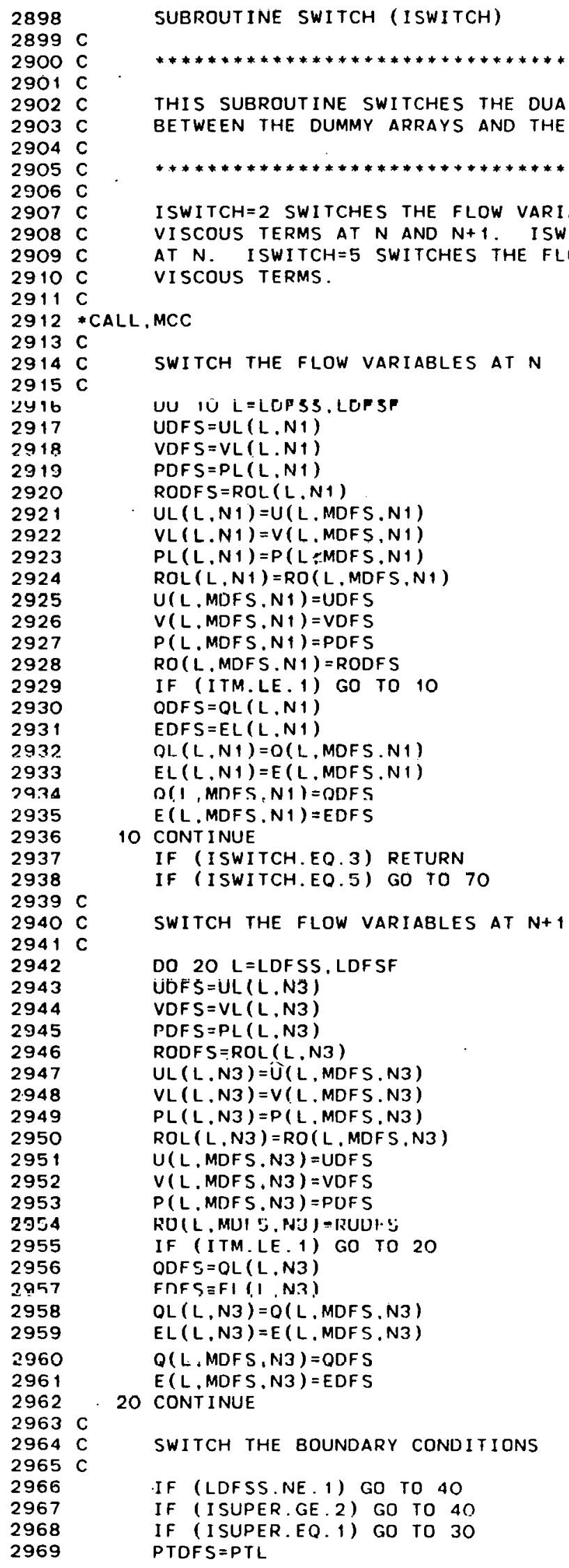




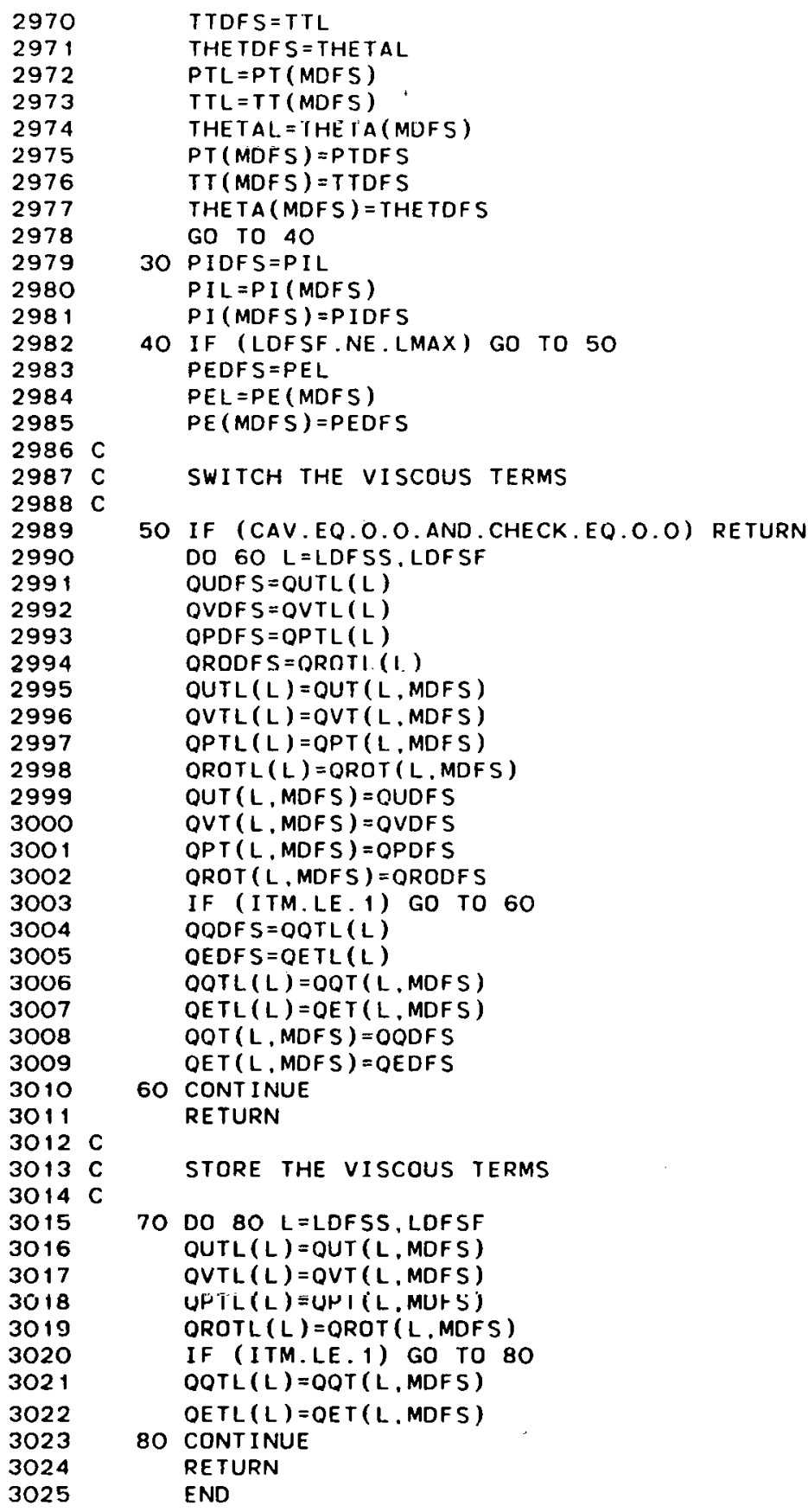




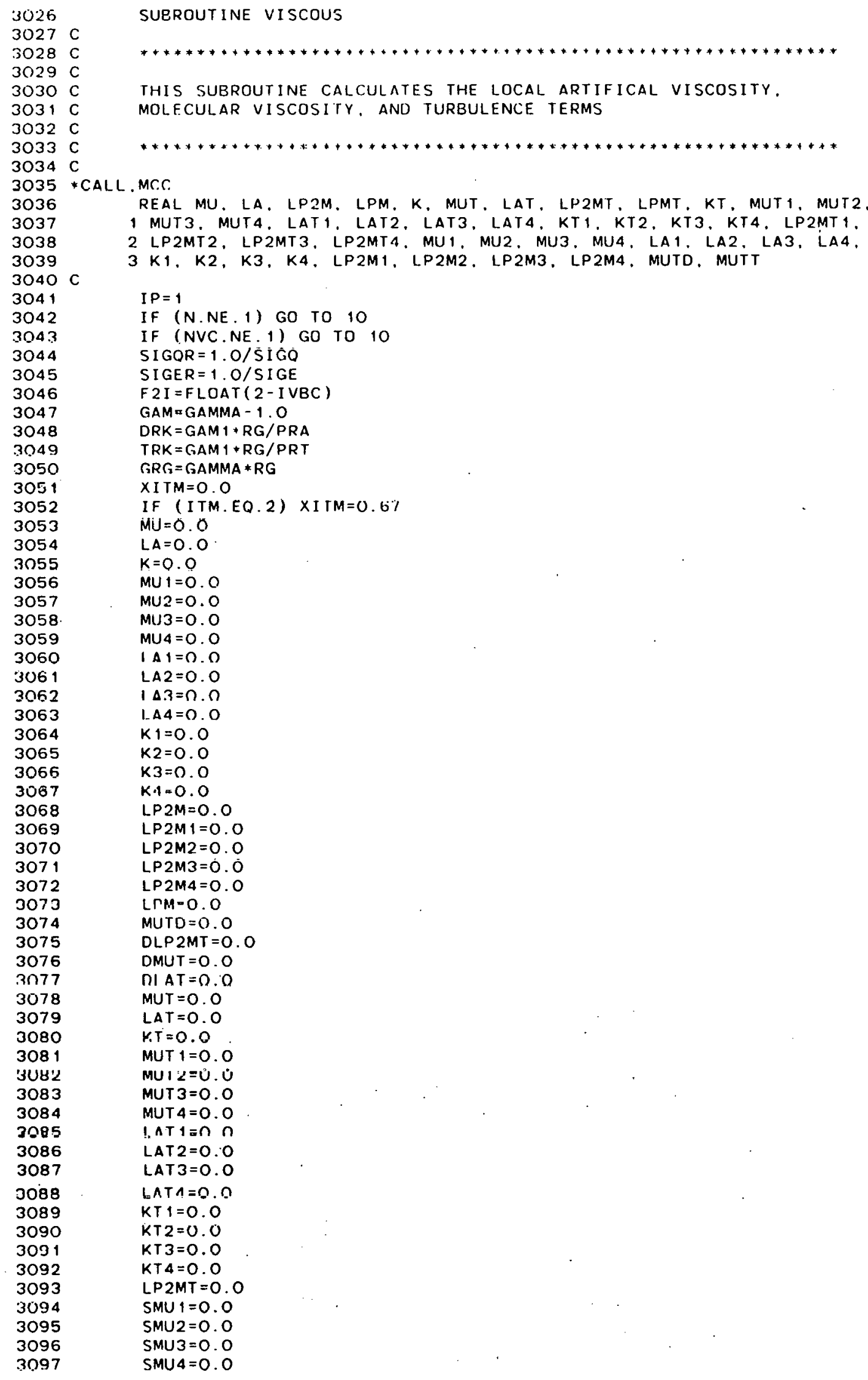




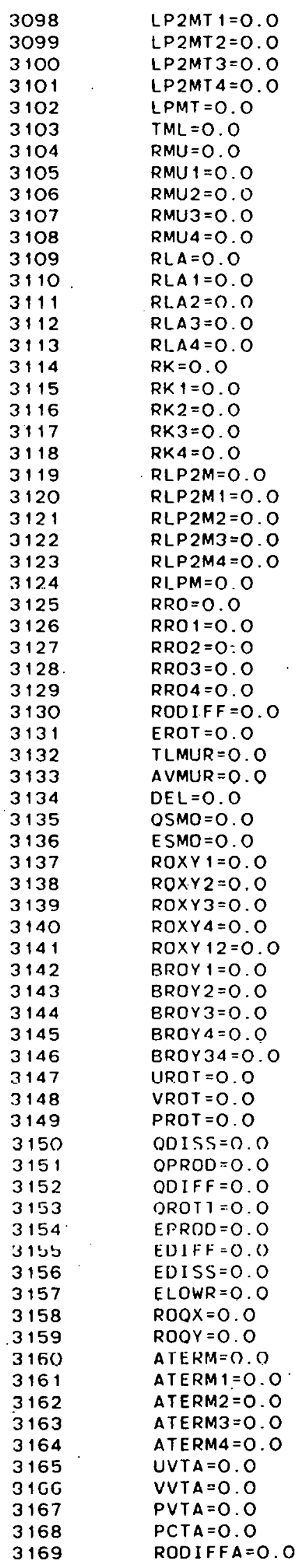




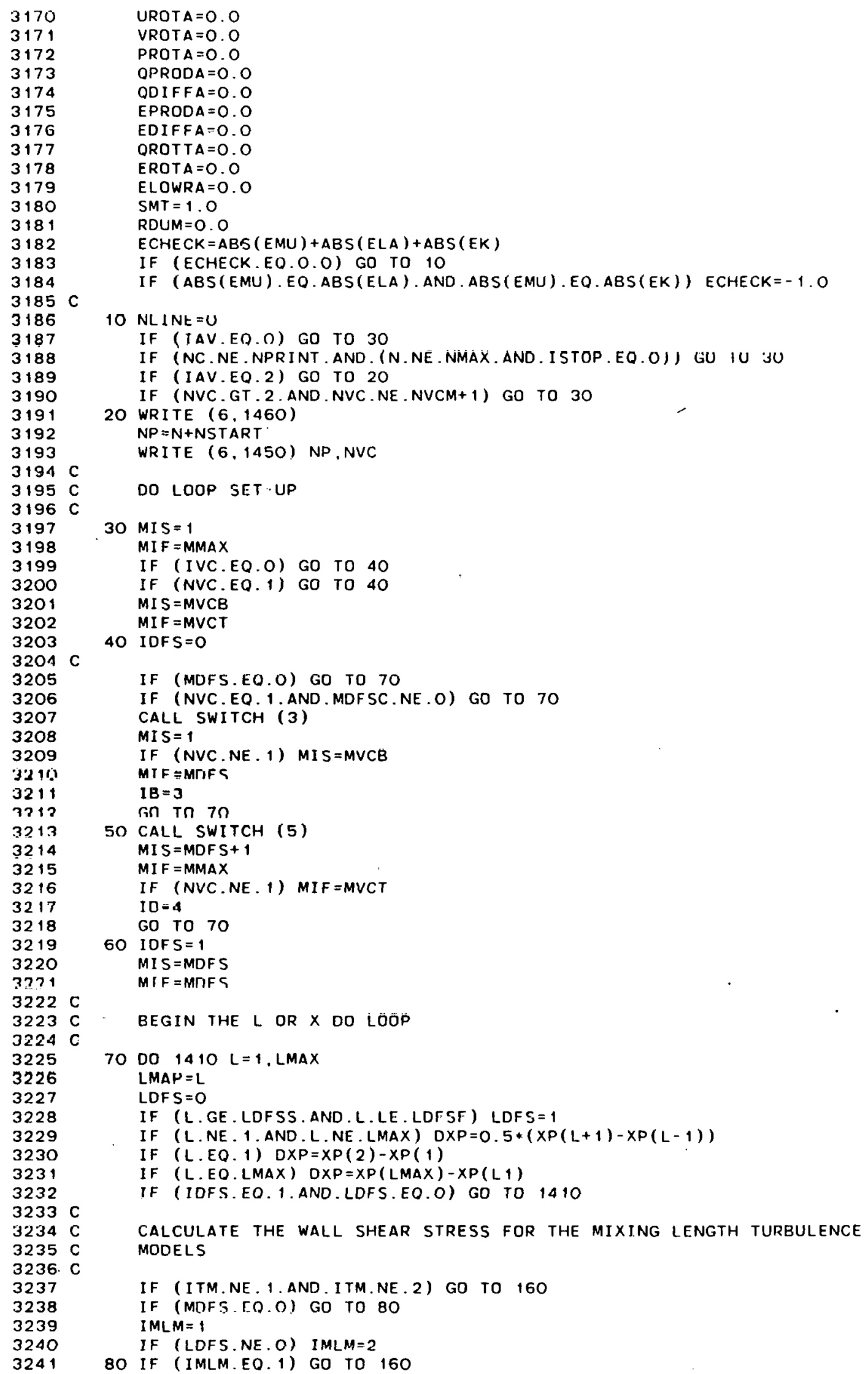




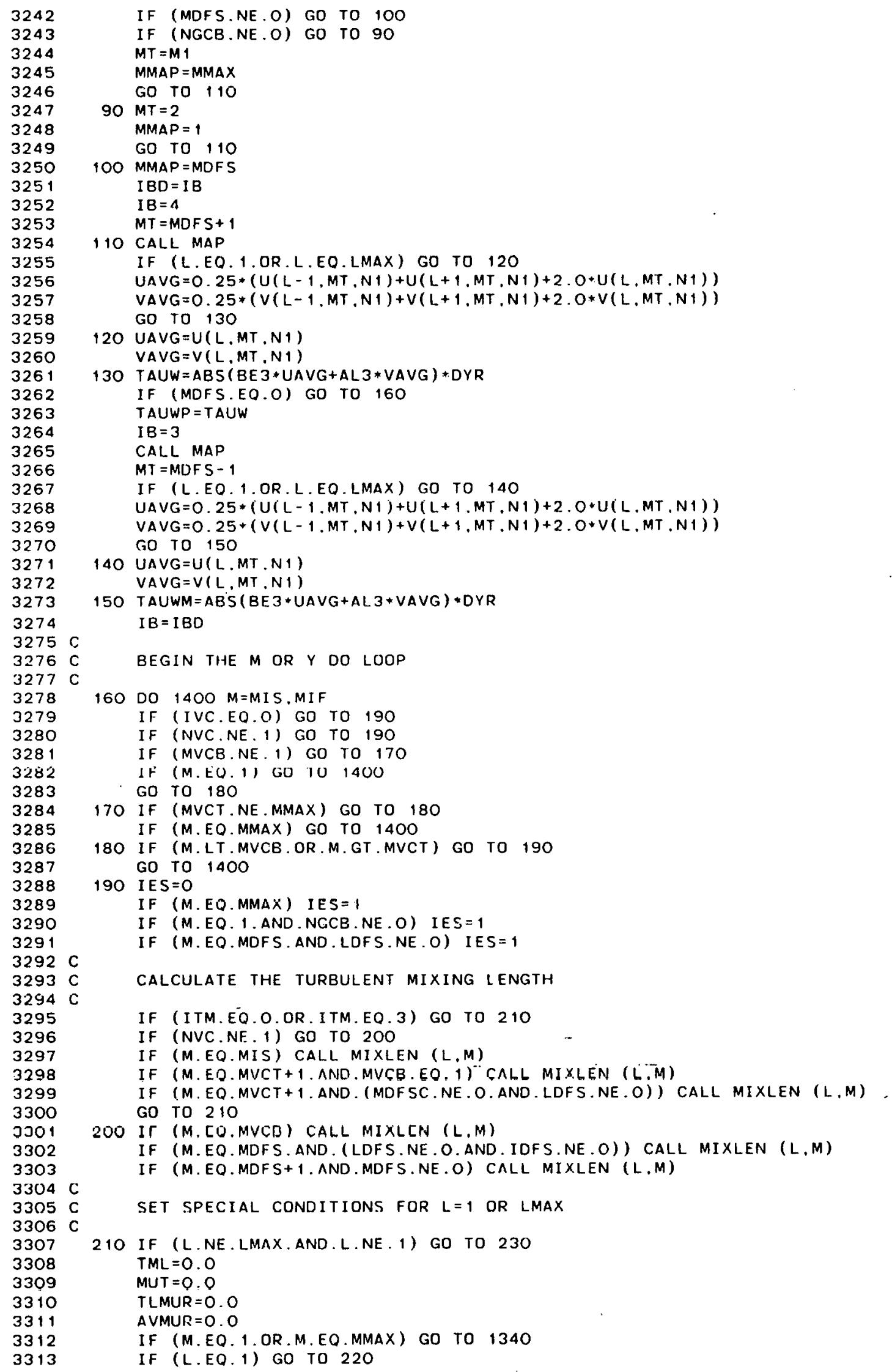




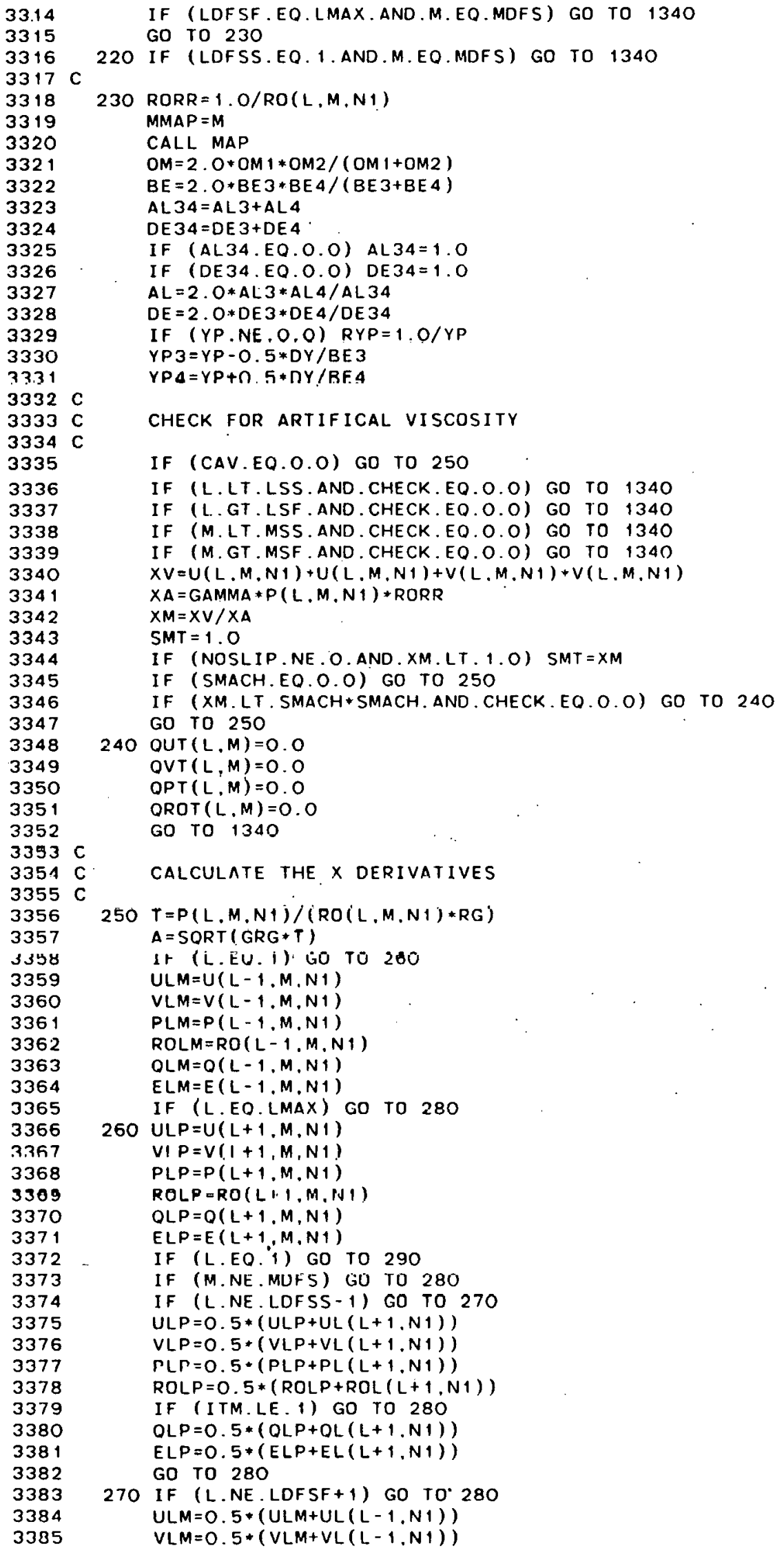




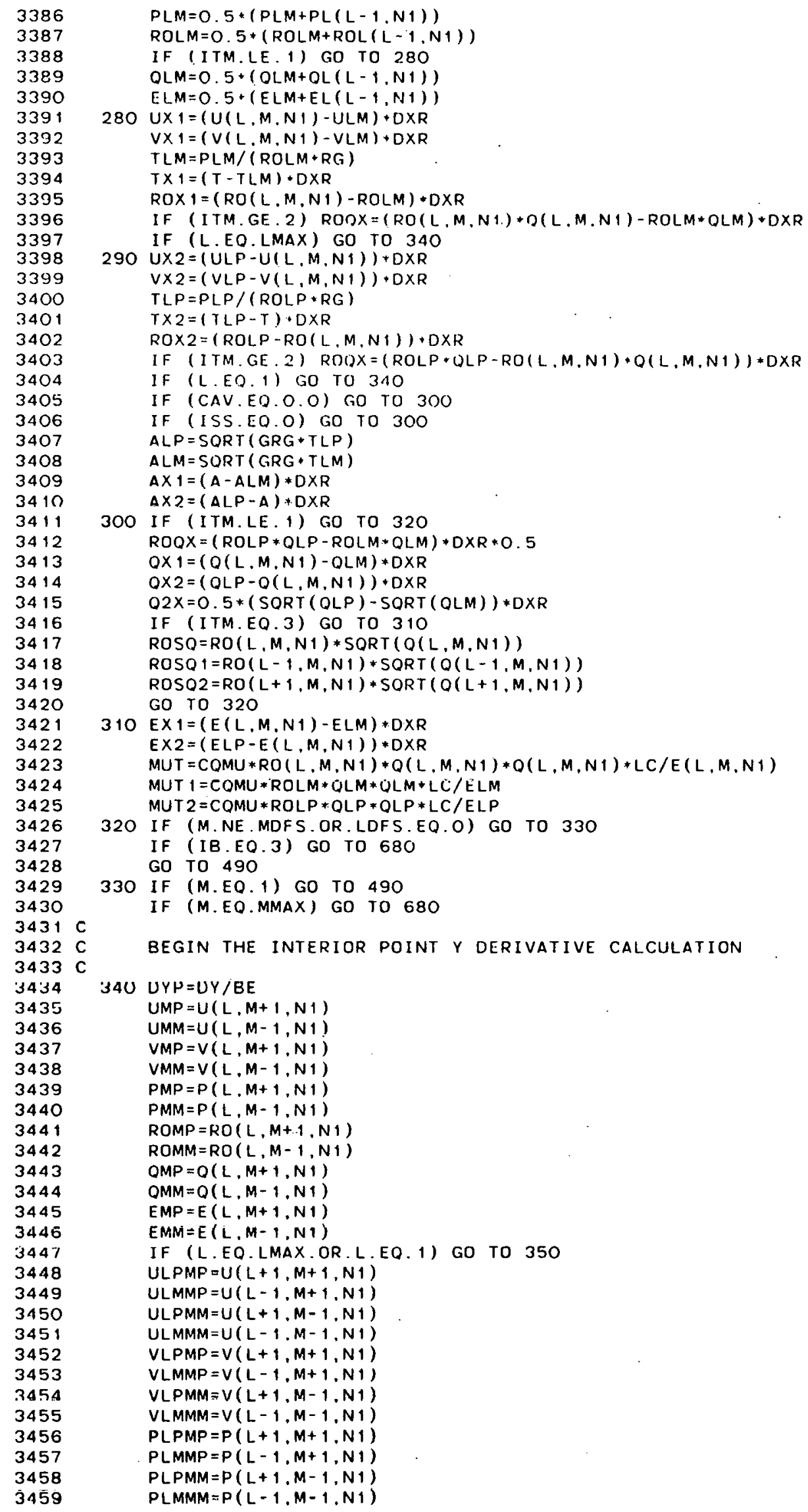




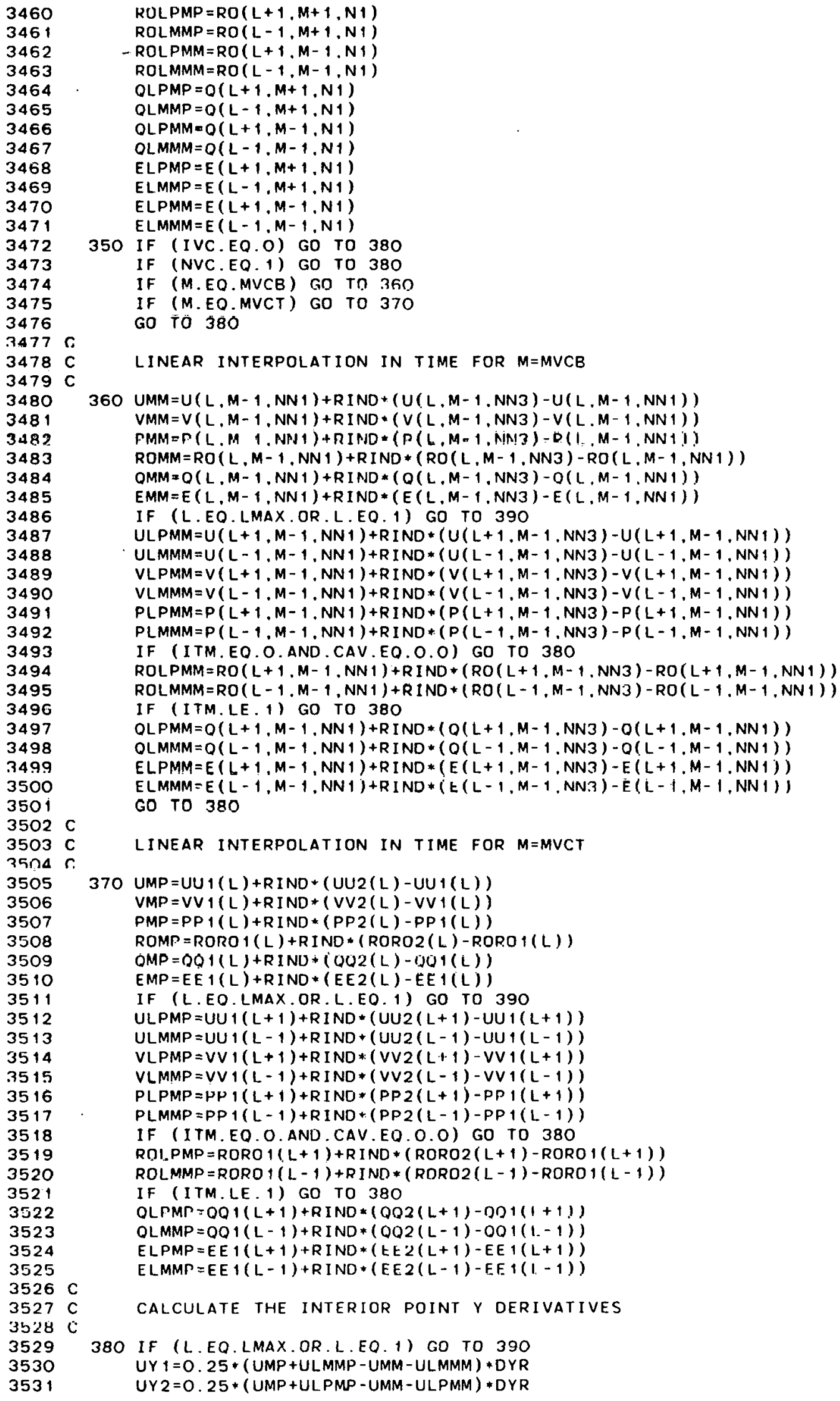

350 IF (IVC.EQ.O) GO TO 380 IF (NVC.EQ.1) GO TO 380

IF (M.EQ.MVCB) GO TO 360

IF (M.EO.MVCT) GO TO 370

GO TOO 380

LINEAR INTERPOLATION IN TIME FOR M=MVCB

$360 U M M=U(L, M-1 . N N 1)+R I N D *(U(L, M-1, N N 3)-U(L, M-1, N N 1))$

$V M M=V(L, M-1, N N 1)+R I N D *(V(L, M-1, N N 3)-V(L, M-1, N N 1))$

$F M M=\Gamma(L, M \quad 1, N A 1)+R I N D=(P(L, M-1, N ! M ! 3)-D(I, M-1, N N 1 I)$

$R O M M=R O(L, M-1, N N 1)+R I N D *(R O(L, M-1, N N 3)-R O(L, M-1, N N 1))$

$Q M M=O(L, M-1 . N N 1)+R I N D *(O(L, M-1, N N 3)-Q(L, M-1, N N 1))$

$E M M=E(L, M-1, N N 1)+R I N D *(E(L, M-1, N N 3)-E(L, M-1, N N 1))$

IF (L.EQ.LMAX.OR.L.EO. 1) GO TO 390

$U L P M M=U(L+1, M-1 . N N 1)+R I N D *(U(L+1, M-1$. NN3 $)-U(L+1, M-1, N N 1))$

$U L M M M=U(L-1, M-1, N N 1)+R I N D *(U(L-1, M-1, N N 3)-U(L-1, M-1, N N 1))$

$V L P M M=V(L+1, M-1, N N 1)+R I N D *(V(L+1, M-1, N N 3)-V(L+1, M-1, N N 1))$

$V L M M M=V(L-1, M-1, N N 1)+R I N D *(V(L-1, M-1, N N 3)-V(L-1, M-1, N N 1))$

$P L P M M=P(L+1 . M-1 . N N 1)+R I N D+(P(L+1, M-1 . N N 3)-P(L+1 . M-1 . N N 1))$

$P L M M M=P(L-1, M-1, N N 1)+R I N D *(P(L-1, M-1 . N N 3)-P(L-1, M-1, N N 1))$

IF (ITM.EO.O.AND.CAV.EQ.O.O) GO TO 380

$R O L P M M=R O(L+1, M-1, N N 1)+R I N D *(R O(L+1, M-1, N N 3)-R O(L+1, M-1, N N 1))$

$R O L M M M=R O(L-1, M-1, N N 1)+R I N D+(R O(L-1, M-1, N N 3)-R O(L-1, M-1, N N 1))$

IF (ITM.LE. 1 ) GO TO 380

$O L P M M=Q(L+1, M-1 . N N 1)+R I N D *(O(L+1, M-1, N N 3)-O(L+1, M-1, N N 1))$

$Q L M M M=Q(L-1, M-1 . N N 1)+R I N D *(Q(L-1, M-1 . N N 3)-Q(L-1, M-1, N N 1))$

$E L P M M=E(L+1, M-1, N N 1)+R I N D *(E(L+1, M-1, N N 3)-E(L+1, M-1, N N 1))$

$E L M M M=E(L-1, M-1, N N 1)+R I N D *(E(L-1, M-1, N N 3)-E(L-1, M-1, N N 1))$ GO TO 380

LINEAR INTERPOLATION IN TIME FOR M=MVCT

370 UMP $=U U_{1}(L)+R I N D+(U U 2(L)-U U 1(L))$

$V M P=V V 1(L)+R I N D *(V V 2(L)-V V 1(L))$

$P M P=P P_{1}(L)+R I N D *\left(P P_{2}(L)-P P_{1}(L)\right)$

ROMP $=$ RORO $1(L)+R I N D *(R O R O 2(L)-R O R O 1(L))$

$O M P=D O 1(L)+R I N U+(002(L)-001(L))$

$E M P=E E 1(L)+R I N D *(E E 2(L)-E E 1(L))$

IF (L.EO.LMAX.OR.L.EO. 1) GO TO 390

$U L P M P=U U 1(L+1)+R 1 N D *(U U 2(L+1)-U U 1(L+1))$

$U L M M P=U U 1(L-1)+R I N D+(U U 2(L-1)-U U 1(L-1))$

$\operatorname{VLPMP}=V V 1(L+1)+R I N D *\left(V_{2}(L+1)-V V 1(L+1)\right)$

$V L M M P=V V 1(L-1)+R I N D *(V \vee 2(L-1)-V V 1(L-1))$

$P L P M P=P P 1(L+1)+R I N O *(P P 2(L+1)-P P 1(L+1))$

$P L M M P=P P 1(L-1)+R 1 N D+(P P 2(L-1)-P P 1(L-1))$

IF (ITM.EO.O.AND.CAV.EO.O.O) GO TO 380

RQI.PMP $=$ RORO $1(L+1)+R I N D *(R O R O 2(L+1)-R O R O 1(L+1))$

$R O L M M P=R O R O 1(L-1)+R I N D *(R O R O 2(L-1)-R O R O 1(L-1))$

IF (ITM.LE. 1) GO TO 380

$O L P M P=O Q 1(L+1)+R I N D *(0 Q 2(L+1)-0 D 1(1+1))$

QLMMP $=0 Q 1(L-1)+R I N D *(Q Q 2(L-1)-O Q 1(L-1))$

$E L P M P=E E 1(L+1)+R I N D *(L E 2(L+1)-E E 1(L+1))$

$E L M M P=E E 1(L-1)+R I N D *\left(E E 2(L-1)-E_{*} 1(1-1)\right)$

CALCULATE THE INTERIOR POINT Y DERIVATIVES

380 If (L.EQ.LMAX.OR.L.EQ.1) GO TO 390

UY $1=0.25 *($ UMP +ULMMP-UMM-ULMMM )*DYR

$U Y 2=0.25 *(U M P+U L P M P-U M M-U L P M M) * D Y R$ 
$V Y 1=0.25 *(V M P+V L M M P-V M M-V L M M M) * D Y R$ $V Y 2=0.25 *(V M P+V L P M P-V M M-V L P M M) * D Y R$ $U \times 3=0.25 *(U L P+U L P M M-U L M-U L M M M) * D X R$ $U \times 4=0.25 *(U L P+U L P M P-U L M-U L M M P) * D \times R$ $V \times 3=0.25 *(V L P+V L P M M-V L M-V L M M M) * D X R$ $V \times 4=0.25 *(V L P+V L P M P-V L M-V L M M P) * D X R$

$390 \vee V Y=(V(L, M, N I)-V M M) * D Y R$ $V Y 4=(V M P-V(L, M, N I))+D Y R$ $U Y 3=(U(L, M, N 1)-U M M) * D Y R$ $U Y 4=(U M P-U(L, M, N 1)) * D Y R$ $T M M=P M M /(R O M M+R G)$ $T M P=P M P /(R O M P * R G)$

$T Y 3=(T-T M M) * D Y R$ $T Y 4=(T M P-T) * D Y R$ ROY $3=(R O(L, M, N 1)-R O M M) * D Y R$ ROY $4=(R O M P-R O(L, M, N 1)) * D Y R$ IF (ITM.LT.2) GO TO 400 ROOY $=(R O M P * O M P-R O M M * Q M M) * D Y R * 0.5$ IF (IOSD.EQ.O.OR.NVC.EQ.1) GO TO 400 IF (M.EQ.MVCB.OR.M.EQ.MVCT) GO TO 400 ROOY $=$ QOT $(L, M)$

400 IF (L.EQ.LMAX,OR.L.EQ. 1) GO TO 4 t0 $T L M M M=P L M M M /(R O L M M M * R G)$ $T L M M P=P L M M P /(R O L M M P * R G)$ $T L P M M=P L P M M /(R O L P M M * R G)$ $T L P M P=P L P M P /(R O L P M P * R G)$ $T Y 1=0.25 *(T M P+T L M M P-T M M-T L M M M) * D Y R$ $T Y 2=0.25 *(T L P M P+T M P-T L P M M-T M M) * D Y R$ $T \times 3=0.25 *(T L P+T L P M M-T L M-T L M M M) * D X R$ $T \times 4=0.25 *(T L P M P+T L P-T L M M P-T L M) * D X R$ IF (ITM.EQ.O. AND.CAV.EQ.O.O) GO TO 450 ROY $1=0.25 *(R O M P+R O L M M P-R O M M-R O L M M M) * D Y R$ ROY $2=0.25 *(R O M P+R O L P M P-R O M M-R O L P M M) * D Y R$ $R O \times 3=0.25 *(R O L P+R O L P M M-R O L M-R O L M M M) * D X R$ $R O \times 4=0.25 *(R O L P+R O L P M P-R O L M-R O L M M P) * D \times R$

410 IF (CAV.EQ.O.O) GO TO 430

IF (NDIM.EQ.O) GO TO 420 $\triangle T E R M=V(L, M, N 1) * R Y P$ $\triangle$ TERM $3=0.5 *(V(L, M, N 1)+V M M) * R Y P$ ATERM4 $=0.5 *(V(L, M, N 1)+V M P) * R Y P$ IF (L.EQ. 1.OR.L.EQ.LMAX) GO TO 420 ATERM $1=0.5 *(V(L, M, N 1)+V(L-1, M, N 1)) * R Y P$ $A T E R M 2=0.5 *(V(L, M, N 1)+V(L+1, M, N 1))+R Y P$

420 IF (ISS.EO.O) GO TO 430 $\triangle M P=S Q R T(G R G * T M P)$ $A M M=S O R T(G R G * T M M)$ $A Y 3=(A-A M M) * D Y R$ AY $4=(A M P-A) * D Y R$

430 IF (ITM.LE. 1) GO TO 450 IF (L.EO.1.OR.L.EO.LMAX) GO TO 450 $Q Y \cdot 1=0.25 *(O M P+Q L M M P-O M M-O L M M M) * D Y R$ $Q Y 2=0.25 *(O M P+Q L P M P-O M M-Q L P M M) * D Y R$ $Q \times 3=0.25 *(O L P+O L P M M-O L M-O L M M M) * D \times R$ $Q \times 4=0.25 *(Q L P+O L P M P-Q L M-O L M M P) * D \times R$ $Q Y 3=(O(L, M, N 1)-O M M)+D Y R$ QY $4=(O M P-O(L, M, N 1)) * D Y R$ Q2Y $=0.5+($ SORT $(O M P)-S Q R T(O M M)) * D Y R$ IF (ITM.EO. 3) GU TO 440 ROSQ3 $=$ ROMM * SORT ( QMM ) ROSQ4 $=$ ROMP + SQRT ( OMP) GO TO 450

$440 E Y 1=0.25 *(E M P+E L M M P-E M M-E L M M M) * D Y R$ $E Y 2=0.25 *(E M P+E L P M P-E M M-E L P M M)+D Y R$ $E X 3=0.25 *(E L P+E L P M M-E L M-E L M M M) * O X R$ $E \times 4=0.25 *(E L P+E L P M P-E L M-E L M M P) * D \times R$ $E Y 3=(E(L, M, N 1)-E M M) * D Y R$ $E Y 4=(E M P-E(L, M, N 1)) * D Y R$ MUT $3=C Q M U * R O M M * Q M M * Q M M+L C / E M M$ MUT $4=C O M U * R O M P * O M P * O M F * L C / E M P$ 


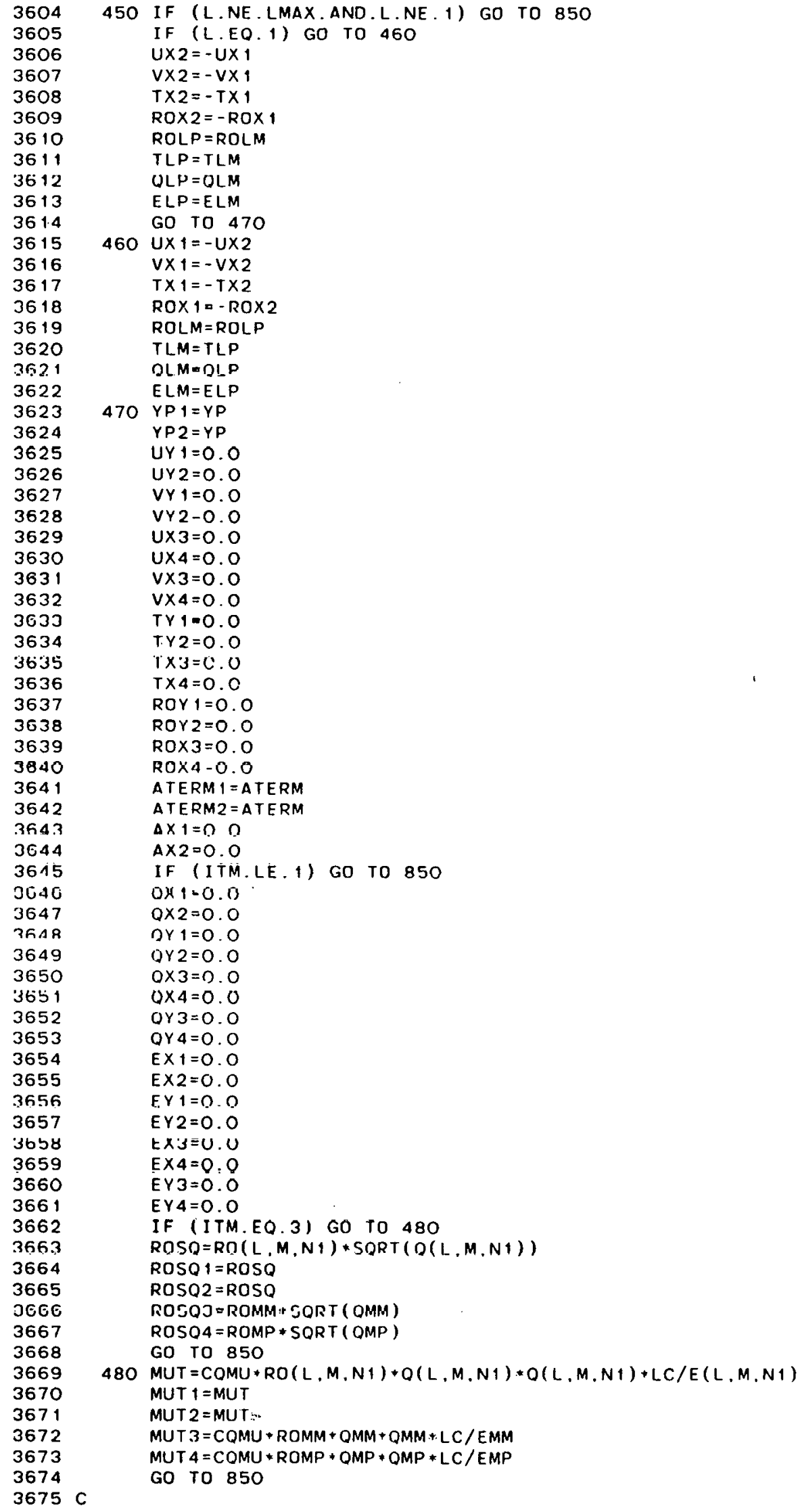




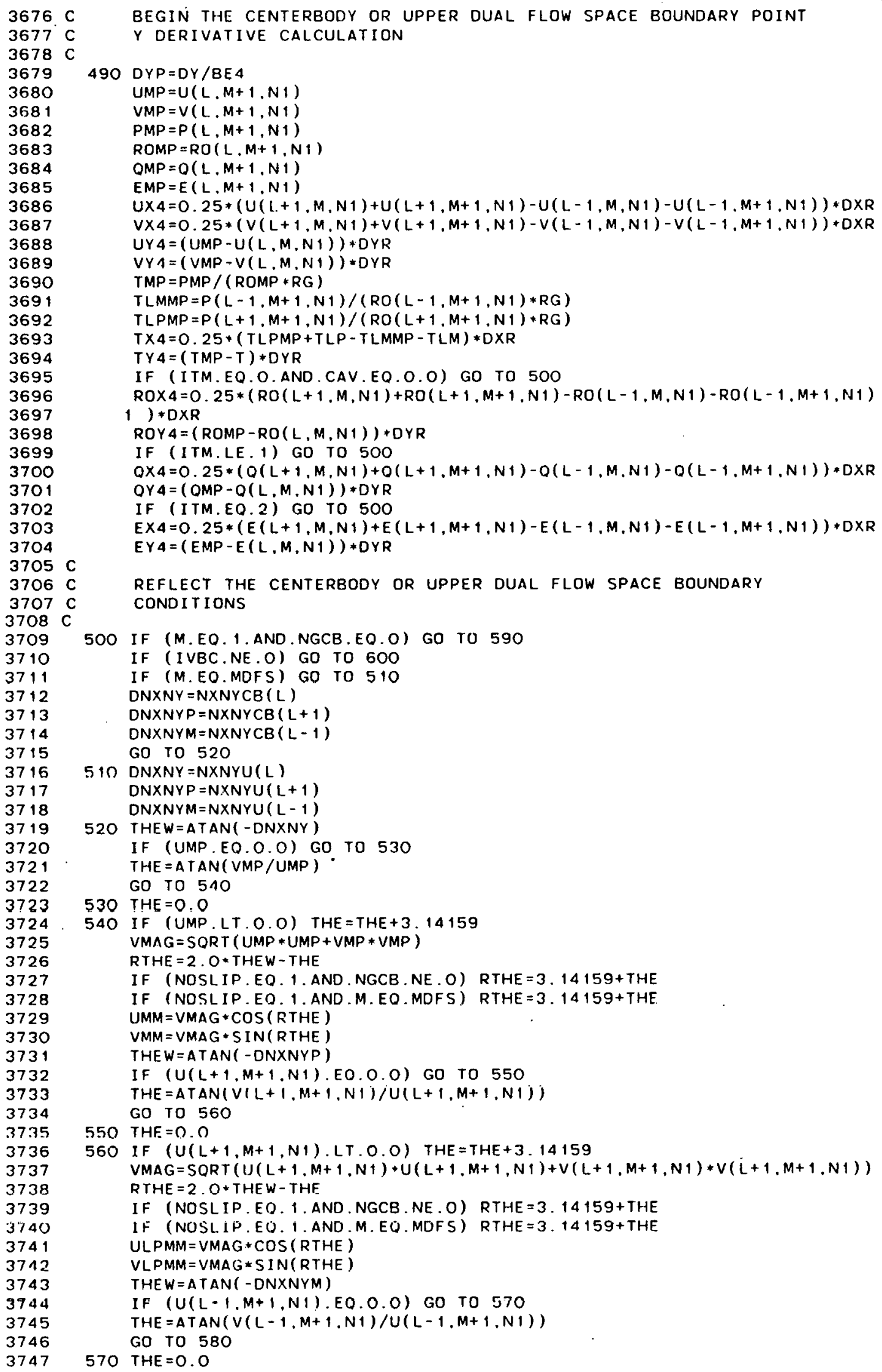




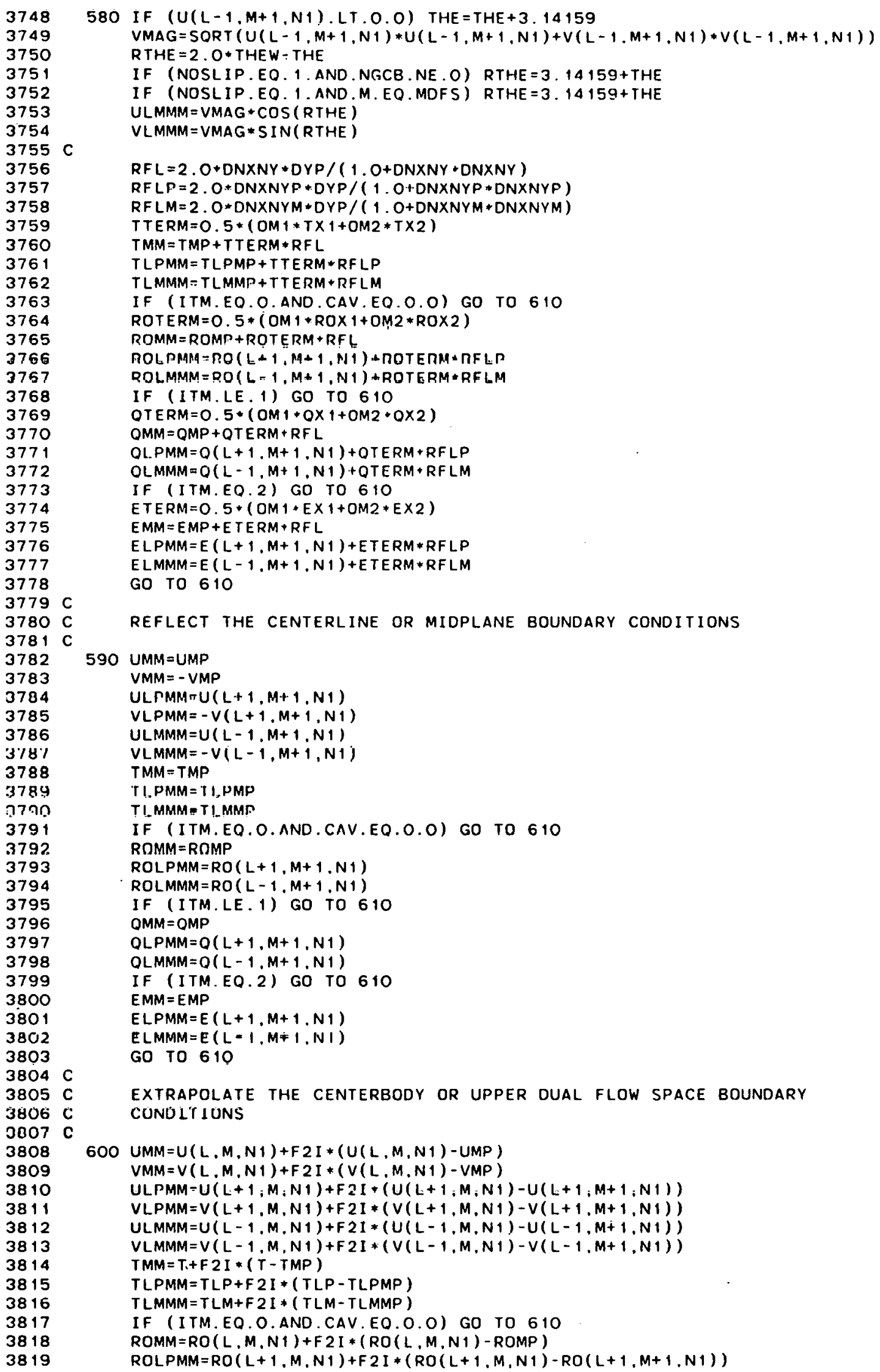


$R O L M M M=R O(L-1, M, N 1)+F 2 I *(R O(L-1, M, N 1)-R O(L-1, M+1, N 1))$

IF (ITM.LE. 1) GO TO 610

$Q M M=Q(L, M, N 1)+F 2 I *(Q(L, M, N 1)-Q M P)$

$Q L P M M=Q(L+1, M, N 1)+F 2 I *(Q(L+1, M, N 1)-Q(L+1, M+1, N 1))$

$Q L M M M=O(L-1, M, N 1)+F 2 I *(Q(L-1, M, N 1)-Q(L-1, M+1, N 1))$

IF (ITM.EQ.2) GO TO 610

$E M M=E(L, M, N 1)+F 2 I *(E(L, M, N 1)-E M P)$

$E L P M M=E(L+1, M, N 1)+F 2 I *(E(L+1, M, N 1)-E(L+1, M+1, N 1))$

$E L M M M=E(L-1, M, N 1)+F 2 I *(E(L-1, M, N 1)-E(L-1, M+1, N 1))$

CALCULATE THE CENTERBODY OR UPPER DUAL FLOW SPACE BOUNDARY DER IVATIVES

610 IF (M.NE.MDFS) GO TO 630

IF (L.NE.LDFSF) GO TO 620

$U L P M M=U(L+1, M-1, N 1)$

$V L P M M=V(L+1 . M-1 . N 1)$

$T L P M M=P(L+1 . M-1 . N 1) /(R O(L+1, M-1, N 1) * R G)$

$R O L P M M=R O(L+1, M-1, N 1)$

IF (ITM.LE. 1) GO TO 630

$Q L P M M=Q(L+1, M-1, N 1)$

$E L P M M=E(L+1 . M-1 . N 1)$

GO TO 630

620 IF (L.NE:LOFSS) GO TO 630

$U L M M M=U(L-1, M-1, N 1)$

$V L M M M=V(L-1, M-1, N 1)$

$T L M M M=P(L-1, M-1 . N 1) /(R O(L-1, M-1, N 1) * R G)$

$R O L M M M=R O(L-1, M-1, N 1)$

IF (ITM.LE. 1) GO TO 630

$O L M M M=O(L-1, M-1 . N 1)$

$E L M M M=E(L-1, M-1, N 1)$

630 UY $1=0.25 *(U M P+U(L-1, M+1, N 1)-U M M-U L M M M) * D Y R$

$V Y 1=0.25 *(V M P+V(L-1 . M+1 . N 1)-V M M-V L M M M) * D Y R$

$U Y 2=0.25 *(U M P+U(L+1, M+1 . N 1)-U M M-U L P M M) * D Y R$

$V Y 2=0.25 *(V M P+V(L+1, M+1, N 1)-V M M-V L P M M) * D Y R$

$U Y 3=(U(L, M, N 1)-U M M) \neq D Y R$

$V Y 3=(V(L, M . N 1)-V M M)+D Y R$

$U \times 3=0.25 *(U(L+1, M . N 1)+U L P M M-U(L-1, M . N 1)-U L M M M)+D \times R$

$V \times 3=0.25 *(V(L+1, M, N 1)+V L P M M-V(L-1, M, N 1)-V L M M M) * D X R$

$T Y 1=0.25 *(T M P+T L M M P-T M M-T L M M M) * D Y R$

$T Y 2=0.25 *(T M P+T L P M P-T M M-T L P M M) * D Y R$

$T \times 3=0.25 *(T L P+T L P M M-T L M-T L M M M) * D X R$

$T Y 3=(T-T M M)+D Y R$

$T M M=T M M$

IF (ITM.EO.O.AND.CAV.EO.O.O) GO TO 850

ROY $1=0.25 *(R O M P+R O(L-1, M+1, N 1)-R O M M-R O L M M M) * D Y R$

$R O Y 2=0.25 *(R O M P+R O(L+1, M+1, N 1)-R O M M-R O L P M M) * D Y R$

ROY $3=(R O(L, M, N 1)-R O M M) * D Y R$

$R O \times 3=0.25+(R O(L+1, M, N I)+R O L P M M-R O(L-I, M, N I)=R O L M M M)=O \times R$

IF (CAV.EO.O.O) GO TO 660

IF (NDIM.EO.O) GO TO 650

IF (M.EQ.1.AND.YCB(L).EO.0.0) GO TO 640

$A T E R M=V(L, M, N 1) * R Y P$

$A \operatorname{TERM} 1=0.5 *(V(L, M, N 1)+V(L-1, M, N 1)) * R Y P$

ATERM $2=0.5 *(V(L, M, N 1)+V(L+1, M, N 1))+R Y P$

$A T E R M 3=0.5 *(V(L . M . N 1)+V M M) * R Y P$

ATERM4 $=0.5 *(V(L, M, N 1)+V M P) * R Y P$

IF (M.EO.MOFS) GO TO 650

IF $(Y C B(L-1)$.EO. O. O) ATERM1 $=0.5 *(B E 4 * V(L-1, M+1, N 1)+D Y R+V(L, M, N 1)$

$1 / Y C B(L))$

IF $(Y C B(L+1) .[0.0 .0)$ ATLRM2-0.5*(BL4+V(LI1,M+1,N1)+DYR+V(L.M.N1)

$1 / Y C B(L))$

IF $(Y C B(L-1) . E O \cdot 0.0 .0 R \cdot Y C B(L+1) . E Q .0 .0)$ ATERM $=0.5 *(A T E R M 1+A T E R M 2)$ GO TO 650

$640 \quad \triangle T E R M=B E * V M P * D Y R$

$A T E R M 1=B E 4 * 0.5 *(V M P+V(L-1, M+1, N 1))+D Y R$

$A T E R M 2=B E 4+0.5+(V M P+V(L+1 . M+1 . N 1))+D Y R$

$\triangle T E R M 4=B E 4 * V M P * D Y R$

$\triangle T E R M 3=A T E R M 4$

IF $(Y C B(L-1) \cdot N E \cdot 0.0)$ ATERM $1=0.5 *(V(L-1, M, N 1) / Y C B(L-1)+B E 4 * V M P * D Y R)$

IF $(Y C E(L+1) . N E . O . O) \quad A T E R M 2=0.5 *(V(L+1, M, N 1) / Y C B(L+1)+B E 4 * V M P * D Y R)$

IF $(Y C B(L-1) \cdot N E \cdot 0 \cdot 0.0 R . Y C B(L+1) . N E .0 .0)$ ATERM $=0.5 *(A T E R M 1+A T E R M 2)$

650 IF (ISS.EO.O) GO TO 660 $\triangle M P=5 U R T(G R G * T M P)$ 


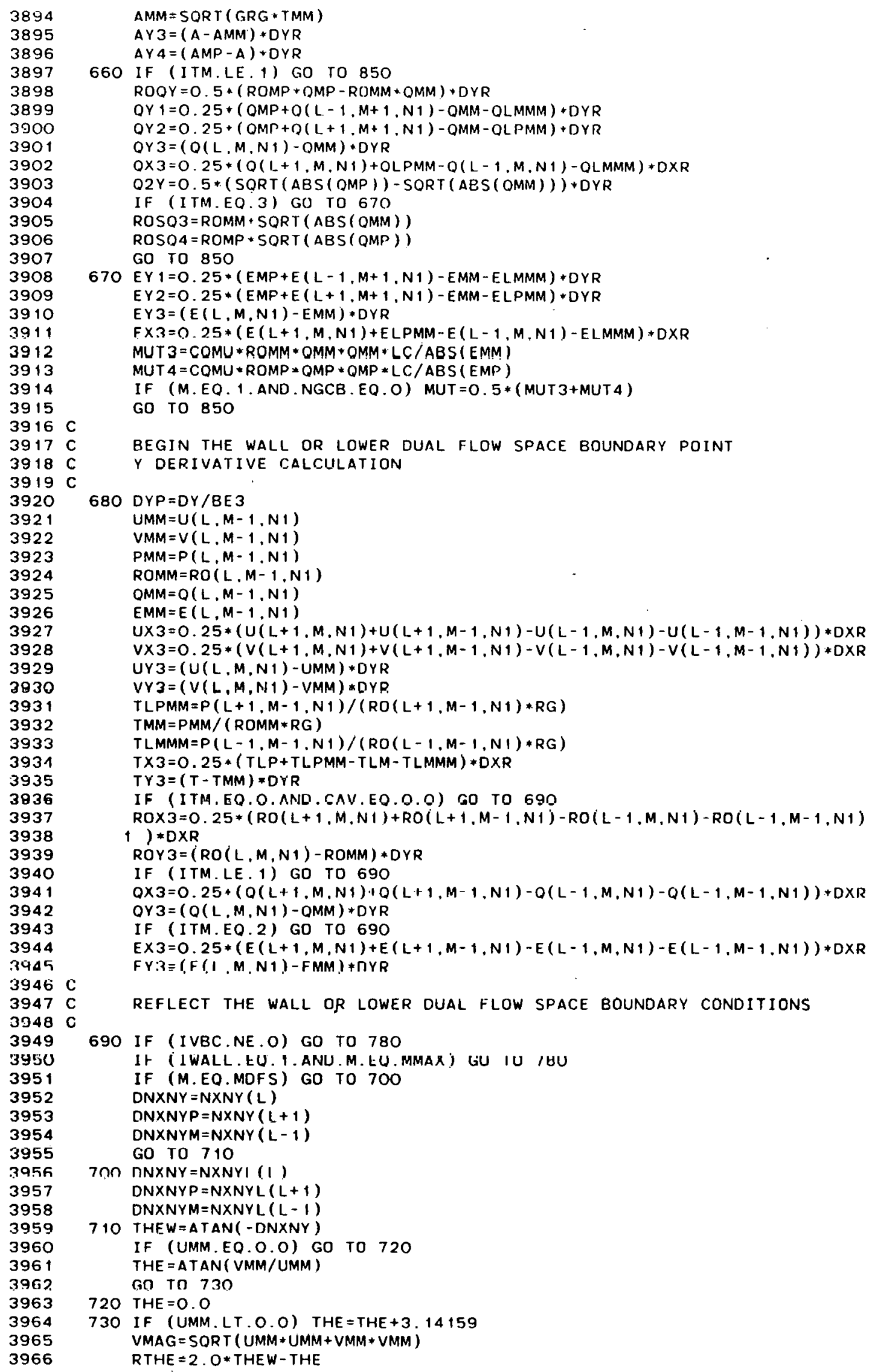

3894

3895

3896

3897

3898

3899

3900

3901

3902

3903

3904

3905

3906

3907

3908

3909

3910

3911

3912

3913

3914

3915

$3916 \mathrm{C}$

$3917 \mathrm{C}$

$3918 \mathrm{C}$

$3919 \mathrm{C}$

3920

3921

3922

3923

3924

3925

3926

3927

3928

3929

3830

3931

3932

3933

3931

3935

3836

3937

3938

3939

3940

3941

3942

3943

3944

3945

$3946 \mathrm{C}$

$3947 \mathrm{C}$

$3348 \mathrm{C}$

3949

3950

3951

3952

3953

3954

3955

39.56

3957

3958

3959

3960

3961

3962

3963

3964

3965

3966

$A M M=S Q R T(G R G * T M M)$

$A Y 3=(A-A M M)+D Y R$

$A Y 4=(A M P-A)+D Y R$

660 IF (ITM.LE. 1) GO TO 850

$R O Q Y=0.5 *(R O M P * D M P-R O M M * Q M M) * D Y R$

QY $1=0.25+(O M P+Q(L-1, M+1, N 1)-O M M-Q L M M M)+D Y R$

$Q Y 2=0.25+(O M P+Q(L+1 . M+1 . N 1)-O M M-Q L P M M)+D Y R$

QY3 $=(Q(L, M, N 1)-Q M M)+D Y R$

$Q \times 3=0.25 *(Q(1+1 . M . N 1)+Q L P M M-O(L-1 . M . N 1)-Q L M M M) * D \times R$

$Q 2 Y=0.5 *(S Q R T(A B S(D M P))-S O R T(A B S(O M M))) * D Y R$

IF (ITM.EO.3) GO TO 670

ROSQ3 $=R O M M+S Q R T(A B S(O M M))$

ROSO4 $=$ ROMP * SQRT $(A B S(Q M P))$

GO TO 850

$670 E Y 1=0.25 *(E M P+E(L-1 . M+1 . N 1)-E M M-E L M M M) * D Y R$

$E Y 2=0.25 *(E M P+E(L+1 . M+1, N 1)-E M M-E L P M M) * D Y R$

$E Y 3=(E(L, M, N 1)-E M M) * D Y R$

$F \times 3=0.25 *(E(L+1, M, N 1)+E L P M M-E(L-1, M, N 1)-E L M M M) * D X R$

MUT $3=C O M U * R O M M * Q M M * Q M M * L C / A B S(E M M)$

MUT $4=C Q M U * R O M P * Q M P * Q M P * L C / A B S(E M P)$

IF (M.EQ.1.AND.NGCB.EQ.O) MUT $=0.5 *(M U T 3+M U T 4)$

GO TO 850

BEGIN THE WALL OR LOWER DUAL FLOW SPACE BOUNDARY POINT

Y DERIVATIVE CALCULATION

$680 D Y P=D Y / 8 E 3$

$U M M=U(L, M-1, N 1)$

$V M M=V(L, M-1, N 1)$

$P M M=P(L, M-1, N 1)$

$R O M M=R O(L, M-1, N 1)$

$Q M M=Q(L, M-1, N 1)$

$E M M=E(L, M-1, N 1)$

$U \times 3=0.25 *(U(L+1, M . N 1)+U(L+1, M-1, N 1)-U(L-1, M, N 1)-U(L-1, M-1, N 1)) * D \times R$

$V \times 3=0.25 *(V(L+1, M, N 1)+V(L+1, M-1, N 1)-V(L-1, M, N 1)-V(L-1, M-1, N 1)) * D \times R$

$U Y 3=(U(L, M, N 1)-U M M) * D Y R$

$V Y 3=(V(L, M, N 1)-V M M) * D Y P$.

$T L P M M=P(L+1, M-1, N 1) /(R O(L+1, M-1, N 1) * R G)$

$T M M=P M M /(R O M M * R G)$

$T L M M M=P(L-1, M-1, N 1) /(R O(L-1, M-1, N 1) * R G)$

$T \times 3=0.25 *(T L P+T L P M M-T L M-T L M M M) * D X R$

$T Y 3=(T-T M M)=D Y R$

IF (ITM.EO.O. AND.GAV.EO.O.O) GO TO 690

$R O \times 3=0.25 *(R O(L+1, M, N 1)+R O(L+1, M-1, N 1)-R O(L-1, M, N 1)-R O(L-1, M-1, N 1)$

$1) * D \times R$

ROY $3=(\operatorname{RO}(L, M, N 1)-R O M M) * D Y R$

IF (ITM.LE.1) GO TO 690

$Q \times 3=0.25+(Q(L+1, M, N 1)+Q(L+1, M-1, N 1)-Q(L-1, M, N 1)-Q(L-1, M-1, N 1)) * D \times R$ OY3 $=(Q(L, M, N 1)-Q M M) * D Y R$

IF (ITM.EQ.2) GO TO 690

$E \times 3=0.25 *(E(L+1, M, N 1)+E(L+1, M-1, N 1)-E(L-1, M, N 1)-E(L-1, M-1, N 1)) * D \times R$

$F Y: 3 \equiv(F(1, M, N 1)-F M M ! * \cap Y R$

REFLECT THE WALL OR LOWER DUAL FLOW SPACE BOUNDARY CONDITIONS

690 IF (IVBC.NE.O) GO TO 780

It (IWALL.EU. 1.ANU.M.LU.MMAX) GU IU IUU

IF (M.EQ.MOFS) GO TO 700

DNXNY $=N \times N Y(L)$

DNXNYP $=$ NXNY $(L+1)$

DNXNYM $=N X N Y(L-1)$

GO TO 710

$7 \cap \cap$ NNXNY $=$ NXNYI (1)

DNXNYP $=$ NXNYL $(L+1)$

DNXNYM $=$ NXNYL $(L-1)$

710 THEW = ATAN ( - DNXNY)

IF (UMM.EQ.O.O) GO TO 720

$T H E=A T A N(V M M / U M M)$

GO TO 730

$720 \mathrm{THE}=0.0$

730 IF (UMM. LT.O.O) THE $=\mathrm{THE}+3.14159$

VMAG $=S Q R T(U M M * U M M+V M M * V M M)$

RTHE $=2$. O* THEW $-T H E$ 


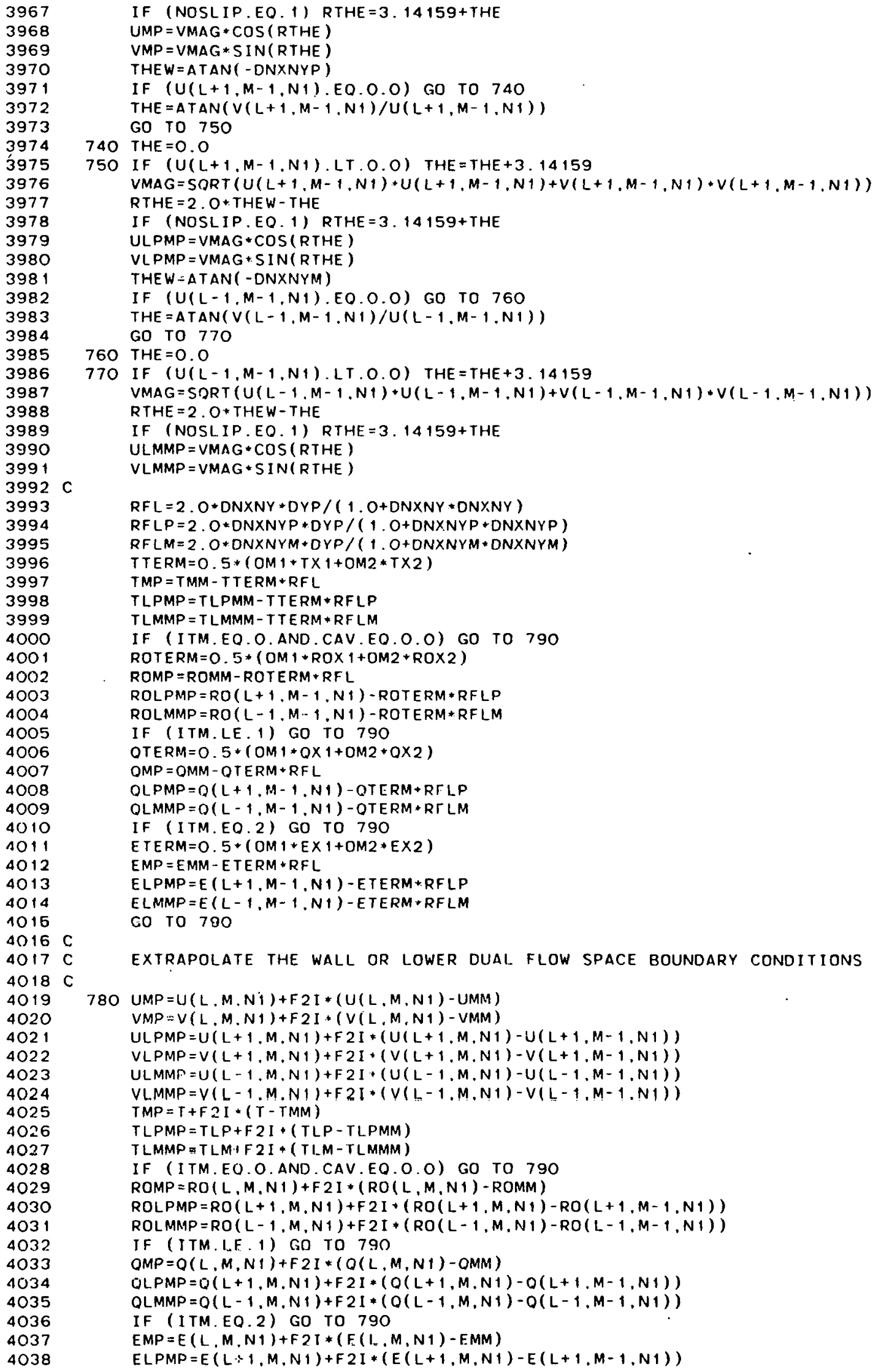

IF (NOSLIP. EO, 1) RTHE $=3,14159+$ THE

$U M P=V M A G * \operatorname{COS}(R T H E)$

$V M P=V M A G * S I N(R T H E)$

THEW = ATAN ( - DNXNYP)

IF $(U(L+1, M-1, N 1) . E 0.0 .0)$ GO TO 740

$T H E=A T A N(V(L+1, M-1, N 1) / U(L+1, M-1, N 1))$

GO TO 750

740 THE $=0.0$

750 IF $(U(L+1, M-1, N 1) . L T .0 . O) \quad T H E=T H E+3.14159$

VMAG $=$ SORT $(U(L+1, M-1, N 1)+U(L+1, M-1 . N 1)+V(L+1, M-1, N 1)+V(L+1, M-1, N 1))$

RTHE $=2$. O + THEW - THE

IF (NOSLIP.EQ.1) RTHE $=3.14159+$ THE

$U L P M P=V M A G * \operatorname{COS}(R T H E)$

$V L P M P=V M A G+S I N(R T H E)$

THEW - ATAN ( - DNXNYM)

IF $(U(L-1, M-1, N 1) . E O .0 .0)$ GO TO 760

THE $=A T A N(V(L-1, M-1, N 1) / U(L-1, M-1, N 1)$

GO TO 770

760 THE $=0.0$

770 IF (U(L-1,M-1,N1).LT.O.O) THE $=T H E+3.14159$

VMAG $=$ SDRT $(U(L-1, M-1 . N 1)+U(L-1, M-1, N 1)+V(L-1, M-1 . N 1)+V(L-1 . M-1 . N 1))$

RTHE $=2.0 *$ THEW - THE

IF (NOSLIP.EO. 1) RTHE $=3.14159+$ THE

$U L M M P=V M A G * \operatorname{COS}(R T H E)$

$V L M M P=V M A G * S I N(R T H E)$

$R F L=2.0 * D N X N Y * D Y P /(1.0+D N X N Y * O N X N Y)$

$R F L P=2.0 * D N X N Y P * D Y P /(1 . O+D N X N Y P * D N X N Y P)$

$R F L M=2.0 *$ ONXNYM+OYP/(1.O+ONXNYM*DNXNYM)

$T T E R M=0.5 *(O M 1+T \times 1+O M 2 * T \times 2)$

$T M P=T M M-T T E R M+R F L$

$T L P M P=T L P M M-T T E R M * R F L P$

$T L M M P=T L M M M-T T E R M+R F L M$

IF (ITM.EO.O.AND.CAV.EO.O.O) GO TO 790

ROTERM $=0.5 *(O M 1+R O \times 1+O M 2+R O \times 2)$

ROMP $=R O M M-R O T E R M+R F L$

$R O L P M P=R O(L+1, M-1, N 1)-R O T E R M * R F L P$

$R O L M M P=R O(L-1, M-1, N 1)-R O T E R M * R F L M$

IF (ITM.LE. 1) GO TO 790

OTERM $=0.5 *(O M 1 * O \times 1+O M 2+Q \times 2)$

$O M P=O M M-Q T E R M+R F L$

$O L P M P=Q(L+1 . M-1 . N 1)-O T E R M+R F L P$

$O L M M P=O(L-1, M-1, N 1)-O T E R M+R T L M$

IF (ITM.EO. 2) GO TO 790

$E T E R M=0.5+(O M 1+E X 1+O M 2 * E \times 2)$

$E M P=E M M-E T E R M+R F L$

$E L P M P=E(L+1, M-1, N 1)-E T E R M+R F L P$

$E L M M P=E(L-1, M-1 . N 1)-E T E R M+R F L M$

GO TO 780

$780 U M P=U(L, M, N i)+F 2 I *(U(L, M, N 1)-U M M)$

$V M P=V(L, M, N 1)+F 2 I *(V(L, M, N 1)-V M M)$

$U L P M P=U(L+1, M, N 1)+F 2 I *(U(L+1, M, N 1)-U(L+1, M-1, N 1))$

$V L P M P=V(L+1, M, N 1)+F 2 I+(V(L+1, M, N 1)-V(L+1, M-1, N 1))$

$U L M M P=U(L-1, M \cdot N 1)+F 2 I \cdot(U(L-1, M, N 1)-U(L-1, M-1, N 1))$

$V L M M P=V(L-1, M \cdot N 1)+F 2 I *(V(L-1, M, N 1)-V(L-1, M-1, N 1))$

$T M P=T+F 2 I *(T-T M M)$

$T L P M P=T L P+F 2 I+(T L P-T L P M M)$

TLMMP TLM+F $2 I$ * (TLM-TLMMM)

IF (ITM.EO.O.AND.CAV.EO.O.O) GO TO 790

$R O M P=R O(L, M, N 1)+F 2 I *(R O(L, M, N 1)-R O M M)$

$R O L P M P=R O(L+1, M, N I)+F 2 I+(R O(L+1, M, N 1)-R O(L+1, M-1, N 1))$

$R O L M M P=R O(L-1, M, N 1)+F 2 I *(R O(L-1, M, N 1)-R O(L-1, M-1, N 1))$

IF (ITM.L.F.1) GO TO 790

$Q M P=Q(L, M, N 1)+F 2 I *(Q(L, M, N 1)-O M M)$

$Q L P M P=Q(L+1, M, N 1)+F 2 I * i(L(L+1, M, N 1)-Q(L+1, M-1, N 1))$

$Q L M M P=Q(L-1, M, N 1)+F 2 I *(Q(L-1, M, N 1)-Q(L-1, M-1, N 1))$

IF (ITM.EQ.2) GO TO 790

$E M P=E(L, M, N 1)+F 2 T *\left(F_{2}\left(I_{0}, M, N 1\right)-E M M\right)$

$E L P M P=E(L+1, M, N 1)+F 2 I *(E(L+1, M, N 1)-E(L+1, M-1, N 1))$ 


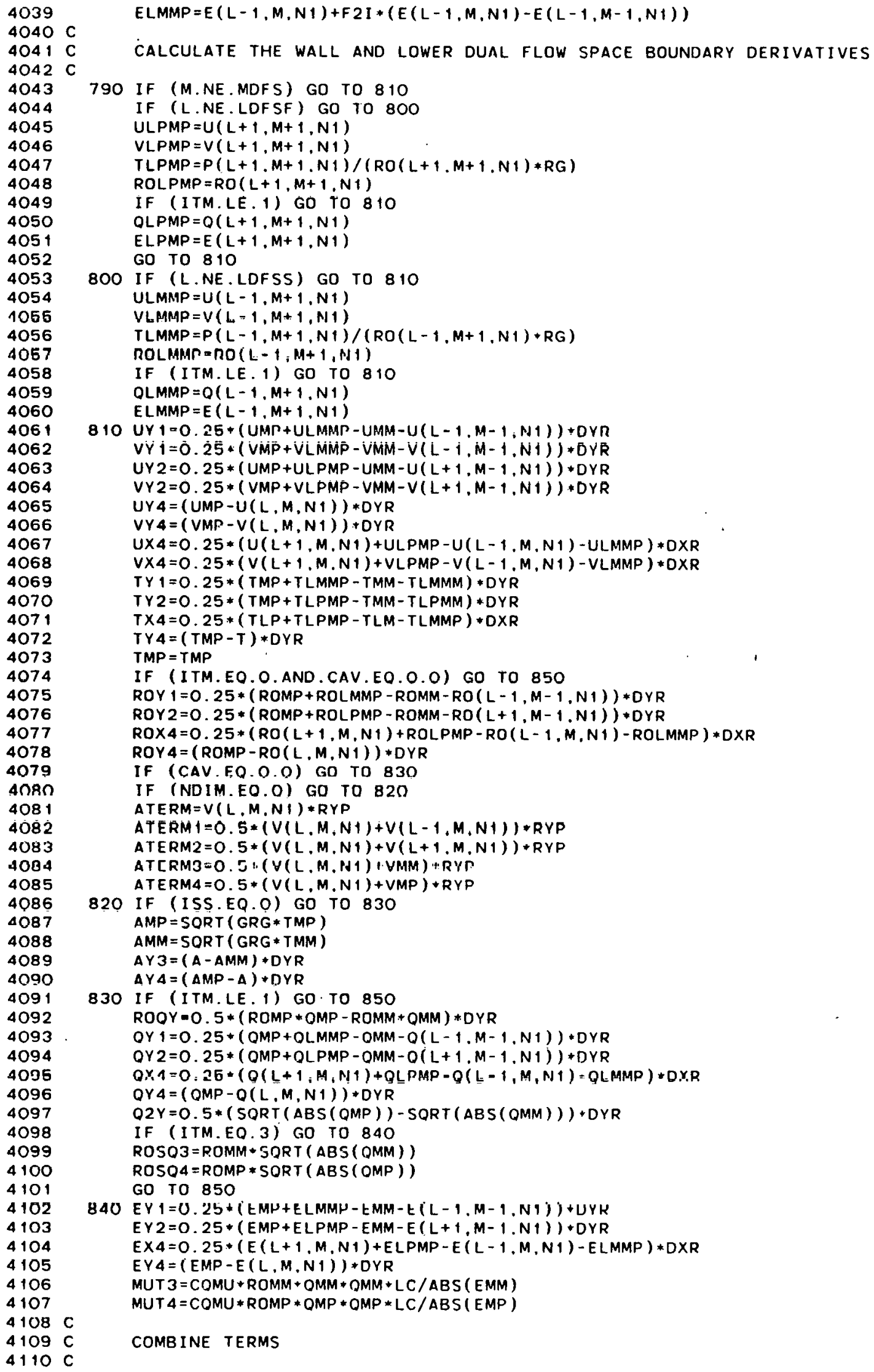

$E L M M P=E(L-1, M, N 1)+F 2 I *(E(L-1, M, N 1)-E(L-1, M-1, N 1))$

CALCULATE THE WALL AND LOWER DUAL FLOW SPACE BOUNDARY DERIVATIVES

790 IF (M.NE.MDFS) GO TO 810

IF ( L.NE.LDFSF) GO TO 800

$U L P M P=U(L+1, M+1, N 1)$

$V L P M P=V(L+1, M+1, N 1)$

$T L P M P=P(L+1 . M+1 . N 1) /(R O(L+1 . M+1 . N 1) * R G)$

$R O L P M P=R O(L+1, M+1, N 1)$

IF (ITM.LE.1) Go To 810

$Q L P M P=Q(L+1, M+1, N 1)$

$E L P M P=E(L+1, M+1, N 1)$

GO TO 810

800 IF (L.NE.LDFSS) GO TO 810

$U L M M P=U(L-1, M+1, N 1)$

$V L M M P=V(L-1, M+1, N 1)$

$T L M M P=P(L-1, M+1, N 1) /(R O(L-1, M+1, N 1)+R G)$

ROLMMP $=n O(L-1 ; M+1, N 1)$

IF (ITM.LE. 1) GO TO 810

$O L M M P=Q(L-1, M+1, N 1)$

$E L M M P=E(L-1, M+1, N 1)$

810 UY $1=0.25 *(U M P+U L$ PAMP $-U M A-U(L-1, M-1, N 1)) * D Y R$

$V Y i=0.25 *(V M M P+V L L M M T \bar{P}-V M M M-V(L-1, M-1, N 1)) \neq O Y R$

$U Y 2=0.25 *(U M P+U L P M P-U M M-U(L+1, M-1, N 1)) * D Y R$

$V Y 2=0.25 *(V M P+V L P M P-V M M-V(L+1, M-1, N 1)) * D Y R$

UY4 $=(U M P-U(L, M, N 1)) * D Y R$

$V Y 4=(V M P-V(L, M, N 1))+D Y R$

$U \times 4=0.25 *(U(L+1, M, N 1)+U L P M P-U(L-1, M, N 1)-U L M M P) * D \times R$

$V \times 4=0.25 *(V(L+1, M . N 1)+V L P M P-V(L-1, M, N 1)-V L M M P) * D \times R$

$T Y 1=0.25 *(T M P+T L M M P-T M M-T L M M M) * D Y R$

$T Y 2=0.25 *(T M P+T L P M P-T M M-T L P M M) * D Y R$

$T X 4=0.25 *(T L P+T L P M P-T L M-T L M M P) * D X R$

$T Y 4=(T M P-T) * D Y R$

$T M P=T M P$

IF (ITM.EO.O.AND.CAV.EQ.O.O) GO TO 850

ROY $1=0.25 *(R O M P+R O L M M P-R O M M-R O(L-1, M-1, N 1)) * D Y R$

$R O Y 2=0.25 *(R O M P+R O L P M P-R O M M-R O(L+1, M-1 . N 1)) * D Y R$

$R O X 4=0.25 *(R O(L+1, M, N 1)+R O L P M P-R O(L-1, M, N 1)-R O L M M P) * D X R$

ROY $4=(R O M P-R O(L, M, N 1)) * D Y R$

IF (CAV.FO.O.O) GO TO 830

IF (NDIM.EO.O) GO TO 820

$\triangle T E R M=V(L, M, N I) * R Y P$

$\triangle T E R M 1=0.5 *(V(L, M, N 1)+V(L-1, M, N 1)) * R Y P$

$\triangle \operatorname{ATERM2}=0.5 *(V(L, M, N 1)+V(L+1, M, N 1)) * R Y P$

ATLRM $=0.5 \div(V(L, M . N 1)+V M M) \backsim R Y \Gamma$

ATERM4 $=0.5 *(V(L, M . N 1)+V M P)+R Y P$

820 IF (ISS.EQ.O) GO TO 830

$A M P=S Q R T(G R G * T M P)$

$A M M=S O R T(G R G * T M M)$

$A Y 3=(A-A M M) * D Y R$

$A Y A=(\triangle M P-A)+D Y R$

830 IF (ITM.LE. 1) GO TO 850

$R O O Y=0.5 *(R O M P * O M P-R O M M * O M M) * D Y R$

OY $1=0.25 *(Q M P+O L M M P-O M M-O(L-1 . M-1, N 1)) * D Y R$

OY $2=0.25 *(O M P+Q L P M P-O M M-O(L+1 . M-1 . N 1))+D Y R$

$Q \times 1=0: 25 *(Q(L+1 ; M, N 1)+Q L P M P-Q(L-1, M, N 1)=Q L M M P) * D, X R$

OY $4=(O M P-O(L, M, N 1)) * D Y R$

$Q 2 Y=0.5 *(\operatorname{SQRT}(A B S(Q M P))-\operatorname{SQRT}(\triangle B S(Q M M))) * D Y R$

IF (ITM.EQ.3) GO TO 840

ROSO3 $=$ ROMM + SORT $(A B S(O M M))$

ROSO4 $=$ ROMP * SORT ( ABS (OMP) ) GO TO 850

$840 E Y 1=0.25 *(E M P+E L M M P-E M M-E(L-1, M-1, N 1))+U Y K$ $E Y 2=0.25+(E M P+E L P M P-E M M-E(L+1, M-1 . N 1))+D Y R$ $E X 4=0.25 *(E(L+1, M, N 1)+E L P M P-E(L-1, M, N 1)-E L M M P) * D X R$ $E Y 4=(E M P-E(L, M, N 1)) * D Y R$ MUT $3=C O M U * R O M M+O M M+O M M+L C / A B S(E M M)$ MUT $4=C O M U * R O M P * Q M P * O M P * L C / A B S(E M P)$

COMBINE TERMS 


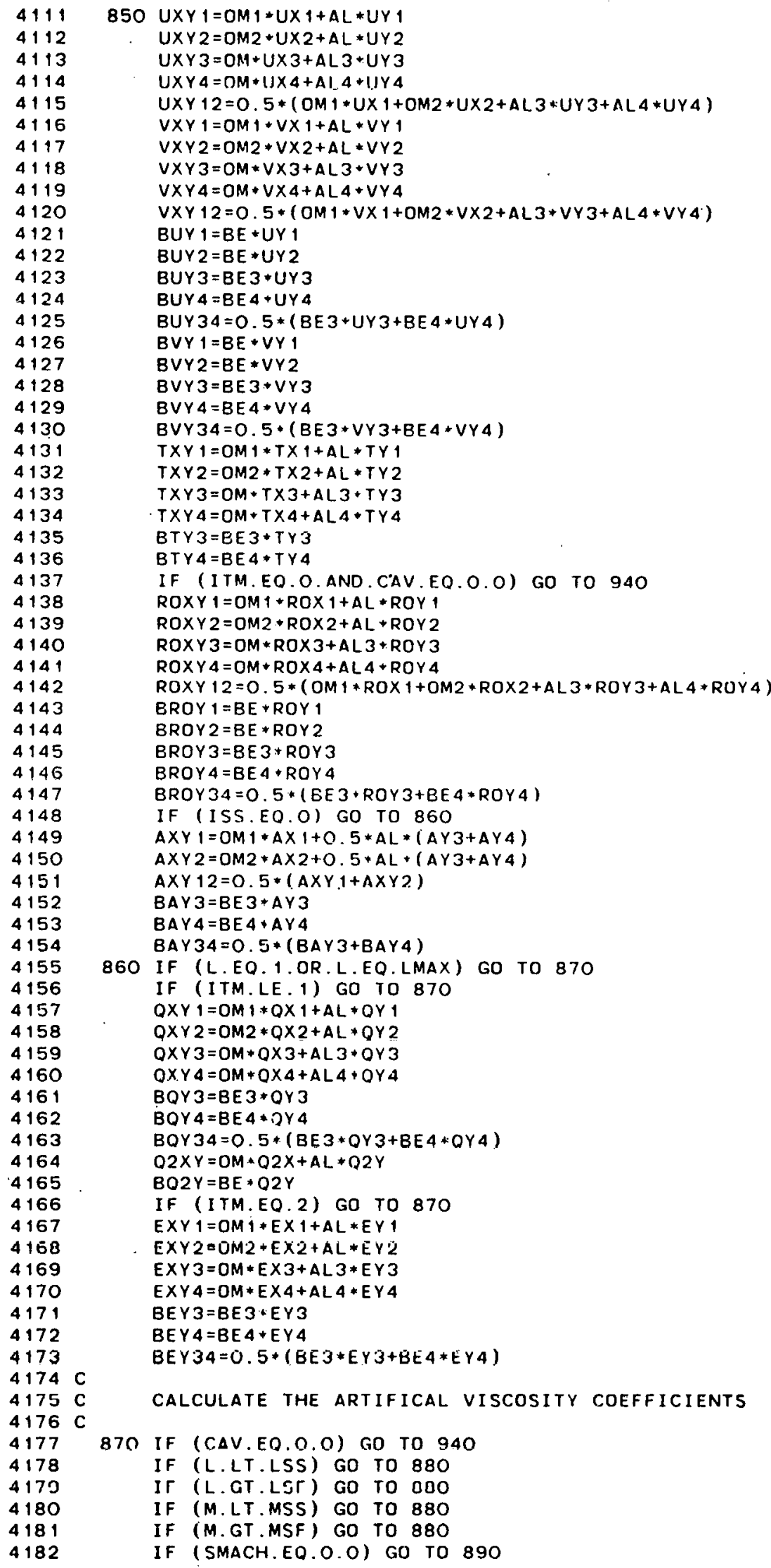

$850 U X Y 1=O M 1 * U X 1+A L * U Y 1$ $U \times Y 2=O M 2+U \times 2+A L * U Y 2$ $U \times Y 3=O M+U \times 3+A L 3+U Y 3$ $U \times Y 4=O M+11 \times 4+A I 4+1 . Y 4$ $U X Y 12=0.5 *(O M 1 * U X 1+O M 2 * U \times 2+A L 3 * U Y 3+A L 4 * U Y 4)$

$V X Y 1=O M 1+V X 1+A L * V Y 1$

$V \times Y 2=O M 2 * V \times 2+A L * V Y 2$

$V \times Y 3=O M * V \times 3+A L 3 * V Y 3$

$V \times Y 4=O M+V \times 4+A L 4+V Y 4$

$V X Y 12=0.5 *(O M 1 * V X 1+O M 2 * V \times 2+A L 3 * V Y 3+A L 4 * V Y 4)$

$B U Y 1=B E+U Y 1$

$B U Y 2=B E * U Y 2$

$B U Y 3=B E 3 * U Y 3$

BUY $4=8 E 4+U Y 4$

$B U Y 34=0.5 *(B E 3+U Y 3+B E 4 * U Y 4)$

$B \vee Y 1=B E+V Y 1$

$B \vee Y 2=B E * V Y 2$

$B \vee Y 3=B E 3+\vee Y 3$

$B \vee Y 4=B E 4 * V Y 4$

$B V Y 34=0.5 *(B E 3 * V Y 3+B E 4 * V Y 4)$

$T X Y 1=O M 1 * T X 1+A L * T Y 1$

$T X Y 2=0 M 2+T \times 2+A L * T Y 2$

$T X Y 3=O M+T \times 3+A L 3+T Y 3$

$T X Y 4=O M+T \times 4+A L 4+T Y 4$

$B T Y 3=B E 3 * T Y 3$

$B T Y 4=B E 4 * T Y 4$

IF (ITM.EO.O.AND.CAV.EO.O.O) GO TO 940

ROXY I $=O M 1+R O X 1+A L * R O Y 1$

$R O X Y 2=O M 2+R O \times 2+A L+R O Y 2$

$R O X Y 3=O M * R O X 3+A L 3+R O Y 3$

$R O X Y 4=O M+R O X 4+A L 4+R O Y 4$

$R O X Y 12=0.5 *(O M 1 * R O X 1+O M 2 * R O \times 2+A L 3 * R O Y 3+A L 4 * R O Y 4)$

$B R O Y 1=B E+R O Y 1$

$B R O Y 2=B E * R O Y 2$

$B R O Y 3=8 E 3 *$ ROY 3

BROY $4=B E 4 *$ ROY 4

BROY $34=0.5 *(E E 3 * \operatorname{ROY} 3+B E 4 * \operatorname{ROY} 4)$

IF (ISS.EQ.O) GO TO 860

$A X Y 1=O M 1 * A X 1+0.5 * A L *(A Y 3+A Y 4)$

$A X Y 2=O M 2 * A X 2+0.5 * A L+(A Y 3+A Y 4)$

$A X Y 12=0.5 *(A X Y .1+A X Y 2)$

$B A Y 3=B E 3 * A Y 3$

$B A Y 4=B E 4+A Y 4$

BAY $34=0.5 *(B A Y 3+B A Y 4)$

860 IF (L.EQ.1.OR.L.EQ.LMAX) GO TO 870

IF (ITM.LE.1) GO TO 870

$Q X Y 1=O M 1 * Q \times 1+A L * O Y 1$

$Q \times Y 2=O M 2 * Q \times 2+A L * Q Y 2$

$Q X Y 3=O M * Q \times 3+A L 3 * O Y 3$

OXY $4=O M * Q \times 4+A L 4+O Y 4$

$B Q Y 3=B E 3 *$ QY 3

BOY $4=B E 4 *$ DY 4

$B Q Y 34=0.5 *(B E 3 * O Y 3+B E 4 * Q Y 4)$

$O 2 X Y=O M * Q 2 X+A L * Q 2 Y$

$B 02 Y=B E * 02 Y$

IF (ITM.EQ.2) GO TO 870

EXY $1=O M 1+E \times 1+A L * E Y 1$

$E X Y 2=O M 2+E X 2+A L+E Y 2$

$E X Y 3=O M * E \times 3+A L 3 * E Y 3$

$E X Y 4=O M * E X 4+A L 4 * E Y 4$

$B E Y 3=B E 3 * E Y 3$

BEY $4=B E 4+E Y 4$

$B E Y 34=0.5 *(B E 3 * E Y 3+B E 4 * E Y 4)$

CALCULATE THE ARTIFICAL VISCOSITY COEFFICIENTS

870 IF (CAV.EO.O.O) GO TO 940

IF (L.LT.LSS) GO TO 880

IT (L.GT.LST) GD TO 000

IF (M.LT.MSS) GO TO 880

IF (M.GT.MSF) GO TO 880

IF (SMACH.EQ.O.O) GO TO 890 


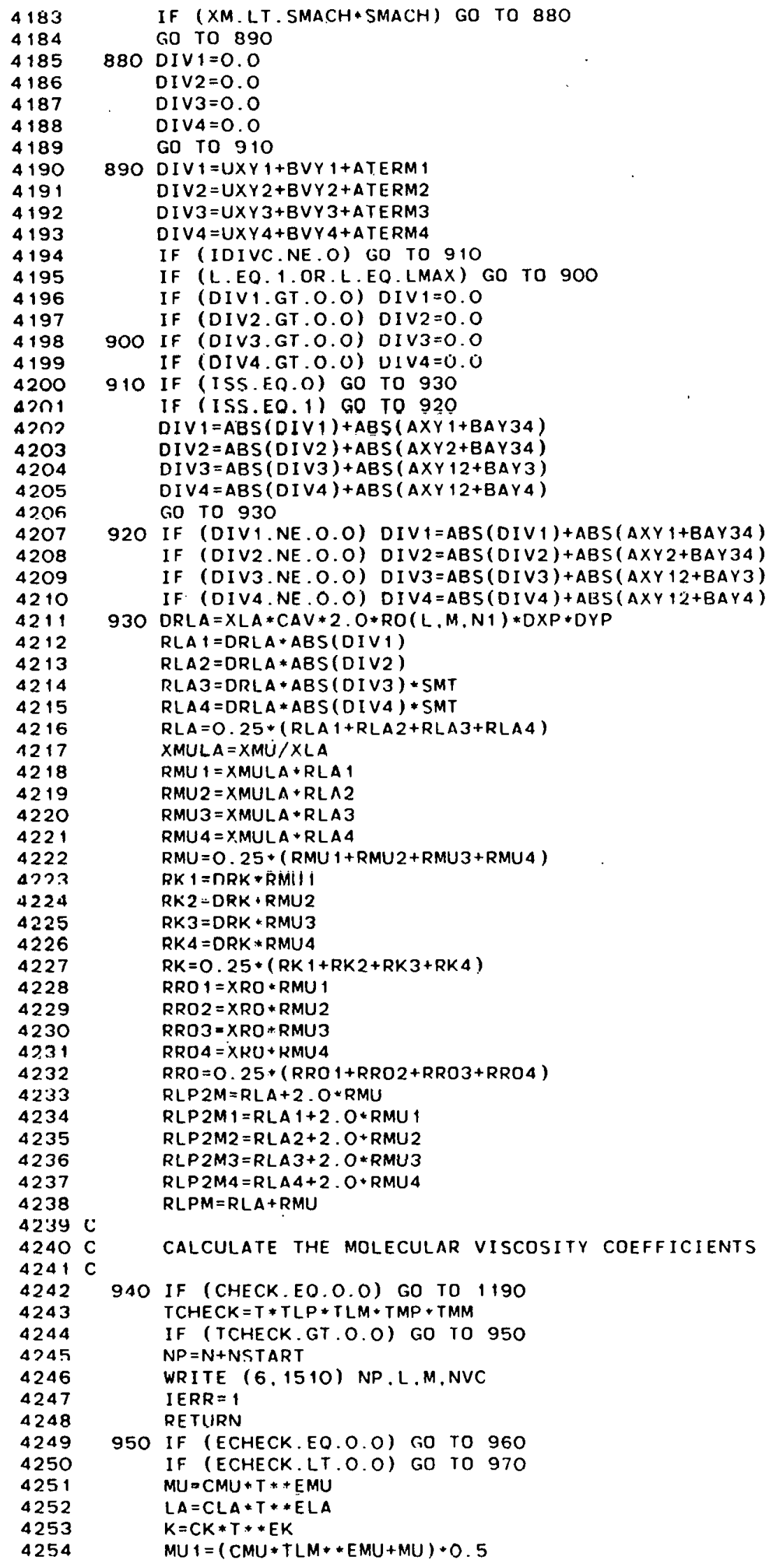




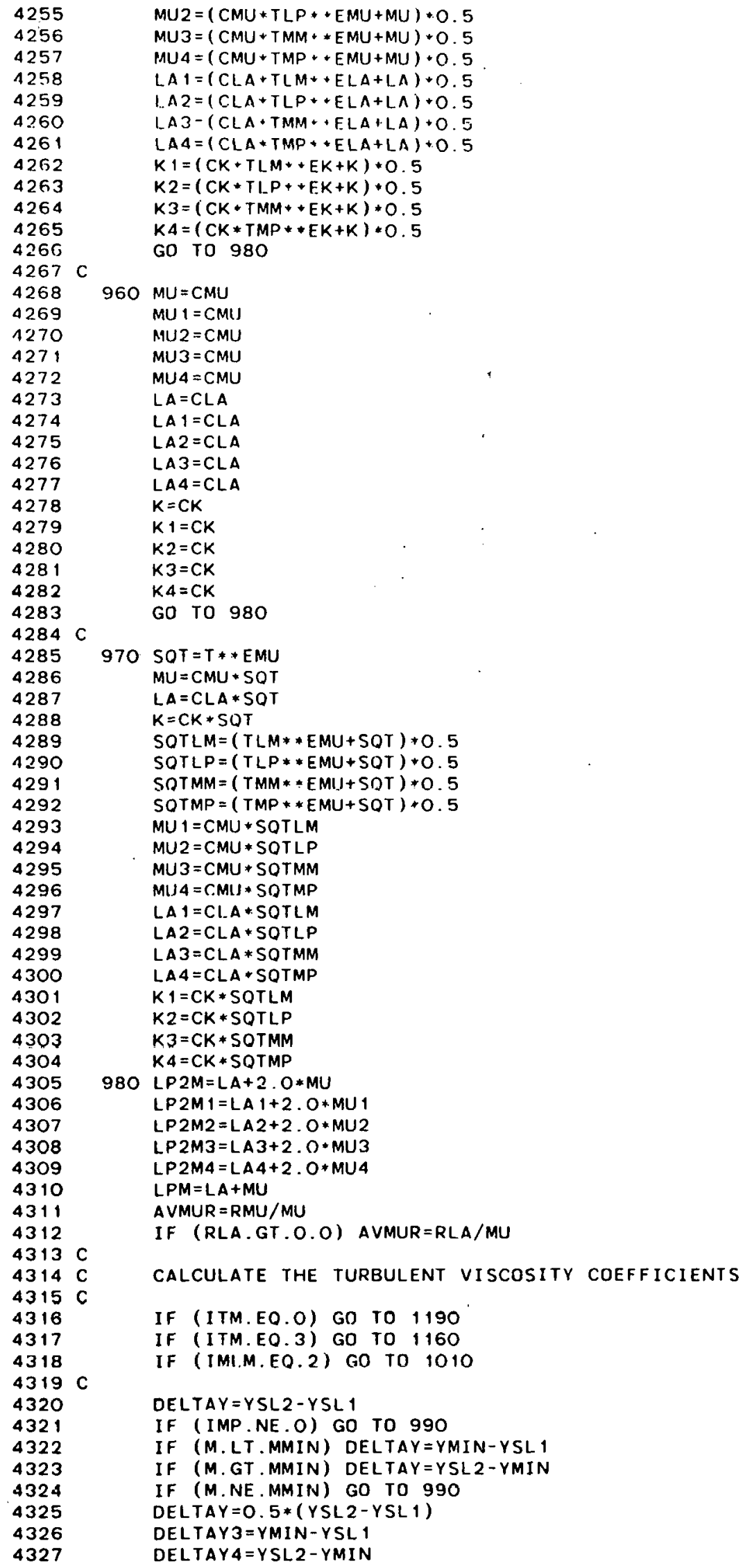




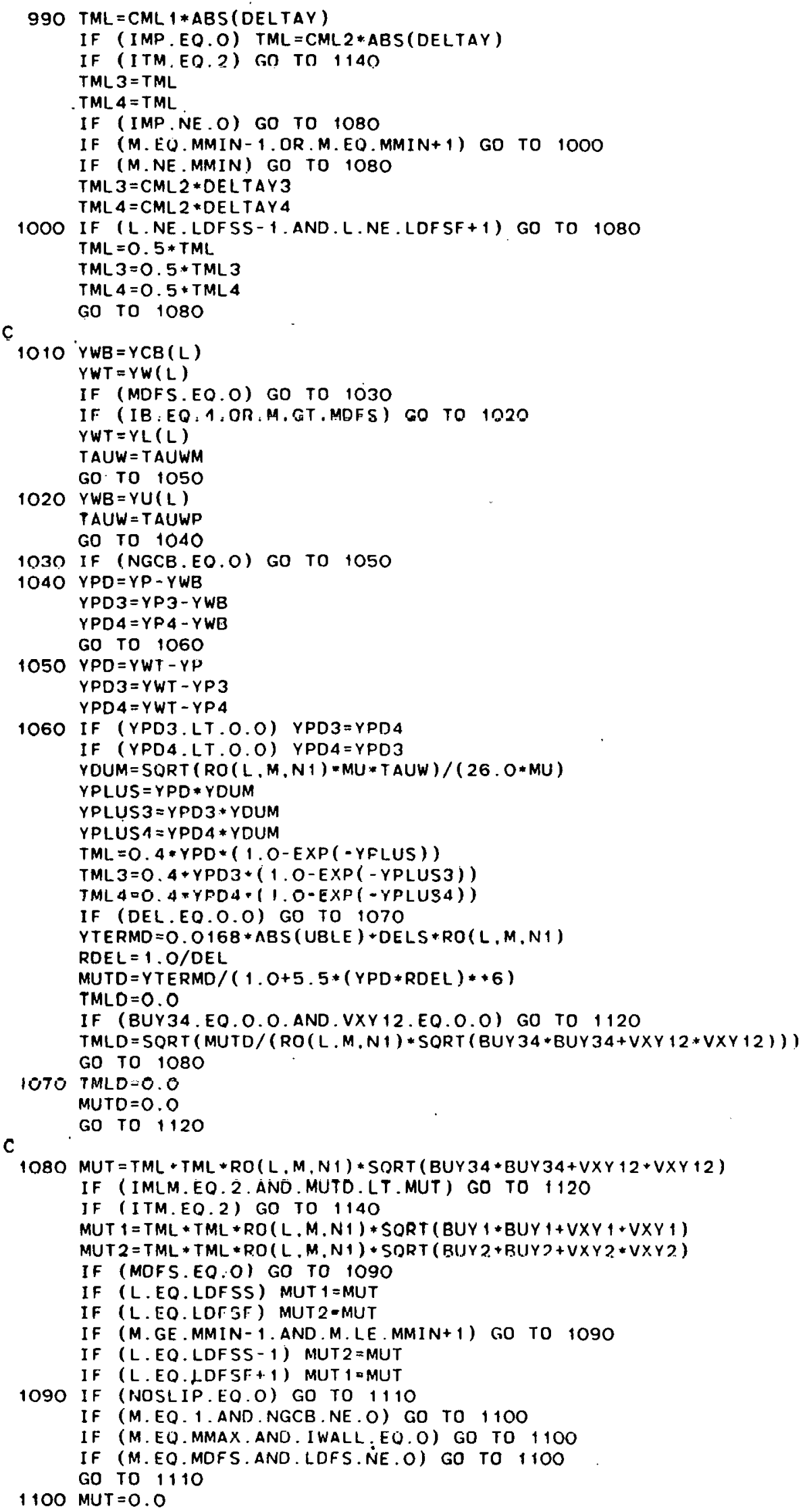




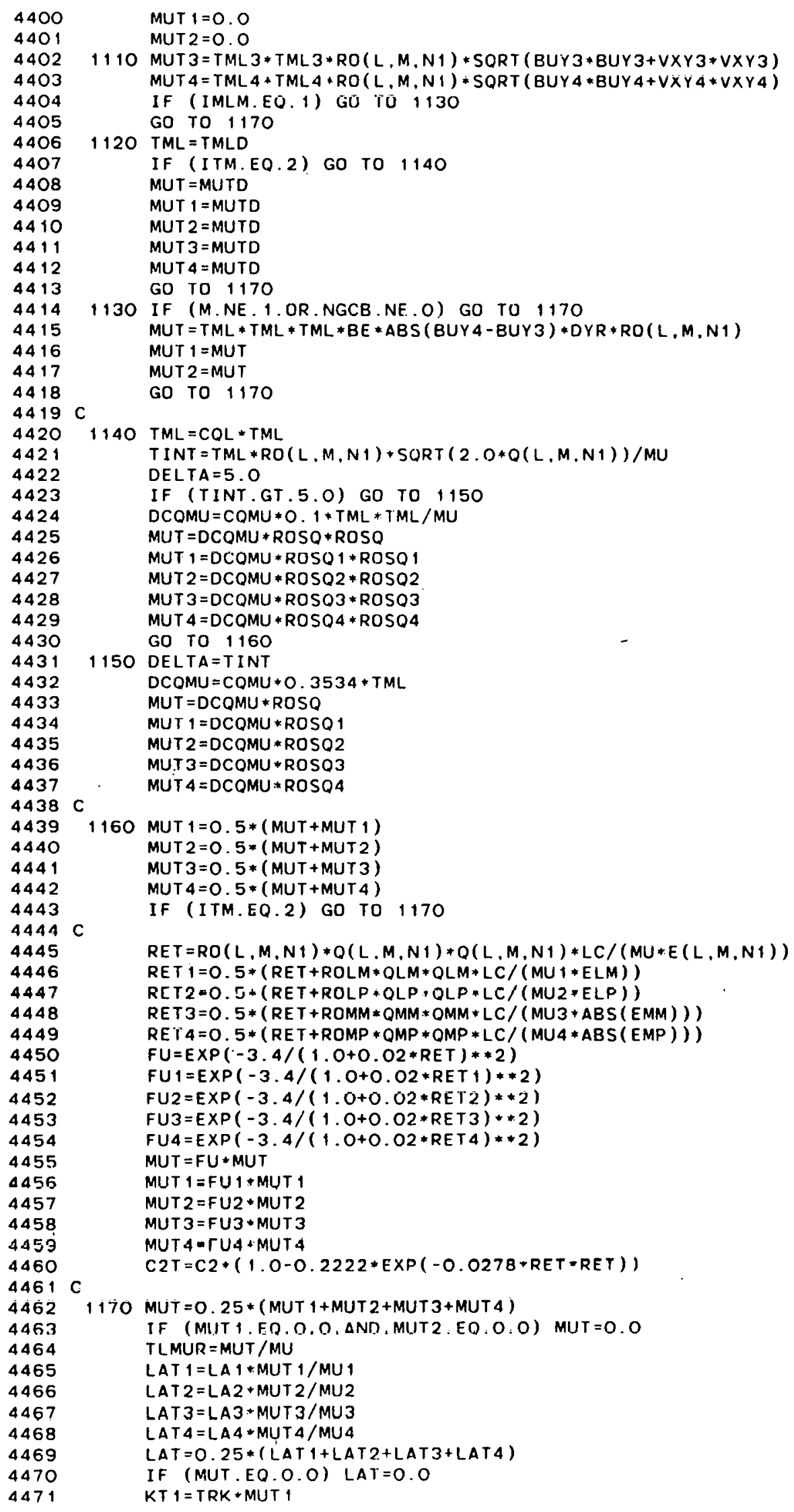




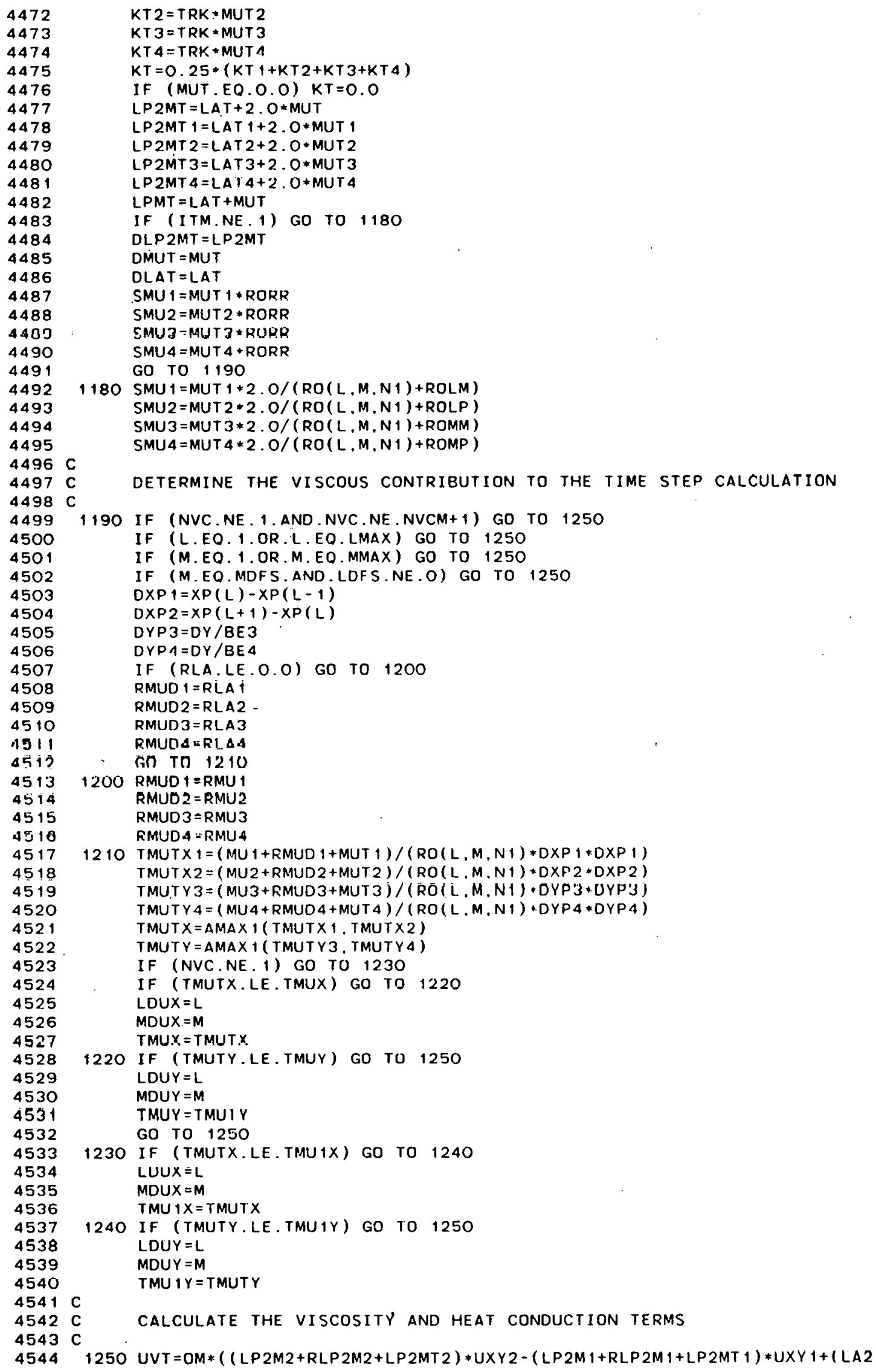


4545

4546

4547

4548

4549

4550

4551

4552

4553

4554

4555

4556

4557

4558

4559

4560

4561

4562

4563

4564

4565

4566

4567

4568

4569

4570

4571

4572

4573

4574

4575

4576

4577

4578

4579

4580

4581

4582

4583

4584

4585

4586

4587

4588

4589

4590

4591

4592

4593

4594

4595

4596

4597

4598

4599

4600

4601

4602

4603

$4604 \mathrm{C}$

$4605 \mathrm{C}$

$4606 \mathrm{C}$

4607

4608

4609

4610

4611

4612

4613

4614

4615

4616

1 +RLA2 +LAT2) *BVY2 - (LA 1+RLA 1+LAT 1)*BVY 1) *DXR+AL * ( (LP2M4+RLP2M4

$2+L P 2 M T 4) * U X Y 4-(L P 2 M 3+R L P 2 M 3+L P 2 M T 3) * U X Y 3+(L A 4+R L A 4+L A T 4) * 6 V Y 4-$

$3($ LA $3+R L A 3+L A T 3) *$ EVY 3$) * D Y R+B E *((M U 4+R M U 4+M U T 4) * V X Y 4-(M U 3+R M U 3+M U T 3$

$4) * V X Y 3+(M U 4+R M U 4+M U T 4) * B U Y 4-($ MU3+RMUं3+MUT3)*BUY3) *DYR

$V V T=O M *((M U 2+R M U 2+M U T 2) *(V X Y 2+B U Y 2)-(M U 1+R M U 1+M U T 1) *(V X Y 1+B U Y 1))$

$1 * D \times R+A L *((M U 4+R M U 4+M U T 4) * V X Y 4-(M U 3+R M U 3+M U T 3) * V X Y 3+(M U 4+R M U 4+M U T 4$

2 ) *BUY 4-(MU3+RMU3+MUT 3)*BUY3)*DYR+BE* ( (LA4+RLA4+LAT4)*UXY4-(LA3

$3+R L A 3+L A T 3) * U X Y 3+(L P 2 M 4+R L P 2 M 4+L P 2 M T 4) * B V Y 4-(L P 2 M 3+R L P 2 M 3+L P 2 M T 3)$

$4 * B \vee Y 3) * D Y R$

$P V T=(L P 2 M+R L P 2 M+D L P 2 M T) *(U \times Y 12 * U X Y 12+B V Y 34 * B V Y 34)+(M U+R M U+D M U T) *$

$1(V X Y 12 * V X Y 12+B U Y 34 * B U Y 34)+2 . O *(L A+R L A+D L A T) * U X Y 12 * B V Y 34+2.0 *(M U$

$2+R M U+D M U T) * B U Y 34 * V X Y 12$

$P C T=O M *((K 2+R K 2+K T 2) * T X Y 2-(K 1+R K 1+K T 1) * T \times Y 1) * D X R+A L *((K 4+R K 4+K T 4)$

$1 * T \times Y 4-(K 3+R K 3+K T 3) * T X Y 3) * D Y R+B E *((K 4+R K 4+K T 4) * B T Y 4-(K 3+R K 3+K T 3)$

$2 * B T Y 3) * D Y R$

IF (ITM.EQ.O.AND.CAV.EQ.O.O) GO TO 1280

$R O D$ I $F F=O M *((C A L * S M U 2+R O R R * R R O 2) * R O \times Y 2-(C A L * S M U 1+R O R R * R R O 1) * R O \times Y 1)$

$1 * D X R+A L *($ ( CAL *SMU4+RORR*RRO4)*ROXY4-(CAL*SMU3+RORR*RRO3)*ROXY3)

2 *DYR+BE * ( (CAL *SMU4+RORR*RRO4)*BROY $4-(C A L * S M U 3+R O R R * R R O 3) * B R O Y 3)$

$3 *$ DYR

IF (ITM.EO.O) GO TO 1280

UROT $=-0.67 *(O M * R O Q X+A L * R O Q Y)+C A L *(U(L, M, N 1) *(O M *(S M U 2 * R O X Y 2-S M U 1$

$1 * R O X Y 1) * D \times R+A L *(S M U 4 * R O X Y 4-S M U 3 * R O X Y 3) * D Y R)+B E * V(L, M, N 1) *(S M U 4$

$2 * R O X Y 4-S M U 3 * R O X Y 3) * D Y R)$

$V R O T=-0.67 * B E * R O Q Y+C A L *(V(L, M, N 1) * B E *(S M U 4 * B R O Y 4-S M U 3 * B R O Y 3) * D Y R+U$

$1(L, M, N 1) *(O M *(S M U 2 * B R O Y 2-S M U 1 * B R O Y 1) * D X R+A L *(S M U 4 * B R O Y 4-S M U 3$

$2 * B R O Y 3) * D Y R)$ )

RODUMT $=O M *(S M U 2+R O X Y 2-S M U 1+R O X Y 1)+D X R+A L *(S M U 4 * R O X Y 4-S M U 3 * R O X Y 3)$

$1 * D Y R+B E *(S M U 4 * B R O Y 4-S M U 3 * B R O Y 3) * D Y R$

$P R O T=-C A L * R G * T * R O D U M T$

IF (IES.NE.O) GO TO 1280

IF (L.EQ.LMAX.OR.L.EQ.1) GO TO 1290

IF (ITM.EQ.1) GO TO 1280

OPROD $=$ LP $2 M T *(U X Y 12 * U X Y 12+B V Y 34 * B V Y 34)+M U T *(V X Y 12 * V X Y 12+B U Y 34 * B U Y 34$

$1)+2 . O+L A T * U X Y 12 * B \vee Y 34+2 . O * M U T * B U Y 34 * V \times Y 12$

ODI FF $=O M *(($ MU2 +MUT 2*SIGOR )*QXY2 $-(M U 1+M U T 1 * S I G Q R) * O \times Y 1) * D \times R+A L *($

1 (MU4+MUT $4 *$ SIGQR)*QXY4-(MU3+MUT3*SIGOR)*QXY3)*DYR+BE*( (MU4+MUT4

2 -SIGQR ) *BOY4-(MU3+MUT3*SIGOR)*BOY3)*DYR

OROTT $=-X I T M * O(L, M, N 1) * R O(L, M, N 1) *(U X Y 12+B V Y 34)$

IF (ITM.EO.3) GO TO 1260

ODI $S S=0.0$

IF (TML.NE.O.O) ODISS $=2 . O * M U * D E L T A * Q(L, M, N 1) /(T M L * T M L)$

GO TO 1280

1260 EPROD $=0.0$

EDISS $=0.0$

IF $(Q(L, M, N 1) . E O . O . O)$ GO 101270

$E P R O D=C 1 * E(L, M, N 1) / O(L, M, N 1) *(L P 2 M T *(U \times Y 12 * U \times Y 12+B V Y 34 * B V Y 34)+M U T *$

$1(V X Y 12 * V X Y 12+B U Y 34+B U Y 34)+2 . O * L A T+U X Y 12 * B V Y 34+2 . O * M U T * B U Y 34 * V X Y 12$ 2)

EDISS $=C 2 T * R O(L, M, N 1) * E(L, M, N 1) *(E(L, M, N 1)-2 . O * M U * R O R R * L C *(Q 2 \times Y$

$1+B Q 2 Y) * * 2) /(Q(L, M, N 1) * L C)$

IF (EOISS.LT.O.O) EDISS $=0.0$

1270 EDIFF $=O M *((M U 2+M U T 2 * S I G E R) * E X Y 2-(M U 1+M U T 1 * S I G E R) * E X Y 1) * D \times R+A L *($ $1($ MU4 + MUT 4*SI GER) *EXY4-(MU3+MUT3*SIGER) *EXY3) *DYR+BE*( (MU4+MUT 4

$2 * S I G E R) * B E Y 4-(M U 3+M U T 3 * S I G E R) * B E Y 3) * D Y R$

$Q D I S S=R O(L, M, N 1) *(E(L, M, N 1)+2 . O * M U * R O R R * L C *(Q 2 X Y+B Q 2 Y)+* 2) / L C$

$E L O W R=2 . O * R O R R * M U * M U T * L C *((O M *(U X Y 2-U X Y 1) * D X R+\Lambda L *(U X Y 4-U X Y 3) * D Y R) *$

$1 * 2+(D M *(V X Y 2-V X Y 1) * D X R+A L *(V X Y 4-V X Y 3) * D Y R) * 2+(B E *(B U Y 4-B U Y 3) * D Y R$

$2) * * 2+(B E *(B \vee Y 4-B \vee Y 3) * D Y R) * * 2)$

- AND E FOURTH ORUER SMUUTHING

IF (STBO.LE.O.O.AND. STBE.LE.O.O) GO TO 1280

$D Q X=Q L P-2 . O+Q(L . M, N 1)+Q L M$

$D Q Y=O M P-2 \cdot O * O(L, M, N 1)+O M M$

$D E X=E L P-2 . O * E(L . M, N I)+E L M$

$D E Y=E M P-2 . O * E(L, M, N 1)+E M M$

$O A V G X=0.25 *(O L P+2.0 * O(L, M, N 1)+Q L M)$

DAVGY $=0.25 *(D M P+2 . O+Q(L, M, N 1)+O M M)$

IF (QAVGX.LE.O.O) QAVGX $=1 . \mathrm{OE}+10$

IF (QAVGY.LE.O.O) OAVGY $=1 . O E+10$

$E A V G X=0.25 *(E L P+2 . O * E(L, M, N 1)+E L M)$ 


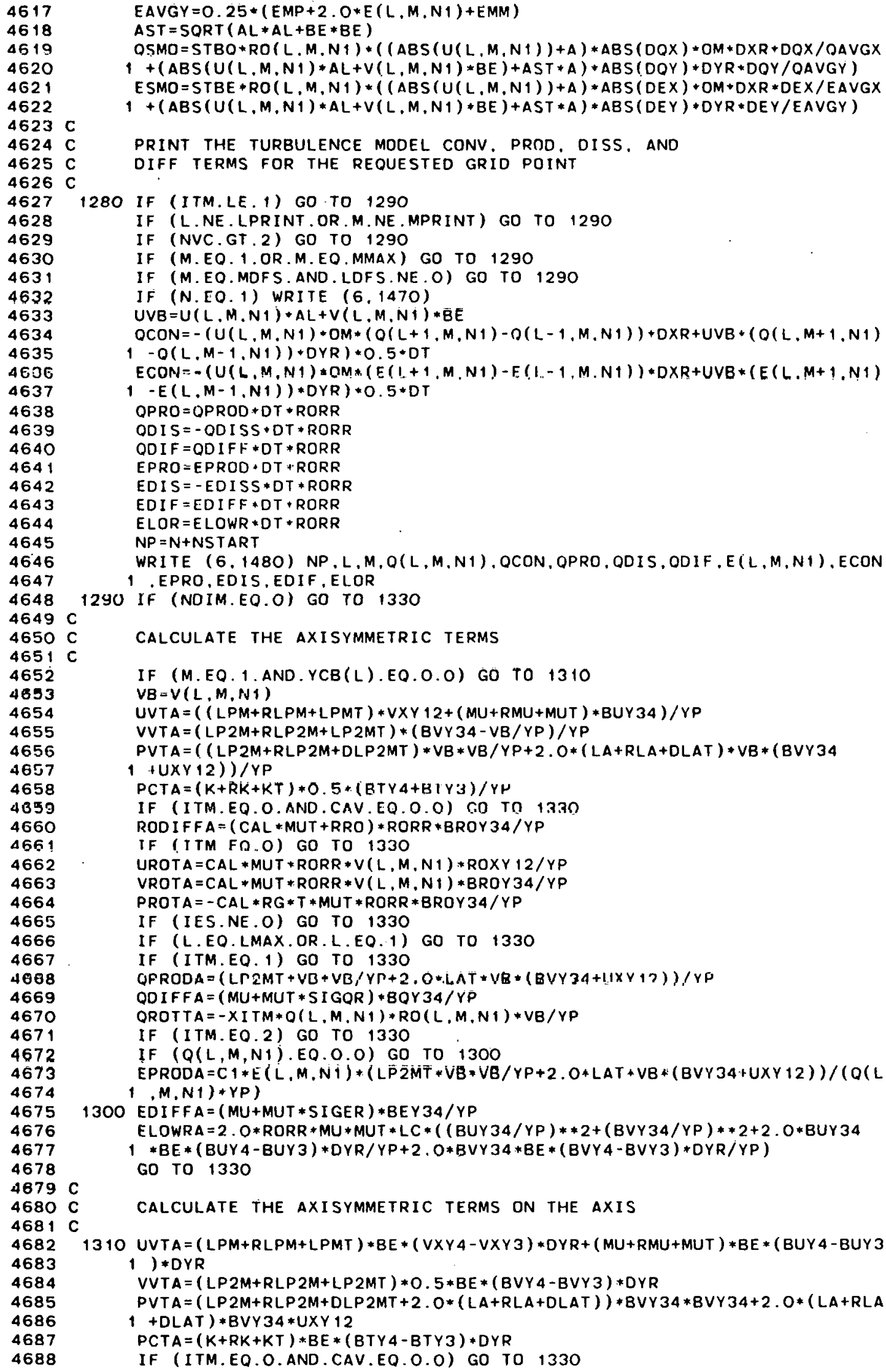




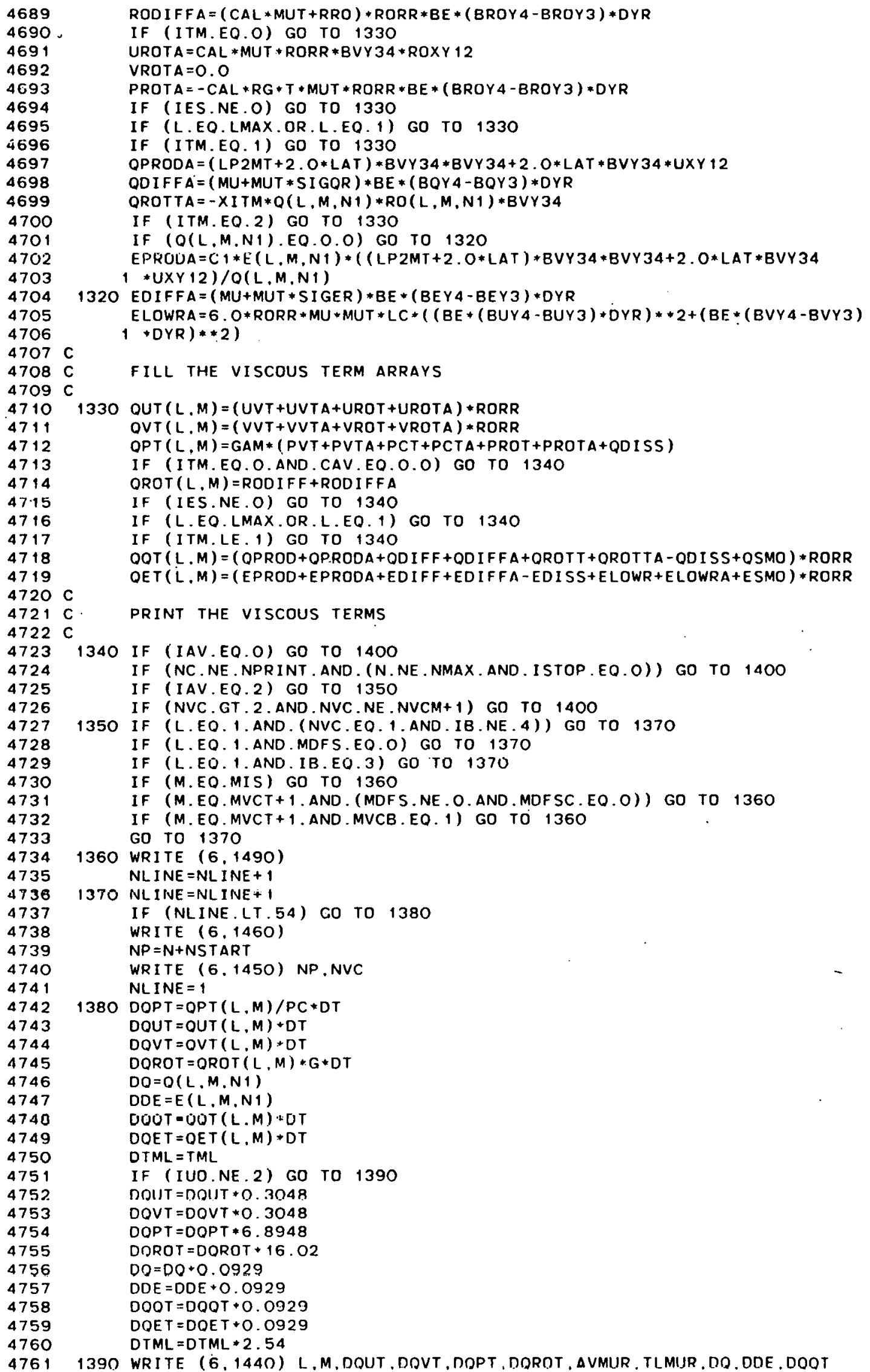




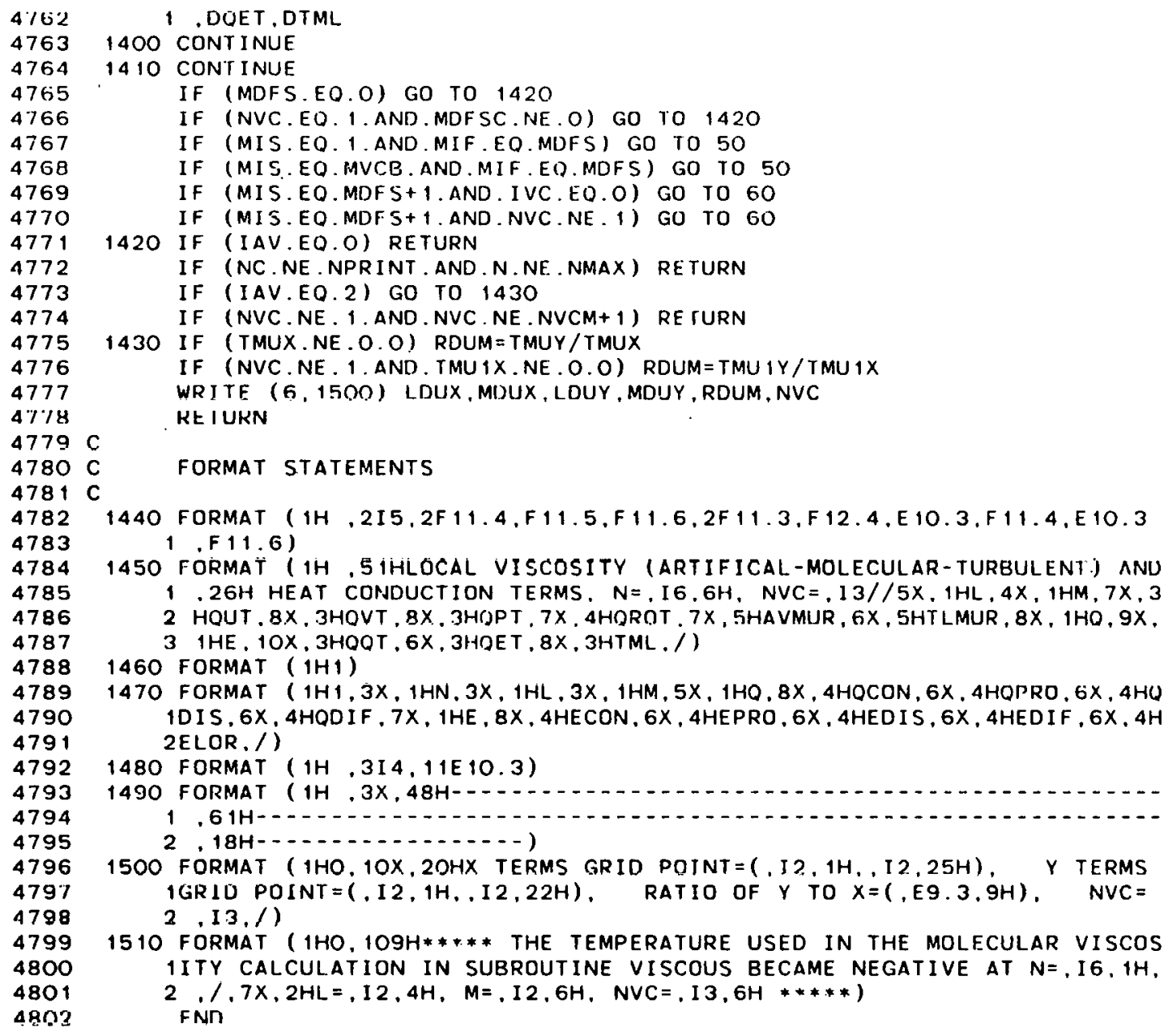

1510 FORMAT (1HO, 109H***** THE TEMPERATURE USED IN THE MOLECULAR VISCOS 1 ITY CALCULATION IN SUBROUTINE VISCOUS BECAME NEGATIVE AT $N=, 16,1 \mathrm{H}$, $2, /, 7 \mathrm{X}, 2 \mathrm{HL}=, 12,4 \mathrm{H}, \mathrm{M}=, 12,6 \mathrm{H}, \mathrm{NVC}=, 13,6 \mathrm{H} * * * * *)$ FNn 


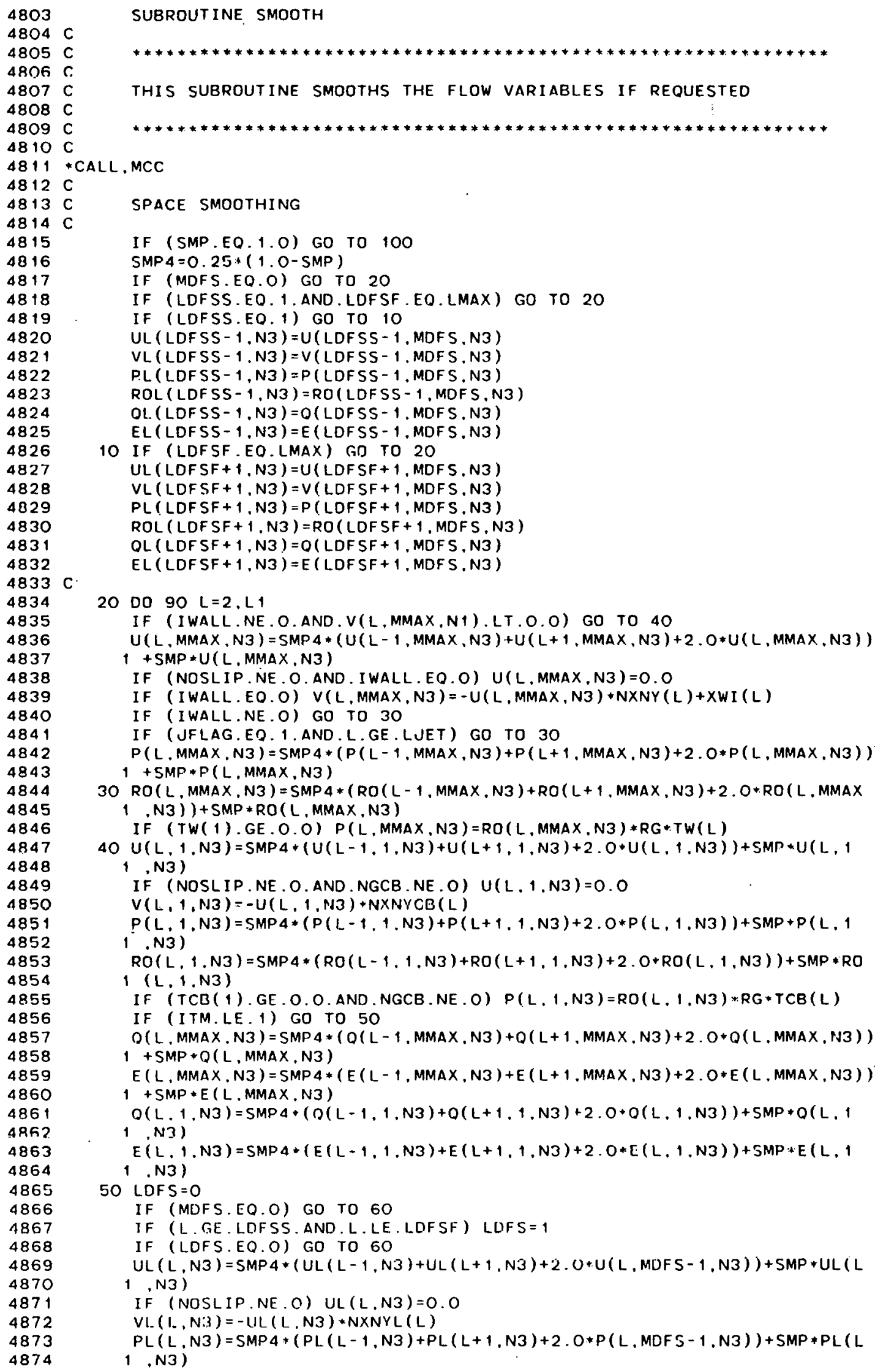


$\operatorname{ROL}(L, N 3)=\operatorname{SMP} 4 *(R O L(L-1 . N 3)+R O L(L+1, N 3)+2.0 * R O(L, M O F S-1, N 3))+S M P$ $1 *$ ROL $(L, N 3)$

IF (TL(1).GE.0.O) PL(L.N3)=ROL (L.N3)*RG*TL(L)

$U(L, M D F S, N 3)=\operatorname{SMP} 4 *(U(L-1, M D F S, N 3)+U(L+1, M D F S, N 3)+2 . O * U(L, M D F S+1, N 3$

$1))+S M P * U(L, M D F S, N 3)$

IF (NOSLIP.NE.O) U(L,MDFS,N3)=0.0

$V(L, M D F S, N 3)=-U(L, M D F S, N 3)=N X N Y U(L)$

$P(L, M D F S, N 3)=S M P 4 *(P(L-1, M D F S, N 3)+P(L+1, M D F S, N 3)+2 . O * P(L, M D F S+1, N 3$ $1))+S M P * P(L, M O F S, N 3)$

$R O(L, M D F S, N 3)=S M P 4 *(R O(L-1, M D F S, N 3)+R O(L+1, M D F S, N 3)+2.0 * R O(L, M D F S+$

$11, N 3))+S M P * R O(L, M D F S, N 3)$

IF (TU( 1).GE .O.O) P(L.MDFS,N3) $=R O(L, M D F S, N 3) * R G * T U(L)$

IF (ITM.LE. 1) GO TO 60

$Q L(L, N 3)=S M P 4 *(Q L(L-1, N 3)+Q L(L+1 . N 3)+2 . O * Q(L . M D F S-1, N 3))+S M P * Q L(L$ 1 N3)

$E L(L, N 3)=\operatorname{SMP} 4 *(E L(L-1, N 3)+E L(L+1, N 3)+2 . O * E(L, M D F S-1, N 3))+S M P * E L(L$

$1, N 3)$

$Q(L, M D F S, N 3)=5 M P 4 *(Q(L-1, M D F S, N 3)+Q(L+1, M D F S, N 3)+2.0 * Q(L, M D F S+1, N 3$

$1)+S M P * O(L, M D F S, N 3)$

$E(L, M D F S, N 3)=S M P 4 *(E(L-1, M D F S, N 3)+E(L+1, M D F S, N 3)+2 . O * E(L, M D F S+1, N 3$

$1))+S M P * E(L, M D F S, N 3)$

60 DO $90 M=2, M 1$

IF (M.EQ.MDFS. AND.LDFS.EQ.1) GO TO 90

IF. (M.NE.MOFS) GO TO 80

IF (L.NE.LDFSS-1. AND.L.NE. LDFSF+1) GO TO 80

IF ( L.NE . LDFSS-1) GO TO 70

$U(L, M, N 3)=5 M P 4+(U(L-1, M, N 3)+U(L, M-1, N 3)+U(L, M+1, N 3)+0.5 *(U)(L+1, M$

$1, N 3)+U L(L+1, N 3)))+S M P+U(L, M, N 3)$

$V(L, M, N 3)=\operatorname{SMn} 4 *(V(L-1, M, N 3)+V(L, M-1, N 3)+V(L, M+1, N 3)+0,5+(V(L+1, M$

$1, N 3)+V L(L+1, N 3)))+S M P \neq V(L, M, N 3)$

$P(L, M, N 3)=S M P 4 *(P(L-1, M, N 3)+P(L, M-1, N 3)+P(L, M+1, N 3)+0.5 *(P(L+1, M$

$1 . N 3)+P L(L+1 . N 3)))+S M P * P(L, M, N 3)$

$R O(L, M, N 3)=\operatorname{SMP} 4 *(R O(L-1, M, N 3)+R O(L, M-1, N 3)+R O(L, M+1, N 3)+O .5 *(R O(L+$

$11, M, N 3)+R O L(L+1, N 3)))+S M P * R O(L, M, N 3)$

IF (ITM.LE. 1) GD TO 90

$Q(L, M, N 3)=S M P 4 *(Q(L-1, M, N 3)+O(L, M-1, N 3)+Q(L, M+1, N 3)+O .5 *(Q(L+1, M$ $1, N 3)+Q L(L+1, N 3)))+S M P * Q(L, M, N 3)$

$E(L, M, N 3)=5 M P 4 *(E(L-1, M, N 3)+E(L, M-1, N 3)+E(L, M+1, N 3)+0.5 *(E(L+1, M$ $1 . N 3)+E L(L+1 . N 3)))+S M P * E(L . M . N 3)$ GO TO 90

$70 U(L, M, N 3)=S M P 4 *(U(L+1, M, N 3)+U(L, M-1, N 3)+U(1 ., M+1, N 3)+U . S+(U(L-1, M$ $1 ; N 3)+U L(L-1 ; N 3)))+\operatorname{SMP} * U(L, M, N 3)$

$V(L, M, N 3)=S M P 4 *(V(L+1, M, N 3)+V(L, M-1, N 3)+V(L, M+1, N 3)+0.5 *(V(L-1, M$

$1 \quad N 3)+V I .(1-1, N 3)))+S M P * V(L, M, N 3)$

$P(L, M, N 3)=S M P 4 *(P(L+1, M, N 3)+P(L, M-1 . N 3)+P(L . M+1 . N 3)+0.5 *(P(L-1, M$ $1 . N 3)+P L(L-1, N 3)))+S M P * P(L, M, N 3)$

$R \dot{O}(L, M, N 3)=\operatorname{SMP} 4 *(R U(L+1, M, N 3)+R O(L, M-1, N 3)+R O(L, M+1, N 3)+O .5 *(R O(L-$ $11, M, N 3)+\operatorname{ROL}(L-1, N 3)))+\operatorname{SMP} * \operatorname{RO}(L, M, N 3)$

IF (ITM.LE. 1) GO TO 90

$Q(L, M, N 3)=S M P 4 *(Q(L+1, M, N 3)+Q(L, M-1, N 3)+Q(L, M+1, N 3)+0.5 *(Q(L-1, M$

$1, N O)+Q L(L=1, N O))) 15 M P+Q(L: M, N 3)$

$E(L, M, N 3)=\operatorname{SMP} 4 *(E(L+1, M, N 3)+E(L, M-1, N 3)+E(L, M+1, N 3)+0.5 *(E(L-1, M$

$1 . N 3)+E L(L-1 . N 3)))+S M P * E(L . M, N 3)$ GO TO 90

$80 U(L . M . N 3)=S M P 4 *(11(1-1, M, N 3)+U(I+1, M, N 3)+U(L, M-1 . N 3)+U(L . M+1 . N 3))$

$1+\operatorname{SMP} * U(L, M, N 3)$

$V(L, M, N 3)=S M P 4 *(V(L-1, M, N 3)+V(L+1, M, N 3)+V(L, M-1, N 3)+V(L, M+1, N 3))$

$1+S M P * V(L, M, N 3)$

$P(L, M, N 3)=S M P A *(P(L-1, M, N J)+P(L+1, M, N 3)+H(L, M=i, N 3)+P(L, M+1, N 3))$

$1+\operatorname{SMP} * \mathrm{P}(\mathrm{L}, \mathrm{M}, \mathrm{N} 3)$

$R O(L, M, N 3)=\operatorname{SMP} 4 *(R O(L-1, M, N 3)+R O(L+1, M, N 3)+R O(L, M-1, N 3)+R O(L, M+1$

$1, N 3))+S M P \times R O(L, M, N 3)$

IF (ITM.LE. 1) GO TO 90

$Q(L, M, N 3)=S M P 4 *(Q(L-1, M, N 3)+Q(L+1, M, N 3)+Q(L, M-1, N 3)+Q(L, M+1, N 3))$

$1+\operatorname{SMP} \% Q(\mathrm{~L}, \mathrm{M}, \mathrm{N} 3)$

$E(L, M, N 3)=S M P 4 *(E(L-1, M, N 3)+E(L+1, M, N 3)+E(L, M-1, N 3)+E(L, M+1, N 3))$

$1+S M P * E(L, M, N 3)$

90 CONT INUE

TIME SMOOTHING (NTST.EO.1) 


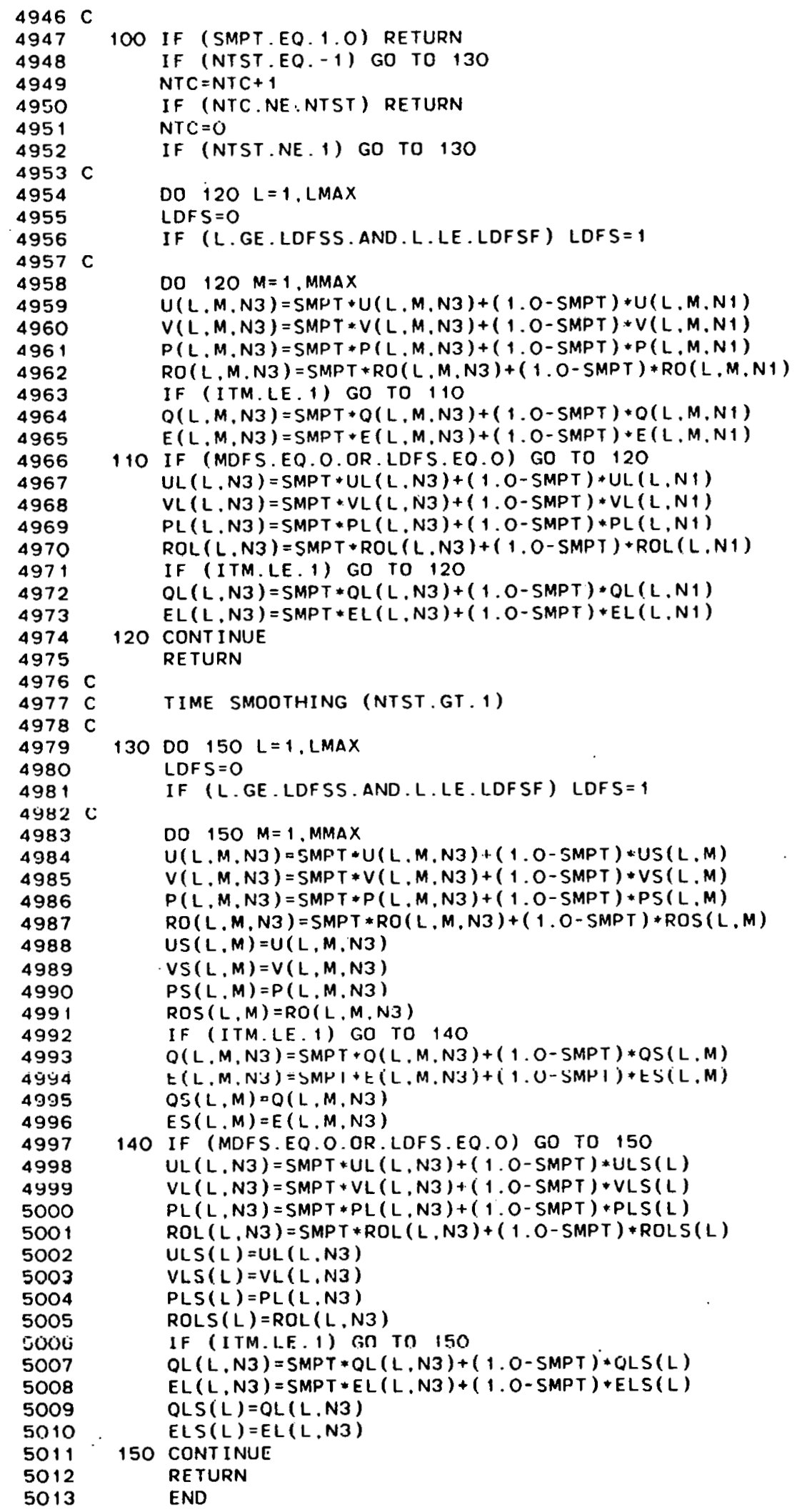




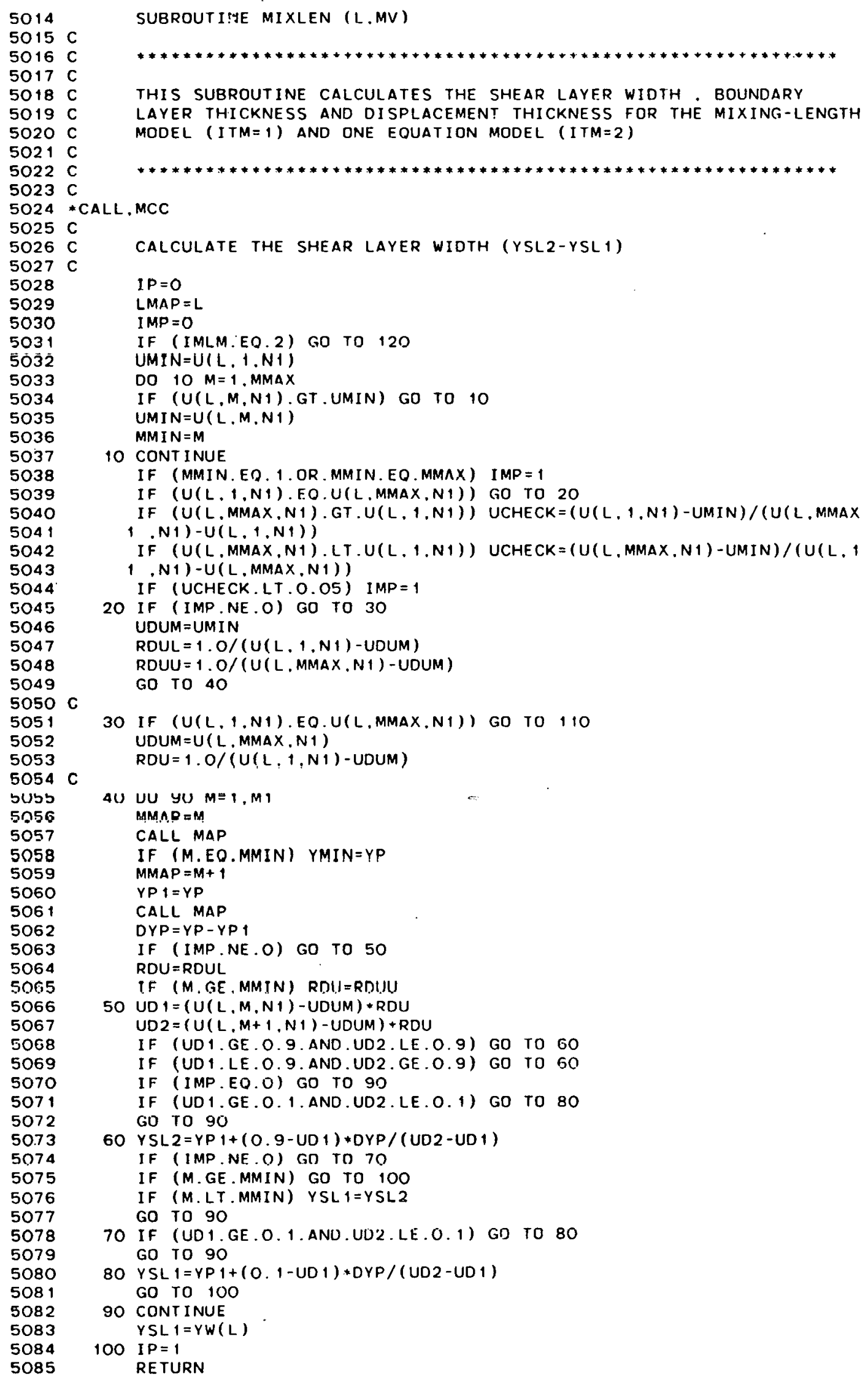




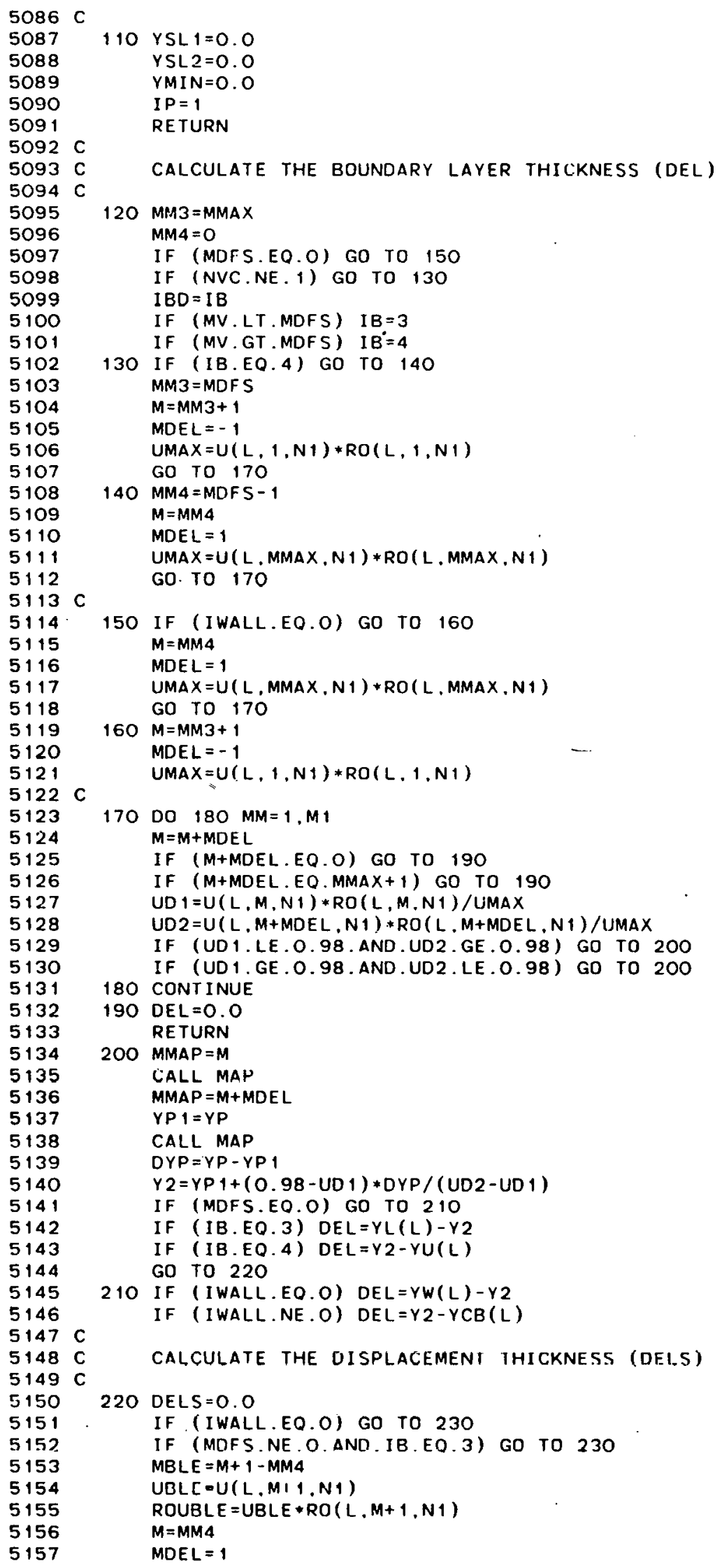




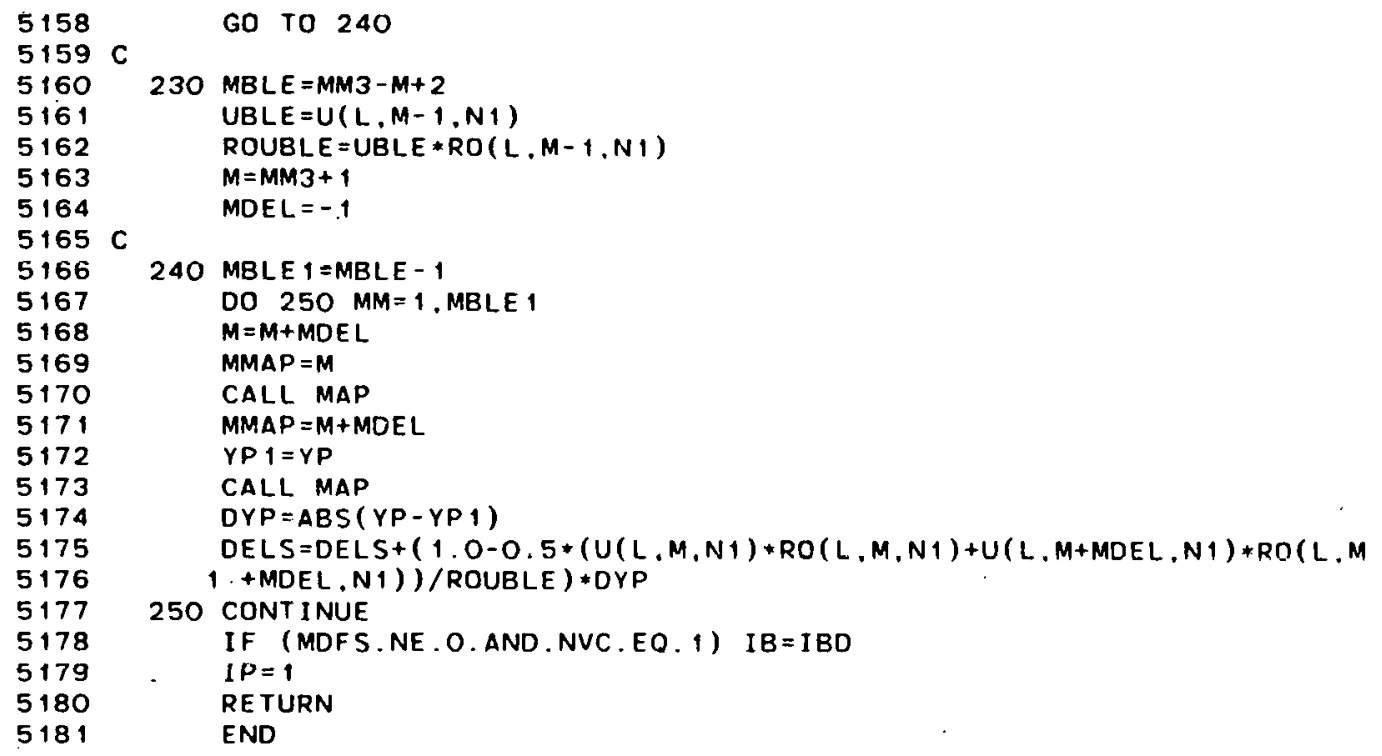




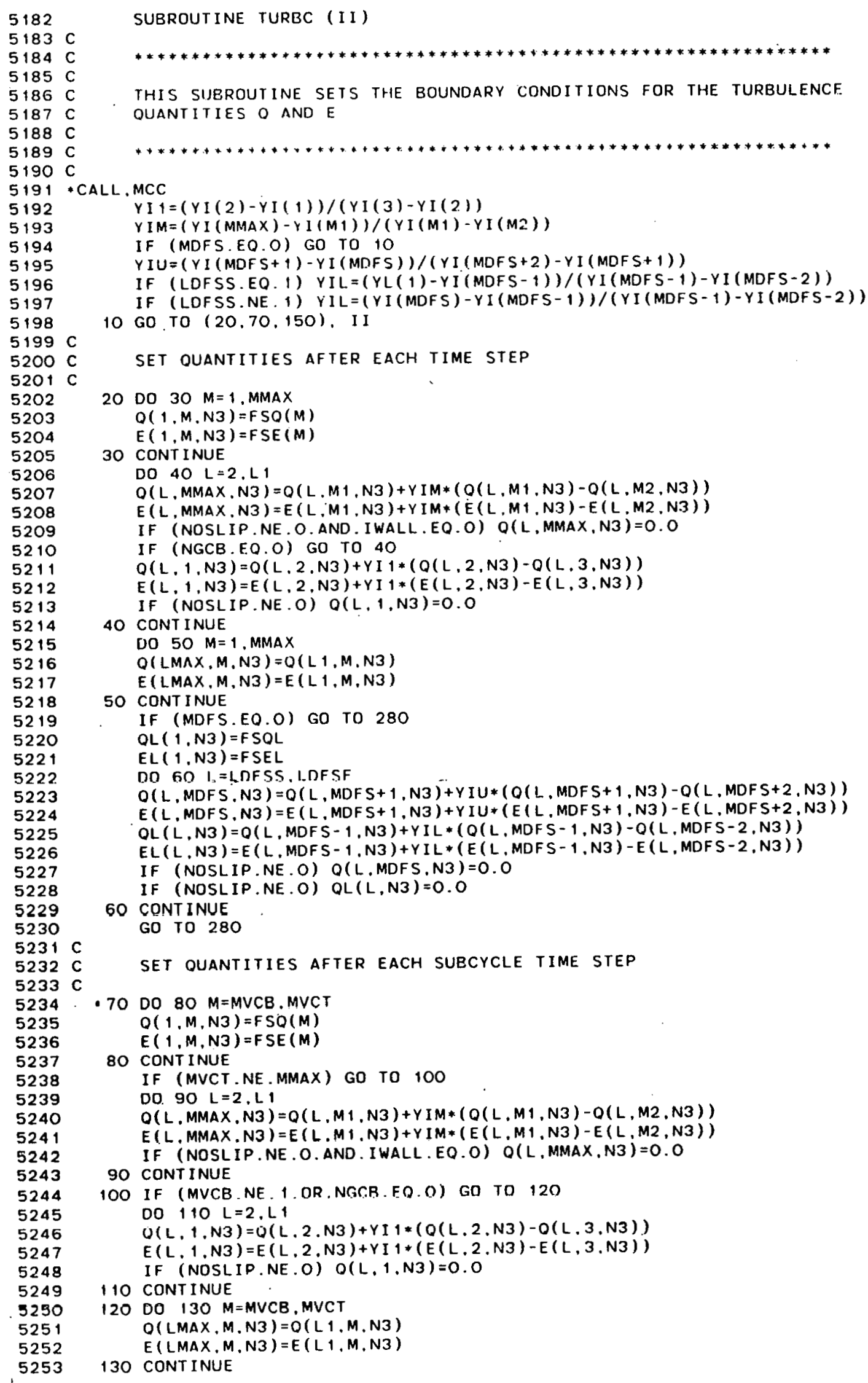

$5195 \quad Y I U=(Y I(M D F S+1)-Y I(M D F S)) /(Y I(M D F S+2)-Y I(M D F S+1))$

THIS SUEROUTINE SETS THE BOUNDARY CONDITIONS FOR THE TURBULENCE QUANTITIES O AND E

If (LDFSS.EQ.1) $Y I L=(Y L(1)-Y I(M D F S-1)) /(Y I(M D F S-1)-Y I(M D F S-2))$

IF (LDFSS.NE.1) YIL=(YI (MDFS)-YI (MDFS-1))/(YI(MDFS-1)-YI(MDFS-2))

10 GO TO $(20,70,150)$. I I

SET QUANTITIES AFTER EACH TIME STEP

20 DO $30 M=1, \operatorname{MMAX}$

$O(1, M, N 3)=F S Q(M)$

$E(1, M, N 3)=F S E(M)$

30 CONT INUE

DO $40 \mathrm{~L}=2$, L 1

$Q(L, M M A X, N 3)=Q(L, M 1, N 3)+Y I M *(Q(L, M 1, N 3)-Q(L, M 2, N 3))$

$E(L, M M A X, N 3)=E(L, M 1, N 3)+Y I M *(E(L, M 1, N 3)-E(L, M 2, N 3))$

IF (NOSLIP.NE.O. AND. IWALL.EQ.O) $Q(L, M M A X, N 3)=0.0$

IF (NGCB.EO.O) GO TO 40

$Q(L, 1, N 3)=Q(L .2 . N 3)+Y I 1 *(Q(L .2, N 3)-Q(L, 3, N 3))$

$E(L, 1, N 3)=E(L, 2, N 3)+Y I 1 *(E(L, 2, N 3)-E(L, 3, N 3))$

IF (NOSLIP.NE.O) $Q(L, 1 . N 3)=0.0$

40 CONT INUE

DO $50 M=1$, MMAX

$Q(L M A X, M, N 3)=Q(L 1, M, N 3)$

$E(L M A X, M, N 3)=E(L 1, M, N 3)$

50 CONT INUE

IF (MDFS.EQ.O) GO TO 280

$\mathrm{QL}(1 . \mathrm{N3})=\mathrm{FSQL}$

$E L(1, N 3)=F S E L$

DO $60 \mathrm{I}_{2}=$ LNFSS, I.DFSF

$Q(L, M D F S . N 3)=Q(L, M D F S+1, N 3)+Y I U *(Q(L, M D F S+1, N 3)-O(L, M D F S+2, N 3))$

$E(L, M D F S, N 3)=E(L, M D F S+1, N 3)+Y I U *(E(L, M D F S+1, N 3)-E(L, M D F S+2, N 3))$

$Q L(L, N 3)=Q(L, M D F S-1, N 3)+Y I L *(Q(L, M D F S-1, N 3)-Q(L, M D F S-2, N 3))$

$E L(L, N 3)=E(L, M D F S-1, N 3)+Y I L *(E(L, M D F S-1, N 3)-E(L, M D F S-2, N 3))$

IF (NOSLIP.NE.O) $O(L . M D F S . N 3)=0.0$

IF (NOSLIP.NE.O) QL $(L, N 3)=0.0$

60 CONT INUE

GO TO 280

- 70 DO 80 M=MVCB, MVCT

SET QUANTITIES AFTER EACH SUBCyCle tIME SteP

$Q(1, M, N 3)=F S Q(M)$

$E(1, M, N 3)=F S E(M)$

80 CONT INUE

IF (MVCT.NE.MMAX) GO TO 100

DO. $90 L=2, L 1$

$Q(L, M M A X, N 3)=Q(L, M 1, N 3)+Y I M *(O(L, M 1, N 3)-Q(L, M 2, N 3))$

$E(L, M M A X . N 3)=E(L, M 1 . N 3)+Y I M *(E(L, M 1, N 3)-E(L, M 2, N 3))$

IF (NOSLIP.NE.O. AND. IWALL.EO.O) O(L.MMAX.N3) =0.O

90 CONTINUE

100 IF (MVCB NE. I. OR. NGC.R.FO.O) GO TO 120

$00110 \mathrm{~L}=2 . \mathrm{L} 1$

$Q(L, 1, N 3)=Q(L, 2 . N 3)+Y I 1 *(Q(L, 2 . N 3)-O(L .3 . N 3))$

$E(L, 1, N 3)=E(L, 2, N 3)+Y I 1 *(E(L, 2 . N 3)-E(L, 3 . N 3))$

IF (NDSLIP.NE.O) $O(L, 1, N 3)=0.0$

110 CONT INUE

120 DO $130 \mathrm{M}=$ MVCB, MVCT

$Q($ LMAX, M.N3 $)=O(L, 1, M, N 3)$

$E(L M A X, M, N 3)=E(L 1, M, N 3)$

130 CONT INUE 


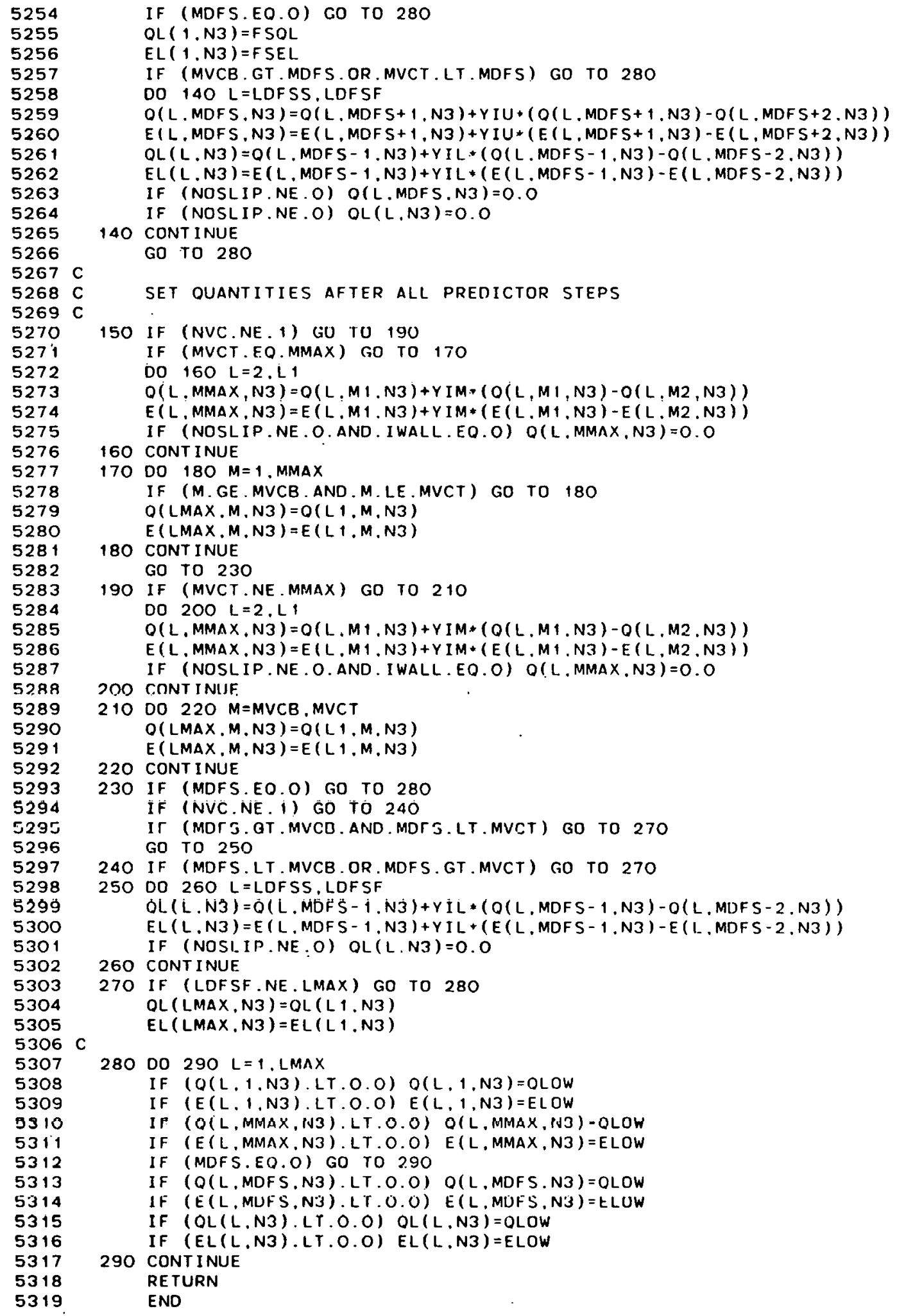




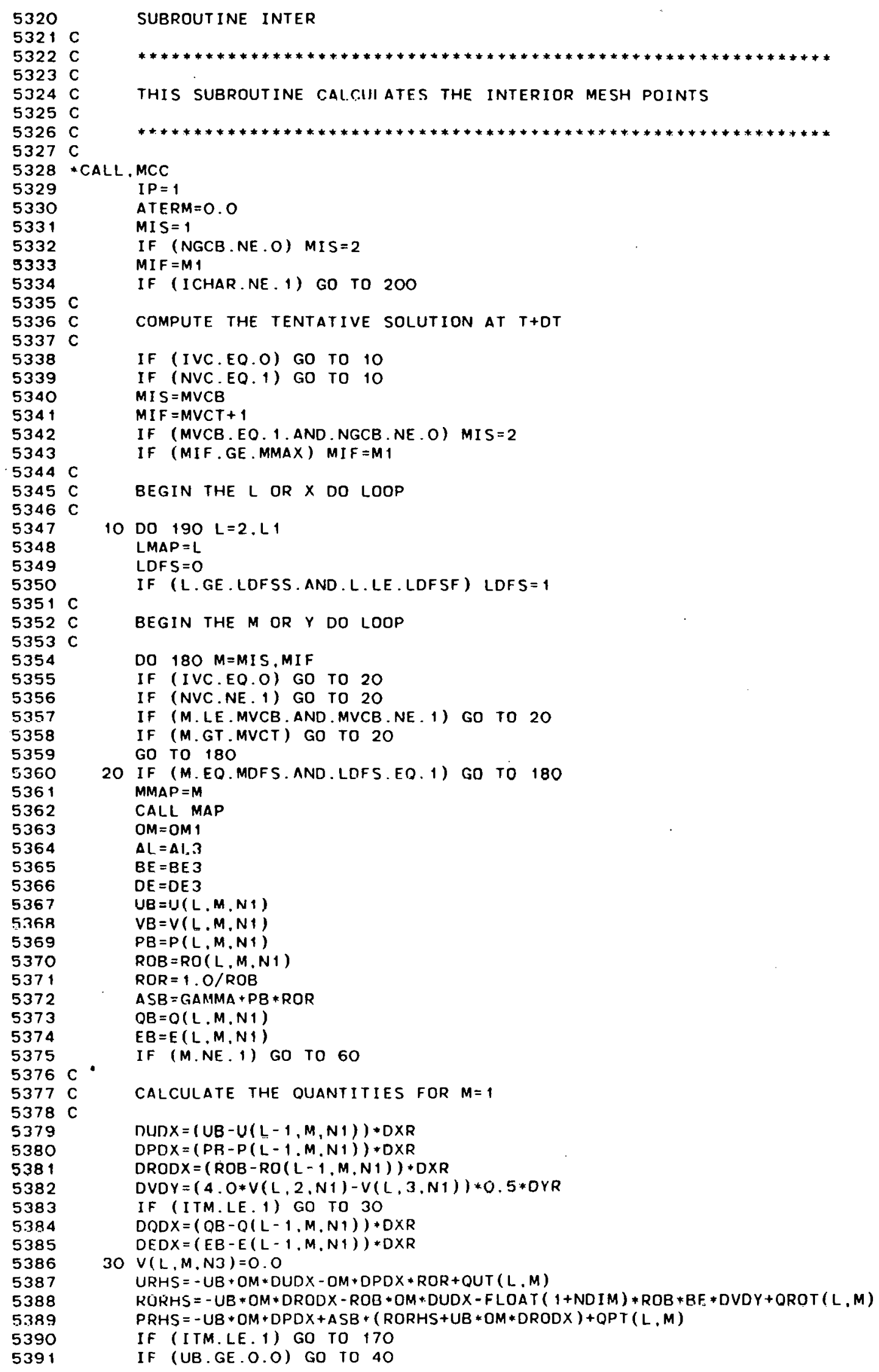




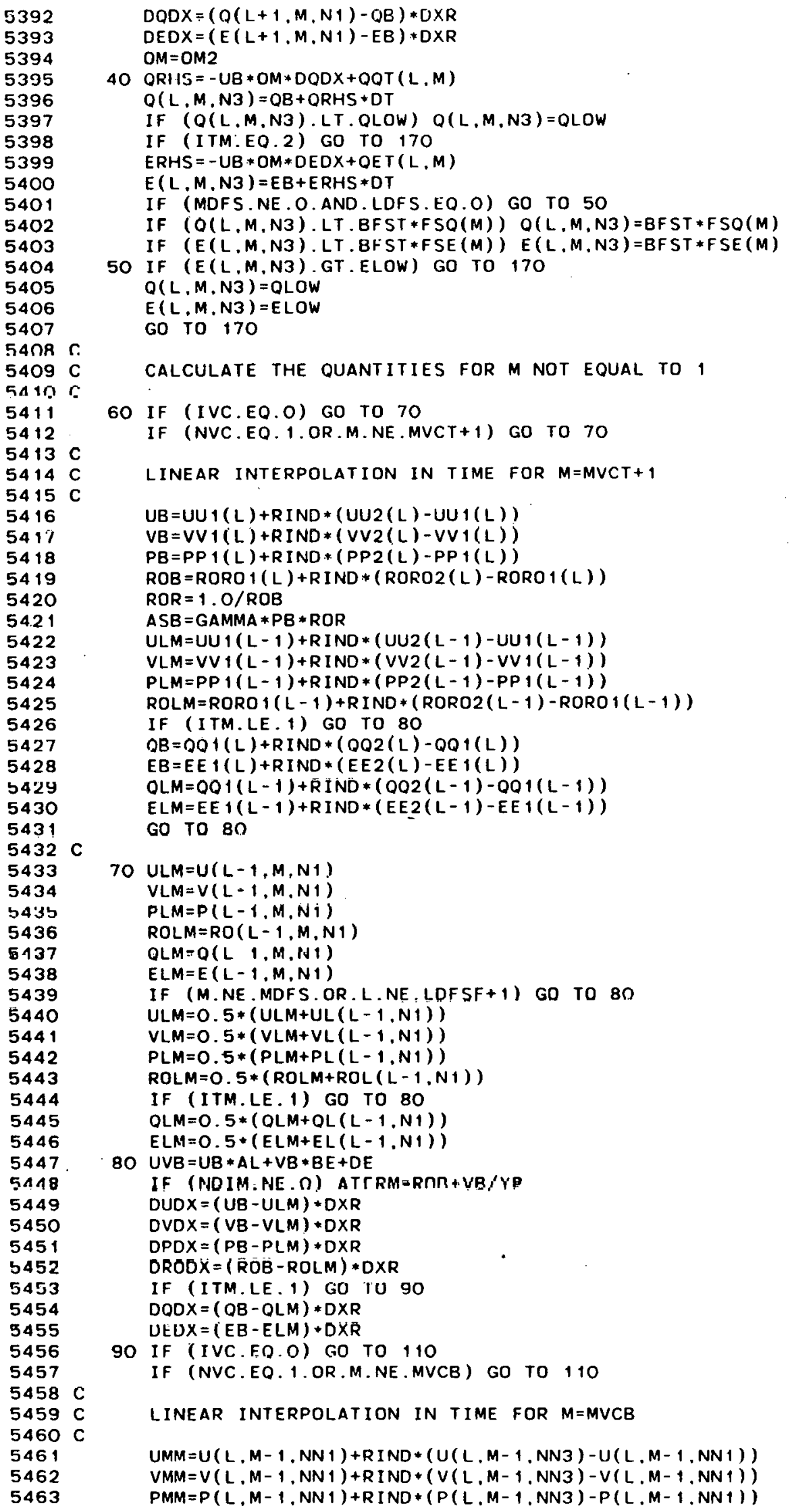




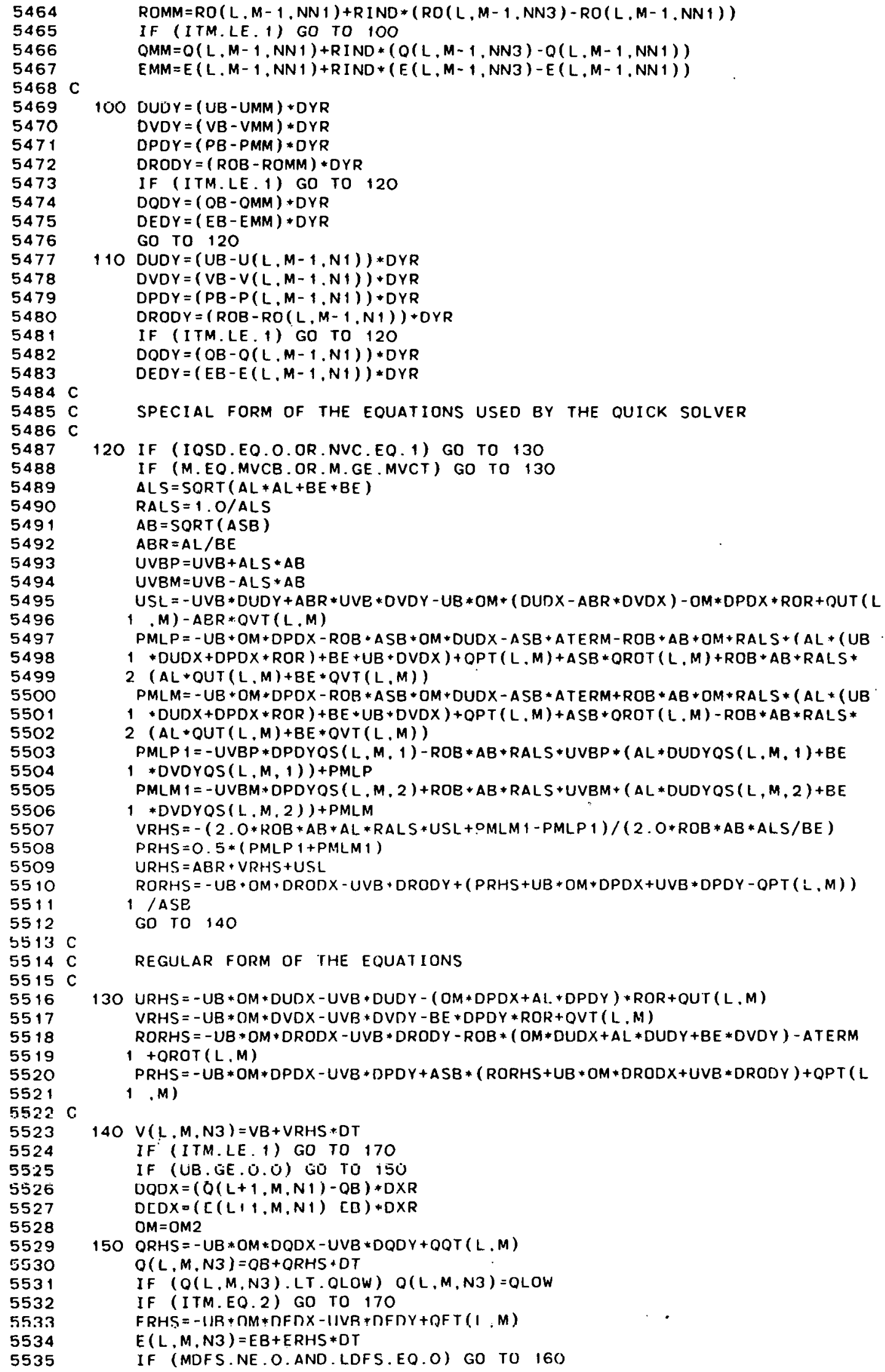




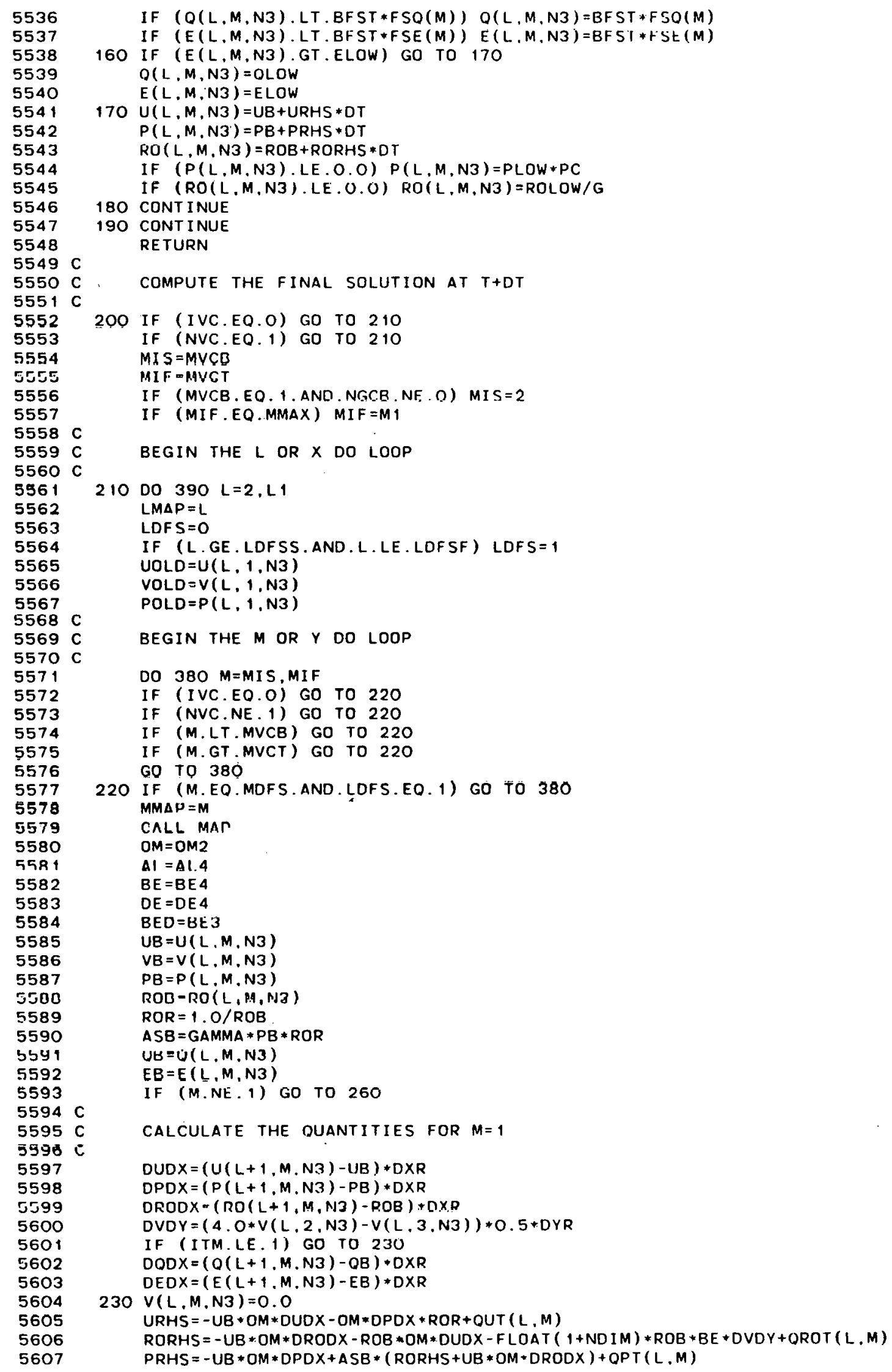




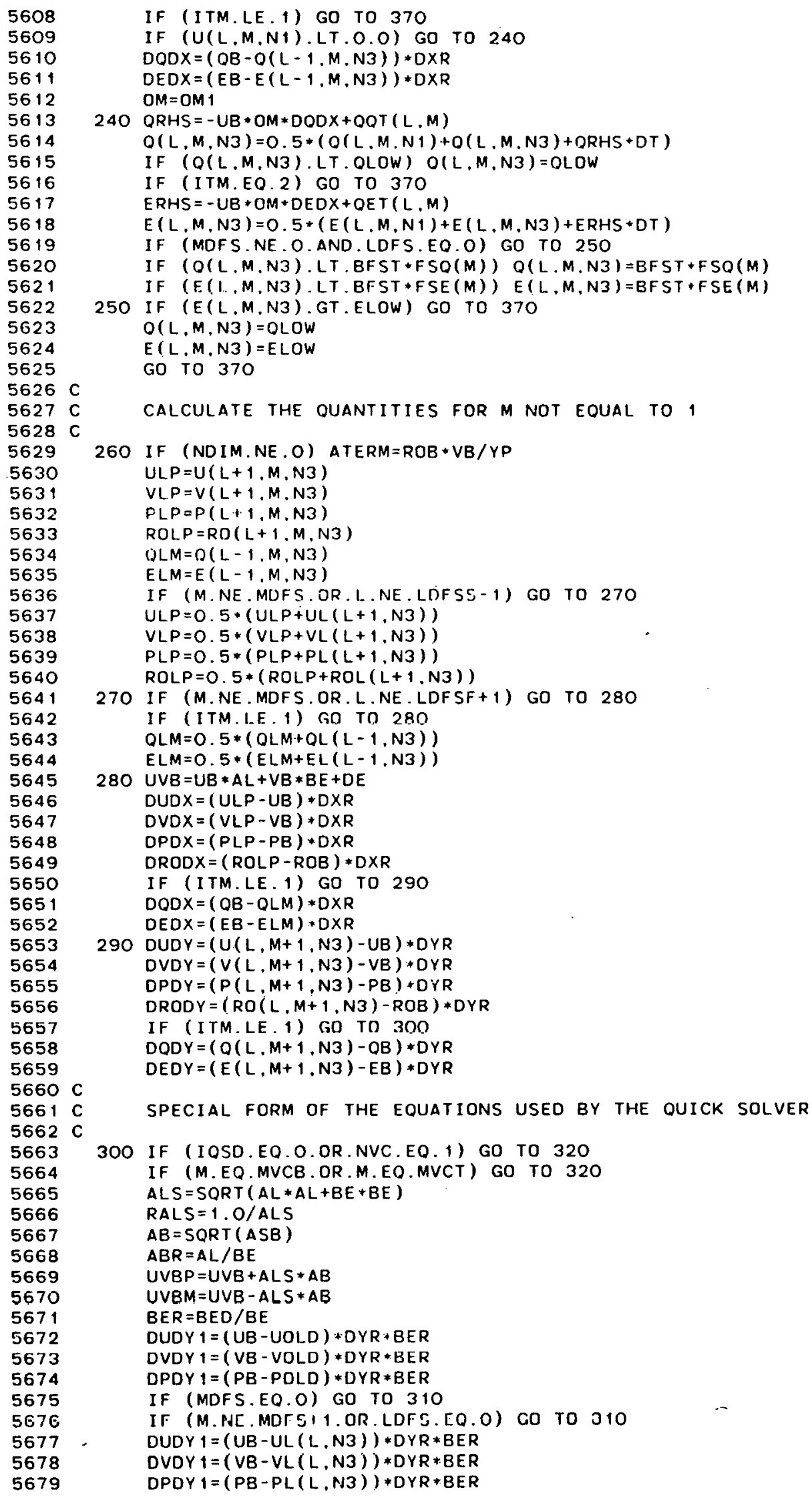




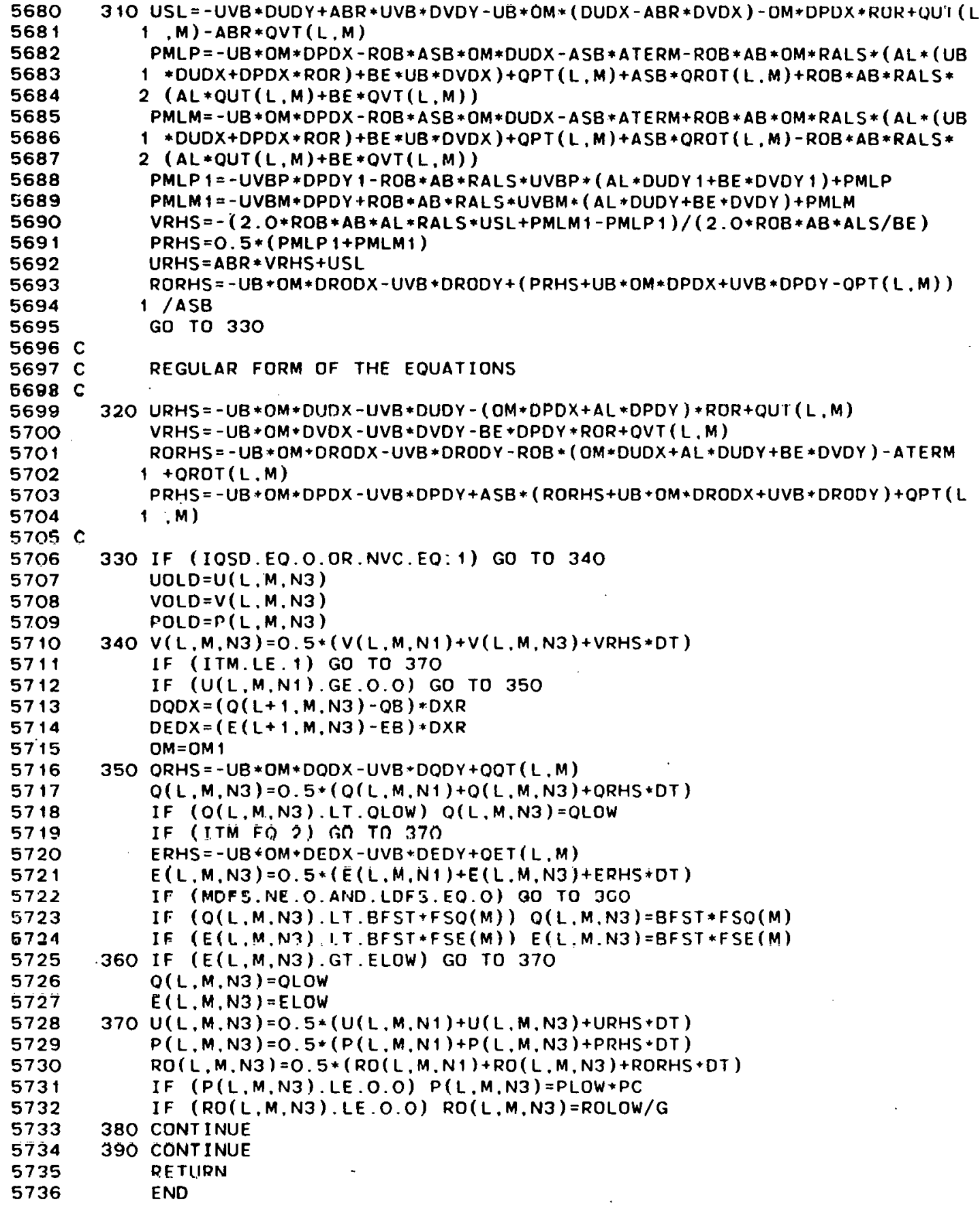




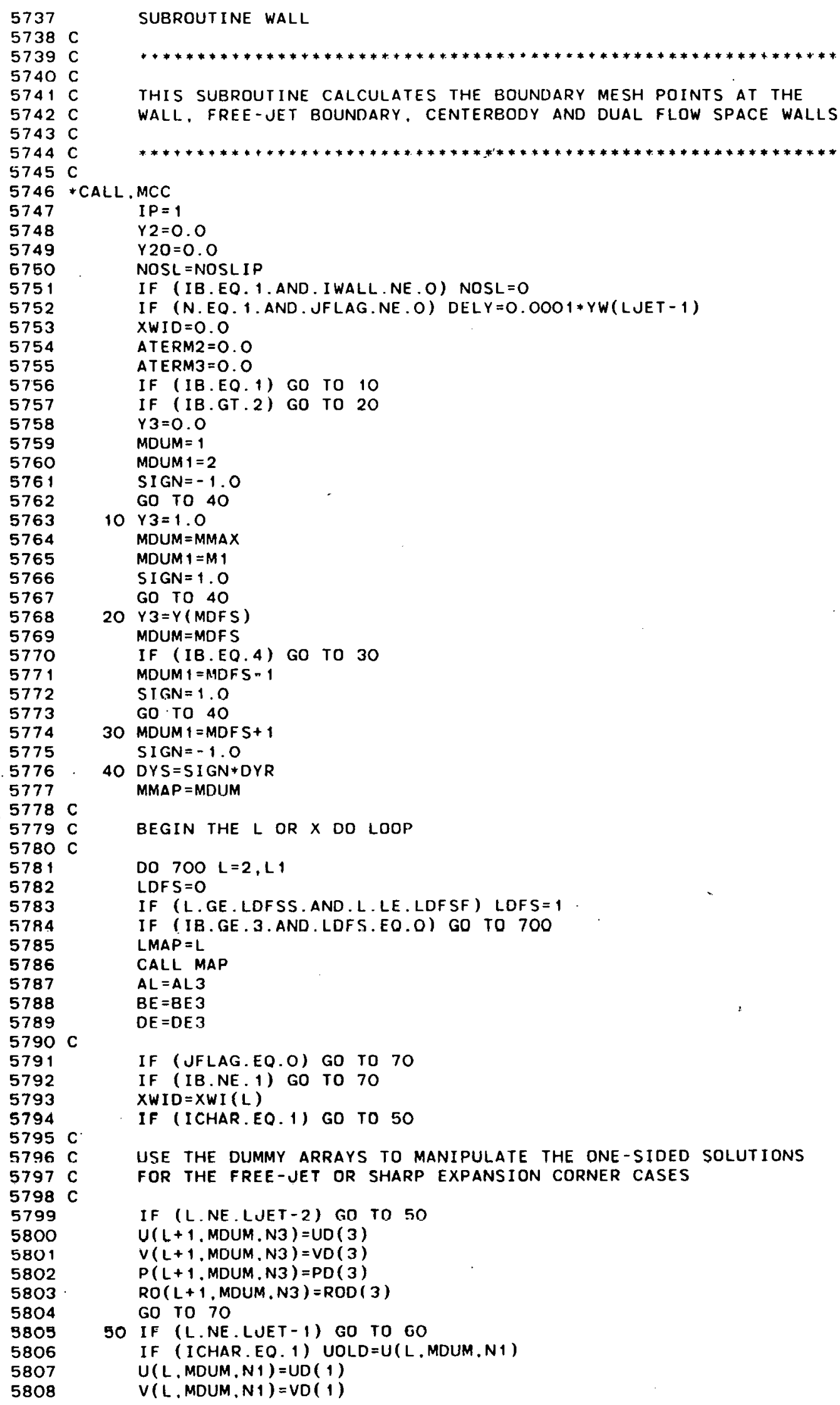

5751 IF (IB.EQ.1. AND. IWALL.NE . O) NOSL=O

THIS SUBROUTINE CALCULATES THE BOUNDARY MESH POINTS AT THE WALL, FREE-JET BOUNDARY, CENTERBODY AND DUAL FLOW SPACE WALLS

IF (N.EO. 1. AND.JFLAG.NE. O) DELY=0.0001*YW(LJET - 1)

$X W I D=0.0$

$\triangle T E R M 2=0.0$

$\triangle T E R M 3=0.0$

IF (IB.EQ.1) GO TO 10

IF (IB.GT.2) GO TO 20

$Y 3=0.0$

MOUM $=1$

MOUM $1=2$

$\operatorname{SIGN}=-1.0$

GO TO 40

$10 Y 3=1.0$

MDUM $=$ MMAX

MDUM $1=M 1$

$S I G N=1.0$

GO TO 40

$20 Y 3=Y($ MDFS $)$

MDUM $=$ MDF $S$

IF (IB.EQ.4) Go TO 30

MDUM $1=$ MDFS -1

ST T.N $=1.0$

GO $\cdot$ TO 40

30 MDUM $1=$ MDF +1

$S I G N=-1.0$

40 DYS $=S I G N * D Y R$

MMAP $=$ MDUM

BEGIN THE L OR $\times$ DO LOOP

DO $700 L=2, L 1$

LDF $S=0$

IF (L.GE. LOFSS. AND. L. LE. LDFSF) LOFS $=1$

IF (IB.GE.3.AND.LDFS.EO.O) GO TO 700

LMAP $=L$

CALL MAP

$A L=A L 3$

$B E=B E 3$

$\mathrm{DE}=\mathrm{DE} 3$

IF (JFLAG.EQ.O) GO TO 70

IF (IB.NE. 1) GO TO 70

$X W I D=X W I(L)$

IF (ICHAR.EO. 1) GO TO 50

USE THE DUMMY ARRAYS TO MANIPULATE THE ONE-SIDED SOLUTIONS

FOR THE FREE-JET OR SHARP EXPANSION CORNER CASES

IF (L.NE. LUET-2) GO TO 50

$U(L+1, M D U M . N 3)=U D(3)$

$V(L+1, M D U M, N 3)=V D(3)$

$P(L+1, M D U M, N 3)=P D(3)$

$R O(L+1, M D U M, N 3)=R O D(3)$

GO TO 70

50 IF (L.NE.LUET-1) GO TO GO

IF (ICHAR.EO.1) UOLD $=U(L . M D U M, N 1)$

$U(L, M D U M, N 1)=U D(1)$

$V(L, M D U M, N 1)=V D(1)$ 


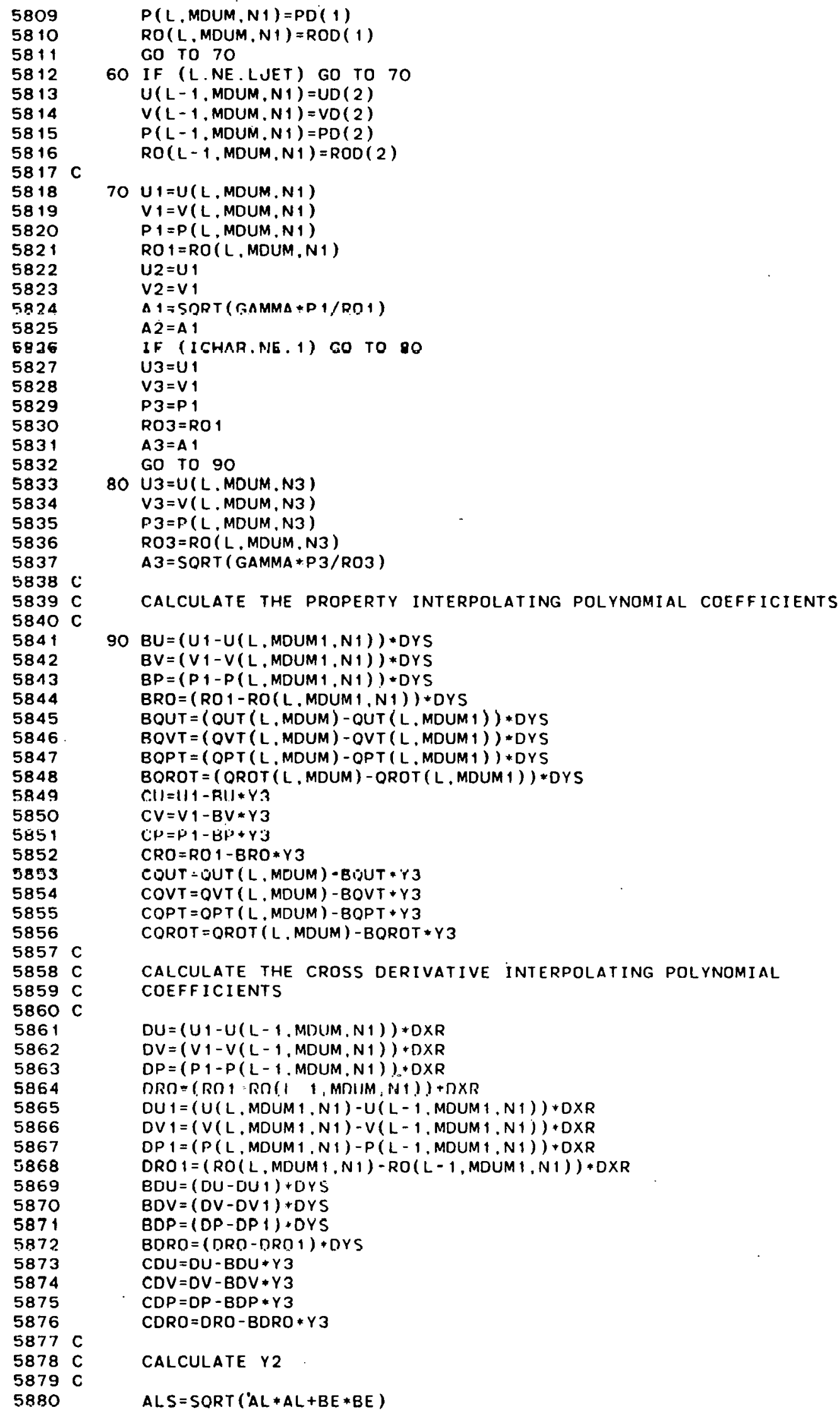




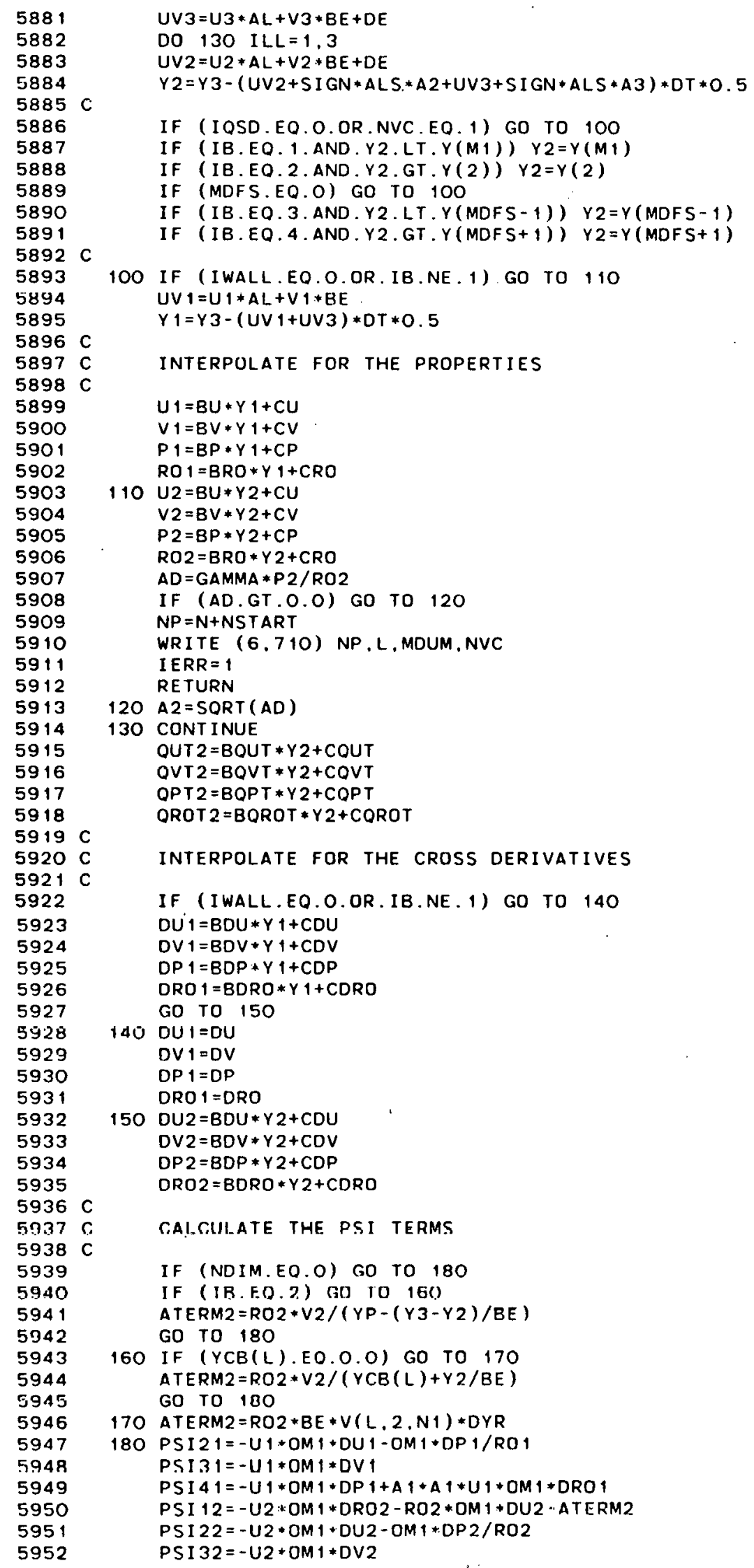




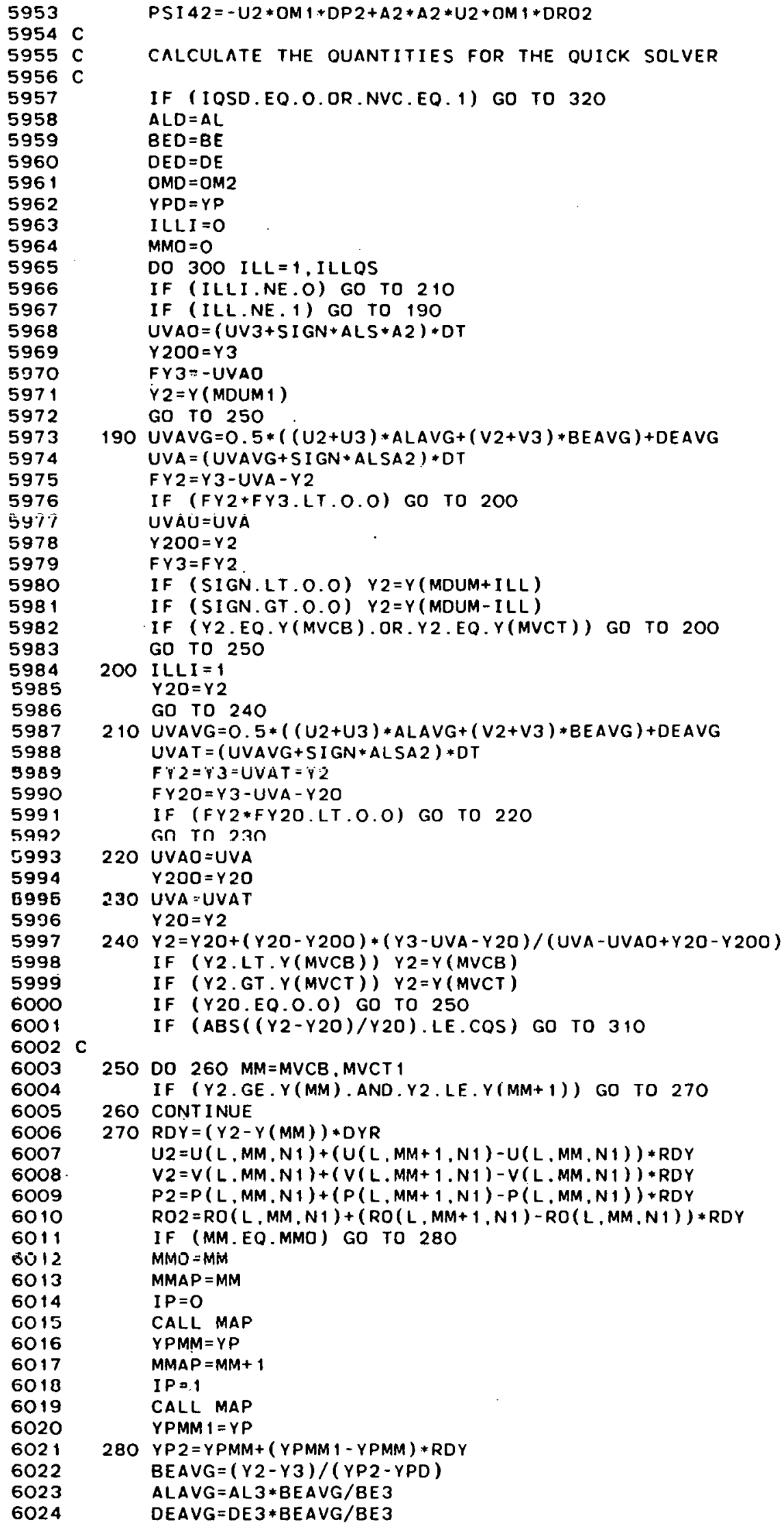




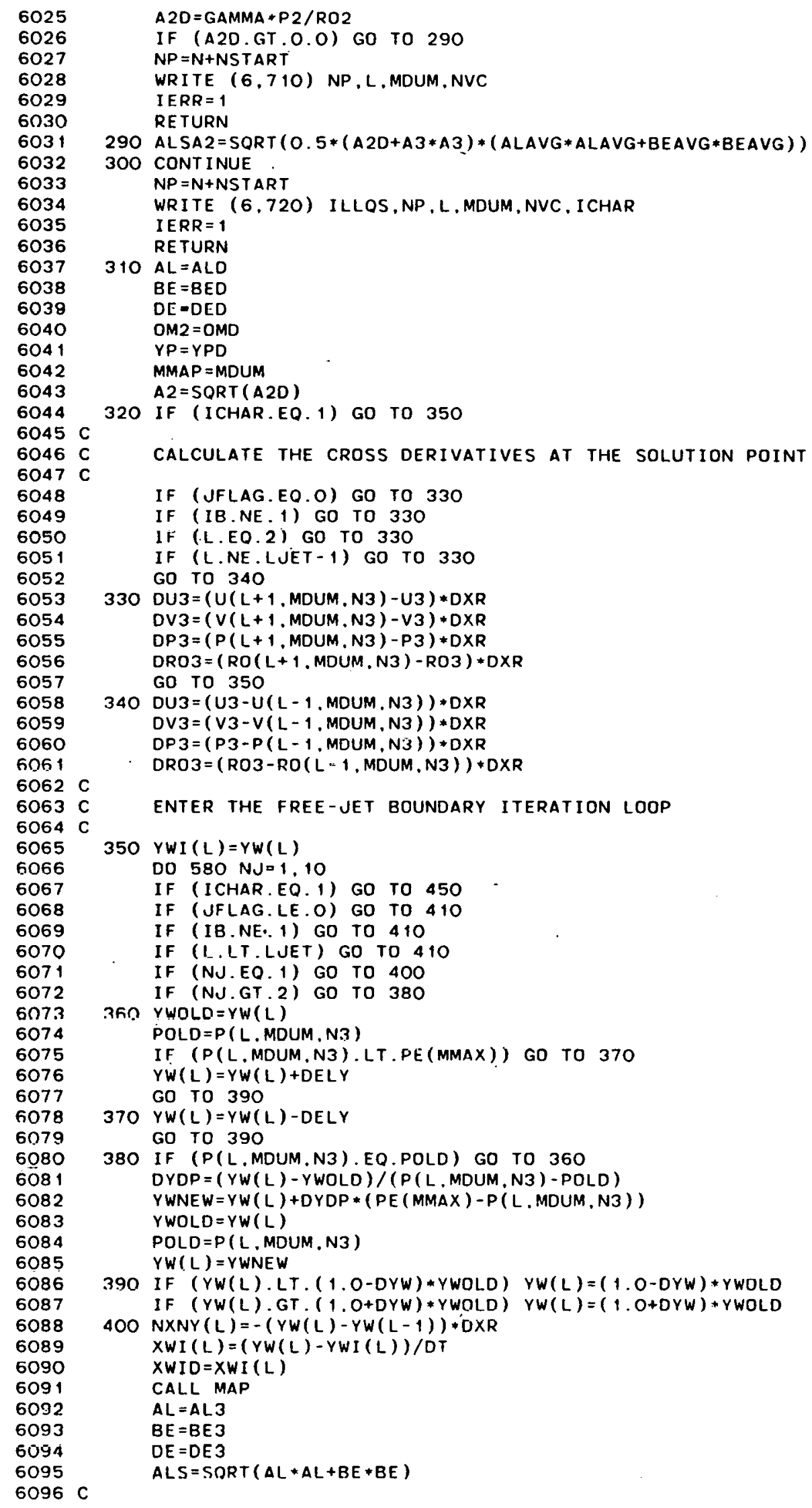




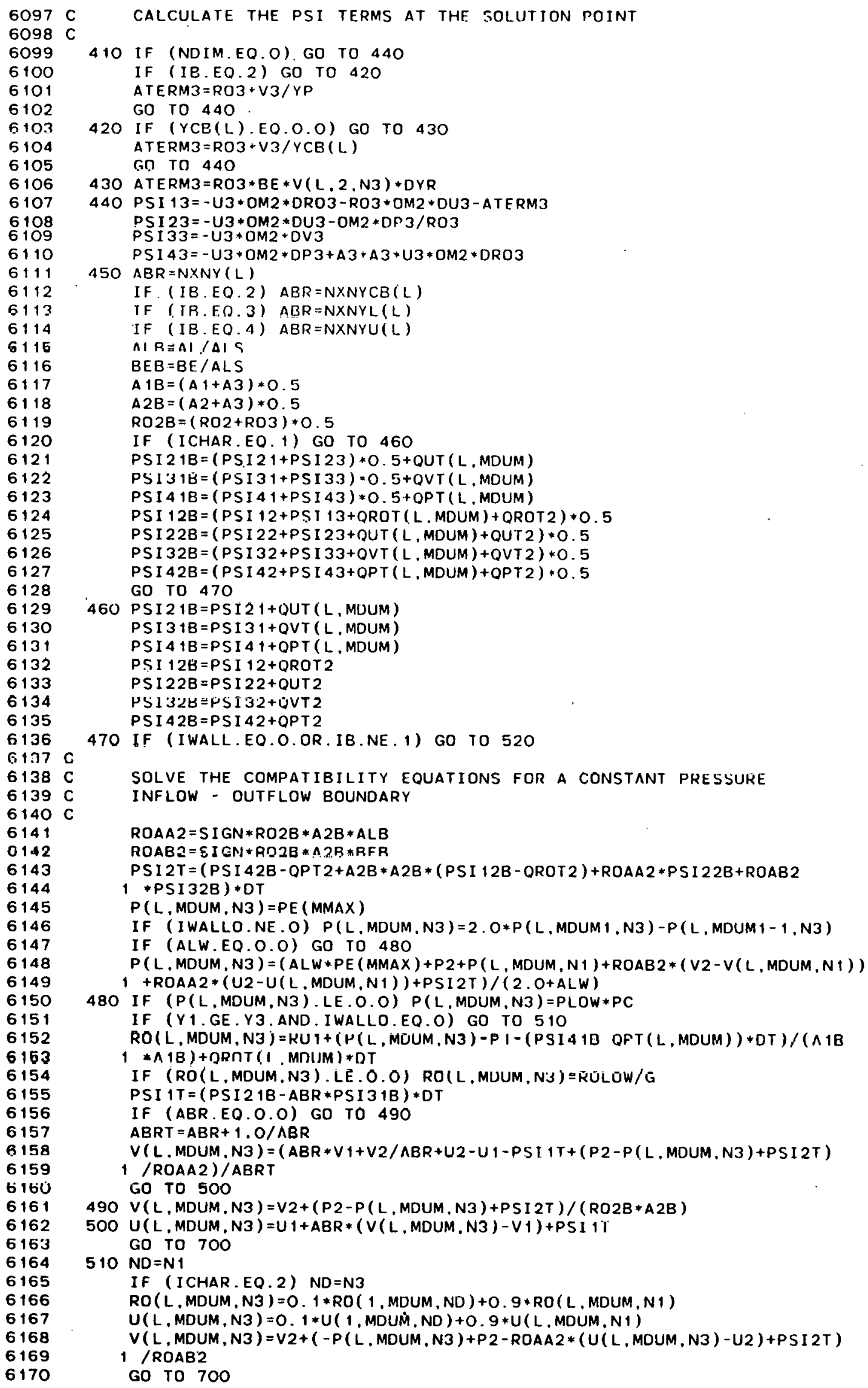




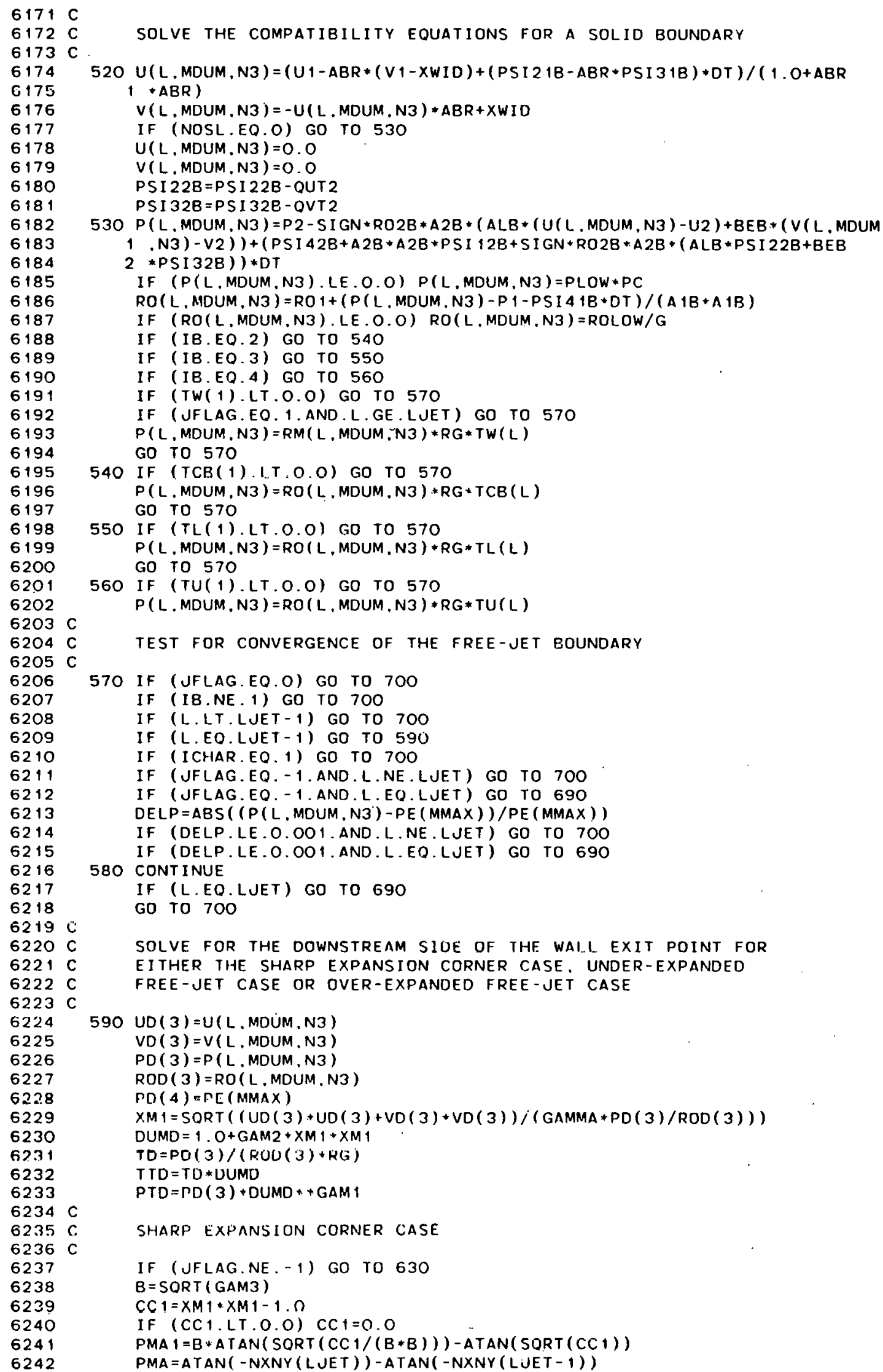




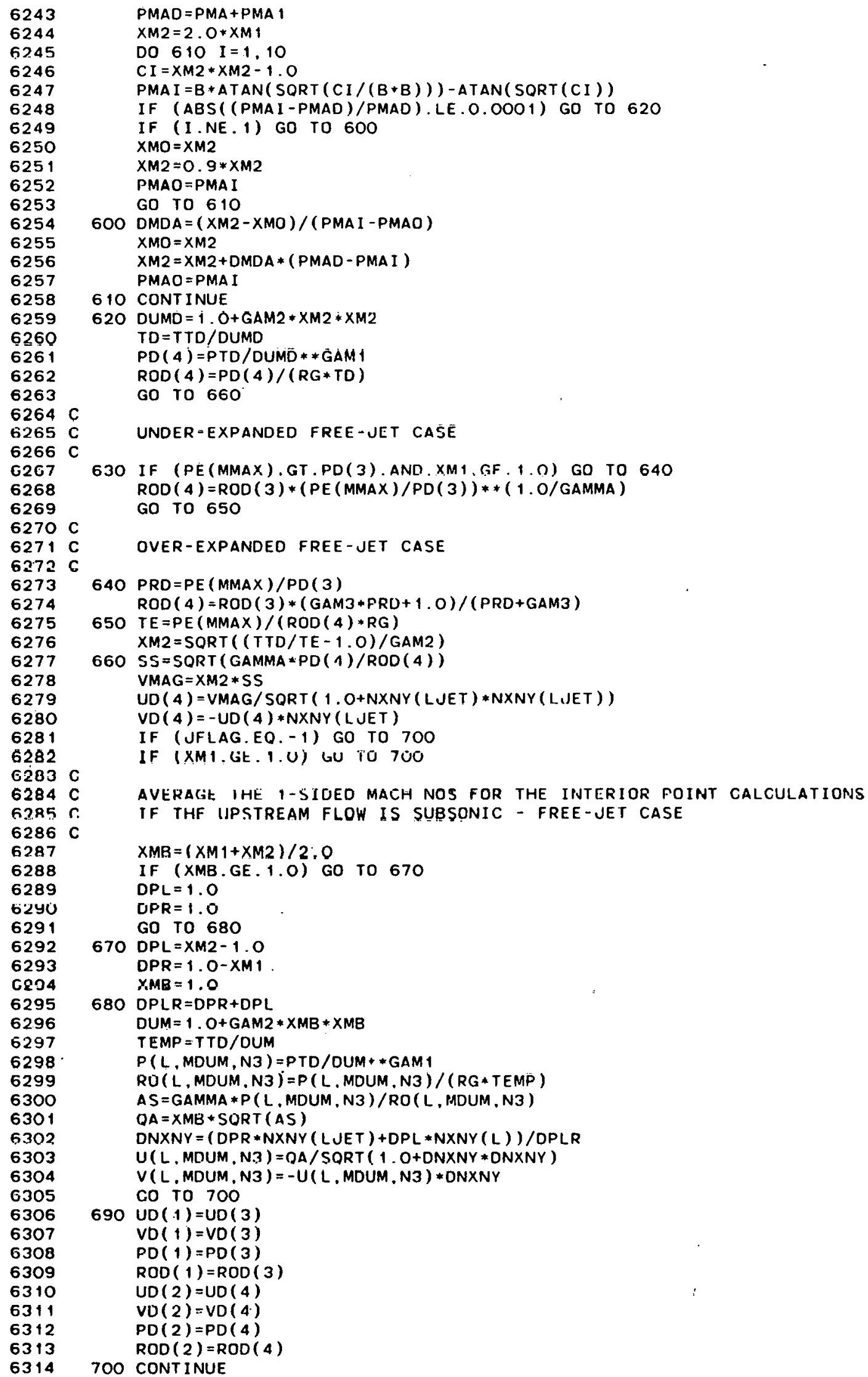




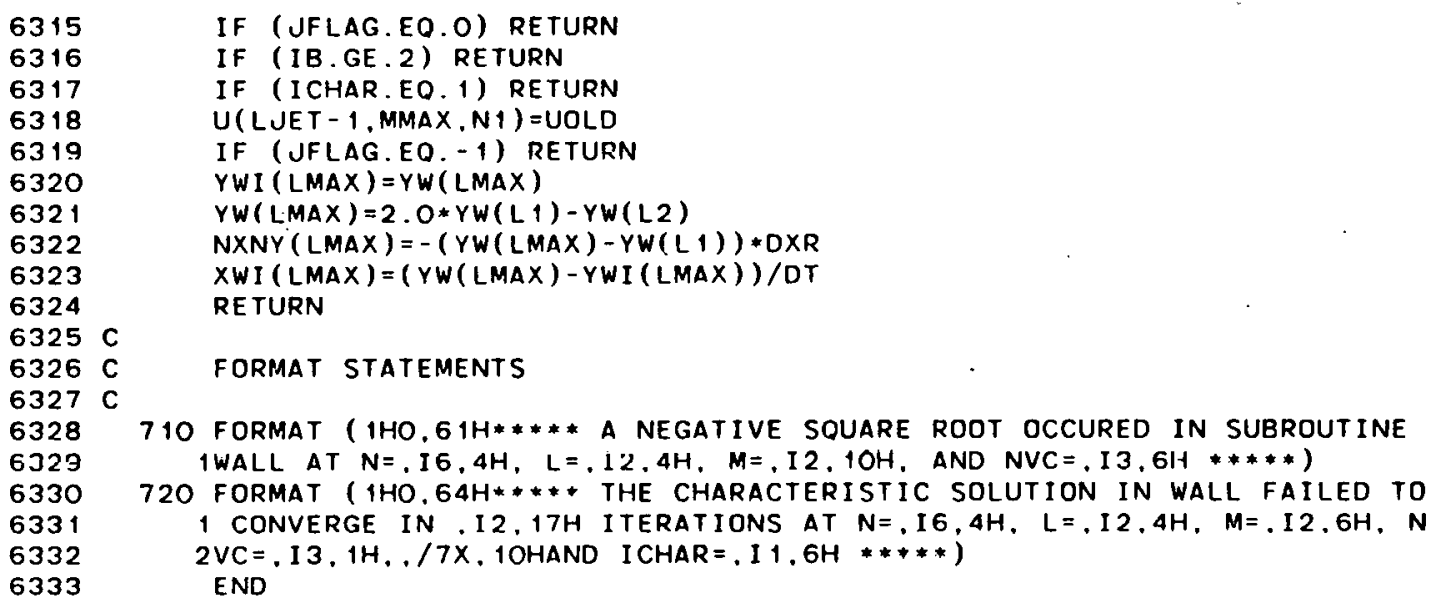




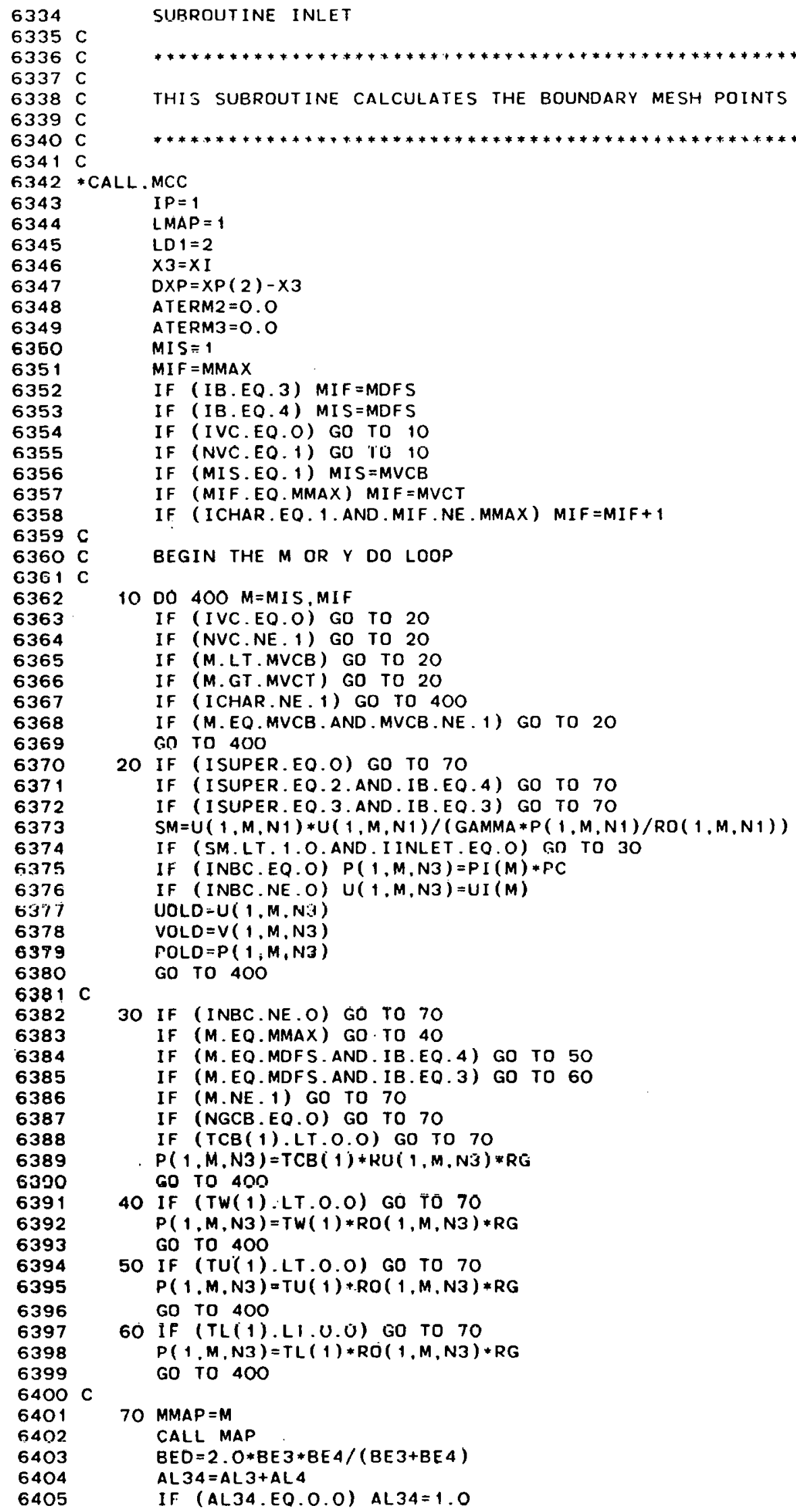




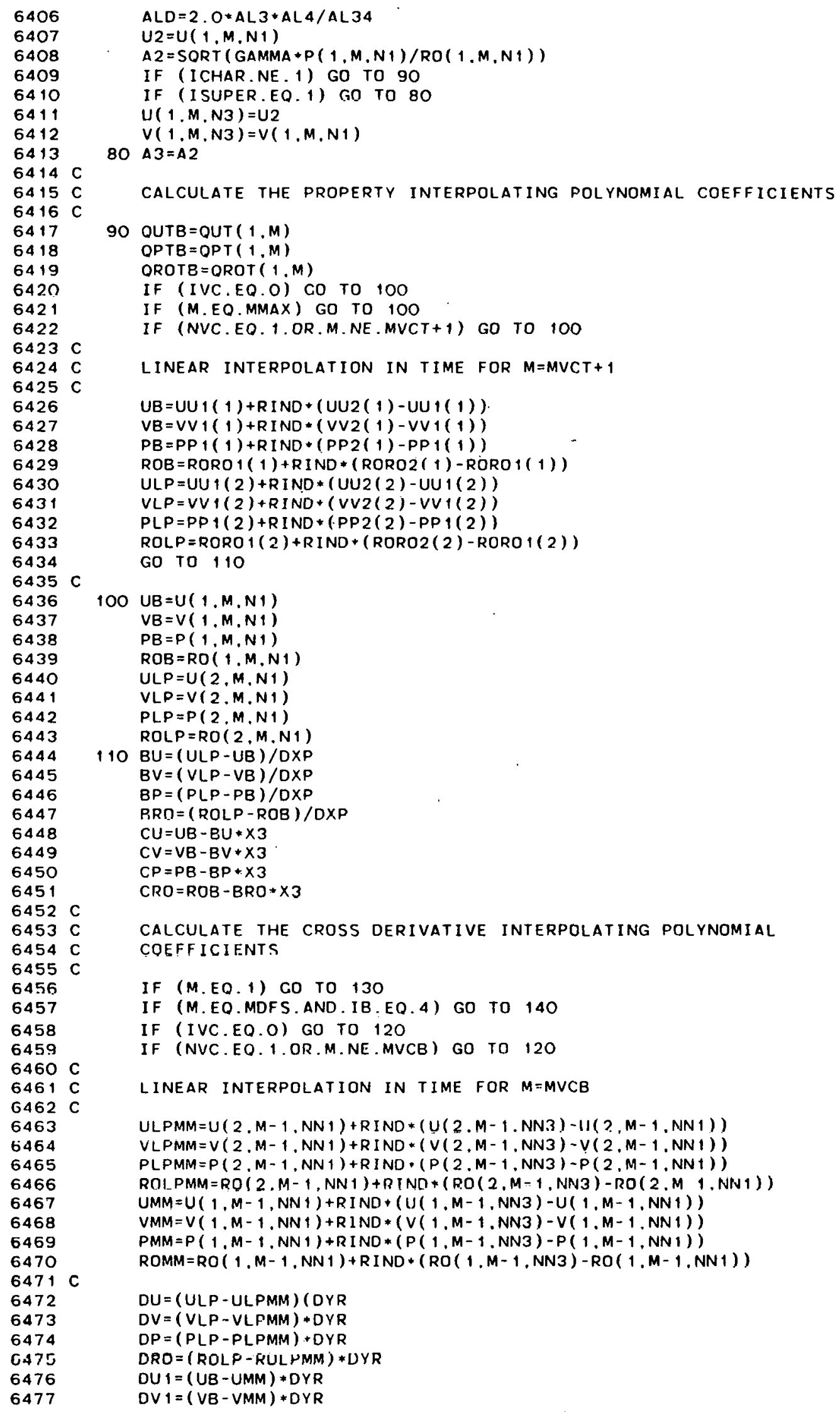




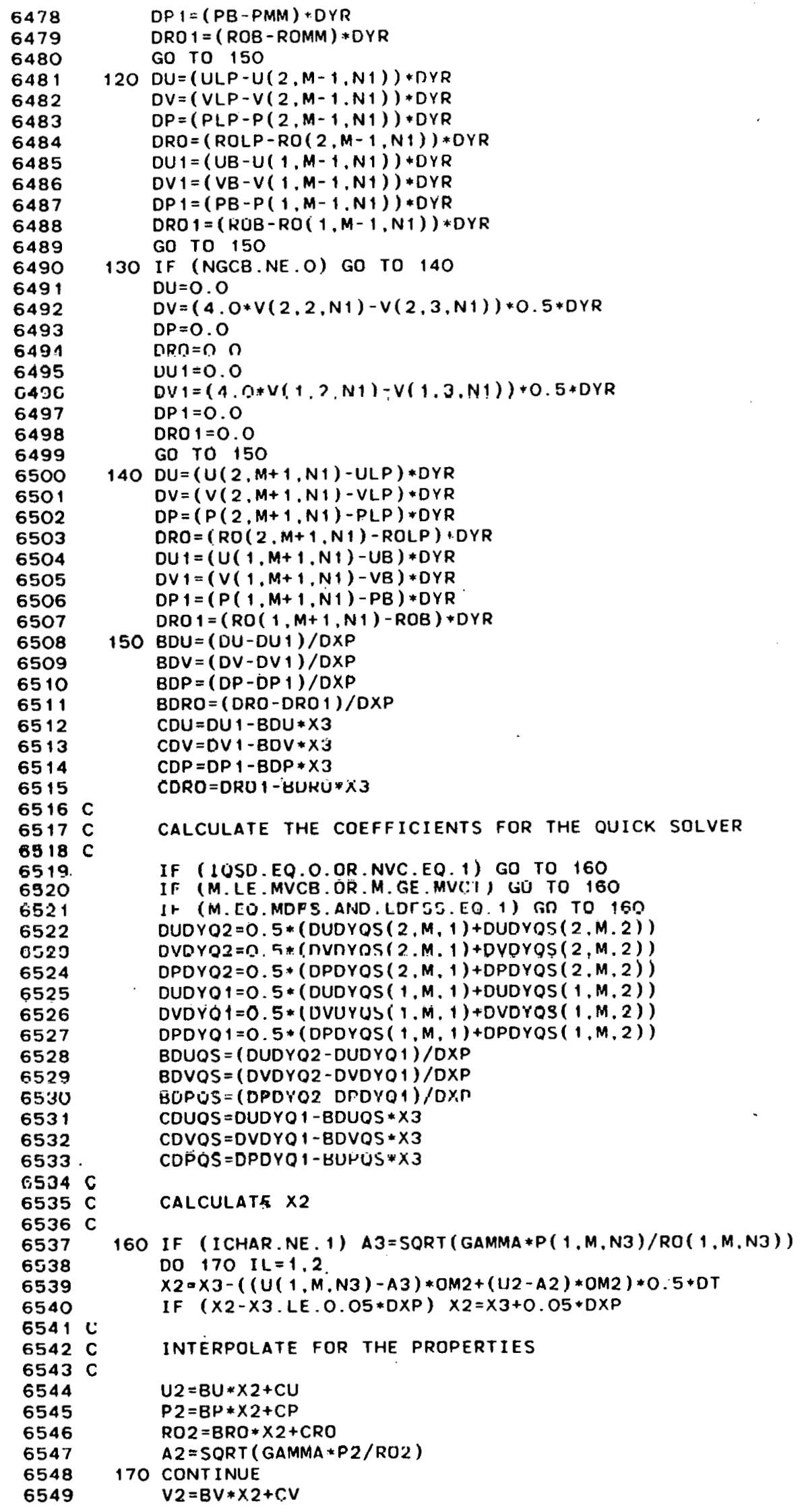




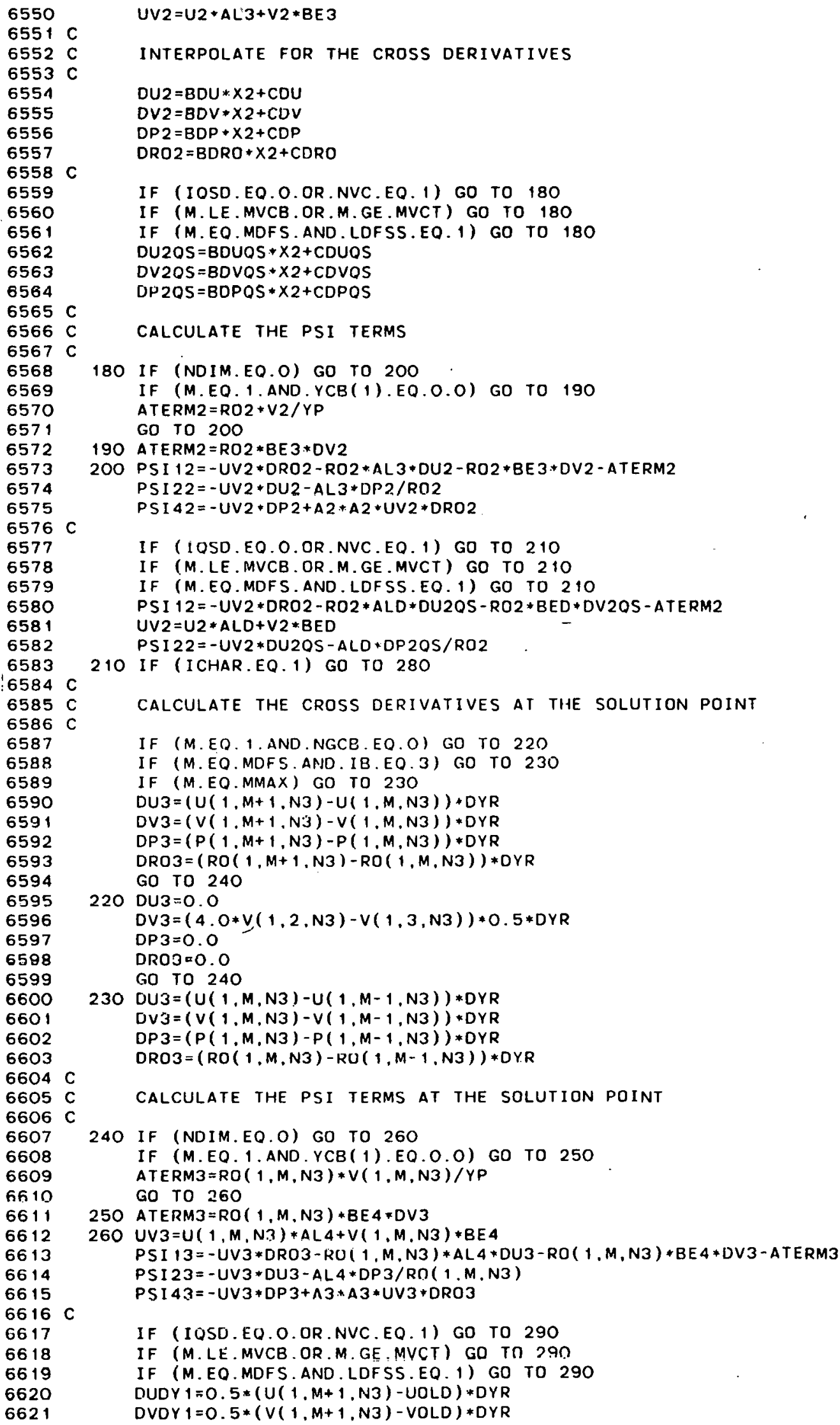




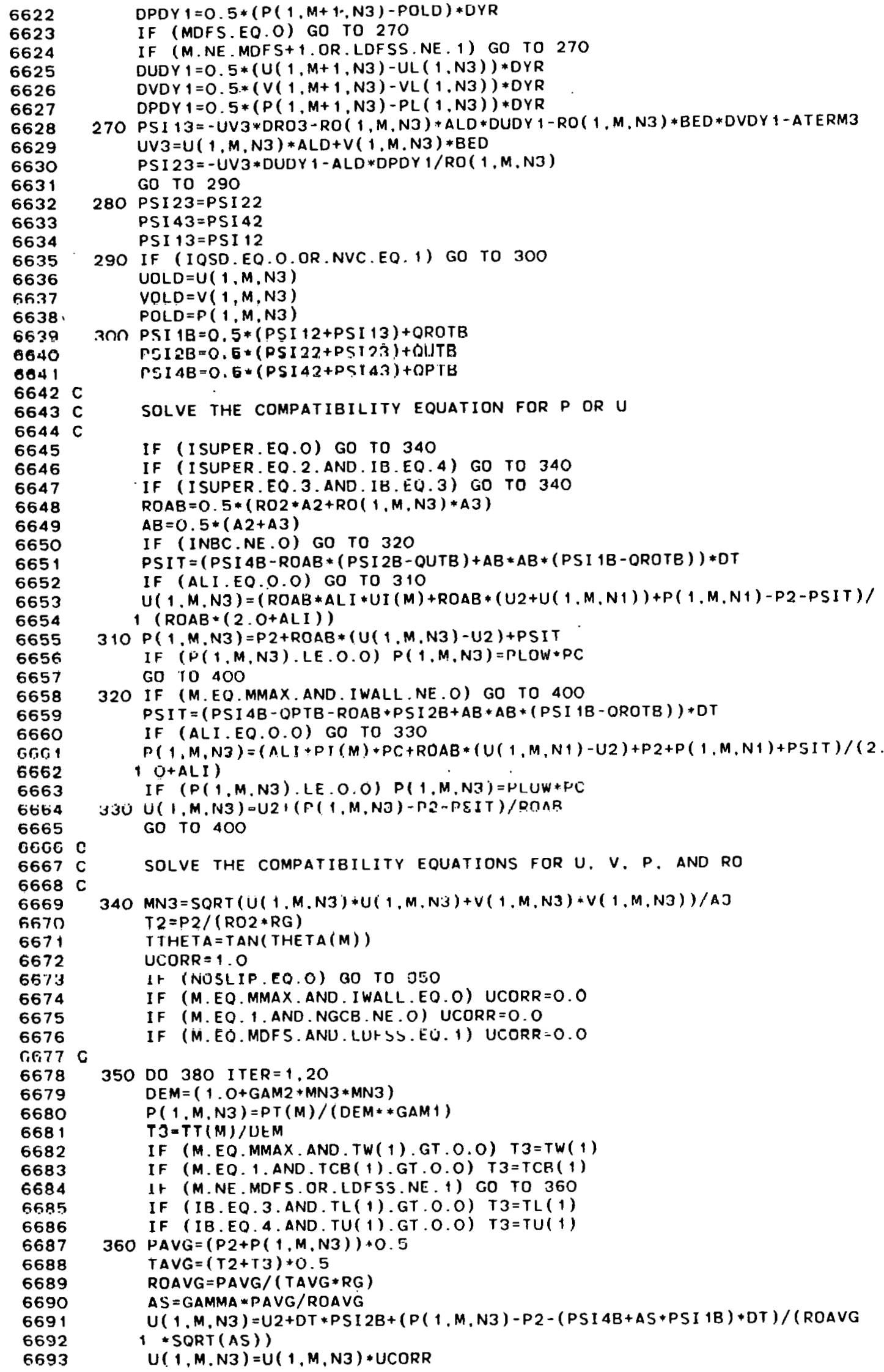




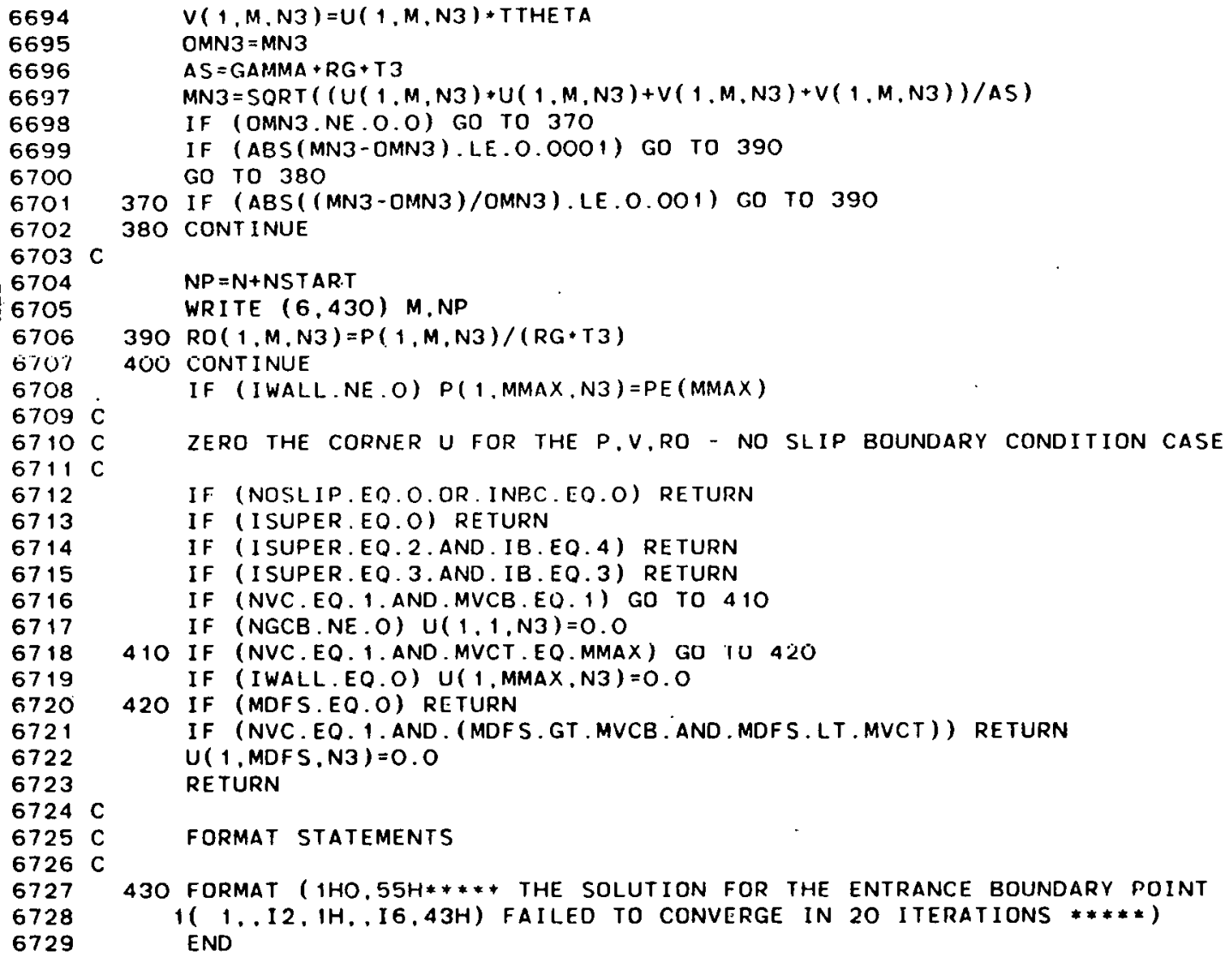




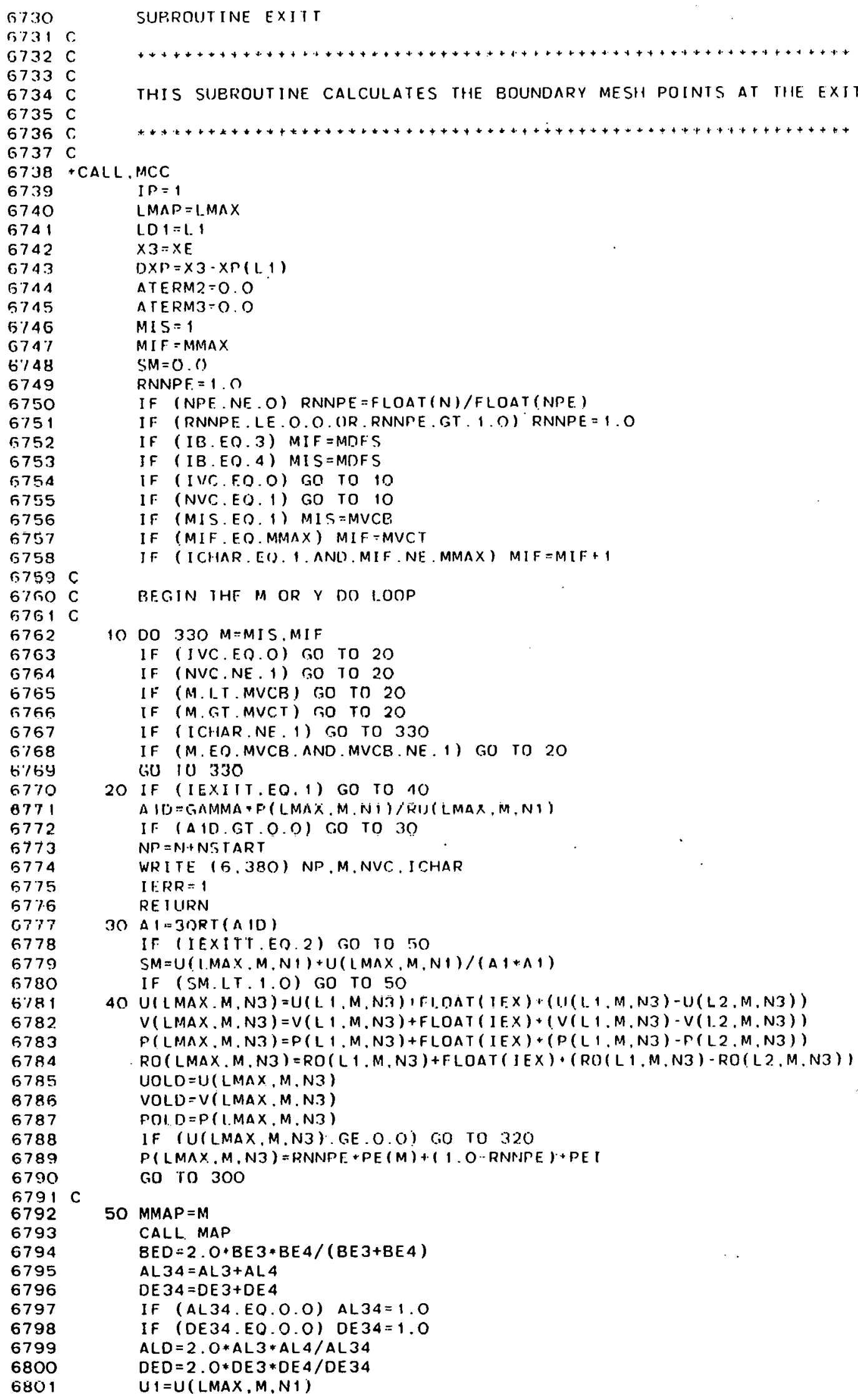




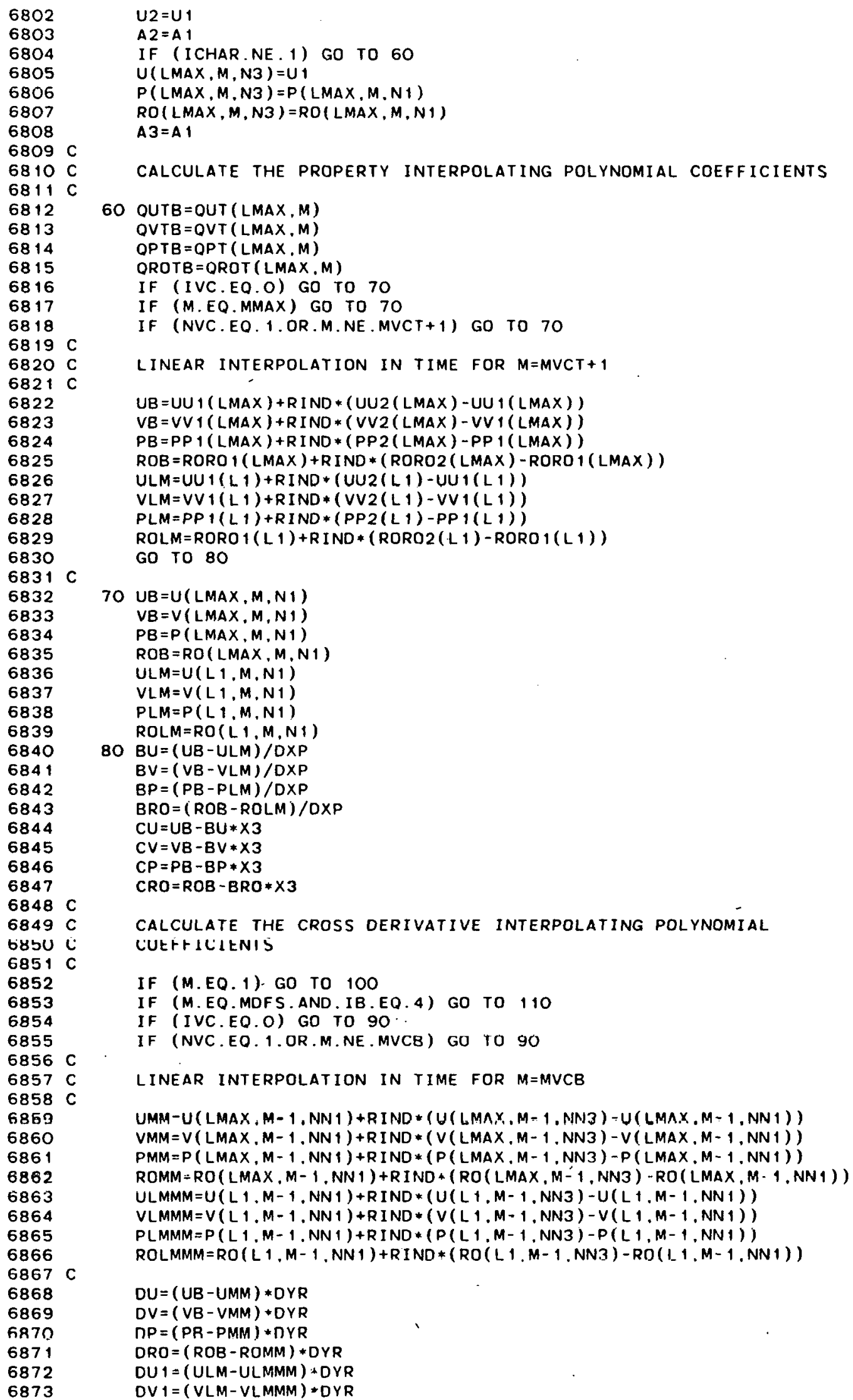




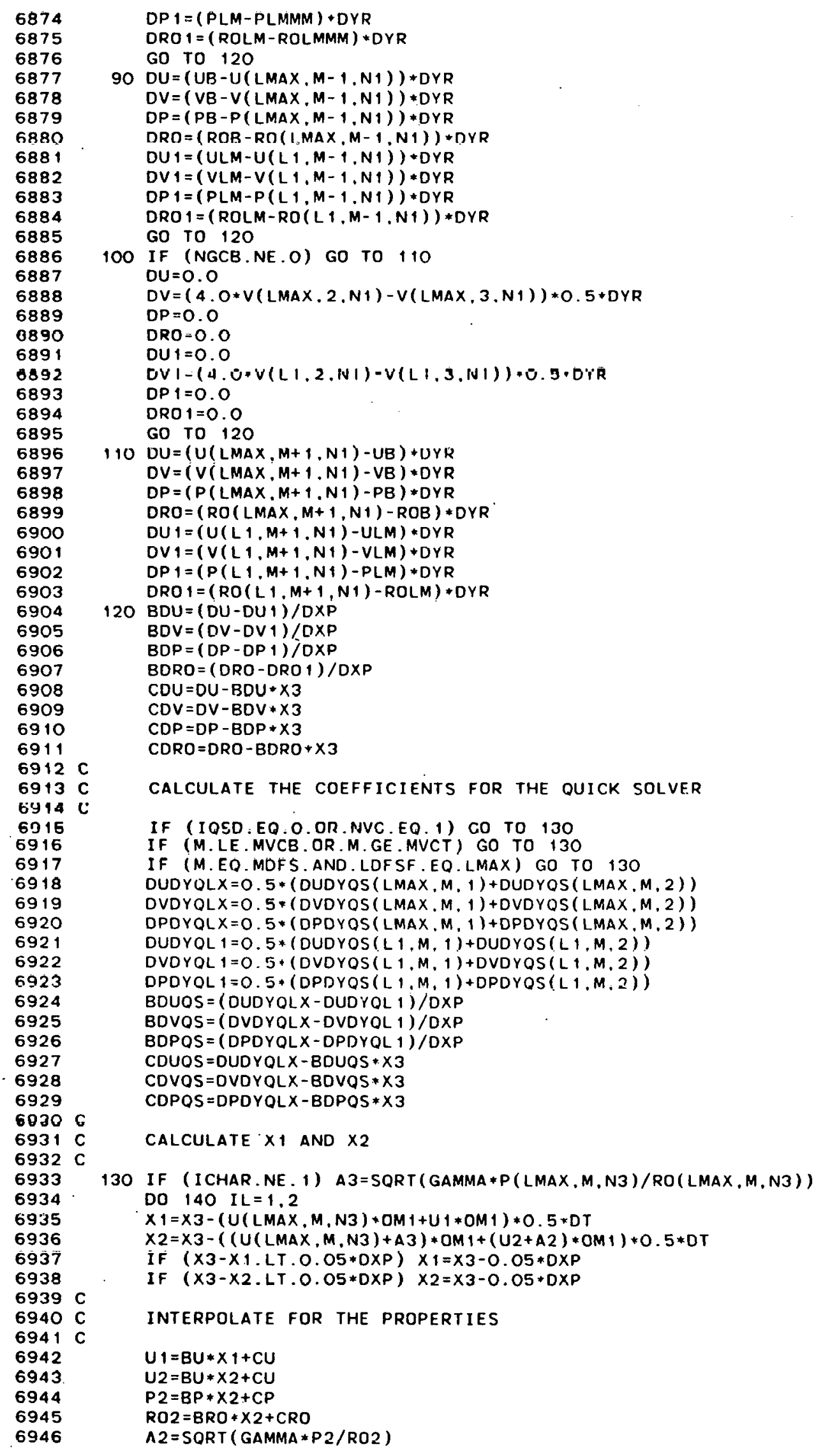




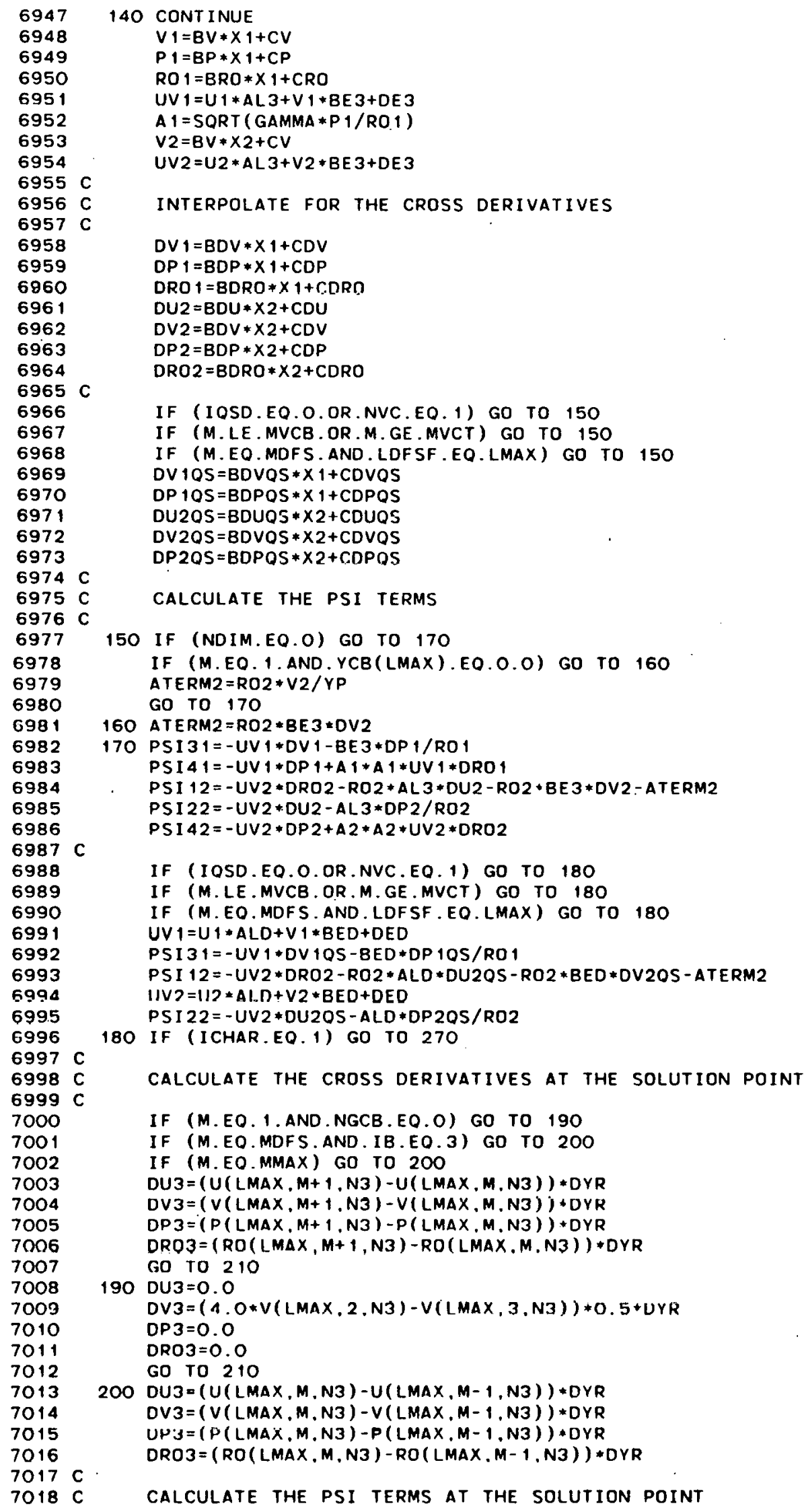




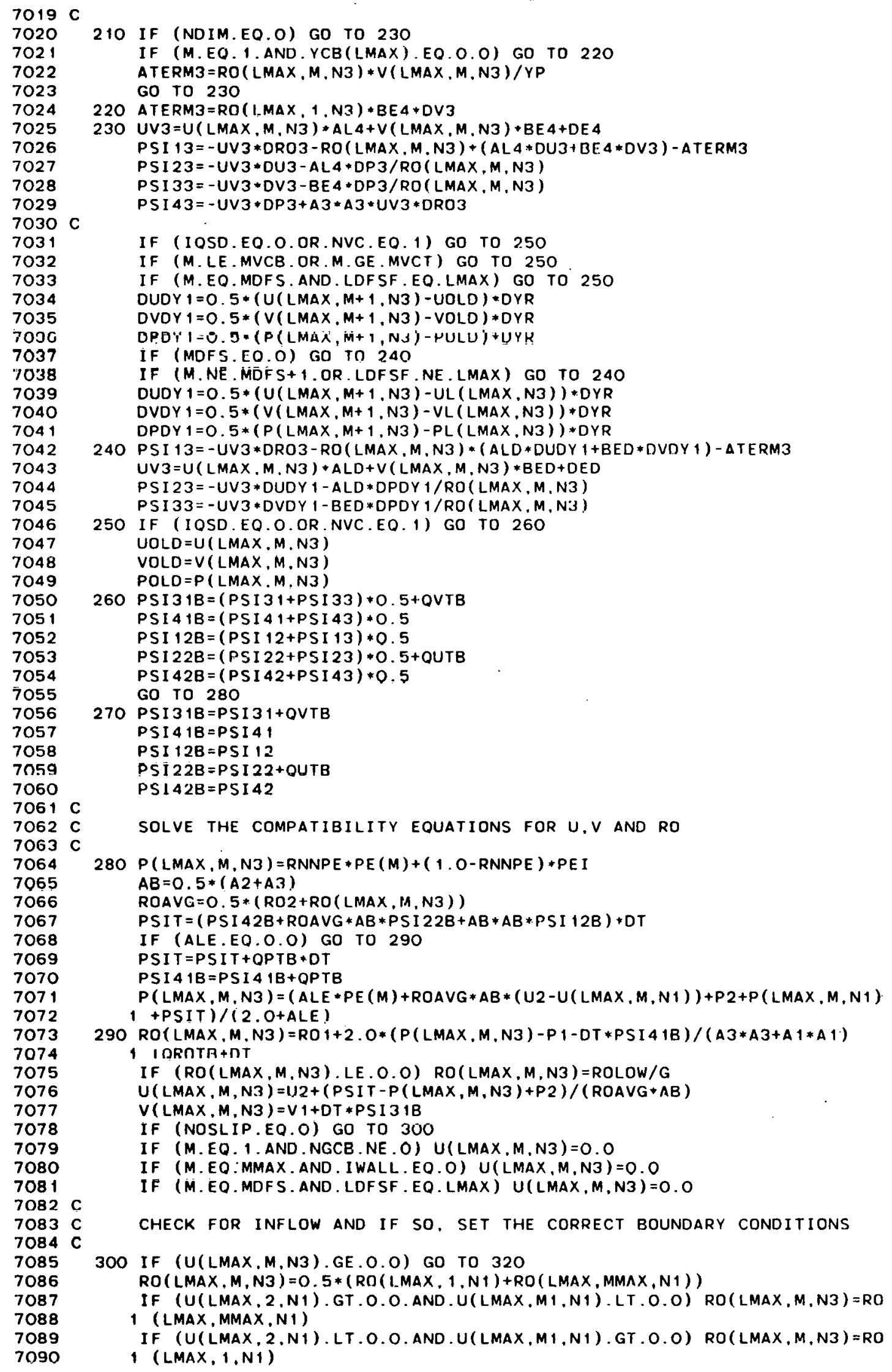




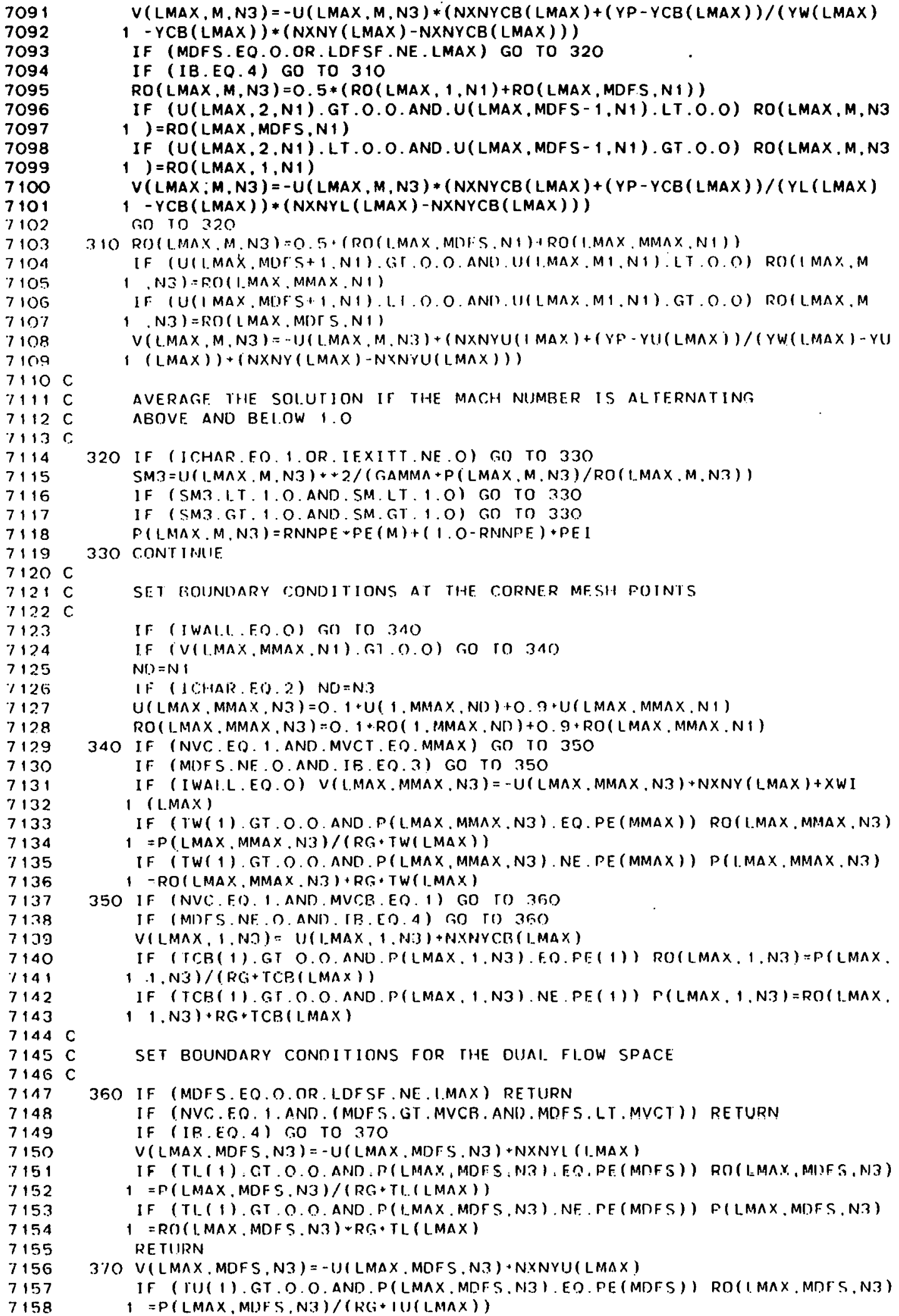


7159 IF (TU(1).GT.O.O.AND.P(LMAX.MUFS.N3).NE.FE(MDFS)) P(LMAX.MDFS.N3) $7160 \quad 1=R O(L M A X, M D F S, N 3)+R G+T U(L M A X)$

7161

$7162 \mathrm{C}$

7163 RE TURN

7164

380 FORMAT (1HO.57H++++ A NEG SOUND SPFED OCCURED IN SURROUTINE FXITT 1 AT $N=, I 6,4 H, M=,\{2,6 H, N V C=, I 3,11 H$ AND ICHAR $=, 11,6 H * * * *$ )

END 


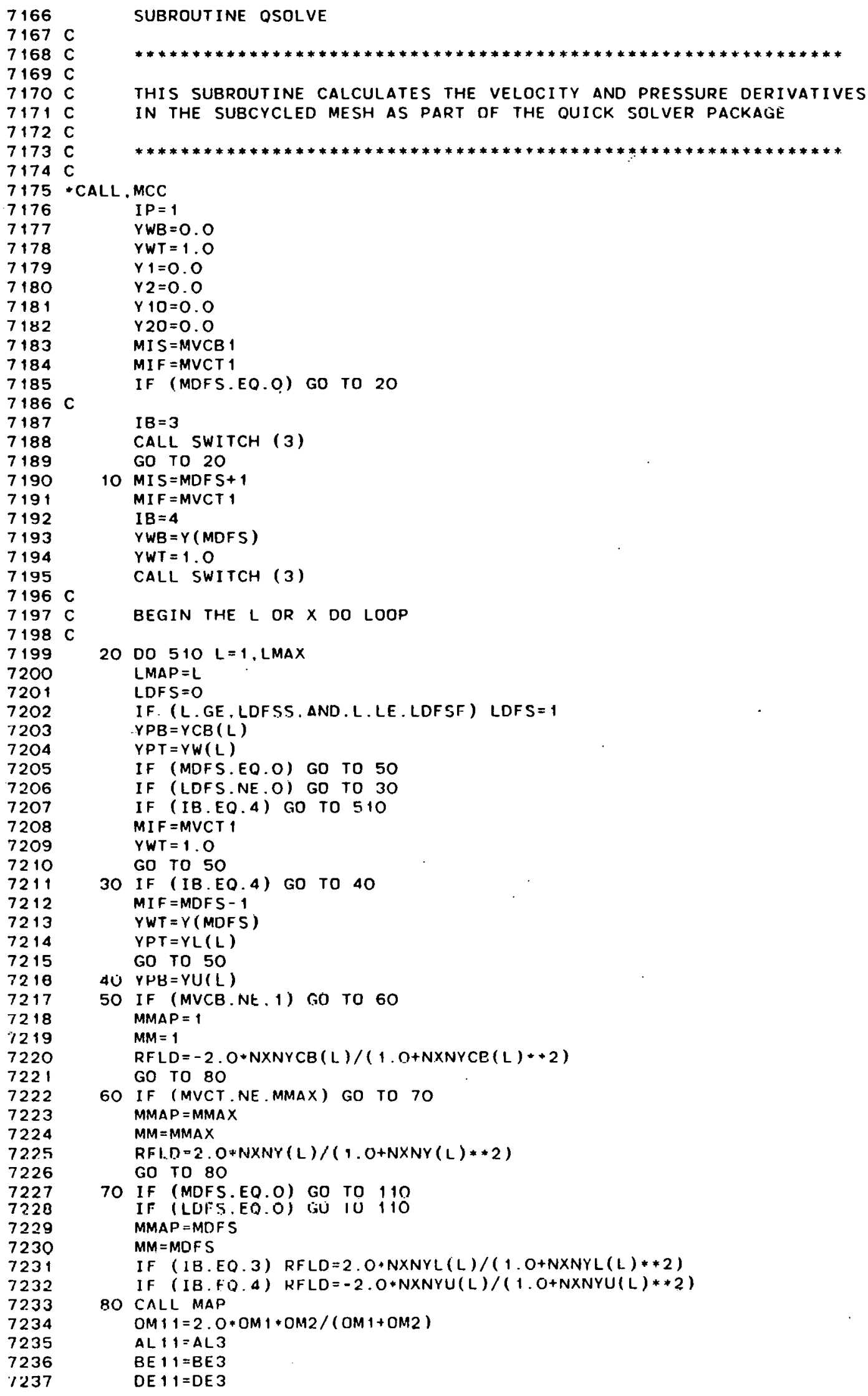




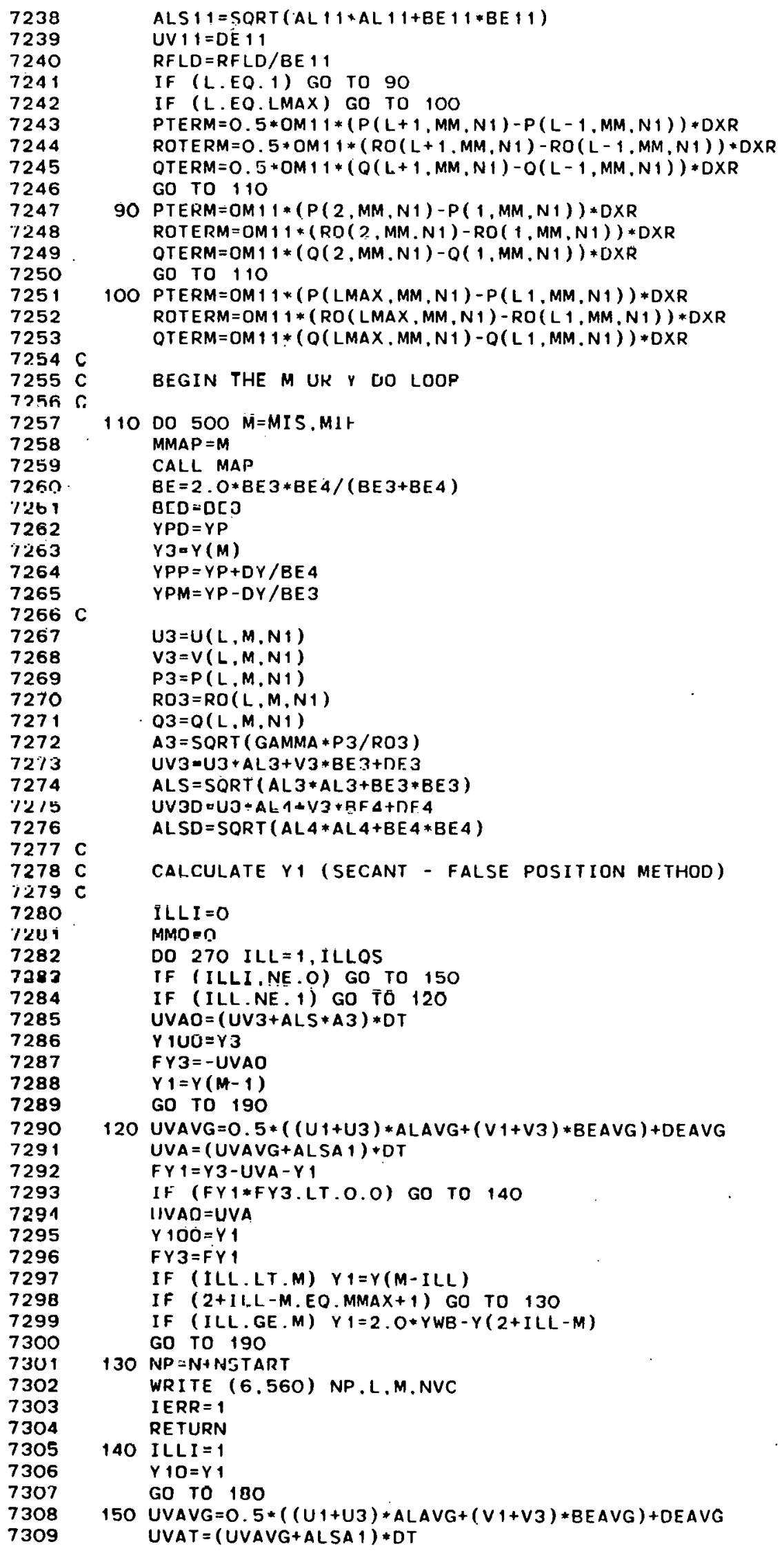




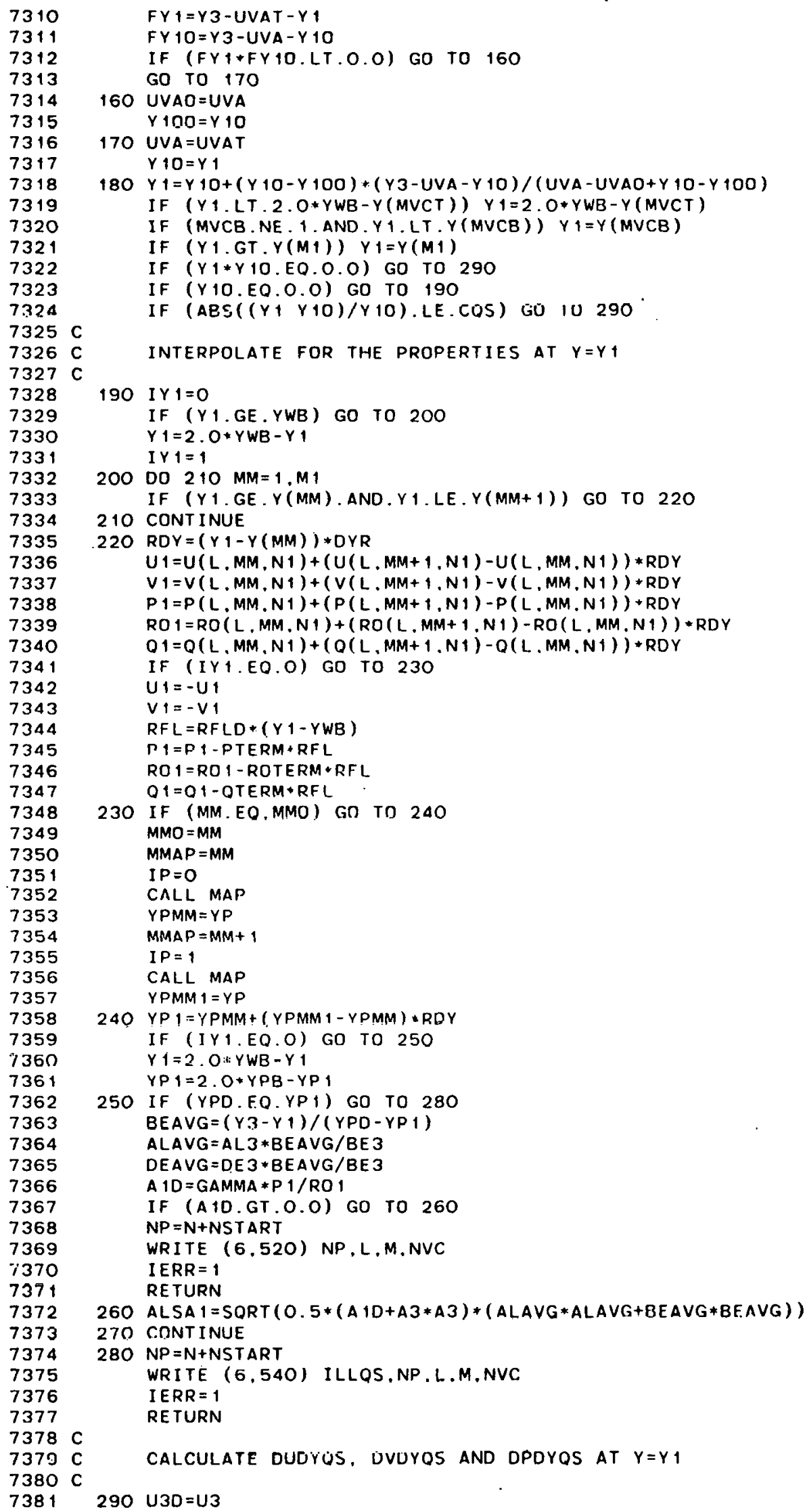




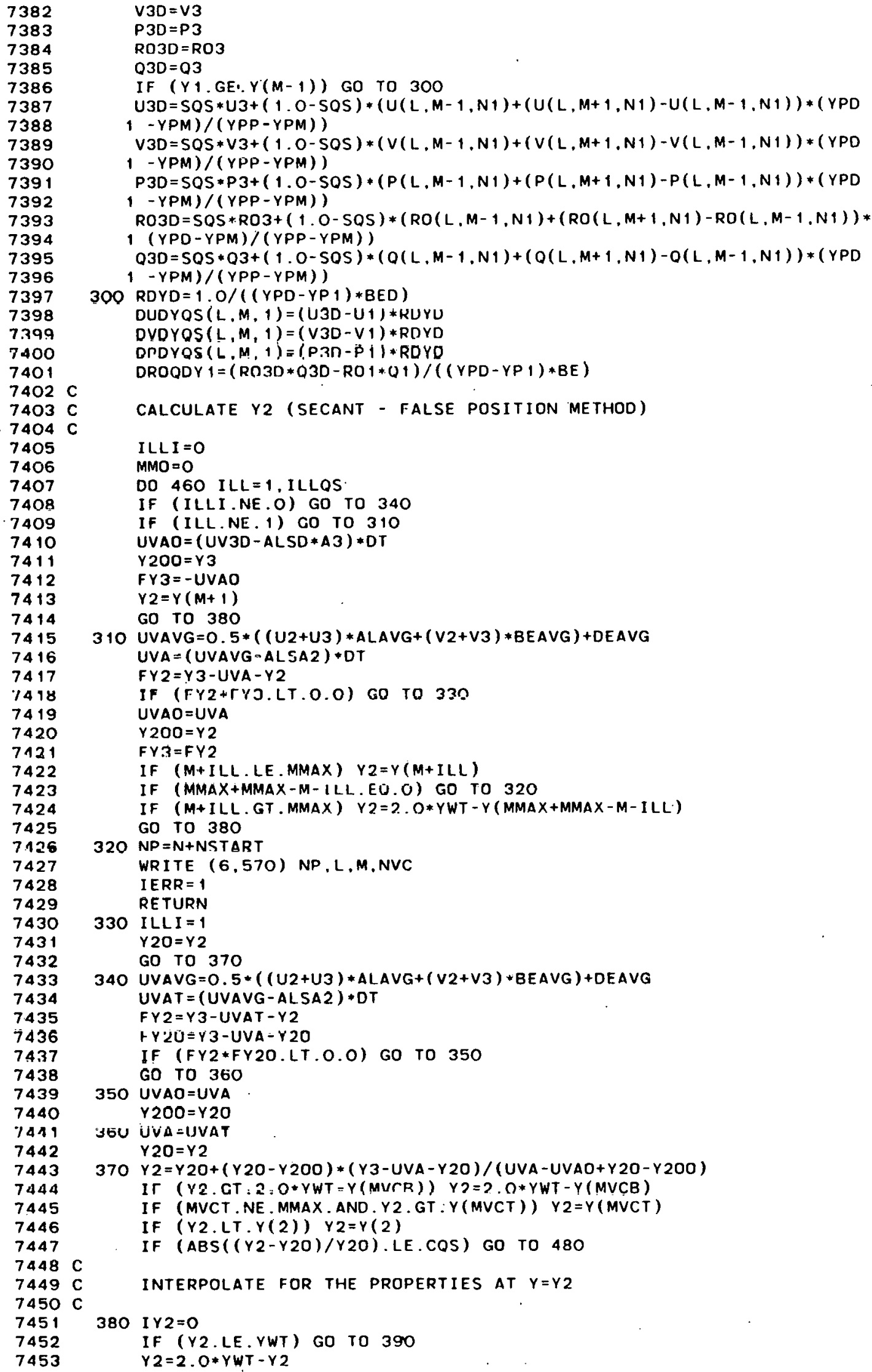

7431

7432

7433

7434

7435

7436

74.37

7438

7439

7440

7411

7442

7443

7444

7445

7446

7447

$7448 \mathrm{C}$

$7449 \mathrm{C}$

$7450 \mathrm{C}$

7451

7452

7453 


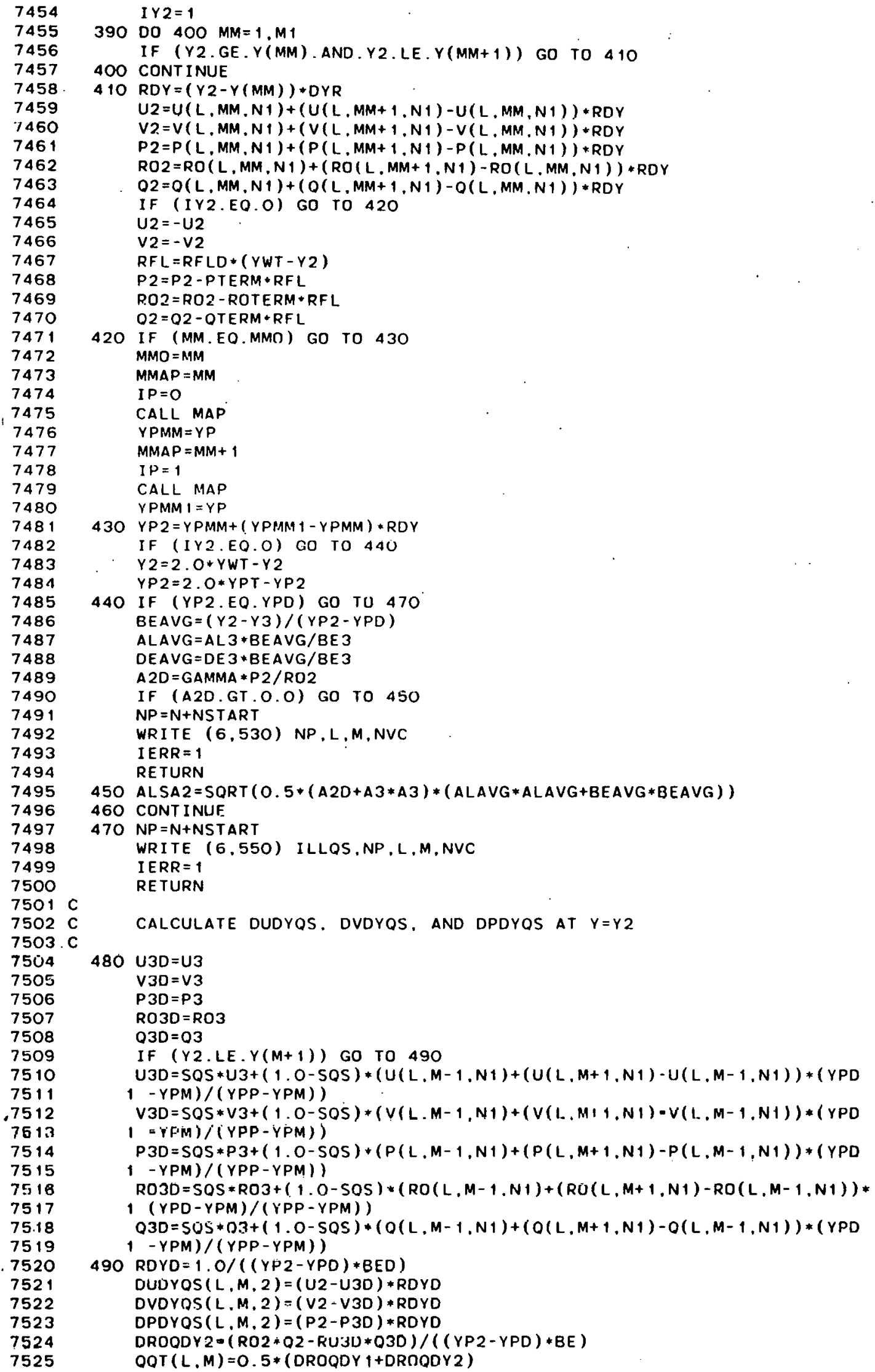



IF (MDFS.NE.O.AND.MIS.EQ.MVCB1) GO TO 10 RE TURN

FORMAT STATEMENTS

520 FORMAT (1HO.63H***** A NEG SOUND SPEED (A1) OCCURED IN SUBROUTINE 10SOLVE AT $N=, 16,4 H, L=, 12,4 H, M=, 12,9 H$ AND $N V C=, 13,6 H * * * * *$ )

530 FORMAT ( 1 HO,63H***** A NEG SOUND SPEED (A2) OCCURED IN SUBROUTINE 10SOLVE AT $\mathrm{N}=, 16,4 \mathrm{H}, \mathrm{L}=.12,4 \mathrm{H}, \mathrm{M}=.12,9 \mathrm{H}$ AND NVC $=13,6 \mathrm{H} * * * * *$ )

540 FORMAT (1HO,84H***** THE CHARACTERISTIC SOLUTION FOR YI IN SUBROUT 1 INE QSOLVE FAILED TO CONVERGE IN . I 2,17H ITERATIONS AT $N=.16,4 \mathrm{H}, L$ $2=.12 .4 \mathrm{H}, M=.12 . /, 7 \times, 6 \mathrm{H}$. NVC $=.13,6 \mathrm{H} * * *+)$

550 FORMAT (1HO,84H***** THE CHARACTERISTIC SOLUTION FOR Y 2 IN SUBROUT 1 INE QSOLVE FAILED TO CONVERGE IN, I2,17H ITERATIONS AT $\mathrm{N}=, 16,4 \mathrm{H}, \mathrm{L}$ $2=, 12,4 \mathrm{H}, M=.12, / .7 \times, 6 \mathrm{H}, \mathrm{NVC}=, 13,6 \mathrm{H} * * * * *)$

560 FORMAT (1HO.59H***** THE SOLUTION FOR Y 1 FAILED IN SUBROUIINE OSOL IVE AT $N=.16,4 \mathrm{H}, \mathrm{L}=.12,4 \mathrm{H}, \mathrm{M}=.12,6 \mathrm{H}$. NVC $=.13,6 \mathrm{H} * * * *)$

570 FORMAT (IHO.59H***** THE SOLUTION FOR Y2 FAILED IN SUBROUTINE OSOL IVE AT $N=, 16,4 \mathrm{H}, \mathrm{L}=, 12,4 \mathrm{H}, M=, 12,6 \mathrm{H}, \mathrm{NVC}=, 13,6 \mathrm{H} * * * * *$ ) END 


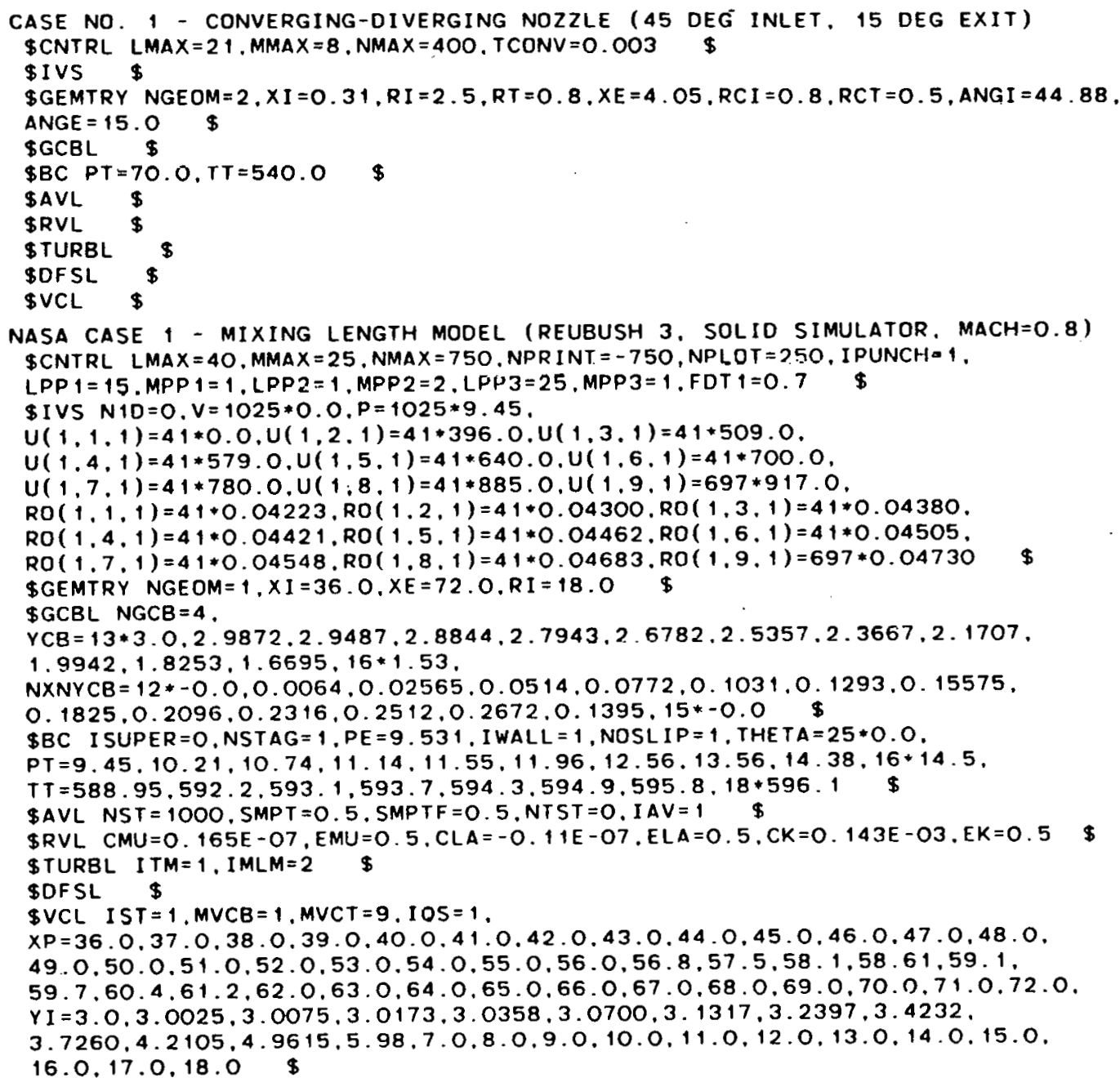


CASE No. 6 - TURBULENT PLANE Jet IN A PARALLEL STREAM - TWO EOUATION \$CNTRL LMAX $=41$, MMAX $=17$. NMAX $=6000 . R G A S=287.0, I U I=2, I U O=2 . N P L O T=500$. NPRINT $=-6000$, FDT $=1,0,1$ PUNCH $=1 \$$ \$IVS $N 1 D=0 . U(1,7,1)=779+7.5895, V=1025 * 0.0, P=1025 * 101,35, R O=1025 * 1,2047$. $U(1,1,1)=47.366,47.0 .46 \cdot 5 \cdot 46 \cdot 0,45 \cdot 5 \cdot 45 \cdot 0.44 \cdot 5 \cdot 44 \cdot 0.43 \cdot 5 \cdot 43 \cdot 0.42 .5$. $42.0 .41 .5 .41 \cdot 0.40 .5 \cdot 40.0 .39 \cdot 5 \cdot 39 \cdot 0.38 \cdot 5 \cdot 38 \cdot 0.37 \cdot 5 \cdot 37 \cdot 0.36 \cdot 5 \cdot 36.0$ $35.5,35 \cdot 0.34 \cdot 5 \cdot 34 \cdot 0.33 \cdot 5 \cdot 33 \cdot 0.32 \cdot 5 \cdot 32 \cdot 0.31 \cdot 5 \cdot 31 \cdot 0.30 .5,30 \cdot 0.29 .5$. $29.0 .28 .5,28 \cdot 0,27.5$

$U(1,2,1)=47.366,46.5 .45 .5,44.5,43.5,43 \cdot 0,42.5,42 \cdot 0,41.5,41.0 .40 .5$. $40.0 .39 \cdot 5 \cdot 39 \cdot 0,38 \cdot 5,38 \cdot 0,37 \cdot 5 \cdot 37 \cdot 0.36 \cdot 5 \cdot 36 \cdot 0.35 \cdot 5 \cdot 35 \cdot 0,34 \cdot 5 \cdot 34 \cdot 0$

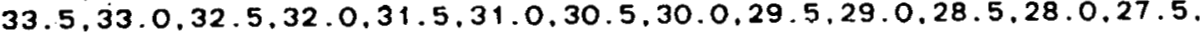
$27.0 .26 \cdot 5 \cdot 26 \cdot 0.25 .5$

$U(1,3,1)=47,366,45,5,43,5,41,5,39.5,39 \cdot 0,38,5,38,0,37.5,37,0,36.5$,

$36.0 .35 \cdot 5 \cdot 35 \cdot 0.34 \cdot 5 \cdot 34 \cdot 0.33 \cdot 5 \cdot 33 \cdot 0.32 \cdot 5 \cdot 32 \cdot 0.31 \cdot 5 \cdot 31 \cdot 0 \cdot 30 \cdot 5 \cdot 30 \cdot 0$.

$29.5,29.0,28.5,28 \cdot 0.27 \cdot 5,27.0 .26 .5,26 \cdot 0.25 \cdot 5 \cdot 25 \cdot 0,24.5,24 \cdot 0,23.5$.

$23.0,22.5,22.0,21.5$

$U(1,4,1)=5 * 0.0,36 * 18,0, U L=5 * 0.0, V L=5 * 0.0 . P L=5 * 101.35, R O L=5 * 1.2047$.

$U(1.5,1)=5 * 7.5859,36 * 15.0$.

$U(1.6,1)=5 * 7.3859,38 * 11.0 \quad \$$

$\$ G E M T R Y$ NDIM=0.NGEOM=1.RI =5.0,XI=-1:9050,XE=38,1 \$

$\$ G C B L \quad \$$

$\$ B C$ I SUPER $=1, P E=101,35, U I L=0.0, V I L=0.0, P I L=101,35, R O I L=1,2047$,

$U I=3 * 47,366,0.0,13 * 7,5895, V I=17 * 0.0 . P I=17 * 101.35, R O I=17 * 1.2047$.

$A L I=0.1 . A L E=0.1, A L W=0.1$, IWALL $=1, N O S L I P=1 \quad \$$

\$AVL I AV $=1$ \$

$\$ R V L$ CMU $=1.813 E-05, C L A=-1.208 E-05$

\$TURBL I TM $=3, F S O L=0.0 . F S E L=10200.0$.

FSO $=0.0,0.0,4 \cdot 4,0.0 .0 .11,12 * 0.0$

$F S E=0.1,0.1,10200,0,18,4,18,4,12 * 0$

$\$ D F S L$ MDF $S=4, L D F S S=1, L D F S F=5, N D F S=2$.

$Y L=5 * 0.47625$. NXNYL $=5 * 0.0, Y U=5 * 0.47625, N X N Y U=5 * 0.0$

$\$ V C L$ IST $=1$.

$X P=-1.9050,-1.4288,-0.9525,-0.47625,0.0,0.47625,0.9525,1.4288,1.9050$.

2.3813,2.8816,3.4072,3.9594.4.5395,5.1489,5.7891,6.4617,7.1683,7.9107.

$8.6905 .9 .5098,10.3704,11.2746,12.2245,13.2224,14: 2708,15.3722,16.5292$,

$17.7447,19.0217,20.3632,21.7725 .23 .2531 .24 .8085,26.4426,28,1592,29.9627$, $31.8573,33.8476 .35 .9386 .38 .1$.

$Y I=0.0,0.15875,0.3175,0.47625,0.635,0.79375,0.9525,1.1375,1.3531$,

$1.6042 .1 .8970 .2 .2380 .2 .6355 .3 .0987 .3 .6384 .4 .2673 .5 .0 \$$ 


\begin{tabular}{|c|c|c|c|c|c|c|c|c|c|c|c|}
\hline \multicolumn{12}{|c|}{$\begin{array}{l}\text { Printed in the United States of America } \\
\text { Available from } \\
\text { National Technical Information Service } \\
\text { US Department of Commerce } \\
\text { 5285 Port Royal Road } \\
\text { Springfield, VA } 22161\end{array}$} \\
\hline Page Range & $\begin{array}{c}\text { Domestic } \\
\text { Price }\end{array}$ & $\begin{array}{c}\text { NTIS } \\
\text { Price Code } \\
\end{array}$ & Page Range & $\begin{array}{c}\text { Domestic } \\
\text { Price }\end{array}$ & $\begin{array}{l}\text { Microfiche } \\
\text { NTIS } \\
\text { Price Code } \\
\end{array}$ & $\begin{array}{l}3.50 \text { (A01) } \\
\text { Page Range } \\
\end{array}$ & $\begin{array}{c}\text { Domestic } \\
\text { Price }\end{array}$ & $\begin{array}{c}\text { NTIS } \\
\text { Price Code } \\
\end{array}$ & Page Range & $\begin{array}{c}\text { Domestic } \\
\text { Price }\end{array}$ & $\begin{array}{c}\text { NTIS } \\
\text { Price Code } \\
\end{array}$ \\
\hline $001-025$ & $\$ 5.00$ & $\mathrm{~A} 02$ & $151-175$ & $\$ 11.00$ & A08 & $301-325$ & $\$ 17,00$ & A 14 & $451-475$ & $\$ 23.00$ & $A 20$ \\
\hline $026-050$ & 6.00 & $\mathrm{~A} 03$ & $176-200$ & 12.00 & A09 & $326-350$ & 18.00 & A15 & $476-500$ & 24.00 & A21 \\
\hline $051-075$ & 7.00 & A04 & 201-225 & 13.00 & A10 & $351-375$ & 19.00 & A16 & $501-525$ & 25.00 & A22 \\
\hline $076-100$ & 8.00 & A05 & $226-250$ & 14.00 & Al1 & $376-400$ & 20.00 & A17 & $526-550$ & 26.00 & A23 \\
\hline $101-125$ & 9.00 & A06 & $251-275$ & 15.00 & A 12 & $401-425$ & 21.00 & A18 & $551-575$ & 27.00 & A24 \\
\hline $126-150$ & 10.00 & A07 & $276-300$ & 16.00 & A 13 & $426-450$ & 22.00 & A19 & $576-600$ & 28.00 & A2S \\
\hline & & & & & & & & & 601-up & $\dagger$ & A99 \\
\hline
\end{tabular}




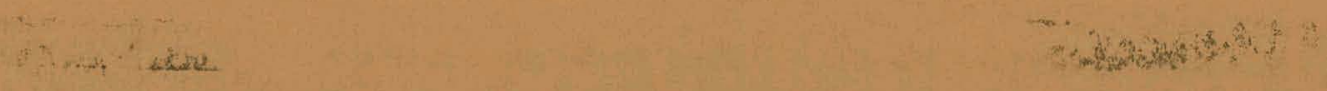

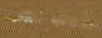

